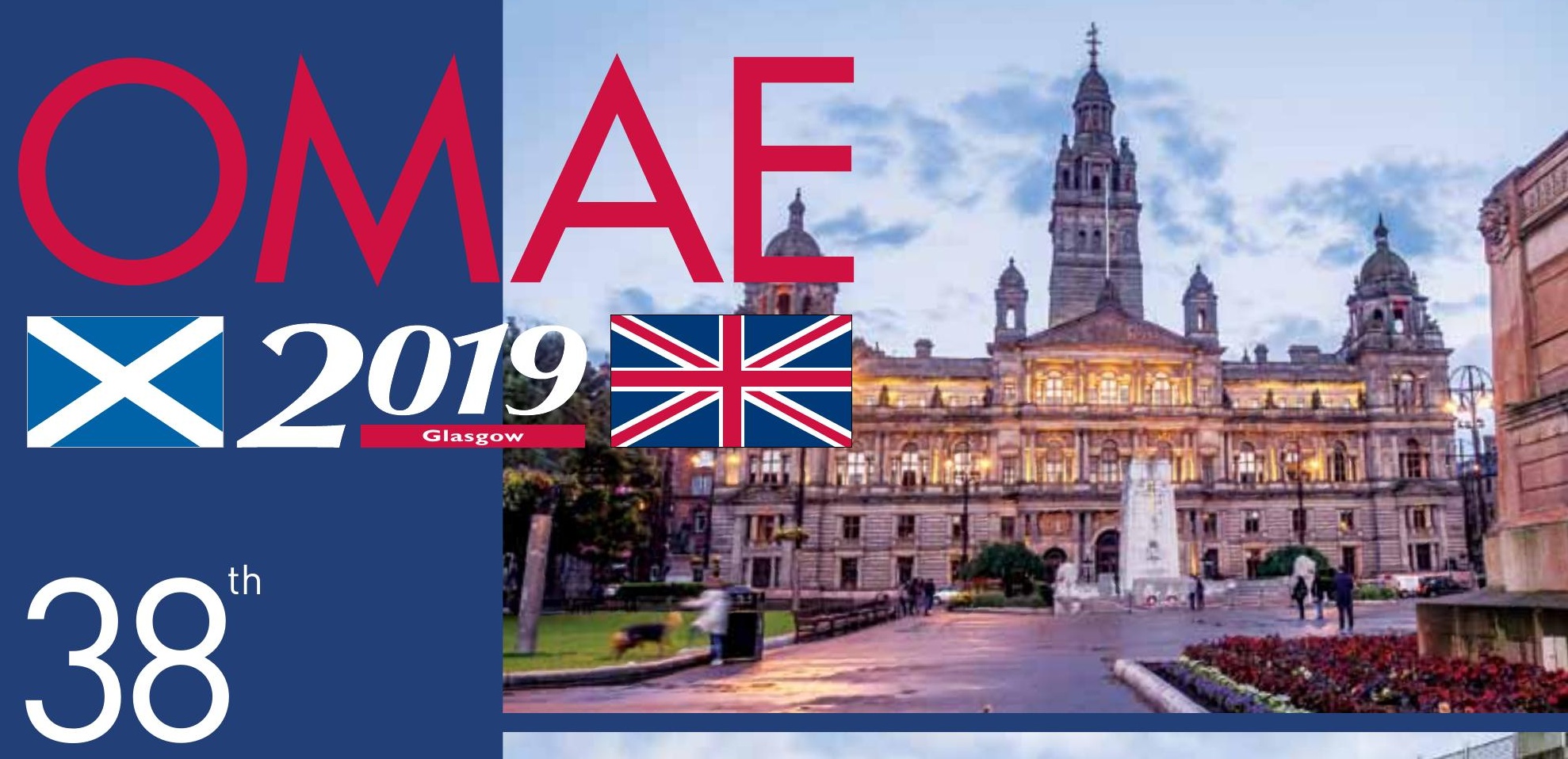

International Conference on Ocean, Offshore and Arctic Engineering

Glasgow, Scotland June 9-14, 2019
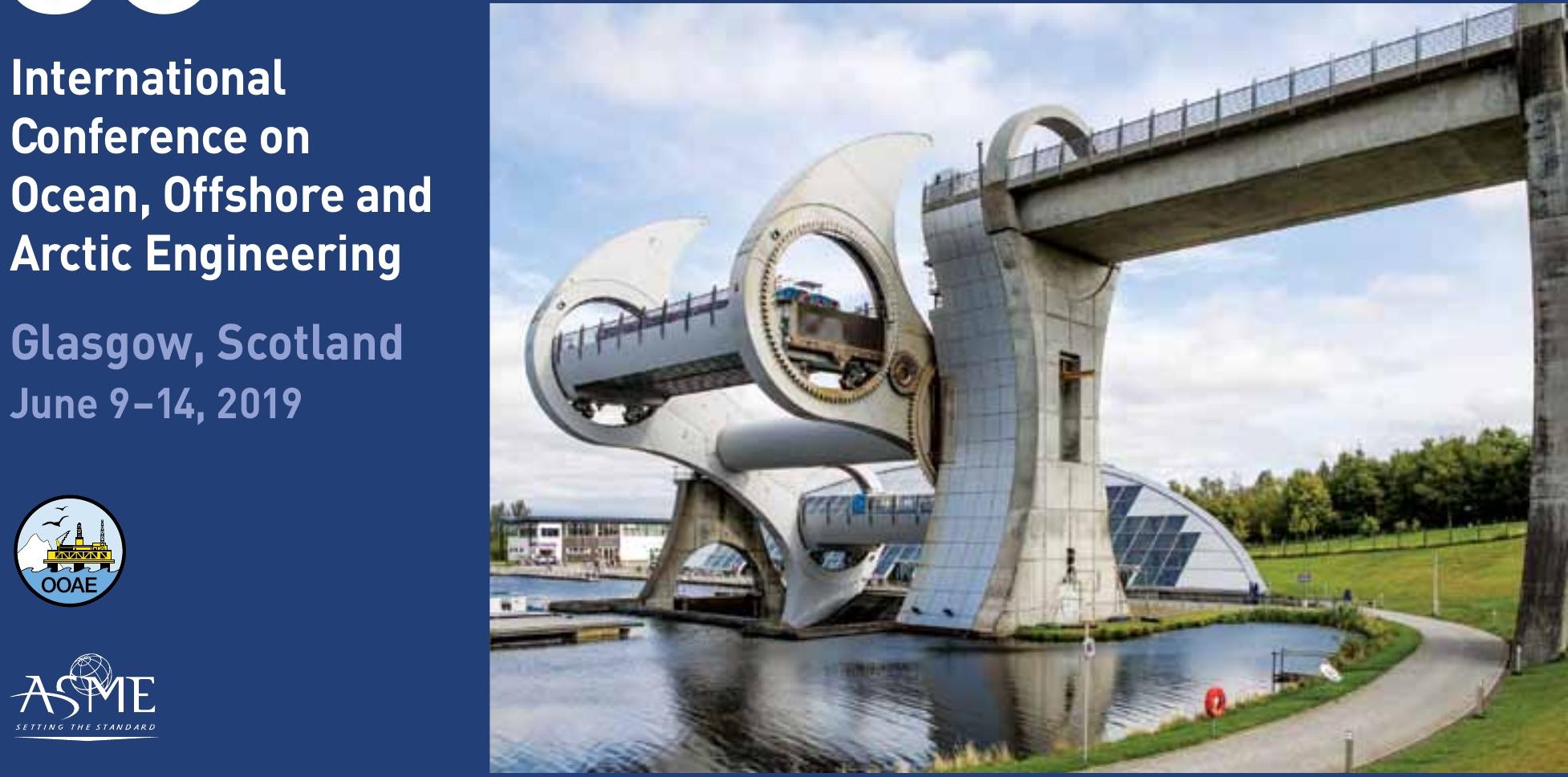

University of -1

Strathclyde Glasgow

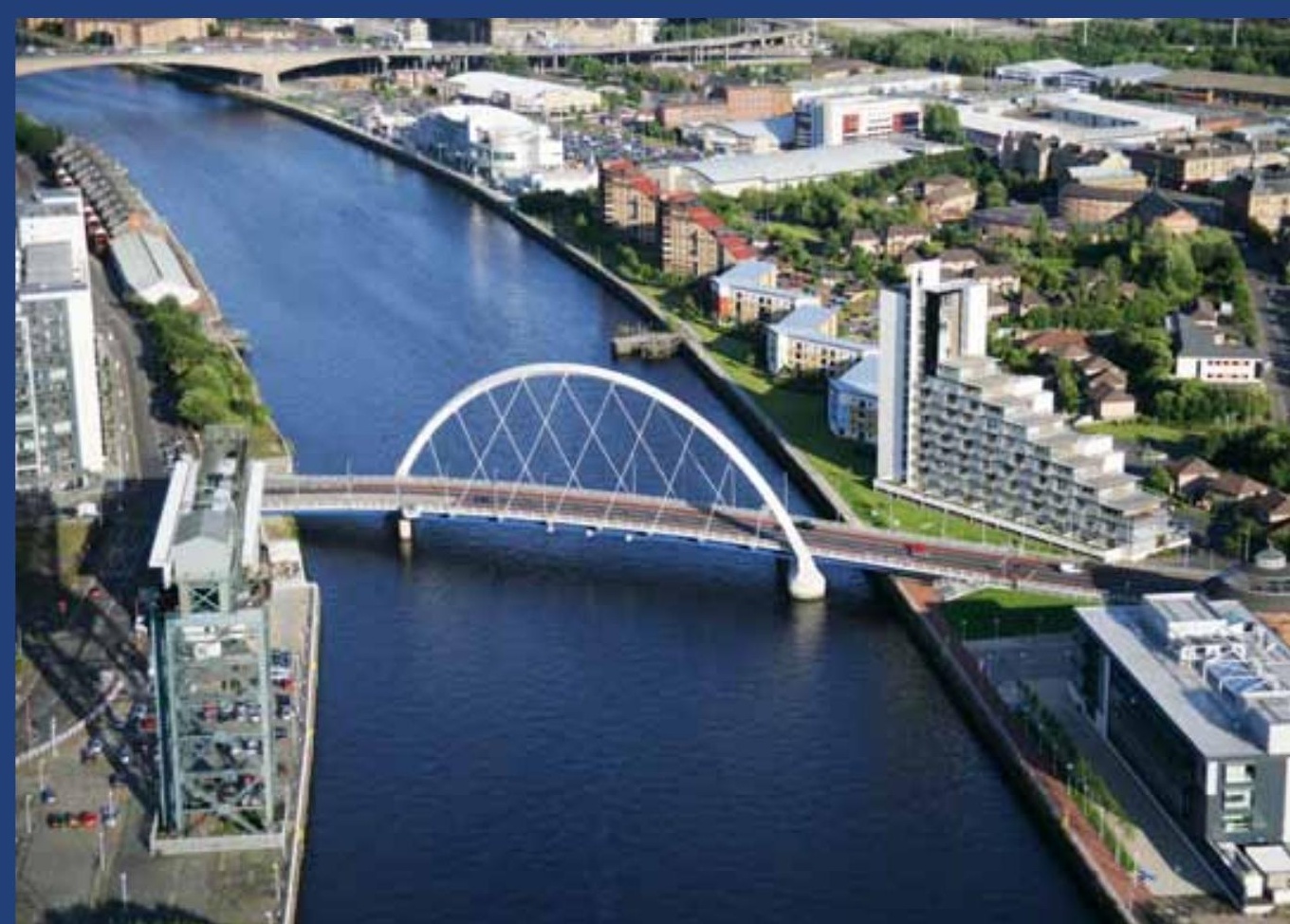




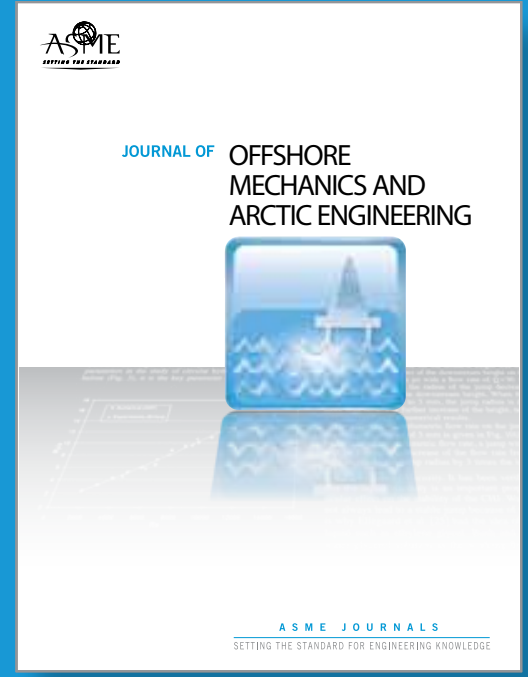

\section{Jourdal of Offshore Mectianjus and Arctic Engyjegring}

EDITOR: Lance Manuel, PhD The University of Texas at Austin, USA

\section{OMAE 2019 Conference Attendees: Access and Download Articles of Interest FREE!}

OMAE 2019 Conference attendees will have access to view and download articles FREE from the Journal of Offshore Mechanics and Arctic Engineering from June 15, 2019 September 15, 2019.

It's Simple, Get Started!

Visit Journal of Offshore Mechanics and Arctic Engineering on The ASME Digital Collection (offshoremechanics.asmedigitalcollection.asme.org) and click on any article.

For queries or support, contact Sharon Giordano:

GiordanoS@asme.org 


\section{YOUR HOSTS}
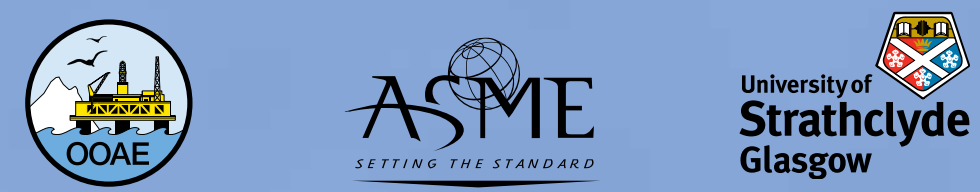

Program at a Glance

2 TECHNICAL PROGRAM

Technical Tours

Floor Plans

$4 \quad$ Honoring Symposia

21

Outreach for Engineers

Welcome Letters

6 Afternoon Lecture Series

22

OMAE 2020

89

Glasgow Map

10

Saturday, June 8

23

Listing of Committees \&

Award Winners

12 Sunday, June 9

24

Organizers

93

Attendee Information

13 Monday, June 10

25

Past \& Future OMAE

Social Events

14 Tuesday, June 11

36

Conferences \& Chairs

Sightseeing Tours

15 Wednesday, June 12

54

Author Index

102

Sponsors \& Exhibitors

16 Thursday, June 13

72

Session Index

CONNECT WITH ASME ONLINE!

Contact ASME asme.org
www.facebook.com/ASME.IPTI
Rasmedotorg

\#OMAE2019

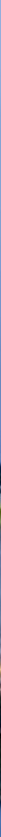


Saturday, June 8 (PG 23)

Short Courses

- Corrosion and Fouling in Marine

Environment

9:00-17:00

Jura (Crowne Plaza)

- Verification \& Validation of Industrial CFD 09:00 - 17:00

Staffa/Shuna (Crowne Plaza)

\section{Outreach}

- Team Building Exercise

17:00 - 19:00

Staffa/Shuna (Crowne Plaza)

- Welcome Dinner

19:00 onwards

Off-site

\section{Sunday, June 9 (PG 24)}

\section{Outreach}

- Welcome \& Introductions

Industry Presentations

08:00 - 17:00

Castle 1 (Crowne Plaza)

\section{Short Courses}

- Offshore Wind Turbines: Dynamic Analysis and Marine Operations

$09.00-17.00$

Jura (Crowne Plaza)

- Introduction to Machine Learning and Data-driven Modelling Methods for Engineering Applications

09:00 - 17:00

Castle 2 (Crowne Plaza)
Monday, June 10 (PG 25)

Opening Ceremony and

Keynote Plenaries 08:30 - 10:00

SEC Armadillo

Welcome and Opening Remarks

Professor Atilla Incecik, Conference Chair

Professor Krish Thiagarajan Sharman, Technical Program Chair

Professor Antonio C. Fernandes, OOAE Division Chair

Professor Sir Jim McDonald, Principal, University of Strathclyde

Bailie Jacqueline McLaren on behalf of the Lord Provost of Glasgow,

Eva Bolander

\section{Keynote Plenary One}

Blue Oceans: Offshore Research for Future Maritime

Challenges

Dr. Bas Buchner, President, MARIN

Awards

\begin{tabular}{lr}
\hline Refreshment Break 10:00 - 10:30 & Hall 5 (SEC) \\
\hline Keymote
\end{tabular}

\section{Keynote Plenary Two}

Advancing a Lower Carbon Future

David Dickson, Vice President, Global Operations, Regions, BP

\section{Keynote Panel}

\section{Offshore Digital}

Moderator: Xiaozhi (Christina) Wang, PhD, Vice President, Global

Marine, American Bureau of Shipping (ABS)

Panelists: Professor Kjetil Skaugset, PhD, Chief Researcher

Upstream and Downstream, Technology, Equinor Expert Centre,

Equinor ASA

Frederic Dabe, Digital Transformation Director, SBM Offshore

OMAE 2020 Presentation

Professor Manhar Dhanak, Conference Chair, OMAE 2020

Professor Ron W. Yeung, Conference Co-Chair, OMAE 2020

Opening Lunch 12:00 - 13:30 Hall 5 (SEC)

Concurrent Sessions 13:30 - 15:00

OFT 1-1-2 FPSO and Arctic Structures

OFT 1-2-1 Dynamic Positioning

SSR 2-10-1 Collision and Crashworthiness I

SSR 2-15-1 Data Driven Models

MAT 3-1-2 Formulation of the Fracture Parameter

PRS 4-1-1 Flexible Pipes

PRS 4-3-1 Collapse

OSU 5-1-1 Marine Utilization and Marine Spatial Planning

OE 6-1-2 Floating Body Technology

OE 6-4-1 Marine Control and Automation

CFD 8-1-1 FSI

ORE 9-1-1 Bottom-Fixed Wind Turbines

OG 10-1-1 Seabed Properties and Processe

PT 11-7-1 Well Drilling Fluids and Hydraulics

HRT 12-1-1 Numerical and Experimental Methods in Hydrodynamics

OFT 13-7-1 Small Vessel and Related Technology

\begin{tabular}{lll}
\hline Refreshment Break $15: 00-15: 30$ \\
\hline \multicolumn{3}{l}{ Concurrent Sessions $15: 30-17: 30$} \\
OFT & $1-4-3$ & Design Optimization \\
OFT & $1-6-1$ & CFD Numerical Waves and Applications \\
SSR & $2-3-1$ & Probabilistic Response Models \\
SSR & $2-10-2$ & Collision and Crashworthiness II \\
MAT & $3-9-1$ & Advances in Materials Characterization \\
PRS & $4-1-4$ & Flexible Pipes IV \\
PRS & $4-2-5$ & SCRs and SLWRs II \\
OSU & $5-6-1$ & High Tide and Tsunamis \\
OE & $6-4-2$ & Marine Operations and Vessel Motions \\
OE & $6-11-1$ & Autonomous Vehicle Technology \\
CFD & $8-1-2$ & Surface Waves \\
ORE & $9-2-1$ & Aerodynamics I \\
OG & $10-3-1$ & Anchors \\
PT & $11-7-3$ & Well Drilling Fluid and Hydraulics III \\
HRT & $12-2-1$ & Multi-Body Hydrodynamics \\
OFT & $13-2-1$ & Numerical Methods
\end{tabular}

ASME \& IMechE

Forth Room

Connect Roundtable 17:00 - 18:15 (SEC Armadillo)

Afternoon Lecture Series 17:40 - 18:10 Lomond Auditorium European Research Council - Funding Opportunities (SEC) for Creative Minds from All Over the World

Dr.-Ing. Luiz Alves dos Santos, Scientific Officer, European Research Council

Afternoon Drinks Reception 18:15 - 19:15 Hall 5 (SEC)
Tuesday, June 11 (PG 36)

Concurrent Sessions $08 \cdot 30$ - 10:00

OFT 1-1-3 Floating Wind Platform

OFT 1-2-2 Mooring System Design and Analysis

SSR 2-4-1 Fatigue and Fracture Reliability I

SSR 2-9-1 Extreme Loading and Responses

SSR 2-12-1 Structural Analysis and Optimization I

MAT 3-1-1 Fracture Toughness Measurement and Assessment

PRS 4-1-2 Flexible Pipes II

PRS 4-3-2 Installation

OSU 5-2-1 Aquaculture I: Design and Modeling I

OE 6-2-1 Coastal Engineering I

OE 6-4-3 Marine Engineering and Applications

CFD 8-2-1 Free Surface Modeling

ORE 9-3-1 Wave Energy Converter Control Systems Competition (WECCCOMP)

OG 10-4-1 Pile Foundations ।

PT 11-7-2 Well Drilling Fluids and Hydraulics II

HRT 12-4-1 Hydrodynamic Aspects of Offshore Renewable Energy

OFT 13-2-2 Experiments and Numerical Validation

Refreshment Break 10:00 - 10:30 Hall 5 (SEC)

Concurrent Sessions 10:30 - 12:00

OFT 1-1-4 Fixed Platforms and Foundations

OFT 1-2-3 Dynamic Positioning II

SSR 2-4-2 Fatigue and Fracture Reliability II

SSR 2-9-2 Extreme Loading and Responses II

SSR 2-12-2 Structural Analysis and Optimization II

MAT 3-4-1 Steel Performance in Sour Environment

PRS 4-1-3 Flexible Pipes III

PRS 4-3-6 ECA

OSU 5-2-2 Aquaculture II: Design and Modeling II

$\mathrm{OE}$ 6-2-2 Coastal Engineering II

OE 6-4-4 Marine Engineering and Applications II

CFD 8-2-2 Free Surface Loading and Structure Interaction I

ORE 9-4-4 Optimization and Load Analysis

OG 10-5-1 Bucket Foundations, Suction Caissons and Spudcans

PT 11-6-1 Integrity of Well Barriers

HRT 12-5-1 Non-Linear Waves and Wave Effects I

OFT 13-2-3 Flow-Induced Motions (FIM)

Lunch $12 \cdot 00-13 \cdot 30$

Hall 5 (SEC)

Concurrent Sessions 13:30 - 15:00

OFT 1-1-5 Artificial Intelligence and Advance Analysis

OFT 1-2-4 Mooring System Design and Analysis II

SSR 2-4-3 Fatigue and Fracture Reliability III

SSR 2-9-3 Extreme Loading and Responses III

SSR 2-12-3 Structural Analysis and Optimization III

MAT 3-3-2 Performance of Mooring Chains

PRS 4-1-5 Flexible Pipes V

PRS 4-2-1 General Design and Analysis

OSU 5-4-1 Underwater Vehicle and Technology

$\mathrm{OE}$ 6-2-3 Coastal Engineering III

OE 6-4-5 Very Large Floating Structures

CFD 8-2-3 Free Surface Loading and Structure Interaction II

ORE 9-5-2 Concepts and Design

OG 10-6-1 Pipeline Geotechnics

PT 11-6-2 Integrity of Well Barriers II

HRT 12-5-2 Non-Linear Waves and Wave Effects II

OFT 13-2-4 Fluid-Structure Interactions (FSI)

Refreshment Break 15:00 - 15:30

Hall 5 (SEC)

Concurrent Sessions $15 \cdot 30-17 \cdot 30$

OFT 1-6-2 Loads and Responses in Current and Wind

SSR 2-8-1 Well Integrity and Reliability Assessment

SSR 2-9-4 Extreme Loading and Responses IV

MAT 3-3-3 Advances on Assessing Performance of Steel

PRS 4-2-2 General Design and Analysis II

PRS 4-5-1 Flow Assurance I

OSU 5-3-1 Development of Deep Sea Mining and Resources

OE 6-4-6 Towed Cables, Ropes and Mooring Systems

OE 6-11-2 Floating Bodies Technology

OE 6-12-1 Ocean Measurement and Data Interpretation

PAT 7-1-1 Arctic Frontiers and Manoeuvring in Ice

CFD 8-5-1 Wave CFD Modeling Applications

ORE 9-2-3 Floating Wind Designs

OG 10-7-1 Pile Foundations II

PT 11-12-1 Cementing I

HRT 12-7-1 Large-Amplitude Non-Linear Ship Motions

OFT 13-2-5 Loads Induced in Floating Systems

Afternoon Lecture Series 17:40 - 18:30 Lomond Auditorium Inspired by Myriad Laughing Waves:

Euler, Navier, Stokes and Others

Professor Rodney Eatock Taylor, Emeritus Professor, University of Oxford 
Wednesday, June 12 (PG 54)

\begin{tabular}{|c|c|c|}
\hline Conc & current & Sessions $08: 30-10: 00$ \\
\hline OFT & $1-5-1$ & FLNG \\
\hline SSR & $2-1-1$ & Abnormal or Rogue Waves I \\
\hline SSR & $2-11-1$ & Ultimate Strength I \\
\hline MAT & $3-6-1$ & Advances in Materials Characterization \\
\hline PRS & $4-1-6$ & Flexible Pipes VI \\
\hline PRS & 4-3-4 & Thermo-Mechanical I \\
\hline OSU & $5-5-1$ & Floating Systems for Renewable Energy \\
\hline $\mathrm{OE}$ & $6-3-1$ & Fluid-Structure Interaction/Hydroelasticity \\
\hline $\mathrm{OE}$ & $6-5-1$ & Advanced Marine Hydrodynamics I \\
\hline $\mathrm{OE}$ & $6-8-1$ & Wave Loads \\
\hline PAT & 7-3-1 & Structures in Ice \\
\hline CFD & 8-3-1 & Data-Driven Modeling and Machine Learning \\
\hline ORE & $9-1-3$ & FWT - Numerical Analysis II \\
\hline PT & $11-15-1$ & Well Abandonment I-Rules and Regulations \\
\hline PT & $11-2-1$ & Drilling Mechanics Session I \\
\hline HRT & $12-1-2$ & $\begin{array}{l}\text { Numerical and Experimental Methods in } \\
\text { Hydrodynamics II }\end{array}$ \\
\hline OFT & $13-1-1$ & Extremes and Environmental Modelling \\
\hline
\end{tabular}

\begin{tabular}{lll}
\hline Refreshment Break $10: 00-10: 30$ & Hall 5 (SEC) \\
\hline \multicolumn{3}{l}{ Concurrent Sessions $10: 30-12: 00$} \\
OFT & $1-4-2$ & Numerical Design and Analysis \\
SSR & $2-1-2$ & Abnormal or Rogue Waves II \\
SSR & $2-11-2$ & Ultimate Strength II \\
MAT & $3-3-1$ & Fatigue Improvement and Repairs \\
PRS & $4-1-7$ & Flexible Pipes VII \\
PRS & $4-3-5$ & Thermo-Mechanical II \\
OSU & $5-1-2$ & Hybrid and Complex Use of Floating Systems I \\
OE & $6-3-2$ & Wave-Body Interactions/CFD \\
OE & $6-5-2$ & Advanced Marine Hydrodynamics II \\
OE & $6-8-2$ & Ship Hydrodynamics \\
PAT & $7-4-1$ & Vessels in Ice and Waves \\
CFD & $8-3-2$ & Code Development and V\&V \\
ORE & $9-2-2$ & Aerodynamics II \\
ORE & $9-3-2$ & Wave Energy: Oscillating Water Column I \\
PT & $11-2-2$ & Drilling Mechanics Session II \\
PT & $11-15-2$ & Well Abandonment II - Research and Operational \\
& & Experiences \\
OFT & $13-1-2$ & Fluid Body Interaction \\
\hline
\end{tabular}

Lunch 12:00-13:30 Hall 5 (SEC)

Concurrent Sessions 13:30 - 15:00

OFT 1-3-1 Nonlinear Wave and Wave Effects

SSR 2-2-1 Probabilistic and Spectral Wave Models I

SSR 2-11-3 Ultimate Strength III

MAT 3-11-1 Developments in BS 7910 and other Fitness-forservice Procedures: Session I

PRS 4-2-3 Drilling Risers I

PRS 4-3-3 Mechanics I

OSU 5-1-3 Hybrid and Complex Use of Floating Systems II

OE 6-15-1 Underwater Vehicles Control

OE 6-3-3 Damping and Viscous Effects

OE 6-5-3 Advanced Marine Hydrodynamics III

PAT 7-11-1 Ice Model Tests and Structure-Ice-Interactions

CFD 8-1-3 Ship Performance I

ORE 9-4-2 Wave Farms and Alternative Markets

ORE 9-6-1 Thermal, Hybrid and Others: Analysis, Design and Prediction

PT 11-3-1 Drilling Geomechanics

PT 11-5-1 Well Inflow Control and Reservoir Management

OFT 13-1-3 Nonlinear Waves ।

Refreshment Break 15:00 - 15:30

Hall 5 (SEC)

Concurrent Sessions 15:30-17:30

OFT 1-3-2 Fluid-Structure Interaction

SSR 2-2-2 Probabilistic and Spectral Wave Models II

SSR 2-6-1 Reliability of Mooring and Riser Systems I

MAT 3-11-2 Developments in BS 7910 and other Fitness-forService Procedures; Session II

PRS 4-2-4 SCRs and SLWRs I

PRS 4-5-2 Flow Assurance II

OE 6-15-2 Underwater Vehicles Design Technology and Hydrodynamics

OE 6-3-4 Wave-body interactions: Special Problems

$\mathrm{OE}$ 6-5-4 Advanced Marine Hydrodynamics IV

PAT 7-12-1 Numerical Ice Modeling

CFD 8-1-4 Ship Performance II

CFD 8-4-1 Cylinder VIV

ORE 9-1-2 FWT- Numerical Analysis I

ORE 9-7-1 Drivetrain Design, Operation and Condition Monitoring

PT 11-4-1 Petroleum Production Systems Design and Operation

PT 11-12-2 Cementing II

OFT 13-1-4 Nonlinear Waves II

Afternoon Lecture Series 17:40 - 18:30 Lomond Auditorium Enjoyable Marine Engineering Researches on Sports, (SEC) Environment, not only Water Wave Engineering, Nonlinear Hydrodynamic Forces and Statistics

Professor Takeshi Kinoshita, Visiting Professor, Nagasaki Institute of Applied Science

Thursday, June 13 (PG 72)

Outreach Breakfast /

Feedback Session 07:30 - 10:00

Concurrent Sessions 08:30 - 10:00

OFT 1-1-1 Semi-Submersibles and TLPs

SSR 2-13-1 Risk Analysis and Management

SSR 2-6-2 Reliability of Mooring and Riser Systems II

MAT 3-2-1 Fabrication and Performance of Clad Pipes

PRS 4-1-10 Umbilicals and Cables I

PRS 4-3-7 Thermo-Mechanical III

OE 6-13-1 Ship Resistance and Wave Loads

OE 6-17-1 Wave Loads on Structures

OE 6-7-1 Regional Metocean I

CFD 8-1-5 Seakeeping

CFD 8-4-2 Risers, Jumpers and Pipelines

ORE 9-1-5 FWT-Mooring Systems

ORE 9-5-3 Numerical Analysis I

PT 11-1-1 General Petrolum Technology

PT 11-13-1 LSU Workshop on Riser Gas Management and Well Control OFT 13-3-2 Wave Energy

Refreshment Break 10:00 - 10:30

Hall 5 (SEC)

Concurrent Sessions 10:30 - 12:00

OFT 1-7-1 Wave Loading and Motions in Extreme Seas I

SSR 2-13-2 Risk Analysis and Management II

SSR 2-6-3 Reliability of Mooring and Riser Systems II

MAT 3-5-1 Fatigue Assessment and Improvement

PRS 4-1-11 Umbilicals and Cables II

PRS 4-3-8 Mechanics II

OE 6-13-2 Ship Manoeuverability and Motion

OE 6-17-2 Nonlinear and Breaking Waves

$\mathrm{OE}$ 6-7-2 Regional Metocean II

CFD 8-1-6 Seakeeping II

CFD 8-4-3 Interference, Proximity and Geometry Effects

ORE 9-1-8 FWT Hydrodynamics

ORE 9-4-3 Advanced Controls

PT 11-1-2 General Petroleum Technology II

PT 11-10-1 New Materials for Well Construction

OFT 13-3-4 Wave Energy II

Technical Session Organizers' Lunch 12:00 - 13:30 Hall 5 (SEC)

Concurrent Sessions 13:30 - 15:00

OFT 1-7-2 Wave Loading and Motions in Extreme Seas II

SSR 2-14-1 Risk Based Maintenance

SSR 2-7-1 Reliability of Renewable Energy Systems

MAT 3-2-2 Analysis and Fatigue Performance of Tubular Joints Subsea Structures I

(John Sharp Honorary Session)

PRS 4-4-1 Subsea Structures I

PRS 4-6-1 Innovative Technologies for Deepwater Low-Cost Production I

OE 6-13-3 Numerical Methods

OE 6-7-3 Metocean Criteria I

CFD 8-1-7 Propulsion

CFD 8-4-4 VIV Suppression and Control

ORE 9-4-1 Power Take-offs and Experiments

ORE 9-5-4 Numerical Analysis II

PT 11-1-3 Genral Petroleum Technology III

PT 11-11-1 Innovations in Drilling, Production and Transport

OFT 13-3-3 Ocean Current Energy, OTEC and Related Technology

Refreshment Break 15:00 - 15:30

Hall 5 (SEC)

Concurrent Sessions 15:30 - 17:30

OFT 1-4-1 Experimental Design and Analysis

SSR 2-5-1 Reliability of Marine Structures

MAT 3-13-1 Dr. John Sharp Honorary Session

PRS 4-4-2 Subsea Structures II

PRS 4-6-2 Innovative Technologies for Deepwater Low-Cost Production II

OE 6-7-4 Metocean Criteria II

CFD 8-5-2 VIVTheory and CFD\&FSI Symposium Workshop

ORE 9-2-6 Hybrid Systems and Farm Analysis

ORE 9-3-3 Wave Energy: CFD Simulations

PT 11-11-2 Innovations in Drilling, Production and Transport

OFT 13-3-1 Wind Energy

Farewell Reception 17:30 - 19:00 Argyll Suite (Crowne Plaza)
Friday, June 14 (PG 87)

Technical Tours

- Technical Tour to Advancing Forming

Research Centre and Falkirk Wheel

- Technical Tour to Subsea 7 and Falkirk

Wheel

\section{Wi Fi Network}

Network at SEC: SEC Wi-Fi

Network at Crowne Plaza:

CrownePlaza

No password required

\section{Registration}

Sunday, June 9

Monday, June 10

Tuesday, June 11

Wednesday, June 12

Thursday, June 13

\section{Exhibition}

Monday, June 10

Tuesday, June 11

Wednesday, June 12

Thursday, June 13
Hall 5 (SEC) 13:00 - 20:00 $07: 00-17: 30$ 08:00 - 17:30 $08: 00-17: 30$ 08:00 - 17:30

Hall 5 (SEC) 08:30 - 19:15 $08: 30-17: 30$ 08:30 - 17:30 $08: 30-15: 30$

\section{Daily Program Handout}

An updated daily program handout will be available at the Registration Desk the mornings of Tuesday, Wednesday and Thursday. The handout will incorporate any last-minute program changes and show the time-synchronized order of presentations in each session for that day. You can use this handout as a general reference and to easily plan your personal attendance schedule for the day. The program changes will also be updated on the ASME Crowd Compass App.

\section{Key to Symposium \\ Abbreviations}

CFD CFD \& FSI

MAT Materials Technology

OE Ocean Engineering

OG Offshore Geotechnics

ORE Ocean Renewable Energy

OFT Offshore Technology

OSU Ocean Space Utilization

PAS Polar and Arctic Sciences and Technology

PRS Pipelines, Risers, and Subsea Systems

PT Petroleum Technology

SSR Structures, Safety and Reliability

ET Professor Rodney Eatock Taylor Honoring Symposium on Marine and Offshore Hydrodynamics

TK Professor Takeshi Kinoshita Honoring Symposium on Offshore Technology

See Detailed Program starting on page 27 for concurrent session room locations. 


\section{Venue Floor Plans}

We are using meeting rooms at the Scottish Event Campus (SEC) and the Crowne Plaza Hotel. There is a walkway/link joining the mezzanine level of the Crowne Plaza Hotel with the upper level of the SEC.

Opening Ceremony (SEC Armadillo)

Exhibits, Refreshment Breaks, Lunches, Registration (Hall 5, SEC)

Concurrent Sessions (Various Rooms, SEC and Crowne Plaza Hotel)

Farewell Reception (Argyll Suite, Ground Level, Crowne Plaza Hotel)

\section{Crowne Plaza Hotel}

Crowne Plaza Hotel, Congress Road, Glasgow, G3 8QT

Tel: +44 (0)8719429091

\section{Ground Level Meeting Rooms}

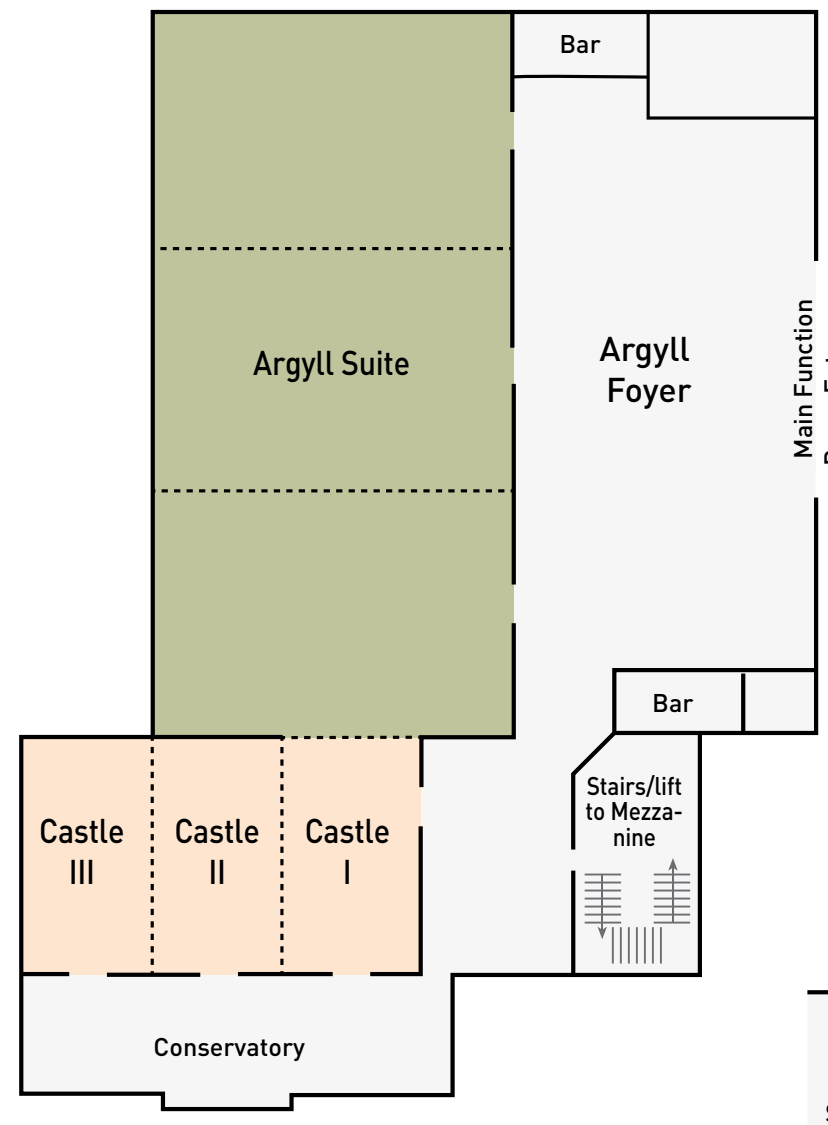

\section{Room Location (Alphabetical)}

\begin{tabular}{l} 
Armadillo \\
Alsh 1 \\
Alsh 2 \\
Argyll Suite \\
Barra \\
Boisdale 1 \\
Boisdale 2 \\
Carron 1 \\
Carron 2 \\
Castle 1 \\
Castle 2 \\
Castle 3 \\
Dochart 1 \\
Dochart 2 \\
Etive \\
Fyne \\
Hall 5 \\
Jura \\
Lomond Auditorium \\
M2 \\
M3 \\
M4 \\
Shuna \\
Staffa \\
\hline
\end{tabular}

SEC-Armadillo SEC-Ground SEC-Ground Crowne Plaza-Ground Crowne Plaza-Mezzanine SEC-Ground SEC-Ground SEC-Upper SEC-Upper Crowne Plaza-Ground Crowne Plaza-Ground Crowne Plaza-Ground SEC-Upper SEC-Upper SEC-Ground SEC-Ground SEC-Ground Crowne Plaza-Mezzanine SEC-Ground/Upper SEC-Upper SEC-Upper SEC-Upper Crowne Plaza-Mezzanine Crowne Plaza-Mezzanine

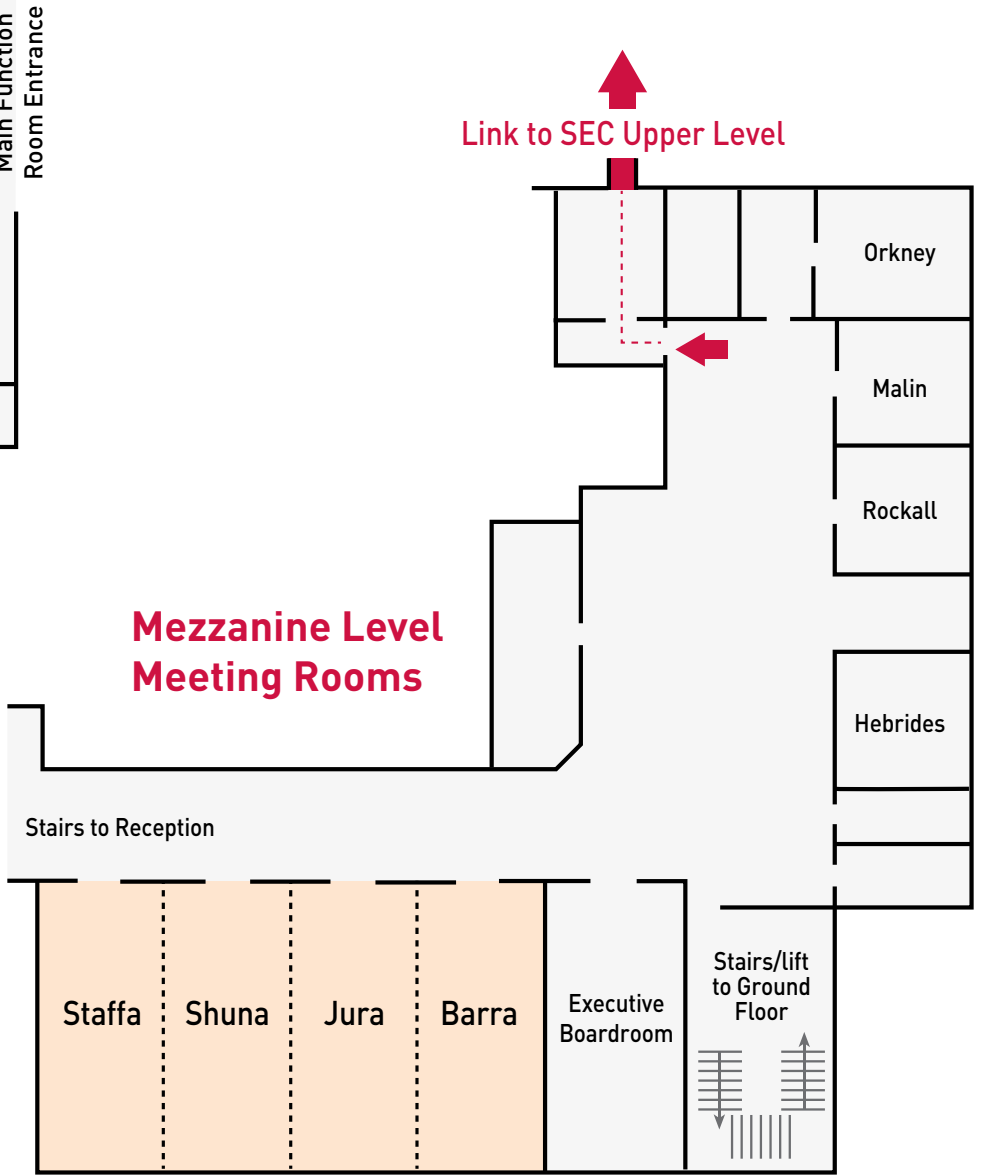




\section{Scottish Event Campus (SEC)}

SEC Centre, Exhibition Way, Glasgow City, UK G3 8YW

\section{ScotRail}

Tel: +44 (0) 1412483000

www.sec.co.uk
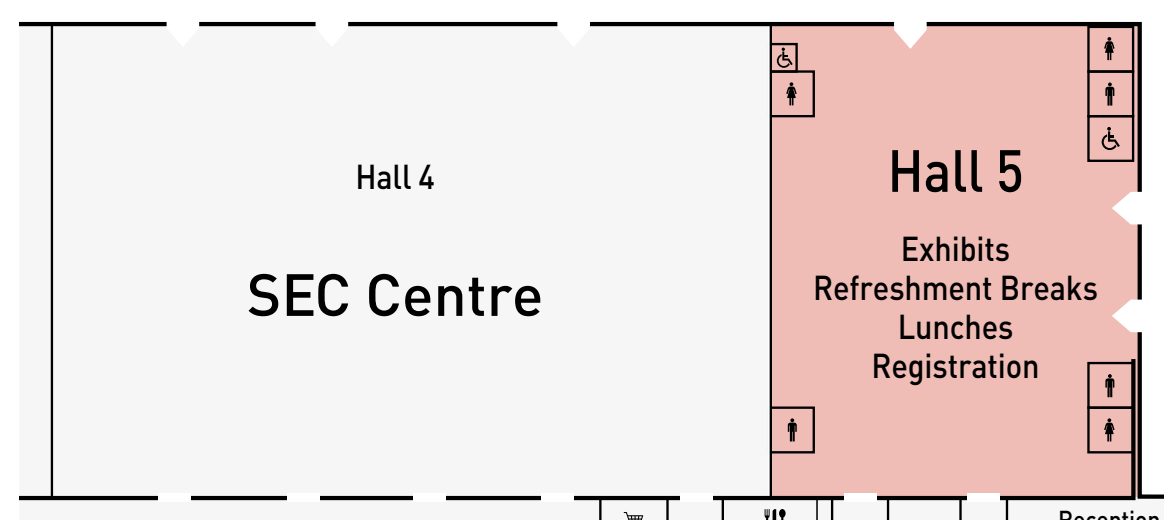

Hall 4

SEC Centre

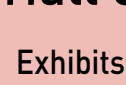

Refreshment Breaks

Lunches

Registration

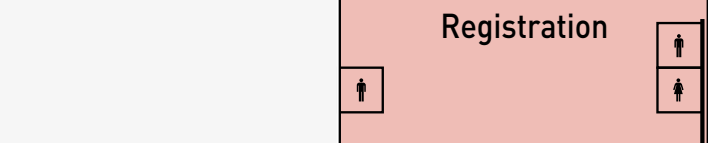

\section{The SSE Hydro}

Exhibition

Centre Station

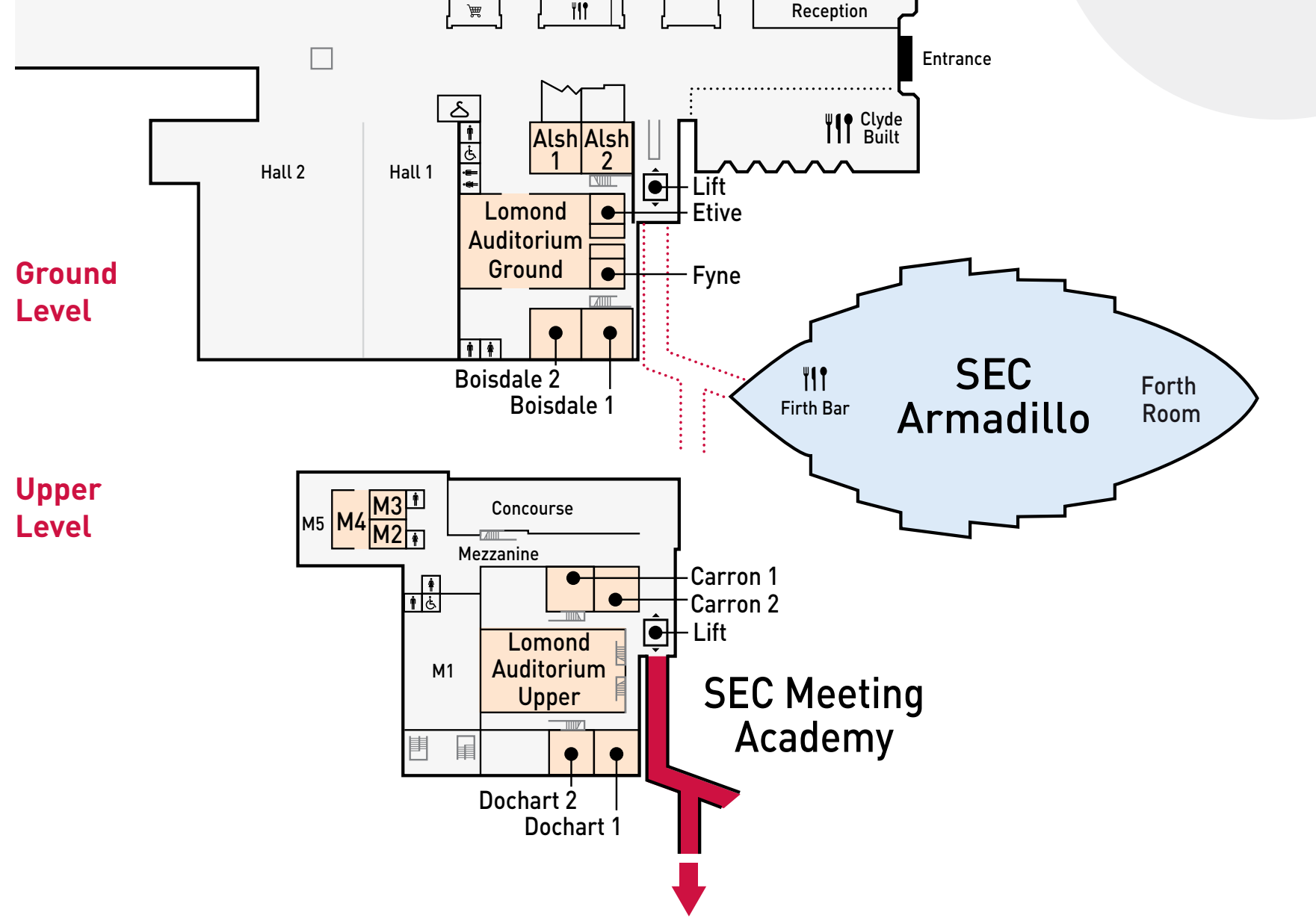

Link to

Crowne Plaza

Meeting Rooms

Mezzanine Level

Crowne Plaza Hotel

Mezzanine Level

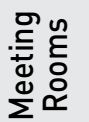




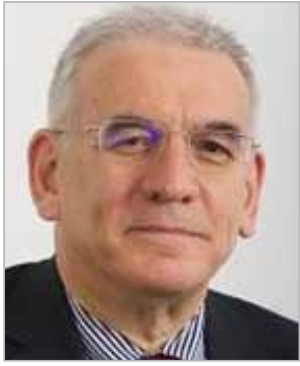

Professor Atilla Incecik

\section{Welcome from the Conference Chair}

$\mathrm{t}$ is my great privilege and honour to welcome you all to the $38^{\text {th }}$ International Conference on Ocean, Offshore and Arctic Engineering (OMAE) in Glasgow, Scotland, from 9 - 14 June 2019.

I am delighted that OMAE 2019 is being hosted by our Department of Naval Architecture, Ocean and Marine Engineering at the University of Strathclyde in Glasgow, Scotland. Established in 1883, and built on Glasgow's rich heritage of naval architecture and shipbuilding, we are one of the oldest Naval Architecture departments in the world. The Department of Naval Architecture, Ocean and Marine Engineering is part of the largest Faculty of Engineering in Scotland, and our University is home to over 22,000 students. Glasgow houses the second highest number of ship management companies after London, and has the largest shipyard in the UK, cementing the fact that Glasgow remains the hub of the UK's shipbuilding industry.

Over 900 papers have been accepted for the conference, organised around 13 symposia which will take place during the week. Monday's Opening Ceremonies will feature welcome speeches from the University of Strathclyde and the City of Glasgow, along with Keynote Presentations from industry. The conference features three afternoon lectures, including a presentation from the European Research Council on the ERC research funding programme. On Tuesday and Wednesday afternoon, we are pleased that our two conference honourees, Professor Rodney Eatock Taylor and Professor Takeshi Kinoshita, will be giving afternoon plenary lectures. Technical tours on Friday include visits to Subsea7 in Glasgow, the University of Strathclyde's Advanced Forming Research Centre and the Falkirk Wheel.
We have an exciting social programme organised for you including the Conference Banquet which will take place in Glasgow's Merchant City, one of Glasgow's oldest quarters dating back to the 1750s. Throughout the conference we will have a truly Scottish theme, introducing you to Scottish food and culture, giving you a real taste of Scotland which you are sure to remember!

Glasgow was this year named in the top ten of Time Out's best cities in the world for 2019, and has previously been named as one of the world's top ten must see cities by various publications including Rough Guides and the Telegraph. National Geographic named Glasgow as one of its 'Best of the World' destinations and we were also voted 'Friendliest City in the World' in Rough Guide 2016 - something I am sure you will agree with after your stay here!

Finally, the Conference could not be a success without the dedication and the hard work of many people. In particular I would like to recognize our Technical Programme Chair, Professor Krish Thiagarajan Sharman, our Local Organising Committee, Mrs Annabel Anderson (Marketing and Events Coordinator of the Faculty of Engineering at the University of Strathclyde), the many symposia and topic organisers, session chairs and reviewers, authors of papers and conference participants, our sponsors and exhibitors, ASME and Sea to Sky Meeting and Association Management staff, and our Volunteers, who will be around all week in the blue shirts to guide and assist you! Thank you all most sincerely.

I wish you all a very productive conference, and a most enjoyable stay in Glasgow.

-Professor Atilla Incecik Conference Chair, OMAE 2019

Professor of Offshore Engineering Associate Principal and Executive Dean of the Faculty of Engineering University of Strathclyde, Scotland, UK 


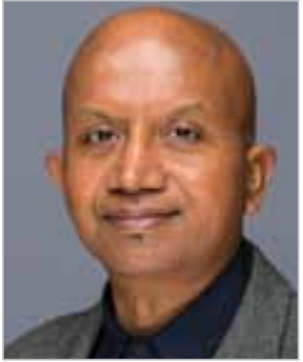

Professor Krish Thiagarajan Sharman

\section{Welcome from the Technical Program Chair}

I $t$ is a great pleasure for me to address you this year as the Technical Program Chair for this year's Ocean Offshore and Arctic Engineering Conference in the city of Glasgow, a city with deep and rich maritime traditions. Most of us attend the OMAE conference every year to delve into the depths of science, technology and engineering as displayed by the technical program. So it was with a sense of trepidation and respect that I took on the role of TPC for 2019.

Our program this year has exceeded our expectations with a large number of submissions. From the 1060 submitted abstracts, 916 technical papers and 38 presentations were accepted for the conference. Over the years, the technical program committee has continually focused on improving quality of the papers, which implied keeping an eye on the rejection rate. This year, the paper rejection rate is close to $6 \%$ of the draft papers that were submitted. You will be able to see improvements in quality as you enjoy the technical sessions. Another aspect we have been working on is to increase the number of technical presentations, which are accompanied by only an abstract. This gives opportunities for our colleagues from industry to present material that they may otherwise not be able to share with others. In consideration of this, please be sensitive about taking pictures of presentations during the sessions without permission from the speaker.

Apart from our regular symposia, this year we are proud to honor two exceptional individuals whose contributions to the fields of offshore engineering and marine hydrodynamics has been legendary. Professor Rodney Eatock-Taylor has a long history of working on complex problems in wave mechanics and offshore platform dynamics. Professor Takeshi Kinoshita has done pioneering work on many aspects of ocean science and offshore engineering. We will hear from them about their life journeys through two afternoon lectures on Tuesday and Wednesday.

My role as this year's TPC would have been daunting if it were not for the tremendous support from all the symposium coordinators, topic and session organizers. I particularly want to acknowledge the support of Dr. Charles Smith, who handled the Ocean Renewable Energy Symposium while I was focused on the TPC role. Working with Professors Atilla Incecik and Antonio Fernandes, the LOC and Executive Committee has been a pleasure. The support given by Stacey Cooper at ASME and the Sea to Sky team has been invaluable.

I hope you will have a great time at OMAE 2019, and I look forward to seeing you again at future OMAE conferences.

- Professor Krish Thiagarajan Sharman

Technical Program Chair, OMAE 2019

Endowed Chair in Renewable Energy and Professor Department of Mechanical and Industrial Engineering University of Massachusetts Amherst, USA

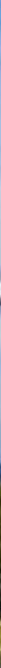




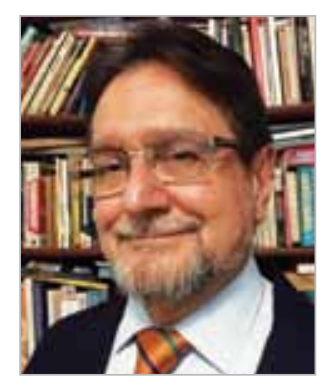

Professor Antonio C. Fernandes

W elcome to the Ocean, Offshore and Arctic Engineering Conference (OMAE), hosted by the American Society of Mechanical Engineers (ASME). This is the 38th edition of the conference, which is held annually around the world. International is one word. The OOAE Division volunteers and ASME staff (Houston and New York) come together each year with truly democratic rules to host this conference, seeking sustainable and safe use of ocean resources.

OMAE is an unusual conference for several reasons. It combines Academia with Industry to adapt scientific achievements into practical applications, leading to tangible technological successes. Industry has an obligation to make it feasible and safe. Academia has an obligation to verify the science. This is a wonderful play well represented in OMAE conferences. Art is another word. OMAE and its volunteers organize comprehensive Symposia, which organize sessions where one can enjoy professional presentations and discussions that lead to networking in the corridors and in the social events.

Starting last July, I had the honor to assume the mandate as Chair of the Executive Committee of the OOAE/ASME division. To put my journey to Chair into perspective, I remember my first OMAE conference in 1985 in Dallas, where I bought a Stetson cowboy hat. The next conference was in Florence, 1996, where I listened to a Bach recital in a medieval church. By then I was convinced, and I have attended every OMAE conference since then 23 years in a row. This includes two conferences I helped organize with my friend Segen Farid Estefen, in 2001 and 2012. I have numerous cities and destinations to reminisce about, without forgetting the approximately 80 papers I have co-authored and presented so far. I strongly recommend participation in OMAE to my students and younger colleagues.

Through my OMAE experiences I have made many friends from all over the world, making me feel that I indeed belong to an international network that makes me a better professional. I profit from this and I can create and provide opportunities in this very stable, very productive community that has contributed to mankind's well-being through quality engineering and sustainable technological achievements.

Rewarding is the final word. Welcome to Glasgow!

-Professor Antonio C. Fernandes OOAE Executive Division Chair Head Ocean Engineering Program of COPPE/UFRJ LOC (Laboratório de Ondas e Correntes - Waves and Currents Laboratory), Coordinator LabOceano, Director for International Affairs

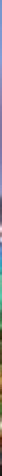




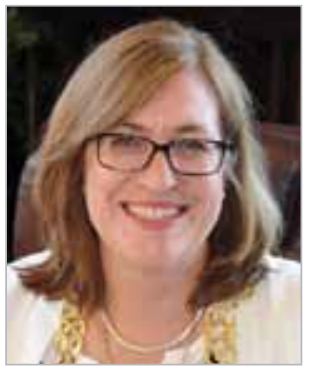

Eva Bolander

\section{Welcome from the Lord Provost of Glasgow}

1

'm delighted to welcome the 38th International Conference on Ocean, Offshore and Arctic Engineering to Glasgow.

Delegates, this is a city with a long and rich seafaring history as well as an unrivalled reputation for world-class shipbuilding and engineering. The term 'Clyde-built' synonymous across the globe, with quality and safety.

A tradition I'm proud to say, continues for Glasgow remains the home of the UK's largest shipyard BAE Systems. While nearby Rosyth boasts the largest engineering dockyard facilities. Both sites involved in delivering the Royal Navy's latest aircraft carriers - built by highly skilled workers BAE Systems Shipyards and assembled by equally accomplished workers at Babcock in Rosyth.

Meanwhile, your conference host, the University of Strathclyde is a leading provider of teaching and research in marine technology. Its Department of Naval Architecture, Ocean and Marine Engineering one of the world's oldest established back in 1883 .

Its enviable status enabling it to forge strong and meaningful links with maritime, offshore and renewable industries across the UK and worldwide.

The Department also has a winning team of postgraduate researchers and academic staff, the largest in Europe, committed to diverse, useful and innovative research, education and knowledge exchange.
It's an institution that is also home to the UK's largest engineering faculty with the capability to attract some of the finest minds to teach and learn.

The decision by the UK government to locate the Renewable Energy Catapult Centre on the university campus also emphasising Strathclyde's significant research capability.

You'll be aware, that Glasgow is also a major renewables hub. With some of the United Kingdom's largest offshore renewable companies choosing to locate here. Making it an obvious destination for conferences like yours.

I'm thrilled to have this opportunity to welcome the conference back to Glasgow and I'm sure you'll have a great experience and an enjoyable stay.

This is a city famed for its friendliness and hospitality. Recently ranked the 8 th best city in the world by Time Out readers. It was also judged -by the same Time Out readers - as the most improved city in the world! That's something we're incredibly proud of.

This forward-thinking, progressive and international city owes its status to the skills and expertise of its citizens. They're our greatest asset and consistently demonstrate that 'People Make Glasgow'.

Friends, I'm confident you'll enjoy your stay here and leave Glasgow with fond memories.

-Councillor Eva Bolander Lord Provost of Glasgow

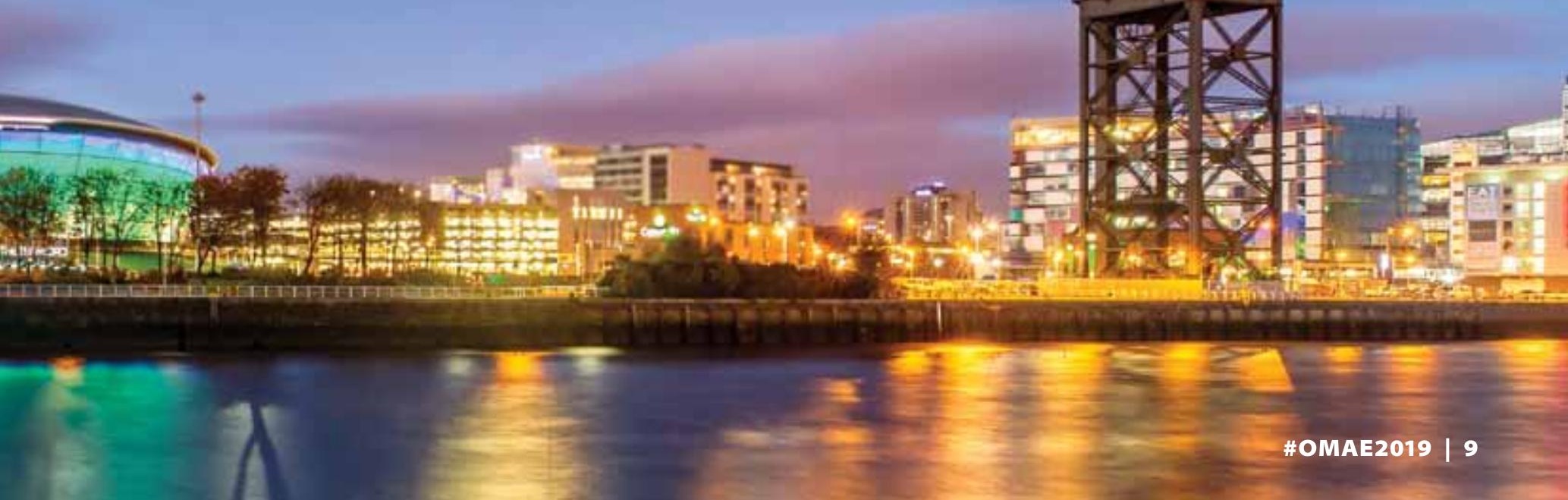




\section{Glasgow City Map}

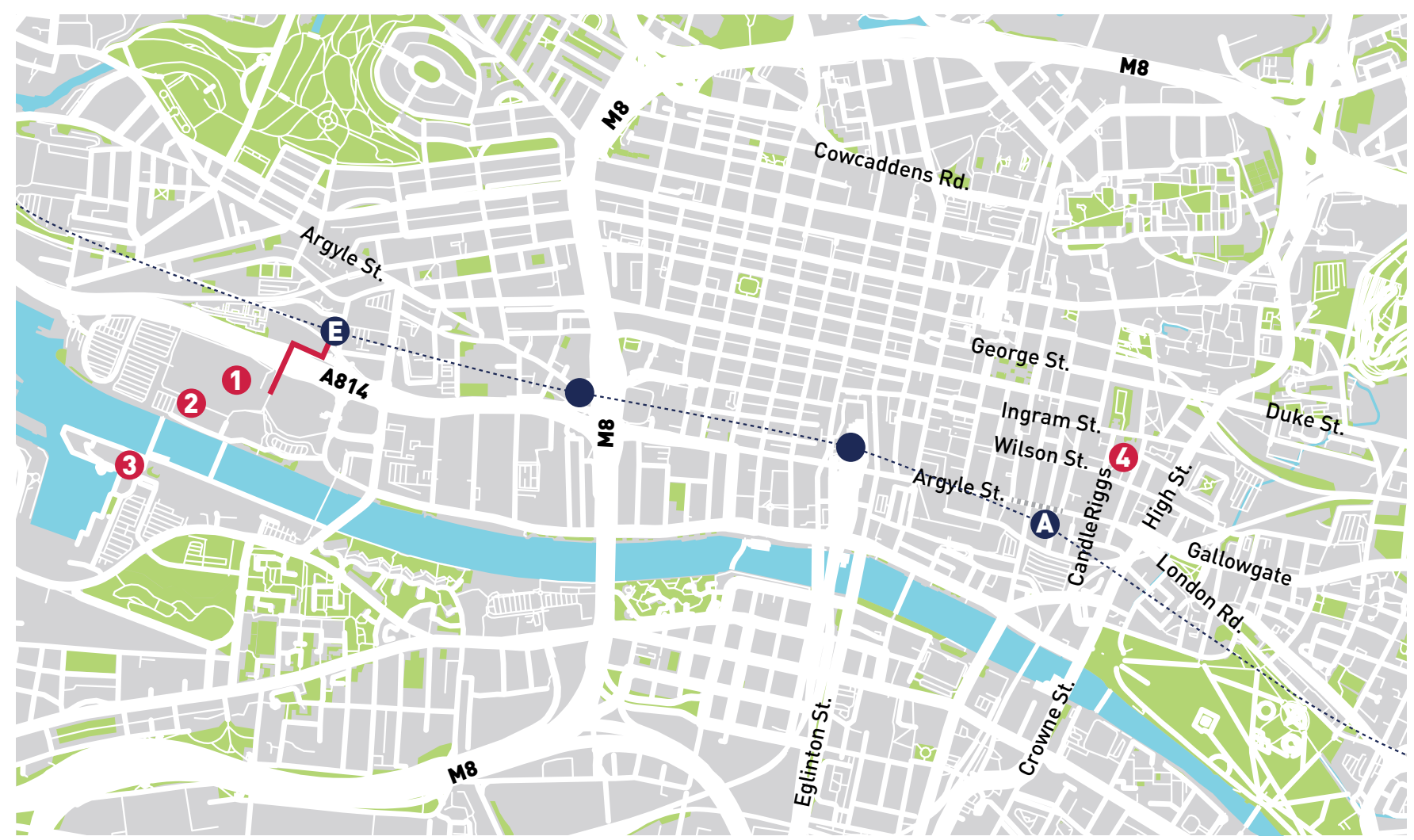

(1) Scottish Event Campus (SEC) (Conference Venue) Exhibition Way, Glasgow, G3 8YW

2 Crowne Plaza Hotel (Breakout Session Rooms, Farewell Reception) Congress Road, Glasgow, G3 8QT

(3) Glasgow Science Centre (Welcome Reception) 50 Pacific Quay, Glasgow, G51 1EA

4 Merchant Square (Conference Banquet) Candleriggs Street, Glasgow

\section{ScotRail}

Covered walkway between SEC and the Exhibition Centre Station

E Exhibition Centre Station

A Argyle Street Station

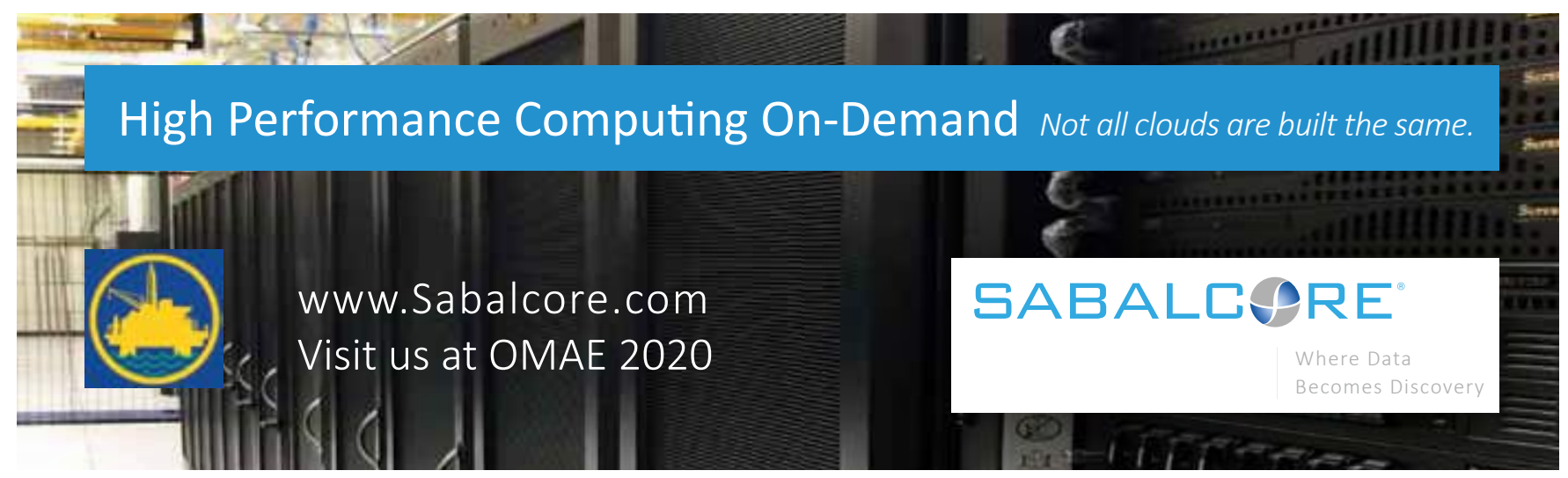




\section{INSIDER NEWS FOR THE OFFSHORE INDUSTRY}

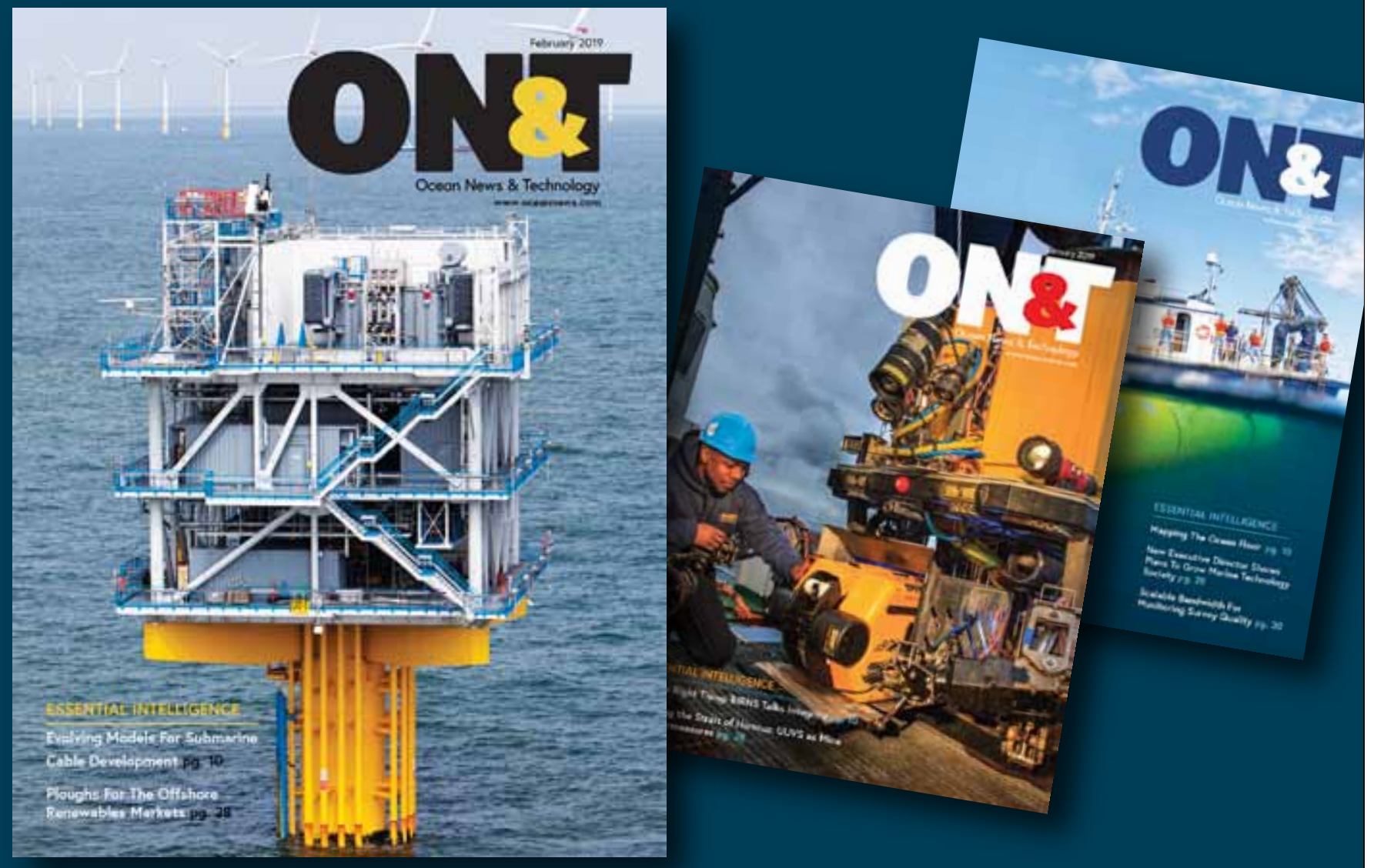

Want to stay up to date on the latest news in the ocean technology industry?

Access industry forecasts, technology developments, and product news for the Offshore Energy, Subsea Intervention and Survey, Maritime Communications, and Defense Industries.

NEWSLETTER

Receive weekly up-to-date information.

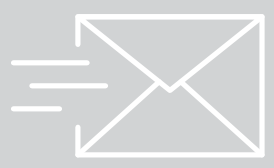

DIRECTLY TO YOUR INBOX

bit.ly/ontnewsletter
MAGAZINE

Essential intelligence with our monthly publication.

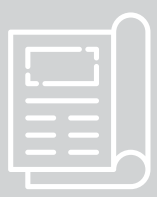

PRINT

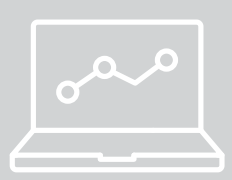

DIGITAL www.oceannews.com/subscribe 


\section{Award Winners}

\section{The Subrata Chakrabarti Young Professional Award}

Dalila Gomes, for her outstanding presentation and paper OMAE2018-77547 “A Transient Flow Model for Investigating Parameters Affecting Kick Behavior in OBM for HPHT Wells and Backpressure MPD Systems"

\section{OMAE 2018 Best Paper Awards}

Offshore Technology Symposium, OMAE2018-78315

"Hydrodynamic Coefficients of Simplified Subsea Structures" by Fredrik Mentzoni, Mia Abrahamsen-Prsic and Trygve Kristiansen

\section{Structures, Safety and Reliability Symposium,}

OMAE2018-77402 "A Study on the Dynamic Ultimate Strength of Global Hull Girder of Container Ships Subjected to Hogging Moment" by Yasuhira Yamada and Kyoko Kameya

\section{Materials Technology Symposium, OMAE2018-78672} "Integrity of Sour Gas Pipeline Despite Local Hard Zones" by Mamdouh M. Salama, Hernan Rincon and Stuart Wilson

\section{Pipelines, Risers, and Subsea Systems Symposium,} OMAE2018-78146 "Equivalent Layer Approaches to Predict the Bisymmetric Hydrostatic Collapse Strength of Flexible Pipes" by José Renato M. de Sousa, Marcelo K. Protasio and Luis V. S. Sagrilo

\section{Ocean Space Utilization Symposium, OMAE2018-77201}

"Validation of Applicability of Low Frequency Motion Analysis Theory Using Observation Data of Floating Offshore Substation" by Haruki Yoshimoto, Hisafumi Yoshida and Ken Kamizawa

Ocean Engineering Symposium, OMAE2018-77238 "SemiSubmersible Floater's VIM Simulation Method for Mooring Line Safety Assessment" by Toshifumi Fujiwara

\section{Polar and Arctic Sciences and Technology Symposium,} OMAE2018-78080 "Investigating the Influence of Bridge Officer Experience on Ice Management Effectiveness Using a Marine Simulator Experiment" by Erik Veitch, David Molyneux, Jennifer Smith, and Brian Veitch
CFD \& FSI Symposium, OMAE2018-78598 "Validation Exercises for a Free Falling Wedge into Calm Water” by João Muralha, Luís Eça, António Maximiano, and Guilherme Vaz

Ocean Renewable Energy Symposium, OMAE2018-77807 "Using Nonlinear Wave Kinematics to Estimate the Loads on Offshore Wind Turbines in 3-hour Sea States" by Tim Bunnik and Erik-jan de Ridder

Offshore Geotechnics Symposium, OMAE2018- 78128 "Simulating the Response of Untrenched Flowlines due to Iceberg-Flowline-Soil Interaction" by Kenton Pike and Andrew Blundon

Petroleum Technology Symposium, OMAE2018-77515 "Experimental and CFD Study of Circulation Efficiency in Simulated Irregular Annulus" by Shreyansh Divyankar, Milad Khatibi, Rune Wiggo Time, Hans Joakim Skadsem and Jan Aage Aasen

Honoring Symposium for Professor Carlos Guedes Soares on Marine Technology and Ocean Engineering, OMAE2018-77672 "Autonomous Ship Navigation under Deep Learning and the Challenges in COLREGs" by L.P. Perera

\section{Honoring Symposium for Professor Bernard Molin} on Marine and Offshore Hydrodynamics, OMAE201878221 "Hydrodynamic Interactions of the Truncated Porous Vertical Circular Cylinder with Water Waves" by Charaf Ouled Housseine and Sime Malenica 


\section{Attendee Information}

\section{Registration}

The Registration Desk is located in Hall 5, and is open during the following hours:

Sunday, June 9: $\quad$ 13:00 $-20: 00$

Monday, June 10: $\quad$ 07:00 - 17:30

Tuesday, June 11: $\quad 08: 00-17: 30$

Wednesday, June 12: $\quad$ 08:00 - 17:30

Thursday, June 13: $\quad$ 08:00 - 17:30

\section{Name Badges}

In addition to being a means of identification to colleagues, you are required to wear your name badge for admission to conference sessions and events. Room monitors will check name badges before allowing anyone into the session or event. Replacement badges are available at the Registration Desk at a cost of $£ 20$ per badge. Attendees who have paid the author/ member, non-member or student registration fee are entitled to admission to all conference sessions, daily refreshment breaks, the Welcome Reception, the Exhibition, the four Lunches, the Conference Banquet and the Farewell Reception. These attendees will also receive a conference bag and a program. Daily Registration: Attendees who have paid the one-day registration fee qualify for the badge representing the day they have selected to attend. Attendees wearing this badge are entitled to the following on their specified day: admission to conference sessions, refreshment breaks, the Exhibition and food and beverage service. The Conference Banquet is excluded from the daily pass. Daily attendees will also receive a conference bag and a program.

Accompanying Person: Guests who have registered as an accompanying person qualify for this badge and are entitled to admission to the Welcome Reception, the Conference Banquet and a special sightseeing tour on Monday.

Exhibitors: Exhibit staff have access to the Exhibition and may participate in the Welcome Reception, the four Lunches, the Conference Banquet, and the Farewell Reception. One representative from each exhibiting company is permitted to attend conference sessions.

Technical Tours and Social Events: Pre-purchased tickets for technical tours and social events are provided with your name badge.

\section{ASME Crowd Compass App}

Engage with sessions, speakers, and organizations, watch social networking in action, including posting on the in-app feed or sharing outside it. Download the Crowd Compass App from your app store. After installation, search for OMAE and download. Once OMAE is downloaded, you can set up a login. You will then receive a verification email with a code you need to enter in the app. Once you have entered the code in the app, this will grant you access to the event.

\section{Author Presentations}

If you are a Presenter, please be in the session room 30 minutes prior to the start of the first presentation of your session in order to upload your presentation. You may also upload your presentation any time prior to your talk on the computer in your session room. Screens aspect ratios are 4:3 in Crowne Plaza and 16:9 in SEC session rooms.

\section{Conference Evaluation}

Our aim is to deliver a conference that is an enjoyable and educational experience. We rely on your full and honest feedback to improve future conferences. An online survey will be emailed to you following the conference and we appreciate your time and assistance in completing the survey and providing your feedback.

\section{Dietary Requirements}

If you advised the Conference Secretariat of your special dietary needs during the registration process, a special meal has been prepared for you. For lunch on Monday, please advise your server of your special requirement. On Tuesday, Wednesday and Thursday, an allergen buffet station will be available where the servers will provide a meal to meet your dietary requirements. Vegetarian meals will be served on the regular buffet stations.

If you did not advise the Conference Secretariat of your special dietary needs during the registration process, advise the staff at the Registration Desk before 18:00 on Sunday, June 9.

\section{First Aid}

For medical first aid assistance, please visit the First Aid Room on the main SEC Centre concourse. The nearest hospital is the Queen Elizabeth University Hospital, a ten-minute drive from the Conference venue. Dial 999 or 112 to contact UK emergency services.

\section{Internet}

Free Wi Fi internet is provided by the conference. The network names are "SEC Wi-Fi" and "CrownePlaza". No password is required.

\section{Lost \& Found}

Should you lose or misplace an item, please go to the Information and Business Centre, located on the public concourse of the SEC Centre.

\section{Meeting Room Protocol}

Every effort will be made to ensure that all sessions start and end on time. Presenters and attendees are asked to work together to achieve this. This may mean having to cut short a valuable discussion; however, conference organizers request your cooperation for the benefit of all attendees. Please turn your cell phone and other noise making devices off or set to silent.

\section{Smoking}

Smoking, including the use of e-cigarettes, is not permitted within the Hotel and SEC Centre. Smoking shelters are located on the campus grounds. 


\section{Social Events}

Welcome Reception

Sunday, June 9, 18:30 - 20:30

Location: Glasgow Science Centre

(50 Pacific Quay, Glasgow)

The Rt Hon The Lord Provost Councillor Eva Bolander is hosting the Welcome / Civic Reception at the Glasgow Science Centre, one of Scotland's most popular visitor attractions. Enjoy appetizers and drinks while exploring interactive science displays including "Powering the Future" and "Quantum Technologies"! The Glasgow Science Centre is a five minute walk from the conference venue.

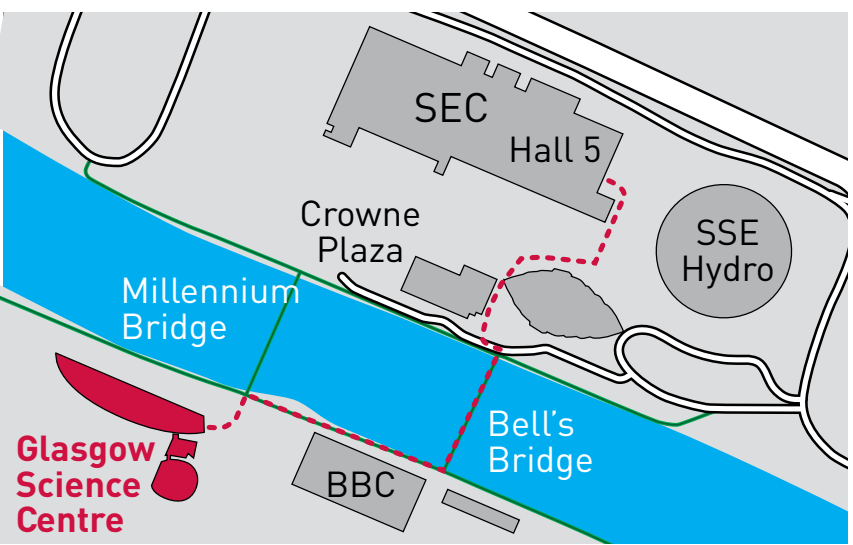

Register in Hall 5 at SEC Centre then follow the walking paths across the River Clyde to the Glasgow Science Centre.

\section{Refreshment Breaks}

Monday, June 10 to Thursday, June 13

Morning: 10:00 - 10:30 / Afternoon: 15:00 - 15:30

Location: Hall 5 (SEC)

Refreshment breaks will take place amongst the exhibits.

\section{Lunches \\ Monday, June 10 to Thursday, June 13 \\ Location: Hall 5 (SEC) \\ Monday: $\quad$ Opening Lunch (12:00 - 13:30) \\ Tuesday: $\quad$ Lunch (12:00 - 13:30) \\ Wednesday: Lunch (12:00 - 13:30) \\ Thursday: Technical Session Organizers Lunch (12:00 - 13:30)}

Lunch is open to all attendees when lunch is included in their fee.

Monday lunch sponsored by Elsevier

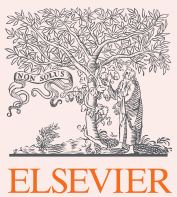

Thursday lunch sponsored by Greater Ft. Lauderdale Convention Bureau

GREATER FORT LAUDERDALE CONVENTION \& VISITORS BUREAU

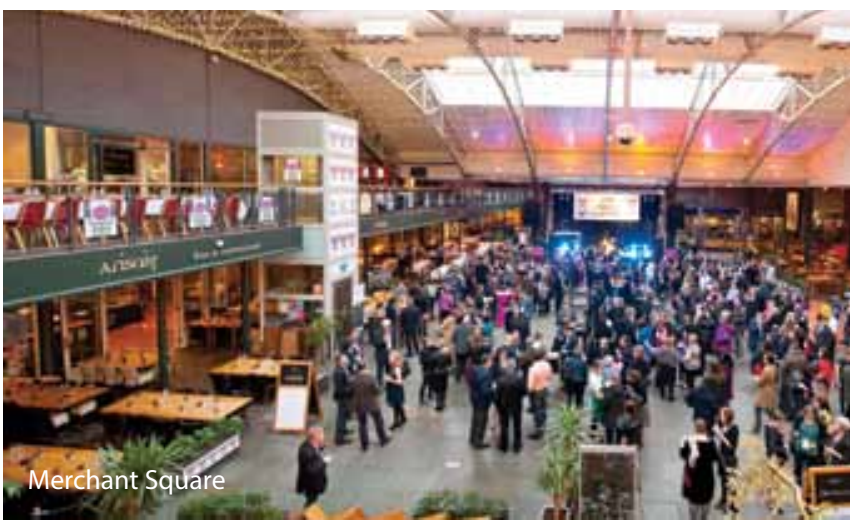

Afternoon Drinks Reception

Monday, June 10, 18:15 - 19:15

Location: Hall 5 (SEC)

Celebrate the first day of the conference with Afternoon Drinks amongst the exhibits.

\section{Conference Banquet}

Wednesday, June 12, 19:00 - 24:00

Location: Merchant Square (Candleriggs Street, Glasgow)

Transportation to and from the banquet venue via ScotRail train: Walk 8 minutes to the Exhibition Centre Station located across the street from The SSE Hydro via a covered walkway, and board the ScotRail train to the Argyle Street Station (three stops). Exit the station and walk east for 5 minutes along Argyle Street. Take a left on Brunswick Street and walk for about 3 minutes then right onto Wilson Street for 2 minutes. The destination will be in front of you. Look for directions and a map to Merchant Square in the conference app or refer to the map on page 10. You will have the option of sampling menus from each of the restaurants and drinks from any of the conveniently located bars. After dinner we'll put on an old fashioned Ceilidh with great music and dancing to the wee hours!

Farewell Reception

Thursday, June 13

17:30 - 19:00

Location: Argyll Suite,

Crowne Plaza Hotel

Hosted by the OMAE 2020 committee, celebrate the end of another amazing conference and find out more about next year's conference in Fort Lauderdale, USA. Get a taste of the tropical beach destination awaiting you in 2020 with Florida themed appetizers, drinks and salsa music provided by "Son Sabroso".

\section{Accompanying Persons Program Monday Tour, June 10 \\ Departure: 08:45}

Departure Point: Registration Desk, Hall 5, SEC Centre

The Accompanying Persons Program includes admission to the Welcome Reception, the Conference Banquet and a tour on Monday, June 10 to Loch Lomond including a boat cruise. 


\section{Sightseeing Tours}

Discounted rates for OMAE 2019 conference attendees and accompanying persons are available for a selection of tours to discover the stunning surroundings of Glasgow and Scotland.

Below is an introduction to the tours available through our partner Rabbie's Tours. OMAE 2019 attendees and accompanying persons receive a $10 \%$ discount on the published rates. A Tour Desk will be available in Hall 5 on Monday, June 10th between 10:00 -15:30. To apply the discount, please enter the tour discount code OMA1466 during the check out process when booking the tour on www.rabbies.com. The code is valid until June 30, 2019.

\section{Loch Lomond \& Whisky Distillery: Half day tour}

Combine a famous loch with a world-renowned whisky on this afternoon trip to the Highlands.

\section{Culzean Castle, Burns Country \& the Ayrshire Coast: 1 day tour}

Discover the landscapes and myths that inspired Scotland's most famous poet, Robert Burns.

Stirling Castle, Loch Lomond \& Whisky: 1 day tour Journey into the Highlands and the heart of Loch Lomond on this tour from Glasgow.

\section{Oban, Glencoe, Highland Lochs \& Castles: 1 day tour}

See epic ruined castles, listen to tales of old clan rivalries, and venture through brilliant mountain scenery on this tour to the Scottish Highlands.

\section{Outlander Adventure: 1 day tour}

Visit three castles and a well-preserved village on this adventure through the sights and stories of Outlander.

\section{Loch Ness, Glencoe \& the Highlands: 1 day tour}

Myths, monsters, and mountains: this tour is the best way to see 'Scotland in a day'.

\section{The Isle of Skye: 3 day tour}

Venture through breath-taking landscapes, quaint villages and epic castles on this journey to the 'Misty Isle'.

\section{Mull \& Iona: 3 day tour}

Journey to Mull and discover a paradise for wildlife enthusiasts, history addicts, and seafood connoisseurs.

Isle of Arran Adventure: 3 day tour

Explore the beautiful Isle of Arran and Robert Burns Country.
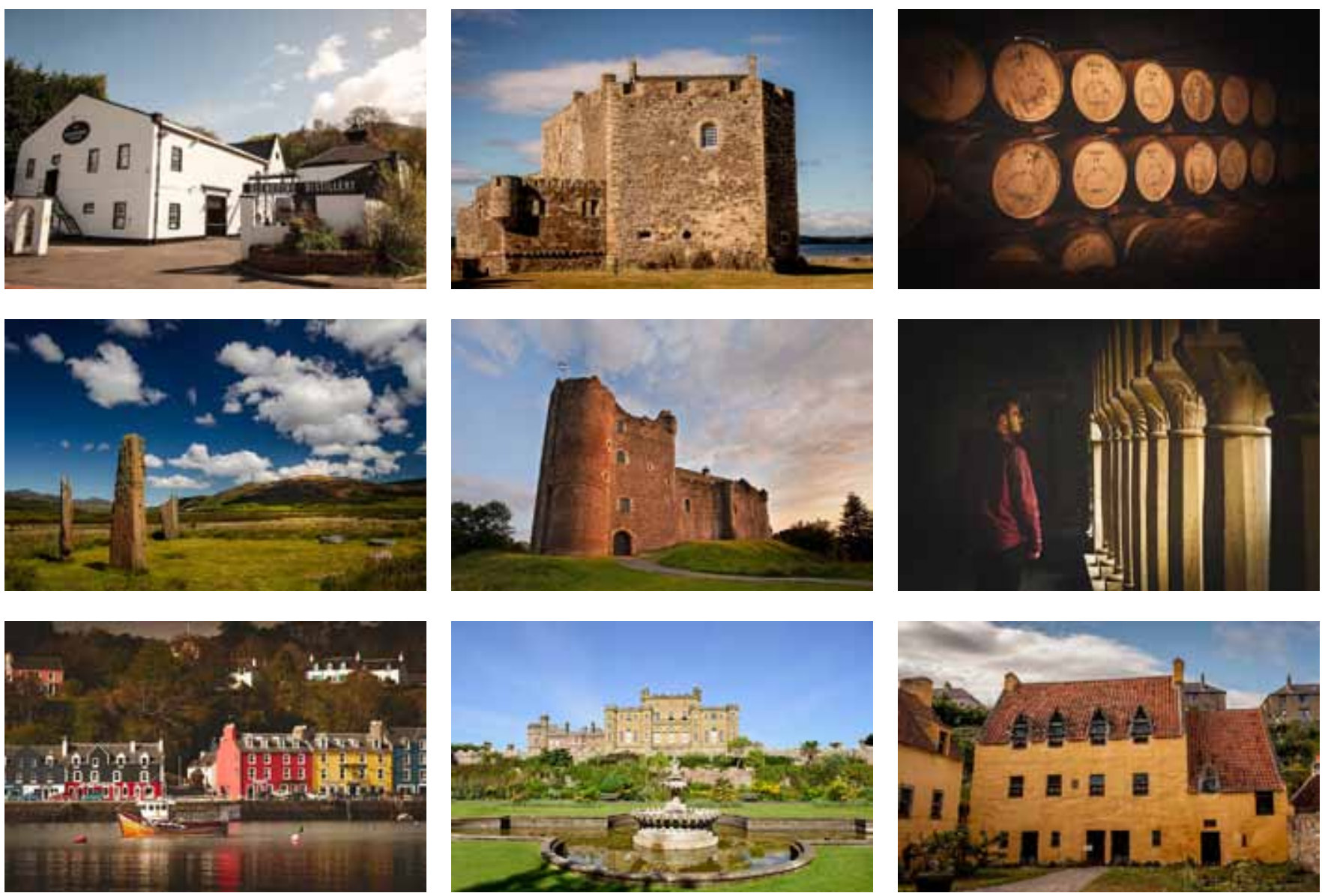


\section{SPONSORS \& EXHIBITORS}

\section{Sponsors \& Exhibitors}

\section{HOST}

\section{University of
Strathclyde Glasgow}

The Department of Naval Architecture, Ocean and Marine Engineering, University of Strathclyde www.strath.ac.uk/engineering/navalarchitecture oceanmarineengineering/

The Department of Naval Architecture, Ocean and Marine Engineering at the University of Strathclyde is one of the premier providers of teaching and research in marine technology. The Department, which is one of the oldest Naval Architecture departments in the world, established in 1883, has strong links with the maritime and offshore industry in the UK and worldwide in research, education and knowledge exchange activities related to marine and offshore hydrodynamics, ship stability and safety, marine and offshore structures, offshore engineering, marine and offshore structures, marine renewable energy, marine engineering and emerging technologies.

\section{CONFERENCE SUPPORTER}

\section{Glasgow \\ Convention \\ Bureau}

\section{GLASGOW \\ CONVENTION BUREAU}

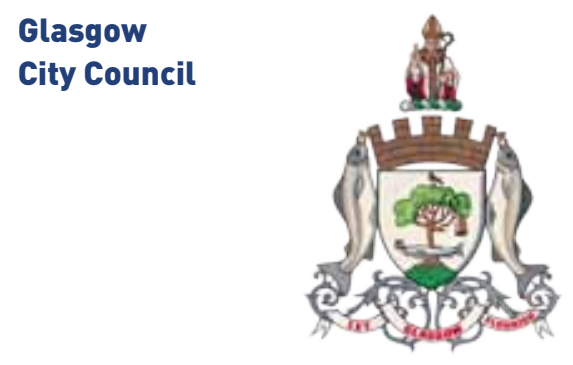

Visit Scotland

\section{Exhibition}

V

isit the exhibits to discover new products and services from some of the industry's leading organizations. Coffee and tea will be served amongst the exhibits during Refreshment Breaks.
Location: Hall 5, SEC

Dates \& Times:

Monday, June $10 \quad$ 08:30 - 19:15

Tuesday, June $11 \quad$ 08:30 - 17:30

Wednesday, June 12 08:30 - 17:30

Thursday, June $13 \quad$ 08:30 - 15:30

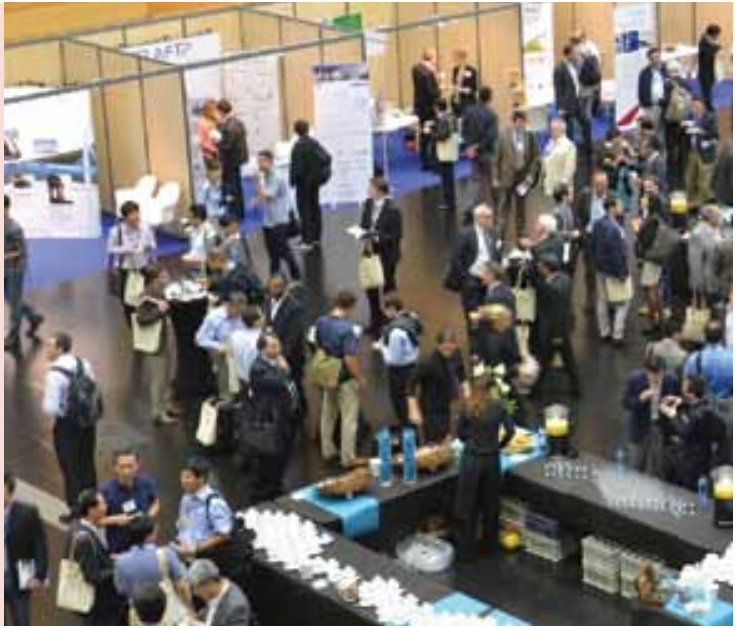




\section{OPENING LUNCH SPONSOR}

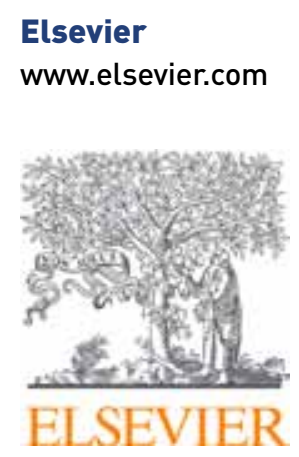

Elsevier is a world-leading provider of scientific, technical and medical information products and services. The company works in partnership with the global science and health communities to publish more than 2,000 journals, including The Lancet, Cell and Ocean Engineering, Applied Ocean Engineering, Coastal Engineering and Marine Structures.

\section{REFRESHMENT BREAK SPONSORS}

\section{TechnipFMC}

www.technipfmc.com

\section{TechnipFMC}

TechnipFMC is a global leader in subsea, onshore, offshore, and surface technologies. With our proprietary technologies and production systems, integrated expertise and comprehensive solutions, we are transforming our clients' projects and unlocking new possibilities for developing their oil and gas resources through innovative technologies and improved efficiencies.

\section{Babcock}

www.babcockinternational.com

\section{babcock}

For more than a century, Babcock, the Aerospace and Defence company, has been trusted to deliver bespoke, highly-skilled engineering services. We help customers in the UK and around the world to improve the capability, reliability and availability of their most critical assets within the four market sectors of Marine, Land, Aviation and Nuclear, underpinned by a deep understanding of technology integration, unique infrastructure and specialist training.

\section{SYMPOSIA SPONSOR}

\section{SYMP 9: Ocean Renewable Energy Principle Power Inc.}

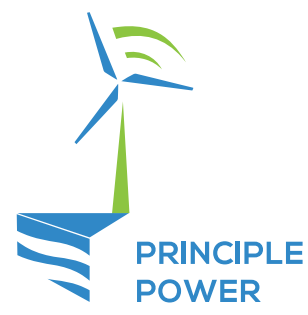

\section{MEDIA SUPPORTERS}

\section{Journal of Marine Science and Engineering}

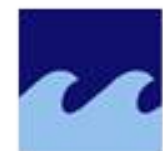

Journal of

Marine Science

and Engineering

an Open Access Journal by MDPI

\section{ON\&T}

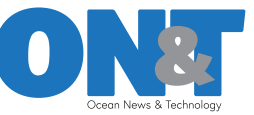

Ship \& Offshore

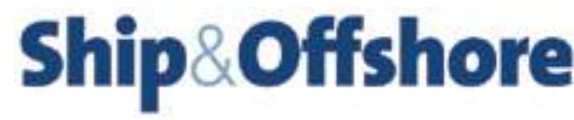

Springer

SPRINGER NATURE

\section{TRAVEL SPONSOR}

\section{Turkish Airlines}

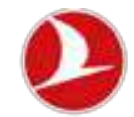

\section{TURKISH AIRLINES}




\section{Exhibitors}

DNV GL

www.dnvgl.com

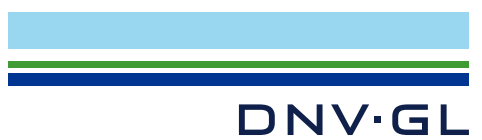

Driven by our purpose of safeguarding life, property and the environment, DNV GL enables organizations to advance the safety and sustainability of their business. Operating in more than 100 countries, our professionals are dedicated to helping our customers in the maritime, oil \& gas, energy and other industries to make the world safer, smarter and greener. DNV GL is the world-leading provider of software for managing risk and improving asset performance in the energy, process and maritime industries. Our solutions support a variety of business critical activities including design and engineering, risk assessment, asset integrity and optimization, QHSE, and ship management.

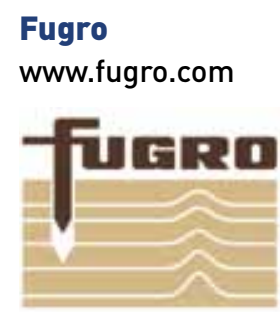

Fugro provide the people, equipment, expertise and technology that support the exploration, development, production and transportation of our world's natural resources. They can provide the technical data and information required to design, construct and maintain client structures and infrastructure in a safe, reliable and efficient manner.

\section{Lankhorst}

www.lankhorst-ep.com

\section{Lankhorst $\mid$ Engineered Products}

Lankhorst provides high quality engineered products for offshore risers and flow lines. With their unique thick-walled plastic production technology, they can supply innovative high performance products to meet the demands of the offshore industry. From piggy-back-blocks and clamps for laying pipelines and vortex-induced-vibration-suppression riser protection, through to bend restrictors, Lankhorst's precision engineered components deliver outstanding performance in the most demanding subsea environments. Clients can rely on their proven track record having delivered hundreds of projects all over the world, from the most northern platforms to the deepest waters.

\author{
Malin Group \\ www.malingroup.com
}

\section{Malin Group}

The Malin Group represents a collection of companies under common ownership that offer a diverse and comprehensive set of services to our clients. Our in house team of naval architects, structural engineers, marine engineers, draughtsmen and project managers have a wealth of practical experience across a broad range of projects, including marine, heavy lift, fabrication and complex transportation projects. Malin are in a unique position where we can offer support for the lifecycle of a project, whether it be at Front-End Engineering Design (FEED) stage, through detailed design, fabrication, delivery and installation.

\section{Orcina}

www.orcina.com

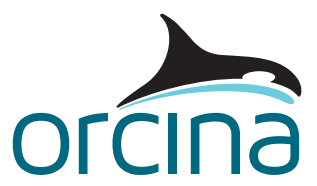

Orcina is a professional engineering software house specialising in the fields of offshore dynamics, risers, moorings, towed systems and installation procedures. We develop and sell leading edge commercial software packages including OrcaFlex (with VIV analysis), OrcaLay and OrcaBend. We also undertake feasibility and design studies, design audit, and engineering systems analysis.

Our main product is OrcaFlex, the market-leading numerical simulation program for modelling flexible and rigid risers, moorings, cable and pipe lay, pipeline pull-in, towed arrays, installation sequences and many other systems. OrcaFlex provides the best-in-class complete design environment for offshore dynamics. 


\section{Siemens}

www.plm.automation.siemens.com/global/en/products/ simcenter/

\section{SIEMENS \\ Ingenuity forlife}

Siemens Industry Software NV (SISW) helps manufacturers worldwide bring innovative products to market faster and with greater confidence, by offering them a comprehensive solutions portfolio, called Simcenter ${ }^{\text {th }}$ solutions. This uniquely combines test, system simulation, 3D CAE, CFD and design space exploration, as well as engineering services.

By employing Simcenter, major industry players can more effectively design and optimize key performance aspects, while dealing with complexities such as ever-increasing mechatronics, additive manufacturing, and concepts like cloud or the internet of things.

SISW runs a business unit of Siemens Product Lifecycle Management (PLM) Software, part of the broader Siemens Digital Factory division.

\section{Springer}

www.springernature.com

\section{SPRINGER NATURE}

Springer Nature is a leading research, educational and professional publisher, providing quality content to our communities through a range of innovative platforms, products and services. Every day, around the globe, our imprints, books, journals and resources reach millions of people - helping researchers, students, teachers and professionals to discover, learn and achieve more.

Through our family of brands, we aim to serve and support the research, education and professional communities by putting them at the heart of all we do, delivering the highest possible standards in content and technology, and helping shape the future of publishing for their benefit and for society overall.

Visit: www.springernature.com/group and follow @SpringerNature.

\section{Supergen ORE}

www.supergen-ore.net

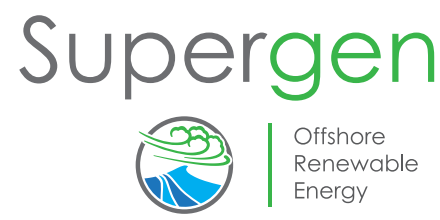

The Supergen Offshore Renewable Energy (ORE) Hub is an Engineering and Physical Sciences Research Council (EPSRC) funded programme that brings together academic and industrial expertise from across the Offshore Renewable Energy
(ORE) sector. The vision of the Hub is to provide research leadership to connect academia, industry, policy and public stakeholders, inspiring innovation and maximising societal value in offshore wind, wave and tidal energy. The Hub is central to the UK ORE community, bringing together shared skills and expertise, transferring fundamental knowledge, and sharing learning and use of resources for inter-disciplinary research, whilst taking a whole systems approach.

\section{TWI}

www.twi-global.com

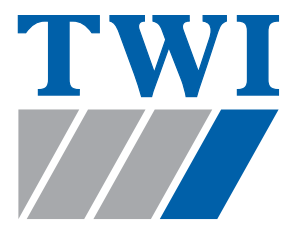

TWI is a world leading research and technology organisation. Bases in the UK and globally see 800 staff provide technical support in joining and technologies such as material science, structural integrity, NDT, surfacing, electronic packaging and cutting. Services include generic research, contract R\&D, technical information, consultancy, standards drafting, training and qualification. TWI offers a single, impartial source of service for joining engineering materials.

TWI is internationally renowned for its multidisciplinary teams that implement established or advanced joining technology solving problems at any stage - from initial design, materials selection, production and quality assurance, through service performance and repair.

\section{Engineering at the University of Strathclyde www.strath.ac.uk/engineering}

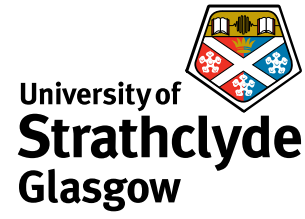

The University of Strathclyde is the third largest university in Scotland and our Faculty of Engineering is largest in Scotland, with eight world-class engineering departments.

We have an established record of research excellence evidenced through our expanding research portfolio of over $£ 100$ million. Much of our engineering research is done with industry and we work to provide specific business solutions for many engineering sectors.

Our Department of Naval Architecture, Ocean and Marine Engineering has Europe's largest team of postgraduate researchers and academic staff to sustain the production of useful and innovative research ideas. We work closely with key UK and global industry partners and take part in many diverse research projects and networks funded by the UK government, the marine and offshore industry and the EU. 


\section{TECHNICAL PROGRAM}

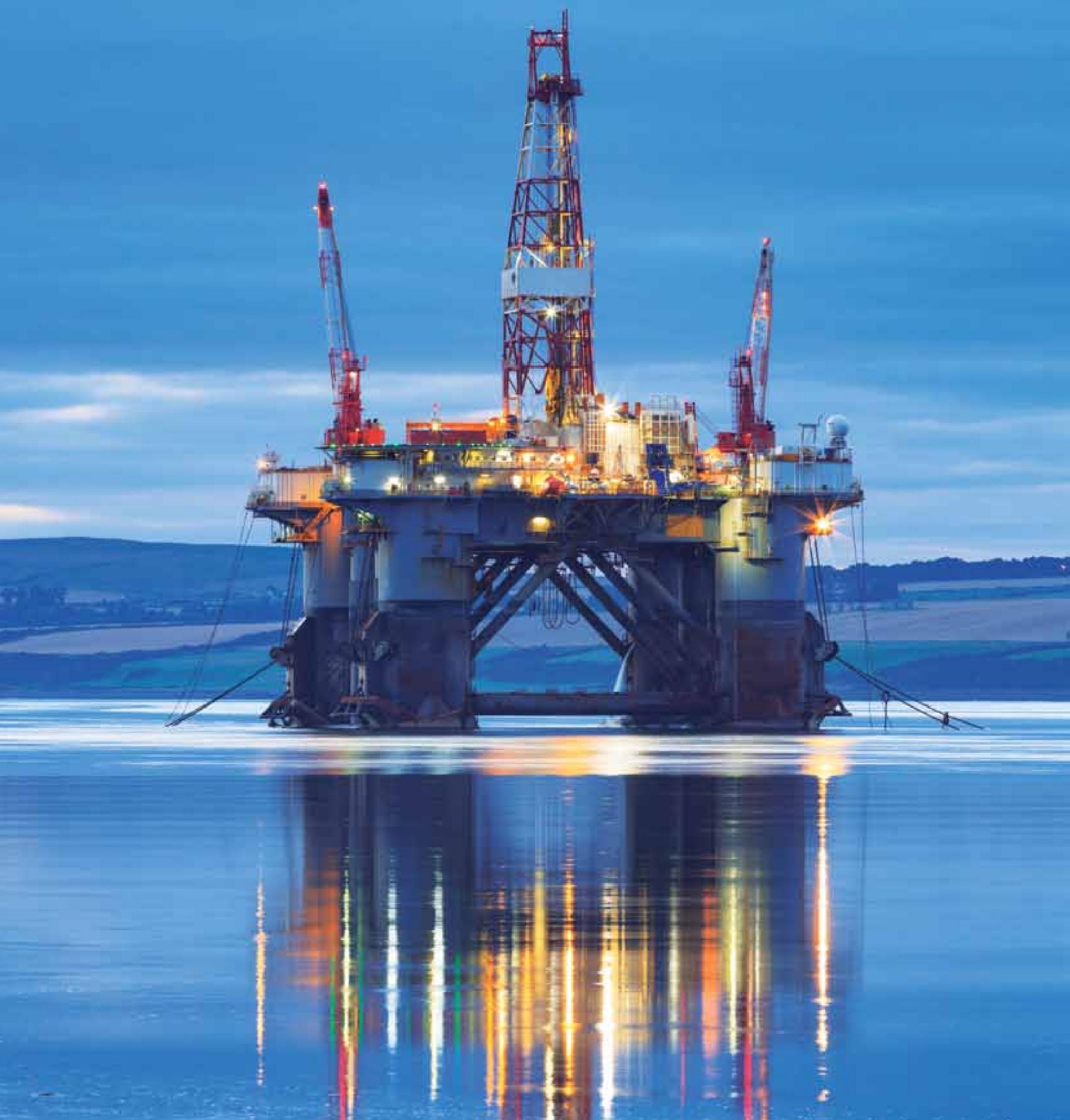




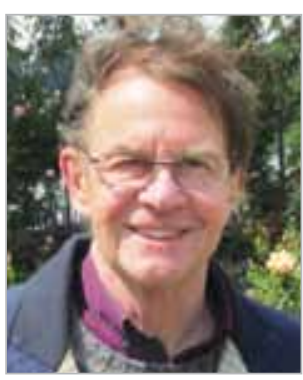

Professor Rodney Eatock Taylor

\section{Professor Rodney Eatock Taylor Honoring Symposium on Marine and Offshore Hydrodynamics}

$\mathbf{T}$ he OMAE 2019 Conference is pleased to dedicate a Special Symposium in honour of Professor Rodney Eatock Taylor. Professor Eatock Taylor has made a significant contribution to the field of Marine and Offshore Hydrodynamics.

Professor Rodney Eatock Taylor (FREng, FRINA, FIMechE) graduated from King's College, Cambridge (Mechanical Sciences followed by Fine Arts, 1965), and obtained his $\mathrm{PhD}$ in Civil Engineering from Stanford University (1968). After two years as a structural engineer with Ove Arup and Partners in London he joined University College London, working on ship structures with Professor R. E. D. Bishop. He became Professor of Ocean Engineering at UCL (1984) and Dean of Engineering (1988-1989). Since then he has been at the University of Oxford as Professor of Mechanical Engineering (now Emeritus), and Head of Engineering Science (1999-2004). His research interests have concerned vibrations of offshore platforms, numerical and analytical hydrodynamics, hydroelasticity of ships and very large floating structures, and marine renewable energy. This work has been published in some 280 journal articles and conference papers.

He was the UK Representative on the Standing Committee of the ISSC (1992-2000). In 2005-06 he was the 28th Weinblum Memorial Lecturer. Elected as a Fellow of the Royal Academy of Engineering in 1990, he was a Vice-President (2004-2007). In 2008 he was made an Honorary Fellow of University College London and an Honorary Professor of Harbin Engineering University. He has been Visiting Professor at Ecole Centrale de Nantes $(1983,1997)$, the Research Institute for Applied Mechanics (RIAM) of Kyushu University (1997), the National University of Singapore (2009-2015); and Adjunct Professor at the University of Western Australia from 2015.

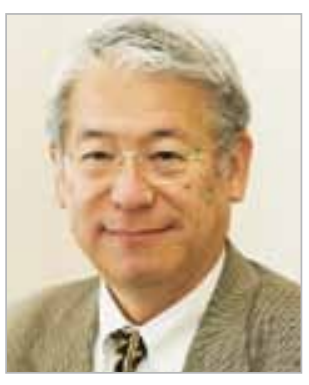

Professor Takeshi Kinoshita

\section{Professor Takeshi Kinoshita Honoring Symposium on Offshore Technology}

$\mathbf{T}$ he OMAE 2019 Conference is pleased to dedicate a Special Symposium in honor of Professor Takeshi Kinoshita. Professor Kinoshita has made a significant contribution to the field of Offshore Technology.

Professor Kinoshita graduated from the department of Naval Architecture of the University of Tokyo with BSc, MSc and PhD.

After his appointments as a lecturer and associate professor in the Department of Naval Architecture at the National Yokohama University Professor Kinoshita was appointed as an associate professor, the Institute of Industrial Science, the University of Tokyo and promoted to a full professor at the same institute.
Professor Kinoshita was seconded to the Japan Society of Promotion of Science as its London Office Director.

After his retirement from the University of Tokyo Professor Kinoshita was appointed as a professor in the Department of Oceanic Architecture, Nihon University.

Professor Kinoshita was the President Nagasaki Institute of Applied Science and is now a visiting Professor at Nagasaki Institute of Applied Science.

Professor Kinoshita received the Appreciation Award, ASME OOAE Division in 2007, and ASME Best Paper Award, ASME OOAE Division in 2010, ASME. 


\section{Afternoon Lecture Series}

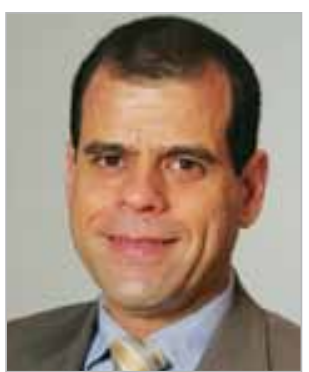

Dr.-Ing. Luiz Alves dos Santos
Monday, June 10

17:40 - 18:10

Location: Lomond Auditorium

European Research

Council - Funding

Opportunities for Creative Minds from all over the World

Dr.-Ing. Luiz Alves dos Santos, Scientific Officer, European Research Council

A presentation about the ERC's mission - to encourage and support the highest quality frontier research in Europe through competitive funding, its funding schemes and opportunities, the evaluation process, and sources of relevant information. Particular remarks would be on the investigator-driven approach of the Work Programme (no thematic specific calls) and on the openness of the programme to non-EU nationals.

L. Santos is a scientific officer at the European Research Council assigned to the management of programmes and projects in the areas of research addressed by the Product and Processes Engineering panel. His technical background is in mechanical engineering and computer sciences, with work experience in the private sector, academia, and public organizations.

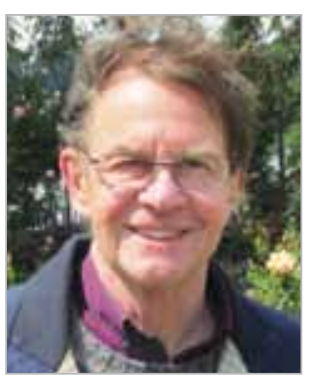

Tuesday, June 11

17:40 - 18:30

Location: Lomond Auditorium

\section{Inspired by Myriad} Laughing Waves: Euler, Navier, Stokes and others

Professor Rodney Eatock Taylor, Emeritus Professor, University of

Professor Rodney Oxford

Who are these people whose names grace the equations many of us use so regularly: Euler, Laplace, Navier, Cauchy, Stokes, Kelvin etc? The lecture will discuss brief aspects of the lives of some of nineteenth century European scientists who were pioneers in the field of hydrodynamics, and some of their interactions.

See page 21 for Professor Rodney Eatock Taylor's bio.

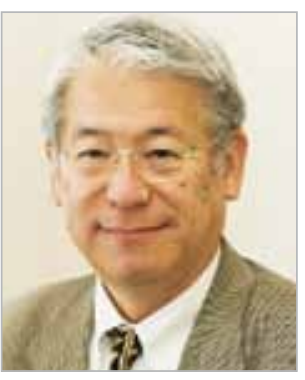

Professor Takeshi Kinoshita
Wednesday, June 12

17:40 - 18:30

Location: Lomond Auditorium

Enjoyable Marine

Engineering Researches

on Sports, Environment,

not only Water Wave

Engineering, Nonlinear Hydrodynamic Forces and Statistics
Professor Takeshi Kinoshita, Visiting Professor, Nagasaki Institute of Applied Science

These several decades we have seen big changes in the field of the ocean technology. In 1983 I firstly studied abroad, in Scotland. At that time the tension leg platform was quite a new concept for deep water structures. Research on wave energy utilization was a kind of fashion for marine hydro dynamists. Offshore oil \& gas industry is now one of the most important worldwide businesses. Marine renewable energies are now recognized as really the most promising energy resource. Ocean technology have to contribute the improvement of their safety, reliability and cost reduction. On the other hand the marine sports engineering is also valuable for life and "enjoyable", and recovering from the environmental damage on ocean and coastal zone is inevitably important and in fact "enjoyable" because of truly interdisciplinary collaboration work with many fields of sciences.

See page 21 for Professor Takeshi Kinoshita’s bio. 


\section{Saturday, June 8}

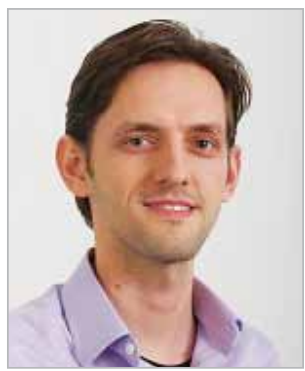

Dr. Tahsin Tezdogan

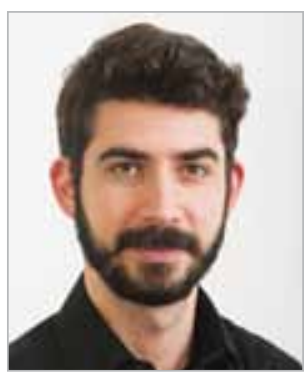

Dr. Yigit Kemal Demirel

\section{Short Course}

\section{Corrosion and Fouling in} Marine Environment

09:00 - 17:00

Location: Jura (Crowne Plaza)

Instructors:

Dr. Tahsin Tezdogan, Senior Lecturer, University of Strathclyde

Dr. Yigit Kemal Demirel, Lecturer, University of Strathclyde

This course is split into two parts, i.e. corrosion and fouling. The first part will cover the corrosion concept in marine environment and the prevention methods. It will focus on the combined use of both cathodic protection (CP) and coatings for ships and offshore structures. A hands-on tutorial will be performed to show the $\mathrm{CP}$ calculation procedure. The second part of the course aims to provide the fundamental concepts of marine biofouling, stateof-the-art fouling control coatings, and the roughness effects of biofouling and coatings on the boundary layer. This module also aims to describe how to estimate the effect of biofouling on the performance of marine vehicles in terms of resistance/power increase through state-of-the-art numerical and experimental approaches.

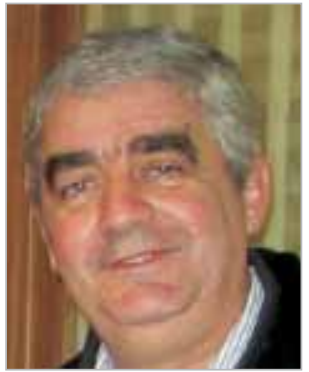

Luís Eça

\section{Short Course}

\section{Verification \& Validation of} Industrial CFD

09:00 - 17:00

Location: Staffa/Shuna

(Crowne Plaza)

Instructor:

Luís Eça, Assistant Professor, IST

CFD simulations have become an engineering tool that complements model testing. As for physical models, such capability requires the assessment of the quality of the results, which depends on the mathematical model (basin for physical models) and its numerical solution (instrumentation for experiments).

This course teaches CFD practitioners to distinguish numerical and modelling errors. It presents the definitions of the different contributions to the numerical error of steady and unsteady flow simulations. Techniques to quantify numerical (Verification) and modelling errors (Validation) in industrial

\begin{tabular}{l:l:l} 
Time & Title & Location \\
\hdashline $09: 00-17: 00$ & $\begin{array}{l}\text { Short Course } \\
\text { Corrosion and Fouling } \\
\text { in Marine Environment }\end{array}$ & $\begin{array}{l}\text { Jura } \\
\text { (Crowne Plaza) }\end{array}$ \\
\cline { 1 - 2 } $09: 00-17: 00$ & $\begin{array}{l}\text { Short Course } \\
\text { Verification \& } \\
\text { Validation of Industrial } \\
\text { CFD }\end{array}$ & $\begin{array}{l}\text { Staffa/Shuna } \\
\text { (Crowne Plaza) }\end{array}$ \\
\hdashline $17: 00-19: 00$ & $\begin{array}{l}\text { Outreach Team } \\
\text { Building Exercise }\end{array}$ & $\begin{array}{l}\text { Staffa/Shuna } \\
\text { (Crowne Plaza) }\end{array}$ \\
\hdashline $19: 00$ onwards & $\begin{array}{l}\text { Outreach Welcome } \\
\text { Dinner }\end{array}$ & Off-site \\
& &
\end{tabular}

CFD Simulations are presented including examples from practical simulations. The course provides a framework for the establishment of the credibility of simulations so that they can be safely used for engineering decisions.

You will learn how to demonstrate the quality of your CFD simulations and evaluate the accuracy of the mathematical models behind those simulations.

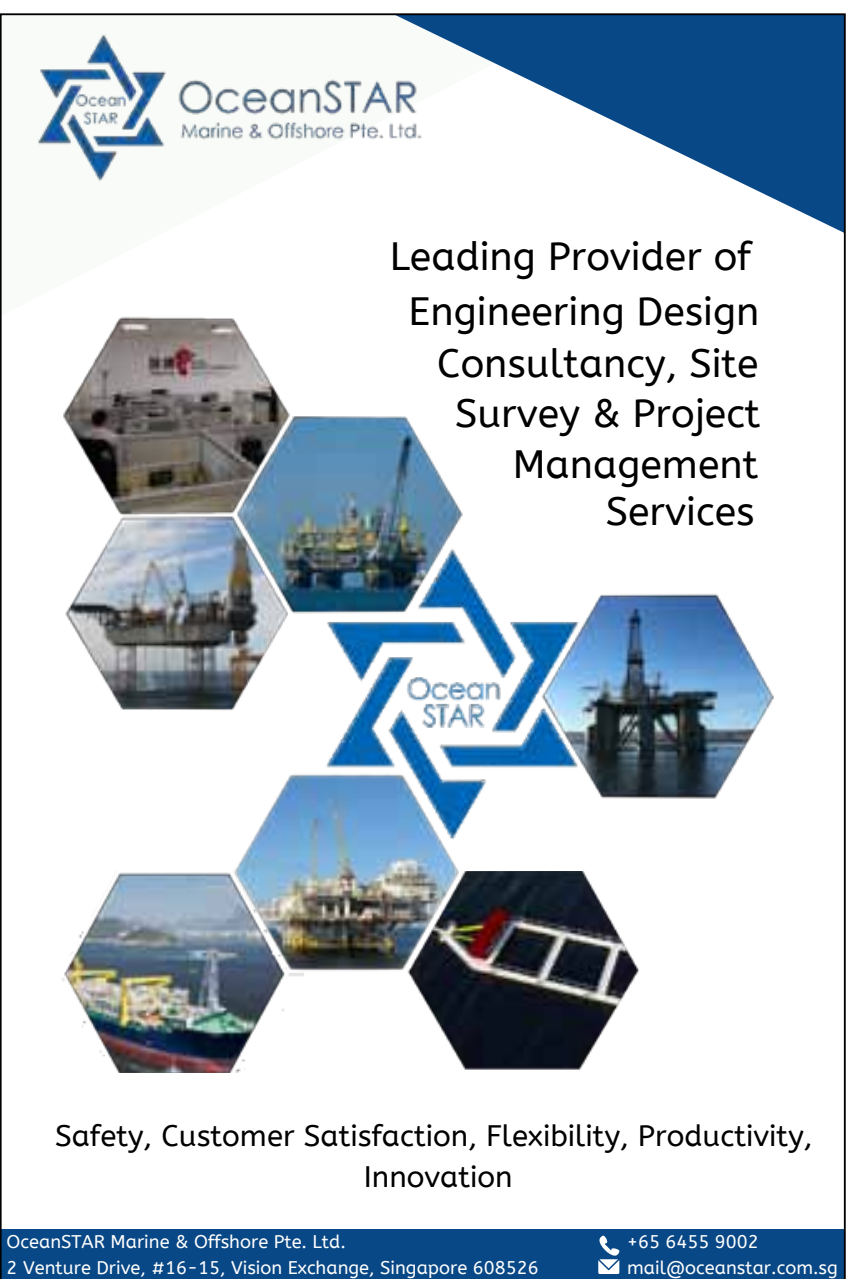




\section{Sunday, June 9}

\begin{tabular}{|c|c|c|}
\hline Time & Title & Location \\
\hline 08:00 - 17:00 & $\begin{array}{l}\text { Outreach Welcome \& } \\
\text { Introductions plus Industry } \\
\text { Presentations }\end{array}$ & Castle 1 \\
\hline 09:00 - 17:00 & $\begin{array}{l}\text { Short Course } \\
\text { Offshore Wind Turbines: } \\
\text { Dynamic Analysis and } \\
\text { Marine Operations }\end{array}$ & Jura \\
\hline 09:00 - 17:00 & $\begin{array}{l}\text { Short Course } \\
\text { Introduction to Machine } \\
\text { Learning and Data-driven } \\
\text { Modelling Methods for } \\
\text { Engineering Applications }\end{array}$ & Castle 2 \\
\hline $18: 30-20: 30$ & Welcome Reception & $\begin{array}{l}\text { Glasgow Science } \\
\text { Centre }\end{array}$ \\
\hline
\end{tabular}

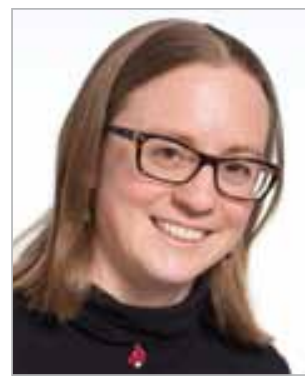

Erin Bachynski

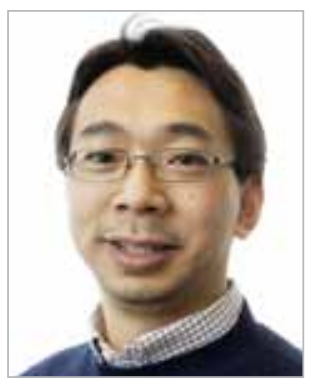

Zhen Gao

\section{Short Course}

\section{Offshore Wind Turbines: Dynamic Analysis and Marine Operations}

09:00 - 17:00

Location: Jura (Crowne Plaza)

Instructors:

Erin Bachynski, Norwegian University of Science and Technology

Zhen Gao, Norwegian University of Science and Technology

This course reviews several considerations related to design and operation of offshore wind turbines. Fundamental concepts in aerodynamic (with focus on blade element/momentum theory) and hydrodynamics (with focus on first and second order radiation-diffraction and Morison-type models) load calculation are presented. The course addresses theoretical background and important practical considerations for structural response analysis considering these load components simultaneously, including wind turbine control, for ULS and FLS design check. A brief review of the state-of-the-art in combined wind-wave testing and the status of validation of the integrated design tools will be provided. Finally, marine operational issues related to transport, installation and access to wind turbines for maintenance and repair, with focus on numerical simulation of onsite installation and weather window analysis, are discussed.

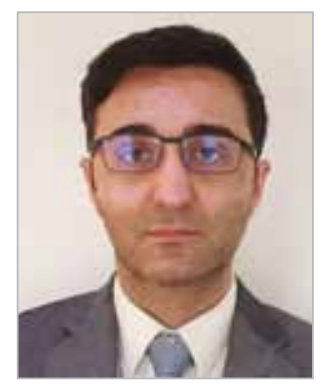

Andrea Coraddu

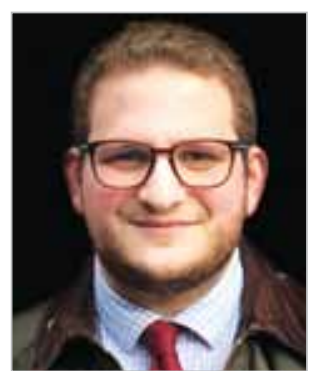

Christos Gkerekos

\section{Short Course}

Introduction to Machine

Learning and Data-driven

Modelling Methods for Engineering Applications

09:00 - 17:00

Location: Castle 2 (Crowne Plaza)

Instructors:

Andrea Coraddu, PhD, Lecturer in Marine Engineering, University of Strathclyde

Christos Gkerekos, MEng, PhD Researcher, University of Strathclyde

The course will focus on Data-driven models for engineering applications, including linear and nonlinear models, model selection and error estimation. Numerical examples and real-life problems will be proposed and analysed, from bearings fault prediction, to fuel consumption optimisation. All course material will be freely available in PDF format for a complete understanding of the related subjects as well as for future consultation. During the afternoon session, a hands-on workshop will be organised with numerical examples focused on various aspects of Data-driven models. The course is designed for professionals who are interested in data analysis and machine learning applications. An engineering background, statistical and numerical skills would be beneficial but not necessary.

\section{Welcome Reception 18:30 - 20:30 Location: Glasgow Science Centre}

Welcome / Civic Reception courtesy of The Rt Hon The Lord Provost Councillor Eva Bolander. See Social Events, page 14 for more details.

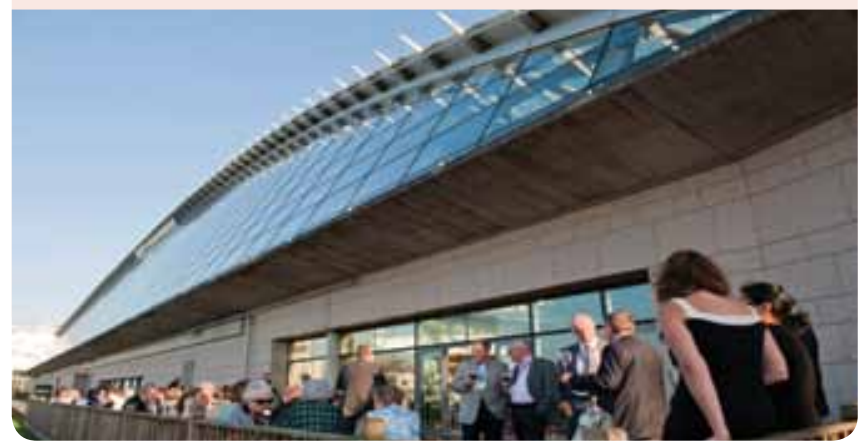




\section{Monday, June 10}

\begin{tabular}{|c|c|c|}
\hline Time & Title & Location \\
\hline $08: 30-19: 15$ & Exhibition open & Hall 5 (SEC) \\
\hline $08: 30-10: 00$ & $\begin{array}{l}\text { Opening Ceremony } \\
\text { and Keynote Plenary } \\
\text { One } \\
\text { Awards Presentations }\end{array}$ & SEC Armadillo \\
\hline $10: 00-10: 30$ & Refreshment Break & Hall 5 (SEC) \\
\hline $10: 30-12: 00$ & $\begin{array}{l}\text { Keynote Plenary Two } \\
\text { Keynote Panel }\end{array}$ & SEC Armadillo \\
\hline $12: 00-13: 30$ & Opening Lunch & Hall 5 (SEC) \\
\hline $13: 30-15: 00$ & Concurrent Sessions & $\begin{array}{l}\text { See pages } 27-31 \\
\text { for session titles, } \\
\text { authors and locations }\end{array}$ \\
\hline $15: 00-15: 30$ & Refreshment Break & Hall 5 (SEC) \\
\hline $15: 30-17: 30$ & Concurrent Sessions & $\begin{array}{l}\text { See pages } 31-35 \\
\text { for session titles, } \\
\text { authors and locations }\end{array}$ \\
\hline $17: 00-18: 15$ & $\begin{array}{l}\text { ASME \& IMechE } \\
\text { Connect Roundtable }\end{array}$ & Forth Room \\
\hline $17: 40-18: 10$ & $\begin{array}{l}\text { Afternoon Lecture } \\
\text { Series }\end{array}$ & $\begin{array}{l}\text { Lomond Auditorium } \\
\text { (SEC) }\end{array}$ \\
\hline $18: 15-19: 15$ & $\begin{array}{l}\text { Afternoon Drinks } \\
\text { Reception }\end{array}$ & Hall 5 (SEC) \\
\hline
\end{tabular}

\section{OPENING CEREMONY AND \\ KEYNOTE PLENARIES \\ 08:30 - 10:00 \\ Location: SEC Armadillo}

\section{Opening Ceremony}

Professor Atilla Incecik, Conference Chair, OMAE 2019

Professor Krish Thiagarajan Sharman, Technical Program

Chair, OMAE 2019

Professor Antonio C. Fernandes, OOAE Division Chair

Professor Sir Jim McDonald, Principal, University of Strathclyde

Bailie Jacqueline McLaren on behalf of the Lord Provost of

Glasgow, Eva Bolander

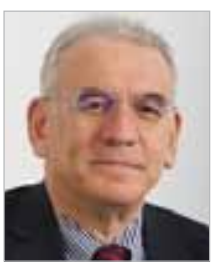

Professor Atilla Incecik

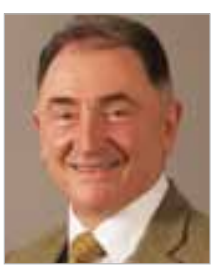

Professor Sir Jim McDonald

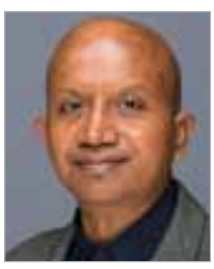

Professor Krish Thiagarajan Sharman

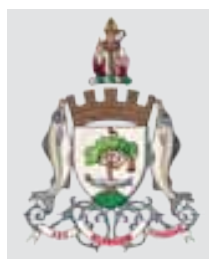

Glasgow City Council

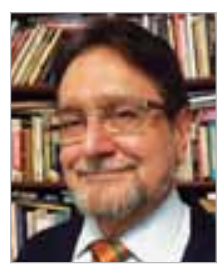

Professor Antonio C Fernandes

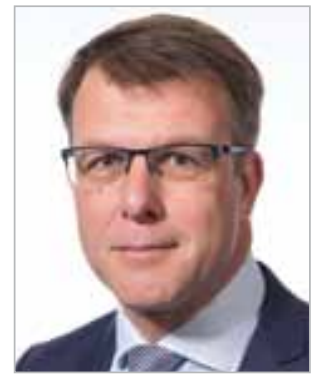

Dr. Bas Buchner

\section{Keynote Plenary One}

Blue Oceans: Offshore Research for Future Maritime Challenges

Dr. Bas Buchner, President, MARIN

What trends do we foresee in offshore energy and transport over sea? What are the related challenges for offshore engineering? Do renewable energy, food from the seas and floating infrastructure provide new opportunities for the offshore industry and its offshore engineers?

Based on the project 'Blueprint 2050: The Maritime World Beyond the Horizon' in The Netherlands, Dr. Bas Buchner (MARIN) will discuss questions such as: Can we use our FPSO knowledge to develop floating energy hubs for zero emission shipping with Hydrogen, liquid Methane or Ammonia? How do we use our offshore platform knowledge to support fixed and floating mega wind turbines? Can we develop floating ports and cities to cope with sea level rise and overpopulation? What is the role of autonomy and digitalisation at sea? What is the role of future Offshore Engineers and what are their tools?

Dr. Bas Buchner studied at Delft University of Technology and graduated in 1991. He joined MARIN and was responsible for many offshore model test and simulation projects related to mooring, platform response, offloading analysis and wave impact loading. He specialised in the topics of extreme waves, green water loading and wave impacts. He completed his PhD on the subject of 'Green Water Loading on Ship Type Offshore Structures' (2002). He was Manager of the MARIN Offshore Department from 2000 to 2010 and was the leader of many Joint Industry Projects (JIP's) in the Offshore sector. He was the Chairman of OMAE 2011 in Rotterdam and has authored more than 50 papers in the field of Offshore Hydrodynamics. Since 2011, he is President of MARIN.

\section{Awards}

The Subrata Chakrabarti Young Professional Award recipient and the OMAE 2018 Best Paper Awards recipients will be recognized. See page 12 for more details.

\section{Refreshment Break 10:00 - 10:30}

Location: Hall 5 (SEC)

Refreshment break sponsored by TechnipFMC

\section{TechnipFMC}




\section{OPENING CEREMONIES AND KEYNOTE \\ PLENARIES (Continued) \\ 10:30 - 12:00}

Location: SEC Armadillo

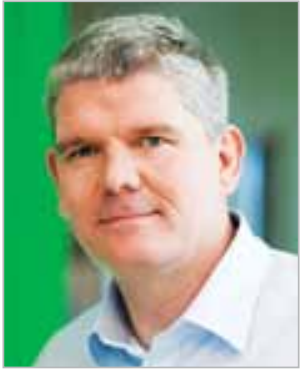

David Dickson

Keynote Plenary Two

\section{Advancing a Lower Carbon Future}

David Dickson, Vice President, Global

Operations, Regions, BP

David Dickson's presentation focuses on: - More energy, fewer emissions; and - Engineering being part of the solution

Dave Dickson joined BP as a Graduate Engineer in 1989.

He is currently the VP Global Operations (Regions), within the upstream segment for BP, with a strong and diverse background in Engineering and Operations, Dave covers upstream production facilities offshore and onshore across the BP portfolio in the Eastern Hemisphere.

He is responsible for the Safe, Reliable operations across a broad range of operating facilities, ensuring conformance to $B P$ 's Operations Management System, it's HSSE standards and expectations, its operating standards and processes.

Dave began his BP career as a Mechanical Engineer in the North Sea and has acquired deep, engineering, operating and HSE experience in Upstream and Downstream assets including assignments in Engineering and Operations Management in both Upstream Oil and Gas Production facilities and downstream Chemical and Refining facilities.

Dave graduated from the University of Strathclyde, Glasgow with a Bachelor of Engineering - Mechanical Engineering. He is a Chartered Engineer with the Institute of Mechanical Engineers and a Fellow of the Institute of Mechanical Engineers.

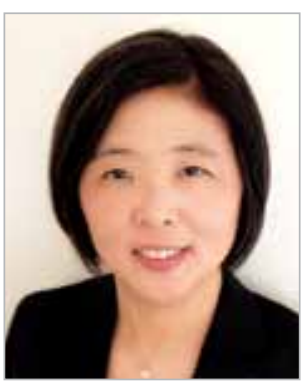

Xiaozhi (Christina) Wang

\section{Keynote Panel}

\section{Offshore Digital Panel}

Moderator: Xiaozhi (Christina) Wang, $\mathrm{PhD}$, Vice President, Global Marine, American Bureau of Shipping (ABS)

Dr. Xiaozhi (Christina) Wang, is currently Vice President of Global Marine American Bureau of Shipping (ABS), focused on strategy and business development. Prior, Dr. Wang held positions as ABS Vice Presidents of Global Engineering and Technology and Advanced Technology and Research, implementing research and development efforts for developing new and innovative technologies.

Dr. Wang received her B.S. in Naval Architecture and Ocean Engineering from Shanghai Jiao Tong University, her MSc and
PhD degrees in Marine Structures from the Norwegian University of Science and Technology. She is a fellow in SNAME and ASME. She also completed the Stanford University Executive Program.

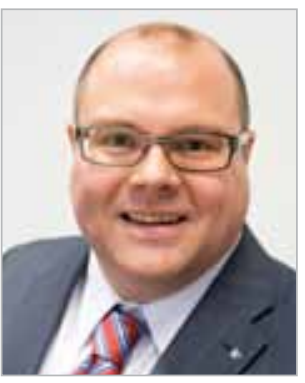

Professor Kjetil Skaugset

\section{Digitalization - Changing the} way to provide energy

Professor Kjetil Skaugset, PhD, Chief Researcher Upstream and Expert Centre, Equinor ASA

Kjetil Skaugset will take the audience through a digital journey seen from an energy company point of view. Downstream, Technology, Equinor

Importance of digital technologies to improve safety, value creation as well as reduction in environmental footprint will be highlighted. Examples will be given across the value chain on present digital initiatives as well as future opportunities offered by a digital transformation.

Kjetil Skaugset studied at Norwegian University of Science and Technology (NTNU) and graduated with a PhD in 2003. He subsequently held post doc positions at Massachusetts Institute of Technology (MIT), and Centre for Ships and Offshore Structures (CeSOS) at NTNU. Kjetil has also worked at the Norwegian Marine Technology Research Institute (MARINTEK) in Trondheim.

Joining Statoil in 2005, he assumed responsibilities for research and development within the area of platform technology. He has since been central in several major field development projects in Statoil. Kjetil has been managing researchers within arctic, pipeline and deep-water technology in Statoil.

The last 7 years (2012-2019) Kjetil has been the Chief Researcher Upstream and Downstream Technology in Equinor. That responsibility entails corporate technical responsibility for all new technologies between wellhead and market in the oil and gas value chain in addition to renewables, new value chains and HSE technologies.

He is currently Board member Centre of Excellence Autonomous Marine Operations and Systems (AMOS) at NTNU, Chairman of the Board of Norwegian Forum for Marine Minerals and heads up the national research strategy OG21 in Norway, technology target area "Future technologies for production, processing and transportation".

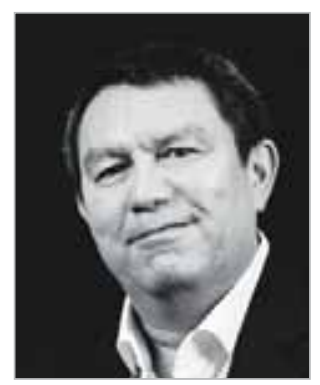

Frederic Dabe
The exciting journey to deliver an As-Built Digital Twin

Frederic Dabe, Digital Transformation Director, SBM Offshore

SBM Offshore believes the oceans will provide the world with safe, sustainable and affordable energy for generations to come. Since many years, the 
company is delivering large floating energy production systems, involving thousands of tons of steels and millions of man-hours.

Digitalization is a key element in SBM Offshore's strategy in order to improve safety, sustainability \& affordability of its solutions. In this era of digital transformation, we now have the opportunity to deliver a digital twin at the same time we are delivering the physical asset.

The potential of a digital twin is impressive and will bring value across the entire life cycle of the asset. It also represents an important paradigm shift for the Engineering, Procurement and Construction value chain both at company level and at the scale of its ecosystem.

Frederic started his career in the naval and nuclear sector as piping production manager and later construction manager. He then spent 6 years developing naval robotics solutions before joining the oil \& gas industry.

Covering the full EPC scope, Frederic has delivered many projects (FPSO, FSRU, TLP, CPP, ...), conducted successful transformations and led business units in Europe, Middle East and Asia.

More recently, Frederic was deeply involved with Industry 4.0 projects, working on transformation roadmap and deployment of digital solutions.

Driven by operational excellence and innovation, Frédéric has joined SBM Offshore to lead the overall digital transformation of the group.

\section{OMAE 2020 Presentation}

Professor Manhar Dhanak, Conference Chair, OMAE 2020

Professor Ron W. Yeung, Conference Co-Chair, OMAE 2020

\section{Opening Lunch \\ 12:00 - 13:30 \\ Location: Hall 5 (SEC) \\ Monday lunch sponsored by Elsevier}

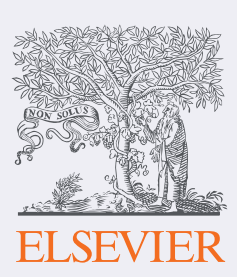

\section{CONCURRENT SESSIONS \\ $13: 30-15: 00$}

\section{Offshore Technology}

\section{1-1-2 FPSO and Arctic Structures}

Monday June 10

Session Chair: Ewoud van Haaften, Shell Global

Solutions International B.V., Netherlands

Session Co-Chair: Anil Sablok, TechnipFMC, USA

Improved Design of Next Generation Hull-platform

"Noah-FPSO Hull" OMAE2019-95269

Shigeru Tanaka, Yasuhiro Sogawa

Mitsui E\&S Shipbuilding (o., Ltd., Tokyo, Japan
Development of Harsh Environment Field with Ice Loadings using Concrete Spar - Variability of Options OMAE2019-96322 Anil Sablok' Erlend Hovland ${ }^{2}$ Svein Ole Strømme ${ }^{3}$ Andrew Blundon ${ }^{4}$ 1. TechnipFMC, Houston, TX, USA; 2. Equinor ASA, Stavanger, Norway; 3. Kvaerner, Lysaker, Norway; 4. TechnipFMC, St. John's, NL, Canada

Conceptual Design of Single Column Drilling Unit for Arctic Climate and Harsh Environment OMAE2019-96474 Mingyuan Sun, Fan Zhang, Lixin Xu China Merchants Offshore Technology Research Center, Haimen, China

Direct Time Domain Simulations for a FPSO Tandem Offloading Operation OMAE2019-96638

Bonjun Koo, Manoj Jegannathan, Johyun Kyoung, Ho-Joon Lim TechnipFMC, Houston, TX, USA

\section{Offshore Technology}

\section{1-2-1 Dynamic Positioning I}

Monday June 10

Room SEC, Dochart 2 | 13:30 - 15:00

Session Chair: Allan Magee, National University of Singapore, Singapore

Session Co-Chair: Anil Sablok, TechnipFMC, USA

Optimal Setpoint Learning of a Thruster-assisted Position

Mooring System with Model-based Acceleration OMAE2019-95215

Bo Li, Lei Wang, Xuefeng Wang

Shanghai Jiao Tong University, Shanghai, China

Dynamic Positioning System: Systematic Weight Assignment for DP Sub-systems using Multi-criteria Evaluation Technique Analytic Hierarchy Process and Validation using DP-RI Tool with Deep Learning OMAE2019-95485 Charles Fernandez ${ }^{1}$ Arun Dev² Rose Norman ${ }^{3}$ Wai Lok Woo ${ }^{3}$ Shashi Kumar ${ }^{1}$ 1. DNV GL Singapore Pte Ltd, Singapore, Singapore; 2. Newcastle University in Singapore, Singapore, Singapore; 3. Newcastle University, Newcastle, United Kingdom

Surge Response Control of FPSO using Multiple Tuned Liquid Dampers - A Study on Effect of Multiple Frequencies in TLD OMAE2019-96062

Saravanan Gurusamy, Deepak Kumar

Indian Institute of Technology Madras, Chennai, India

Estimating Second Order Wave Drift Forces and Moments for Calculating DP Capability Plots OMAE2019-96307 Saeed Barzegar Valikchali' ${ }^{1}$ Mitchell Anderson ${ }^{1}$ David Molyneux ${ }^{1}$ Dean Steinke ${ }^{2}$ 1. Memorial University of Newfoundland, St. John's, NL, Canada; 2. Dynamic Systems Analysis, Victoria, BC, Canada

\section{Structures, Safety and Reliability}

\section{2-10-1 Collision and Crashworthiness I}

Monday June 10

Room Crowne Plaza, Castle 1 | 13:30 - 15:00

Session Chair: Sören Ehlers, Hamburg University of Technology, Germany

Session Co-Chair: Zhiqiang Hu, Newcastle University, United Kingdom

Dynamic Responses Prediction for a Spar-type Offshore Floating

Wind Turbine under Ship Collision Scenarios OMAE2019-95094

Yichi Zhang, Zhiqiang Hu

Newcastle University, Newcastle upon Tyne, United Kingdom 
Numerical Study on the Dynamical Characteristic and Impact Force between Vessel with Rake Bow and Bridge Pier OMAE2019-95602 Ming Cai Xu' Zi Xuan Zhang Xiao Qiang Zhang ${ }^{2}$ Jin Pan ${ }^{3}$ Yi Fei Huang ${ }^{3}$ 1. Huazhong University of Science and Technology, Wuhan, China; 2. School of Naval Architecture and Ocean Engineering, Huazhong University of Science \& Technology, Wuhan, China; 3. Wuhan University of Technology, Wuhan, China

Mechanical Modeling of the Polymeric Coating

on a Subsea Pipeline OMAE2019-95920

Ole Vestrum, Magnus Langseth, Tore Børvik

Norwegian University of Science and Technology, Trondheim, Norway

\section{Structures, Safety and Reliability}

\section{2-15-1 Data Driven Models}

Monday June $10 \quad$ Room Crowne Plaza, Castle 2 | 13:30-15:00 Session Chair: YeongAe Heo, Case Western Reserve University, USA

Session Co-Chair: Bernt Leira, Norwegian University

of Science and Technology, Norway

Motion and Load Prediction of Floating Platform in South China Sea using Deep Learning and Prototype Monitoring Information OMAE2019-95412

Ji Yao, Wenhua Wu, Zishu Zhao

Dalian University of Technology, Dalian, China

Power Spectrum for Surface Description of Corroded

Ship Structure from Laser Scan 0MAE2019-95907

Karoline Mali Neumann' Sören Ehlers ${ }^{2}$

1. Wood, Sandefford, Norway; 2. Hamburg University of Technology, Hamburg, Germany

The State-of-the-Art in Structural Integrity Management:

A Review and Proposed Data-driven Approach OMAE2019-96396

YeongAe Heo

Case Western Reserve University, Cleveland, OH, USA

Squall Detection and Analysis from Historical

Satellite Data 0MAE2019-96549

Laury Renac' David Hurdle' Francois Enet ${ }^{1}$ Joris de Vroom ${ }^{2}$

1. Aktis Hydraulics, Zwolle, Netherlands; 2. Argoss, Houten, Netherlands

\section{Materials Technology}

\section{3-1-2 Formulation of the Fracture Parameter}

Monday June 10

Room SEC, Boisdale 1 | 13:30 - 15:00

Session Chair: Carey L. Walters, Delft University of Technology, Netherlands

Session Co-Chair: Koji Gotoh, Kyushu University, Japan

Evaluations of Ductile and Cleavage Fracture using Coupled GTN and Beremin Model in API X70 Pipelines Steel 0MAE2019-96483

Youn-Young Jang Ji-Hee Moon ${ }^{1}$ Nam-Su Huh' Ki-Seok Kim²

Woo-Yeon Cho ${ }^{2}$ Myeong-Woo Lee ${ }^{3}$ Yun-Jae Kim ${ }^{3}$

1. Seoul National University of Science and Technology, Seoul, Korea;

2. POSCO, Incheon, Korea; 3. Korea University, Seoul, Korea
Parameter Calibration for Continuum Damage Mechanics Models to Simulate Ductile Fracture of High Strength Pipeline Steels OMAE2019-96316 Filip Van den Abeele, ArcelorMittal Global R\&D, Zwijnaarde, Belgium

Numerical Investigation of Ductile Crack Growth Behavior at Different Locations of Weld Joint for X80 Pipeline Steel OMAE2019-95517

Bin Qiang, Xin Wang

Carleton University, Ottawa, ON, Canada

Recent Developments and Challenges of Cleavage Fracture Modelling in Steels: Aspects on Microstructural Mechanics and Local Approach Methods OMAE2019-95464 Quanxin Jiang' ${ }^{1}$.M. Bertolo ${ }^{1}$ V.A. Popovich ${ }^{1}$ Carey L. Walters ${ }^{2}$ 1. Delft University of Technology, Delft, Netherlands; 2. TNO, Delft, Netherlands

\section{Pipelines, Risers, and Subsea Systems}

\section{4-1-1 Flexible Pipes I}

Monday June $10 \quad$ Room Crowne Plaza, Staffa / Shuna | 13:30 - 15:00 Session Chair: Svein Saevik, Norwegian University of Science and Technology, Norway Session Co-Chair: Zhimin Tan, Baker Bughes, a GE company, USA

Analytical Methodology to Evaluate Flexible Risers

Fatigue Lives at the Top Region OMAE2019-96372

Fernando Sousa ${ }^{1}$ Marcos Queija de Siqueira ${ }^{2}$ José Renato M. de Sousa ${ }^{1}$ George Campello ${ }^{3}$

1. Federal University of Rio de Janeiro, Rio de Janeiro, RJ, Brazil; 2. UFRJ/COPPE/

LACEO, Rio de Janeiro, RJ, Brazil; 3. Petrobras, Rio de Janeiro, RJ, Brazil

Study of the Vortex-induced Vibration of the Marine

Risers with the Buoyancy 0MAE2019-96824

Lin Zhao, Hang Su, Yanju Yin

Ocean University of China, Qingdao, China

Methodology Proposal for Corrosion Fatigue Assessment for Flexible Pipes Tensile Armour in Aggressive Environments OMAE2019-96423 Marcelo Favaro Borges, Mariana dos Reis Tagliari,

Rafaela Gonçalves, Carlos Eduardo Fortis Kwietniewski UFRGS, Porto Alegre, RS, Brazil

Frequency Domain Fatigue Analysis for a Unbonded Flexible Riser Damage Induced by Dynamic Tension OMAE2019-95118

Jiabei Yuan, Yucheng Hou, Zhimin Tan

Baker Hughes, a GE Company, Houston, TX, USA

\section{Pipelines, Risers, and Subsea Systems}

\section{4-3-1 Collapse}

Monday June 10 Room Crowne Plaza, Castle 3 | 13:30 - 15:00 Session Chair: Ilson Pasqualino, COPPE/UFRJ, Brazil

Session Co-Chair: Spyros A. Karamanos, University of Thessaly, Greece

Pure Collapse Behavior of Pipelines With D/t Ratio Below 10:

Real Scale Experimental Tests and Numerical Studies OMAE2019-95145 Ana Paula França de Souza ${ }^{1}$ Ana Carolina Vilas Boas ${ }^{2}$

Rafael F. Solano ${ }^{3}$ Gabriel Jorge ${ }^{4}$ Júlio Márcio Silveira e Silva ${ }^{2}$

1. LTS/COPPE, Niterói, RJ, Brazil;2. Vallourec, Belo Horizonte, MG, Brazil; 3. Petrobras,

Rio de Janeiro, RJ, Brazil; 4. Vallourec Competence Center Rio, Rio de Janeiro, RJ, Brazil

Pipe Development ERW/HFIW Casing API5CT

Grade P110 High Collapse 0MAE2019-95363

Luis Melo, Wilson Cordeiro, Marcus Ferreira

Apolo Tubulars S/A, Lorena, SP, Brazil 
Influence of Residual Curvature in the Pipeline

Pure Collapse OMAE2019-95768

Ana Paula França de Souza' Theodoro Netto ${ }^{2}$ Carlos Abad Estrada Quispe ${ }^{3}$

1. LTS/COPPE, Niterói, RJ, Brazil; 2. COPPE/Universidade Federal do Rio de Janeiro,

Rio de Janeiro, RJ, Brazil; 3. COPPE/LTS/UFRJ, Rio de Janeiro, RJ, Brazil

Efficiency of Carbon Fibre Buckle Arrestors

for Subsea Pipelines OMAE2019-95821

Hassan Karampour ${ }^{1}$ Mahmoud Alrsai ${ }^{2}$ Wayne Hall ${ }^{3}$

1. Griffith School of Engineering, Gold Coast, QLD, Australia;

2. Griffith University, Gold Coast, QLD, Australia; 3. Griffith School of

Engineering and Built Environment, Gold Coast, QLD, Australia

\section{Ocean Space Utilization}

\section{5-1-1 Marine Utilization and Marine Spatial Planning}

Monday June 10

Room SEC, Dochart 1 | 13:30 - 15:00

Session Chair: Kazuhiro lijima, Dept of NAOE, Osaka University, Japan

Impacts of Educational Outreach on Envisioning Marine

Cities of the Future Development Project OMAE2019-95431

Ikuo Yoshida

Shimizu Corporation, Tokyo, Japan

Trend of Utilization of Ocean Space according to Structural

Form of Oceanic Architectures OMAE2019-96453

Ryo Sugahara' ${ }^{1}$ Akio Kuroyanagi ${ }^{2}$

1. Nihon University, Chiba, Japan; 2. Nihon University, Funabashi-shi, Japan

The Application of Nearshore Risk Assessment of

Hazard and Vulnerability in Marine Resources Area

for National Spatial Planning OMAE2019-96706

Lien-Kwei Chien ${ }^{1}$ Chi-Wen Huang ${ }^{1}$ Wei-Po Huang' Cheng-Yu Ku' Chih Hsin Chang ${ }^{2}$

1. National Taiwan Ocean University, Keelung, Taiwan; 2. National Science

and Technology Center for Disaster Reduction, New Taipei City, Taiwan

\section{Ocean Engineering}

\section{6-1-2 Floating Body Technology}

Monday June 10

Room SEC, M4 | 13:30 - 15:00

Session Chair: Wei Qiu, Memorial University of Newfoundland, Canada

Virtual Prototyping and Simulation of Multibody Marine

Operations using Web-based Technologies OMAE2019-96051

Ícaro A. Fonseca, Felipe F. de Oliveira, Henrique M. Gaspar

Norwegian University of Science and Technology, Ålesund, Norway

A Study on Motions and Connector Loads for a New Type

of Two-module Semi-submersible OMAE2019-96771

Jun Ding, Chao Tian, Yuji Miao, Zhengwei Zhang, Zhanhua Zhao, Xinyun Ni

China Ship Scientific Research Center, Wuxi, China

Evaluation and Optimization of Trimaran Configurations

using Deep Neural Network OMAE2019-96832

Dongchi Yu, Lu Wang, Qian Zhong, Ronald W. Yeung

University of California, Berkeley, Berkeley, CA, USA

Penetration of Annular and General Jets into

Underwater Plates OMAE2019-96805

Zhifan Zhang, Haoliang Hu, Cheng Wang

Beijing Institute of Technology, Beijing, China

\section{Ocean Engineering}

\section{6-4-1 Marine Control and Automation}

Monday June 10

Room SEC, M2 \& M3 | 13:30 - 15:00

Session Chair: Lokukaluge Prasad Perera, UiT The Arctic University of Norway, Norway Session Co-Chair: Yihan Xing, University of Stavanger, Norway

Development of a Control Strategy for Underway

Tandem-like 0il Transfer Operation between a

Conventional and a DP Tanker OMAE2019-96335

Felipe Moreno ${ }^{1}$ José Amendola ${ }^{1}$ Eduardo Tannuri ${ }^{2}$ Marcos Ferreira ${ }^{3}$

1. Universidade de São Paulo, São Paulo, SP, Brazil; 2. Numerical Offshore Tank-

Universidade de São Paulo, São Paulo, SP, Brazil; 3. Petrobras, Rio de Janeiro, RJ, Brazil

Dynamic Positioning Observer Design using

Exogenous Kalman Filter OMAE2019-96490

Song An, Dengshuo Chen, Yong Bai

Southern University of Science and Technology, ShenZhen, China

Port Channel Navigation Subjected to Environmental

Conditions using Reinforcement Learning OMAE2019-96120

José Amendola ${ }^{1}$ Eduardo Tannuri2 Fábio Cozmann Anna Reali Costa ${ }^{1}$

1. University of São Paulo, São Paulo, SP, Brazil; 2. Numerical Offshore

Tank - University of São Paulo, São Paulo, SP, Brazil

The Modelling and Optimal Control of a Hybrid Propulsion

System for an Ice-capable Ship OMAE2019-95142

Yi Zhou' Kayvan Pazouki' Rose Norman'

1. Newcastle University, Newcastle, United Kingdom; 2. Marine, Offshore and Subsea

Technology Group, School of Engineering, Newcastle upon Tyne, United Kingdom

\section{CFD \& FSI}

\section{8-1-1 FS}

Monday June 10

Room SEC, Lomond Auditorium | 13:30 - 15:00

Session Chair: Allan Magee, National University of Singapore, Singapore

Session Co-Chair: Hyunchul Jang, TechnipFMC, USA

CFD for VIM and Line Forces of a Floating Caisson

with Complex Geometry OMAE2019-95789

Boudewijn Decrop, Rohit Kulkarni, Alexander Breugem, Damian Villaverde Vega

IMDC, Antwerp, Belgium

Prediction of Hydrodynamic Damping of Moored

Offshore Structures using CFD OMAE2019-95935

Changqing Jiang, Ould el Moctar, Thomas Schellin

University of Duisburg-Essen, Duisburg, Germany

The Vortex and Wall Fluctuating Pressure around

Submarine Sail based on DDES Method OMAE2019-96018

Rui Luo' Yue Sun ${ }^{2}$ Hang Zhang ${ }^{2}$ Jin Zhan ${ }^{3} \mathrm{Xiao}_{\mathrm{Cai}}{ }^{2}$

1. China Ship Design and Development Center, Wuhan, China; 2. Huazhong University

of Science and Technology, Wuhan, China; 3. School of Naval Architecture \& Ocean

Engineering Huazhong University of Science and Technology, Wuhan, China

Boundary Layer Effects in the Modeling of

Semi-submersible Columns OMAE2019-96684

Samuel Holmes

Red Wing Engineering, Inc, Palo Alto, CA, USA 


\section{Ocean Renewable Energy}

\section{9-1-1 Bottom-fixed Wind Turbines}

Monday June 10

Room SEC, Carron 1 | 13:30 - 15:00

Session Chair: Wojciech Popko, Fraunhofer Institute

for Wind Energy Systems IWES, Germany

Session Co-Chair: Tonio Sant, Dept of Mechanical

Engineering,University of Malta, Malta

Dynamic Response of a Large-diameter Monopile considering 35-hour Storm Conditions OMAE2019-95170

Erin E. Bachynski' Ana Page ${ }^{2}$ George Katsikogiannis'

1. Norwegian University of Science and Technology, Trondheim,

Norway; 2. Norwegian Geotechnical Institute, Oslo, Norway

Validation of Numerical Models of the Offshore Wind

Turbine from the Alpha Ventus Wind Farm against Full-scale

Measurements within OC5 Phase III OMAE2019-95429

Wojciech Popko ${ }^{1}$ Amy Robertson ${ }^{2}$ Jason Jonkman ${ }^{2}$ Fabian Wendt ${ }^{2}$ Philipp Thomas ${ }^{1}$

Kolja Müller ${ }^{3}$ Matthias Kretschmer ${ }^{4}$ Torbjørn Ruud Hagen ${ }^{5}$ Christos Galinos ${ }^{6}$

Jean-Baptiste Le Dreff' Philippe Gilbert ${ }^{8}$ Bertrand Auriac ${ }^{9}$ Sho $0 \mathrm{~h}^{10} \mathrm{Jacob}$ Qvist ${ }^{11}$

Stian Høegh Sørum ${ }^{12}$ Loup Suja-Thauvin ${ }^{13}$ Hyunkyoung Shin ${ }^{14}$ Climent Molins $^{15}$

Pau Trubat ${ }^{15}$ Paul Bonnet ${ }^{16}$ Roger Bergua ${ }^{17}$ Kai Wang ${ }^{17}$ Pengcheng Fu ${ }^{18}$

Jifeng Cai ${ }^{18}$ Zhisong Cai ${ }^{18}$ Armando Alexandre ${ }^{19}$ Robert Harries ${ }^{20}$

1. Fraunhofer IWES (Fraunhofer Institute for Wind Energy Systems), Bremerhaven, Germany;

2. National Renewable Energy Laboratory, Golden, CO, USA; 3. University of Stuttgart,

Stuttgart, Germany; 4. University of Stuttgart, Stuttgart Wind Energy, Stuttgart, Germany;

5. OWEC Tower AS, Oslo, Norway; 6. Technical University of Denmark - Department of

Wind Energy, Roskilde, Denmark; 7. Electricité de France, Recherche et Développement,

Palaiseau, France; 8. IFP Energies Nouvelles, Solaize, France; 9. Principia, La Ciotat, France;

10. ClassNK, Chiyodaku, Japan; 11. Subsea, Nesbru, Norway; 12. Norwegian University of

Science and Technology, Department of Marine Technology, Trondheim, Norway; 13. Simis

AS, Trondheim, Norway; 14. University of Ulsan, Ulsan, Korea; 15. Universitat Politècnica

de Catalunya, Barcelona, Spain; 16. Siemens Industry Software, Cornellà de Llobregat,

Spain; 17. Envision Energy Limited, Shanghai, China; 18. China General Certification,

Beijing, China; 19. DNV GL, Bristol, United Kingdom; 20. DNV GL, Zaragoza, Spain

REDWIN Foundation Models for Integrated Dynamic

Analyses of Offshore Wind Turbines OMAE2019-96168

Ana Page, Karin Norén-Cosgriff, Kristoffer S. Skau, Amir M. Kaynia

Norwegian Geotechnical Institute, Oslo, Norway

Development of Offshore Structure Analysis Software

X-SEA Coupled with FAST OMAE2019-96778

Ki-Du Kim' Sorrasak Vachirapanyakun' Pasin Plodpradit ${ }^{1}$ Van Nguyen Dinh ${ }^{2}$ Jin Ho Park $^{3}$

1. Konkuk University, Seoul, Korea; 2. MaREI Centre, University College

Cork, Cork, Ireland; 3. Korean Register, Busan, Korea

\section{Offshore Geotechnics}

\section{0-1-1 Seabed Properties and Processes}

Monday June 10

Room Crowne Plaza, Jura | 13:30-15:00

Session Chair: Henry Milewski, TechnipFMC, United Kingdom

Influence of Suction Dredging on the Failure

Mechanism of Sandy Submarine Slopes: Revisited with

a Coupled Numerical Approach OMAE2019-95151

Manuela Kanitz, Jürgen Grabe

Hamburg University of Technology, Hamburg, Germany
Effect of Stress History and Shallow Embedment on Centrifuge Cone Penetration Tests in Sand OMAE2019-95393 Anamitra Roy, Shiao Huey Chow, Conleth O' Loughlin, Mark F. Randolph University of Western Australia, Perth, WA, Australia

On the Selection of an Appropriate Consolidation Coefficient for Offshore Geotechnical Design 0MAE2019-95800 David JWhite Jinbo Chen ${ }^{2}$ Susan Gourvenec ${ }^{3}$ Conleth $0^{\prime}$ Loughlin ${ }^{4}$ 1. University of Southampton, Southampton, United Kingdom; 2. Shell Global Solutions (US), Inc., Houston, TX, USA; 3. University of Southampton/ Southampton Marine and Maritime Institute, Southampton, United Kingdom; 4. University of Western Australia, Perth, WA, Australia

Fundamental Engineering Characteristics of Cohesive Sediments in the Northern Region of South China Sea OMAE2019-96599 Shuzhao Li, Xu Jia, Zhigang Li, Jiagang Li CNOOC Research Institute, Beijing, China

\section{Petroleum Technology}

\section{1-7-1 Well Drilling Fluids and Hydraulics I}

Monday June 10 Room Crowne Plaza, Barra | 13:30 - 15:00

Session Chair: Arild Saasen, UiS, Norway

Session Co-Chair: Ergun Kuru, University of Alberta, Canada

Modelling of the Movement of a Prolate Particle in the Steady State Flow of a Non-Newtonian Fluid in an Inclined Annulus with Inner String Rotation OMAE2019-95049 Eric Cayeux, NORCE, Stavanger, Norway

A New Three-layer Model for Gravel Packing Applications OMAE2019-95164 Alireza Sarraf Shirazi, lan Frigaard University of British Columbia, Vancouver, $B C$, Canada

Cuttings Transport Simulation in Large-diameter Inclined Borehole OMAE2019-95228 Yaroslav Ignatenko ${ }^{1}$ Andrey Gavrilov' ${ }^{2}$ leg Bocharov' ${ }^{1}$ Roland May ${ }^{3}$ 1. Baker Hughes, Novosibirsk, Russia; 2. Institute of Thermophysics of SB RAS, Krasnoyarsk branch, Krasnoyarsk, Russia; 3. Baker Hughes, a GE company, Celle, Germany

How does a Stationary Sand Bed affect the Flow Dynamics in an Eccentric Annulus? OMAE2019-96338 Majid Bizhani' Ergun Kuru²

1. University of British Columbia, Edmonton, $A B$, Canada;

2. University of Alberta, Edmonton, $A B$, Canada

\section{Rodney Eatock Taylor Honouring Symposium on Marine and Offshore Hydrodynamics}

\section{2-1-1 Numerical and Experimental Methods in Hydrodynamics I}

Monday June 10

Room SEC, Carron 2 | 13:30 - 15:00

Session Chair: Paul Taylor, University of Oxford, United Kingdom

Session Co-Chair: Kie Hian Chua, Technology Centre

for Offshore and Marine, Singapore

Numerical and Experimental Modelling of Wave

Loads on Thin Porous Sheets OMAE2019-95148

Edward Mackay' Lars Johanning ${ }^{1}$ Dezhi Ning ${ }^{2}$ Dongsheng Qiao ${ }^{2}$

1. University of Exeter, Penryn, United Kingdom;

2. Dalian University of Technology, Dalian, China 
Application of 4-phase Decomposition to the Analysis of Random Time Series from Wave Basin Tests OMAE2019-95172 Thomas A.A. Adcock ${ }^{1}$ Xingya Feng ${ }^{1}$ Tianning Tang ${ }^{1}$ Ton S. van den Bremer ${ }^{1}$ Sandy Day ${ }^{2}$ Saishuai Dai ${ }^{2}$ Ye Li Zhiliang Lin ${ }^{3}$ Wentao $\mathrm{Xu}^{3}$ Paul Taylor ${ }^{1}$ 1. University of Oxford, Oxford, United Kingdom; 2. University of Strathclyde, Glasgow, United Kingdom; 3. Shanghai Jiao Tong University, Shanghai, China

Irregular Frequency Removal and Convergence in Higher-order Bem for Wave Diffraction/Radiation Analysis OMAE2019-95482 Tomoaki Utsunomiya, Kyushu University, Fukuoka, Japan

Numerical Study on Seakeeping Performance of a Damaged Ship 0MAE2019-96193 Luning Cui' Yi Zheng Yinggang Li Ling Zhu' Mingsheng Chen ${ }^{2}$

1. Naval Research Academy, Beijing, China; 2. Wuhan University of Technology, Wuhan, China

\section{Takeshi Kinoshita Honoring Symposium on Offshore Technology}

\section{3-7-1 Small Vessel and Related Technology}

Monday June 10

Room SEC, Boisdale 2 | 13:30 - 15:00

Session Chair: Daisuke Kitazawa, University of Tokyo, Japan

Session Co-Chair: Yasunori Nihei, Osaka Prefecture University, Japan

Design Methodology and Development of an Independently

Rotating Multi-hull Vessel OMAE2019-95401

Yasunori Nihei' Sharath Srinivasamurthy' Hiroshi Sakamoto²

Norikazu Masuda ${ }^{3}$ Naoyuki Hara' ${ }^{1}$

1. Osaka Prefecture University, Osaka, Japan; 2. Fractaly, Osaka, Japan;

3. Nippon Kaiko, Hyogo, Japan

Numerical Hull Resistance and Hydrodynamic Characteristics of an Independently Rotating Multi-hull Vessel OMAE2019-95403

Sharath Srinivasamurthy' Hiroshi Sakamoto ${ }^{2}$ Tatsuo Nishikawa ${ }^{3}$ Yasunori Nihei ${ }^{1}$

1. Osaka Prefecture University, Osaka, Japan; 2. Fractaly, Osaka, Japan;

3. Shipbuilding Research Centre of Japan, Tokyo, Japan

Study on Attitude Control of a Cabin-suspended Catamaran by using a Double-Ioop Control System OMAE2019-95827 Jialin Han' Sota Kanno ${ }^{2}$ Akito Mochizuki ${ }^{2}$ Daisuke Kitazawa ${ }^{2}$ Teruo Maeda $^{3}$ Hiroshi Itakura $^{2}$ 1. Osaka Prefecture University, Osaka, Japan;2. The University of Tokyo,

Kashiwa, Japan; 3. Management Strategy Corporation, Yokohama, Japan

\section{REFRESHMENT BREAK}

15:00 - 15:30

Location: Hall 5 (SEC)

\section{CONCURRENT SESSIONS}

$$
15: 30-17: 30
$$

\section{Offshore Technology}

\section{1-4-3 Design Optimization}

Monday June 10

Room SEC, Alsh 1 | 15:30 - 17:30

Session Chair: Binbin Zhao, Harbin Engineering University, China

Session Co-Chair: Masoud Hayatdavoodi, University of Dundee, United Kingdom

How Emulation Improves Offshore Operations OMAE2019-95178

Leslie McGuire, Subsea7, Aberdeen, United Kingdom
Machine Learning to Predict Mooring Line Tensions OMAE2019-96488 Hema Wadhwa

INTECSEA, Perth, WA, Australia

Efficient Anchoring System for FPSO in Arbritary Waters OMAE2019-96575 Jairo Araujo ${ }^{1}$ Antonio Carlos Fernandes ${ }^{2}$ Joel S. Sales Junior ${ }^{3}$ Mario Santos ${ }^{4}$ Ana Thurler ${ }^{2}$ 1. ATNAV, Rio de Janeiro, RJ, Brazil; 2. COPPE/Universidade Federal do Rio de Janeiro, Rio De Janeiro, RJ, Brazil; 3. Laboratory of Waves and Current - LOC - Universidade Federal do Rio de Janeiro, Rio de Janeiro, RJ, Brazil; 4. NAVIUM, Rio de Janeiro, RJ, Brazil

The Use of Ensemble Forecast in Defining Offshore Installation Operability: A Case Study on Umbilical Shore Float-in Operations OMAE2019-96137 Francisco Tinoco, Kee Chien Ting, Kishor Chavan Subsea 7, Sutton, United Kingdom

Trajectory Prediction of Moored Vessels with Reduced Station Keeping Capability due to Exceeded Anchor Load Limits OMAE2019-96145 Michal Josten Hamburg University of Technology, Hamburg, Germany

\section{Offshore Technology}

\section{1-6-1 CFD Numerical Waves and Applications}

Monday June 10 Room SEC, Dochart 2 | 15:30 - 17:30 Session Chair: Csaba Pakozdi, SINTEF Ocean, Norway

Thorough Verification and Validation of CFD Simulation for FPSO Roll Damping OMAE2019-95046

Donghwan Lee, Zhenjia (Jerry) Huang

ExxonMobil Upstream Research Company, Spring, TX, USA

Dynamic Response of Monopile Wind Turbine in Large Waves OMAE2019-95288 Sopheak Seng' Charles Monroy ${ }^{2}$ Sime Malenica

1. Bureau Veritas, Marine \& Offshore, Paris, France; 2. Bureau Veritas, Paris, France

Wave Propagation in CFD-based Numerical Wave Tanks OMAE2019-96460 Jang Kim, Aldric Baquet, Hyunchul Jang TechnipFMC, Houston, TX, USA

CFD-based Numerical Wave Basin for FPSO

in Irregular Waves OMAE2019-96838

Aldric Baquet ${ }^{1}$ Hyunchul Jang ${ }^{1}$ Ho-Joon Lim ${ }^{1}$ Johyun Kyoung ${ }^{1}$

Nicolas Tcherniguin ${ }^{2}$ Timothee Lefebvre ${ }^{2}$ Jang Kim ${ }^{1}$

1. TechnipFMC, Houston, TX, USA;2. TechnipFMC, Paris, France

Simulation of Irregular Wave Impact on Semi-submersible Platform based on Coupled GN-CFD Method OMAE2019-95675 Kangping Liao ${ }^{1}$ Wenyang Duan' Qingwei Ma' Binbin Zha0 ${ }^{1}$ Rong-Gui Han² Jang Kim³ 1. Harbin Engineering University, Harbin, China; 2. Yantai CIMC Raffles Offshore Limited, Yantai, China; 3. TechnipFMC, Houston, TX, USA

\section{Structures, Safety and Reliability}

\section{2-3-1 Probabilistic Response Models}

Monday June 10

Room Crowne Plaza, Castle 2 | 15:30 - 17:30

Session Chair: Lance Manuel, University of Texas at Austin, USA

Session Co-Chair: Sverre K. Haver, University of Stavanger, Norway

Air - Gap Assessment of Semi-submersible Accounting for

Simultaneous Occurrence of Wind Sea and Swell OMAE2019-95144

Sverre K. Haver ${ }^{1}$ Julio Patino ${ }^{2}$

1. University of Stavanger, Stavanger, Norway; 2. Subsea 7, Røyneberg, Norway 


\author{
A Monte Carlo Based Simulation Method for \\ Damage Stability Problems OMAE2019-95295 \\ Stefan Krueger' Hendrik Dankowski \\ 1. Hamburg University of Technology, Hamburg, Germany; \\ 2. Flensburger Schiffbau Gesellschaft, Flensburg, Germany
}

Optimal Control for Response Reduction Of Single Hinged

Articulated Tower using MR-damper 0MAE2019-96076

Kushal Solomon, Deepak Kumar

Indian Institute of Technology Madras, Chennai, India

Extended Kalman Filtering for Estimating Drag and Inertia

Coefficients for Slender Offshore Structures OMAE2019-96630

Dhruv Bhagtani, Nilanjan Saha

Indian Institute of Technology Madras, Chennai, India

\section{Structures, Safety and Reliability}

\section{2-10-2 Collision and Crashworthiness II}

Monday June 10

Room Crowne Plaza, Castle 1 | 15:30 - 17:30

Session Chair: Zhiqiang Hu, Newcastle University, United Kingdom

Session Co-Chair: Sören Ehlers, Hamburg University of Technology, Germany

3D Printing Miniature Marine Structures Models for

Structural Analysis Purpose: Is it Possible? OMAE2019-95772

Miguel Angel Calle Gonzales, Pentti Kujala

Aalto University, Espoo, Finland

Enhancement of Structural Redundancy of Hull Structure in

Accidental Condition by Applying Highly Ductile Steel OMAE2019-95912

Shin Nakayama' Keiji Ueda ${ }^{2}$ Masahiro Aoki ${ }^{3}$ Kazuyuki Matsumoto ${ }^{4}$

1. Mitsubishi Ship Building, Nagasaki, Japan; 2. JfE Steel Corporation, Kurashiki, Japan;

3. JFESteel Corporation, Tokyo, Japan; 4. Nippon Kaiji Kyokai (ClassNK), Tokyo, Japan

\section{Materials Technology}

\section{3-9-1 Advances in Materials Characterization}

Monday June 10

Room SEC, Boisdale 1 | 15:30 - 17:30

Session Chair: Sheng Bao, Zhejiang University, China

Session Co-Chair: Yanhui Zhang, TWI Ltd, United Kingdom

Corrosion Behaviour of Cupronickel 90/10 Alloys in

Arabian Sea Conditions and its Effect on Maintenance

of Marine Structures OMAE2019-96227

Muntazir Abbas, Mahmood Shafiee, Nigel Simms

Cranfield University, Bedford, United Kingdom

A Comparative Study of Mechanical Properties of Biodegradable PBSAT and PA Gillnets in Norwegian Coastal Waters OMAE2019-95350 Biao Su, Heidi Moe Føre, Eduardo Grimaldo

SINTEF Ocean, Trondheim, Norway

Material Design of Offshore Linepipe Steels for

Ultra Deep Water Application OMAE2019-95863

Kyono Yasuda' Junji Shimamura' Satoshi Igji ${ }^{2}$ Ryuji Muraoka ${ }^{1}$

1. JFE Steel Corporation, Fukuyama, Japan; 2. JFE Steel Corporation, Kurashiki, Japan

Improvement on Toughness of Weld Heat Affected Zone of Cu-containing Low Alloy Steel of Long Part Forging for Offshore Applications by Optimizing Chemical Composition OMAE2019-95816 Yuta Honma ${ }^{1}$ Gen Sasaki' Kunihiko Hashi' Fumiyoshi Minami ${ }^{2}$

1. The Japan Steel Works, Ltd., Muroran, Japan; 2. Osaka University, Ibaraki, Japan
Development of YS 500MPA Thick Steel Plate with Weld Joint CTOD Property for Offshore Structures OMAE2019-95465 Yusuke Terazawa' Katsuyuki Ichimiya' Keiji Ueda' Satoshi Igi' Toshitaka Tanaka² Akiyoshi Tsuji ${ }^{2}$ Minoru Suwa ${ }^{3}$

1. JFE Steel Corporation, Kurashiki, Japan; 2. JFE Steel Corporation,

Fukuyama, Japan; 3. JFE Steel Corporation, Tokyo, Japan

Effect of Tensile Pre-strain on Collapse Pressure

of Coated Linepipe OMAE2019-95923

Takahiro Sakimoto ${ }^{1}$ Tsunehisa Handa ${ }^{1}$ Hisakazu Tajika ${ }^{1}$ Yoshiaki Murakami' Joe Kondo ${ }^{2}$

1. JFE Steel Corporation, Chiba, Japan; 2. JFE Steel Corporation, Tokyo, Japan

\section{Pipelines, Risers, and Subsea Systems}

\section{4-1-4 Flexible Pipes IV}

Monday June $10 \quad$ Room Crowne Plaza, Staffa/Shuna | 15:30 - 17:30

Session Chair: Anh Tuan Do, TechnipFMC, France

Session Co-Chair: Murilo Augusto Vaz, COPPE/UFRJ, Brazil

Lean Global Analysis of Marine Slender Structures

with Machine Learning OMAE2019-95147

Vinicius Ribeiro Machado da Silva, Matheus Santos, Mario Vignoles

TechnipFMC, Rio de Janeiro, RJ, Brazil

Non-linearly Restoring Performance and its Hysteresis

Behavior of Dynamic Catenary OMAE2019-95651

Yilun Li' Shuangxi Guo ${ }^{2}$ Yue Kong 'Min Li' Weimin Chen ${ }^{2}$

1. Beijing University of Aeronautics and Astronautics, Beijing, China;

2. Institute of Mechanics, Chinese Academy of Sciences, Beijing, China

Flexible Riser Top Connection Analysis with I-Tube Interface and Bending Hysteresis Effect OMAE2019-95826 Yangye He' Hailong Lu' Murilo Augusto Vaz ${ }^{2}$ Marcelo Caire' 1. Universidade Federal do Rio de Janeiro, Rio de Janeiro, RJ, Brazil; 2. COPPE/ Universidade Federal do Rio de Janeiro, Rio de Janeiro, RJ, Brazil

Sensitivity Studies on Offshore Submarine Hoses on CALM Buoy with Comparisons for Chinese-Lantern and Lazy-S Configuration OMAE2019-96755

Chiemela Victor Amaechi' Jianqiao Ye' Xiaonan Hou' ${ }^{1}$ a-Cheng Wang ${ }^{2}$

1. Lancaster University, Lancaster, United Kingdom; 2. Tsinghua University, Beijing, China

Investigation on Mechanical Properties of Fiberglass Reinforced Flexible Pipes under Bending OMAE2019-95457

Yifan Gao' Shan Jin ${ }^{2}$ Peng Cheng ${ }^{1}$ Peihua Han ${ }^{1}$ Yong Bai ${ }^{3}$

1. Zhejiang University, Hangzhou, China; 2. Zhejiang University, College of Civil Engineering and Architecture, Hangzhou, China; 3. Zhejiang University, Zhejiang, China

\section{Pipelines, Risers, and Subsea Systems}

\section{4-2-5 SCRs and SLWRs II}

Monday June 10 Room Crowne Plaza, Castle 3 | 15:30 - 17:30 Session Chair: Olav Fyrileiv, DNV GL, Norway

Strength and Fatigue Performance of Steel Lazy Wave Risers with Change in Configuration Parameters OMAE2019-95135

Mayank Lal, Feng Wang, Xiaohua Lu, Abhilash Sebastian Genesis Oil and Gas Consultants, Houston, TX, USA

Improved Fatigue Design of SCR-modified Miner's Rule OMAE2019-95344 Hans Olav Knagenhjelm ${ }^{1}$ Mons Hauge ${ }^{2}$ Bård Nyhus $^{3}$ 1. Equinor ASA, Fornebu, Norway; 2. Equinor ASA, Ranheim, Norway; 3. SINTEF, Trondheim, Norway 
Finite Element Analysis of Seafloor-SCR Interaction

in Touchdown Zone OMAE2019-95830

Zhang Wei' Peng Peng ${ }^{2}$

1. Tianjn University, Tianjin, China; 2. Hunan Hydro and

Power Design Institute, Changsha, China

A Fracture Mechanics-based Feasibility Study of Damped Steel Catenary Risers for Pre-salt Field Developments OMAE2019-96297 Alexandre G. Garmbis ${ }^{1}$ Petrônio Zumpano Jr.' Ludimar L. Aguiar ${ }^{2}$

Raphael M. Brito ${ }^{2}$ Domingos A. Rade ${ }^{3}$

1. Petrobras, São José dos Campos, SP, Brazil; 2. Petrobras, Rio de Janeiro, RJ,

Brazil; 3. Aeronautics Institute of Technology, São José dos Campos, SP, Brazil

Study on the Design Method of Deepwater

Steel Lazy Wave Riser OMAE2019-95631

Zhao Wang 'Wei Qin ${ }^{1}$ Xiaojie Zhang' Jiannan Zhao ${ }^{1}$ Yong Bai ${ }^{2}$

1. Southern University of Science and Technology, Shenzhen,

China; 2. Zhejiang University, Zhejiang, China

\section{Ocean Space Utilization}

\section{5-6-1 High Tide and Tsunamis}

Monday June 10

Room SEC, Dochart 1 | 15:30 - 17:30

Session Chair: Koichi Masuda, Nihon University, Japan

Session Co-Chair: Koji Takahashi, Japan Port Consultants, Ltd., Japan

Introduction of Virtual Structural Boundary for Collision Force

Analysis of Tsunami Drifting Objects in Particle Method OMAE2019-95408

Yasuhiro Aida' Koichi Masuda ${ }^{2}$ Tomoki lkoma $^{2}$ Hiroaki Eto ${ }^{2}$

1. Nihon University, Chiba, Japan; 2. Nihon University, Funabashi, Japan

Study on Characteristic and Problem of Water Utilization and

Management by Floating Residence in the United States OMAE2019-95881

Daisuke Dobashi' Akio Kuroyanagi² Ryo Sugahara'

1. Nihon University, Chiba, Japan; 2. Nihon University, Funabashi-shi, Japan

A Research on Predicting Method of the Damage by

Tsunami Drifting Objects in Urban Port OMAE2019-95927

Koichi Masuda ${ }^{1}$ Tomoki lkoma ${ }^{1}$ Yasuhiro Aida ${ }^{2}$ Masayuki Takada ${ }^{3}$ Yuta Fukunaga ${ }^{4}$

1. Nihon University, Funabashi, Japan; 2. Nihon University, Chiba, Japan;

3. Tohoku Regional Bureau Ministry of Land Infrastructure Transport and

Tourism, Sendai, Japan; 4. Nihon University, Hunabashi, Japan

A Fundamental Study on Tsunami Protection Measures for a Vessel Moored at a Wharf considering the Backwash Influence OMAE2019-96159 Mitsuhiro Masuda' Kiyokazu Minami' Koichi Masuda²

1. Tokyo University of Marine Science and Technology, Tokyo,

Japan; 2. Nihon University, Funabashi, Japan

Improving the Productivity and Sustainability of Port

Management against High Tide and Tsunamis OMAE2019-96406

Koji Takahashi

Japan Port Consultants, Ltd., Shinagawa, Japan

\section{Ocean Engineering}

\section{6-4-2 Marine Operations and Vessel Motions}

Monday June 10 Room SEC, M2 \& M3 | 15:30 - 17:30

Session Chair: Lin Li, University of Stavanger, Norway

Session Co-Chair: Shuzheng Sun, Harbin Engineering University, China

Generic On-board Decision Support System Framework

for Marine Operations OMAE2019-95146

Stian Skjong' Lars T. Kyllingstad' Karl J. Reite' Joakim Haugen ${ }^{1}$

Jarle Ladstein ${ }^{1}$ Karl Gunnar Aarsaether ${ }^{2}$

1. SINTEF Ocean, Trondheim, Norway; 2. SINTEF Ocean, Tromsoe, Norway

A Simulation Program for Load-out Operation using

Self-propelled Modular Transporters OMAE2019-95673

Zunfeng Du, Haiming Zhu, Dong Xu

Tianjin University, Tianjin, China

Seismic RTDT - Real-time Digital Twin for Boosting

Performance of Seismic Operations OMAE2019-95885

Severin Sadjina' Stian Skjong ${ }^{2}$ Armin Pobitzer' Lars T. Kyllingstad²

Roy-Jostein Fiskerstrand ${ }^{3}$ Sverre Torben ${ }^{3}$ Jason D. D. A. Granholt ${ }^{4}$

1. SINTEF Ålesund, Ålesund, Norway; 2. SINTEF Ocean, Trondheim, Norway;

3. Rolls-Royce Marine AS, Aalesund, Norway; 4. CGG Services AS, OSlo, Norway

Impact of the Uncertainties of the RAOs of a

Semi-submersible Platform on the Performance of a

Motion-based Wave Inference Method OMAE2019-96670

Jordi Mas Soler ${ }^{1}$ Pedro C. de Mello2 Eduardo Tannuri ${ }^{3}$

Alexandre N. Simos ${ }^{2}$ Antonio Souto-Iglesias ${ }^{1}$

1. Technical University of Madrid (UPM), Madrid, Spain; 2. Universidade de São Paulo, São Paulo, SP, Brazil; 3. Numerical Offshore Tank - University of São Paulo, São Paulo, SP, Brazil

Downtime Technique using Artificial Intelligence:

A Case Study for an Exposed Berthing Facility 0MAE2019-95312

Ghassan El Chahal' Peter Morel2 Sindhu Mole ${ }^{3}$ Nadjib Saadali ${ }^{3}$

1. COWI A/S, Kongens Lyngby, Denmark; 2. COWI A/S, Århus, Denmark;

3. COWI A/S, Dubai, United Arab Emirates

\section{Ocean Engineering}

\section{6-11-1 Autonomous Vehicle Technology}

Monday June 10

Room SEC, M4 | 15:30-17:30

Session Chair: Celso Pesce, University of S. Paulo - Escola Politecnica, Brazil

Session Co-Chair: Daniel Costa, COPPE/UFRJ, Brazil

Path Following and Collision Avoidance of Underactuated

Marine Vessels based on MPC Design OMAE2019-95081

Guoping Zheng, Cheng Liu, Cheng Li

Dalian Maritime University, Dalian, China

Situation Awareness of Autonomous Ship Navigation in a Mixed Environment with Advanced Ship Predictor OMAE2019-95571

Lokukaluge Prasad Perera, Brian Murray

Uit The Arctic University of Norway, Tromso, Norway

Time-varying Vector Field Guidance Law for Path

Following and Obstacle Avoidance Control for

Underactuated Autonomous Vehicles OMAE2019-96618

Haitong $\mathrm{Xu}^{1}$ Miguel Hinostroza ${ }^{2}$ Carlos Guedes Soares ${ }^{1}$

1. Instituto Superior Técnico, Universidade de Lisboa, Lisbon, Portugal; 2. Centre

for Marine Technology and Ocean Engineering (CENTEC), Lisbon, Portugal 
An AIS-based Multiple Trajectory Prediction Approach for Collision Avoidance in Future Vessels OMAE2019-95963 Brian Murray, Lokukaluge Prasad Perera UiT The Arctic University of Norway, Tromso, Norway

Towards the Development of an Ocean Engineering Library for OpenModelica OMAE2019-95054 Savin Viswanathan, Christian Holden Dept. Mechanical and Industrial Engineering, Norwegian University of Science and Technology, Trondheim, Norway

\section{CFD \& FSI}

\section{8-1-2 Surface Waves}

Monday June $10 \quad$ Room SEC, Lomond Auditorium | 15:30 - 17:30 Session Chair: Luis Eca, Technical University of Lisbon, Portugal Session Co-Chair: Owen H. Oakley, Jr, Retired, USA

Numerical Simulation of the Effect About Speed and Pitch Angle of Initial Stage on a Airplane Landing in Smooth Water OMAE2019-95438 Yong Ding, Shengcang Li, Kaiye Hu, Mo Chen Harbin Engineering University, Harbin, China

Numerical Investigations of Ship Parametric Rolling

in Regular Head Waves of DTC OMAE2019-95515

Qing Wang

Huazhong University of Science and Technology, Wuhan, China

\section{Numerical Manoeuvrable Tank on Wave Based}

Moving Domain OMAE2019-95714

Dakui Feng' Xiao Cai' Yue Sun' ZhiGuo Zhang' XiaoWei Huang ${ }^{2}$

1. Huazhong University of Science and Technology, Wuhan, China;

2. China Ship Design and Development Center, Wuhan, China

Assessment of LNG Pump Tower Loads OMAE2019-96138

Michael Thome, Jens Neugebauer, Ould el Moctar

University of Duisburg-Essen, Duisburg, Germany

Numerical Simulation of Damaged Ship's

Motion in Beam Waves OMAE2019-96791

Qing Wang ${ }^{1}$ Xuanshu Chen ${ }^{1}$ Liwei Liu' Xianzhou Wang ${ }^{1}$ Mingling Liu ${ }^{2}$

1. Huazhong University of Science and Technology, Wuhan, China;

2. China Ship Design and Development Center, Wuhan, China

\section{Ocean Renewable Energy}

\section{9-2-1 Aerodynamics I}

Monday June 10

Room SEC, Carron 1 | 15:30 - 17:30

Session Chair: Jason Jonkman, National Renewable Energy Laboratory, USA

Impact of Rotor Misalignment due to Platform Motions on Floating Offshore Wind Turbine Blade Loads OMAE2019-95759 Rachael E. Smith, Ajit C Pillai, Gavin Tabor, Philipp R. Thies, Lars Johanning University of Exeter, Exeter, United Kingdom

An Experimental Apparatus for Investigating the Unsteady Aerodynamics of a Floating Wind Turbine OMAE2019-95915 Binrong Wen, Qi Zhang, Haoxue Liu, Xinliang Tian, Xingjian Dong,

Zhi-Ke Peng, Yongsheng Zhao, Yufeng Kou

Shanghai Jiao Tong University, Shanghai, China
A 6-DOFs Hardware-in-the-loop System for Wind Tunnel Tests of Floating Offshore Wind Turbines OMAE2019-95967 Alessandro Fontanella ${ }^{1}$ IImas Bayati ${ }^{2}$ Federico Taruffi' Francesco La Mura ${ }^{1}$ Alan Facchinetti ${ }^{1}$ Marco Belloli ${ }^{1}$

1. Politecnico di Milano, Milano, Italy; 2. MARIN, Wageningen, Netherlands

Numerical and Experimental Wind Tunnel Analysis of Aerodynamic Effects on a Semi-submersible Floating Wind Turbine Response OMAE2019-95976 Alessandro Fontanella ${ }^{1}$ IImas Bayati ${ }^{2}$ Federico Taruffi' Alan Facchinetti' Marco Belloli 1. Politecnico di Milano, Milano, Italy; 2. MARIN, Wageningen, Netherlands

Aerodynamic Analysis of a Wind Turbine with Elevated Inflow Turbulence and Wake using Harmonic Method OMAE2019-96769 Shine Win Naung, Mohammad Rahmati, Hamed Farokhi Northumbria University, Newcastle upon Tyne, United Kingdom

\section{Offshore Geotechnics}

\section{0-3-1 Anchors}

Monday June 10 Room Crowne Plaza, Jura | 15:30-17:30

Session Chair: Fu-Ping Gao, Institute of Mechanics,

Chinese Academy of Sciences, China

CFD Analysis on Hydrodynamic Characteristics for Optimizing Torpedo Anchors OMAE2019-95778

Jing Sun, Haixiao Liu

Tianjin University, Tianjin, China

CFD Analysis on Similarity Criteria of Hydrodynamic Characteristics for Gravity-installed Anchors OMAE2019-95960 Jiancai Gao, Haixiao Liu Tianjin University, Tianjin, China

Effect of Seabed Trenching on Holding Capacity of Suction Anchors in Deepwater Gulf of Guinea Clays - A Numerical Study 0MAE2019-96096 Pablo Castillo Garcia' Regis Wallerand ${ }^{2}$ Dinh Hong Doan ${ }^{1}$ 1. Subsea7 France, Suresnes, France; 2. Total, La Défense, France

Combined Wave-current Induced Instantaneous Liquefaction of a Sandy Seabed OMAE2019-96655 Lijing Yang' Chang-Fei Li' Jun-Qin Wang ${ }^{2}$ Fu-Ping Gao ${ }^{1}$ 1. Institute of Mechanics, Chinese Academy of Sciences, Beijing, China; 2. China National Offshore Oil Company (CNOOC) Research Institute, Beijing, China

\section{Petroleum Technology}

\section{1-7-3 Well Drilling Fluid and Hydraulics III}

Monday June 10 Room Crowne Plaza, Barra | 15:30 - 17:30

Session Chair: Ergun Kuru, University of Alberta, Canada

Rheology of Brine-based Fuzzy-ball Drilling Fluids

in Deepwater Drilling OMAE2019-96094

Zhaochuan Li' Lihui Zheng' Panfeng Wei' Xiaojuan Dai ${ }^{2}$ Weian Huang 1. China University of Petroleum, Beijing, China; 2. Jilin University, Jilin, China; 3. China University of Petroleum (East China), Qingdao, China

A Field Study on the Marine Environmental Impact of the Drilling Fluid's Discharge 0MAE2019-96231 Meirong Jiang ${ }^{1}$ Xiaohan $\mathrm{He}^{2}$ 1. CNOOC Research Institute, Beijing, China;2. China MEHECO Corporation, Beijing, China 
Viscosity Models for Drilling Fluids - Viscosity

Parameters and their Use 0MAE2019-96595

Arild Saasen ${ }^{1}$ Jan David Ytrehus ${ }^{2}$

1. UiS, Gullaug, Norway; 2. SINTEF, Trondheim, Norway

Rheological Properties of Water Based Drilling Fluids in Deep Offshore Conditions with Large Temperature Difference and High Pressure OMAE2019-96719 Qian Ding, Baojiang Sun, Zhiyuan Wang, Yonghai Gao,

Yu Gao, Yongqiang Liao, Di Wang, Andi Xia

China University of Petroleum, Qingdao, China

Effect of LCM Fibers on the Rate of THF-water Clathrate Hydrate

Growth in Water-based Drilling Fluids OMAE2019-96682

James L. Nielsen' Syed Y. Nahri' Panfeng Wei' Wei Zhao ${ }^{3}$ Yuanhang Chen ${ }^{1}$

1. Louisiana State University, Baton Rouge, LA, USA; 2. Beijing Lihuilab Energy

Technology Co.,Ltd Plugging Fluid Technology Research and Development

Branch, Beijing, China; 3. China University of Petroleum, Beijing, China

\section{Rodney Eatock Taylor Honouring Symposium on Marine and Offshore Hydrodynamics}

\section{2-2-1 Multi-Body Hydrodynamics}

Monday June 10

Room SEC, Carron 2 | 15:30 - 17:30

Session Chair: Wei Bai, Manchester Metropolitan University, United Kingdom

Session Co-Chair: Wei Qiu, Memorial University of Newfoundland, Canada

Model Experiments of Floating Side-by-side Barges OMAE2019-95238

Kie Hian Chua' Pedro de Mello ${ }^{2}$ Kazuo Nishimoto ${ }^{3} Y_{00}$ Sang Choo ${ }^{4}$

1. Technology Centre for Offshore and Marine, Singapore, Singapore; 2. Tanque de Provas (TPN-USP), São Paulo, SP, Brazil; 3. University of São Paulo, São Paulo,

$S P$, Brazil; 4. National University of Singapore, Singapore, Singapore

Numerical Modelling of Wave Resonance in a Narrow Gap between Two Floating Bodies in Close Proximity using a Hybrid Model OMAE2019-95247 Shiqiang Yan, Qingwei Ma, Junxian Wang, Jinghua Wang

City, University of London, London, United Kingdom

Unified One-fluid Approach for Multi-body

Hydrodynamics OMAE2019-96814

Liang Yang, Christopher C Pain

Imperial College London, London, United Kingdom

Numerical Analysis of GBS Float-over Deck Installation

at Docking and Undocking Stages based on a Coupled

Heave-Roll-Pitch Impact Model OMAE2019-95717

Mingsheng Chen, Meiyan Zou, Ling Zhu, Liang Sun

Wuhan University of Technology, Wuhan, China

\section{Takeshi Kinoshita Honoring} Symposium on Offshore Technology

\section{3-2-1 Numerical Methods}

Monday June 10

Room SEC, Boisdale 2 | 15:30 - 17:30

Session Chair: Celso Morooka, University of Campinas, Brazil

Session Co-Chair: Yuri Coelho Del Sarto, Universidade Estadual de Campinas, Brazil

Investigation on the Effect of DNVGL OTG 13 on Air Gap

Assessment of a Semi-submersible Unit OMAE2019-95072

Youwei Kang' Bing Wang 'Lei Li' Zhao Ziguang

1. CIMC Offshore Co. Ltd., Shenzhen, China; 2. COOEC Subsea Technology. Ltd, Shenzhen, China
Time Domain Simulations of Ship Maneuvering and Roll Motion in Regular Waves based on a Hybrid Method OMAE2019-95562

Chengqian Ma, Ning Ma, Xiechong Gu

Shanghai Jiao Tong University, Shanghai, China

Study on the Influence of Mesh Grouping on Numerical Simulation Results of Fish Cages OMAE2019-95706

Liuyi Huang ${ }^{1}$ Yuyan Li' Yi Ni ${ }^{1}$ Hui Cheng ${ }^{2}$ Xinxin Wang ${ }^{1}$ Gang Wang ${ }^{1}$ Fenfang Zhao ${ }^{1}$ 1. Ocean University of China, Qingdao, China; 2. University of Stavanger, Stavanger, Norway

The Analysis of the Joint Limitation Condition of Wave Height-period on the Floating Crane Lifting Operation OMAE2019-96461

Xue-gang Wang, Ying Zong-quan, Chen Ze-cong

cccCFourth Harbor Engineering Institute Co., Ltd, Guangzhou, China

Nonlinear Analysis of an Oscillating Water Column Wave Energy Device in Frequency Domain via Statistical Linearization OMAE2019-96727 Leandro Souza Pinheiro da Silva' Celso Pesce ${ }^{2}$ Hélio Morishita' ${ }^{1}$ Rodolfo T. Gonçalves ${ }^{3}$ 1. University of São Paulo, São Paulo, SP, Brazil; 2. University of São Paulo - Escola Politecnica, São Paulo, SP, Brazil; 3. University of Tokyo, Bunkyo, Japan

\section{ASME \& IMECHE CONNECT ROUNDTABLE 17:00 - 18:15 \\ Location: Forth Room}

\section{Afternoon Lecture}

17:40 - 18:10

Location: Lomond Auditorium (SEC)

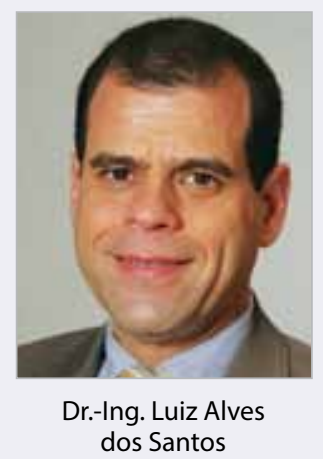

European Research Council - Funding Opportunities for Creative Minds from All Over the World

Dr.-Ing. Luiz Alves dos Santos, Scientific Officer, European Research Council dos Santos

See Afternoon Lecture Series, page 22 for more details.

\section{AFTERNOON DRINKS RECEPTION}

18:15 - 19:15

Location: Hall 5 (SEC) 


\section{Tuesday, June 11}

\begin{tabular}{|c|c|c|}
\hline Time & Title & Location \\
\hline $08: 30-17: 30$ & Exhibition open & Hall 5 (SEC) \\
\hline $08: 30-10: 00$ & $\begin{array}{l}\text { Concurrent } \\
\text { Sessions }\end{array}$ & $\begin{array}{l}\text { See pages } 36-40 \text { for } \\
\text { session titles, authors } \\
\text { and locations }\end{array}$ \\
\hline $10: 00-10: 30$ & $\begin{array}{l}\text { Refreshment } \\
\text { Break }\end{array}$ & Hall 5 (SEC) \\
\hline $10: 30-12: 00$ & $\begin{array}{l}\text { Concurrent } \\
\text { Sessions }\end{array}$ & $\begin{array}{l}\text { See pages } 40-44 \text { for } \\
\text { session titles, authors } \\
\text { and locations }\end{array}$ \\
\hline $12: 00-13: 30$ & Lunch & Hall 5 (SEC) \\
\hline $13: 30-15: 00$ & $\begin{array}{l}\text { Concurrent } \\
\text { Sessions }\end{array}$ & $\begin{array}{l}\text { See pages } 44-48 \text { for } \\
\text { session titles, authors } \\
\text { and locations }\end{array}$ \\
\hline $15: 00-15: 30$ & $\begin{array}{l}\text { Refreshment } \\
\text { Break }\end{array}$ & Hall 5 (SEC) \\
\hline $15: 30-17: 30$ & $\begin{array}{l}\text { Concurrent } \\
\text { Sessions }\end{array}$ & $\begin{array}{l}\text { See pages } 49-53 \text { for } \\
\text { session titles, authors } \\
\text { and locations }\end{array}$ \\
\hline $17: 40-18: 30$ & $\begin{array}{l}\text { Afternoon } \\
\text { Lecture Series }\end{array}$ & Lomond Auditorium (SEC) \\
\hline
\end{tabular}

\section{CONCURRENT SESSIONS}

08:30 - 10:00

\section{Offshore Technology}

\section{1-1-3 Floating Wind Platforms}

\section{Tuesday June 11}

Room SEC, Alsh 1 | 08:30 - 10:00

Session Chair: Bonjun Koo, TechnipFMC, USA

Session Co-Chair: Mareike Leimeister, Fraunhofer IWES

(Fraunhofer Institute for Wind Energy Systems), Germany

Larger MW-class Floater Designs without Upscaling? A Direct Optimization Approach OMAE2019-95210

Mareike Leimeister ${ }^{1}$ Athanasios Kolios ${ }^{2}$ Maurizio Collu ${ }^{2}$ Philipp Thomas 1. Fraunhofer IWES (Fraunhofer Institute for Wind Energy Systems), Bremerhaven, Germany; 2. University of Strathclyde, Glasgow, United Kingdom

Effect of Wind Turbulence on Extreme Load Analysis of an Offshore Wind Turbine OMAE2019-95634

Xiaolu Chen ${ }^{1}$ Zhiyu Jiang ${ }^{2}$ Qinyuan Lij ${ }^{3}$ Le Li ${ }^{1}$

1. Shanghai Jiao Tong University, Shanghai, China; 2. University of Agder, Grimstad, Norway; 3. Norwegian University of Science and Technology, Trondheim, Norway

Baseline Design of the Deep Turbine Installation-Floating, a New Floating Wind Concept OMAE2019-95477

Jordi Serret ${ }^{1}$ Tahsin Tezdogan $^{2}$ Tim Stratford $^{3}$ Philipp R. Thies ${ }^{4}$ Vengatesan Venugopa ${ }^{3}$ 1. University of Edinburgh - IDCORE, Edinburgh, United Kingdom; 2. University of Strathclyde, Glasgow, United Kingdom; 3. University of Edinburgh, Edinburgh, United Kingdom; 4. University of Exeter, Penryn, United Kingdom

\section{Offshore Technology}

\section{1-2-2 Mooring System Design and Analysis I}

Tuesday June 11

Session Chair: Djoni Sidarta, TechnipFMC, USA

Session Co-Chair: Jaakko Lehtonen, TechnipFMC Genesis, USA

Snap Load Induced by Slack-taut Process in a

Taut Mooring Line OMAE2019-95016

Dongsheng Qiao ${ }^{1}$ Wei Tang ${ }^{1}$ Yunfei Suo ${ }^{2}$ Jun Yan' Yugang Li' Daocheng Zhou ${ }^{1}$

1. Dalian University of Technology, Dalian, China;

2. CCCC-FHDI Engineering Company, Guangzhou, China

Spring-Dashpot Simulations of Polyester Ropes -

Validation of the Syrope Model OMAE2019-95469

Erik Falkenberg, Limin Yang, Vidar R. Åhjem

DNV GL, Høvik, Norway

Three-Dimensional Dynamic Analysis Method of Multi-component Mooring Lines OMAE2019-96056

Yuda Apri Hermawan, Yoshitaka Furukawa

Kyushu University, Fukuoka, Japan

\section{Structures, Safety and Reliability}

\section{2-4-1 Fatigue and Fracture Reliability I}

Tuesday June 11

Room SEC, Alsh 2 | 08:30 - 10:00

Session Chair: Marcelo Igor Lourenço Souza, UFRJ, Brazil

Session Co-Chair: Fredhi Agung Prasetyo, Research \& Development

Division, Biro Klasifikasi Indonesia, Indonesia

Inner Bend Cracks in Mooring Chain - Investigation of Cracks

Observed on Chains Taken Out of Service OMAE2019-95084

Øystein Gabrielsen ${ }^{1}$ Inge Morten Kulbotten ${ }^{2}$ Imanol Martinez Perez ${ }^{3}$ Lars Håskoll ${ }^{4}$

1. Equinor, Trondheim, Norway; 2. Equinor ASA, Trondheim, Norway;

3. Principia, La Ciotat, France; 4. Equinor ASA, Stjordal, Norway

Fracture Mechanics Based Mooring Fatigue Analysis for a Semi-submersible Subjected to Triple

Narrow-band Loading Processes OMAE2019-95108

Xutian Xue' Nianzhong Chen $^{2}$ Yongchang Pu ${ }^{3}$

1. Newcastle University, Newcastle upon Tyne, United Kingdom; 2. Tianjin

University, Tianjin, China; 3. Marine, Offshore and Subsea Technology Group,

School of Engineering, Newcastle upon Tyne, United Kingdom

Computational Fatigue Assessment of Mooring Chains

Working in Twisted Conditions OMAE2019-96000

Imanol Martinez Perez ${ }^{1} \emptyset$ ystein Gabrielsen²

1. Principia, La Ciotat, France; 2. Equinor, Trondheim, Norway

Predictions of Tensile Strain Capacity for Strain-based Pipelines with a Circumferential and Internal Surface Flaw 0MAE2019-96480 Youn-Young Jang' Ju-Yeon Kang ${ }^{1}$ Nam-Su Huh ${ }^{1}$

Ik-Joong Kim² Cheol-Man Kim² Young-Pyo Kim²

1. Seoul National University of Science and Technology, Seoul, Korea; 2. KOGAS, Ansan, Korea

A Review of Offshore Structures Science and Engineering for Future Floating Wind Platforms OMAE2019-95795

Maria Lourdes Jalon Ramirez, Feargal Brennan

University of Strathclyde, Glasgow, United Kingdom 


\section{Structures, Safety and Reliability}

\section{2-9-1 Extreme Loading and Responses I}

Tuesday June 11

Room Crowne Plaza, Castle 1 | 08:30 - 10:00

Session Chair: Carlos Guedes Soares, Instituto Superior

Técnico, Universidade de Lisboa, Portugal

Session Co-Chair: Luis V.S. Sagrilo, LACEO/COPPE/

Federal University of Rio De Janeiro, Brazil

Elementary Loading Processes and Scale Effects involved in Wave-in-Deck Type of Loading - A Summary of the BreaKin JIP OMAE2019-95004

Jule Scharnke

MARIN, Wageningen, Netherlands

Numerical Simulation and Analysis of Phase Focused

Breaking and Non-breaking Wave Impact on Fixed

Offshore Platform Deck OMAE2019-95193

Rameeza Moideen ${ }^{1}$ Manasa Ranjan Behera' Arun Kamath² Hans Bihs ${ }^{3}$

1. Indian Institute of technology Bombay, Mumbai, India;

2. Norwegian University of Science and Technology, Trondheim, Norway;

3. Norwegian University of Science and Technology, Sør-Trøndelag, Norway

The Impact of Climate Change on the Long-term Response of Offshore Structures: A Study Case OMAE2019-95261

Irvin Alberto Mosquera' Luis V.S. Sagrilo² Paulo M. Videiro²

1. Universidade Federal do Rio de Janeiro, Rio de Janeiro, RJ, Brazil; 2. LACEO/

COPPE/Universidade Federal do Rio de Janeiro, Rio de Janeiro, RJ, Brazil

Study of Uncertainties in Laboratory Wave Impact

Measurements on Dike Mounted Walls due to

Non-repeatability, Scale- and Model-effects OMAE2019-96703

Maximilian Streicher' ${ }^{1}$ Andreas Kortenhaus ${ }^{1}$ Corrado Altomare' Steven Hughes ${ }^{2}$ Krasimir Marinov ${ }^{3}$ Bas Hofland ${ }^{4}$ Xuexue Chen ${ }^{5}$ Tomohiro Suzuki $^{6}$ Lorenzo Cappietti $^{7}$

1. Ghent University, Ghent, Belgium; 2. Colorado State University, Fort Collins, CO, USA;

3. University of Architecture, Civil Engineering and Geodesy Sofia, Sofia, Bulgaria; 4. TU Delft, Delft, Netherlands; 5. Haskoning DHV Nederland B.V., Rotterdam, Netherlands; 6 . Flanders Hydraulics Research, Antwerp, Belgium; 7. Universita degli Studi di Firenze, Florence, Italy

\section{Structures, Safety and Reliability}

\section{2-12-1 Structural Analysis and Optimization I}

Tuesday June 11

Room Crowne Plaza, Castle 2 | 08:30 - 10:00

Session Chair: Jonas W. Ringsberg, Chalmers University of Technology, Sweden

Session Co-Chair: Paulo M. Videiro, LACEO/COPPE/

Federal University of Rio De Janeiro, Brazil

Analytical Method for Preliminary Design of Anchor

Flanges for Subsea Structures OMAE2019-95051

Sabesan Rajaratnam ${ }^{1}$ Sriskandarajah Thurairajah' Daryl Clayton' ${ }^{1}$

Graeme Roberts ${ }^{1}$ Vincent Loentgen² Carlos Charnaux ${ }^{1}$

1. Subsea 7, Sutton, United Kingdom; 2. Subsea7, Paris, France

Simplifying Methods for Fatigue Analysis of Risers OMAE2019-95386 Luiz Otavio C. M. Pereira' Paulo M. Videiro² Luis V.S. Sagrilo²

1. Federal University of Rio de Janeiro, Rio de Janeiro, RJ, Brazil;

2. LACEO/COPPE/Federal University of Rio de Janeiro, Rio de Janeiro, RJ, Brazil

Four-point Bending of Metallic I-Core Sandwich

Beams with Longitudinal Girder OMAE2019-95491

Wenwei Hu, Jun Liu, Pan Zhang, Yuansheng Cheng

Huazhong University of Science and Technology, Wuhan, China
Validation of External Moment Determination for the

Shaft-line of the SA Agulhas II OMAE2019-96746

Brendon Nickerson, Annie Bekker

Stellenbosch University, Department of Mechanical and

Mechatronic Engineering, Stellenbosch, South Africa

\section{Materials Technology}

\section{3-1-1 Fracture Toughness Measurement and Assessment}

Tuesday June 11

Room SEC, Boisdale 1 | 08:30 - 10:00

Session Chair: Xin Wang, Carleton University, Canada

Session Co-Chair: Myung-Hyun Kim, Pusan National University, Korea

Influence of Microstructural Variation in Thick Section

Steels on the Characterisation of Fracture Toughness

using Sub-size Specimens OMAE2019-96010

Philippa Moore ${ }^{1}$ Borislava Yordanova ${ }^{2} Y_{\text {ong }}$ Lu $^{2}$ Yin Jin Janin $^{1}$

1. TWI Ltd, Cambridge, United Kingdom;

2. University of Edinburgh, Edinburgh, United Kingdom

Correlation between Steel Microstructural Characteristics and the Initiation and Arrest Toughness Determined from Small-scale Mechanical Testing OMAE2019-95290 Jessica Taylor' ${ }^{1}$ Philippa Moore ${ }^{2}$ Ali Mehmanparast ${ }^{1}$ Rob Kulka $^{2}$

1. Cranfield University, Cranfield, United Kingdom;2. TWI Ltd, Cambridge, United Kingdom

CTOD Fracture Toughness Assessment under Different Notch

Type (Fatigue Pre-cracking and EDM) OMAE2019-95130

Israel Marines-Garcia' Aaron Aguilar' Kristian Carreon ${ }^{1}$ Philippe Darcis ${ }^{2}$

1. Tenaris TTSA, Veracruz, Mexico; 2. Dalmine S.p.A., Dalmine, Italy

Scaling of Pop-ins during Brittle Fracture Testing OMAE2019-95368 Okko J Coppejans ${ }^{1}$ Carey L. Walters ${ }^{2}$

1. TNO, Delft, Netherlands; 2. Delft University of Technology, Delft, Netherlands

Scatter in Charpy Data Considered as a

Transferrable Parameter OMAE2019-96748

William Mohr, Neal Birchfield

EWI, Columbus, OH, USA

\section{Pipelines, Risers, and Subsea Systems}

\section{4-1-2 Flexible Pipes II}

Tuesday June $11 \quad$ Room Crowne Plaza, Staffa / Shuna | 08:30 - 10:00 Session Chair: Zhimin Tan, Baker Bughes (a GE company), USA

Session Co-Chair: Adrian Connaire, Wood, Ireland

Session Co-Chair: Svein Saevik, Norwegian University

of Science and Technology, Norway

A Symbolic Regression Formulation to Estimate the Lateral Buckling Resistance of the Tensile Armors in Flexible Pipes OMAE2019-95510

Gabriel Gonzalez ${ }^{1}$ José Renato M. de Sousa' Luis V.S. Sagrilo²

Ricardo R. Martins ${ }^{3}$ Djalene Rocha ${ }^{3}$

1. Federal University of Rio de Janeiro, Rio de Janeiro, RJ, Brazil; 2. LACEO/COPPE/Federal

University of Rio de Janeiro, Rio de Janeiro, RJ, Brazil; 3. Petrobras, Rio de Janeiro, RJ, Brazil

An Application of Fault Tree Analysis for Decommissioning

of Subsea Flexible Pipeline in Brazil OMAE2019-96730

Rafaela Ramos ${ }^{1}$ Ison Pasqualino ${ }^{2}$ Marcelo Igor Lourenço Souza ${ }^{3}$ Eduardo Ribeiro Nicolosi ${ }^{4}$

1. Fundação - COPPETEC - Federal University of Rio de Janeiro, Rio de Janeiro, RJ, Brazil;

2. COPPE/Universidade Federal do Rio de Janeiro, Rio de Janeiro, RJ, Brazil; 3. Universidade

Federal do Rio de Janeiro, Rio de Janeiro, RJ, Brazil; 4. Petrobras, Rio de Janeiro, RJ, Brazil 
Tie in of a Rigid Pipeline to a Flexible Riser - Design and Installation - Challenges and Lessons Learned OMAE2019-95057 Curti Gianbattista ${ }^{1}$ François Lirola ${ }^{2}$ Pirinu Gianluigi ${ }^{3}$ Pavone Diego ${ }^{1}$ Perrin Frederic ${ }^{3}$ 1. Saipem, Fano, Italy; 2. Saipem, Montigny le Bretonneux, France; 3. Saipem, Saint Quentin Yvelines, France

\section{Pipelines, Risers, and Subsea Systems}

\section{4-3-2 Installation}

Tuesday June 11

Room Crowne Plaza, Castle 3 | 08:30 - 10:00

Session Chair: Julian Hallai, ExxonMobil, USA

Session Co-Chair: Chris Timms, C-FER Technologies, Canada

Improved Pipelay Equipment Settings Methodology

for Rigid Pipes OMAE2019-95475

Geoffrey Marmonier, Andrew Harrop, Ludovic Lacan

TechnipFMC, Westhill, United Kingdom

\section{Prediction of Liner Wrinkling during High Strain}

Bending of Mechanically Lined Pipe OMAE2019-95511

Aurelien Pepin ${ }^{1}$ Tomasz Tkaczyk ${ }^{2}$ Martinez Michael ${ }^{3}$ Noel 0 'Dowd ${ }^{4}$ Kamran Nikbin $^{5}$

1. Technip UK/Imperial College London, Aberdeen, United Kingdom;2. TechnipFMC,

Westhill, United Kingdom; 3. IFP Energies Nouvelles, Solaize, France; 4. University of

Limerick, Limerick, Ireland; 5. Imperial College London, London, United Kingdom

Influence of Lined-pipe Fabrication on Liner Wrinkling OMAE2019-95743

Ilias Gavriilidis' Spyros A. Karamanos ${ }^{2}$

1. School of Engineering, Edinburgh, United Kingdom;

2. The University of Edinburgh, Edinburgh, United Kingdom

Polymer Liner Collapse Design Model OMAE2019-96219

Scott Mathieson, Colin Jones, Allan Feeney

Swagelining, Glasgow, United Kingdom

\section{Ocean Space Utilization}

\section{5-2-1 Aquaculture I: Design and Modeling I}

Tuesday June 11

Room SEC, Dochart 1 | 08:30 - 10:00

Session Chair: Muk Chen Ong, University of Stavanger, Norway

Session Co-Chair: Lin Li, University of Stavanger, Norway

\section{A Multipurpose Framework for Modelling and Simulation of Marine Aquaculture Systems OMAE2019-95414 Biao Su' Karl J. Reite' Martin Føre ${ }^{2}$ Karl Gunnar Aarsaether ${ }^{3}$ Morten Omholt Alver $^{2}$ Per Christian Endresen ${ }^{1}$ David Kristiansen' Joakim Haugen ${ }^{1}$ Walter Caharija ${ }^{1}$ Andrei Tsarau 1. SINTEF Ocean, Trondheim, Norway; 2. Norwegian University of Science and Technology, Trondheim, Norway; 3. SINTEF Ocean, Tromsoe, Norway \\ Coupled Motion and Sloshing Analysis of a Cylindrical Closed Fish Cage in Regular Waves 0MAE2019-96002 Yuelin $\operatorname{Tan}^{1}$ Yanlin Shao ${ }^{2}$ Robert Read ${ }^{3}$ 1. Technical University of Denmark, Vanløse, Denmark; 2. Technical University of Denmark, Kongens Lyngby, Denmark; 3. Delft University of Technology/Mechanical Engineering, Lyngby, Denmark}

Hydrodynamic Load Modeling for Offshore Free-floating Macroalgal Aquaculture under Extreme Environmental Conditions OMAE2019-96803 Ming Chen ${ }^{1}$ Solomon Yim ${ }^{1}$ Daniel Cox Taiping Wang ${ }^{2}$ Michael Huesemann ${ }^{2}$ Zhaoging Yang ${ }^{3}$ Thomas Mumford ${ }^{4}$ Geoffrey Wood 1. Oregon State University, Corvallis, OR, USA; 2. Pacific Northwest National Laboratory, Sequim, WA, USA; 3. Pacific Northwest National Laboratory, Seattle, WA, USA; 4. Marine Agronomics, LLC, Olympia, WA, USA; 5. Composite Recycling Technology Center, Port Angeles, WA, USA
Current Induced Drag Forces on Cultivated Sugar Kelp OMAE2019-96375 Per Christian Endresen, Carina Norvik, David Kristiansen, Jens Birkevold, Zsolt Volent SINTEF Ocean, Trondheim, Norway

\section{Ocean Engineering}

\section{6-2-1 Coastal Engineering I}

Tuesday June 11 Room SEC, M4 | 08:30 - 10:00 Session Chair: Masoud Hayatdavoodi, University of Dundee, United Kingdom

Numerical Study on Influence of Width of Vegetated

Zone on Wave Attenuation OMAE2019-95713

Jun Tang, Yongming Shen

Dalian University of Technology, Dalian, China

Wave Response of a Novel Floating Breakwater-windbreak with Oscillating Water Columns OMAE2019-95860 Mengmeng Han' Chien Ming Wang Wenhui Duan ${ }^{2}$ 1. University of Queensland, Brisbane, QLD, Australia; 2. Monash University, Clayton, VIC, Australia

Solitary Wave Interaction with Vertical Porous Barriers OMAE2019-95194 Vivek Francis' Balaji Ramakrishnan² Murray Rudman ${ }^{3}$ 1. IITB-Monash Research Academy, Mumbai, India; 2. IIT Bombay, Mumbai, India; 3. Monash University, Melbourne, VIC, Australia

Bore Pressure on Horizontal and Vertical Surfaces OMAE2019-96013 Jiaqi Liu' Masoud Hayatdavoodi' R. Cengiz Ertekin² 1. University of Dundee, Dundee, United Kingdom; 2. University of Hawaii, Township of Washington, NJ, USA

Effect of the Wind Drag Estimation Methods on Numerical Storm Surge Modeling 0MAE2019-95895 C. Gowri Shankar, Manasa Ranjan Behera Indian Institute of Technology Bombay, Mumbai, India

\section{Ocean Engineering}

\section{6-4-3 Marine Engineering and Applications I}

Tuesday June 11 Room SEC, M2 \& M3 | 08:30 - 10:00

Session Chair: Muk Chen Ong, University of Stavanger, Norway Session Co-Chair: Guang Yin, University of Stavanger, Norway

Multi Objective Design of Ships: A Pareto Procedure OMAE2019-96643 Sander Calisal

University of British Columbia, Vancouver, BC, Canada

Noise Reduction of Bio-inspired Marine Propeller based on Serrated Trailing Edge OMAE2019-96782

Wencai Zhu, Hongtao Gao

Dalian Maritime University, Dalian, China

Experimental and Numerical Study Propeller

Shaft Oil Whip OMAE2019-95331

Adarsh D' Kiran Vijayan² Kartheek Amaroju'

1. OENA, IIT Kharagpur, Kharagpur, India; 2. IIT Kharagpur, Kharagpur, India

Openmodelica Modelling of the Thruster in a Compact Work-class Remotely Operated Vehicle 0MAE2019-96839

Yihan Xing ${ }^{1}$ Kristian Fotland ${ }^{2}$ Muk Chen Ong ${ }^{1}$

1. University of Stavanger, Stavanger, Norway; 2. IKM Technology AS, Bryne, Norway 


\section{CFD \& FSI}

\section{8-2-1 Free Surface Modeling}

Tuesday June 11

Room SEC, Lomond Auditorium | 08:30 - 10:00

Session Chair: Hans Bihs, Norwegian University of Science and Technology, Norway

Session Co-Chair: Arun Kamath, Norwegian University

of Science and Technology, Norway

Numerical Simulation of Solitary Wave Breaking with Adaptive Mesh Refinement OMAE2019-95224

Yunxing Zhang, Wenyang Duan, Kangping Liao, Shan Ma, Guihua Xia

Harbin Engineering University, Harbin, China

\section{REEF3D Wave Generation Interface for}

Commercial CFD Codes OMAE2019-95921

Csaba Pakozdi ${ }^{1}$ Hans Bihs ${ }^{2}$ Arun Kamath ${ }^{3}$ Elin Marita H. Hermundstad

1. SINTEF Ocean, Trondheim, Norway; 2. Norwegian University of Science and Technology, Sør-

Trøndelag, Norway; 3. Norwegian University of Science and Technology, Trondheim, Norway

Numerical Study on the Temporal Discretization Schemes

in Two-phase Wave Simulation OMAE2019-96278

Young Jun Kim ${ }^{1}$ Benjamin Bouscasse' ${ }^{1}$ Sopheak Seng ${ }^{2}$ David Le Touze ${ }^{1}$

1. Ecole Centrale de Nantes, Nantes, France; 2. Bureau Veritas, Marine \& Offshore, Paris, France

REEF3D::FNPF - A Flexible Fully Nonlinear Potential

Flow Solver on Fixed Grids OMAE2019-96524

Hans Bihs' Weizhi Wang ${ }^{2}$ Tobias Martin² Arun Kamath²

1. Norwegian University of Science and Technology, Sør-Trondelag, Norway;

2. Norwegian University of Science and Technology, Trondheim, Norway

\section{Ocean Renewable Energy}

\section{9-3-1 Wave Energy Converter Control Systems Competition (WECCCOMP)}

Tuesday June 11

Room SEC, Carron 1 | 08:30 - 10:00

Session Chair: John Ringwood, Maynooth University, Ireland

\section{The WECCCOMP Wave Energy Control}

Competition - Overview OMAE2019-95216

John Ringwood ${ }^{1}$ Francesco Ferri' ${ }^{2}$ Nathan Tom ${ }^{3}$ Kelley Rueh ${ }^{4}$

Nicolás Faedo ${ }^{5}$ Giorgio Bacelli ${ }^{4}$ Yi-Hsiang Yu ${ }^{3}$ Ryan $\mathrm{Coe}^{4}$

1. Maynooth University, County Kildare, Ireland; 2. Dept. of Civil

Engineering, Aalborg University, Aalborg, Denmark; 3. National Renewable

Energy Laboratory, Golden, CO, USA; 4. Sandia National Laboratories,

Albuquerque, NM, USA; 5. Maynooth University, Maynooth, Ireland

An Energy-maximising MPC Solution to the WEC

Control Competition OMAE2019-95197

Paolino Tona, Guillaume Sabiron, Hoai-Nam Nguyen

IFP Energies Nouvelles, Solaize, France

Development of a Model Predictive Controller for the Wave

Energy Converter Control Competition OMAE2019-95544

Bradley A. Ling

Northwest Energy Innovations, Portland, OR, USA

Learning a Predictionless Resonating Controller

for Wave Energy Converters OMAE2019-95619

Shuo Shi, Ron Patton, Mustafa Abdelrahman, Yanhua Liu

University of Hull, hull, United Kingdom

\section{Offshore Geotechnics}

\section{0-4-1 Pile Foundations I}

Tuesday June 11

Room Crowne Plaza, Jura | 08:30 - 10:00

Session Chair: Susan Gourvenec, University of Southampton/

Southampton Marine and Maritime Institute, United Kingdom

Method Evaluating Axial Response of Vertically-loaded

Piles during Spudcan Penetration OMAE2019-95422

Yifei Fan, Jianhua Wang

Tianjin University, Tianjin, China

Feasibility Study of an Innovative Large Open-ended Monopile

Foundation for Offshore Wind Turbine OMAE2019-95641

Jiale Li' Xuefei Wang ${ }^{1}$ Xiong Yu$^{2}$ Yougang Tang ${ }^{3}$

1. Hebei University of Technology, Tianjin, China; 2. Case Western Reserve

University, Cleveland, OH, USA; 3. Tianjin University, Tianjin, China

Evaluation of Uncertainty of Damage Results in Experimental

Modelling of Monopile Foundation Scour Protection OMAE2019-95793

Minghao Wu' Jonas Arnout ${ }^{1}$ Josep Molina Ruiz ${ }^{1}$

Carlos Arboleda Chavez' Vasiliki Stratigaki ${ }^{2}$ Peter Troch ${ }^{1}$

1. Ghent University, Zwijnaarde, Belgium; 2. Ghent University, Ghent, Belgium

Application of Friction Fatigue Pile Driving

Models in GRLWEAP OMAE2019-95944

Henry Milewski, Justin Kennedy

TechnipFMC, Westhill, United Kingdom

\section{Petroleum Technology}

\section{1-7-2 Well Drilling Fluids and Hydraulics II}

Tuesday June 11

Room Crowne Plaza, Barra | 08:30 - 10:00

Session Chair: Ergun Kuru, University of Alberta, Canada

Session Co-Chair: Majid Bizhani, University of British Columbia, Canada

Hydraulic Behaviour in Cased and Open Hole Sections

in Highly Deviated Wellbores OMAE2019-96347

Jan David Ytrehus' Bjørnar Lund' Ali Taghipour' Birgitte Ruud Kosberg'

Luca Carazza ${ }^{2}$ Knud Richard Gyland ${ }^{3}$ Arild Saasen ${ }^{4}$

1. SINTEF, Trondheim, Norway; 2. Aker BP, Stavanger, Norway; 3. M-I Swaco

Schlumberger Fluids, Stavanger, Norway; 4. UiS, Gullaug, Norway

Use of Tracer Particles for Tracking Fluid Interfaces

in Primary Cementing OMAE2019-96400

Amir Taheri' Jan David Ytrehus ${ }^{2}$ Ali Taghipour ${ }^{3}$ Bjørnar Lund ${ }^{2}$

Alexandre Lavrov² Malin Torsæter ${ }^{2}$

1. Norwegian University of Science and Technology, Trondheim, Norway; 2. SINTEF,

Trondheim, Norway; 3. Sintef Petroleum Research AS, Trondheim, Norway

Hole Cleaning Related Stuck Pipe Analysis during

Extended Reach Drilling by using a Transient Cuttings

Transport Model - A Case Study OMAE2019-96617

Hao Zeng ${ }^{1}$ Yijin Zeng ${ }^{1}$ Feifei Zhang ${ }^{2}$ Guang Yang ${ }^{3} Y_{\text {uezhi Wang }}{ }^{2} \mathrm{X}_{\text {Wang }}{ }^{2}$

1. Sinopec, Beijing, China; 2. Yangtze University, Wuhan, China; 3. CNPC, Beijing, China

Mechanical Friction in Well Construction and Laboratory

Testing of Friction Coefficients OMAE2019-96594

Parisa Ghaedi ${ }^{1}$ Mahmoud Khalifeh ${ }^{1}$ Arild Saasen ${ }^{2}$ Helge Hodne ${ }^{1}$

Tor Henry Omland ${ }^{3}$ Farzad N. Shogh ${ }^{3}$

1. University of Stavanger, Stavanger, Norway; 2. University of Stavanger,

Gullaug, Norway; 3. Equinor ASA, Stavanger, Norway 


\section{Rodney Eatock Taylor Honouring Symposium on Marine and Offshore Hydrodynamics}

\section{2-4-1 Hydrodynamic Aspects of Offshore Renewable Energy}

Tuesday June 11

Room SEC, Carron 2 | 08:30 - 10:00

Session Organizer: Xingya Feng, University of Oxford, United Kingdom

Session Co-Chair: Deborah Greaves, University of Plymouth, United Kingdom

Experimental and Numerical Investigations on Wave Dynamics

of a Dual-chamber OWC Wave Energy Device OMAE2019-95165

Dezhi Ning ${ }^{1}$ Rongquan Wang ${ }^{1}$ Qingping Zou ${ }^{2}$ Bin Teng ${ }^{1}$

1. Dalian University of Technology, Dalian, China,

2. Heriot-Watt University, Edinburgh, United Kingdom

Evaluation of the Performance of an Integrated WEC

Type of Breakwater System OMAE2019-95739

Haoyu Ding Jun Zang' Dezhi Ning ${ }^{2}$ Xuanlie Zhao ${ }^{2}$

Qiang Chen ${ }^{1}$ Chris Blenkinsopp Junliang Gao ${ }^{3}$

1. University of Bath, Bath, United Kingdom; 2. Dalian University of Technology,

Dalian, China; 3. Jiangsu University of Science and Technology, Zhenjiang, China

Rigid and Flexible Inter-connection of Arrays of Oscillating Water Column Wave Energy Converters:

Findings from the WETFEET Project OMAE2019-96573

Keri Collins ${ }^{1}$ Deborah Greaves ${ }^{2}$ Martyn Hann ${ }^{1}$ Ben Howey ${ }^{1}$

Rui P. F. Gomes ${ }^{3}$ Joao C.C. Henriques ${ }^{3}$

1. School of Engineering, University of Plymouth, Plymouth, United

Kingdom;2. University of Plymouth, Plymouth, United Kingdom; 3. IDMEC,

Instituto Superior Técnico, Universidade de Lisboa, Lisboa, Portugal

Time-domain Diffraction Modelling with Mean Force

Effects and Experimental Comparison with Slack-moored

M4 Wave Energy Converter OMAE2019-96756

Peter Stansby, Efrain Carpintero Moreno

University of Manchester, Manchester, United Kingdom

\section{Takeshi Kinoshita Honoring Symposium on Offshore Technology}

\section{3-2-2 Experiments and Numerical Validation}

Tuesday June 11

Room SEC, Boisdale 2 | 08:30 - 10:00

Session Chair: Marcio Yamamoto, National Maritime Research Institute, Japan

Session Co-Chair: Celso Morooka, University of Campinas, Brazil

Ship as a Wave Buoy - Estimating Relative Wave

Direction from In-service Ship Motion Measurements

using Machine Learning OMAE2019-96201

Bart Mak, Bulent Duz

Maritime Research Institute in the Netherlands (MARIN), Wageningen, Netherlands

Ship as a Wave Buoy - Using Simulated Data to Train Neural Networks for Real Time Estimation of Relative Wave Direction OMAE2019-96225 Bart Mak, Bulent Duz

Maritime Research Institute in the Netherlands (MARIN), Wageningen, Netherlands
An Experimental Study of Snap Loads on a Vertical Hanging Cable System OMAE2019-96424

Wei-Ting Hsu' Tzu-Ching Chuang ${ }^{2}$ Wen-Yang Hsu ${ }^{3}$ Krish Sharman $^{4}$ Ray-Yeng Yang ${ }^{2}$ 1. Energo Engineering, a KBR Comapny, Houston, TX, USA; 2. National Cheng Kung University, Tainan, Taiwan; 3. Tainan Hydraulics Laboratory, National Cheng Kung University, Tainan, Taiwan; 4. University of Massachusetts Amherst, Amherst, MA, USA

Experimental and Numerical Study of Motion of Rotating Drill Pipe Owing to Magnus Effect OMAE2019-96602

Tomoya Inoue ${ }^{1}$ Hiroyoshi Suzuki ${ }^{2}$ Tokihiro Katsui ${ }^{3}$ Keita Tsuchiya ${ }^{2}$ Yusuke Notani ${ }^{3}$

1. JAMSTEC, Yokosuka, Japan; 2. Osaka University, Suita, Japan; 3. Kobe University, Kobe, Japan

\section{Refreshment Break}

10:00 - 10:30

Location: Hall 5 (SEC)

Sponsored by Babcock

\section{babcock}

\section{CONCURRENT SESSIONS \\ 10:30 - 12:00}

\section{Offshore Technology}

\section{1-1-4 Fixed Platforms and Foundations}

Tuesday June 11

Room SEC, Alsh 1 | 10:30 - 12:00

Session Chair: Partha Chakrabarti, Zentech Inc, USA

Co-Chair: Marc Cahay, TechnipFMC

Punch-through Structural Analysis of Jack-up Rigs during

Preloading of the Foundations OMAE2019-95537

Partha Chakrabarti, Abhijeet Chawan

Zentech, Inc., Houston, TX, USA

Critical Review of Early Age Cycling Effects on the Capacity of Pile to Sleeve Grouted Connections as Treated in ISO 19902 OMAE2019-95626 Andi Merxhani' Jacob Fisker Jensen ${ }^{2} J o a 0$ Caetano ${ }^{2}$ Casper Klintø Christiansen ${ }^{2}$ 1. Ramboll Energy, Copenhagen, Denmark; 2. Ramboll Offshore Wind, Copenhagen, Denmark

Effects of Cap Bottom Elevation on Wave Loads on the Piles under the Cap OMAE2019-96777

Jifu Zhou, Xu Wang

Institute of Mechnaics, Chinese Academy of Sciences, Beijing, China

\section{Offshore Technology}

\section{1-2-3 Dynamic Positioning II}

Tuesday June 11 Room SEC, Dochart 2 | 10:30 - 12:00

Session Chair: Xinshu Zhang, Shanghai Jiao Tong University, China

Session Co-Chair: Xinliang Tian, Shanghai Jiao Tong University, China

Session Co-Chair: Dimitris Chalkias, GustoMSC, Netherlands

Learning from Our Dynamic Positioning Events OMAE2019-96710

Arne Kvitrud

Petroleum Safety Authority (PSA), Stavanger, Norway 
QUAD Lift: Enabling Lifting of Larger Integrated Topsides OMAE2019-95375 Ivan van Winsen, Radboud van Dijk

Heerema Marine Contractors, Leiden, Netherlands

Can the Shore Tension System Reduce Sloshing during

LNG Terminal Loading Operations OMAE2019-96548

Olger Koop

Aktis Hydraulics, Zwolle, Netherlands

Realistic Adaptive DP Controller for Flotel Operating in Side-by-side Configuration with FPSO OMAE2019-96577

Anurag Yenduri' Allan Magee ${ }^{1}$ Jing Liu' ${ }^{2} W_{\text {Wei }} \mathrm{u}^{2}$ Ankit Choudhary $^{2}$ Anis Altaf Hussain ${ }^{2}$

1. National University of Singapore, Singapore, Singapore;

2. Keppel Offshore and Marine Technology Centre, Singapore, Singapore

\section{Structures, Safety and Reliability}

\section{2-4-2 Fatigue and Fracture Reliability II}

Tuesday June 11

Room SEC, Alsh 2 | 10:30 - 12:00

Session Chair: Nianzhong Chen, Tianjin University, China

Session Co-Chair: Fang Wang, Shanghai Ocean University, China

Low-cycle-fatigue Crack Closure Effect of Ship Cracked Plate considering the Accumulative Plastic Damage OMAE2019-95230 Yuelin Song, Ping Yang, Ziya Peng, Wei Jiang, Kang Hu Wuhan University of Technology, Wuhan, China

Multiobjective Reliability-based Design of Ship Structures Subjected to Fatigue Damage and Compressive Collapse OMAE2019-96666 Yordan Garbatov, Huang Yingcai University of Lisbon, Lisboa, Portugal

Comparative Study on Fatigue Damage Assessment of a Structure Member in a Bulk Carrier using Various Environmental Conditions OMAE2019-96760

Fredhi Agung Prasetyo' Naoki Osawa² Mohammad Arif Kurniawan' Siti Komariyah' 1. Research \& Development Division, Biro Klasifikasi Indonesia, Jakarta, Indonesia; 2. Osaka University, Suita, Japan

Fatigue Behavior of Large, Rolled-after-heat-treatment and Hot-dip Galvanized HT Bolts OMAE2019-96808 Julian Unglaub, Klaus Thiele Institute of Steel Structures, TU Braunschweig, Braunschweig, Germany

\section{Structures, Safety and Reliability}

\section{2-9-2 Extreme Loading and Responses II}

Tuesday June 11

Room Crowne Plaza, Castle 1 | 10:30 - 12:00

Session Chair: Kazuhiro lijima, Dept of NAOE, Osaka University, Japan

Session Co-Chair: Hans Bihs, Norwegian University of Science and Technology, Norway

Comparison of the Environmental Contour Method and

Response-based Analysis using Response Emulator for

Estimating Extreme Ship Responses OMAE2019-95098

Erik Vanem, Bingjie Guo

DNV GL, Høvik, Norway

Long-term Extreme Response Analysis for a Straight Floating Bridge Across the Bjørnafjord OMAE2019-95212 Finn-Idar G. Giske ${ }^{1}$ Arnt G. Fredriksen ${ }^{2}$

1. Multiconsult, Oslo, Norway; 2. Multiconsult, Tromsø, Norway
A New Approach for Environmental Contour and Multivariate De-clustering OMAE2019-95993 Quentin Derbanne ${ }^{1}$ Guillaume de Hauteclocque ${ }^{2}$

1. Bureau Veritas, Marine \& Offshore, Paris, France; 2. Bureau Veritas, Paris, France

Experimental Assessment of Vertical Shear Force and

Bending Moment in Severe Sea Conditions OMAE2019-96272

Boris Horel' Benjamin Bouscasse ${ }^{2}$ Arnaud Merrien ${ }^{1}$ Guillaume de Hauteclocque 1. Ecole Centrale Nantes, LHEEA res. dept. (ECN and CNRS), Nantes, France;

2. Ecole Centrale de Nantes, Nantes, France; 3. Bureau Veritas, Paris, France

\section{Structures, Safety and Reliability}

\section{2-12-2 Structural Analysis and Optimization II}

Tuesday June 11

Room Crowne Plaza, Castle 2 | 10:30 - 12:00

Session Chair: Paulo M. Videiro, LACEO/COPPE/

Federal University of Rio De Janeiro, Brazil

Session Co-Chair: Jonas W. Ringsberg, Chalmers University of Technology, Sweden

Numerical Simulation of Container Stacks Dynamics

under Typical Motion Excitation OMAE2019-95644

Chuntong Li, Deyu Wang, Jiaqi Liu

Shanghai Jiao Tong University, shanghai, China

Dynamic Response of Metallic Y-type Core Sandwich Panels Subjected to Air Blast Loading - Numerical Investigation OMAE2019-96628 Ting Liu, Yuansheng Cheng, Jun Liu, Ganchao Chen, Changhai Chen, Pan Zhang Huazhong University of Science and Technology, Wuhan, China

Quasi-static and Dynamic Compressive Behaviors of Closed-cell Stochastic Foams based on Voronoi Model OMAE2019-95924 Jianyong Chen, Jun Liu, Yuansheng Cheng, Pan Zhang Huazhong University of Science and Technology, Wuhan, China

\section{Materials Technology}

\section{3-4-1 Steel Performance in Sour Environment}

Tuesday June 11 Room SEC, Boisdale 1 | 10:30 - 12:00

Session Chair: Agnes Marie Horn, DNV GL, Norway

Session Co-Chair: Carol Johnston, TWI Ltd, United Kingdom

Fatigue Performance and Crack Growth Assessments of Riser Welds in Mild Sour Environment OMAE2019-96329 Rupak Ghosh ${ }^{1}$ Robert Aune ${ }^{2}$ Carl Popelar $^{3}$ 1. ExxonMobil, Spring, TX, USA; 2. ExxonMobil Production Company, Spring, TX, USA; 3. Southwest Research Institute, San Antonio, TX, USA

Local Hard Zones in Sour Service Steels OMAE2019-96593 Doug Fairchild' Brian Newbury' Tim Anderson' Neeraj Thirumalai' 1. ExxonMobil Production Company, Spring, TX, USA;

2. Exxonmobil Research and Engineering $(0$, Annandale, NJ, USA

Qualification of TMCP Pipe for Sour Service: Mitigation of Local Hard Zones OMAE2019-96614 Brian Newbury ${ }^{1}$ Doug Fairchild ${ }^{1}$ Andrew Prescott ${ }^{1}$ Andrew Wasson ${ }^{2}$ Tim Anderson $^{1}$ 1. Exxonmobil Production Company, Spring, TX, USA;

2. Exxonmobil Upstream Research Company, Spring, TX, USA 


\author{
An Investigation Concerning the Sulfide Stress \\ Cracking of TMCP Steels OMAE2019-96556 \\ Xin Yue ${ }^{1}$ Andrew Wasson' David Fischer' Tim Anderson ${ }^{2}$ \\ Brian Newbury ${ }^{2}$ Weiji Huang ${ }^{2}$ Doug Fairchild ${ }^{2}$ \\ 1. Exxonmobil Upstream Research Company, Spring, TX, USA; \\ 2. ExxonMobil Production Company, Spring, TX, USA
}

\section{Pipelines, Risers, and Subsea Systems}

\section{4-1-3 Flexible Pipes III}

Tuesday June $11 \quad$ Room Crowne Plaza, Staffa / Shuna | 10:30-12:00

Session Chair: Murilo Augusto Vaz, COPPE/UFRJ, Brazil

Session Co-Chair: Anh Tuan Do, TechnipFMC, France

Evaluation of the Temperature Effect on the Viscoelastic

Responses of Flexible Risers OMAE2019-95141

Junpeng Liu' Jinsheng Ma ${ }^{2}$ Murilo Augusto Vaz ${ }^{3}$ Menglan Duan'

1. China University of Petroleum, Beijing, China; 2. Fudan University, Shanghai,

China; 3. COPPE/Universidade Federal do Rio de Janeiro, Rio de Janeiro, RJ, Brazil

Study on Mechanical Behavior of Tensile Armor Wires of Marine

Flexible Pipes and Cables during Winding Process OMAE2019-95426

Qingzhen Lu' Shanghua Wu ${ }^{2}$ Dong Wang Zhixun Yang ${ }^{2}$

Yuanchao Yin² Haitao Hü Jun Yan² Qianjin Yue

1. Dalian University of Technology, Panjin, China; 2. Dalian

University of Technology, Dalian, China

Recent Advances in the Prediction and Mitigation of Flow Induced

Pulsations in Flexible Risers and Flowlines OMAE2019-95906

Stefan Belfroid, Nestor Gonzalez Diez, Harry Korst

TNO, Delft, Netherlands

Effect of Local Model Dynamics on Flexible Riser Tensile

Armor Wire Stress Predictions OMAE2019-95303

Gabriel Rombado ${ }^{1}$ Krassimir Doynov² Nathan Cooke ${ }^{3}$ Arya Majed $^{4}$

1. ExxonMobil Production Company, Spring, TX, USA; 2. ExxonMobil Upstream Integrated

Solutions, Spring, TX, USA; 3. INTECSEA, St. John's, NL, Canada; 4. INTECSEA, Houston, TX, USA

\section{Pipelines, Risers, and Subsea Systems}

\section{4-3-6 ECA}

Tuesday June 11

Room Crowne Plaza, Castle 3 | 10:30 - 12:00

Session Chair: Bostjan Bezensek, Shell, United Kingdom

Session Co-Chair: Andrew Cosham, Ninth Planet Engineering Ltd, United Kingdom

Fatigue Life Assessment for Pipeline Dents

under Highway Crossings OMAE2019-95450

M Liu' Colin Cross ${ }^{2}$ Jason Brown

1. Aker Solutions, Windsor, United Kingdom; 2. Aker Solutions, London,

United Kingdom; 3. Aker Solutions, Aberdeen, United Kingdom

Integrity Assessment of Subsea Pipeline Dent /

Buckle using ILI Data OMAE2019-95470

Gurumurthy Kagita, Gudimella G. S., Achary, Mahesh Babu Addala, Balaji Srinivasan,

Penchala S. K., Pottem, Deepak Gupta, Subramanyam V. R., Sripada

Engineers India Limited, Gurugram, India

ECAs and Lateral Buckling OMAE2019-95529

Andrew Cosham ${ }^{1}$ Malcolm Carr ${ }^{2}$ lan MacRae ${ }^{3}$ Kenneth Macdonald ${ }^{4}$

1. Ninth Planet Engineering Ltd, Newcastle upon Tyne, United Kingdom;

2. Crondall Energy, Cramlington, United Kingdom; 3. Crondall Energy, Aberdeen,

United Kingdom; 4. University of Stavanger, Hafrsjord, Norway
Dented Externally-pressurised Pipes Subjected

to Cyclic Axial Loading OMAE2019-95814

Konstantinos Chatziioannou, Yuner Huang, Spyros A. Karamanos

The University of Edinburgh, Edinburgh, United Kingdom

\section{Ocean Space Utilization}

\section{5-2-2 Aquaculture II: Design and Modeling II}

Tuesday June 11

Room SEC, Dochart 1 | 10:30 - 12:00

Session Chair: Yanlin Shao, Technical University of Denmark, Denmark

Session Co-Chair: Xu Xiang, Norwegian Public Roads Administration, Norway

Numerical Modelling of Net Motion in Waves

and Current using CFD OMAE2019-95154

Tobias Martin ${ }^{1}$ Arun Kamath ${ }^{1}$ Hans Bihs ${ }^{2}$

1. Norwegian University of Science and Technology, Trondheim, Norway;

2. Norwegian University of Science and Technology, Sør-Trøndelag, Norway

Numerical Study of a Single-point Mooring Gravity Fish Cage with

Different Deformation Suppression Methods OMAE2019-96079

Hui Cheng' Karl Gunnar Aarsaether ${ }^{2}$ Lin Li' Muk Chen Ong ${ }^{1}$

1. University of Stavanger, Stavanger, Norway; 2. SINTEF Ocean, Tromsoe, Norway

Experimental Study on the Motion of a Flexible Hose Net used in Automated Net-hauling System OMAE2019-95670 Yue Li' ${ }^{1}$ Yoichi Mizukami ${ }^{2}$ Takero Yoshida ${ }^{2}$ Qiao Li $^{2}$ Jialin Han² Daisuke Kitazawa ${ }^{3}$ 1. The University of Tokyo, Tokyo, Japan; 2. Institute of Industrial Science, The University of Tokyo, Kashiwa, Japan; 3. The University of Tokyo, Kashiwa, Japan

Three-Dimensional Physical Environment Modelling for Integrated Multi-trophic Aquaculture (IMTA)

Implementation in Onagawa Bay, Japan OMAE2019-95672

Jinxin Zhou' Takero Yoshida ${ }^{2}$ Junbo Zhang ${ }^{3}$ Sanggyu Park' Daisuke Kitazawa ${ }^{4}$ 1. Institute of Industrial Science, The University of Tokyo, Chiba, Japan; 2. Institute of Industrial Science, The University of Tokyo, Kashiwa, Japan; 3. College of Marine Sciences, Shanghai Ocean University, Shanghai, China; 4. The University of Tokyo, Kashiwa, Japan

\section{Ocean Engineering}

\section{6-2-2 Coastal Engineering II}

Tuesday June 11 Room SEC, M4 | 10:30-12:00

Session Chair: Neelamani Subramaniam, Kuwait Institute for Scientific Research, Kuwait Session Co-Chair: Ghassan El Chahal, COWI A/S, Denmark

Effect of Reynolds Number on Local Scour around a

Monopile in Steady Current OMAE2019-96735

Xiaofan Lou, Kaibing Zhang, Zhenhong Chen

Dalian University of Technology, Dalian, China

Bulk and LNG Terminals: An Advanced Approach for Downtime Estimate and Breakwater Optimization OMAE2019-95313 Ghassan El Chahal COWI A/S, Kongens Lyngby, Denmark

Numerical Simulation of Consecutive Multiple Lateral Impact on the Reinforced Concrete Pier OMAE2019-96508 Shuai Yang ${ }^{1}$ Xiaozhou Xia ${ }^{2}$ Qing Zhang ${ }^{2}$ Xue-gang Wang ${ }^{1}$ Ying Zong-quan ${ }^{1}$ 1. CCCC Fourth Harbor Engineering Institute Co., Ltd., Guangzhou, China; 2. Hohai University, Nanjing, China 
Coastal Engineering Analysis, Field Measurments, Numerical Modeling and Design for the Optimized Extension of the Beach in Ras AI-Ardh Area, Salmiya, Kuwait OMAE2019-95236 Neelamani Subramaniam, Bassam Shuhaibar, Khaled Al-SAlem, Yousef Al-Osairi, Qusaie E. Karam, Dana Al-Houti, Noor Al-Anjari Kuwait Institute for Scientific Research, Shuwaikh, Kuwait

\section{Ocean Engineering}

\section{6-4-4 Marine Engineering and Applications II}

Tuesday June 11 Room SEC, M2 \& M3 | 10:30 - 12:00

Session Chair: Muk Chen Ong, University of Stavanger, Norway

Session Co-Chair: Zhenhui Liu, Aker Solutions AS, Norway

A Broadband Underwater Acoustic Signal Array Signal Processing Method based on the Joint Sparsity

of Signal Spatial Domain OMAE2019-95530

Daqian He, Dahai Zhang, Congying Wang, Xirui Peng

China Ship Development and Design Center, Wuhan, China

Research on Characteristics of Removing Particles in Ship

Exhaust Gas by Charged Droplets OMAE2019-96716

Lei Jiao ${ }^{1}$ Zhaohui Qian' Dekai Huang' Peilin Zhou ${ }^{2}$ Pengfei Chen ${ }^{1}$ Lida Meng $^{1}$

1. Zhejiang University, Zhoushan, China; 2. University of Strathclyde, Glasgow, United Kingdom

Design of Performance Monitoring System for Diesel

Generators of Offshore Drilling Platform OMAE2019-96726

Lei Hu, Jianguo Yang

Wuhan University of Technology, Wuhan, China

The Design and Simulation of Hull Segmentation Docking

and Correcting Hydraulic System OMAE2019-95926

Yuhao Zeng, Zuyao Yu, Tang Xu

Huazhong University of Science and Technology, Wuhan, China

\section{CFD \& FSI}

\section{8-2-2 Free Surface Loading and Structure Interaction I}

Tuesday June 11

Room SEC, Lomond Auditorium | 10:30 - 12:00

Session Chair: Tim Bunnik, MARIN, Netherlands

Session Co-Chair: Csaba Pakozdi, SINTEF Ocean, Norway

A Ghost Cell Method Based FDM-FEM Model for Free-surface

Flow Interactions with Deformable Structures OMAE2019-95209

Xizeng Zhao, Zhijian Yang, Kaiyuan Zheng, Songchang Duan

Zhejiang University, Zhoushan, China

Numerical Prediction of the Ship Resistance and Vertical

Motions in Regular Head Waves OMAE2019-95237

Adham S. Bekhit, Adrian Lungu

"Dunărea de Jos" University of Galati, Galati, Romania

Unsteady Numerical Simulation of the Behavior of

a Ship Moving in Head Sea OMAE2019-95239

Adrian Lungu

"Dunărea de Jos" University of Galati, Galati, Romania

Numerical Investigation of the Roll Decay of a Container Ship

Moving with Forward Speed in Calm Water OMAE2019-95240

Adrian Lungu

"Dunărea de Jos" University of Galati, Galati, Romania

\section{Ocean Renewable Energy}

\section{9-4-4 Optimization and Load Analysis}

Tuesday June 11

Room SEC, Carron 1 | 10:30 - 12:00

Session Chair: Yi-Hsiang Yu, National Renewable Energy Laboratory, USA

Making Effective WEC Design Choices based on

Simulation and Analysis OMAE2019-95138

Charlene Vance, Jonas W. Ringsberg, Shun-Han Yang

Chalmers University of Technology, Gothenburg, Sweden

Fluid-Structure-Soil Interaction of a Moored

Wave Energy Device OMAE2019-95419

Joe Tom ${ }^{1}$ Dirk Rijnsdorp ${ }^{1}$ Raffaele Ragni' David J White ${ }^{2}$

1. University of Western Australia, Perth, WA, Australia; 2. University

of Southampton, Southampton, United Kingdom

Shape Optimization of a Submerged Pressure Differential

Wave Energy Converter for Load Reductions OMAE2019-96390

Michael Kelly, Mohammad-Reza Alam

University of California, Berkeley, Berkeley, CA, USA

\section{Offshore Geotechnics}

\section{0-5-1 Bucket Foundations, Suction Caissons and Spudcans}

Tuesday June 11

Room Crowne Plaza, Jura | 10:30 - 12:00

Session Chair: Tulio Quiroz, Fraunhofer Institute for

Wind Energy Systems IWES, Germany

Protection of Pipelines and Cables with a Combination

of Soil and Rock Cover OMAE2019-95262

Damian R Morrow ${ }^{1}$ Andrew A Small ${ }^{2}$

1. Marine Geoengineering Ltd, Aberdeen, United Kingdom;

2. Xodus Group, Aberdeen, United Kingdom

Simplified Numerical Simulation of the Dense Sand Progressive Failure involved in Spudan Punch-through Failure OMAE2019-95911 Jun Zhao, Futai Sun, Wenbo Jin

Xi'an Shiyou University, Xi'an, China

Bearing Capacities of Shallow Skirted Foundations after the Action of Multi-directional Cyclic Displacements considering Soil Degradation OMAE2019-96036

Zhong Xiao, Donghai Zhang, Haixiao Liu, Ying Liu

Tianjin University, Tianjin, China

Cone Penetration Test in Stiff Over Soft Clay

in Centrifuge Test OMAE2019-96698

Qiang Xie ${ }^{1}$ Yuxia Hu' Mark J. Cassidy ${ }^{2}$ Alireza Salehi ${ }^{1}$

1. University of Western Australia, Perth, WA, Australia;

2. University of Melbourne, Parkville, VIC, Australia 


\section{Petroleum Technology}

\section{1-6-1 Integrity of Well Barriers I}

Tuesday June 11

Room Crowne Plaza, Barra | 10:30 - 12:00

Session Chair: Jan David Ytrehus, SINTEF, Norway

Numerical Modelling and Sensitivity Analysis of Gas Kick

Migration and Unloading of Riser OMAE2019-95214

Dalila Gomes ${ }^{1}$ Knut Bjørkevoll12 Kjell Kåre Fjelde Johnny Froyen ${ }^{2}$

1. University of Stavanger, Stavanger, Norway; 2. SINTEF Petroleum, Bergen, Norway

Nano-modified Rock-based Geopolymers as Supplement to

Portland Cement for Oil Well Cementing OMAE2019-95380

Mahmoud Khalifeh ${ }^{1}$ Saeed Salehi ${ }^{2}$ Aleksandra Jamrozik ${ }^{3}$

Raymos Kimanzi ${ }^{4}$ Saeid Abdollahpour ${ }^{5}$

1. UiS, Stavanger, Norway; 2. Mewbourne School of Petroleum and Geological Engineering,

Norman, OK, USA; 3. AGH University of Science and Technology, Krakow, Poland; 4. The

Oklahoma University, Norman, OK, USA; 5. University of Stavanger, Stavanger, Norway

Improved Model for Tubular Burst OMAE2019-95819

Bjorn Brechan, Sigbjørn Sangesland, Stein Dale

Norwegian University of Science and Technology, Trondheim, Norway

Next Generation Well Design and Integrity Digital Tools -

Boosting Drilling Systems Automation (DSA) OMAE2019-95995

Bjorn Brechan, Stein Dale, Sigbjørn Sangesland

Norwegian University of Science and Technology, Trondheim, Norway

\section{Rodney Eatock Taylor Honouring Symposium on Marine and Offshore Hydrodynamics}

\section{2-5-1 Non-Linear Waves and Wave Effects I}

Tuesday June 11

Room SEC, Carron 2 | 10:30 - 12:00

Session Chair: Ronald W. Yeung, University of California, Berkeley, USA

Session Co-Chair: Mamoun Naciri, Single Buoy Moorings Inc, Monaco

Numerical Analysis of Nonlinear Wave Loads on an

Offshore Wind Turbine Monopile OMAE2019-95161

Xingya Feng' Richard H.J. Willden ${ }^{1}$ Binzhen Zhou ${ }^{2}$ Thomas A.A. Adcock ${ }^{1}$

1. University of Oxford, Oxford, United Kingdom;

2. Harbin Engineering Technology, Harbin, China

Nonlinear Wave Loads on Offshore Wind Turbines:

Extreme Statistics and Fatigue OMAE2019-96679

Yu Zhang, Paul Sclavounos

MIT, Cambridge, MA, USA

Numerical Simulation of Multidirectional Waves with

Full-spectrum using DualSPHysics OMAE2019-96405

Taiga Kanehira' Hidemi Mutsuda' Samuel Draycott² David Ingram² Yasuaki Doi'

1. Hiroshima University, Higashi-Hiroshima, Japan;

2. The University of Edinburgh, Edinburgh, United Kingdom

Recreating the Draupner Wave in the Laboratory OMAE2019-96817 Mark McAllister' Sam Draycott ${ }^{2}$ Thomas A.A. Adcock' Paul Taylor' Ton van den Bremer ${ }^{1}$ 1. University of Oxford, Oxford, United Kingdom;

2. University of Edinburgh, Edinburgh, United Kingdom
Takeshi Kinoshita Honoring Symposium on Offshore Technology

\section{3-2-3 Flow-Induced Motions (FIM)}

Tuesday June 11

Room SEC, Boisdale 2 | 10:30 - 12:00

Session Chair: Rodolfo T. Gonçalves, University of Tokyo, Japan

Session Co-Chair: Longfei Xiao, Shanghai Jiao Tong University, China

Experimental Study of the Effect of the Pontoon Presence

on the Flow-induced Motions of a Semi-submersible

Platform with Four Square Columns OMAE2019-95250

Rodolfo T. Gonçalves' Hideyuki Suzuki ${ }^{2}$ Fredi Cenci ${ }^{3}$

André L. C. Fujarra ${ }^{3}$ Shinichiro Hirabayashi ${ }^{4}$

1. University of Tokyo, Bunkyo, Japan; 2. University of Tokyo, Tokyo, Japan; 3. Federal

University of Santa Catarina, Joinville, SC, Brazil; 4. The University of Tokyo, Chiba, Japan

Hydrodynamics Interactions on Vortex-induced Motions

of a Multi-body Floating System 0MAE2019-95597

Yibo Liang, Longbin Tao

University of Strathclyde, Glasgow, United Kingdom

Numerical Study on Vortex-induced Motions of Semi-submersibles with Three Columns with Different Sections Types OMAE2019-95601 Chenling Tian, Longfei Xiao, Mingyue Liu, Lijun Yang, Jing Liu Shanghai Jiao Tong University, Shanghai, China

Analysis of Wake Interaction of Oscillating

Platform with Four Columns OMAE2019-95749

Shinichiro Hirabayashi ${ }^{1}$ Murilo M. Cicolin ${ }^{2}$ Rodolfo T. Gonçalves ${ }^{3}$

Gustavo R. S. Assi ${ }^{2}$ Hideyuki Suzuki ${ }^{4}$

1. The University of Tokyo, Chiba, Japan; 2. Univeristy of Sao Paulo, São Paulo, SP, Brazil;

3. The University of Tokyo, Bunkyo, Japan; 4. The University of Tokyo, Tokyo, Japan

\section{Lunch}

12:00 - 13:30

Location: Hall 5 (SEC)

\section{CONCURRENT SESSIONS}

$13: 30-15: 00$

\section{Offshore Technology}

\section{1-1-5 Artificial Intelligence and Advance Analysis}

Tuesday June 11 Room SEC, Alsh 1 | 13:30 - 15:00

Session Chair: Allan Magee, National University of Singapore, Singapore

Session Co-Chair: Erwan Auburtin, TechnipFMC, France

Detection of Mooring Line Failure of a Spread-moored FPSO,

Part 1: Development of an Artificial Neural

Network Based Model OMAE2019-96288

Djoni Sidarta' Ho-Joon Lim' Johyun Kyoung' Nicolas Tcherniguin ${ }^{2}$

Timothee Lefebure ${ }^{2} \mathrm{Jim}$ O'Sullivan' $^{1}$

1. TechnipFMC, Houston, TX, USA;2. TechnipFMC, Paris, France 
Detection of Mooring Line Failure of a Spread-moored FPSO, Part 2: Global Performance Analysis using MLTSIM OMAE2019-96339 Johyun Kyoung ${ }^{1}$ Ho-Joon Lim Djoni Sidarta ${ }^{1}$ Nicolas Tcherniguin ${ }^{2}$ Timothee Lefebvre $^{2}$ 1. TechnipFMC, Houston, TX, USA; 2. TechnipFMC, Paris, France

Response Based Time Domain Structural Analysis on Floating Offshore Platform OMAE2019-96139 Johyun Kyoung ' Sagar Samaria' Jang Kim' Brian Duffy ${ }^{2}$ 1. TechnipFMC, Houston, TX, USA; 2. TechnipFMC/Genesis, Houston, TX, USA

A Study of Trajectory based on AIS Positions by Genetic Algorithm OMAE2019-95879 Hitoi Tamaru, Ruri Shoji

Tokyo University of Marine Science and Technology, Tokyo, Japan

\section{Offshore Technology}

\section{1-2-4 Mooring System Design and Analysis II}

Tuesday June 11

Room SEC, Dochart 2 | 13:30 - 15:00

Session Chair: David Molyneux, Memorial University of Newfoundland, Canada

Session Co-Chair: Allan Magee, National University of Singapore, Singapore

Study on Anchor Leg Installation Sequence of Nanhai

Shengli FPSO under Typhoon Season OMAE2019-96650

Hui Shen ${ }^{1}$ Huoping Wang ${ }^{2}$ Weiquan Zhu' Deyang Wang ${ }^{2}$

1. COTEC Offshore Engineering Services(Beijing), Beijing,

China; 2. CNOOC China Ltd., Shenzhen, China

A New Fully Detailed Finite Element Model of Wire Rope for

Fatigue Life Estimate of a Mooring Line OMAE2019-96165

Federico Bussolati' Martin Guiton' Pierre-Alain Guidault ${ }^{2}$

Yann Poirette ${ }^{1}$ Martinez Michael ${ }^{1}$ Olivier Allix ${ }^{2}$

1. IFP Energies Nouvelles, Solaize, France; 2. ENS-Paris-Saclay, Cachan, France

Fatigue Performance of Mooring Chains Subjected

to Wear Degradation OMAE2019-96386

Gilang Muhammad Gemilang' Philippa Reed ${ }^{2}$ Adam Sobey ${ }^{2}$

1. University of Pertamina and University of Southampton, Southampton, United

Kingdom; 2. University of Southampton, Southampton, United Kingdom

\section{Structures, Safety and Reliability}

\section{2-4-3 Fatigue and Fracture Reliability III}

Tuesday June 11

Room SEC, Alsh 2 | 13:30 - 15:00

Session Chair: Yordan Garbatov, University of Lisbon, Portugal

Session Co-Chair: Myung-Hyun Kim, Pusan National University, Korea

On Calculating the Crack Growth within a Single

Load-Dwell-Unload Cycle for Metal Structures OMAE2019-95327

Fang Wang, Xuezhong Zhang, Zhe Jiang, Weicheng Cui

Shanghai Ocean University, Shanghai, China

Two-parameter J-A Estimation for Weld Centerline Cracks in Welded Single Edge Cracked Plate under Tensile Loading OMAE2019-95392

Chuanjie Duan, Shuhua Zhang

Hohai University, Nanjing, China

Study on Fatigue Crack of Marine Typical

Sandwich Composite Joint OMAE2019-95630

Luo Bailu, Shaowen Zheng

China Ship Development and Design Center, Hubei, China
Study on Mechanical Behaviors of Low-cycle Fatigue Crack Tip for Notch Cracked Plate under Variable Amplitude Loading 0MAE2019-96052 $\mathrm{BoDu}$

Qinzhou University, Qinzhou, China

\section{Structures, Safety and Reliability}

\section{2-9-3 Extreme Loading and Responses III}

Tuesday June 11

Room Crowne Plaza, Castle 1 | 13:30 - 15:00

Session Chair: Erik Vanem, DNV GL, Norway

Session Co-Chair: Guillaume de Hauteclocque, Bureau Veritas, France

Wave Load and Response Predictions combining HOSM, CFD and Machine Learning OMAE2019-95352 Jan Oberhagemann ${ }^{1}$ Anna Kringlen Ervik ${ }^{2}$ Odin Gramstad ${ }^{2}$ Jan Kaufmann ${ }^{1}$ Jens B. Helmers' ${ }^{2}$ Francois-Xavier Sireta ${ }^{3}$ 1. DNV GL, Hamburg, Germany; 2. DNV GL, Høvik, Norway; 3. DNV GL, Singapore, Singapore

Experimental Validation of FORM-based Approach for Predicting Extreme Value Distribution of Hull Girder Bending Moment in a Ship OMAE2019-95389 Tomoki Takami ${ }^{1}$ Yusuke Komoriyama ${ }^{1}$ Takahiro Ando ${ }^{1}$ Kazuhiro lijima ${ }^{2}$ 1. National Institute of Maritime, Port and Aviation Technology, Mitaka, Japan; 2. Department of NAOE, Osaka University, Osaka, Japan

Evaluation of an Equivalent Design Wave Method to Define Lifetime Combined Loading Scenarios for Trimarans OMAE2019-95497 Harleigh C Seyffert, Austin Kana Technical University Delft, Delft, Netherlands

Dynamic Load Inversion Method of Ship Body based on Influence Coefficient Matrix OMAE2019-95777 Huilong Ren, Guoqing Feng, Hao Liu, Xuecong Hu, Jian Zou Harbin Engineering University, Harbin, China

Analysis of Fatigue Life of Ship Structure under the Non-linear Slamming Load OMAE2019-95781

Huilong Ren, He Ma

Harbin Engineering University, Harbin, China

\section{Structures, Safety and Reliability}

\section{2-12-3 Structural Analysis and Optimization III}

Tuesday June 11

Room Crowne Plaza, Castle 2 | 13:30 - 15:00

Session Chair: Jonas W. Ringsberg, Chalmers University of Technology, Sweden

Session Co-Chair: Paulo M. Videiro, LACEO/COPPE/

Federal University of Rio De Janeiro, Brazil

Study on Impact Resistance of Ship Arrow-shaped Negative Poisson Ratio Honeycomb Pedestal OMAE2019-95745

Haoran Wu, Xiaobin Li, Jie Zhang

Wuhan University of Technology, Wuhan, China

Study on the Effect of Impact Load Generated from Pile Driving on Aged Berthing Structure OMAE2019-96092 Jeena Mary John' Nilanjan Saha ${ }^{2}$ Ranganathan Sundaravadivelu ${ }^{2}$ 1. Department of Ocean Engineering, Chennai, India; 2. Indian Institute of Technology Madras, Chennai, India 
Impacts of Condensate Storage on the Hull Structure Design of Semi-submersible Platform OMAE2019-96601 Jiaguo Feng ${ }^{1}$ Yi Yu$^{2}$ Da Li' Bin Xie' Wenhui Xie' Haishan Zhu' Min Wu 1. CNOOC Research Institute, Beijing, China; 2. CNOOC, Ltd., Beijing, China; 3. SBM Offshore USA, Inc., Houston, TX, USA

\section{Materials Technology}

\section{3-3-2 Performance of Mooring Chains}

Tuesday June 11

Room SEC, Boisdale 1 | 13:30 - 15:00

Session Chair: Jens Tronskar, Det Norske Veritas Pte Ltd, Singapore

Session Co-Chair: Koji Gotoh, Kyushu University, Japan

Testing and Modeling of Mooring Chains Subjected

to Cyclic Out-of-plane Bending OMAE2019-95369

Edgar Mamiya ${ }^{1}$ Fabio Castro ${ }^{1}$ Lucival Malcher ${ }^{1}$ Guilherme Ferreira ${ }^{1}$ Eduardo Nunes Filho ${ }^{1}$

Raniere Neves ${ }^{1}$ Felipe Canut ${ }^{1}$ Carlos Augusto ${ }^{2}$ Mario Ribeiro ${ }^{3}$ Pedro Teixeira $^{3}$

1. Universidade de Brasilia, Brasilia, DF, Brazil; 2. Petrogal Brasil, Rio

de Janeiro, RJ, Brazil; 3. Galp Energia, Lisbon, Portugal

A Comprehensive Set of Round-bar Stress Intensity Factor Solutions for ECA of Mooring Shackle and Chain Components OMAE2019-96631

Pingsha Dong ${ }^{1}$ Jean-Michel Aubert ${ }^{2}$ Jean-Pierre Sauvage ${ }^{3}$

1. University of Michigan, Ann Arbor, MI, USA;2. Total,

Paris, France; 3. Bureau Veritas, Paris, France

Wear Performance of Mooring Chain in Wet Environment

with Substitute Ocean Water 0MAE2019-95822

Koji Gotoh, Tetsuya Ueda, Koji Murakami, Tomoaki Utsunomiya

Kyushu University, Fukuoka, Japan

Development of a New Material Technology for Offshore

Mooring Chains - High Manganese Steel OMAE2019-95541

Neerav Verma' Andrew Wasson' Zhen Li Harpreet Sidhar ${ }^{2} \mathrm{Haiping}^{1} \mathrm{He}^{2}$

Hyunwoo Jin ${ }^{3}$ HyunJo Jun ${ }^{2}$ Adnan Ozekcin² Shiun Ling ${ }^{3}$

1. ExxonMobil Upstream Research Company, Spring, TX, USA; 2. ExxonMobil, Spring,

TX, USA; 3. Exxonmobil Research and Engineering Co, Annandale, NJ, USA

\section{Pipelines, Risers, and Subsea Systems}

\section{4-1-5 Flexible Pipes V}

Tuesday June 11

Room Crowne Plaza, Staffa / Shuna | 13:30 - 15:00

Session Chair: Krassimir Doynov, ExxonMobil Upstream Integrated Solutions, USA

Session Co-Chair: Lin Zhao, Ocean university of China, China

Qualification of Thermoplastic Composite Pipe Risers -

Combined Pressure and Bending Loading OMAE2019-95274

Ali Bahtui, Karl Kidykas, Josh Richardson, Jonathan Wilkins

Magma Global, Portsmouth, United Kingdom

Prediction and Design of Internal Pressure

of Flexible Pipe OMAE2019-95458

Qiangqiang Shao ${ }^{1}$ Ting Liu² Shuai Yuan² Peihua Han ${ }^{1}$ Yong Bai ${ }^{2}$

1. Zhejiang University, Hangzhou, China; 2. Zhejiang University, Zhejiang, China

Analysis of Polyester Reinforced Flexible Composite

Pipe under Internal Pressure OMAE2019-95903

Xinyu Sun ${ }^{1}$ Yong Bai' Xiaojie Zhang ${ }^{1}$ Chang Liu' Jiannan Zhao

1. Southern University of Science and Technology, Shenzhen,

China; 2. Zhejiang University, Zhejiang, China
Research on Fatigue Life Assessment of Fiber Glass

Reinforced Flexible Pipe OMAE2019-95676

Jiannan Zhao ${ }^{1}$ Xiaojie Zhang ${ }^{1}$ Xinyu Sun' Zhao Wang ${ }^{1}$ Yong Bai ${ }^{2}$

1. Southern University of Science and Technology, Shenzhen,

China; 2. Zhejiang University, Zhejiang, China

\section{Pipelines, Risers, and Subsea Systems}

\section{4-2-1 General Design and Analysis I}

Tuesday June 11

Room Crowne Plaza, Castle 3 | 13:30 - 15:00

Session Chair: Vishnu Vijayaraghavan, Aker Solutions Inc., USA

The Branched Riser Systems - Concept Development OMAE2019-95160 Achoyamen M. Ogbeifun' Selda Oterkus Julia Race ${ }^{1}$

Naik Harit' Decnop Eduardo ${ }^{2}$ Moorthy Dakshina ${ }^{3}$

1. University of Strathclyde, Glasgow, United Kingdom; 2. McDermott International,

Surrey, United Kingdom; 3. McDermott International, Houston, TX, USA

Corrosion-fatigue Crack Growth Performance of Titanium

Grade 29 Welds in Tapered Stress Joints OMAE2019-95175

Gabriel Rombado ${ }^{1}$ David A. Baker ${ }^{2}$ Lars M. Haldorsen ${ }^{3}$

Pedro Craidy ${ }^{4}$ Jim H. Feiger ${ }^{5}$ Stephen J. Hudak, Jr. ${ }^{6}$

1. ExxonMobil Production Company, Spring, TX, USA; 2. Exxon Mobil Upstream Research Co,

Spring, TX, USA; 3. Equinor, Forus, Norway; 4. Petrobras R\&D Center, Rio de Janeiro, RJ, Brazil;

5. Southwest Research Institute, San Antonio, TX, USA; 6. Consultant, San Antonio, TX, USA

Vessel Interface Considerations for Ultra-deepwater

Intervention Risers OMAE2019-95519

Rohit Vaidya ${ }^{1}$ Mahesh Sonawane ${ }^{1}$ Ben Toleman ${ }^{1}$ Elaine Whiteley ${ }^{2}$ Jonathan Rourke ${ }^{3}$

1. 2H Offshore inc, Houston, TX, USA;2. 2H Offshore Engineering Ltd,

Bridge of Don, United Kingdom; 3. Helix, Houston, TX, USA

Life Extension of Deepwater Risers used for a Spar

Application in Gulf of Mexico OMAE2019-95804

Yongming Cheng ${ }^{1}$ Chenteh Alan Y $\mathrm{U}^{2}$ Guangqiang Yang ${ }^{3}$ Manuel Carballo ${ }^{4}$

1. FloaTec, A Company of Keppel, Houston, TX, USA; 2. ABS, Spring, TX, USA;

3. Exxon Mobil Company, Spring, TX, USA; 4. ExxonMobil, Spring, TX, USA

\section{Ocean Space Utilization}

\section{5-4-1 Underwater Vehicle and Technology}

Tuesday June 11

Room SEC, Dochart 1 | 13:30 - 15:00

Session Chair: Yoshitaka Watanabe, JAMSTEC, Japan

Session Co-Chair: Tomoya Inoue, JAMSTEC, Japan

A Low Cost Autonomous Underwater Vehicle for

Irrigation Canal Monitoring OMAE2019-95134

Mamoon Masud, Suleman Mazhar

Information Technology University, Lahore, Pakistan

On the Use of Consumer-grade Remotely Piloted Aircraft Systems for Monitoring Shallow Coral Reefs in Colombia:

Case Old Providence Island OMAE2019-95385

Manuela Lopera-Gil, Rafael E. Vasquez, Carlos A. Zuluaga, Paula Andrea Zapata-Ramírez Universidad Pontificia Bolivariana, Medellin, Colombia

Integrated Acoustic Communication and Positioning System

between an Autonomous Surface Vehicle and Multiple

Autonomous Underwater Vehicles OMAE2019-96623

Yoshitaka Watanabe, Koji Meguro, Mitsuyasu Deguchi, Yukihiro Kida, Takuya Shimura

JAMSTEC, Yokosuka, Japan 


\section{Ocean Engineering}

\section{6-2-3 Coastal Engineering III}

Tuesday June 11 Room SEC, M4 | 13:30-15:00

Session Chair: Ian Robertson, University of Hawaii, USA

Study of Extreme Waves Propagating over Reefs

in Large Wave Flume OMAE2019-95039

Songgui Chen ' Zeming Wang ${ }^{2}$ Jinhai Zheng ${ }^{2}$ Chi Zhang ${ }^{2} \mathrm{Ke} \mathrm{Hu}^{1}$

1. Tianjin Research Institute of Water Transport Engineering,

Tianjin, China; 2. HoHai University, Nanjing, China

Numerical Simulation of Drifting and Run-up Ice Floes

driven by Tsunami OMAE2019-95901

Shinji Kioka' ${ }^{1}$ Maiko Ishida ${ }^{1}$ Takahiro Takeuchi ${ }^{2}$

1. Civil Engineering Research Institute for Cold Region Public Works Research,

Sapporo, Japan; 2. Hachinohe Institute of Technology, Hachinohe, Japan

Designing Coastal Structures for Tsunami Loads

per ASCE 7-16 OMAE2019-95101

Ian Robertson, Jacob McKamey

University of Hawaii, Honolulu, HI, USA

\section{Ocean Engineering}

\section{6-4-5 Very Large Floating Structures}

Tuesday June 11

Room SEC, M2 \& M3 | 13:30 - 15:00

Session Chair: Zhengshun Cheng, Shanghai Jiao Tong University, China

Session Co-Chair: Xu Xiang, Norwegian Public Roads Administration, Norway

Numerical Study into Site-specific Effect on the

Response of Sea-crossing Bridge under Correlated

Wind and Wave Loadings OMAE2019-95687

Kai Wei' Saad Riaz' Jin Zhu' ${ }^{1}$ Hasan Imani2 Jiarui Zhang ${ }^{1}$

1. Southwest Jiaotong University, Chengdu, China;

2. Sharif Univeristy of Technology, Tehran, Iran

Dynamics of an Array of Submersible Mussel Rafts

in Waves and Current OMAE2019-96388

Tobias Dewhurst ${ }^{1}$ Spencer Hallowell ${ }^{2}$ Carter Newell ${ }^{3}$

1. Maine Marine Composites, Portland, ME, USA;2. Independent Author, West

Bath, ME, USA; 3. Pemaquid Mussel Farm, Damariscotta, ME, USA

Extreme Response Analysis of an End-anchored

Floating Bridge OMAE2019-96793

Zhengshun Cheng ${ }^{1}$ Zhen Gao ${ }^{2}$ Torgeir Moan $^{3}$

1. Shanghai Jiao Tong University, Shanghai, China; 2. Norwegian University of Science and Technology, Trondheim, Norway; 3. Center For Ships \& Ocean Structures, Trondheim, Norway

Experimental and Numerical Investigation on

Planar Motion Responses of a Single Point Moored

Shuttle Tanker in Waves OMAE2019-95251

Ning He Cheng Zhang ${ }^{2}$ Zhuang Kang ${ }^{2}$ Youwei Kang ${ }^{3}$ Changhong Wang ${ }^{2}$

1. China Offshore Oil Engineering Co. (COOEC), Tianjin, China; 2. Harbin Engineering

University, Harbin, China; 3. CIMC Offshore Co. Ltd., Shenzhen, China

Dynamic Response of Spar Wind Turbine Moored by Dynamic Catenaries under Random Wind and Wave Loads OMAE2019-95658 Yilun Li' Shuangxi Guo ${ }^{2}$ Yue Kong ${ }^{1}$ Weimin Chen ${ }^{2}$ Min Li ${ }^{1}$

1. Beijing University of Aeronautics and Astronautics, Beijing, China;

2. Institute of Mechanics, Chinese Academy of Sciences, Beijing, China

\section{CFD \& FSI}

\section{8-2-3 Free Surface Loading and Structure Interaction II}

Tuesday June 11

Room SEC, Lomond Auditorium | 13:30 - 15:00 Session Chair: Arun Kamath, Norwegian University of Science and Technology, Norway Session Co-Chair: Csaba Pakozdi, SINTEF 0cean, Norway

Multi-phase Simulation of Droplet Trajectories of

Wave-impact Sea Spray Over a Vessel OMAE2019-95799

Shafiul Mintu, David Molyneux, Bruce Colbourne

Memorial University of Newfoundland, St. John's, NL, Canada

Wave Impact Loads Prediction with Compressible

Air Effects using CFD OMAE2019-96026

Inno Gatin' Shengnan Liu' ${ }^{2}$ Nikola Vladimir ${ }^{1}$ Hrvoje Jasak $^{3}$

1. University of Zagreb, Zagreb, Croatia; 2. University of Stavanger,

Stavanger, Norway; 3. Wikki Ltd, London, United Kingdom

CFD Analyis of a Captive Bullet Entry in Calm Water:

Turbulence Modelling OMAE2019-96099

René Bettencourt Rauffus ${ }^{1}$ António Maximiano ${ }^{2}$ Luis Eca $^{3}$ Guilherme Vaz ${ }^{4}$

1. Instituto Superior Técnico, Lisboa, Portugal; 2. WavEC - Offshore

Renewables, Lisboa, Portugal; 3. Technical University of Lisbon,

Lisbon, Portugal; 4. MARIN, Wageningen, Netherlands

Computational Methods for Moving and Deforming

Objects in Extreme Waves OMAE2019-96321

Arthur Veldman' ${ }^{1}$ Henk Seubers ${ }^{1}$ Matin Hosseini ${ }^{2}$ Xing Chang $^{2}$

Peter Wellens ${ }^{2}$ Peter van der Plas Joop Helder ${ }^{3}$

1. University of Groningen, Groningen, Netherlands;

2. TU Delft, Delft, Netherlands; 3. MARIN, Wageningen, Netherlands

\section{Ocean Renewable Energy}

\section{9-5-2 Concepts and Design}

Tuesday June 11

Room SEC, Carron 1 | 13:30 - 15:00

Session Chair: Madjid Karimirad, Queen's University Belfast, United Kingdom

Session Co-Chair: Wei Shi, Dalian University of Technology, China

Feasibility Study of Mooring Lines Design for a Tidal Turbine

Platform using Floating Double Hull OMAE2019-95998

Nu Rhahida Arini' Philipp R. Thies ${ }^{1}$ Lars Johanning ${ }^{1}$ Edward Ransley ${ }^{2}$

Scott Brown ${ }^{3} \mathrm{Nan} \mathrm{Xie}^{3}$ Deborah Greaves ${ }^{3}$

1. University of Exeter, Penryn, United Kingdom; 2. Plymouth University, Plymouth, United Kingdom; 3. University of Plymouth, Plymouth, United Kingdom

Synergistic Flow Induced Vibration of Multiple Cylinders in Harvesting Marine Hydrokinetic Energy OMAE2019-96671 Hai Sun ${ }^{1}$ Michael Bernitsas ${ }^{2}$ Chen Zhiyun ${ }^{1}$

1. Harbin Engineering University, Harbin, China; 2. University of Michigan, Michigan, MI, USA

FIV Energy Harvesting from Sharp-edge Oscillators OMAE2019-95227

Vahid Tamimi ${ }^{1}$ Milad Armin² Selda Shahvaghar-As ${ }^{3}$

Seyed Taghi Omid Naeeni' Mostafa Zeinoddini ${ }^{4}$

1. University of Tehran, Tehran, Iran; 2. Liverpool John Moores University,

Liverpool, United Kingdom; 3. Sharif University of Technology, Tehran,

Iran; 4. K.N.Toosi University of Technology, Tehran, Iran 
Implementation of Tidal Stream Turbines and Tidal Barrage Structures in DG-SWEM OMAE2019-95767 Andrea M. Schnabl' Tulio M. Moreira ${ }^{2}$ Dylan Wood ${ }^{3}$ Ethan J. Kubatko ${ }^{3}$ Guy T. Houlsby' Ross A. McAdam ${ }^{4}$ Thomas A.A. Adcock' 1. University of Oxford, Oxford, United Kingdom; 2. Federal University of Minas Gerais (UFMG), Belo Horizonte, MG, Brazil; 3. The Ohio State University, Columbus, OH, USA; 4. Department of Engineering Science, University of Oxford, Oxford, United Kingdom

\section{Offshore Geotechnics}

\section{0-6-1 Pipeline Geotechnics}

Tuesday June 11

Room Crowne Plaza, Jura | 13:30-15:00

Session Chair: Borana Kullolli, Bundesanstalt für

Materialforschung -und prüfung, Germany

Axial Resistance of Smooth Polymer Pipelines on Sand OMAE2019-95938 Henry Milewski ${ }^{1}$ Matt Dietz ${ }^{2}$ Andrea Diambra ${ }^{2}$ Lawrence de Leeuw ${ }^{2}$ 1. TechnipFMC, Westhill, United Kingdom; 2. University of Bristol, Bristol, United Kingdom

Drained Lateral Breakout Resistance of Subsea Pipelines OMAE2019-96174 Jean-Christophe Ballard' Zack Westgate ${ }^{2}$

1. Fugro Geoconsulting, Brussels, Belgium; 2. Fugro USA Marine, Inc., Houston, TX, USA

Centrifuge Modelling of Skirted Spudcan

Penetration in Layered Soil OMAE2019-96541

Conleth 0' Loughlin' Christophe Gaudin ${ }^{1}$ Matthew Quah ${ }^{2}$ Michael Perry ${ }^{2}$

1. University of Western Australia, Perth, WA, Australia; 2. Keppel, Singapore, Singapore

FEA Based Simplified Integrated Analysis

for Mudmat Design OMAE2019-96754

Srikanth Srigiriraju, Arindam Chakraborty, Burak Ozturk, Devvrat Rathore

Virtual Integrated Analytics Solutions, Houston, TX, USA

\section{Petroleum Technology}

\section{1-6-2 Integrity of Well Barriers II}

Tuesday June 11 Room Crowne Plaza, Barra | 13:30-15:00

Session Chair: Jan David Ytrehus, SINTEF, Norway

Numerical Modeling of Radial Fracturing of Cement

Sheath Caused by Pressure Tests OMAE2019-96319

Sohrab Gheibi' Sigbjørn Sangesland' Torbjorn Vralstad ${ }^{2}$

1. Norwegian University of Science and Technology, Trondheim,

Norway; 2. SINTEF, Trondheim, Norway

Effect of Rock on Cement Sheath Integrity:

Shale vs. Sandstone OMAE2019-96738

Ragnhild Skorpa, Benjamin Werner, Torbjorn Vralstad

SINTEF, Trondheim, Norway

A Discussion on Different Types of Cement Bond Strength OMAE2019-96773 Nils Opedal' Pierre Cerasi ${ }^{2}$ Torbjorn Vralstad ${ }^{2}$

1. SINTEF Industry, Trondheim, Norway; 2. SINTEF, Trondheim, Norway

Comparative Evaluation of Elastomer Seal Energization in

Conventional and Expandable Hanger Assembly OMAE2019-96776

Harshkumar Patel' Saeed Salehi'

1. University of Oklahoma, Norman, OK, USA; 2. Mewbourne School

of Petroleum and Geological Engineering, Norman, OK, USA

\section{Rodney Eatock Taylor Honouring Symposium on Marine and Offshore Hydrodynamics}

\section{2-5-2 Non-Linear Waves and wave Effects II}

Tuesday June 11

Room SEC, Carron 2 | 13:30 - 15:00

Session Chair: Robert Beck, University of Michigan, USA

CFD Investigations of 2D Greenwater Overtopping of

a Freely Floating Offshore Vessel OMAE2019-95865

Xiantao Zhang' Scott Draper ${ }^{2}$ Hugh Wolgamot ${ }^{2}$ Wenhua Zhao ${ }^{2}$ Lifen Chen ${ }^{2}$ Liang Cheng ${ }^{2}$

1. Shanghai Jiao Tong University, Shanghai, China;

2. University of Western Australia, Perth, WA, Australia

Development of 3-Dimensional Fully Nonlinear Potential

Flow Wave Tank in Framework of Openfoam OMAE2019-96098

Zaibin Lin, Ling Qian, Wei Bai, Zhihua Ma, Hao Chen, Jian Guo Zhou

Manchester Metropolitan University, Manchester, United Kingdom

A 2D Nonlinear Numerical Wave Tank with a

Moored Floating Body OMAE2019-96669

Hui Sun' Jens B. Helmers ${ }^{2}$

1. DNL GL, Høvik, Norway; 2. DNV GL, Høvik, Norway

Wave Interaction with a Shallowly Submerged Step in 2D OMAE2019-95933 Guy McCauley, Hugh Wolgamot, Scott Draper, Jana Orszaghova

University of Western Australia, Perth, WA, Australia

\section{Takeshi Kinoshita Honoring Symposium on Offshore Technology}

\section{3-2-4 Fluid-Structure Interactions (FSI)}

Tuesday June 11

Room SEC, Boisdale 2 | 13:30 - 15:00

Session Chair: Longfei Xiao, Shanghai Jiao Tong University, China

Session Co-Chair: Shinichiro Hirabayashi, University of Tokyo, Japan

Force Measurements and Stationarity Analysis on the Flow around a Single Square Column with Rounded Edges OMAE2019-95353

Dennis Gambarine ${ }^{1}$ Arjen Koop ${ }^{2}$ Gustavo R. S. Assi ${ }^{3}$

Fabiano Rampazzo ${ }^{1}$ Rodolfo T. Gonçalves ${ }^{4}$

1. Technomar Engenharia, São Paulo, SP, Brazil; 2. MARIN, Wageningen, Netherlands;

3. Univeristy of São Paulo, São Paulo, SP, Brazil; 4. University of Tokyo, Bunkyo, Japan

Hydrodynamics around a Deep-draft Semi-submersible with Biomimetic Tubercle Corner Design OMAE2019-95607

Yibo Liang, Weichao Shi, Longbin Tao

University of Strathclyde, Glasgow, United Kingdom

Investigation of the 2D Behavior of a Rotating Cylinder in Flow using the Discrete Vortex Method OMAE2019-95841

Changkyu Rheem

The University of Tokyo, Tokyo, Japan

\section{REFRESHMENT BREAK}

15:00 - 15:30

Location: Hall 5 (SEC) 


\section{CONCURRENT SESSIONS \\ 15:30 - 17:30}

\section{Offshore Technology}

\section{1-6-2 Loads and Responses in Current and Wind I}

Tuesday June 11

Room SEC, Alsh 1 | 15:30 - 17:30

Session Chair: Arjen Koop, MARIN, Netherlands

Session Co-Chair: António Maximiano, WavEC - Offshore Renewables, Portugal

Thorough Verification and Validation of CFD Prediction of FPSO

Current Load for Confident Applications OMAE2019-95017

Wei Xu' Zhenjia (Jerry) Huang ${ }^{2}$ Hyun Joe Kim ${ }^{3}$

1. ExxonMobil, Spring, TX, USA; 2. Exxonmobil Upstream Research Company,

Spring, TX, USA; 3. Samsung Heavy Industries, Daejeon, Korea

Numerical Investigation for Vortex-induced Vibrations of Steel-Lazy-Wave-Risers, Part I: CFD Validation against

Forced Oscillation Model Test OMAE2019-96401

Hyunchul Jang, Jang Kim

TechnipFMC, Houston, TX, USA

Thorough Verification and Validation of CFD Prediction of FPSO Wind Load for Confident Applications OMAE2019-95018 Wei Xu' Zhenjia (Jerry) Huang ${ }^{2}$ Hyun Joe Kim ${ }^{3}$

1. ExxonMobil, Spring, TX, USA; 2. Exxonmobil Upstream Research Company, Spring, TX, USA; 3. Samsung Heavy Industries, Daejeon, Korea

Numerical Investigation of Scour around Subsea

Pipelines near the Seabed OMAE2019-96069

Guang Yin ${ }^{1}$ Zhen Cheng ${ }^{2}$ Shengnan Liu' Muk Chen Ong ${ }^{1}$

1. University of Stavanger, Stavanger, Norway; 2. Applied Ocean Physics and

Engineering, Woods Hole Oceanographic Institution, Woods Hole, MA, USA

Numerical Modeling Practice and Verification of the Wind Load Estimation for FPSO and Semi-submersible OMAE2019-96429 SeongMo Yeon ${ }^{1}$ Hyunchul Jang ${ }^{2}$ Jang Kim² Joo-Sung Kim ${ }^{3}$ Bo Woo Nam ${ }^{4}$ Zhenjia (Jerry) Huang ${ }^{5}$ Jim 0'Sullivan ${ }^{2}$ Hyun Joe Kim ${ }^{3}$ Sa Young Hong ${ }^{4}$ 1. Ship and Offshore Performance Research Center, Daejeon, Korea; 2. TechnipFMC, Houston, TX, USA; 3. Samsung Heavy Industries, Daejeon, Korea; 4. Korea Research Institute of Ships and Ocean Engineering, Daejeon, Korea; 5. Exxonmobil Upstream Research Company, Spring, TX, USA

\section{Structures, Safety and Reliability}

\section{2-8-1 Well Integrity and Reliability Assessment}

Tuesday June 11 Room Crowne Plaza, Castle 2 | 15:30 - 17:30

Session Chair: Max Russo, Konsberg, Norway

Tethered Bops - Performance and Monitoring OMAE2019-95523 Stuart Killbourn' Elizbar B Kebadze ${ }^{2}$ James Maher ${ }^{3}$

1. Fugro GB Marine, Glasgow, United Kingdom; 2. BP Exploration Operating Company, Sunbury-on-Thames, United Kingdom; 3. Trendsetter Vulcan Offshore, Houston, TX, USA

Wellhead Fatigue: Benefits of Structural Reliability

Analysis Applied to Groups of Wells OMAE2019-96214

Torfinn Horte ${ }^{1}$ Michael Macke ${ }^{2}$ Andreas Buvarp Aardal ${ }^{1}$

Lorents Reinas ${ }^{3}$ Paal Bjonnes ${ }^{3}$ Erik Frimanslund ${ }^{3}$

1. DNV GL, Høvik, Norway; 2. DNV GL, Oslo, Norway; 3. Equinor, Stavanger, Norway
Well Integrity: Preliminary Risk Analysis for Different

Well Life Cycle Phases OMAE2019-96280

Danilo T. M. P. Abreu' Carlos H. B. Morais' Joaquim Santos ${ }^{2}$

Danilo Colombo ${ }^{3}$ Marcelo Ramos Martins ${ }^{1}$

1. LabRisk - Analysis, Evaluation and Risk Management Laboratory -

University of São Paulo, São Paulo, SP, Brazil; 2. University of São Paulo,

São Paulo, SP, Brazil; 3. Petrobras, Rio de Janeiro, RJ, Brazil

\section{Structures, Safety and Reliability}

\section{2-9-4 Extreme Loading and Responses IV}

Tuesday June 11

Room Crowne Plaza, Castle 1 | 15:30 - 17:30

Session Chair: YeongAe Heo, Case Western Reserve University, USA

Session Co-Chair: Deyu Wang Shanghai Jiao Tong University, China

A Calculation Method for the Quasi-stationary Pressure in Cabin Explosion with Venting OMAE2019-95776

Pengduo Zhao ${ }^{1}$ Haojie Wang ${ }^{2}$ Zhipeng Du' Xiaobin Li ${ }^{2}$

1. Naval Research Academy, Beijing, China; 2. Wuhan University of Technology, Wuhan, China

Explosion Response of Cold Bond Corrosion Repairs

Applied to Offshore Living Quarters OMAE2019-95810

Trey Turner, Abhimanyu Kumar

Atkins, Houston, TX, USA

Localisation Analysis in an X65 Offshore Pipeline Steel OMAE2019-96786 Martin Kristoffersen, David Morin, Odd Sture Hopperstad, Tore Børvik Norwegian University of Science and Technology, Trondheim, Norway

\section{Materials Technology}

\section{3-3-3 Advances on Assessing Performance of Steel}

Tuesday June 11

Room SEC, Boisdale 1 | 15:30 - 17:30

Session Chair: Carol Johnston, TWI Ltd, United Kingdom

Session Co-Chair: Jens Tronskar, Det Norske Veritas Pte Ltd, Singapore

Life Extension of Environmental Assisted Cracking of High

Strength Subsea Material due to CP OMAE2019-96685

Agnes Marie Horn ${ }^{1}$ Erling Øbtby ${ }^{2}$ Viggo Roneid ${ }^{2}$ Finn Kirkemo ${ }^{3}$

1. DNV GL, Oslo, Norway; 2. DNV GL, Høvik, Norway; 3. Equinor, Tranby, Norway

Investigation of Strain-based Failure Assessment based on Reference Strain Method for Welded Pipes OMAE2019-96489

Jae Sung Lee, Myung-Hyun Kim

Pusan National University, Busan, Korea

Accurate Closed-form SIF Determination and Fatigue Life Investigation on Ship Construction Model OMAE2019-95268

Benqiang Lou

Jiangsu University of Science and Technology, Zhenjiang, China

Magnetoelastic Characteristics of Pipeline Steel

under Tensile Stress OMAE2019-95275

Sheng Ba0 ${ }^{1}$ Pengfei Jin ${ }^{1}$ Ashri Mustapha ${ }^{2}$ Zhengye Zhao ${ }^{1}$

1. Zhejiang University, Hangzhou, China; 2. Petronas, Kuala Lumpur, Malaysia

Material Property Requirements for High Strength

Steels used in Mobile Offshore Units OMAE2019-96844

Rolf $\mathrm{H}$. Hinderaker

Petroleum Safety Authority, Stavanger, Norway 


\section{Pipelines, Risers, and Subsea Systems}

\section{4-2-2 General Design and Analysis II}

Tuesday June 11

Room Crowne Plaza, Castle 3 | 15:30 - 17:30

Session Chair: Olav Fyrileiv, DNV GL, Norway

Multi-pronged Approach for the Design of HP/HT

Deepwater Steel Catenary Risers OMAE2019-96249

Gurudutt Bangalore, Yongming Cheng, Surya Banumurthy

KeppelFloaTEC, Houston, TX, USA

Seismic Design Challenges of High Pressure Riser

Systems on Gravity Based Structures OMAE2019-96409

Mahesh Sonawane ${ }^{1}$ Rohit Vaidya' Ronak Kadakia ${ }^{2}$ Hunter Haeberle ${ }^{2}$ Phil Ward $^{3}$

1. 2 H Offshore Inc, Houston, TX, USA; 2. Baker Hughes, a GE Company, Houston,

TX, USA; 3. 2H Offshore Engineering Ltd, Bridge of Don, United Kingdom

Flow Past a Forced Oscillating Cylinder:

A Three-Dimensional Numerical Study OMAE2019-96477

Huan Ping, Yan Bao, Dai Zhou, Zhaolong Han

Shanghai Jiao Tong University, ShangHai, China

A Numerical Investigation on the Effect of Heave Motion

Frequency in the Deep Sea Mining System OMAE2019-95292

Qi Wu, Jianmin Yang, Haining Lu, Wenyue Lu, Tao Peng, Jun Li

Shanghai Jiao Tong University, Shanghai, China

\section{Pipelines, Risers, and Subsea Systems}

\section{4-5-1 Flow Assurance I}

Tuesday June 11

Room Crowne Plaza, Staffa / Shuna | 15:30 - 17:30

Session Organizer: Daniel Carneiro, Wood, Brazil

Session Co-Chair: Paulo Paz, Federal University of Rio de Janeiro, Brazil

Session Co-Chair: Hualei Yi, CNOOC Research Institute Co., Ltd, China

Flow-induced Vibration Analysis of a Water Injection System

at Elevated Flow Rates of an FPSO OMAE2019-95019

Nestor Gonzalez Diez ${ }^{1}$ Oluwaseun M. Awe ${ }^{2}$ Pieter Van Beek' Can Tümer' Juan Pontaza ${ }^{3}$

1. TNO, Delft, Netherlands; 2. Shell Nigeria Exploration \& Production

Company, Ltd., Lagos, Nigeria; 3. Shell, Houston, TX, USA

On Deriving High Pressure Empirical Multiphase Forcing

Functions from CFD Analysis OMAE2019-96155

Olivier Macchion' Stefan Belfroid ${ }^{2}$ Leszek Stachyra ${ }^{1}$ Atle Jensen ${ }^{3}$

1. TechnipFMC, Lysaker, Norway; 2. TNO, Delft, Netherlands; 3. University of Oslo, Oslo, Norway

Examination and Analysis of Four-phase Four-fluid Flow Techniques

in Offshore Pipelines OMAE2019-95005

Mohamed Odan' Faraj Ben Rajeb' Mohammad Azizur Rahman²

Amer Aborig' Syed Imtiaz' ${ }^{1}$ Yan Zhang ${ }^{1}$ M. M. Awad ${ }^{3}$

1. Memorial University of Newfoundland, St. John's, NL, Canada; 2. Texas A\&M

University at Qatar, Doha, Qatar; 3. Mansoura University, Mansoura, Egypt

Computational Investigation of Oil Accumulation

in a Subsea Deadleg OMAE2019-96804

Egemen Caglar, Yi Zeng, Jeyhoon Khodadadi

Auburn University, Auburn, AL, USA

Automated Subsea Architecture Optimization using

Low-dimensional Multiphase Flow Models OMAE2019-96293

Zurwa Khan ${ }^{1}$ Amine Meziou ${ }^{2}$ Reza Tafreshi' ${ }^{1}$ Matthew Franchek ${ }^{2}$ Karolos Grigoriadis ${ }^{2}$

1. Texas A\&M University at Qatar, Doha, Qatar; 2. University of Houston, Houston, TX, USA

\section{Ocean Space Utilization}

\section{5-3-1 Development of Deep Sea Mining and Resources}

Tuesday June 11 Room SEC, Dochart 1 | 15:30 - 17:30

Session Chair: Yoshiyasu Watanabe, Tokai University, Japan

Session Co-Chair: Marcio Yamamoto, National Maritime Research Institute, Japan

Experimental Study on Bubble Size Measurement for Development of Seafloor Massive Sulfides OMAE2019-95186 Seira Imai' Yasuharu Nakajima² ${ }^{2}$ Motohiko Murai ${ }^{1}$

1. Yokohama National University, Yokohama, Japan; 1. Yokohama National University, Yokohama, Japan; 2. National Maritime Research Institute, Mitaka, Japan; 2. National Maritime Research Institute, Mitaka, Japan

Study on Pipe Wear Evaluation based on Large Scale Experiment for Deep Sea Mining OMAE2019-95270 Satoru Takano ${ }^{1}$ Hirotaka Sato ${ }^{2}$ Takashi Terao ${ }^{3}$ Sotaro Masanobu ${ }^{4}$ Seiya Kawano $0^{5}$ 1. National Maritime Research Institute, Mitaka, Japan; 2. Nippon Steel \& Sumikin Engineering (0., Ltd., Futtsu-city, Japan; 3. Mitsui Miike Machinery Co., Ltd., Omuta-city, Japan; 4. National Maritime Research Institute, Tokyo, Japan; 5. Japan Oil, Gas and Metals National Corporation, Meguro-ku, Japan

Research and Development on a Self-walking Vertical Mining System using DTH for Seafloor Mining and Sampling OMAE2019-95394 Yoshiyasu Watanabe ${ }^{1}$ Keisuke Watanabe ${ }^{1}$ Hideyuki Suzuki ${ }^{2}$ Teruo Ooshima ${ }^{3}$ Yoshiaki Tsukamoto ${ }^{3}$ 1. Tokai University, Shizuoka-shi Shizuoka, Japan; 2. University of Tokyo, Tokyo, Japan; 3. Furukawa Co., Ltd.,, Tsukuba-Shi Ibaraki-Ken, Japan

Experimental Analysis of Reduced-scale Jumper for Deep-sea Mining OMAE2019-95990 Marcio Yamamoto, Tomo Fujiwara, Shigeo Kanada, Masao 0no, Satoru Takano, Joji Yamamoto National Maritime Research Institute, Mitaka, Japan

Development of Elemental Technologies for Seafloor Mineral Processing of Seafloor Massive Sulfides OMAE2019-96040 Yasuharu Nakajima ${ }^{1}$ Joji Yamamoto ${ }^{1}$ Tomoko Takahashi ${ }^{2}$ Blair Thornton ${ }^{3}$ Yuta Yamabe ${ }^{4}$ Gjergj Dodbiba ${ }^{4}$ Toyohisa Fujita ${ }^{5}$ 1. National Maritime Research Institute, Mitaka, Japan; 2. Institute of Industrial Science, The University of Tokyo, Meguro-ku, Japan; 3 . Institute of Industrial Science, The University of Tokyo, and University of Southampton, Meguro-ku, Japan; 4. University of Tokyo, Bunkyo, Japan; 5. University of Tokyo, Meguro-ku, Japan

\section{Ocean Engineering}

\section{6-4-6 Towed Cables, Ropes and Mooring Systems}

Tuesday June 11 Room SEC, M2 \& M3 | 15:30 - 17:30

Session Chair: Muk Chen Ong, University of Stavanger, Norway Session Co-Chair: Zhiyu Jiang, University of Agder, Norway

Application for Improved Awareness of Cable Geometry during Seismic Survey Operation OMAE2019-95038

Jan Vidar Grindheim ${ }^{1}$ Ken Welker ${ }^{1}$ Inge Revhaug ${ }^{2}$ 1. Geograf AS, Stavanger, Norway; 2. NMBU, REALTEK, Ås, Norway

Sensitivity Analysis of Different Parameters of Taut Mooring System of a Truss Spar OMAE2019-95490

Zhuang Kang' Rui Chang' Youwei Kang ${ }^{2}$ Shanchuan Liu'

1. Harbin Engineering University, Harbin, China; 2. IIMC Offshore Co. Ltd., Shenzhen, China 
Experimental Validation of Towed Underwater

Cable Codes OMAE2019-96349

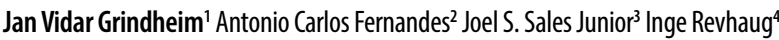

1. Geograf AS, Stavanger, Norway; 2. UFRJ/COPPE, Rio de Janeiro, RJ, Brazil;

3. Laboratory of Waves and Current - LOC - Universidade Federal do Rio de

Janeiro, Rio de Janeiro, RJ, Brazil; 4. NMBU, REALTEK, Ås, Norway

Tension Based Heading Control Strategy of the Arctic FPSO

with DP Assisted Mooring System OMAE2019-96557

Jaeyong Lee' Sol-Mi Choi² Seung Jae Lee² Kwang Hyo Jung ${ }^{3}$

1. Dong-eui University, Busan, Korea; 2. Korea Maritime and Ocean

University, Busan, Korea; 3. Pusan National University, Busan, Korea

Experimental Investigation of Stresses in Winch Drums subjected to Multilayer Spooling Loads from Synthetic Fibre Ropes OMAE2019-95283 Reidar André Skarbøvik ${ }^{1,2}$ Henry Piehl2 ${ }^{2}$ Sverre Torben ${ }^{1}$

Mette Lokna Nedreberg ${ }^{3}$ Vilmar Æsøy ${ }^{2}$

1. Rolls-Royce Marine AS, Ålesund, Norway; 2. Norwegian University of Science and

Technology, Ålesund, Norway; 3. Rolls-Royce Marine AS, Ulsteinvik, Norway

\section{Ocean Engineering}

\section{6-11-2 Floating Bodies Technology}

Tuesday June 11

Room SEC, Dochart 2 | 15:30 - 17:30

Session Chair: Joel S. Sales Junior, Laboratory of Waves and Current -

LOC - Universidade Federal do Rio de Janeiro, Brazil

Session Co-Chair: Milad shadman, COPPE/UFRJ, Brazil

Experimental and Numerical Study on Dynamic Responses of

FSRU-LNGC Side-by-side Mooring System OMAE2019-95473

Jingxia Yue (Le) ' Weili Kang ${ }^{1}$ Wengang Mao ${ }^{2}$ Pengfei Chen ${ }^{3}$ Xi Wang $^{4}$

1. Wuhan University of Technology, Wuhan, China; 2. Chalmers University of Technology,

Gothenburg, Sweden; 3. Shanghai Investigation, Design \& Research Institute Co.,

Ltd., Shanghai, China; 4. cCS Wuhan Rules \& Research Institute, Wuhan, China

Ship Manoeuvring Model Parameter Identification using Intelligent Machine Learning Method and the

Beetle Antennae Search Algorithm OMAE2019-95565

Changyuan Chen ${ }^{1}$ Manases Tello Ruiz ${ }^{1}$ Evert Lataire ${ }^{1}$ Guillaume Delefortrie ${ }^{2}$

Marc Mansuy ${ }^{1}$ Tianlong Mei ${ }^{3}$ Marc Vantorre ${ }^{1}$

1. Ghent University, Ghent, Belgium; 2. Flanders Hydraulics Research,

Antwerp, Belgium; 3. Shanghai Jiao Tong University, Shanghai, China

Study on the Multi-body Dynamic Characteristics of FPSO Soft Yoke

Mooring System based on Symplectic Algorithm OMAE2019-96464

Wenhua Wu' Baicheng Lyu' Ji Yao ${ }^{1}$ Qianjin Yue ${ }^{2}$ Zhang Yantao ${ }^{3}$ Xinglin Guo ${ }^{1}$

1. Dalian University of Technology, Dalian, China; 2. Dalian University

of Technology, Panjin, China; 3. CNOOC, Tianjin, China

Effect of Irregular Seabed on the Dynamic Response of

Spar-type Floating Offshore Platform OMAE2019-96816

Atul Krishna Banik, Shovan Roy

National Institute of Technology, Durgapur, India

Experimental Study on the Mechanics of a Coiled

Tubing Working within a Marine Riser under the

Affection of Marine Loads OMAE2019-95840

Yingchun Chen ' Chang Wang' Xinhua Wang ${ }^{1}$ Wenming Wang ${ }^{2}$ Wenda Wang ${ }^{3}$

1. Beijing University of Technology, Beijing, China; 2. China University of Petroleum-Beijing,

Beijing, China; 3. China HuanQiu Contracting \& Engineering Corp.,Ltd, Beijing, China

\section{Ocean Engineering}

\section{6-12-1 Ocean Measurement and Data Interpretation}

Tuesday June 11

Room SEC, M4 | 15:30 - 17:30

Session Chair: Gus Jeans, Oceanalysis Ltd, United Kingdom

Developments in Metocean HF Radar Technology,

Applications and Accuracy OMAE2019-95202

Lucy Wyatt ${ }^{1}$ M.D. Moorhead ${ }^{2}$ I.A. Fairley ${ }^{3}$

1. University of Sheffield, Sheffield, United Kingdom; 2. Neptune Radar Ltd,

Gloucester, United Kingdom; 3. Swansea University, Swansea, United Kingdom

Studies Toward the Development of Accurate Directional Spectrum Estimation Method using Field Observation Data OMAE2019-95220

Noriaki Hashimoto ${ }^{1}$ Masao Mitsui ${ }^{2}$ Koji Kawaguchi ${ }^{3}$ Takashi Fujiki ${ }^{3}$

1. Kyushu University, Fukuoka, Japan; 2. Sonic Corporation, Tokyo, Japan;

3. Port and Airport Research Institute, kanagawa, Japan

Effective Harmonic analysis with Spectrum

Filtering Technique OMAE2019-96021

Zhong Peng, Hazel Grant, Richard Sproson

Fugro GB Marine Limited, Wallingford, United Kingdom

\section{Polar and Arctic Sciences and Technology}

\section{7-1-1 Arctic Frontiers and Manoeuvring in Ice}

Tuesday June 11 Room SEC, Alsh 2 | 15:30 - 17:30

Session Chair: Walter Kuehnlein, sea2ice Ltd. \& Co. KG, Germany

Session Co-Chair: Sören Ehlers, Hamburg University of Technology, Germany

Introduction to Polar and Arctic Sciences and

Technology Symposium OMAE2019-96846

Sören Ehlers

Hamburg University of Technology, Hamburg, Germany

Numerical Simulation of Ice Load of a Ship Turning in Level Ice considering Fluid Effects OMAE2019-95694

Baoyu Ni' Zhipeng Li' Fan Jiang ${ }^{2}$ Meng Wu' ${ }^{3}$ Yanzhuo Xue

1. Harbin Engineering University, Harbin, China; 2. Jiujiang Vocational and Technical College, Jiujiang, China; 3. Guangzhou Marine Engineering Corporation, Guangzhou, China

A Voyage Planning Tool for Arctic Transit of Cargo Ships 0MAE2019-95128 Zhiyuan Li, Jonas W. Ringsberg, Francisco Afonso Rita

Chalmers University of Technology, Gothenburg, Sweden

The Calving Events of Petermann Glacier from 2008 to 2012:

Ice Island Drift Characteristics, Assessment of Fracture

Events, and Geographical Data Analysis OMAE2019-96732

Reza Zeinali Torbati' lan Turnbull2 Rocky Taylor' Derek Mueller ${ }^{3}$

1. Memorial University of Newfoundland, St. John's, NL, Canada; 2. Captain Robert A. Bartlett Building, C-CORE, St. John's, NL, Canada; 3. Carleton University, Ottawa, ON, Canada 


\section{CFD \& FSI}

\section{8-5-1 Wave CFD Modeling Applications}

Tuesday June 11

Room SEC, Lomond Auditorium | 15:30 - 17:30

Session Chair: Madhusuden Agrawal, BP, USA

Session Co-Chair: Yuwang Xu, Shanghai Jiao Tong University, China

Numerical and Mechanistic Modelling of Two-phase Liquid-gas

Flow's Pressure Drop across Sharp-edged Orifices OMAE2019-96305

Zurwa Khan' Reza Tafreshi' Matthew Franchek ${ }^{2}$ Karolos Grigoriadis ${ }^{2}$

1. Texas A\&M University at Qatar, Doha, Qatar; 2. University of Houston, Houston, TX, USA

Numerical Simulation of Ship-Ship Interactions in Waves OMAE2019-95737 Xueshen Xie' Yuxiang Wan' ${ }^{2}$ Qing Wang ${ }^{2}$ Hao Liu $^{2}$ Dakui Feng ${ }^{2}$

1. China Ship Design and Development Center, Wuhan, China; 2. Huazhong

University of Science and Technology, Wuhan, China

Numerical Convergence on the Hydroelasticity

of a Large Containership OMAE2019-95200

Ye Lu' ${ }^{1}$ Pandeli Temarel ${ }^{2}$ Qiu Jin ${ }^{2}$ Yousheng Wu' Xinyun Ni ${ }^{1}$ Chao Tian

1. China Ship Scientific Research Center, Wuxi, China;

2. University of Southampton, Southampton, United Kingdom

Two-phase MPS Method for Dam-break Flows OMAE2019-95518

Xiao Wen, Decheng Wan

Shanghai Jiao Tong University, Shanghai, China

Numerical Investigations on the Flow Past an Inclined

Thin Square Plate at $\mathbf{R e}=\mathbf{3 0 0}$ OMAE2019-95744

Yakun Zhao, Xinliang Tian, Xia Wu, Xiantao Zhang, Xin Li

Shanghai Jiao Tong University, Shanghai, China

\section{Ocean Renewable Energy}

\section{9-2-3 Floating Wind Designs}

Tuesday June 11

Room SEC, Carron 1 | 15:30 - 17:30

Session Chair: Amy Robertson, National Renewable Energy Laboratory, USA

Performance of a Passive Tuned Liquid Column Damper

for Floating Wind Turbines OMAE2019-96360

Wei Yu, Frank Lemmer, Po Wen Cheng

University of Stuttgart, Stuttgart, Germany

A Novel Semi-submersible Floating Wind Turbine Platform

Design based on Tuned Liquid Column Dampers OMAE2019-95945

Baijin Mao, Jili Sun, Zecheng Tang, Bo Feng, Weijie Zhang, Dahai Zhang, Yulin Si

Zheliang University, Zhoushan, China

Bottom Supported Tension Leg Tower with Inclined

Tethers for Offshore Wind Turbines OMAE2019-95014

Mohd Ishtiyak, Arunjyoti Sarkar

Indian Institute of Technology, Kharagpur, India

Coupled Numerical Analysis of a Concept TLB Type

Floating Offshore Wind Turbine OMAE2019-95244

Iman Ramzanpoor, Martin Nuernberg, Longbin Tao

University of Strathclyde, Glasgow, United Kingdom

Dynamic Response of a Conceptual Designed

Articulated Offshore Wind Turbine OMAE2019-95635

Yan Li, Zheng Liu, Yougang Tang, Xiyang Zhu, Ruoyu Zhang

Tianjin University, Tianjin, China

\section{Offshore Geotechnics}

\section{0-7-1 Pile Foundations II}

Tuesday June 11

Room Crowne Plaza, Jura | 15:30 - 17:30

Session Chair: Manuela Kanitz, Hamburg University of Technology, Germany

Assessment of Offshore Wind Turbine with Hybrid Monopile Foundation under Lateral Load using Centrifuge Tests OMAE2019-95637 Xuefei Wang ${ }^{1}$ David Zeng ${ }^{2}$ Jiale Li' Yougang Tang ${ }^{3}$

1. Hebei University of Technology, Tianjin, China; 2. Case Western Reserve University, Cleveland, OH, USA; 3. Tianjin University, Tianjin, China

Model Tests and Numerical Simulation on Effect of Spudcan Penetration on P-Delta of an Adjacent Pile OMAE2019-95752 Jianhua Wang, Yifei Fan, Dong Guo

Tianjin University, Tianjin, China

An Enhanced Interface Model for Friction Fatigue Problems of Axially Loaded Piles OMAE2019-96078 Borana Kullolli ${ }^{1}$ Matthias Baessler' Pablo Cuellar' Shilton Rica ${ }^{2}$ Frank Rackwitz ${ }^{3}$ 1. Bundesanstalt für Materialforschung und -prüfung, Berlin, Germany;

2. University of Luxembourg, Luxembourg, Luxembourg; 3. Technische Universitat Berlin, Faculty Planning Building Environment, Berlin, Germany

Influence of Different Pile Installation Methods

on Dense Sand OMAE2019-96109

Severin Spill, Tulio Quiroz, Aligi Foglia

Fraunhofer Institute for Wind Energy Systems IWES, Hanover, Germany

\section{Petroleum Technology}

\section{1-12-1 Cementing I}

Tuesday June 11 Room Crowne Plaza, Barra | 15:30 - 17:30 Session Chair: Ian Frigaard, University of British Columbia, Canada

Exchange Flow in Well Abandonment Operations OMAE2019-95131 Mônica Naccache ${ }^{1}$ Priscilla Varges ${ }^{1}$ Paulo de Souza Mendes ${ }^{1}$ Bruno Fonseca' Gabriella Cavalcante ${ }^{2}$ Cristiane Miranda ${ }^{2}$

1. Pontificia Universidade Católica do Rio de Janeiro, Rio de Janeiro,

RJ, Brazil; 2. Petrobras, Rio de Janeiro, RJ, Brazil

A Fast 3D Model for Annular Flows of Wellbore

Completion Fluids OMAE2019-95133

Philippe M.J. Tardy

Schlumberger Technology Corp., Sugar Land, TX, USA

Efficient Fluid-Fluid Displacement of Yield Stress Fluids in Axially Rotating Pipes OMAE2019-95382 Shan Lyu, Seyed Mohammad Taghavi Université Laval, Québec, OC, Canada

Effect of Buoyancy and Inertia on Viscoplastic Fluid-Fluid Displacement in an Eccentric Annulus with an Irregular Section, Part 2: Displacements in Vertical Annulus OMAE2019-95700 Hans Joakim Skadsem, Steinar Kragset Norwegian Research Centre AS, Stavanger, Norway

Enhanced Cement Composition for Preventing Annular Gas Migration OMAE2019-95589 Mustafa Al Ramadan' Saeed Salehi² Catalin Teodoriu² George Kwatia' 1. University of Oklahoma, Norman, OK, USA; 2. Mewbourne School of Petroleum and Geological Engineering, Norman, OK, USA 


\section{Rodney Eatock Taylor Honouring Symposium on Marine and Offshore Hydrodynamics}

\section{2-7-1 Large-Amplitude Non-Linear Ship Motions}

Tuesday June 11

Room SEC, Carron 2 | 15:30-17:30

Session Chair: Allan Magee, National University of Singapore, Singapore

Session Co-Chair: Longbin Tao, University of Strathclyde, United Kingdom

Experimental Study on a Relation between Nonlinear Hydrodynamic Forces and Wave-induced Ship Motions OMAE2019-95555

Masakazu Taguchi, Masashi Kashiwagi

Osaka University, Osaka, Japan

An Improved Body-exact Method to Predict the

Maneuvering of Ships in a Seaway OMAE2019-96441

Rahul Subramanian' Robert Beck

1. Texas A\&M University, Galveston, TX, USA; 2. University of Michigan, Ann Arbor, MI, USA

Solving 2-D Slamming Problems by the Higher-order MPS Method with an Improved Pressure Gradient Model OMAE2019-96775

Ruosi Zha, Heather Peng, Wei Qiu

Memorial University of Newfoundland, St. John's, NL, Canada

CFD Modelling to Investigate Design of a Whaleback-type

Forecastle for Greenwater Protection OMAE2019-95198

Lifen Chen ${ }^{1}$ Xiantao Zhang ${ }^{1}$ Paul Taylor ${ }^{2}$ Scott Draper ${ }^{1}$ Hugh Wolgamot ${ }^{1}$

1. University of Western Australia, Perth, WA, Australia;

2. University of Oxford, Oxford, United Kingdom

\section{Takeshi Kinoshita Honoring Symposium on Offshore Technology}

\section{3-2-5 Loads Induced in Floating Systems}

Tuesday June 11

Room SEC, Boisdale 2 | 15:30 - 17:30

Session Chair: Shinichiro Hirabayashi, University of Tokyo, Japan

Evaluation of Contact Forces in the Vertical Connection of a

Flexible Riser in the Subsea Equipment OMAE2019-95204

Yuri Coelho Del Sarto ${ }^{1}$ Ricardo Franciss ${ }^{2}$ Celso Morooka'

1. Universidade Estadual de Campinas, Campinas, SP, Brazil;

2. University of Petropolis, Petropolis, RJ, Brazil
Effects of Euler Angles of Vertical Cambered Otter Board on Hydrodynamics based on Response Surface Methodology and MOGA OMAE2019-95308

Gang Wang' Rong Wan' Liuyi Huang ${ }^{1}$ Fenfang Zhao ${ }^{1}$ Xinxin Wang ${ }^{1}$ Wenbin Zhu' Lei Wang ${ }^{3}$ Qing Chang Xu' Yuyan Li

1. Ocean University of China, Qingdao, China; 2. Marine Fisheries Research Institute of Zhejiang, Zhejiang, China; 3. East China Sea Fisheries Research Institute, Chinese Academy of Fishery Sciences, Shanghai, China

Fundamental Study on Structural Strength of Large-scale Floating Coal Transshipment Station OMAE2019-96482 Hiroaki Eto' Koji lizuka' Ryo Nishigochi' Tomoki lkoma' Yasuhiro Aida ${ }^{2}$ Koichi Masuda ${ }^{1}$ 1. Nihon University, Funabashi, Japan; 2. Nihon University, Chiba, Japan

\section{On GIS Based Facility Scale and Selection of Suitable Site of} Floating Medical Support System on Big Disaster OMAE2019-96493 Hiroaki Eto ${ }^{1}$ Sachio Togawa' ${ }^{1}$ Morikazu Yamamoto ${ }^{2}$ Shintaro Miyasawa ${ }^{3}$ Junko Yamaguchi ${ }^{4}$ Tomoki Ikoma ${ }^{1}$ Yasuhiro Aida ${ }^{5}$ Koichi Masuda' Sena Shimomoto ${ }^{1}$ Yuichi Kitabatake ${ }^{6}$ 1. Nihon University, Funabashi, Japan; 2. Nihon University, Tokorozawa, Japan; 3. SMusic and Express, Setagaya, Japan; 4. Nihon University, Itabashi, Japan; 5. Nihon University, Chiba, Japan; 6. Penta-Ocean Construction Co., Ltd., Bunkyo, Japan

\section{Afternoon Lecture Series 17:40 - 18:30 \\ Location: Lomond Auditorium}

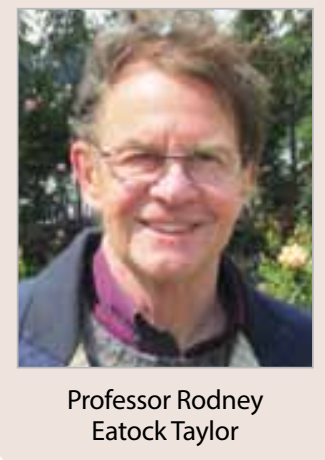

Inspired by Myriad Laughing Waves: Euler, Navier, Stokes and Others Professor Rodney Eatock Taylor, Emeritus Professor, University of Oxford See Afternoon Lecture Series, page 22 for more details.
Eatock Taylor 


\section{Wednesday, June 12}

\begin{tabular}{|c|c|c|}
\hline Time & Title & Location \\
\hline $08: 30-17: 30$ & Exhibition open & Hall 5 (SEC) \\
\hline $08: 30-10: 00$ & $\begin{array}{l}\text { Concurrent } \\
\text { Sessions }\end{array}$ & $\begin{array}{l}\text { See pages } 54-58 \text { for } \\
\text { session titles, authors } \\
\text { and locations }\end{array}$ \\
\hline $10: 00-10: 30$ & $\begin{array}{l}\text { Refreshment } \\
\text { Break }\end{array}$ & Hall 5 (SEC) \\
\hline $10: 30-12: 00$ & $\begin{array}{l}\text { Concurrent } \\
\text { Sessions }\end{array}$ & $\begin{array}{l}\text { See pages } 58-62 \text { for } \\
\text { session titles, authors } \\
\text { and locations }\end{array}$ \\
\hline $12: 00-13: 30$ & Lunch & Hall 5 (SEC) \\
\hline $13: 30-15: 00$ & $\begin{array}{l}\text { Concurrent } \\
\text { Sessions }\end{array}$ & $\begin{array}{l}\text { See pages } 63-66 \text { for } \\
\text { session titles, authors } \\
\text { and locations }\end{array}$ \\
\hline $15: 00-15: 30$ & $\begin{array}{l}\text { Refreshment } \\
\text { Break }\end{array}$ & Hall 5 (SEC) \\
\hline $15: 30-17: 30$ & $\begin{array}{l}\text { Concurrent } \\
\text { Sessions }\end{array}$ & $\begin{array}{l}\text { See pages } 67-71 \text { for } \\
\text { session titles, authors } \\
\text { and locations }\end{array}$ \\
\hline $17: 40-18: 30$ & $\begin{array}{l}\text { Afternoon } \\
\text { Lecture Series }\end{array}$ & $\begin{array}{l}\text { Lomond Auditorium } \\
\text { (SEC) }\end{array}$ \\
\hline $19: 00-24: 00$ & $\begin{array}{l}\text { Conference } \\
\text { Banquet }\end{array}$ & $\begin{array}{l}\text { Offsite: } \\
\text { Merchant Square }\end{array}$ \\
\hline
\end{tabular}

\section{CONCURRENT SESSIONS \\ 08:30 - 10:00}

\section{Offshore Technology}

\section{1-5-1 FLNG}

Wednesday June 12

Room SEC, Alsh 1 | 08:30 - 10:00

Session Chair: Wenhua Zhao, University of Western Australia, Australia

Session Co-Chair: Marc Cahay, TechnipFMC, France

Support of Operational Decisions for Prelude's

Side-by-side LNG Offloading OMAE2019-96090

Erwan Auburtin ${ }^{1}$ Thiago Miliante ${ }^{1}$ Ewoud van Haaften ${ }^{2}$ Finlay McPhail ${ }^{2}$

1. TechnipFMC, La Defense Cedex, France; 2. Shell Global

Solutions International B.V., Rijswijk, Netherlands

\section{A Simple Conceptual Methodology for the Operability Analysis of a Floating Liquefied Natural Gas (FLNG) \\ Unit in Small Production Fields OMAE2019-96135 \\ Lionel Wamba' Zhiming Yuan ${ }^{2}$ \\ 1. University of Strathclyde, Glasgow, United Kingdom; 2. University \\ of Strathclyde, NAOME, Glasgow, United Kingdom}

ARCOS: Advancing Ship-to-Ship LNG Transfer Solutions OMAE2019-96840 Raphael Poichot, Stephane Paquet

TechnipFMC, Sens, France

\section{Structures, Safety and Reliability}

\section{2-1-1 Abnormal or Rogue Waves I}

Wednesday June 12 Room Crowne Plaza, Castle 1 | 08:30 - 10:00 Session Chair: Elzbieta M. Bitner-Gregersen, DNV GL, Norway

Session Co-Chair: Alexander V. Babanin, University of Melbourne, Australia

\section{Identifying Higher-order Interactions in Wave Time-series} OMAE2019-95378

Kevin Ewans' Marios Christou² Suzana llic ${ }^{3}$ Philip Jonathan ${ }^{4}$

1. MetOcean Research Ltd, New Plymouth, New Zealand; 2. Imperial College

London, London, United Kingdom; 3. Lancaster University, Lancaster,

United Kingdom; 4. Shell Research Ltd., London, United Kingdom

\section{Extending Integrability of Nonlinear Water Wave Equations: Nonlinear Fourier Analysis of Breather Packets and Rogue Waves at Higher Order OMAE2019-95543 Alfred R. Osborne \\ Nonlinear Wave Research Corporation, Alexandria, VA, USA}

\section{Quantification of Predicted Wave Forces from Distant}

Elevation Measurements OMAE2019-96289

Spencer Hallowell' Sanjay R. Arwade ${ }^{2}$ Hannah Johlas ${ }^{2}$ Pedro Lomonaco ${ }^{3}$ Andrew T. Myers ${ }^{4}$ 1. Independent Author, West Bath, ME, USA; 2. University of Massachusetts Amherst, Amherst, MA, USA; 3. O.H. Hinsdale Wave Research Laboratory, Corvallis, OR, USA; 4. Northeastern University, Boston, MA, USA

Nonlinear Airy Wave Pulses on the Sea Surface OMAE2019-96298 Igor Shugan' Sergei Kuznetsov ${ }^{2}$ Yana Saprykina ${ }^{3}$ Y.-Y. Chen ${ }^{1}$ 1. National Sun Yat-Sen University, Kaoshiung, Taiwan;

2. Shirshov Institute of Oceanology of the Russian Academy of Sciences, Moscow, Russia; 3. Shirshov Institute of Oceanology, Moscow, Russia

\section{Structures, Safety and Reliability}

\section{2-11-1 Ultimate Strength I}

Wednesday June 12

Room Crowne Plaza, Castle 2 | 08:30 - 10:00

Session Chair: Masahiko Fujikubo, Osaka University, Japan

Session Co-Chair: Deyu Wang Shanghai Jiao Tong University, China

Numerical and Experimental Research on Residual Ultimate Strength of Hull Plates under Uniaxial Cyclic Loads OMAE2019-95226 Tian Xia' Ping Yang ' Cui Cong ${ }^{2}$ Ziya Peng ${ }^{1}$ Li Ma'

1. Wuhan University of Technology, Wuhan, China;

2. Xinlian College of Henan Normal University, Zhengzhou, China

An Empirical Formula for Predicting Elastic Ultimate Buckling Strength of Flat-bar Stiffened Panels with Initial Imperfections OMAE2019-95683 Hongyuan Mei, Deyu Wang

Shanghai Jiao Tong University, Shanghai, China 


\section{Materials Technology}

\section{3-6-1 Advances in Materials Characterization}

Wednesday June 12 Room SEC, Boisdale 1 | 08:30 - 10:00

Session Chair: Agnes Marie Horn, DNV GL, Norway

Session Co-Chair: Xin Wang, Carleton University, Canada

Safe Operations of Bolted Connections in the

Oil and Gas Industries OMAE2019-95260

Morten Langøy, Rolf H. Hinderaker, Terje L. Andersen

Petroleum Safety Authority, Stavanger, Norway

An Improved Methodology to Assess Weldability

of Line Pipe Steel OMAE2019-95953

Laura Alleva' ${ }^{1}$ Mauro Monti ${ }^{1}$ Emanuele Paravicini Bagliani ${ }^{2}$

Alessandro Paggi ${ }^{3}$ Philippe Darcis ${ }^{2}$

1. Rina Consulting Centro Sviluppo Materiali, Roma, Italy;

2. Dalmine S.p.A., Dalmine, Italy; 3. Tenaris, Dalmine, Italy

A Study on Unification of Welding Consumables in Construction of Chemical Cargo Tanker Made of Duplex Stainless Steel OMAE2019-95818 Takayuki Yotsuzuka' Yusuke Endo ${ }^{1}$ Eiji Niino ${ }^{1}$ Koji Gotoh ${ }^{2}$

1. Shin Kurushima Dockyard Co., Ltd., Imabari, Japan;2. Kyushu University, Fukuoka, Japan

Standardisation on Measurement and Interpretation

of Residual Stress Data 0MAE2019-96615

Ali Mirzaee Sisan' P John Bouchard² Foroogh Hosseinzadeh ${ }^{2}$

1. AMS Energy Solutions, Barnet, United Kingdom;

2. The Open University, Milton Keynes, United Kingdom

Small-scale HISC Testing of a Superduplex Stainless

Steel Welded Joint: The Impact and Interaction of

Testing and Residual Stresses OMAE2019-95053

Lisa Blanchard' Kasra Sotoudeh ${ }^{2}$ Tyler London ${ }^{3}$ Saurabh Kabra ${ }^{4}$

1. University of Leicester, Leicester, United Kingdom;2. TWI Ltd, Cambridge,

United Kingdom; 3. TWI Ltd, Middlesbrough, United Kingdom; 4. Science

and Technology Facilities Council, Didcot, United Kingdom

\section{Pipelines, Risers, and Subsea Systems}

\section{4-1-6 Flexible Pipes VI}

Wednesday June $12 \quad$ Room Crowne Plaza, Staffa / Shuna | 08:30 - 10:00 Session Chair: Lin Zhao, Ocean university of China, China

Session Co-Chair: Krassimir Doynov, ExxonMobil Upstream Integrated Solutions, USA

Theoretical Modeling of Steel Strip Reinforced Flexible

Pipe With Swaging End Fitting by Taking Into Account

Stress Concentration Effect OMAE2019-95462

Yifan Gao ${ }^{1}$ Wei Chen ${ }^{1}$ Yong Bai ${ }^{2}$

1. Zhejiang University, Hangzhou, China; 2. Zhejiang University, Zhejiang, China

Controlling Factors of Carcass Fatigue in

Unbonded Flexible Pipes OMAE2019-96310

Upul Fernando ${ }^{1}$ Andrew Roberts ${ }^{1}$ Michelle Davidson ${ }^{2}$

1. Baker Hughes, a GE Company, Newcastle upon Tyne, United

Kingdom; 2. GE, Newcastle upon Tyne, United Kingdom

Assessment the Carcass Role in Designing

Deepwater Riser Connectors OMAE2019-96597

Mohsen Saneian' Yong Bai ${ }^{2}$

1. Zhejiang University, Hangzhou, China; 2. Zhejiang University, Zhejiang, China
Mechanical Analysis of Fiber Glass Reinforced Bonded

Flexible Pipe under External Pressure OMAE2019-95692

Xiaojie Zhang, Yong Bai, Chang Liu, Zhao Wang, Jiannan Zhao

Southern University of Science and Technology, Shenzhen, China

\section{Pipelines, Risers, and Subsea Systems}

\section{4-3-4 Thermo-Mechanical I}

Wednesday June 12

Room Crowne Plaza, Castle 3 | 08:30 - 10:00

Session Chair: Rafael F. Solano, Petrobras, Brazil

Session Co-Chair: Celso Morooka, University of Campinas, Brazil

Analytical Study for Lateral Buckling of Imperfect Pipelines with Distributed Buoyancy Section OMAE2019-95031

Zhenkui Wang' G.H.M. van der Heijden ${ }^{2}$ Yougang Tang ${ }^{1}$

1. Tianjin University, Tianjin, China; 2. University College London, London, United Kingdom

Large Diameter Deepwater Gas Pipelines Subjected

to Global Buckling Behavior OMAE2019-95343

Bruno R. Antunes, Rafael F. Solano, Carlos 0. Cardoso

Petrobras, Rio de Janeiro, RJ, Brazil

Controlled Lateral Buckling of a Pipeline on the Seabed by Residual Curvature Imperfections OMAE2019-96694 Weihan Zhang, Stelios Kyriakides

University Texas at Austin, Austin, TX, USA

Application of the MCC Model on 2-D Finite Element Analyses for the Assessment of Pipe-Soil Lateral Response OMAE2019-95577

Tianna Thomaz ${ }^{1}$ Daniel Carneiro ${ }^{2}$ Gilberto Bruno Ellwanger ${ }^{3}$ Leonardo Nascimento ${ }^{4}$

1. University of Edinburgh, Edinburgh, United Kingdom;2. Wood Group,

Rio de Janeiro, RJ, Brazil; 3. Universidade Federal do Rio de Janeiro, Rio

de Janeiro, RJ, Brazil; 4. Bureau Veritas, Rio de Janeiro, RJ, Brazil

\section{Ocean Space Utilization}

\section{5-5-1 Floating Systems for Renewable Energy}

Wednesday June 12

Room SEC, Dochart 1 | 08:30 - 10:00

Session Chair: Motohiko Murai, Yokohama National University, Japan

Session Co-Chair: Qiao Li, Institute of Industrial Science, the University of Tokyo, Japan

Validation of the Motion Analysis Method of Floating

Offshore Wind Turbines using Observation Data Acquired

by Full Scale Demonstration Project OMAE2019-95828

Haruki Yoshimoto, Ken Kamizawa

Japan Marine United Corporation, Yokohama, Japan

Quantitative Wear Estimation for Mooring Chain of

Floating Structures and its Validation OMAE2019-96750

Takaaki Takeuchi' Tomoaki Utsunomiya' Koji Gotoh' Iku Sato ${ }^{2}$

1. Kyushu University, Fukuoka, Japan; 2. Toda Corporation, Tokyo, Japan

Development of Wireless Control System with Underwater

Fish Eye Video Camera to Monitor Fish at the Test Site

of Marine Renewable Energy 0MAE2019-95978

Takero Yoshida' Yoichi Mizukami' Jinxin Zhou ${ }^{2}$ Daisuke Kitazawa ${ }^{3}$

1. Institute of Industrial Science, The University of Tokyo, Kashiwa,

Japan; 2. Institute of Industrial Science, The University of Tokyo,

Chiba, Japan; 3. The University of Tokyo, Kashiwa, Japan 
Investigation on the Relationship between Rotor Speed and Flow Rate of Rotary Energy Recovery Device OMAE2019-96715 Lei Jiao, Dekai Huang, Zhaohui Qian, Tianzhuang Ye, Ming Sheng, Han Ge Zhejiang University, Zhoushan, China

\section{Ocean Engineering}

\section{6-3-1 Fluid-Structure Interaction/Hydroelasticity}

Wednesday June 12

Room SEC, M4 | 08:30 - 10:00

Session Organizer: Pierre Ferrant, Ecole Centrale De Nantes/CNRS, France

A Fluid-structure Interaction Study on a Passively

Deformed Fish Fin OMAE2019-95578

Yang Luo' Qing Xiao' Guangyu Shi' Li Wen ${ }^{2}$ Zhiming Yuan ${ }^{3}$

1. University of Strathclyde, Glasgow, United Kingdom; 2. Beihang University,

Beijing, China; 3. University of Strathclyde, NAOME, Glasgow, United Kingdom

Impact of a Plate on an Asymmetric Water Wedge OMAE2019-95556

Xueliang Wen, Peiqing Liu, Qiulin Qu, Qingchuan Liu

Beihang University, Beijing, China

The Development of 3D Hydroelastic Software and

its Application on Platform OMAE2019-96122

Xinyun Ni, Zhengwei Zhang, Chao Tian, Ye Lu, Jun Ding

China Ship Scientific Research Center, Wuxi, China

Air-gap Analyses of a Semi-submersible considering

Full Second Order Effects OMAE2019-95300

Zhiyuan Pan, Torgeir Kirkhorn Vada, Arne Nestegaard

DNV GL, Høvik, Norway

A Numerical Evaluation of the Quadratic Transfer

Function for a Floating Structure OMAE2019-95620

Zhitian Xie, Yujie Liu, Jeffrey Falzarano

Texas A\&M University, College Station, TX, USA

\section{Ocean Engineering}

\section{6-5-1 Advanced Marine Hydrodynamics I}

Wednesday June 12

Room SEC, M2 \& M3 | 08:30 - 10:00

Session Chair: Gregor Macfarlane, Australian Maritime

College, University of Tasmania, Australia

Session Co-Chair: Sanne van Essen, MARIN, Netherlands

Validation of Hydrodynamic Loads on a Large-diameter

Monopile in Regular Waves OMAE2019-95929

Fatemeh H. Dadmarzi' Maxime Thys ${ }^{2}$ Erin E. Bachynski ${ }^{1}$

1. Norwegian University of Science and Technology, Trondheim,

Norway; 2. SINTEF Ocean AS, Trondheim, Norway

Improvement of Wave Loads Estimation using Spatial

Pressure Distribution on Ship Hull OMAE2019-95273

Kurniawan T. Waskito, Masashi Kashiwagi

Osaka University, Osaka, Japan

Large Amplitude Time Domain Seakeeping Simulations of KVLCC2 in Head Seas taking into account Forward Speed Effect OMAE2019-95316

Christos Pollalis' Evangelos Boulougouris' ${ }^{2}$ sman Turan' Olgun Hizir ${ }^{1}$

1. University of Strathclyde, Glasgow, United Kingdom;

2. University of Strathclyde, MSRC, Glasgow, United Kingdom
On the Hydrodynamic Interaction between Ship and Free-surface Motions on Vessels with Moonpools OMAE2019-95932 Senthuran Ravinthrakumar' ${ }^{1}$ Trygve Kristiansen' ${ }^{1}$ Babak $0 \mathrm{mmani}^{2}$ 1. Norwegian University of Science and Technology, Trondheim, Norway; 2. SINTEF Ocean, Trondheim, Norway

\section{Ocean Engineering}

\section{6-8-1 Wave Loads}

Wednesday June 12

Room SEC, Dochart 2 | 08:30 - 10:00

Session Chair: Longbin Tao, University of Strathclyde, United Kingdom

Preliminary Experimental Study on the Influence of the Local Wind Field on Forces from Breaking

Waves on a Circular Cylinder OMAE2019-95179

Julie Caroee Kristoffersen ${ }^{1}$ Henrik Bredmose ${ }^{2}$ Christos Thomas Georgakis ${ }^{1}$ Longbin Ta $0^{3}$

1. Aarhus University, Aarhus, Denmark; 2. Delft University of Technology wind,

Copenhagen, Denmark; 3. University of Strathlyde, Glasgow, United Kingdom

A Method for Designing the Backbone for the Segmented Model of an Ultra-large Container Carrier OMAE2019-96136 Hui Li' Jian Zou' Weijia Sheng ${ }^{2}$ Xuecong Hu' Wenjia Hu' 1. Harbin Engineering University, Harbin, China; 2. Haerbin Engineering University, Harbin, China

Study on Flow Field and Shielding Effect of Semi-sphere Artificial Reef and other Submarine Structures OMAE2019-96444 Lin Zhao, Junwei Tan

Ocean University of China, Qingdao, China

Experimental Evaluation of Hydrodynamic

Loads on Marine Risers OMAE2019-96569

Vinicius Vileti' Paulo de Tarso T. Esperança' Marcelo A. Vitola ${ }^{2}$ Mario Vignoles ${ }^{3}$

1. LabOceano/COPPE/Universidade Federal do Rio de Janeiro, Rio de

Janeiro, RJ, Brazil; 2. LabOceano - Universidade Federal do Rio de Janeiro,

Rio de Janeiro, RJ, Brazil; 3. TechnipFMC, Rio de Janeiro, RJ, Brazil

Numerical and Experimental Study on the Dynamics of a

High Compressed Air Generated Bubble OMAE2019-95232

Shiping Wang, Xiugang Lu, Aman Zhang

Harbin Engineering University, Harbin, China

\section{Polar and Arctic Sciences and Technology}

\section{7-3-1 Structures in Ice}

Wednesday June 12 Room SEC, Alsh 2 | 08:30 - 10:00 Session Chair: Michael Huisman, Hamburg University of Technology, Germany Session Co-Chair: Walter Kuehnlein, sea2ice Ltd. \& Co. KG, Germany

Wear Amount of Steel Structure in Ice-infested

Sea by Sliding Wear Test OMAE2019-95654

Takahiro Takeuchi' Shinji Kioka

1. Hachinohe Institute of Technology, Hachinohe, Japan; 2. Civil Engineering Research Institute for Cold Region Public Works Research, Sapporo, Japan

Validation of Pack Ice Resistance in Oblique Condition by the Comparison with Ice Model Test Results OMAE2019-95689 HyunSoo Kim Erinc Ozden ${ }^{2}$ Jae-bin Lee ${ }^{2}$

1. Inha Technical College, Incheon, Korea; 2. South Korea /Inha university, Incheon, Korea 
Hydrodynamic Modelling and Estimating Response of Glacial Ice Near a Drilling Rig OMAE2019-95798

Babak Ommani' Petter Andreas Berthelsen' Halvor Lie ${ }^{1}$ Vegard Aksnes ${ }^{1}$ Geir Løland ${ }^{2}$ 1. SINTEF Ocean, Trondheim, Norway; 2. Petroleum Safety

Authority Norway, Stavanger, Norway

Eliminating the Uncertainties in Hydraulic and Ice

Loads on Berm Breakwaters OMAE2019-95139

Maria Pontiki' Bernt Leira ${ }^{2}$ Knut Vilhelm Høyland ${ }^{2}$

1. University of Delaware, Department of Civil and Environmental Engineering, Newark, DE, USA; 2. Norwegian University of Science and Technology, Trondheim, Norway

\section{CFD \& FSI}

\section{8-3-1 Data-Driven Modeling and Machine Learning}

Wednesday June 12 Room SEC, Lomond Auditorium | 08:30 - 10:00 Session Chair: Rajeev Kumar Jaiman, University of British Columbia, Canada Session Co-Chair: Luis Eca, Technical University of Lisbon, Portugal

Numerical Study of Breaking Waves and Associated Wave Forces on a Jacket Substructure for Offshore Wind Turbines OMAE2019-95233 Ankit Aggarwal, Tobias Martin, Seimur Shirinov, Hans Bihs, Arun Kamath Norwegian University of Science and Technology, Trondheim, Norway

Three-Dimensional Numerical Analysis of Horizontal and Vertical Coalescence of Bubbles at Two Submerged Horizontal Orifices on the Wall OMAE2019-95850

Zhipeng Li, Longquan Sun, Xiongliang Yao, Yi Piao Harbin Engineering University, Harbin, China

A Hybrid Data-driven Deep Learning Technique for Fluid-Structure Interaction 0MAE2019-95870 Tharindu Pradeeptha Miyanawala' Rajeev Kumar Jaiman² 1. National University of Singapore, Singapore, Singapore; 2. University of British Columbia, Vancouver, BC, Canada

Reduced Order Model for Unsteady Fluid Flows via Recurrent Neural Networks OMAE2019-96543 Sandeep Bukka Reddy ${ }^{1}$ Allan Magee ${ }^{1}$ Rajeev Kumar Jaiman ${ }^{2}$ Jing Liu ${ }^{3}$ Wei X $u^{3}$ Ankit Choudhary ${ }^{3}$ Anis Altaf Hussain ${ }^{3}$ 1. National University of Singapore, Singapore, Singapore; 2. University of British Columbia, Vancouver, BC, Canada;

3. Keppel Offshore and Marine Technology Centre, Singapore, Singapore

\section{Ocean Renewable Energy}

\section{9-1-3 FWT - Numerical Analysis II}

Wednesday June 12 Room SEC, Carron 1 | 08:30 - 10:00 Session Chair: Carlos Souza, Norwegian University of Science and Technology, Norway Session Co-Chair: Wei Shi, Dalian University of Technology, China

Numerical Simulations of OC3 Spar and OC4 Semi-submersibleType Platforms under Extreme Conditions in the East Sea, Korea OMAE2019-95919 Hyunkyoung Shin, Youngjae Yu, Thanh Dam Pham, Junbae Kim, Rupesh Kumar University of Ulsan, Ulsan, Korea

A Comparative Study on the Dynamic Response of Three Semi-submersible Floating Offshore Wind Turbines OMAE2019-96221 Wei Shi' 'Lixian Zhang' Dezhi Ning' Zhiyu Jiang ${ }^{2}$ Constantine Michailides ${ }^{3}$ Madjid Karimirad ${ }^{4}$ 1. Dalian University of Technology, Dalian, China; 2. University of Agder, Grimstad, Norway; 3. Cyprus University of Technology, Limassol, Cyprus; 4. Queen's University Belfast, Belfast, United Kingdom
Effects of Platform Mounting Orientations on the Long-term Performance of a Semi-submersible Floating Wind Turbine OMAE2019-96240 Shengtao Zhou' Chao Li' Yiqing Xiao ${ }^{1}$ Frank Lemmer ${ }^{2}$ Wei Y $u^{2}$ Po Wen Cheng ${ }^{2}$ 1. Harbin Institute of Technology, Shenzhen, China; 2. University of Stuttgart, Stuttgart, Germany

Dynamic Response of Spar-type Floating Offshore Wind Turbine in Freak Wave OMAE2019-95638 Yougang Tang ${ }^{1}$ Yan Li' Peng Xie ${ }^{1}$ Xiaoqi $\mathrm{Qu}^{1}$ Bin Wang ${ }^{2}$ 1. Tianjin University, Tianjin, China; 2. CCS, Tianjin, China

\section{Petroleum Technology}

\section{1-15-1 Well Abandonment I - Rules and Regulations}

Wednesday June 12 Room Crowne Plaza, Jura | 08:30 - 10:00 Session Chair: Mahmoud Khalifeh, UiS, Norway

Well Abandonments in British Columbia OMAE2019-95163 Majid Bizhani' Elizabeth Trudel lan Frigaard ${ }^{2}$ 1. University of British Columbia, Edmonton, $A B$, Canada; 2. University of British Columbia, Vancouver, $B C$, Canada

A Critical Review of Rules and Regulations for Permanently Plugged and Abandoned Wells OMAE2019-95330 Mahmoud Khalifeh ${ }^{1}$ Babak Akbari² Ahsan Khan' Daniel Braga ${ }^{2}$ 1. UiS, Stavanger, Norway; 2. Louisiana State University, Baton Rouge, LA, USA

Robust Leakage Modeling for Plug \& Abandonment Applications OMAE2019-95612 Mustafa Al Ramadan' Saeed Salehi Catalin Teodoriu ${ }^{2}$ 1. University of Oklahoma, Norman, OK, USA; 2. Mewbourne School of Petroleum and Geological Engineering, Norman, OK, USA

Fate of Hydrocarbon Leaks from Plugged and Abandoned Wells Compared to Natural Seepages OMAE2019-95674 Mari R. Tveit ${ }^{1}$ Mahmoud Khalifeh' ${ }^{1}$ Tor Nordam² Arild Saasen ${ }^{3}$ 1. UiS, Stavanger, Norway; 2. SINTEF Ocean AS, Trondheim, Norway; 3. UiS, Gullaug, Norway

\section{Petroleum Technology}

\section{1-2-1 Drilling Mechanics Session I}

Wednesday June 12 Room Crowne Plaza, Barra | 08:30 - 10:00 Session Chair: Jorge H B Sampaio Jr., Colorado School of Mines, USA

On the Importance of the Coupling between Transient Mechanical, Hydraulic and Thermal Effects for the Modelling of Real-time Drilling Operations OMAE2019-95062 Erik W. Dvergsnes ${ }^{1}$ Eric Cayeux ${ }^{2}$

1. NORCE, Kristiansand, Norway; 2. NORCE, Stavanger, Norway

Experimental Study of Drillstring Dynamics using a High-speed Camera as a Non-invasive Motion Sensor OMAE2019-95217 Ekaterina Wiktorski, Milad Khatibi, Suranga Geekiyanage, Dan Sui, Rune Wiggo Time University of Stavanger, Stavanger, Norway

Modal Interactions in Drillstring Borehole Interactions OMAE2019-95871 Kartheek Amaroju' Kiran Vijayan² Michael Friswell ${ }^{3}$

1. OENA, IIT Kharagpur, Kharagpur, India; 2. IIT Kharagpur, Kharagpur, India; 3. Swansea University, Bay Campus, Swansea, United Kingdom 
The Characteristics of Numerical Solution of NDDE to

Solve the Drill Pipe Stick-slip OMAE2019-95883

Tokihiro Katsui' Tomoya Inoue ${ }^{2}$ Daisuke Sogawa' Yusuke Notani ${ }^{1}$

1. Kobe University, Kobe, Japan; 2. JAMSTEC, Yokosuka, Japan

\section{Rodney Eatock Taylor Honouring Symposium on Marine and Offshore Hydrodynamics}

\section{2-1-2 Numerical and Experimental Methods in Hydrodynamics II}

Wednesday June 12 Room SEC, Carron 2 | 08:30 - 10:00

Session Chair: Arne Løken, Dr. Ing. Arne E. Løken Engineering \& Consulting, Norway Session Co-Chair: Xinshu Zhang, Shanghai Jiao Tong University, China

Hydro-elastic Analysis and Validation of an End-anchored

Floating Bridge under Wave and Current Loads OMAE2019-95114

Xu Xiang ${ }^{1}$ Arne Løken ${ }^{2}$

1. Norwegian Public Roads Administration, Oslo, Norway;

2. Dr. Ing. Arne E. Løken Engineering \& Consulting, Lier, Norway

Development of an Experimental System for the Twin-lift Decommissioning Operation OMAE2019-96739

Xin Li' Zhihuan Hu' ${ }^{1}$ Andy Wang ${ }^{2}$ Weidong Zhang ${ }^{1}$ Xiao Wu' Xinliang Tian' ${ }^{1}$ Hao Sun ${ }^{3}$

1. Shanghai Jiao Tong University, Shanghai, China; 2. DNV GL - Oil \& Gas China,

Shanghai, China; 3. COSCO Shipping Specialized Carriers Co. Ltd, Guangzhou, China

Edge Effect on Numerical Calculation of Nonlinear

Radiation Forces for a Submerged Body OMAE2019-95421

Jianye Yang, Bin Teng, Ying Gou

Dalian University of Technology, Dalian, China

Hydrodynamic Investigation of a Novel Concept of

OWC Type Wave Energy Converter Device OMAE2019-96510

Kourosh Rezanejad' Carlos Guedes Soares ${ }^{2}$

1. Centre for Marine Technology and Ocean Engineering (CENTEC),

Instituto Superior Técnico(IST), Lisboa, Portugal; 2. Instituto Superior

Técnico, Universidade de Lisboa, Lisboa, Portugal

\section{Takeshi Kinoshita Honoring Symposium on Offshore Technology}

\section{3-1-1 Extremes and Environmental Modelling}

Wednesday June 12

Room SEC, Boisdale 2 | 08:30 - 10:00

Session Chair: Kevin Ewans, Met0cean Research Ltd, New Zealand

Estimation of Expected Loss by Storm Surges

along Tokyo Bay Coast OMAE2019-95336

Rikito Hisamatsu' Sooyoul Kim² Shigeru Tabeta'

1. The University of Tokyo, Kashiwa, Japan; 2. Tottori University, Tottori, Japan

Estimating Extreme Waves in Gulf of Mexico using a

Simple Spatial Extremes Model OMAE2019-95442

Ryota Wada' Philip Jonathan ${ }^{2}$ Takuji Waseda' Shejun Fan ${ }^{3}$

1. The University of Tokyo, Kashiwa, Japan; 2. Shell Research Ltd., London,

United Kingdom; 3. Shell Oil Company, Houston, TX, USA
Environmental Restoration for a Small-scale Beach

"Heda-Mihama Project" OMAE2019-95596

Shigeru Tabeta ${ }^{1}$ Ken 0 kamoto ${ }^{2}$ Takayoshi Kato ${ }^{2}$ Rikito Hisamatsu' Hiroaki Muto

Akinori Hino ${ }^{3}$ Motohiko Murai ${ }^{4}$ Sho Ito ${ }^{5}$ Daisuke Kitazawa' Takeshi Kinoshita ${ }^{6}$

1. The University of Tokyo, Kashiwa, Japan; 2. The University of Tokyo, Tokyo, Japan; 3. Marine Ecology Research Institute, Tokyo, Japan; 4. Yokohama National University, Yokohama, Japan; 5. Nichimo Co.Ltd, Tokyo, Japan; 6. Nagasaki Institute of Applied Science, Nagasaki, Japan

Application of the Spectral Nudging on Global Tides towards a Global Total Water Level Prediction System OMAE2019-95842

Tsubasa Kodaira' Natacha Bernier2 Keith Thompson ${ }^{3}$

1. The University of Tokyo, Kashiwa, Japan; 2. Environment and Climate Change

Canada, Dorval, QC, Canada; 3. Dalhousie University, Halifax, NS, Canada

\section{REFRESHMENT BREAK \\ 10:00 - 10:30 \\ Location: Hall 5 (SEC)}

\section{CONCURRENT SESSIONS \\ 10:30 - 12:00}

\section{Offshore Technology}

\section{1-4-2 Numerical Design and Analysis}

Wednesday June 12

Room SEC, Alsh 1 | 10:30 - 12:00

Session Chair: Yanlin Shao, Technical University of Denmark, Denmark

Session Co-Chair: Xu Xiang, Norwegian Public Roads Administration, Norway

Calculation of the Dynamic Positioning Capability

of an Offshore Wind Farm Vessel during the Jack-up

Process in the Early Design Stage OMAE2019-95248

Maximilian Liebert

Hamburg University of Technology, Hamburg, Germany

Evaluation of Impact Loads on Offshore Jacket Platform during Float-over Mating Operation OMAE2019-95467 Gurumurthy Kagita, Mahesh Babu Addala, Gudimella G. S.,

Achary, Subramanyam V. R., Sripada

Engineers India Limited, Gurugram, India

Component-based Modeling and Simulation of Nonlinear Drill-string Dynamics OMAE2019-95474 Njaal Tengesdal' Christian Holden ${ }^{1}$ Eilif Pedersen ${ }^{2}$ 1. Dept. Mechanical and Industrial Engineering, Norwegian University of Science and Technology, Trondheim, Norway; 2. Norwegian University of Science and Technology, Trondheim, Norway

Use of 3D Scan of Weld Joint in Finite Element Analysis and Stochastic Analysis of Hot-spot Stresses in Tubular Joint for Fatigue Life Estimation OMAE2019-95704 Mikkel L. Larsen ${ }^{1}$ Vikas Arora ${ }^{2}$ Marie Lützen ${ }^{2}$ Ronnie R. Pedersen ${ }^{3}$ Eric Putnam ${ }^{4}$ 1. Ramboll Offshore Wind, København S, Denmark; 2. University of Southern Denmark, Odense, Denmark; 3. Ramboll Offshore Wind, Esbjerg, Denmark; 4. FORCE Technology, Munkebo, Denmark

Asymmetrical Twin-hull Crane Vessel Global Performance Study OMAE2019-96603 Joe Zhou, Kai Huang, Jinguang Wang, Indra Datta, Lixin Xu China Merchants Offshore Technology Research Center, Haimen, China 


\section{Structures, Safety and Reliability}

\section{2-1-2 Abnormal or Rogue Waves II}

Wednesday June 12

Room Crowne Plaza, Castle 1 | 10:30 - 12:00

Session Chair: Alexander V. Babanin, University of Melbourne, Australia

Session Co-Chair: Elzbieta M. Bitner-Gregersen, DNV GL, Norway

Comparison of Temporal and Spatial Statistics

of Nonlinear Waves OMAE2019-95357

Elzbieta M. Bitner-Gregersen, Odin Gramstad

DNV GL, Høvik, Norway

Effect of Spectrum Tail Length on Modulational Instability and

Freak Wave Occurrence in JONSWAP Sea States OMAE2019-95740

Cagil Kirezci, Alexander V. Babanin

University of Melbourne, Melbourne, VIC, Australia

Predicting Extreme Waves from Wave Spectral

Properties using Machine Learning 0MAE2019-96061

Odin Gramstad, Elzbieta M. Bitner-Gregersen

DNV GL, Høvik, Norway

"Three Sisters" Measured as a Triple Rogue Wave Group 0MAE2019-96837

Anne Karin Magnusson ${ }^{1}$ Karsten Trulsen ${ }^{2}$ Elzbieta M. Bitner-Gregersen ${ }^{3}$

Ole Johan Aarnes ${ }^{1}$ Mika Malila ${ }^{4}$

1. Norwegian Meteorological Institute, Bergen, Norway; 2. University

of Oslo, Oslo, Norway; 3. DNV GL AS, Høvik, Norway; 4. Norwegian

Meteorological Institute/University of Bergen, Bergen, Norway

\section{Structures, Safety and Reliability}

\section{2-11-2 Ultimate Strength II}

Wednesday June 12

Room Crowne Plaza, Castle 2 | 10:30 - 12:00

Session Chair: Deyu Wang Shanghai Jiao Tong University, China

Session Co-Chair: Masahiko Fujikubo, Osaka University, Japan

Evaluation of Hull Girder Capacity considering the Effects of

Lateral Pressure and Transverse Stresses OMAE2019-95867

Karan Doshi, Yogendra Parihar, Saikat Dan

Indian Register of Shipping, Mumbai, India

Experimental Study on Ultimate Strength of Thin-walled

Square Tube under Axial Compression OMAE2019-96134

Hanwei Zhou' ${ }^{1}$ Ling Zhu' Shengming Zhang ${ }^{2}$ Tongxi Yu ${ }^{3}$

1. Wuhan University of Technology, Wuhan, China; 2. Lloyd's Register,

Southampton, United Kingdom; 3. Hong Kong University of Science

\& Technology, Clear Water Bay-kowloon, Hong Kong

Ultimate Compressive Strength of Eccentrically Loaded Stiffened

Panels in Ship Structures: A Computational Study 0MAE2019-96708

Konstantinos Anyfantis

National Technical University of Athens, Zografou, Greece

\section{Materials Technology}

\section{3-3-1 Fatigue Improvement and Repairs}

Wednesday June 12

Room SEC, Boisdale 1 | 10:30 - 12:00

Session Chair: Yanhui Zhang, TWI Ltd, United Kingdom

Session Co-Chair: Myung-Hyun Kim, Pusan National University, Korea

A Study on Improvement of Fatigue Performance by

Shot Blasted Surface Treatment OMAE2019-95817

Toshihiro Fujii' Koji Gotoh ${ }^{2}$ Junichi Deguchi' Koji Murakami

1. Oshima Shipbuilding Co., Ltd., Saikai, Japan; 2. Kyushu University, Fukuoka, Japan

Fatigue Life Evaluation for the Repaired Methods

of High Pressure Gas Pipeline OMAE2019-95281

Woo Sik Kim

KOGAS, Ansan, Korea

World First Fatigue S-N Curve for Bonded Repairs

and FPSO Application OMAE2019-96239

Hamza Abbad el Andaloussi' Luc Mouton² Firas Sayed Ahmad'

Stéphanie Maherault-Mougin ${ }^{3}$ Stéphane Paboeuf ${ }^{2}$ Xabier Errotabehere ${ }^{1}$

1. COLD PAD, Paris, France; 2. Bureau Veritas, Nantes, France; 3. Bureau Veritas, Puteaux, France

Numerical Investigation on Surface Crack Growth in Steel Plates

Repaired with Carbon Fiber-reinforced Polymer OMAE2019-95746

Zongchen LI, Xiaoli Jiang, Hans Hopman

Delft University of Technology, Delft, Netherlands

\section{Pipelines, Risers, and Subsea Systems}

\section{4-1-7 Flexible Pipes VII}

Wednesday June $12 \quad$ Room Crowne Plaza, Staffa / Shuna | 10:30 - 12:00 Session Chair: José Renato M. de Sousa, Federal University of Rio de Janeiro, Brazil Session Co-Chair: Farzan Parsinejad, Chevron, USA

Development of Flexible Composite Pipe Cross-section Design Software based on Visual Basic OMAE2019-95916 Xinyu Sun 'Yong Bai' Jiannan Zhao' Chang Liu' Xiaojie Zhang' 1. Southern University of Science and Technology, Shenzhen,

China; 2. Zhejiang University, Zhejiang, China

Thermal and Mechanical Coupled Analysis of Marine Composite Cryogenic Pipeline OMAE2019-96144

Haitao Hu' Jun Yan' Baoshun Zhou' Zhixun Yang ${ }^{1}$ Liang Yang ${ }^{2}$ Jiakun Fan ${ }^{2}$ 1. Dalian University of Technology, Dalian, China; 2. CNNOC Gas \& Co., Ltd, Beijing, China

A Three-Dimensional FE Approach for the Stress Analysis of Tensile Armors inside End Fittings OMAE2019-95506

Marcelo Miyazaki' José Renato M. de Sousa ${ }^{2}$ Gilberto Bruno Ellwanger ${ }^{2}$

1. Technip FMC, Rio de Janeiro, RJ, Brazil; 2. Federal University

of Rio de Janeiro, Rio de Janeiro, RJ, Brazil

An Analytical Approach for Predicting the Collapse Pressure of the Flexible Risers with Initial Ovalization and Gap OMAE2019-95642 Xiao Li' Xiaoli Jiang ${ }^{2}$ Hans Hopman²

1. Delft University of Technology, Delft, Netherlands; 2. TU Delft, Delft, Netherlands 


\section{Pipelines, Risers, and Subsea Systems}

\section{4-3-5 Thermo-Mechanical II}

Wednesday June 12

Room Crowne Plaza, Castle 3 | 10:30 - 12:00

Session Chair: Daniel Carneiro, Wood, Brazil

Session Co-Chair: Rafael F. Solano, Petrobras, Brazil

Overview of the Lateral Buckling and Walking Designs of

Deepwater Pipelines in Offshore Brazil OMAE2019-95289

Rafael F. Solano, Carlos 0. Cardoso, Bruno R. Antunes

Petrobras, Rio de Janeiro, RJ, Brazil

Lessons Learned from Operational Integrity of HP/HT

Deepwater Pipelines OMAE2019-95298

Carlos 0. Cardoso, Rafael F. Solano, Bruno R. Antunes

Petrobras, Rio de Janeiro, RJ, Brazil

Walking Anchors - When to Fix One or Both Ways? OMAE2019-95359

Daniel Carneiro ${ }^{1}$ Luciano Franco ${ }^{2}$

1. Wood, Rio de Janeiro, RJ, Brazil; 2. McDermott, Rio de Janeiro, RJ, Brazil

Challenges and Lessons Learnt from the Design, Fabrication, and Installation of Pipe Walking Mitigations OMAE2019-95055

Curti Gianbattista ${ }^{1}$ Pavone Diego ${ }^{1}$ Marchionni Lorenzo ${ }^{1}$

Guyon Vivien² Perrin Frederic ${ }^{2}$ Pirinu Gianluigi ${ }^{2}$

1. Saipem, Fano, Italy; 2. Saipem, Saint Quentin Yvelines, France

\section{Ocean Space Utilization}

\section{5-1-2 Hybrid and Complex Use of Floating Systems I}

Wednesday June 12

Session Chair: Fonseca Nuno, SINTEF Ocean, Norway

Session Co-Chair: Tomoki lkoma, Nihon University, Japan

New Engineering Approach for the Development and Demonstration of a Multi-purpose Platform for the Blue Growth Economy 0MAE2019-96104 Fabrizio Lagasco ${ }^{1}$ Maurizio Collu ${ }^{2}$ Alessandra Mariotti ${ }^{3}$ Elchanan Safier ${ }^{4}$ Felice Arena ${ }^{5}$ Timothy H. Atack ${ }^{6}$ Giulio Brizzi ${ }^{7}$ Paul Tett ${ }^{8}$ Anita Santoro ${ }^{9}$ Sylvain Bourdier ${ }^{10}$

Fernando Salcedo Fernandez ${ }^{11}$ Muggiasca Sara ${ }^{12}$ Ibon Larrea ${ }^{13}$

1. RINA Consulting S.p.A., Genova, Italy; 2. University of Strathclyde, Glasgow, United Kingdom; 3. Fincosit s.r.l., Genova, Italy; 4. Safier Ingenierie SAS, Serris, France;

5. Mediterranea University, Reggio Calabria, Italy; 6. Sagro Aquaculture Ltd, Limassol, Cyprus; 7. Chlamys s.r.l., Trani, Italy; 8. The Scottish Association For Marine Science LBG, Dunbeg Oban, United Kingdom; 9. Wavenergy.It srl, Reggio Calabria, Italy; 10. Ecole Centrale de Nantes, Nantes, France; 11. Fundacion Tecnalia Research \& Innovation, Derio Bizkaia, Spain; 12. Politecnico di Milano, Milano, Italy; 13. Ditrel Industrial S.L., Vitoria, Spain

Frequency Domain Analysis of a Hybrid Aquaculture-Wind Turbine Offshore Floating System OMAE2019-96171

Abhinav K A ${ }^{1}$ Maurizio Collu² Sun Ke ${ }^{3}$ Zhou Bin Zhen ${ }^{3}$

1. Dept. of Naval Architecture, Ocean and Marine Engineering, University of

Strathclyde, Glasgow, United Kingdom; 2. University of Strathclyde, Glasgow,

United Kingdom; 3. College of Shipbuilding Engineering, Harbin, China

Analysis of the Coupled Dynamics of an Offshore Floating Multi-purpose Platform, Part A: Rigid Body Analysis OMAE2019-96212 Liang Li' Maurizio Collu' Carlo Ruzzo² Giuseppe Failla² KA Abhinav' Felice Arena ${ }^{2}$ 1. University of Strathclyde, Glasgow, United Kingdom;

2. Mediterranea University, Reggio Calabria, Italy
Analysis of the Coupled Dynamics of an Offshore Floating Multi-purpose Platform, Part B: Hydro-elastic Analysis with Flexible Support Platform 0MAE2019-96282 Carlo Ruzzo ${ }^{1}$ Giuseppe Failla ${ }^{1}$ Felice Arena ${ }^{1}$ Maurizio Collu ${ }^{2}$ Liang Lii Alessandra Mariotti ${ }^{3}$ 1. Mediterranea University, Reggio Calabria, Italy; 2. University of Strathclyde, Glasgow, United Kingdom; 3. Fincosit s.r.l., Genova, Italy

\section{Ocean Engineering}

\section{6-3-2 Wave-Body Interactions/CFD}

Wednesday June 12 Room SEC, M4 | 10:30 - 12:00 Session Chair: Jeffrey Falzarano, Texas A\&M University, USA

Interaction of Fixed Cylinder with Waves through Weakly Coupled FNPT and Lagrangian Navier-stokes OMAE2019-96745 Shagun Agarwal, Sriram Venkatachalam, K. Murali Indian Institute of Technology Madras, Chennai, India

Numerical Simulation of Fully Nonlinear Interaction between Regular and Irregular Waves and a 2D Floating Body 0MAE2019-96680 Haoran Li, Erin E. Bachynski Norwegian University of Science and Technology, Trondheim, Norway

Gap Resonance of Fixed Floating Multi Caissons OMAE2019-96383 Limin Chen 1 Guanghua He ${ }^{2}$ Harry B. Bingham ${ }^{3}$ Yanlin Sha ${ }^{4}$ 1. Harbin Institute of Technology, Harbin, China; 2. Harbin Institute of Technology, Weihai, China; 3. Delft University of Technology, Lyngby, Denmark; 4. Technical University of Denmark, Kongens Lyngby, Denmark

A Numerical Study of Wave Impacts on a

Semi-submersible OMAE2019-95070

Yanfei Deng, Wei Feng, Lei Li, Youwei Kang, Xiqia Chen CIMC Offshore Co. Ltd., Shenzhen, China

\section{Ocean Engineering}

\section{6-5-2 Advanced Marine Hydrodynamics II}

Wednesday June 12

Session Chair: Ilmas Bayati, MARIN, Netherlands

Numerical Modelling of Wave Interaction with an FPSO under Different Incident Wave Conditions OMAE2019-96004 Arun Kamath' Tobias Martin ${ }^{1}$ Hans Bihs ${ }^{2}$

1. Norwegian University of Science and Technology, Trondheim, Norway; 2. Norwegian University of Science and Technology, Sør-Trondelag, Norway

Parametric Rolling in Regular Head Waves of the KRISO Container Ship (KCS): Numerical and Experimental Investigation in Shallow Water OMAE2019-96341 Manases Tello Ruiz ${ }^{1}$ Jose Villagomez ${ }^{2}$ Guillaume Delefortrie ${ }^{2}$ Evert Lataire ${ }^{1}$ Marc Vantorre 1. Ghent University, Ghent, Belgium; 2. Flanders Hydraulics Research, Antwerp, Belgium

Wave Effects on the Turning Circle Ability of an Ultra Large Container Ship in Shallow Water OMAE2019-96346 Manases Tello Ruiz' ${ }^{1}$ Marc Mansuy' ${ }^{1}$ Luca Donatini' Jose Villagomez ${ }^{2}$ Guillaume Delefortrie ${ }^{2}$ Evert Lataire ${ }^{1}$ Marc Vantorre 1. Ghent University, Ghent, Belgium; 2. Flanders Hydraulics Research, Antwerp, Belgium

System Identification of Abkowitz Model for Ship Manoeuvring Motion based on Epsilon-support Vector Regression OMAE2019-96699 Bin Liu' Yuting Jin ${ }^{1}$ Allan Magee' Lucas J. Yiew' Shanli Zhang ${ }^{2}$

1. Technology Centre for Offshore and Marine Singapore, Singapore, Singapore; 2. TCOMS, Singapore, Singapore 


\section{Ocean Engineering}

\section{6-8-2 Ship Hydrodynamics}

Wednesday June 12

Room SEC, Dochart 2 | 10:30 - 12:00

Session Chair: Claudio A. Rodriguez C., Universidade Federal de Rio de Janeiro, Brazil

Uncertainty Analysis of Free Running Manoeuvring Model Tests

on a Modern Ferry, with Emphasis on Heel Angles OMAE2019-95513

Anton Kisjes' Frans H. H. A. Quadvlieg ${ }^{2}$ Victor Ferrari²

1. MARIN, Overveen, Netherlands; 2. MARIN, Wageningen, Netherlands

Biofouling Characterization and its Effect on

Resistance of Surface Ship OMAE2019-96220

Della Thomas, S. Surendran, Nilesh J. Vasa

Indian Institute of Technology Madras, Chennai, India

Estimation of Roll Damping Coefficients based on Model

Tests Responses of a FPSO in Waves OMAE2019-96334

Claudio Alexis Rodríguez' Paulo de Tarso T. Esperança ${ }^{2}$ Mauro C. de Oliveira ${ }^{3}$

1. LabOceano - Universidade Federal do Rio de Janeiro, Rio De Janeiro, RJ,

Brazil; 2. LabOceano/COPPE/Universidade Federal do Rio de Janeiro, Rio de

Janeiro, RJ, Brazil; 3. CENPES/PETROBRAS, Rio de Janeiro, RJ, Brazil

Experimental and Numerical Investigation on the Hydrodynamic

Performance of a CRP Propulsor OMAE2019-95311

Zheng Huang, Shancheng LI, Peng Xi, Ying Xiong

NUE, WuHan, China

Outcomes of Recent Work on the Optimisation of

Ride Control Operation to Reduce Motions and Loads

of High-speed Catamarans OMAE2019-96562

Javad Mehr' Jason Lavroff ${ }^{2}$ Damien Holloway ${ }^{2}$ Michael Davis ${ }^{2}$ Giles Thomas ${ }^{3}$

1. University of Tasmania, Launceston, TAS, Australia; 2. University of Tasmania,

Hobart, TAS, Australia; 3. University College London, London, United Kingdom

\section{Polar and Arctic Sciences and Technology}

\section{7-4-1 Vessels in Ice and Waves}

Wednesday June 12

Room SEC, Alsh 2 | 10:30 - 12:00

Session Chair: Sören Ehlers, Hamburg University of Technology, Germany

Session Co-Chair: Walter Kuehnlein, sea2ice Ltd. \& Co. KG, Germany

Numerical Simulation of Ship-Ice Interaction OMAE2019-96740

Michael Huisman, Sandro Erceg, Rüdiger Ulrich Franz Von Bock Und Polach,

Thomas Rung, Sören Ehlers

Hamburg University of Technology, Hamburg, Germany

Numerical Simulation of Ice-Wave Interaction

by Coupling DEM-CFD OMAE2019-95105

Lu Tengchao, Zaojian Zou

Shanghai Jiao Tong University, Shanghai, China

Numerical Study on Nonlinear Wave-Ice-Interaction OMAE2019-95116

Moritz Hartmann' Rüdiger Ulrich Franz Von Bock Und Polach'

Sören Ehlers ${ }^{1}$ Norbert Hoffmann ${ }^{1}$ Miguel Onorato ${ }^{2}$ Marco Klein ${ }^{1}$

1. Hamburg University of Technology, Hamburg, Germany; 2. Universita di Torino, Torino, Italy

\section{CFD \& FSI}

\section{8-3-2 Code Development and V\&V}

Wednesday June 12

Room SEC, Lomond Auditorium | 10:30 - 12:00 Session Chair: Luis Eca, Technical University of Lisbon, Portugal Session Co-Chair: Rajeev Kumar Jaiman, University of British Columbia, Canada

Plastic Accumulation in Front of a Plate in Cross Flow Model Scale Test and CFD-DEM Modeling OMAE2019-96095 Hendrik Wrenger ${ }^{1}$ Bruno Sainte-Rose ${ }^{1}$ Christoph Goniva ${ }^{2}$ Renan Hilbert ${ }^{3}$ 1. The Ocean Cleanup, Rotterdam, Netherlands;

2. DCS Computing, Linz, WA, Austria; 3. Y-plus, Paris, France

Comparisons between the Particle-based Methods Smoothed Particle Hydrodynamics (SPH) and Moving Particle Semi-implicit (MPS) to Model Dam Breaking Event OMAE2019-95102 Sergey Buruchenko' Rubens Augusto Amaro Jr. ${ }^{2}$ Liang-Yee Cheng ${ }^{2}$ 1. South Ural State University, Chelyabinsk, Russia;

2. University of São Paulo, São Paulo, SP, Brazil

Numerical Study on Vortex Induced Motion of Circular Cylinder with Low Aspect Ratio in Currents OMAE2019-95525

Jiawei He, D.C. Wan

Shanghai Jiao Tong University, Shanghai, China

Understanding the Capability of RANS Based Turbulence

Models on Fully Turbulent Channel Flow OMAE2019-96290

Yasin Kaan Ilter, Ugur Oral Unal

Istanbul Technical University, Istanbul, Turkey

\section{Ocean Renewable Energy}

\section{9-2-2 Aerodynamics II}

Wednesday June 12 Room SEC, Carron 1 | 10:30 - 12:00 Session Chair: Kurt Delpeche, Pacifico Energy K.K., Japan Session Co-Chair: Nigel Terry, Marine Renewable Engineering, United Kingdom

The Aerodynamic Performance of Offshore Twin Vertical Axis Wind Turbines with Deflector OMAE2019-95104 Yichen Jiang, Peidong Zhao, Li Zou, Guiyong Zhang, Zhi Zong Dalian University of Technology, Dalian, China

The Aerodynamic Analysis of Helical-type VAWT with Semi Empirical and CFD Method OMAE2019-95207 Ying Guo, Liqin Liu, Xinxin Lv, Yougang Tang Tianjin University, Tianjin, China

Analyzing the Effect of Shaft and End-plates of a Newly Developed Elliptical-bladed Savonius Rotor from Wind Tunnel Tests OMAE2019-95570 Nur Alom ${ }^{1}$ Nitish Kumar ${ }^{2}$ Ujjwal K. Saha'

1. Indian Institute of Technology Guwahati, Guwahati, India;

2. Larsen and Toubro, Mumbai, India

Aeromechanical Analysis of Wind Turbines using Non-linear Harmonic Method OMAE2019-96256 Shine Win Naung, Mohammad Rahmati, Hamed Farokhi Northumbria University, Newcastle upon Tyne, United Kingdom

State of the Art and Knowledge Gaps on Modelling

Structures in Cold Regions OMAE2019-95085

Rüdiger Ulrich Franz Von Bock Und Polach, Marco Klein,

Jan Kubiczek, Leon Kellner, Moritz Braun, Hauke Herrnring

Hamburg University of Technology, Hamburg, Germany 


\section{Ocean Renewable Energy}

\section{9-3-2 Wave Energy: Oscillating Water Column I}

Wednesday June 12

Room SEC, Carron 2 | 10:30 - 12:00

Session Chair: Kourosh Rezanejad, Centre for Marine Technology and

Ocean Engineering (CENTEC), Instituto Superior Técnico(IST), Portugal

Session Co-Chair: Alessandra Romolo, University Mediterranea, Italy

Geometrical Optimization of U-Oscillating Water

Columns in Random Waves 0MAE2019-95973

Andrea Scialò, Giovanni Malara, Felice Arena

Mediterranea University, Reggio Calabria, Italy

Oscillating Water Column Motion inside Circular

Cylindrical Structures OMAE2019-96048

Daniel de Oliveira Costa Joel S. Sales Junior ${ }^{1}$ Antonio Carlos Fernandes ${ }^{2}$

1. Laboratory of Waves and Current - LOC - Universidade Federal do Rio de

Janeiro, Rio de Janeiro, RJ, Brazil; 2. UFRJ/COPPE, Rio de Janeiro, RJ, Brazil

The Impact of Modelling Air Compressibility in the Selection of Optimal OWC Design Parameters in Site Specific Wave Conditions OMAE2019-96123 Irene Simonetti, Lorenzo Cappietti

University of Florence, Florence, Italy

Improving the Hydrodynamic Performance of OWC Wave

Energy Converter by Attaching a Step 0MAE2019-96408

Kourosh Rezanejad' Jorge Filipe Marques Gadelho ${ }^{1}$ Ivan López ${ }^{2}$

Rodrigo Carballo ${ }^{2}$ Carlos Guedes Soares ${ }^{3}$

1. Centre for Marine Technology and Ocean Engineering (CENTEC), Instituto Superior

Técnico (IST), Lisboa, Portugal; 2. Hydraulic Engineering Area, Universidade de Santiago de

Compostela, Lugo, Spain; 3. Instituto Superior Técnico, Universidade de Lisboa, Lisboa, Portugal

\section{Petroleum Technology}

\section{1-2-2 Drilling Mechanics Session II}

Wednesday June $12 \quad$ Room Crowne Plaza, Barra | 10:30 - 12:00

Session Chair: Jorge H B Sampaio Jr., Colorado School of Mines, USA

Fatigue Evaluation of Drill Pipes for Scientific Drilling Program

by Applying Non-stop Driller Concept OMAE2019-95896

Tomoya Inoue ${ }^{1}$ Masahiko Fujikubo ${ }^{2}$ Kenji Nakano ${ }^{2}$ Noriaki Sakurai ${ }^{3}$

1. JAMSTEC, Yokosuka, Japan; 2. Osaka University, Suita, Japan; 3. JAMSTEC, Yokohama, Japan

A Random Method for Calculation of Hoisting Drag OMAE2019-96170

Hongyuan Qiu, Jianming Yang, Geoff Rideout, Stephen Butt

Memorial University of Newfoundland, St. John's, NL, Canada

Selecting the Best 3D Wellbore Trajectory using a Fast Stiff

Semi-analytical 3D Torque\&Drag Simulator OMAE2019-96385

Jorge H B Sampaio Jr., Ahmed Mansour

Colorado School of Mines, Golden, CO, USA

A Simplified Fatigue Assessment Procedure for Drilling

Pipes of Scientific Vessels OMAE2019-96639

Martina Aguiari' Cesare Mario Rizzo ${ }^{2}$ Tomoya Inoue ${ }^{3}$

1. University of Genoa - DITEN, Genova, Italy; 2. Universita Degli Studi

di Genova, Genova, Italy; 3. JAMSTEC, Yokosuka, Japan

\section{Petroleum Technology}

\section{1-15-2 Well Abandonment II - Research and Operational Experiences}

Wednesday June 12

Room Crowne Plaza, Jura | 10:30 - 12:00

Session Chair: Mahmoud Khalifeh, UiS, Norway

Cement Plug Sealing Studies of Silica Cement System OMAE2019-95928

Anisa Noor Corina' Nils Opedal ${ }^{2}$ Torbjorn Vralstad ${ }^{3}$ Sigbjørn Sangesland'

1. Norwegian University of Science and Technology, Trondheim, Norway;

2. SINTEF Industry, Trondheim, Norway; 3. SINTEF, Trondheim, Norway

Time-to-failure Estimation of Barrier Systems in Permanently

Plugged and Abandoned Wells OMAE2019-96546

Øystein Arild' Hans Petter Lohne' Hans Joakim Skadsem ${ }^{1}$

Eric Patrick Ford' Jon Tømmerås Selvik ${ }^{2}$

1. NORCE, Stavanger, Norway; 2. University of Stavanger, Stavanger, Norway

Experimental Study of Pipe Pulling through

Settled Barite OMAE2019-96547

Ali Taghipour, Torbjorn Vralstad, Ragnhild Skorpa,

Mohammad Hossain Bhuiyan, Jan David Ytrehus, Anna Stroisz

SINTEE, Trondheim, Norway

Effect of Well Construction on Efficient P\&A Process OMAE2019-96607

Farzad N. Shoghl ${ }^{1}$ Arild Saasen ${ }^{2}$ Mahmoud Khalifeh ${ }^{3}$

1. Equinor ASA, Stavanger, Norway; 2. UiS, Gullaug, Norway; 3. UiS, Stavanger, Norway

\section{Takeshi Kinoshita Honoring Symposium on Offshore Technology}

\section{3-1-2 Fluid Body Interaction}

Wednesday June 12

Room SEC, Boisdale 2 | 10:30 - 12:00

Session Organizer: Takanori Hino, Yokohama National University, Japan

Evaluation of Flow Field in the Layouts of

Cross-shaped Artificial Reefs OMAE2019-95192

YanLi Tang, Qi Hu, Xinxin Wang, Fenfang Zhao, Liuyi Huang, Tao Xie

Ocean University of China, Qingdao, China

Nonlinear Wave Surface Elevation Characteristic Analysis around

a Multi-body Offshore Platforms System OMAE2019-95203

Xiudi Ren, Yibo Liang, Longbin Tao

University of Strathclyde, Glasgow, United Kingdom

Two-Dimensional Numerical Simulation of Vortex

Shedding of Multiple Stranded Rope OMAE2019-95225

Xinxin Wang, Liuyi Huang, YanLi Tang, Fenfang Zhao, Peng Sun

Ocean University of China, Qingdao, China

Experimental Study on the Hydrodynamic

Characteristics of Artificial Reefs OMAE2019-96019

Fenfang Zhao ${ }^{1}$ Muk Chen Ong ${ }^{2}$ YanLi Tang ${ }^{1}$ Xinmeng Wang ${ }^{1}$

1. Ocean University of China, Qingdao, China; 2. University of Stavanger, Stavanger, Norway

\section{Lunch}

12:00 - 13:30

Location: Hall 5 (SEC) 


\section{CONCURRENT SESSIONS}

13:30 - 15:00

\section{Offshore Technology}

\section{1-3-1 Nonlinear Wave and Wave Effects}

Wednesday June 12 Room SEC, Alsh 1 | 13:30 - 15:00

Session Chair: Longfei Xiao, Shanghai Jiao Tong University, China

Session Co-Chair: Zhenhua Huang, University of Hawaii at Manoa, USA

Behaviour of a Suspended Wellbay Module and Flare Tower in Waves during Transit to Shore OMAE2019-95001 Hoi-Sang Chan, Evren Armaoglu, Matthew Thomson, Alistair Garner Saipem Ltd, Kingston-upon-Thames, United Kingdom

Slamming Force Contributions due to Plunging Breakers on Different Geometrical Cylinders OMAE2019-95126 Xin Wang ${ }^{1}$ Arun Dev' Longbin $\mathrm{TaO}^{2}$ DW Chia ${ }^{3}$ Yali Zhang ${ }^{3}$ 1. Newcastle University, Singapore, Singapore; 2. University of Strathclyde, Glasgow, United Kingdom; 3. Lloyd's Register Singapore Pte Ltd, Singapore, Singapore

Hydrodynamic Analysis of Deep-water Fish Cage based on Two Different Methods in Waves OMAE2019-96486 Yihou Wang, Yuwang Xu, Shuai Li, Haojie Ren, Shixiao Fu Shanghai Jiao Tong University, Shanghai, China

Experimental Research of Wave Transformation on Porous Coral Reef OMAE2019-96582 Gancheng Zhu' Bing Ren ${ }^{2}$ Yongxue Wang ${ }^{1}$ Chao Wang ${ }^{2}$ 1. State Key Laboratory of Coastal and Offshore Engineering, Dalian University of Technology, Dalian, China; 2. Dalian University of Technology, Dalian, China

\section{Structures, Safety and Reliability}

\section{2-2-1 Probabilistic and Spectral Wave Models I}

Wednesday June 12

Room Crowne Plaza, Castle 1 | 13:30 - 15:00

Session Chair: Carlos Guedes Soares, Instituto Superior

Técnico, Universidade de Lisboa, Portugal

Session Co-Chair: Felice Arena, Univ Mediterranea, Italy

Nonlinear Fourier Analysis Algorithm and Models for

Water Waves in Terms of Surface Elevation, Amplitude

Modulations and Frequency Modulations OMAE2019-95546

Alfred R. Osborne

Nonlinear Wave Research Corporation, Alexandria, VA, USA

Optimal Methods for Estimating the JONSWAP

Spectrum Peak Enhancement Factor from Measured

and Hindcast Wave Data OMAE2019-95451

Kevin Ewans ${ }^{1}$ Jason McConochie ${ }^{2}$

1. MetOcean Research Ltd, New Plymouth, New Zealand;

2. Shell Development Australia, Perth, WA, Australia

A Benchmarking Exercise on Estimating Extreme Environmental

Conditions for Engineering Design OMAE2019-96523

Andreas F Haselsteiner ${ }^{1}$ Ryan $\mathrm{Coe}^{2}$ Lance Manuel $^{3}$

Phong T. T. Nguyen ${ }^{3}$ Nevin Martin ${ }^{4}$ Aubrey Eckert-Gallup ${ }^{4}$

1. University of Bremen, Bremen, Germany; 2. Sandia National

Laboratories, Albuquerque, NM, USA; 3. University of Texas at Austin,

Austin, TX, USA; 4. Sandia National Labs, Albuquerque, NM, USA

\section{Structures, Safety and Reliability}

\section{2-11-3 Ultimate Strength III}

Wednesday June 12 Room Crowne Plaza, Castle 2 | 13:30 - 15:00 Session Chair: Masahiko Fujikubo, Osaka University, Japan Session Co-Chair: Deyu Wang Shanghai Jiao Tong University, China

Analysis of Strain Characteristic of Carbon Filament-wound Composite Cylinders under Hydrostatic Pressure 0MAE2019-95106 Ke Chun Shen, Guang Pan, Ran Feng Wei, Zhun Li Northwestern Polytechnical University, Xi'an, China

Study on Residual Strength of Egg-shaped Pressure

Shell with Local Damage OMAE2019-95456

Siming Yuan, He Gao

Naval Research Academy, PLA, Beijing, China

Axial and Moment Carrying Capacity of Split Sleeve Grouted

Connection for Tubular Members OMAE2019-96267

Vignesh Chellappan N, Nallayarasu Seeninaidu

Indian Institute of Technology Madras, Chennai, India

\section{Materials Technology}

\section{3-11-1 Developments in BS 7910 and other Fitness-for-service Procedures: Session I}

Wednesday June 12

Room SEC, Boisdale 1 | 13:30 - 15:00

Session Chair: Bostjan Bezensek, Shell Global Solutions UK, United Kingdom

Session Co-Chair: Isabel Hadley, TWI Ltd, United Kingdom

A Brief Guide to BS 7910 OMAE2019-96619

Isabel Hadley

TWI Ltd, Cambridge, United Kingdom

Emerging Technology in Fitness-for-service

Assessment of Crack-like Flaws OMAE2019-96415

Ted Anderson

TL Anderson Consulting, Longmont, CO, USA

Estimating Fracture Toughness from Charpy Data 0MAE2019-95787

Henryk Pisarski' Bostjan Bezensek²

1. Independent Consultant, Cambridge, United Kingdom;

2. Shell, Laurencekirk, United Kingdom

Residual Stress in Girth Welds: Seeing Measurement

Data Differently OMAE2019-96622

Ali Mirzaee Sisan' Guiyi Wu ${ }^{2}$

1. AMS Energy Solutions, Barnet, United Kingdom; 2. TWI Ltd, Cambridge, United Kingdom

\section{Pipelines, Risers, and Subsea Systems}

\section{4-2-3 Drilling Risers I}

Wednesday June 12 Session Chair: Chenteh Alan Yu, ABS, USA

Emergency Disconnect and Storm Hang-off - Reducing Risk

for Drilling Risers in Harsh Environments OMAE2019-95213

Conor Gallagher, Dara Williams

Wood PLC, Galway, Ireland 
Mitigated Riser Response during Submerged BOP Move through use of Drillstring Active Heave Compensation System OMAE2019-95809 Lucas Sevillano ${ }^{1}$ Celso Morooka ${ }^{1}$ Sigbjørn Sangesland ${ }^{2}$

1. University of Campinas, Campinas, SP, Brazil;

2. Norwegian University of Science and Technology, Trondheim, Norway

Advances in Riser Management Technology Enabling Improved Efficiency for Deepwater and Harsh Environment Drilling 0MAE2019-96261 Donogh Lang ${ }^{1}$ Paul Bohan ${ }^{1}$ Victor Gomes ${ }^{2}$ Germain Venero ${ }^{2}$ Hugues Corrignan ${ }^{2}$ 1. Wood PLC, Galway, Ireland; 2. Wood, Rio de Janeiro, RJ, Brazil

Field Trial of Vortex-induced Vibration Suppression Technology for Drilling Riser Buoyancy OMAE2019-96426 Phillip P. Kurts ${ }^{1}$ Hayden Marcollo ${ }^{2}$ Andrew A. Kilner ${ }^{1}$ Daniel Johnstone ${ }^{2}$ Andrew E. Potts ${ }^{2}$ Peter Pezet ${ }^{3}$ Tricia Hill $^{4}$ 1. AMOG Consulting, Houston, TX, USA; 2. AMOG Consulting, Notting Hill, VIC, Australia; 3. Matrix Composites and Engineering, Henderson, WA, Australia; 4. Matrix Composites and Engineering, Houston, TX, USA

\section{Pipelines, Risers, and Subsea Systems}

\section{4-3-3 Mechanics I}

Wednesday June $12 \quad$ Room Crowne Plaza, Staffa/Shuna | 13:30-15:00 Session Chair: Theodoro Netto, COPPE/UFRJ, Brazil

Session Co-Chair: Duane DeGeer, INTECSEA, USA

A Method to Calculate the Multi-axial Fatigue of Subsea Rigid Jumper due to VIV OMAE2019-96332 Kunal Kapoor ${ }^{1}$ Zhenhui Liu ${ }^{2}$ Muk Chen Ong ${ }^{3}$ 1. University of Stavanger, Delhi, India; 2. Aker Solutions AS, Trondheim, Norway; 3. University of Stavanger, Stavanger, Norway

Dynamic Behaviour of Subsea Pipeline Crossings under Compressive Axial Loads OMAE2019-96662 Abdul El-Chayeb, Don Wang, Faris Kamal, Oussama Takieddine National Petroleum and Construction Company, Abu Dhabi, United Arab Emirates

\footnotetext{
A Design Practice for Subsea Pipeline Subjected

to UXO Hazards OMAE2019-96343

Zhenhui Liu' ${ }^{1}$ Ragnar Igland ${ }^{1}$ Sindre Bruaseth ${ }^{1}$ Luca Ercoli-Malacari ${ }^{2}$ Odd Arne Lillebø ${ }^{3}$ 1. Aker Solutions AS, Trondheim, Norway; 2. Equinor AS, Trondheim, Norway; 3. Equinor AS, Stavanger, Norway
}

Fatigue Assessment of Dented Rigid Risers OMAE2019-96741 Elvis J Osorio Santander' Bianca Pinheiro ${ }^{2}$ Carlos Magluta ${ }^{2}$ Ney Roitman' 1. Universidade Federal do Rio de Janeiro, Rio de Janeiro, RJ, Brazil; 2. COPPE/ Universidade Federal do Rio de Janeiro, Rio de Janeiro, RJ, Brazil

\section{Ocean Space Utilization}

\section{5-1-3 Hybrid and Complex Use of Floating Systems II}

Wednesday June 12

Room SEC, Dochart 1 | 13:30 - 15:00

Session Chair: Maurizio Collu, University of Strathclyde, United Kingdom

Session Co-Chair: Tomoki Ikoma, Nihon University, Japan

Experimental and Numerical Study on the Hydrodynamic Properties of a Simplified Floating Hydrocarbon Storage Facility OMAE2019-96753 Chi Zhang ${ }^{1}$ Fonseca Nuno ${ }^{2}$ Allan Magee ${ }^{1}$ Nianxin Ren ${ }^{1}$

1. National University of Singapore, Singapore, Singapore;

2. SINTEF Ocean, Trondheim, Norway
Hydrodynamic Model Tests with a Large Floating Hydrocarbon Storage Facility OMAE2019-96761 Fonseca Nuno ${ }^{1}$ Chi Zhang ${ }^{2}$ José Miguel Rodrigues Nianxin Ren ${ }^{2}$ Oyvind Hellan ${ }^{1}$ Allan Magee $^{2}$ 1. SINTEF Ocean, Trondheim, Norway; 2. National University of Singapore, Singapore, Singapore

Effect of Special Outermost Module Designs on the Hydrodynamic Responses of a Modular Multi-purpose Floating Structure System OMAE2019-96789 Nianxin Ren' Chi Zhang' Allan Magee' Xiao Liu' Oyvind Hellan² Kok Keng Ang ${ }^{1}$ 1. National University of Singapore, Singapore, Singapore; 2. SINTEF Ocean, Trondheim, Norway

Design and Inclusion of a Desalination System in a Floating Offshore Wind Farm OMAE2019-95024 Davide Miriello ${ }^{1}$ Michael Walker ${ }^{2}$ Loris Canizares ${ }^{2}$ Aaron Smith ${ }^{3}$ Dominique Roddier ${ }^{2}$ 1. Principle Power, Inc., Italy; 2. Principle Power, Inc., Emeryville, CA, USA; 3. Principle Power, Lisbon, Portugal

\section{Ocean Engineering}

\section{6-15-1 Underwater Vehicles Control}

Wednesday June $12 \quad$ Room SEC, Dochart 2 | 13:30 - 15:00

Session Organizer: Eduardo Tannuri, Numerical Offshore Tank-

University of São Paulo, Brazil

Session Co-Chair: Zhe Jiang, Shanghai Ocean University, China

Session Co-Chair: Alexandre Immas, University of California, Berkeley, USA

Development of an Image Processing Module for Autonomous Underwater Vehicles through Integration of Object Recognition with Stereoscopic Image Reconstruction OMAE2019-95321

Yu-Hsien Lin, Shao-Yu Chen

National Cheng-Kung University, Tainan, Taiwan

Development of a PID Control Strategy for a Compact

Autonomous Underwater Vehicle OMAE2019-95345

Avilash Sahoo' Santosha K. Dwivedy ${ }^{2}$ P. S. Robi

1. National Institute of Technology Meghalaya, Shillong, India;

2. Indian Institute of Technology Guwahati, Guwahati, India

High-bandwidth Underwater Wireless Communication using a Swarm of Autonomous Underwater Vehicles OMAE2019-96270 Alexandre Immas, Mohsen Saadat, Jesus Navarro, Matthew Drake, Julie Shen, Mohammad-Reza Alam University of California, Berkeley, Berkeley, CA, USA

Optimization of a Swarm of Autonomous Underwater Vehicles for High-bandwidth Underwater Wireless Communication OMAE2019-96285 Alexandre Immas, Mohammad-Reza Alam University of California, Berkeley, Berkeley, CA, USA

\section{Ocean Engineering}

\section{6-3-3 Damping and Viscous Effects}

Wednesday June 12 Room SEC, M4 | 13:30 - 15:00 Session Organizer: Torgeir Kirkhorn Vada, DNV GL, Norway

Vortex Shedding and Roll Damping from Hulls with Rounded Bilges OMAE2019-95629 lan A Milne' ${ }^{1}$ Feifei Tong J. Michael R. Graham ${ }^{2}$ 1. University of Western Australia, Perth, WA, Australia; 2. Imperial College London, London, United Kingdom 
A Semi-analytical Method for Calculating the Hydrodynamic Force on Perforated Plates in Oscillating Flow OMAE2019-95093 Fredrik Mentzoni, Trygve Kristiansen Norwegian University of Science and Technology, Trondheim, Norway

An Experimental and Numerical Study of Added Mass and Damping for Side by Side Plates in Oscillating Flow OMAE2019-96008 Frøydis Solaas' Fredrik Mentzoni ${ }^{2}$ Mia Abrahamsen-Prsic ${ }^{2}$ Trygve Kristiansen $^{2}$ 1. SINTEF Ocean, Trondheim, Norway; 2. Norwegian University of Science and Technology, Trondheim, Norway

The Hydrodynamic Reflection Characteristic Study on the Wave Dissipating and Protecting System with a Submerged Structure Before the Vertical Wall OMAE2019-95942 Zhenglin Tian, Zhaochen Sun, Shuxiu Liang The State Key Laboratory of Coastal and Offshore Engineering, Dalian University of Technology, Dalian, China

\section{Ocean Engineering}

\section{6-5-3 Advanced Marine Hydrodynamics III}

Wednesday June 12

Room SEC, M2 \& M3 | 13:30 - 15:00

Session Chair: Mohammad Rahmati, Northumbria University, United Kingdom

Session Co-Chair: Joop Helder, MARIN, Netherlands

The Increase in Wave Wake Characteristics of Marine

Vessels when Accelerating OMAE2019-95337

Gregor Macfarlane' Keegan Graham-Parker ${ }^{2}$ Michael Connellan ${ }^{1}$

1. Australian Maritime College, University of Tasmania, Launceston,

TAS, Australia; 2. ASC Pty Ltd, Henderson, WA, Australia

Achieving a High Accuracy Numerical Simulations of

the Flow around a Full Scale Ship 0MAE2019-95769

Blanca Pena' ${ }^{1}$ Ema Muk-Pavic ${ }^{1}$ Dmitriy Ponkratov ${ }^{2}$

1. University College London, London, United Kingdom;

2. JoRes Joint Research Project, London, United Kingdom

Numerical Simulation of the Ducted Propeller and

Application to a Semi-submerged Vehicle OMAE2019-96799

Guoge Tan ${ }^{1}$ Jin Zou' Jie Xu ${ }^{2}$

1. Harbin Engineering University, Harbin, China;

2. Chongqing Changan Automobile Co., Ltd, Chongqing, China

Research on the Estimated Error of Wave Action by using Wave Elevation Data OMAE2019-96657

Jiabin Liu, Anxin Guo

Harbin Institute of Technology, Harbin, China

\section{Polar and Arctic Sciences and Technology}

\section{7-11-1 Ice Model Tests and Structure-Ice-Interactions}

Wednesday June 12 Room SEC, Alsh 2 | 13:30 - 15:00

Session Chair: Walter Kuehnlein, sea2ice Ltd. \& Co. KG, Germany

Session Co-Chair: Sören Ehlers, Hamburg University of Technology, Germany

Design Optimization of Ship's Bow Sailing in

Kara Sea and Barents Sea OMAE2019-95586

Jianfei Liu, Guoqing Feng, Huilong Ren, Wenjia Hu, Yuwei Sun

Harbin Engineering University, Harbin, China
Ice Model Tests for Semi-submersible Platforms

in Pack Ice Conditions OMAE2019-95786

Luping Liu, Xin Li, Xiao Wu, Bo Wu

Shanghai Jiao Tong University, Shanghai, China

Experimental and Theoretical Investigations on the Characteristics of Ice Floes Broken by Ships Sailing Ahead in Level Ice OMAE2019-95936 Daniela Myland

HSVA, Hamburg, Germany

Anti-icing and De-icing of Pipe Structures on Marine Vessels using Waste Heat Recovery OMAE2019-96689 Lene Aesoy ${ }^{1}$ Henry Piehl ${ }^{2}$ Palmar Bjornoy ${ }^{3}$

1. Norwegian University of Science and Technology, Trondheim, Norway; 2. Norwegian University of Science and Technology, Ålesund, Norway; 3. Ulmatec Pyro, Gamlem, Norway

\section{CFD \& FSI}

\section{8-1-3 Ship Performance I}

Wednesday June 12 Room SEC, Lomond Auditorium | 13:30-15:00 Session Chair: Ould el Moctar, University of Duisburg-Essen, Germany Session Co-Chair: Prasanta Sahoo, Florida Institute of Technology, USA

A Study of Ship's Frictional Resistance in

Extremely Shallow Water OMAE2019-95076

Qingsong Zeng, Robert Hekkenberg, Cornel Thill

Delft University of Technology, Delft, Netherlands

Added Resistance CFD Analysis of the KVLCC2

with the Naval Hydro Pack OMAE2019-95293

Vuko Vukcevic Inno Gatin ${ }^{2}$ Geon-Hong Kim ${ }^{3}$ Hrvoje Jasak $^{4}$

1. Faculty of Mechanical Engineering and Naval Architecture, Zagreb, Croatia;

2. University of Zagreb, Zagreb, Croatia; 3. Hyundai Heavy Industries, Hyundai

Maritime Research Institute, Ulsan, Korea; 4. Wikki Ltd, London, United Kingdom

Numerical Simulation of Trim Optimization on Resistance Performance based on CFD Method OMAE2019-96181

Wenyang Duan, Hongsen Zhang, Limin Huang, Jianyu Liu,

Wenbo Shao, Guanzhou Cao, Zhang Shi

Harbin Engineering University, Harbin, China

Fundamental CFD Study on the Hydrodynamic Performance of the DARPA Suboff Submarine OMAE2019-96190

Kenshiro Takahashi, Prasanta Sahoo

Florida Institute of Technology, Melbourne, FL, USA

\section{Ocean Renewable Energy}

\section{9-4-2 Wave Farms and Alternative Markets}

Wednesday June 12 Room SEC, Carron 1 | 13:30 - 15:00

Session Chair: Kelley Ruehl, Sandia National Laboratories, USA

Session Co-Chair: Ryan Coe, Sandia National Laboratories, USA

Wave-powered AUV Recharging: A Feasibility Study OMAE2019-95383

Blake Driscol' Andy Gish ${ }^{1}$ Ryan Coe ${ }^{2}$

1. US Naval Academy, Annapolis, MD, USA; 2. Sandia

National Laboratories, Albuquerque, NM, USA

Development Demonstration of an Automated, Economic Patented Modular Industrial, Environmentally Friendly Multi-functional Platform for Open Sea Farm Installations OMAE2019-95900

Elchanan Safier

Safier Ingenierie SAS, Serris, France 
Analysis of WEC Array and Influence of Plant Level

Power Management Control OMAE2019-96466

Jeremy Stefek, Dominique Bain, Yi-Hsiang Yu, Dale Jenne, Greg Stark

National Renewable Energy Laboratory, Golden, $\mathrm{CO}$, USA

\section{Ocean Renewable Energy}

\section{9-6-1 Thermal, Hybrid and Others: Analysis, Design and Prediction}

Wednesday June 12

Room SEC, Carron 2 | 13:30-15:00

Session Chair: Fabio Licheri, University of Cagliari, Italy

Impact of the Swansea Bay Lagoon on Storm

Surges in the Bristol Channel OMAE2019-95075

Qian Ma' Tulio M. Moreira ${ }^{2}$ Thomas A.A. Adcock

1. University of Oxford, Oxford, United Kingdom; 2. Federal University

of Minas Gerais (UFMG), Belo Horizonte, Brazil

Numerical Study on Aero-hydrodynamics with Inter-turbine Spacing Variation for Two Floating Offshore Wind Turbines OMAE2019-95520

Yang Huang, Decheng Wan

Shanghai Jiao Tong University, Shanghai, China

Evaluation of Entropy Generation Methods

in Wells Turbines OMAE2019-96513

Fabio Licheri' Tiziano Ghisu' Irene Virdis' Pierpaolo Puddu' ${ }^{1}$ Francesco Cambuli ${ }^{2}$

1. University of Cagliari, Cagliari, Italy; 2. University of Cagliari, DIMCM, Cagliari, Italy

Failure Estimation of Offshore Renewable Energy Devices based on Hierarchical Bayesian Approach OMAE2019-95099 Mohammad Mahdi Abaei, Nu Rhahida Arini, Philipp R. Thies, Lars Johanning University of Exeter, Penryn, United Kingdom

\section{Petroleum Technology}

\section{1-3-1 Drilling Geomechanics}

Wednesday June 12 Room Crowne Plaza, Barra | 13:30 - 15:00

Session Chair: Arash Dahi Taleghani, Pennsylvania State University, USA

Experimental Study on Conductivity of Regional

Sand and its Field Application OMAE2019-95624

Jin Wang, Fujian Zhou, Lufeng Zhang, Minghui Li, Yuechun Wang

China University of Petroleum-Beijing, Beijing, China

Additives to Enhance Cement Sheath Durability OMAE2019-96421

Maryam Tabatabaei, Arash Dahi Taleghani, Nasim Alem

Pennsylvania State University, University Park, PA, USA

An Experimental Investigation on Filtercake Reinforced

Wellbore Strengthening and Fracture Sealing OMAE2019-96675

Mingzheng Yang Y Yuanhang Chen' Frederick B Growcock ${ }^{2}$ Feifei Zhang ${ }^{3}$

1. Louisiana State University, Baton Rouge, LA, USA; 2. Occidental Oil and

Gas Corp, Houston, TX, USA; 3. Yangtze University, Jingzhou, China

Quantification of the Effects of Filtercake on Wellbore Strengthening: Filtercake Rupture Resistance and Fracture Sealing Time 0MAE2019-96676 Mingzheng Yang ' Yuanhang Chen ${ }^{1}$ Frederick B Growcock ${ }^{2}$

1. Louisiana State University, Baton Rouge, LA, USA

2. Occidental Oil and Gas Corp, Houston, TX, USA

\section{Petroleum Technology}

\section{1-5-1 Well Inflow Control and Reservoir Management}

Wednesday June 12

Room Crowne Plaza, Jura | 13:30 - 15:00

Session Chair: Bernt Aadnoy, University of Stavanger, Norway

Probabilistic Estimation of Recovery from Naturally Fractured Bottom-water Reservoir with Uncertain Well

Placement in Fracture Network OMAE2019-96836

Samir Prasun, Andrew Wojtanowicz

Louisiana State University, Baton Rouge, LA, USA

Visualization Study on Plugging Mechanism of Fibers and

Particles in Rough and Tortuous Fracture OMAE2019-95480

Fan Fan, Fujian Zhou, Lishan Yuan, Xuda Yang

China University of Petroleum-Beijing, Beijing, China

Increased Recovery using Autonomous

Inflow Management OMAE2019-96003

Bernt Aadnoy' Beder Al Furati ${ }^{2}$

1. University of Stavanger, Stavanger, Norway; 2. Equinor AS, Bergen, Norway

A Pilot Study on Time-dependent Dissolution of $\mathrm{CO}_{2}$ in Oil for Prediction of Gas Kick Behaviors in Non-aqueous Fluids OMAE2019-96678 Mahendra Kunju' James L. Nielsen ${ }^{1}$ Yuanhang Chen ${ }^{1}$ Otto Santos' Wesley Williams ${ }^{1}$ Paulo Ribeiro ${ }^{2}$ Felipe Chagas ${ }^{2}$

1. Louisiana State University, Baton Rouge, LA, USA;

2. Universidade Estadual de Campinas, Campinas, SP, Brazil

\section{Takeshi Kinoshita Honoring Symposium on Offshore Technology}

\section{3-1-3 Nonlinear Waves I}

Wednesday June 12

Room SEC, Boisdale 2 | 13:30 - 15:00

Session Organizer: Alessandro lafrati, CNR-INM (INstitute of Marine Engineering), Italy

On Signatures and Features of Modulational

Instability in Ocean Waves OMAE2019-95633

Alexander V. Babanin

University of Melbourne, Melbourne, VIC, Australia

Data Assimilation of the Stereo Reconstructed Wave Fields to a Nonlinear Phase Resolved Wave Model OMAE2019-95949 Shogo Watanabe ${ }^{1}$ Wataru Fujimoto ${ }^{2}$ Takehiko Nose' $^{1}$ 'subasa Kodaira' Graham Davies ${ }^{3}$ Daniel Lechner ${ }^{4}$ Takuji Waseda ${ }^{1}$

1. The University of Tokyo, Kashiwa, Japan; 2. MS\&AD InterRisk Research \& Consulting, Inc., Tokyo, Japan; 3. Waseda University, Tokyo, Japan; 4. Karlsruhe Institute of Technology, Karlsruhe, Germany

On the Rogue Wave Occurrence in Crossing Wave Fields 0MAE2019-96029 Shuai Liu, Xinshu Zhang, Xingyu Song, Ke Chen Shanghai Jiao Tong University, Shanghai, China

Generalized Nonlinear Fourier Analysis for Water Waves OMAE2019-96613 Alfred R. Osborne

Nonlinear Wave Research Corporation, Alexandria, VA, USA

\section{REFRESHMENT BREAK}

15:00 - 15:30

Location: Hall 5 (SEC) 


\section{CONCURRENT SESSIONS}

15:30 - 17:30

\section{Offshore Technology}

\section{1-3-2 Fluid-Structure Interaction}

Wednesday June 12

Room SEC, Alsh 1 | 15:30 - 17:30

Session Chair: Tahsin Tezdogan, University of Strathclyde, United Kingdom

Session Co-Chair: Yibo Liang, University of Strathclyde, United Kingdom

Hydrodynamic Forces on Intermittently Spanning

Pipelines in Steady Currents OMAE2019-95585

Yunfei Teng' Liang Cheng ${ }^{2}$ Hongwei $\mathrm{An}^{2}$ Feifei Tong ${ }^{2}$

Terry Griffiths ${ }^{2}$ Wei Sun ${ }^{2}$ Jiawei Chi $^{2}$ Zhijian Xiong ${ }^{2}$

1. Dalian University of Technology, Dalian, China;

2. University of Western Australia, Perth, WA, Australia

The Effect of Aspect Ratio on the Drag of Bare Cylinders OMAE2019-96431 Douglas A. Potts ${ }^{1}$ Jonathan R. Binns ${ }^{1}$ Andrew E. Potts ${ }^{2}$ Hayden Marcollor 1. University of Tasmania Australian Maritime College, Launceston, TAS, Australia; 2. AMOG Consulting, Notting Hill, VIC, Australia

Hydrodynamics of Towed Vertical Surface-piercing

Cylinders OMAE2019-95109

Douglas A. Potts' Jonathan R. Binns' Hayden Marcollo ${ }^{2}$ Andrew E. Potts ${ }^{2}$

1. University of Tasmania Australian Maritime College, Launceston, TAS,

Australia; 2. AMOG Consulting, Notting Hill, VIC, Australia

Experimental and Numerical Study of Horizontal Wave Impact Loads for a Semi-submersible Drilling Unit OMAE2019-96236 Joo-Sung Kim, Seon Oh Yoo, Hyun Joe Kim, Jong Hun Lee, So Lyoung Han, DongYeon Lee Samsung Heavy Industries, Daejeon, Korea

An Iterative Updating Method for Dynamic Responses of a Floating Platform under Action of Internal Solitary Waves OMAE2019-96553 Junrong Wang, Junfeng Du, Min Zhang, Anteng Chang Ocean University of China, Qingdao, China

\section{Structures, Safety and Reliability}

\section{2-2-2 Probabilistic and Spectral Wave Models II}

Wednesday June 12 Room Crowne Plaza, Castle 1 | 15:30 - 17:30

Session Chair: Felice Arena, Univ Mediterranea, Italy

Session Co-Chair: Carlos Guedes Soares, Instituto Superior

Técnico, Universidade de Lisboa, Portugal

Quantitative Evaluation of Ship Operational Effect in

Actually Encountered Sea States OMAE2019-95121

Rei Miratsu, Tsutomu Fukui, Toshiyuki Matsumoto, Tingyao Zhu

Nippon Kaiji Kyokai (ClassNK), Tokyo, Japan

Estimation and Comparison of Accuracy in Various Data Resolutions on Optimal Ship Routing across the North Pacific Ocean OMAE2019-95173 Kenji Sasa' Takuya Fujimatsu' Chen Chen' Ruri Shoji 1. Kobe University, Kobe, Japan; 2. Tokyo University of Marine Science and Technology, Tokyo, Japan

Comparison of VOS and ERA-Interim Wave Data OMAE2019-95287 Roberto Vettor ${ }^{1}$ Carlos Guedes Soares ${ }^{2}$

1. Centre for Marine Technology and Ocean Engineering, Instituto Superior Técnico, Universidade de Lisboa, Lisbon, Portugal; 2. Instituto Superior Técnico, Universidade de Lisboa, Lisboa, Portugal
A Parameterization of DNV Storm Profile for Long-term Analysis of Ocean Storms: Equivalent Trapezoidal Storm Model OMAE2019-95880 Valentina Laface' Elzbieta M. Bitner-Gregersen ${ }^{2}$ Felice Arena ${ }^{1}$ Alessandra Romolo ${ }^{1}$ 1. University Mediterranea, Reggio Calabria, Italy; 2. DNV GL AS, Høvik, Norway

\section{Structures, Safety and Reliability}

\section{2-6-1 Reliability of Mooring and Riser Systems I}

Wednesday June 12

Room Crowne Plaza, Castle 2 | 15:30 - 17:30

Session Chair: Ying Min Low, National University of Singapore, Singapore Session Co-Chair: Luis V.S. Sagrilo, LACEO/COPPE/

Federal University of Rio De Janeiro, Brazil

An Efficient System Reliability Approach against

Mooring Overload Failures OMAE2019-95048

Darrell Leong' Ying Min Low' Youngkook Kim²

1. National University of Singapore, Singapore, Singapore;

2. Lloyd's Register, Singapore, Singapore

Numerical Modelling of the Mooring Line Failure Induced Performance Changes of a Marine Fish Cage in Irregular Waves and Currents OMAE2019-95730 Hung-Jie Tang' Ray-Yeng Yang' Chai-Cheng Huang ${ }^{2}$ 1. National Cheng Kung University, Tainan, Taiwan;

2. National Sun Yat-sen University, Kaohsiung, Taiwan

Assessment of the Reliability of the Moorings of a Floating Structure against the Extreme Cyclone Hazard OMAE2019-96032 Mark Manzocchi' Vikas Kejriwal ${ }^{2}$ Eric $\mathrm{HoO}^{2}$

1. Atkins Energy, Edinburgh, United Kingdom; 2. Atkins - SNC Lavalin, Perth, WA, Australia

Extreme Value Estimation of Mooring Lines Top Tension OMAE2019-96210 Marina L. Simão ${ }^{1}$ Paulo M. Videiro ${ }^{2}$ Mauro C. de Oliveira ${ }^{3}$ Luis V.S. Sagrilo ${ }^{2}$ 1. LACEO/Federal University of Rio de Janeiro, Rio de Janeiro, RJ, Brazil; 2. LACEO/COPPE/Federal University of Rio de Janeiro, Rio de Janeiro, RJ, Brazil; 3. CENPES/PETROBRAS, Rio de Janeiro, RJ, Brazil

Application of Machine Learning Techniques as a Means of Mooring Integrity Monitoring OMAE2019-96411

Jonathan M. Gumley, Hayden Marcollo, Stuart Wales, Andrew E. Potts, Christopher J. Carra AMOG Consulting, Notting Hill, VIC, Australia

\section{Materials Technology}

\section{3-11-2 Developments in BS 7910 and other Fitness-for-service Procedures; Session II}

Wednesday June 12

Room SEC, Boisdale 1 | 15:30 - 17:30

Session Chair: Isabel Hadley, TWI Ltd, United Kingdom

Session Co-Chair: Bostjan Bezensek, Shell Global Solutions UK, United Kingdom

Proposed Updates to the Buried-to-Surface Flaw Recharacterization Rules in the Annex E of BS 7910 OMAE2019-96327

Bostjan Bezensek' Yuri Tkach (WG) ${ }^{2}$ John Sharples ${ }^{3}$ Harry Coules $^{4}$

1. Shell, Laurencekirk, United Kingdom; 2. Wood PLC, Aberdeen, United Kingdom;

3. Wood PLC, Warrington, United Kingdom; 4. University of Bristol, Bristol, United Kingdom

The Assessment of Locally Thinned Areas subject to a Hoop Stress and an Axial Stress: Background to the Guidance given in Annex G of BS 7910:2013 OMAE2019-95532

Andrew Cosham ${ }^{1}$ Robert Andrews ${ }^{2}$

1. Ninth Planet Engineering Ltd, Newcastle upon Tyne, United Kingdom;

2. ROSEN Group, Newcastle upon Tyne, United Kingdom 
Optimising Fracture Assessment of Welded Structures using BS 7910, R6 and FEA OMAE2019-95934

Isabel Hadley ${ }^{1}$ Tyler London ${ }^{2}$

1. TWI Ltd, Cambridge, United Kingdom; 2. TWI Ltd, Middlesbrough, United Kingdom

Review of Available Probabilistic Models of the Crack

Growth Parameters in Paris Equation OMAE2019-96161

Peyman Amirafshari' Alexander Stacey ${ }^{2}$

1. University of Strathclyde, Glassgow, United Kingdom; 2. Energy

Division, Health \& Safety Executive, London, United Kingdom

BS7910 Procedure for Probabilistic Fracture

Mechanics Assessment OMAE2019-96843

Alexander Stacey

Energy Division, Health \& Safety Executive, London, United Kingdom

\section{Pipelines, Risers, and Subsea Systems}

\section{4-2-4 SCRs and SLWRs I}

Wednesday June $12 \quad$ Room Crowne Plaza, Castle 3 | 15:30 - 17:30

Session Chair: Alan Whooley, Wood PIc, USA

Impact of Soil Modeling on Fatigue Design of

Lazy Wave Riser Systems OMAE2019-96156

Rupak Ghosh ${ }^{1}$ Haydar Arslan²

1. ExxonMobil, Spring, TX, USA; 2. ExxonMobil Production Company, Spring, TX, USA

Design of Steel Lazy Wave Riser for External

Turret Moored FPSO OMAE2019-96422

Jingyun Cheng, Peimin Cao

SBM Offshore, Houston, TX, USA

Fatigue Assessment of SLWR Riser in Brazilian Pre-salt: The Impact of Slope Changing Point in SN Curve OMAE2019-96592 Stael Ferreira Senra' ${ }^{1}$ Ludimar L. Aguiar' Eduardo Hippert ${ }^{1}$ Alexandre G. Garmbis ${ }^{2}$ Marcelo Dos Santos ${ }^{3}$ Marcos Andre Duarte Martins' Luis Manoel Paiva Nunes ${ }^{1}$ 1. Petrobras, Rio de Janeiro, RJ, Brazil; 2. Petrobras, São José dos Campos, SP, Brazil; 3. Petrobrás (cenpes), Rio de Janeiro, RJ, Brazil

The Impact of Second-order FPSO Motions on the Fatigue Performance of Large Diameter SCRs OMAE2019-96451 Rasoul Hejazi, Andrew Grime, Mark F. Randolph, Mike Efthymiou University of Western Australia, Perth, WA, Australia

Effect of Flexible Joint Modelling Method on Deep Water Catenary Riser Hang-off Fatigue Response 0MAE2019-96826 Guanyu Hu' Chaojun Huang' Fengjie Yin' Mark Cerkovnik' Guangqiang Yang ${ }^{2}$ 1. 2 H Offshore Inc., Houston, TX, USA; 2. ExXon Mobil Company, Spring, TX, USA

\section{Pipelines, Risers, and Subsea Systems}

\section{4-5-2 Flow Assurance II}

Wednesday June 12

Room Crowne Plaza, Staffa / Shuna | 15:30 - 17:30

Session Organizer: Daniel Carneiro, Wood, Brazil

Session Co-Chair: Jongbae Kim, University of Ulsan, Korea

Session Co-Chair: Nestor Gonzalez Diez, TNO, Netherlands

A Study of Hydrate Inhibition for Deepwater

Gas Field Development OMAE2019-95177

Hualei Yi ${ }^{1}$ Yun Hao ${ }^{1}$ Xiaohong Zhou ${ }^{2}$

1. CNOOC Research Institute, Beijing, China; 2. CNOOC, Beijing, China
Numerical Simulation of the Fluid-Solid Two-phase in the Horizontal Pipe based on DEM-CFD Coupling Method OMAE2019-95455

J,S., Pu' Yongping Chen ${ }^{2}$ Peng Yao ${ }^{3}$

1. Hohai University, NanJing, China; 2. Hohai University, Hon,

China; 3. Sun yat-sen University, Guangdong, China

Introducing a Novel MEG/EtOH Mixture to Improve Gas Hydrate Blockages Removal during Offshore

Oil and Gas Production OMAE2019-95808

Paulo Paz ${ }^{1}$ Theodoro Netto ${ }^{2}$

1. Federal University of Rio de Janeiro, Rio de Janeiro, RJ, Brazil;

2. COPPE/Federal University of Rio de Janeiro, Rio de Janeiro, RJ, Brazil

Analysis of Heat Transfer Performance for Deepwater

Phase Change Material Sandwich Pipes OMAE2019-95876

Chen An, Hui Wang, Menglan Duan

China University of Petroleum, Beijing, China

Optimization Design of FPSO Central Cooling Water System based on Pipe Network Fluid Analysis OMAE2019-95966

Huan Zhang, Yuan Hongtao, Wang Chao, Kong Weiwen, Xu Jiangguo, Wang Zhaogiang Shanghai Waigaoqiao Shipbuilding (0., Ltd., Shanghai, China

\section{Ocean Engineering}

\section{6-15-2 Underwater Vehicles Design Technology and Hydrodynamics}

Wednesday June 12 Room SEC, Dochart 2 | 15:30 - 17:30

Session Organizer: Celso Morooka, University of Campinas, Brazil Session Co-Chair: Zhe Jiang, Shanghai Ocean University, China Session Co-Chair: Alexandre Immas, University of California, Berkeley, USA

A Preliminary Study on the Development of a Novel Marine Growth Cleaning Robot for Jacket Platforms OMAE2019-95176 Zhe Jiang, Tao Sun, Gaosheng Luo, Biao Wang, Wei Guo Shanghai Ocean University, Shanghai, China

Analysis of the Pitting Corrosion's Effect on the Residual Strength of Submerged Pressure Shell OMAE2019-96001 Weijun Xu, Tianyi Chen, Chenfeng Li, Xueqian Zhou, Feng Liu Harbin Engineering University, Harbin, China

Hydrodynamic Design of a Morphic Autonomous Underwater Vehicle using Neural Networks OMAE2019-96469 Quentin Becker, Mohammad-Reza Alam, Alexandre Immas University of California, Berkeley, Berkeley, CA, USA

Experimental Study on Hydrodynamic Performance of Mini-AUV in Non-uniform Flow Field OMAE2019-96835 Jiayuan Zhuang ' Cao Jian' Yumin Su' Lei Zhang ${ }^{1}$ Xianzhao Yu 1. Harbin Engineering University, Harbin, China; 2. China Shipbuilding Information Center, beijing, China

A Study on Piping Design Neutral File to Convert Augmented Reality Model in Real-time 0MAE2019-96053 Jung Min Lee, Kyung Ho Lee, Young Soo Han Inha University, Incheon, Korea 


\section{Ocean Engineering}

\section{6-3-4 Wave-Body Interactions: Special Problems}

Wednesday June 12

Room SEC, M4 | 15:30-17:30

Session Organizer: Fonseca Nuno, SINTEF Ocean, Norway

Experimental Investigation of Wave Runup on Offshore Intake

Wells in a Random Wave Environment OMAE2019-96505

Venkatraman Prabu Kumar, Ranganathan Sundaravadivelu, K. Murali

Indian Institute of Technology Madras, Chennai, India

Efficacy of Analysis Techniques in Assessing Broken Wave

Loading on a Cylinder Upon a Shoal OMAE2019-96262

Darshana T. Dassanayake, Alessandro Antonini, Alison Raby

Plymouth University, Plymouth, United Kingdom

Piston-modal Resonance in a Box-wall System OMAE2019-95006

Sheng-Chao Jiang ${ }^{1}$ Yu-Xin Huang ${ }^{2}$ Baolei Geng ${ }^{2}$

1. Dalian University of Technology, Dalian, China; 2. Tianjin Research

Institute for Water Transport Engineering, Tianjin, China

Dynamic Response of a Gangway between Two Floating

Bodies in a Side-by-side Configuration OMAE2019-96578

Qing Dong ${ }^{1}$ Jianmin Yang ${ }^{2}$ Haining L Lu² Xin Li Wenyue Lu Lei Liu ${ }^{2}$

1. State Key Laboratory of Ocean Engineering, Shanghai Iiao Tong Uneiversity,

Shanghai, China; 2. Shanghai Jiao Tong University, Shanghai, China

Water Wave Scattering by Two Circular-arc-shaped Thin

Plates with Non-uniform Permeability 0MAE2019-95498

Rupanwita Gayen, Sourav Gupta

Indian Institute of Technology, Kharagpur, India

\section{Ocean Engineering}

\section{6-5-4 Advanced Marine Hydrodynamics IV}

Wednesday June $12 \quad$ Room SEC, M2 \& M3 | 15:30 - 17:30

Session Chair: Jule Scharnke, MARIN, Netherlands

Session Co-Chair: Joost Sterenborg, MARIN, Netherlands

Influence of Mixed Flows on Ship Hydrodynamics

in Dredged Channels OMAE2019-95445

Momchil Terziev, Tahsin Tezdogan, Atilla Incecik

University of Strathclyde, Glasgow, United Kingdom

Big Data Analytics as a Tool to Monitor Hydrodynamic

Performance of a Ship OMAE2019-95815

Prateek Gupta' ${ }^{1}$ Sverre Steen ${ }^{1}$ Adil Rasheed ${ }^{2}$

1. Department of Marine Technology, Norwegian University of Science and

Technology (NTNU), Trondheim, Norway; 2. Department of Engineering Cybernetics,

Norwegian University of Science and Technology (NTNU), Trondheim, Norway

An Investigation of the Effect of Biomimetic

Tubercles on a Flat Plate OMAE2019-96276

Alessandro Marino, Mehmet Atlar, Yigit Demirel

University of Strathclyde, Glasgow, United Kingdom

Research on the Probability Distribution of the Underwater Moving of the Wrecked Targets OMAE2019-96581

Wenyang Duan, Zhang Shi, Yunsai Chen, Li Min Huang, Guanzhou Cao, Hongsen Zhang

Harbin Engineering University, Harbin, China

\section{Polar and Arctic Sciences and Technology}

\section{7-12-1 Numerical Ice Modeling}

Wednesday June 12 Room SEC, Alsh 2 | 15:30 - 17:30 Session Chair: Sören Ehlers, Hamburg University of Technology, Germany Session Co-Chair: Walter Kuehnlein, sea2ice Ltd. \& Co. KG, Germany

\section{A Numerical Method for Ice Resistance Calculation of Polar Ships} Navigating in Floating Ice Region OMAE2019-96131

Hui Li, Yuan Qian, Yan Feng, Weilia Sheng, Hao Jin Li

Haerbin Engineering University, Harbin, China

Investigation of the Effect of Block Size, Shape and Freeze-bond Strength on Flexural Failure of Freshwater Ice Rubble using the Discrete Element Method OMAE2019-96722 Soroosh Afzali' Rocky Taylor' Eleanor Bailey ${ }^{2}$ Robert Sarracino² Marjan Taghi Boroojerdi 1. Memorial University of Newfoundland, St. John's, NL, Canada; 2. C-CORE, St. John's, NL, Canada

Optimization of Insulation and Heating System of Derrick for Arctic Mobile Offshore Drilling Unit OMAE2019-95930 Dahui Liu' Zhiyuan Wang ${ }^{2}$ KaiBo Zheng ${ }^{3}$

1. CIMC Raffles, Yantai, China; 2. University of Petroleum East China, Qingdao, China; 3. China University of Petroleum (East China), Qingdao, China

Closing Remarks OMAE2019-96845

Walter Kuehnlein

seazice Ltd. \& Co. KG, Hamburg, Germany

\section{CFD \& FSI}

\section{8-1-4 Ship Performance II}

Wednesday June $12 \quad$ Room SEC, Lomond Auditorium | 15:30 - 17:30 Session Chair: Prasanta Sahoo, Florida Institute of Technology, USA Session Co-Chair: Ould el Moctar, University of Duisburg-Essen, Germany

Evaluation of a Practical Approach to Numerical Propulsion Tests OMAE2019-95339 Andreas Giannoulis, Karl Halse

Norwegian University of Science and Technology, Aalesund, Norway

Studies About Design of Rear Stator of Ducted Propeller using CFD OMAE2019-96020

Dakui Feng' Hang Zhang' Yue Sun' Qing Wang' Xiaofei Hu ${ }^{2}$

1. Huazhong University of Science and Technology, Wuhan, China;

2. China Ship Design and Development Center, Wuhan, China

Strategies to Minimise Numerical Ventilation in CFD Simulations of High-speed Planing Hulls OMAE2019-95784 Angus G. D. Gray-Stephens, Tahsin Tezdogan, Sandy Day University of Strathclyde, Glasgow, United Kingdom

Shipboard Fuel Consumption Reduction by Air Lubrication and Trim Optimization OMAE2019-96770

Waleed Yehia' ${ }^{1}$ M. M. Moustafa' Adel A. Tawfik' ${ }^{1}$ A. Nasef ${ }^{2}$

1. Port Said University, Port Said, Egypt; 2. Egyptian Authority for Maritime Safety, Suez, Egypt

Numerical Study on the Effect of Stern Flap for Hydrodynamic

Performance of Catamaran OMAE2019-96819

Peng Zhou' Liwei Liu Lixiang Guo ${ }^{2}$ Qing Wang ${ }^{2}$ Xianzhou Wang ${ }^{2}$

1. Shanghai Division, China Ship Development and Design Center, Shanghai,

China; 2. Huazhong University of Science and Technology, Wuhan, China 


\section{CFD \& FSI}

\section{8-4-1 Cylinder VIV}

Wednesday June 12

Room SEC, Dochart 1 | 15:30 - 17:30

Session Chair: Michael Bernitsas, University of Michigan, USA

Session Co-Chair: Long Ge, BP, USA

Cross-flow VIV Simulation of a Circular Cylinder under

Oscillatory Flow with Different KC Number OMAE2019-95271

Kunpeng Wang, Qinghai Chi, Yizhao Zhang

Jiangsu University of Science and Technology, Zhenjiang, China

Vortex Induced Vibration of a Steel Catenary Riser under

Out-of-plane Current: An Experimental Study OMAE2019-96112

Yuwang $\mathrm{Xu}^{1}$ Jungao Wang ${ }^{2}$ Haojie Ren ${ }^{1}$ Mengmeng Zhang ${ }^{1}$ Shixiao $\mathrm{Fu}^{1}$

1. Shanghai Jiao Tong University, Shanghai, China;

2. Norwegian Public Roads Administration, Stavanger, Norway

In-line VIV based on Forced-vibration Tests OMAE2019-95972

Decao Yin' Jie Wu' Elizabeth Passano ${ }^{1}$ Halvor Lie ${ }^{1}$ Ralf Peek ${ }^{2}$

0ctavio Sequeiros ${ }^{3}$ Sze Yu Ang ${ }^{3}$ Chiara A. Bernardo ${ }^{4}$ Meliza Atienza ${ }^{4}$

1. SINTEF Ocean, Trondheim, Norway; 2. Peek Solutions, St. Andreu de

Llavaneres, Spain; 3. Shell Global Solutions International B.V., Rijswijk,

Netherlands; 4. Shell Philippines Exploration B.V., Manila, Philippines

Numerical Investigation for Vortex-induced Vibrations of Steel-Lazy-Wave-Risers, Part II: CFD Study on Long Flexible Riser OMAE2019-96404

Hyunchul Jang, Jang Kim

TechnipFMC, Houston, TX, USA

Vortex-induced Vibration of a Flexible Cylinder Experiencing Oscillatory Flow with Different Aspect Ratios OMAE2019-95522 Di Deng, Lei Wu, Decheng Wan

Shanghai Jiao Tong University, Shanghai, China

\section{Ocean Renewable Energy}

\section{9-1-2 FWT - Numerical Analysis I}

Wednesday June 12

Room SEC, Carron 1 | 15:30 - 17:30

Session Chair: Alessandro Fontanella, Politecnico di Milano, Italy

Session Co-Chair: Imas Bayati, MARIN, Netherlands

Impact of Simulation Duration Analysis for Offshore Floating Wind

Turbines using a Coupled FAST-OrcaFlex Model OMAE2019-95159

Ajit C Pillai, Philipp R. Thies, Lars Johanning

University of Exeter, Penryn, United Kingdom

Upgrading the Numerical Analysis of the Mooring System and the Aerodynamics of the TELWIND Platform OMAE2019-96380

Tommaso Battistella José Armesto ${ }^{1}$ Álvaro Rodriguez Luis ${ }^{1}$

Lucia Meneses ${ }^{1}$ Bernardino Couñago ${ }^{2}$ José Serna ${ }^{2}$ Raúl Guanche ${ }^{1}$

Joaquin Urbano ${ }^{2}$ Sergio Hernandez $z^{2}$ José Fernandez ${ }^{2}$

1. Environmental Hydraulics Institute of Cantabria,

Santander, Spain; 2. Esteyco SAP, Madrid, Spain

Second-order Responses of a 10 MW Floating Wind Turbine, considering the Full QTF OMAE2019-95661

Qun Cao, Longfei Xiao, Xiaoxian Guo, Mingyue Liu

Shanghai Jiao Tong University, Shanghai, China
Impact of High Order Wave Loads on a 10MW Tension-leg Platform Floating Wind Turbine at Different Tendon Inclination Angles

OMAE2019-96243

Daniel Milano ${ }^{1}$ Christophe Peyrard ${ }^{2}$ Matteo Capaldo ${ }^{3}$

David Ingram ${ }^{4}$ Qing Xiao ${ }^{5}$ Lars Johanning ${ }^{6}$

1. University of Edinburgh, Edinburgh, United Kingdom; 2. EDFR\&D, Chatou, France; 3. EDF Lab Saclay, Paris, France; 4. The University of Edinburgh, Edinburgh, United Kingdom; 5. University of Strathclyde, Glasgow, United Kingdom; 6. University of Exeter, Penryn, United Kingdom

Hybrid Model Testing of Floating Wind Turbines: Test Bench for System Identification and Control Performance Assessment OMAE2019-96374

Vincent Arnal, Felicien Bonnefoy, Jean-Christophe Gilloteaux, Sandrine Aubrun Ecole Centrale de Nantes, Nantes, France

\section{Ocean Renewable Energy}

\section{9-7-1 Drivetrain Design, Operation and Condition Monitoring I}

Wednesday June 12

Room SEC, Carron 2 | 15:30 - 17:30

Session Chair: Amir R. Nejad, Norwegian University of Science and Technology, Norway Session Co-Chair: Jan Helsen, Vrije Universiteit Brussel, Belgium

On Digital Twin Condition Monitoring System

for Drivetrains OMAE2019-95152

Sigrid Siksjø Johansen, Amir R. Nejad

Norwegian University of Science and Technology, Trondheim, Norway

Gaining Insights in Wind Turbine Drivetrain Dynamics by Means of Automatic Operational Modal Analysis combined with Machine Learning Algorithms OMAE2019-96731 Nicoletta Gioia' Roberto Medico ${ }^{2}$ Pieter-Jan Daems ${ }^{1}$ Cédric Peeters ${ }^{1}$ Dirk Deschrijver ${ }^{2}$ Tom Dhaene ${ }^{2}$ Patrick Guillaume ${ }^{1}$ Jan Helsen ${ }^{1}$ 1. Vrije Universiteit Brussel, Brussel, Belgium; 2. Ghent University, Gent, Belgium

The Effect of Operational Parameters on Vibration Signals of Wind Turbine Gearboxes OMAE2019-96720 Sofia Koukoura' ${ }^{1}$ Eric Bechhoefer ${ }^{2}$ James Carroll' Alasdair McDonald 1. University of Strathclyde, Glasgow, United Kingdom; 2. GPMS, Cornwall, VI, USA

Wind Turbine Planetary Gear Fault Identification using Statistical Condition Indicators and Machine Learning 0MAE2019-96713 Cédric Peeters, Timothy Verstraeten, Ann Nowé, Jan Helsen Vrije Universiteit Brussel, Brussel, Belgium

On Design and Analysis of a Drivetrain Test Rig for Wind Turbine Health Monitoring OMAE2019-96721 Lorenzo Balestra' Amir R. Nejad ${ }^{2}$ Giovanni Naldi ${ }^{3}$ 1. University of Bologna, Cervia, Italy; 2. Norwegian University of Science and Technology, Trondheim, Norway; 3. University of Bologna, Bologna, Italy 


\section{Petroleum Technology}

\section{1-4-1 Petroleum Production Systems Design and Operation}

Wednesday June 12

Room Crowne Plaza, Jura | 15:30-17:30

Session Organizer: Sergio Bordalo, Unicamp, Brazil

Session Co-Chair: Juliana Baioco, LAMCSO/COPPE/UFRJ, Brazil

Session Co-Chair: Lucas Sevillano, State University of Campinas, UNICAMP, Brazil

CFD Simulation of Two-phase Vertical Annular Flow in Both Upward and Downward Direction in a Small Pipe OMAE2019-96311 Ekhwaiter Abobaker' Abadelhalim Elsanoose' John Shirokoff' Mohammad Rahman ${ }^{2}$ 1. Memorial University of Newfoundland, St. John's, NL, Canada;

2. Texas A\&M University at Qatar, Doha, Qatar

Operational Safety Risk Assessment in Offshore Oil Wells OMAE2019-95069 Marcelo A. Jaculli' Danilo Colombo ${ }^{2}$ José Ricardo P. Mendes ${ }^{1}$

Cinara F. G. Marculino ${ }^{1}$ Beethoven G. S. Costa ${ }^{1}$

1. University of Campinas, Campinas, SP, Brazil; 2. Petrobras, Rio de Janeiro, RJ, Brazil

Optimizing Production Facilities using a Transient

Multiphase Flow Simulator OMAE2019-95002

Abdulaziz AlQasim ${ }^{1}$ Fahad AlMudairis ${ }^{2}$ Abdulrahman Bin 0 mar $^{1}$ Abdullatif Omair ${ }^{1}$

1. Saudi Aramco, Dhahran, Saudi Arabia; 2. Kuwait University, Kuwait, Kuwait

Uncertainty Analysis in the Multi-objective

Optimization of Hydraulic Fracture OMAE2019-96103

Juliana Baioco ${ }^{1}$ Breno Jacob ${ }^{2}$ Luis Felipe Mazadiego ${ }^{3}$

1. LACEO/COPPE/Federal University of Rio de Janeiro, Rio de Janeiro, RJ,

Brazil; 2. Federal University of Rio de Janeiro, Rio de Janeiro, RJ, Brazil;

3. UPM - Polytechnic University of Madrid, Madrid, Spain

\section{Petroleum Technology}

\section{1-12-2 Cementing II}

Wednesday June 12

Room Crowne Plaza, Barra | 15:30 - 17:30

Session Chair: Ian Frigaard, University of British Columbia, Canada

Session Co-Chair: Majid Bizhani, University of British Columbia, Canada

Laminar Displacement Flows in Vertical Eccentric Annuli:

Experiments and Simulations OMAE2019-95180

Ali Etrati, lan Frigaard

University of British Columbia, Vancouver, BC, Canada

Removal of Viscoplastic Gels from Conduits OMAE2019-95218

Kamran Alba' Olamide Oladosu' Paris Brown' Jai Bhakta' lan Frigaard ${ }^{2}$

1. University of Houston, Houston, TX, USA;

2. University of British Columbia, Vancouver, $B C$, Canada

Real-time Measurement of Eccentricity in Primary

Cementing of Oil and Gas Wells OMAE2019-95415

Amir Maleki, lan Frigaard

University of British Columbia, Vancouver, BC, Canada

\section{Developing Sensitivity Response Curves to Evaluate}

Mechanical Integrity of Set Cement OMAE2019-95588

Harshkumar Patel' Saeed Salehi ${ }^{2}$ Catalin Teodoriu ${ }^{2}$

1. University of Oklahoma, Norman, OK, USA; 2. Mewbourne School

of Petroleum and Geological Engineering, Norman, OK, USA

Real Time Cementing Hydraulics Simulations

Bring Risk Down OMAE2019-95100

Sviatoslav Pelipenko ${ }^{1}$ Nicolas Flamant ${ }^{2}$ Simon Impey $^{1}$

1. Oxford Numerics, London, United Kingdom;2. Schlumberger, Sugar Land, TX, USA

\section{Takeshi Kinoshita Honoring Symposium on Offshore Technology}

\section{3-1-4 Nonlinear Waves II}

Wednesday June 12

Room SEC, Boisdale 2 | 15:30 - 17:30

Session Organizer: Takuji Waseda, University of Tokyo, Japan

Second Order Wave Propagating Along VLFS OMAE2019-95132

Kazuhiro lijima' ${ }^{1}$ Chong $\mathrm{Ma}^{2}$

1. Dept of NAOE, Osaka University, Osaka, Japan;

2. National Maritime Research Institute, Tokyo, Japan

Nonlinear Generation of Narrow-banded Wave Trains OMAE2019-95364

Anatoliy Khait, Lev Shemer

Tel Aviv University, Tel Aviv, Israel

Analysis of the Momentum Transfer Operated by the Breaking in

Modulated Wave Trains in Wind and No-wind Conditions OMAE2019-95756

Alessandro lafrati, Massimo Falchi

CNR-INM (INstitute of Marine Engineering), Roma, Italy

CFD Simulations of Breaking Stokes Waves OMAE2019-95946

Takanori Hino, Harushi Ikenoue, Youhei Takagi

Yokohama National University, Yokohama, Japan

Performance of a Two-phase Flow Solver for the

Simulation of Breaking Waves OMAE2019-96326

Qiu Jin, Dominic A. Hudson, Pandeli Temarel, W. Geraint Price

University of Southampton, Southampton, United Kingdom

\section{Afternoon Lecture Series \\ 17:40 - 18:30 \\ Location: Lomond Auditorium (SEC)}

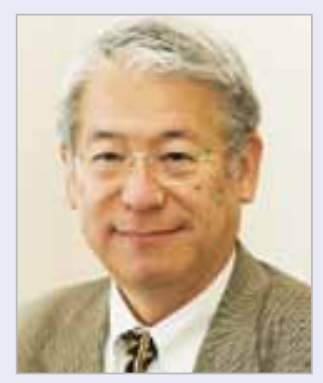

Professor Takeshi Kinoshita
Enjoyable Marine

Engineering Researches on Sports, Environment, not only Water Wave Engineering, Nonlinear Hydrodynamic Forces and Statistics

Professor Takeshi Kinoshita, Visiting Professor, Nagasaki Institute of Applied Science

See Afternoon Lecture Series, page 22 for more details.

\section{Conference Banquet}

Banquet: 19:00 - 24:00

Location: Merchant Square

See Social Events, page 14 for more details. 


\section{Thursday, June 13}

\begin{tabular}{|c|c|c|}
\hline Time & Title & Location \\
\hline $07: 30-10: 00$ & $\begin{array}{l}\text { Outreach Breakfast / } \\
\text { Feedback Session }\end{array}$ & Alsh 2 (SEC) \\
\hline $08: 30-15: 30$ & Exhibition open & Hall 5 (SEC) \\
\hline $08: 30-10: 00$ & Concurrent Sessions & $\begin{array}{l}\text { See pages } 72-75 \\
\text { for session titles, } \\
\text { authors and locations }\end{array}$ \\
\hline $10: 00-10: 30$ & Refreshment Break & Hall 5 (SEC) \\
\hline $10: 30-12: 00$ & Concurrent Sessions & $\begin{array}{l}\text { See pages } 76-80 \\
\text { for session titles, } \\
\text { authors and locations }\end{array}$ \\
\hline $12: 00-13: 30$ & $\begin{array}{l}\text { Technical Session } \\
\text { Organizers' Lunch }\end{array}$ & Hall 5 (SEC) \\
\hline $13: 30-15: 00$ & Concurrent Sessions & $\begin{array}{l}\text { See pages } 80-84 \\
\text { for session titles, } \\
\text { authors and locations }\end{array}$ \\
\hline $15: 00-15: 30$ & Refreshment Break & Hall 5 (SEC) \\
\hline $15: 30-17: 30$ & Concurrent Sessions & $\begin{array}{l}\text { See pages } 84-86 \\
\text { for session titles, } \\
\text { authors and locations }\end{array}$ \\
\hline $17: 30-19: 00$ & Farewell Reception & $\begin{array}{l}\text { Argyll Suite } \\
\text { (Crowne Plaza) }\end{array}$ \\
\hline
\end{tabular}

\section{CONCURRENT SESSIONS}

$08: 30-10: 00$

\section{Offshore Technology}

\section{1-1-1 Semi-submersibles and TLPs}

Thursday June 13

Room SEC, Alsh 1 | 08:30 - 10:00

Session Chair: Johyun Kyoung, TechnipFMC, USA

Session Co-Chair: Sandeep Bukka Reddy, National University of Singapore, Singapore

Global Performance Analysis of Deep Draft Semi-submersible

Designed for Standard GoM Application OMAE2019-95272

Raehyoung Yuck Daehoon Kang ${ }^{1}$ Eung-su Kim² Munsung Kim²

Tae-min Kim² Hyun Joe Kim ${ }^{1}$ DongYeon Lee ${ }^{1}$ Young-chan Park ${ }^{3}$

1. Samsung Heavy Industries, Daejeon, Korea; 2. Samsung Heavy

Industries, Seongnam, Korea; 3. Williams, Houston, TX, USA

\begin{abstract}
A Conjoint Analysis of the Stability and Time-domain Analysis on
Floating Platform during Mooring Line Breaking OMAE2019-96661

Jiaguo Feng ${ }^{1} Y_{i} Y_{u^{2}}$ Yan $Q u^{3}$ Wenhui Xie ${ }^{1} \mathrm{Min} \mathrm{Wu}^{4}$ Jingrui Zhao ${ }^{5}$

1. CNOOC Research Institute, Beijing, China; 2. CNOOC, Ltd., Beijing, China;

3. Southern University of Science and Technology, Shenzhen, China; 4. SBM Offshore

USA, Inc., Houston, TX, USA; 5. CNOOC Research Institute, Shenzhen, China
\end{abstract}

First in Place Replacement of a TLP Top Tendon

Connector Flex Bearing OMAE2019-96232

Jeffrey D. Otten ${ }^{1}$ Vinu Kuriakose' Shakib Amini

1. SBM Offshore USA, Inc., Houston, TX, USA; 2. Baker Hughes a GE Company, Houston, TX, USA

Concept Design of Very Large Floating Structures and

Laboratory-scale Physical Modelling 0MAE2019-96259

Lorenzo Cappietti, Irene Simonetti, Ilaria Crema

University of Florence, Florence, Italy

\section{Structures, Safety and Reliability}

\section{2-13-1 Risk Analysis and Management I}

Thursday June 13

Room Crowne Plaza, Castle 2 | 08:30 - 10:00

Session Chair: Marcelo Ramos Martins, LabRisk - Analysis, Evaluation

and Risk Management Laboratory - University of São Paulo, Brazil

Session Co-Chair: Amir R. Nejad, Norwegian University

of Science and Technology, Norway

The Influence of Non-prescriptive Legislation in the Evolution of Offshore Well Integrity Practices: An Exploratory Review OMAE2019-96269

Carlos H. B. Morais' Danilo T. M. P. Abreu' Joaquim Santos ${ }^{2}$

Marcos C. Maturana ${ }^{3}$ Danilo Colombo ${ }^{4}$ Marcelo Ramos Martins ${ }^{1}$

1. LabRisk - Analysis, Evaluation and Risk Management Laboratory - University of São Paulo, São Paulo, SP, Brazil; 2. University of São Paulo, São Paulo, SP, Brazil; 3. Centro Tecnologico da Marinha em Sao Paulo, São Paulo, SP, Brazil; 4. Petrobras, Rio de Janeiro, RJ, Brazil

On Disaster Risk Reduction in Norwegian 0il \& Gas Industry

through Life-cycle Perspective OMAE2019-95622

Michaela Ibrion, Nicola Paltrinieri, Amir R. Nejad

Norwegian University of Science and Technology, Trondheim, Norway

Towards Implementing Condition-based Maintenance (CBM) Policy for Offshore Blowout Preventer (BOP) System OMAE2019-95539

Tobiloba Elusakin, Mahmood Shafiee, Tosin Adedipe

Cranfield University, Bedford, United Kingdom

Subsea Spares Analysis Optimisation OMAE2019-96100 Tiarnan Coffey, Christopher Rai, John Greene, Stephen 0'Brien Bromley Wood, Galway, Ireland

Research on Assessment Method for Bridge Pier Foundation Stability using Non-destructive Tests OMAE2019-95845 Mintaek Yoo Korea Railroad Research Institute, Ui Wang, Korea

\section{Structures, Safety and Reliability}

\section{2-6-2 Reliability of Mooring and Riser Systems II}

Thursday June $13 \quad$ Room Crowne Plaza, Castle 1 | 08:30 - 10:00 Session Chair: Luis V.S. Sagrilo, LACEO/COPPE/

Federal University of Rio De Janeiro, Brazil

Session Co-Chair: Ying Min Low, National University of Singapore, Singapore

Mean Load Impact on Mooring Chain Fatigue Capacity - Lessons Learned from Full Scale Fatigue Testing of Used Chains OMAE2019-95083 Øystein Gabrielsen ${ }^{1}$ Kjell Larsen ${ }^{2}$ Oddgeir Dalane ${ }^{3}$ Hans B. Lie ${ }^{4}$ Svein-Arne Reinholdtsen ${ }^{5}$ 1. Equinor, Trondheim, Norway; 2. Equinor ASA, Trondheim, Norway; 3. Equinor ASA, Stavanger, Norway; 4. Equinor ASA, Sandsli, Norway; 5. Equinor ASA, Stjordal, Norway

Finite Element Analysis of the Effect of Twist on Chain Fatigue Performance OMAE2019-95276 Justin Jones

Petrofac, Woking, United Kingdom

Fatigue Tests on Corroded Mooring Chains Retrieved from Various Fields in Offshore West Africa and the North Sea OMAE2019-95618 Kai-tung $\mathrm{Ma}^{1}$ Øystein Gabrielsen ${ }^{2}$ Zhen Li David A. Baker ${ }^{4}$ Aifeng Yao ${ }^{1}$ Pedro Vargas ${ }^{5}$ Meng Lu ${ }^{6}$ Amir Izadparast $^{7}$ Alberto Arredondo ${ }^{8}$ 
Linfang Zhu ${ }^{9}$ Nina Sverdlova ${ }^{10}$ Ingrid Skutle Høgsæt ${ }^{11}$

1. Chevron, Houston, TX, USA; 2. Equinor, Trondheim, Norway; 3. ExxonMobil, Spring, TX, USA; 4. Exxon Mobil Upstream Research Co, Spring, TX, USA; 5. Chevron Energy Technology Company, Houston, TX, USA; 6. Shell International Exploration and Production Inc., Houston, TX, USA; 7. Sofec, Houston, TX, USA; 8. Vicinay Marine Innovación, Leioa, Spain; 9. ZhengMao Group, Zhenjiang, China; 10. Feubo, Hattingen, Germany; 11. DNV GL, Høvik, Norway

Fatigue Performance of High Strength and Large Diameter Mooring Chain in Seawater OMAE2019-95984 Yanhui Zhang' ${ }^{1}$ Philip Smedley ${ }^{2}$

1. TWI Ltd, Cambridge, United Kingdom; 2. BP, London, United Kingdom

Fatigue Assessment of "Corroded" Mooring Chain 0MAE2019-96191 David A. Baker' Zhen Li ${ }^{2}$ Sue Wang ${ }^{3}$ Xiying Zhang ${ }^{3}$ Yunliang Sha0 ${ }^{4}$ Harry Li Xiaoqin Zhan ${ }^{6}$ Linfang Zhu ${ }^{6}$ Xing Ta0 ${ }^{7}$

1. Exxon Mobil Upstream Research Co, Spring, TX, USA;2. ExxonMobil, Spring,

TX, USA; 3. ABS, Houston, TX, USA; 4. Jiangsu Asian Star Anchor Chain Co., Ltd., Jingjiang City, China; 5. Asian Star Anchor Chain, Jiangjiang, China; 6. Zhengmao Group, Zhenjiang, China; 7. Asian Star Anchor Chain, Jiangsu, China

\section{Materials Technology}

\section{3-2-1 Fabrication and Performance of Clad Pipes}

Thursday June 13

Session Chair: Agnes Marie Horn, DNV GL, Norway

Session Co-Chair: Koji Gotoh, Kyushu University, Japan

Review of the In-air Fatigue Behaviour of CRA

Clad and Lined Pipe OMAE2019-96233

Carol Johnston, Jennifer Crump

TWI Ltd, Cambridge, United Kingdom

\section{Fatigue Crack Growth Rate Testing for Clad and}

Lined Pipe Girth Weld OMAE2019-95500

Zhengmao Yang ${ }^{1}$ Youyou $\mathrm{Wu}^{2}$ Jens Tronskar ${ }^{3}$ Daqin $\mathrm{Xu}^{2}$

1. DNV GL, Singapore, Singapore; 2. DNV GL Laboratory, Singapore,

Singapore; 3. Det Norske Veritas Pte Ltd, Singapore, Singapore

Recent Developments on Welding NDT and ECA of Clad and

Lined Offshore Pipelines OMAE2019-96358

Petrônio Zumpano Jr.' Alexandre G. Garmbis' Diogo 0. Moraes' ${ }^{1}$

Fausto Hirata' Bruno R. M. Cunha ${ }^{2}$ Eduardo Hippert ${ }^{2}$ Marcelo Paes ${ }^{2}$

1. Petrobras, São José dos Campos, SP, Brazil; 2. Petrobras, Rio de Janeiro, RJ, Brazil

Assessment of Weld Overlays in a Cladded Piping

Systems with Varied Thicknesses OMAE2019-96348

Bridget Kogo ${ }^{1}$ Bin Wang ${ }^{2}$ Luiz Wrobel $^{2}$ Mahmoud Chizari ${ }^{2}$

1. Brunel University London, Worcester, United Kingdom;

2. Brunel University London, London, United Kingdom

\section{Pipelines, Risers, and Subsea Systems}

\section{4-1-10 Umbilicals and Cables I}

Thursday June $13 \quad$ Room Crowne Plaza, Staffa / Shuna | 08:30 - 10:00

Session Chair: Jun Yan, Dalian University of Technology, China

Session Co-Chair: Alan Dobson, Technip Umbilicals, United Kingdom

Section Translational Movement Imposition

for Macroelements OMAE2019-95033

Rodrigo Provasi, Clovis de Arruda Martins

University of São Paulo, São Paulo, SP, Brazil
Extreme and Fatigue Analysis of a Dynamic

Subsea Power Umbilical OMAE2019-95123

Yan Qu $\mathbf{u}^{1}$ Hong Guo ${ }^{2}$ Lei Zhang ${ }^{3}$ Zhenqin Yuan ${ }^{4}$ Juyue Li $^{4}$

1. Southern University of Science and Technology, Shenzhen, China;

2. CNOOC Research Institute, Beijing, China; 3. Hengtong Submarine Cable Ltd.,

Changshu, China; 4. Hengtong Submarine Cable Ltd., Jiangshu, China

Effect of Weld Porosity on Super Duplex Stainless

Steel Umbilical Tubes under Hydrogen Induced Stress

Cracking Exposure Condition OMAE2019-95986

Mariana Socariceanu, Xiaoxue An

TechnipFMC Umbilicals, Newcastle upon Tyne, United Kingdom

The Design and Installation of Wet Park Configuration for Dynamic Umbilicals in Ultra-deep Environment OMAE2019-96006 Perdinan Reagan, Graeme Lovie

Subsea 7, Sutton, United Kingdom

\section{Pipelines, Risers, and Subsea Systems}

\section{4-3-7 Thermo-Mechanical III}

Thursday June 13 Room Crowne Plaza, Castle 3 | 08:30 - 10:00

Session Chair: Celso Morooka, University of Campinas, Brazil

Session Co-Chair: Daniel Carneiro, Wood, Brazil

Effect of Seabed Imperfection on the Buckling of Buried

Pipelines Subjected to Wave-induced Loads OMAE2019-95409 Duggivalasa Suresh Kumar, Mohammed Rabius Sunny, Trilochan Sahoo Indian Institute of Technology Kharagpur, Kharagpur, India

Pipeline Rockberm Design Principles for UHB Mitigation OMAE2019-95444 M Liu' Colin Cross

1. Aker Solutions, Windsor, United Kingdom; 2. Aker Solutions, London, United Kingdom

UHB Design Approach for Multiple Pipelines

Installed in Shared Trench OMAE2019-95448

M Liu' Colin Cross ${ }^{2}$

1. Aker Solutions, Windsor, United Kingdom; 2. Aker Solutions, London, United Kingdom

Design of Buried Pipelines in Soft Clay: A Case Study 0MAE2019-95505 Martin Gallegillo ${ }^{1}$ Michele Cerulli' Ali Haghighi Justin Kennedy ${ }^{2}$

1. Genesis, London, United Kingdom; 2. TechnipFMC, Westhill, Scotland

\section{Ocean Engineering}

\section{6-13-1 Ship Resistance and Wave Loads}

Thursday June 13 Room SEC, M2 \& M3 | 08:30 - 10:00 Session Chair: Atilla Incecik, University of Strathclyde, United Kingdom

Validation of a Multi-level Approach to the Prediction of the Added Resistance and Powering of Ships in Waves OMAE2019-95113 Shukui Liu' Apostolos Papanikolaou ${ }^{2}$ Peiyuan Feng ${ }^{3}$ Sheming Fan ${ }^{3}$ 1. Nanyang Technological University, Singapore, Singapore; 2. National Technical University of Athens, Athens, Greece; 3. Marine Design and Research Institute of China, Shanghai, China

Study of Wave Added Resistance and Motions of KCS in Waves with Different Wave Lengths OMAE2019-95526 Hao Guo, D.C. Wan Shanghai Jiao Tong University, Shanghai, China

An Experimental Investigation of the Trim Effect on the Behaviour of a Containership in Shallow Water OMAE2019-95790 Khaled Elsherbiny' Tahsin Tezdogan' Mohamed Kotb² Atilla Incecik' Sandy Day' 1. University of Strathclyde, Glasgow, United Kingdom; 2. Arab Academy for Science, Technology and Maritime Transport, Alexandria, Egypt 
Capturing Two Consecutive Green Water

Events by Convolution OMAE2019-96747

Jassiel V. Hernández-Fontes, Rodolfo Silva-Casarín, Edgar Mendoza

Universidad Nacional Autónoma de México, Mexico City, DF, Mexico

\section{Ocean Engineering}

\section{6-17-1 Wave Loads on Structures}

Thursday June 13

Session Chair: Sanne van Essen, MARIN, Netherlands

Systematic Experimental Validation of High-order Spectral

Method for Deterministic Wave Prediction 0MAE2019-95063

Marco Klein ${ }^{1}$ Matthias Dudek ${ }^{2}$ Günther Clauss ${ }^{3}$

Norbert Hoffmann ${ }^{1}$ Jasper Behrendt ${ }^{1}$ Sören Ehlers ${ }^{1}$

1. Hamburg University of Technology, Hamburg, Germany; 2. Neue Warnow Design \&

Technology GmbH, Rostock, Germany; 3. Technische Universität Berlin, Berlin, Germany

Variability in Encountered Waves during Deterministically

Repeated Seakeeping Tests at Forward Speed OMAE2019-95065

Sanne van Essen, MARIN, Wageningen, Netherlands

Multi-focused Wave Groups in Wave Flume OMAE2019-95831

Qinghe Fang ${ }^{1}$ Cunbao Zhao ${ }^{2}$ Anxin Guo ${ }^{1}$

1. Harbin Institute of Technology, Harbin, China; 2. Shijiazhuang

Tiedao University, Shijiazhuang, China

Experimental Study of Wave Loading by Internal Solitary

Waves on a Semi-submersible Platform OMAE2019-95891

Jingjing Zhang, Ke Chen, Yunxiang You, Xinshu Zhang

Shanghai Jiao Tong University, Shanghai, China

Linear Evolution of a Narrow-banded Surface Gravity

Wavepacket Over an Infinite Step OMAE2019-96082

Yan Li, Thomas A.A. Adcock, Ton S. van den Bremer

University of Oxford, Oxford, United Kingdom

Numerical Simulation of the Loads on Cylinders

Exerted by Internal Wave Trains OMAE2019-96828

Xu Wang, Jifu Zhou

Institute of Mechnaics, Chinese Academy of Sciences, Beijing, China

\section{Ocean Engineering}

\section{6-7-1 Regional Metocean I}

Thursday June 13

Room SEC, M4 | 08:30 - 10:00

Session Chair: Gus Jeans, Oceanalysis Ltd, United Kingdom

Session Co-Chair: Kevin Ewans, Met0cean Research Ltd, New Zealand

Study on Gust Parameters and Wind Spectrum

of South China Sea OMAE2019-95779

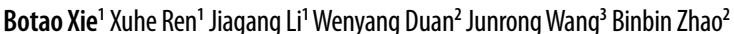

1. CNOOC Research Institute, Beijing, China; 2. Harbin Engineering University,

Harbin, China; 3. Ocean University of China, Qingdao, China

Statistical Analysis for the Duration and Time Intervals

of Tropical Cyclones, Hong Kong OMAE2019-95791

Shanshan Tao, Jialing Song, Zhifeng Wang, Yong Liu, Sheng Dong

Ocean University of China, Qingdao, China

Evaluation of the Ocean Circulation for the Solomon Sea using

the Regional Ocean Modelling System (ROMS) OMAE2019-96179

A. Rute Bento, Henrique Coelho, Chunxue Yang

Fugro GB Marine Ltd, Wallingford, United Kingdom
A Probabilistic Approach to Tropical Cyclone Modelling OMAE2019-96245

Stephen Grey, Ye Liu

HR Wallingford Ltd, Wallingford, United Kingdom

\section{CFD \& FSI}

\section{8-1-5 Seakeeping I}

Thursday June 13

Room SEC, Dochart 1 | 08:30 - 10:00

Session Chair: Sandy Day, University of Strathclyde, United Kingdom

Session Co-Chair: Kie Hian Chua, Technology Centre

for Offshore and Marine, Singapore

URANS Predictions of Low-frequency Viscous

Damping of a LNGC OMAE2019-95171

Frédérick Jaouën ${ }^{1}$ Arjen Koop ${ }^{1}$ Lucas Vatinel ${ }^{2}$

1. MARIN, Wageningen, Netherlands; 2. MARIN Academy, Wageningen, Netherlands

Predicting Roll Damping for Barge-type FPSO using CFD OMAE2019-95306 Arjen Koop ${ }^{1}$ Frédérick Jaouën ${ }^{1}$ Xavier Wadbled ${ }^{2}$ Erwan Corbineau $^{2}$ 1. MARIN, Wageningen, Netherlands; 2. MARIN Academy, Wageningen, Netherlands

Numerical Simulations of KCS Parametric

Rolling in Head Waves OMAE2019-95563

Shuang Wang ${ }^{1}$ Junkai Wei' Xuanshu Chen ' Liwei Liu' ZhiGuo Zhang

1. Huazhong University of Science and Technology, Wuhan, China;

2. China Ship Design and Development Center, Wuhan, China

URANS Prediction of ONR Tumblehome Parametric

Rolling Characteristics in Waves OMAE2019-96425

ZhiGuo Zhang' Lixiang Guo' Shuang Wang' Ye Yuan ${ }^{2}$ Can Chen $^{2}$

1. Huazhong University of Science and Technology, Wuhan, China;

2. China Ship Design and Development Center, Wuhan, China

\section{CFD \& FSI}

\section{8-4-2 Risers, Jumpers and Pipelines}

Thursday June 13 Room SEC, Lomond Auditorium | 08:30 - 10:00 Session Chair: Hai Sun, Harbin Engineering University, China Session Co-Chair: Aravind Nair, DNV GL, USA

Flow-induced Vibration Screening of a Thermoplastic Composite Pipe Water Injection Jumper OMAE2019-95030 Juan Pontaza ${ }^{1}$ Meng Luo ${ }^{2}$ Varadarajan Nadathur' John Rosche ${ }^{1}$ 1. Shell, Houston, TX, USA; 2. Shell International Exploration and Production Inc., Houston, TX, USA

Vertical Riser under VIV: A Numerical Assessment of Experimental Results using a Reduced Order Model OMAE2019-95150 Renato Orsino ${ }^{1}$ Guilherme Lopes ${ }^{1}$ Celso Pesce ${ }^{2}$ Guilherme Franzini ${ }^{1}$ Fernanda Takafuji' 1. University of São Paulo, São Paulo, SP, Brazil;

2. University ofS. Paulo - Escola Politecnica, São Paulo, SP, Brazil

Three-Dimensional Numerical Simulations of Severe

Gas-Liquid Slugging Flows in S-shaped Riser OMAE2019-95459

Youn-Wook Moon ${ }^{1}$ Narakorn Srinil' Jong-Chun Park ${ }^{2}$

1. Newcastle University, Newcastle upon Tyne, United Kingdom;

2. Pusan National University, Busan, Korea

Fluid Dynamics Numerical Assessment to Evaluate the

Ice Formation around the Pipeline OMAE2019-95528

Giuseppe Blasioli, Furio Marchesani

Saipem S.p.A., Fano, Italy 


\section{Ocean Renewable Energy}

\section{9-1-5 FWT - Mooring Systems}

Thursday June 13

Room SEC, Carron 1 | 08:30 - 10:00

Session Chair: Tonio Sant, Dept of Mechanical Engineering,University of Malta, Malta Session Co-Chair: Erin E. Bachynski, Norwegian

University of Science and Technology, Norway

Mooring Line Dynamics of a Semi-submersible Wind Energy Platform: Cross Validation of Two Commercial Numerical

Codes with Experimental Data OMAE2019-96295

Rachel Chester' Cian Desmond' Jimmy Murphy' Simon J. Watson²

1. University College Cork, Cork, Ireland; 2. TU Delft, Delft, Netherlands

Force Dynamics and Stationkeeping Costs for Multiline Anchor Systems in Floating Wind Farms with

Different Spatial Parameters OMAE2019-96395

Casey M. Fontana' Sanjay R. Arwade ${ }^{1}$ Don J. Degroot ${ }^{1}$ Spencer Hallowell ${ }^{2}$

Melissa E. Landon ${ }^{3}$ Charles Aubeny ${ }^{4}$ Brian Diaz ${ }^{4}$ Andrew T. Myers ${ }^{5}$ Senol Ozmutlu ${ }^{6}$

1. University of Massachusetts Amherst, Amherst, MA, USA; 2. Independent Author, West Bath, ME, USA; 3. University of Maine, Orono, ME, USA; 4. Texas A\&M University, College Station, TX, USA; 5. Northeastern University, Boston, MA, USA; 6. Vryhof Anchors, Schiedam, Netherlands

Analysis on Hydrodynamic Responses of a Spar Offshore Wind Turbine with an Innovative Type of Mooring System OMAE2019-96759 Yuan Ma, Chaohe Chen, Xinkuan Yan, Yijun Shen, Tianhui Fan South China University of Technology, Guangzhou, China

Modeling a Non-linear Mooring System for Floating Offshore

Wind using a Hydraulic Piston Analogy OMAE2019-96080

Magnus Harrold' Philipp R. Thies' David Newsam²

Claudio Bittencourt Ferreira ${ }^{3}$ Lars Johanning ${ }^{1}$

1. University of Exeter, Penryn, United Kingdom; 2. Teqniqa Systems Ltd.,

Lavister, United Kingdom; 3. DNV GL, London, United Kingdom

\section{Ocean Renewable Energy}

\section{9-5-3 Numerical Analysis I}

Thursday June 13

Session Chair: Marc Cahay, TechnipFMC, France

Room SEC, Carron 2 | 08:30 - 10:00

Session Co-Chair: Qing Xiao, University of Strathclyde, United Kingdom

CFD-based Study of a Tidal Current Turbine in a

Horizontal Axis under Regular Waves OMAE2019-95231

Jing Liu' Longfei Xia0 ${ }^{1}$ Fengmei Jing ${ }^{2}$

1. Shanghai Jiao Tong University, Shanghai, China; 2. Harbin

Engineering University, Harbin, China

Local Blockage Effects for Idealised Turbines

in Tidal Channels OMAE2019-95347

Lei Chen ${ }^{1}$ Paul A.J. Bonar ${ }^{2}$ Christopher Vogel ${ }^{1}$ Thomas A.A. Adcock ${ }^{1}$

1. University of Oxford, Oxford, United Kingdom;

2. University of Edinburgh, Edinburgh, United Kingdom

Numerical Analysis of Tidal Turbine Performance

for Floating Platform OMAE2019-95884

Xiuqing Xing' Chang Wei Kang ' George Xu' Jing Lou' Ken Takagi' Jarrod Sinclair ${ }^{3}$

1. Institute of High Performance, $A^{*}$ STAR, Singapore, Singapore; 2. The University

of Tokyo, Tokyo, Japan; 3. Mako Turbines Asia Pte. Ltd, Singapore, Singapore

Effects of Tubercles on Blade and Wake of HAMCT in Post

Stall Regimes - Linear Cascade Study OMAE2019-96287

Varun Dondapati, K. Murali

Indian Institute of Technology Madras, Chennai, India

\section{Petroleum Technology}

\section{1-1-1 General Petroleum Technology - Production Enhancement}

Thursday June 13 Room Crowne Plaza, Jura | 08:30 - 10:00 Session Chair: Stephen Butt, Memorial University of Newfoundland, Canada Session Co-Chair: Mohammad Rahman, Texas A\&M University at Qatar, Qatar

$\mathrm{CO}_{2}$-Foam Rheology Behavior under Reservoir Conditions OMAE2019-95191 Abdulaziz AlQasim, Sunil Kokal, Fawaz AlOtaibi

Saudi Aramco, Dhahran, Saudi Arabia

Experimental and Numerical Investigation of Gas-yield Power-law Fluids in a Horizontal Pipe OMAE2019-95219 Abdalsalam Ihmoudah' M. M. Awad ${ }^{2}$ Mohammad Azizur Rahman ${ }^{3}$ Stephen Butt ${ }^{1}$ 1. Memorial University of Newfoundland, St. John's, NL, Canada; 2. Mansoura University, Mansoura, Egypt; 3. Texas A\&M University at Qatar, Doha, Qatar

Software Simulation and Experimental Study on Wax Deposition Pattern of Deep Condensate Gas OMAE2019-95326 Jie Wang' Fujian Zhou' Erdong Yao' Fan Fan' Lishan Yuan' Yanpeng Xue ${ }^{2}$ 1. China University of Petroleum-Beijing, Beijing, China; 2. Tarim Oilfield, CNPC, Korla, China

Experimental Investigation on Stress Sensitivity Behavior for Tight Naturally Fractured Sandstone Reservoirs OMAE2019-95774 Y.C Wang, Fujian Zhou, Lufeng Zhang, Jin Wang China University of Petroleum, Beijing, China

\section{Petroleum Technology}

\section{1-13-1 LSU Workshop on Riser Gas Management and Well Control}

Thursday June 13 Room Crowne Plaza, Barra | 08:30 - 10:00 Session Chair: Yuanhang Chen, Louisiana State University, USA Session Co-Chair: Kjell Kåre Fjelde, University of Stavanger, Norway

Buoyancy Induced Convection of Riser Gas in Deepwater Drilling Operations OMAE2019-96649 Syed Y. Nahri' Yuanhang Chen 'Wesley Williams' ${ }^{1}$ Otto Santos ${ }^{1}$ Ting Sun ${ }^{2}$ 1. Louisiana State University, Baton Rouge, LA, USA;

2. China University of Petroleum, Beijing, China

Numerical Simulations of Riser Gas Behavior in Non-aqueous Muds using a Modified Drift Flux Model OMAE2019-96672 Nnamdi Nwaka, Yuanhang Chen Louisiana State University, Baton Rouge, LA, USA

Well Control Simulation with Non-aqueous Drilling Fluids OMAE2019-96736 Felipe Chagas ${ }^{1}$ Paulo Ribeiro' 0 tto Santos ${ }^{2}$

1. Universidade Estadual de Campinas, Campinas, SP, Brazil;

2. Louisiana State University, Baton Rouge, LA, USA

Experimental Investigation of Absorption and Desorption of Gas in Riser during MPD Well Control OMAE2019-96767 James L. Nielsen' Mahendra Kunju' Yuanhang Chen' Ting Sun ${ }^{2}$ 1. Louisiana State University, Baton Rouge, LA, USA; 2. China University of Petroleum, Beijing, China 


\section{Takeshi Kinoshita Honoring Symposium on Offshore Technology}

\section{3-3-2 Wave Energy I}

Thursday June 13

Room SEC, Boisdale 2 | 08:30 - 10:00

Session Organizer: Tomoki lkoma, Nihon University, Japan

Session Co-Organizer: Yukitaka Yasuzawa, Kyushu University, Japan

Session Co-Organizer: Changkyu Rheem, University of Tokyo, Japan

Study on the Generated Power Changes by the Relation between an Arrangement of an Array of Point Absorber Type WECs and an Incident Wave Angle OMAE2019-95400 Motohiko Murai, Qiao Li, Junki Funada

Yokohama National University, Yokohama, Japan

Nonlinear Analysis of a Heaving Point Absorber in Frequency Domain via Statistical Linearization OMAE2019-95785 Leandro Souza Pinheiro da Silva ${ }^{1}$ Hélio Morishita ${ }^{1}$ Celso Pesce ${ }^{2}$ Rodolfo T. Gonçalves $^{3}$ 1. University of São Paulo, São Paulo, SP, Brazil; 2. University of São Paulo - Escola Politecnica, São Paulo, SP, Brazil; 3. University of Tokyo, Bunkyo, Japan

A Strategy of a Control Algorism for a Point Absorber Wave Energy Converter OMAE2019-95836 Qiao Li, Motohiko Murai

Yokohama National University, Yokohama, Japan

Fundamental Study on Development on Numerical Method for Evaluation of Wave Power Generating Systems with Pendulum Type by the Particle Method OMAE2019-95869 Kazuki Murata' Changkyu Rheem² Tomoki Ikoma ${ }^{3}$

1. Institute of Industrial Science, The University of Tokyo, Meguro-ku, Japan;

2. The University of Tokyo, Tokyo, Japan; 3. Nihon University, Funabashi, Japan

\section{REFRESHMENT BREAK}

10:00 - 10:30

Location: Hall 5 (SEC)

\section{CONCURRENT SESSIONS}

10:30 - 12:00

\section{Offshore Technology}

\section{1-7-1 Wave Loading and Motions in Extreme Seas I}

Thursday June 13 Room SEC, Alsh 1 | 10:30 - 12:00

Session Chair: Limin Yang, DNV GL, Norway

Experimental Evaluation of Wave Impact Loads on Semi-submersible Structure according to Trim Angle 0MAE2019-95406

Min-Guk Seo ${ }^{1}$ Yoon-Jin Ha' Nam-Woo Kim' Bo Woo Nam Kang-Su Lee ${ }^{2}$ 1. Korea Research Institute of Ships and Ocean Engineering, DaeJeon, Korea; 2. Korea Institute of Ocean Science and Technology, Daejeon, Korea

Efficient Indicators for Screening of Random Waves for Wave Impacts on a Jacket Platform and a Fixed Offshore Wind Turbine OMAE2019-95481 Tim Bunnik, Jule Scharnke, Erik-Jan de Ridder MARIN, Wageningen, Netherlands

Effect of Non-Gaussian Distribution in Fully-nonlinear Waves on Offshore Platform Motion Responses OMAE2019-96465

Aldric Baquet, Ho-Joon Lim, Jang Kim

TechnipFMC, Houston, TX, USA

\section{Structures, Safety and Reliability}

\section{2-13-2 Risk Analysis and Management II}

Thursday June 13

Room Crowne Plaza, Castle 2 | 10:30 - 12:00 Session Chair: Marcelo Ramos Martins, LabRisk - Analysis, Evaluation and Risk Management Laboratory - University of São Paulo, Brazil Session Co-Chair: Mahmood Shafiee, Cranfield University, United Kingdom

A Hybrid Methodology for Maritime Accident Analysis: The Case of Ship Collision OMAE2019-96663 Ludfi Pratiwi Bowo, Ramdhani Eka Prilana, Masao Furusho Kobe University, Kobe, Japan

\section{Human Reliability Analysis of Ship Maneuvers} in Harbor Areas OMAE2019-96251

Danilo T. M. P. Abreu' Marcos C. Maturana ${ }^{2}$ Marcelo Ramos Martins' Siegberto R. Schenk Jr. ${ }^{3}$ 1. LabRisk - Analysis, Evaluation and Risk Management Laboratory - University of São Paulo, São Paulo, SP, Brazil; 2. Centro Tecnologico da Marinha em Sao Paulo, São Paulo, SP, Brazil; 3. Brazilian Maritime Pilots Association (CONAPRA), Rio de Janeiro, RJ, Brazil

Analysing Dependent Failures in a Bayesian

Belief Network 0MAE2019-95853

Mei Ling Fam ${ }^{1}$ Dimitrios Konovessis ${ }^{2}$ Xuhong He ${ }^{3}$ Lin Seng Ong ${ }^{1}$ Hoon Kiang Tan ${ }^{4}$ 1. Nanyang Technological University, Singapore, Singapore; 2. Singapore Institute of Technology, Singapore, Singapore; 3. Lloyd's Register Consulting - Energy AB, Sundbyberg, Sweden; 4. Global Technology Centre, Lloyd?s Register Singapore,, Singapore, Singapore

\section{Risk Assessment of Ship Systems based on}

Forward FTF Method OMAE2019-95320

SongYan Mai' Ji Zeng' Qi Feng ${ }^{2}$ Renan Liu ${ }^{3}$ Yan Chen ${ }^{2}$

1. Shanghai Maritime University, Shanghai, China;

2. Shanghai Honghua Ocean Oil \& Gas Equipment Co., Ltd., Shanghai, China;

3. AFFCO Flow Control (Shanghai) Co., Ltd, Shanghai, China

Risk Assessment in Offshore Salt Caverns to Store $\mathrm{CO}_{2}$ OMAE2019-96250 Marco A. Pestana' ${ }^{1}$ Carlos H. B. Morais ${ }^{1}$ Alvaro M. Costa ${ }^{2}$ Camila Brandão ${ }^{3}$ Marcelo Ramos Martins ${ }^{1}$

1. LabRisk - Analysis, Evaluation and Risk Management Laboratory - University of São Paulo, São Paulo, SP, Brazil; 2. MODECOM - Tecnologia em Geomecânica e Modelagem Coputacional., Rio de Janeiro, RJ, Brazil; 3. Shell Brasil Ltda., Rio de Janeiro, RJ, Brazil

\section{Structures, Safety and Reliability}

\section{2-6-3 Reliability of Mooring and Riser Systems III}

Thursday June 13

Room Crowne Plaza, Castle 1 | 10:30 - 12:00

Session Chair: Ying Min Low, National University of Singapore, Singapore

Session Co-Chair: Luis V.S. Sagrilo, LACE0/COPPE/

Federal University of Rio De Janeiro, Brazil

Review and Comparison of Collated Offshore Mooring Chain Fatigue Test Data OMAE2019-95875

Gary H. Farrow' Andrew E. Potts ${ }^{1}$ Andrew A. Kilner' Eric Jal' Nicholas D'Arcy Evans ${ }^{1}$ 1. AMOG Consulting, Notting Hill, VIC, Australia; 2. AMOG Consulting Inc, Houston, TX, USA

\section{Development of a New, Correlated FEA Method of}

Assessing Mooring Chain Fatigue OMAE2019-95882

Gary H. Farrow ${ }^{1}$ Andrew E. Potts ${ }^{1}$ Andrew A. Kilner ${ }^{2}$

Phillip P. Kurts ${ }^{2}$ Simon Dimopoulos ${ }^{1}$ Eric Jal'

1. AMOG Consulting, Notting Hill, VIC, Australia; 2. AMOG Consulting, Houston, TX, USA 
Numerical Methods for Interlink Stiffness Formulations and Parameters Sensitivity of Out-of-plane Bending Fatigue Failure in Mooring Chains OMAE2019-96042 Ceasar Edward, Arun Dev Newcastle University in Singapore, Singapore, Singapore

Fatigue of Mooring Chains Connected to Offshore Floating Structures considering Out-of-plane Bending Effects OMAE2019-96114 Vidar Hellum' Songxiong Ding' Tom Lassen ${ }^{2}$

1. University of Agder, Grimstad, Norway;2. APL/NOV, Arendal, Norway

\section{Materials Technology}

\section{3-5-1 Fatigue Assessment and Improvement}

Thursday June 13

Room SEC, Boisdale 1 | 10:30 - 12:00

Session Chair: Carol Johnston, TWI Ltd, United Kingdom

Session Co-Chair: David A. Baker, Exxon Mobil Upstream Research Co, USA

Fatigue Life Estimation for HFMI Treated Weldments considering Weld Toe Magnification Factor OMAE2019-95910 Dong Yub Kim ${ }^{1}$ Myung-Hyun Kim²

1. Dept. of Naval Architecture and Ocean Engineering; Pusan National

University, Busan, Korea; 2. Pusan National University, Busan, Korea

Girth Weld Joints from Long Upset Pipe Ends for Improving

Fatigue Strength of Offshore Oil \& Gas Pipelines OMAE2019-96345

Israel Marines-Garcia' Aaron Aguilar' Ramon Aguilar ${ }^{2}$ Mauricio Pelcastre ${ }^{3}$ Philippe Darcis ${ }^{4}$

1. Tenaris TTSA, Veracruz, VER, Mexico; 2. Testing and Technical Solutions

S.A De C.V, Veracruz, VER, Mexico; 3. Tubos de Acero de Mexico S.A,

Veracruz, VER, Mexico; 4. Dalmine S.p.A., Dalmine, Italy

Process-Structure-Property Fatigue Characterisation for

Welding of X100 Steel Catenary Risers OMAE2019-96516

Ronan J. Devaney' Adrian Connaire ${ }^{2}$ Padraic E. O'Donoghue ${ }^{\prime}$ Sean B. Leen ${ }^{1}$

1. NUI Galway, Galway, Ireland; 2. Wood, Galway, Ireland

Evolution of the Stress-induced Magnetic Field of

Pipeline Steel due to Fatigue Loading OMAE2019-95547

Sheng Bao, Zhengye Zhao, Qiang Luo, Yibin Gu

Zhejiang University, Hangzhou, China

Data Mining for Estimating Fatigue Strength based on

Composition and Process Parameters OMAE2019-95155

Arvind Keprate, R.M. Chandima Ratnayake

University of Stavanger, Stavanger, Norway

\section{Pipelines, Risers, and Subsea Systems}

\section{4-1-11 Umbilicals and Cables II}

Thursday June $13 \quad$ Room Crowne Plaza, Staffa / Shuna | 10:30-12:00

Session Chair: Alan Dobson, Technip Umbilicals, United Kingdom

Session Co-Chair: Jun Yan, Dalian University of Technology, China

Analysis of Mechanical Properties of Carbon Fiber

Reinforced Spiral Rod in Umbilical OMAE2019-95988

Yu Zhang' Peng Zhang ${ }^{2}$ Ningyi Cheng' Yi Zhao ${ }^{1}$

1. China University of Petroleum, Beijing, China; 2. Fudan University, Shanghai, China

Coupled Thermo-elastic Analysis on Cross-section of Umbilical Cables OMAE2019-96195

Jun Yan' Haitao Hu' Qi Su' Qingzhen Lu² Zhixun Yang' Qianjin Yue ${ }^{2}$

1. Dalian University of Technology, Dalian, China; 2. Dalian

University of Technology, Panjin, China
Experimental Study on Friction of Steel Wires of Dynamic Umbilical for Fatigue Life Analysis OMAE2019-96491

Yuanchao Yin ${ }^{1}$ Qingzhen Lu ${ }^{2}$ Shanghua Wu' Jun Yan ${ }^{1}$ Qianjin Yue ${ }^{2}$ Jinlong Chen ${ }^{3}$

1. Dalian University of Technology, Dalian, China; 2. Dalian University

of Technology, Panjin, China; 3. Panjin Institute of Industrial

Technology, Dalian University of Technology, Panjin, China

Analysis of Subsea Umbilical Mechanical Behavior under Simultaneous Bending, Tension and Torsion OMAE2019-96596 Mohsen Saneian' Yifan Gao ${ }^{1}$ Yong Bai' Ting Liu'

1. Zhejiang University, Hangzhou, China; 2. Zhejiang University, Zhejiang, China

\section{Pipelines, Risers, and Subsea Systems}

\section{4-3-8 Mechanics II}

Thursday June 13 Room Crowne Plaza, Castle 3 | 10:30 - 12:00

Session Chair: Doug Swanek, C-FER Technologies, Canada Session Co-Chair: Corey Drake, C-FER Technologies, Canada

Parametric Resonance Analysis of a Submerged Floating

Pipeline Considering Uncertainties OMAE2019-95266

Hezhen Yang ${ }^{1}$ Fei Xiao ${ }^{2}$

1. University of Glasgow, Glasgow, United Kingdom; 2. Nanyang

Technological University, Singapore, Singapore

Case Study: Non-conventional Remaining Life Assessment of a Significant Dent in a Subsea Pipeline Scheduled for Decommissioning OMAE2019-95277 Alex Brett, Andy Russell

Rosen UK, Newcastle upon Tyne, United Kingdom

Hydrodynamic Forces on Near-bed Small Diameter Cables and Pipes in Currents, Waves and Combined Flow OMAE2019-95557 Terry Griffiths' Yunfei Teng ${ }^{2}$ Liang Cheng ${ }^{1}$ Hongwei An ${ }^{1}$ Scott Draper ${ }^{1}$ Henning Mohr ${ }^{1}$ Antonino Fogliani' Alessio Mariani ${ }^{3}$ David J White ${ }^{4}$ 1. University of Western Australia, Crawley, WA, Australia; 2. Dalian University of Technology, Dalian, China; 3. Woodside Energy Ltd, Perth, WA, Australia; 4. University of Southampton, Southampton, United Kingdom

Out-of-roundness of the Turkstream Project Line Pipe 0MAE2019-96154 Ruud Selker ${ }^{1}$ Ping Liu' ${ }^{1}$ Erich Jurdik² Jay Chaudhuri ${ }^{2}$ 1. INTECSEA, Delft, Netherlands; 2. South Stream Transport B.V., Amsterdam, Netherlands

\section{Ocean Engineering}

\section{6-13-2 Ship Manoeuverability and Motion}

Thursday June 13 Room SEC, M2 \& M3 | 10:30 - 12:00 Session Chair: Atilla Incecik, University of Strathclyde, United Kingdom

Hybrid Method for Predicting Ship Manoeuvrability

in Regular Waves OMAE2019-95249

Tianlong Mei ${ }^{1} Y_{i} L_{i}{ }^{2}$ Manases Tello Ruiz ${ }^{3}$ Marc Vantorre ${ }^{3}$

Evert Lataire ${ }^{3}$ Changyuan Chen ${ }^{3}$ Zaojian Zou'

1. Shanghai Jiao Tong University, Shanghai, China; 2. Marine Design and Research Institute of China, Shanghai, China; 3. Ghent University, Ghent, Belgium

The Hydrodynamic Analysis of Dolphin Fluke Motion with a Flexible Tail OMAE2019-95727

Xi Chen ${ }^{1}$ Wenjing Yang ${ }^{2}$ Jiawei Y $\mathrm{u}^{2}$ Dakui Feng ${ }^{2}$ Yongfeng W ${ }^{2}$

1. Shanghai Division China Ship Development and Design Center Shanghai, Shanghai,

China; 2. Huazhong University of Science and Technology, Wuhan, China 
System Based Prediction of Ship's Manoeuverability in Varying Water Depth Area OMAE2019-95868 Shi He ${ }^{1}$ Atilla Incecik ${ }^{2}$ Zhiming Yuan ${ }^{3}$ Paula Kellett ${ }^{4}$ 1. Shanghai Maritime University, Shanghai, China; 2. University of Strathlyde, Glasgow, United Kingdom; 3. University of Strathclyde, NAOME, Glasgow, United Kingdom; 4. European Marine Board, Ostend, Belgium

Research of Wind Resistance and Flow Field

of Container Ship OMAE2019-96798

Zhiyuan Sun, Hanbing Sun, Ping Li

Harbin Engineering University, Harbin, China

\section{Ocean Engineering}

\section{6-17-2 Nonlinear and Breaking Waves}

Thursday June 13

Room SEC, Dochart 2 | 10:30 - 12:00

Session Chair: Thomas A. A. Adcock, University of Oxford, United Kingdom

The Average Shape of Large Waves in the Norwegian Sea -

Is Non-linear Physics Important? OMAE2019-95068

Tianning Tang' Margaret J. Yelland ${ }^{2}$ Thomas A. A. Adcock'

1. University of Oxford, Oxford, United Kingdom; 2. National Oceanography

Centre, Southampton |NOCS, Southampton, United Kingdom

Nonlinear Evolution of a Steep, Focusing Wave Group in Deep

Water Simulated with OceanWave3D OMAE2019-95299

Dylan Barratt ${ }^{1}$ Harry B. Bingham ${ }^{2}$ Thomas A. A. Adcock'

1. University of Oxford, Oxford, United Kingdom; 2. Delft

University of Technology, Lyngby, Denmark

Numerical Simulation of Water Wave Propagation over Porous

Slope Bottom by using Two-domain Method OMAE2019-95664

Eun-Hong Min, Weoncheol Koo

Inha University, Incheon, Korea

Characteristics of Transforming Waves Breaking

over a Fringing Reef OMAE2019-96674

Fuxian Gong ${ }^{1}$ Manhar Dhanak ${ }^{2}$

1. Florida Atlantic University, Boca Raton, FL, USA; 2. Florida

Atlantic University, Dania Beach, FL, USA

Numerical Modeling of the Nonlinear Standing Waves in a

Rectangular Tank and Damping Devices OMAE2019-96790

Xin Jin' Pengzhi Lin ${ }^{2}$

1. Chengdu University of Technology, Chengdu, China; 2. Sichuan university, Chengdu, China

\section{Ocean Engineering}

\section{6-7-2 Regional Metocean II}

Thursday June 13 Room SEC, M4 | 10:30-12:00

Session Chair: Gus Jeans, Oceanalysis Ltd, United Kingdom

Session Co-Chair: Kevin Ewans, Met0cean Research Ltd, New Zealand

The Wave Climate of the Southern Ocean OMAE2019-95168

lan Young

The University of Melbourne, Melbourne, VIC, Australia

The Increasing Prevalence of High Frequency Internal Waves in an Arctic Ocean with Declining Sea Ice Cover OMAE2019-96621

Tom Rippeth ${ }^{1}$ Vasyl Vlasenko ${ }^{2}$ Igor Kozlov ${ }^{3}$ Nataliya Stashchuk ${ }^{4}$

Brian Scannell ${ }^{5}$ Mattias Green ${ }^{1}$ Ben Lincoln ${ }^{6}$ Yueng-Djern Lenn ${ }^{7}$

1. Bangor University, Menai Bridge, United Kingdom;2. School of Biological and Marine

Sciences, Plymouth, United Kingdom; 3. Russian State Hydrometeorological University,
St Petersburg, Russia; 4. Plymouth University, Plymouth, United Kingdom; 5. School of Ocean Sciences, Ynys Mon, United Kingdom; 6. Bangor University, School of Ocean Sciences, Bangor, United Kingdom; 7. School of Ocean Sciences, Menai Bridge, United Kingdom

Extreme Wind and Wave Predictability from Operational

Forecasts at Drake Passage OMAE2019-96626

Ricardo Campos ${ }^{1}$ Andressa D'Agostini² Leandro Machado Cruz $^{2}$

Bruna Reis Leite França ${ }^{3}$ Carlos Guedes Soares ${ }^{4}$

1. CENTEC - Instituto Superior Técnico, Universidade de Lisboa, Lisbon, Portugal; 2. Centro de Hidrografia da Marinha CHM, Seção de Modelagem Oceanográfica/REMO, Marinha do Brasil, Niteroi, RJ, Brazil; 3. Oceanographic Modeling and Observation Network (REMO), Niteroi, RJ, Brazil; 4. Instituto Superior Técnico, Universidade de Lisboa, Lisboa, Portugal

Global Assessments of Surface Winds and Waves from an Ensemble Forecast System using Satellite Data OMAE2019-96627 Ricardo Campos' Carlos Guedes Soares ${ }^{2}$

1. CENTEC - Instituto Superior Técnico, Universidade de Lisboa, Lisbon, Portugal;

2. Instituto Superior Técnico, Universidade de Lisboa, Lisboa, Portugal

\section{CFD \& FSI}

\section{8-1-6 Seakeeping II}

Thursday June 13 Room SEC, Dochart 1 | 10:30 - 12:00

Session Chair: Steve Cosgrove, Altair Engineering, Inc., USA

Session Co-Chair: Samuel Holmes, Red Wing Engineering, Inc, USA

Development and Validation of CFD Analysis Procedure for

Predicting Wind Load on Commercial Ships OMAE2019-95410

Sang-Hun Lee' Sei-Hwan Kim² Deok-Su Kim ${ }^{1}$ Young-Bum Lee ${ }^{2}$

1. Daewoo Shipbuilding and Marine Engineering CO., Ltd., Geoje-si, Korea;

2. Daewoo Shipbuilding and Marine Engineering CO., Ltd., Seoul, Korea

Wind and Current Loads on Barges and Ships OMAE2019-95716 Ould el Moctar, Thomas Schellin, Jens Neugebauer University of Duisburg-Essen, Duisburg, Germany

Cargo Liquefaction and Influence on Ship Stability OMAE2019-96448 Kie Hian Chua' Yali Zhang ${ }^{2}$ Dimitrios Konovessis ${ }^{3}$

1. Technology Centre for Offshore and Marine, Singapore, Singapore;

2. Lloyd's Register Singapore Pte Ltd, Singapore, Singapore;

3. Singapore Institute of Technology, Singapore, Singapore

Numerical Study on Scale Effect of KCS OMAE2019-96831

Yujie Zhou' Liwei Liu' Xiao Cai' Dakui Feng' Bin Guo ${ }^{2}$

1. Huazhong University of Science and Technology, Wuhan, China;

2. China Ship Design and Development Center, Wuhan, China

\section{CFD \& FSI}

\section{8-4-3 Interference, Proximity and Geometry Effects}

Thursday June 13

Room SEC, Lomond Auditorium | 10:30 - 12:00

Session Chair: Rajeev Kumar Jaiman, University of British Columbia, Canada

Session Co-Chair: Mohammed Abdul Hannan, Newcastle

University, UK (singapore Campus), Singapore

Numerical Investigation on Vortex Dynamics around Vibrant Monopile Regarding Cross-sectional Shape and Keulegan-Carpenter Number OMAE2019-96827 Mohammad Mohammad Beigi Kasvaei ${ }^{1}$ Mohammad Hossein Kazeminezhad' Abbas Yeganeh-Bakhtiary ${ }^{2}$ 1. Iranian National Institute for Oceanography and Atmospheric Science, Tehran, Iran; 2. Iran University of Science and Technology, Tehran, Iran 
Numerical Investigation of Steady Flow Induced Rotary Response of Circular Cylinder with Splitter Plate OMAE2019-95584

Dan Pang, Huili Xi, Zhongbing Zhou, Guoqiang Tang, Lin Lu

Dalian University of Technology, Dalian, China

The Evolutionary Geometric Physics of

Vortex-induced Vibrations OMAE2019-95548

Robert Zueck

US Navy-NAVFACEXWC, Port Hueneme, CA, USA

Three-Dimensional Direct Numerical Simulations of Flows Past an Inclined Cylinder Near a Plane Boundary OMAE2019-95466

Chunning Ji' Zhimeng Zhang ${ }^{1}$ Dong X $\mathrm{u}^{1}$ Narakorn Srinil ${ }^{2}$

1. Tianjin University, Tianjin, China; 2. Newcastle University,

Newcastle upon Tyne, United Kingdom

\section{Ocean Renewable Energy}

\section{9-1-8 FWT Hydrodynamics I}

Thursday June 13

Room SEC, Carron 1 | 10:30 - 12:00

Session Chair: Jean-Christophe Gilloteaux, Ecole Centrale de Nantes, France

Investigation of Focused Wave Impact on Floating Platform for Offshore Floating Wind Turbine - A CFD Study OMAE2019-96043

Yang Zhou' Qing Xiao' Yuanchuan Liu' Atilla Incecik' Christophe Peyrard²

1. University of Strathclyde, Glasgow, United Kingdom;2. EDF R\&D, Chatou, France

Development of a Panel Cutting Method Coupled with a Time-domain Potential Flow Model based on the Weak-scatterer Approximation OMAE2019-96296 Pierre-Yves Wuillaume ${ }^{1}$ Aurelien Babarit ${ }^{2}$ Mattias Lynch ${ }^{3}$ Pierre Ferrant ${ }^{4}$ 1. LHEEA Ecole Centrale de Nantes /INNOSEA, Nantes, France; 2. Centrale Nantes, Nantes, France; 3. INNOSEA, Nantes, France; 4. Ecole Centrale de Nantes/CNRS, Nantes, France

Hydrodynamic Analysis of a Suspended Cylinder under Regular Wave Loading based on Computational Fluid Dynamics OMAE2019-95533 Philipp Mucha, Amy Robertson, Jason Jonkman, Fabian Wendt National Renewable Energy Laboratory, Golden, CO, USA

On Motion and Hydroelastic Analysis of a Floating Offshore Wind Turbine OMAE2019-96034

Azin Lamei' Masoud Hayatdavoodi' Carlos Wong² Bin Tang ${ }^{3}$

1. University of Dundee, Dundee, United Kingdom; 2. CBJ Ocean Engineering

Corp., Zhuhai, China; 3. Harbin Engineering University, Harbin, China

\section{Ocean Renewable Energy}

\section{9-4-3 Advanced Controls}

Thursday June 13

Room SEC, Carron 2 | 10:30 - 12:00

Session Chair: Ryan Coe, Sandia National Laboratories, USA

Study on a Wave Energy Converter with Tension Leg

Mooring under Optimal Control OMAE2019-95650

Jun Umeda ${ }^{1}$ Tomoki Taniguchi ${ }^{2}$ Toshifumi Fujiwara ${ }^{2}$

1. National Maritime Research Institute, Mitaka, Japan;

2. National Maritime Research Institute, Tokyo, Japan

Assessment of Latching Control of Hemispherical Heaving Buoy

Point Absorber with Nonlinear Froude-Krylov Force OMAE2019-96055

Sung-Jae Kim, Weoncheol Koo, Chul Hee Jo

Inha University, Incheon, Korea
Load Reduction for a WEC via PTO Control OMAE2019-96382

Ryan Coe, Giorgio Bacelli, Steven J. Spencer, Hancheol Cho, Victor Nevarez Sandia National Laboratories, Albuquerque, NM, USA

Modeling and Analysis of Nonlinear Effects of Ocean Waves and Power-take-off Control on Wave Energy Conversion System Dynamics OMAE2019-96802 Solomon Yim ${ }^{1}$ Nasim Adami' Bret Bosma' Ted Brekken ${ }^{1}$ Ming Chen ${ }^{1}$ Leila Ghorban Zadeh ${ }^{1}$ David Glennon ${ }^{1}$ Yushun Lian' Pedro Lomonaco ${ }^{2}$ Ali Mohtat' Tuba Ozkan-Haller' Jim Thomson ${ }^{3}$

1. Oregon State University, Corvallis, OR, USA; 2. O.H. Hinsdale Wave Research Laboratory, Corvallis, OR, USA; 3. University of Washington, Seattle, WA, USA

\section{Petroleum Technology}

\section{1-1-2 General Petroleum Technology - Production and Drilling Enhancement}

Thursday June 13

Room Crowne Plaza, Jura | 10:30 - 12:00

Session Chair: Stephen Butt, Memorial University of Newfoundland, Canada Session Co-Chair: Mohammad Rahman, Texas A\&M University at Qatar, Qatar

Evaluation Adaptability of Nano Wetting Fluid for Releasing Tight Sandstone Gas Reservoir Water Locking Effect OMAE2019-95402 Erdong Yao ${ }^{1}$ Jie Wang ${ }^{1}$ Yanpeng Xue ${ }^{2}$ Fujian Zhou' ${ }^{1}$ Le Zhang ${ }^{1}$ Yafei Li' 1. China University of Petroleum-Beijing, Beijing, China; 2. Tarim Oilfield, CNPC, Korla, China

Study of the influence of Controlled Axial Oscillations of pVARD on Generating Downhole Dynamic WOB and Improving Coring and Drilling Performance in Shale OMAE2019-96189 Abdelsalam Abugharara, John Molgaard, Charles Hurich, Stephen Butt Memorial University of Newfoundland, St. John's, NL, Canada

An Experimental Development to Characterise the Flow Phenomena at the Near-wellbore Region OMAE2019-96373 Mohammad Ahammad' Mohammad Rahman' ${ }^{2}$ Stephen Butt' Jahrul Alam 1. Memorial University of Newfoundland, St. John's, NL, Canada;

2. Texas A\&M University at Qatar, Doha, Qatar

Understanding the Phenomenon of Dissolved Gas Migration of Gas in Riser during Drilling Operations OMAE2019-96683 Syed Y. Nahri ${ }^{1}$ Jianjun Zhu ${ }^{2}$ Wesley Williams ${ }^{1} 0$ tto Santos ${ }^{1}$ Louis Thibodeaux' ${ }^{1}$ Yuanhang Chen

1. Louisiana State University, Baton Rouge, LA, USA;2. University of Tulsa, Tulsa, OK, USA

\section{Petroleum Technology}

\section{1-10-1 New Materials for Well Construction}

Thursday June 13 Room Crowne Plaza, Barra | 10:30 - 12:00 Session Chair: Mihail Minescu, University Oil And Gas Ploiesti, Romania Session Co-Chair: Catalin Teodoriu, Mewbourne School of Petroleum and Geological Engineering, Norman, OK, USA

Potentials of Nano-designed Plugs: Implications for Short and Long Term Well Integrity OMAE2019-95614 Raymos Kimanzi ${ }^{1}$ Harshkumar Patel' Mahmoud Khalifeh ${ }^{2}$ Saeed Salehi ${ }^{3}$ Catalin Teodoriu ${ }^{3}$ 1. University of Oklahoma, Norman, OK, USA; 2. UiS, Stavanger, Norway;

3. Mewbourne School of Petroleum and Geological Engineering, Norman, OK, USA

Efficiency Assessment of the Composite Materials Repair Systems Intended for Corrosion Damaged Pipelines OMAE2019-96279 Andrei Dumitrescu, Alin Dinita

Petroleum-Gas University of Ploiesti, Ploiesti, Romania 
Assessment of Variations in the Physico-mechanical Properties of Fiberglass Tubing Working in Different Environments OMAE2019-96283 Alin Dinita ${ }^{1}$ Mihail Minescu ${ }^{2}$ Andrei Dumitrescu ${ }^{1}$ Catalin Teodoriu ${ }^{3}$ Codrut-Stefan Sararu ${ }^{4}$ 1. Petroleum-Gas University of Ploiesti, Ploiesti, Romania; 2. University Oil And Gas Ploiesti, Ploiesti, Romania; 3. Mewbourne School of Petroleum and Geological Engineering, Norman, OK, USA; 4. OMV Petrom SA, Bucharest, Romania

How Heuristics and Biases Impact Judgment and Decision Making in Well Integrity Operations OMAE2019-96820 Catalin Teodoriu, Saeed Salehi

Mewbourne School of Petroleum and Geological Engineering, Norman, OK, USA

\section{Takeshi Kinoshita Honoring Symposium on Offshore Technology}

\section{3-3-4 Wave Energy II}

Thursday June 13

Room SEC, Boisdale 2 | 10:30 - 12:00

Session Chair: Yasunori Nihei,

Osaka Prefecture University, Japan

\begin{abstract}
A Basic Study on Influence of Airchamber Volume on OWC Models to Power Conversion Performance OMAE2019-95925 Tomoki Ikoma ' Yoshiyuki Kihara' Shota Hirai' Yasuhiro Aidaa Koichi Masuda' Hiroaki Eto ${ }^{1}$ 1. Nihon University, Funabashi, Japan; 2. Nihon University, Chiba, Japan
\end{abstract}

Numerical Modelling of a Relatively Small Floating Body's Wave and Low Frequency Motion Response, Compared with Observational Data OMAE2019-96443 Christopher Wright ${ }^{1}$ Haruki Yoshimoto ${ }^{2}$ Ryota Wada ${ }^{3}$ Ken Takagi ${ }^{3}$ 1. The University of Tokyo, Chiba, Japan; 2. Japan Marine United Corporation, Yokohama, Japan; 3. The University of Tokyo, Kashiwa, Japan

Observation of Waves in Naru Strait, Goto, Nagasaki, a Tidal Current Test Site 0MAE2019-96652

Yusaku Kyozuka

Nagasaki University, Nagasaki, Japan

\section{Technical Session Organizers' Lunch 12:00 - 13:30 \\ Location: Hall 5 (SEC)}

Thursday lunch sponsored by Greater Fort Lauderdale Convention \& Visitors Bureau

\section{GREATER FORT LAUDERDALE} CONVENTION \& VISITORS BUREAU

\section{CONCURRENT SESSIONS \\ 13:30 - 15:00}

\section{Offshore Technology}

\section{1-7-2 Wave Loading and Motions in Extreme Seas II}

Thursday June 13

Room SEC, Alsh 1 | 13:30 - 15:00

Session Chair: Babak 0mmani, SINTEF Ocean, Norway

Wave Impact Load and Corresponding Nonlinear

Response of a Semi-submersible OMAE2019-95693

Yinghao Guo ${ }^{1}$ Longfei Xiao ${ }^{1}$ Handi Wei ${ }^{2}$ Lei Li ${ }^{3}$ Yanfei Deng $^{3}$

1. Shanghai Jiao Tong University, Shanghai, China; 2. State Key

Laboratory of Ocean Engineering, Shanghai Jiao Tong University,

Shanghai, China; 3. CIMC Offshore Co. Ltd., Shenzhen, China

The Ocean Cleanup System 001 Performance during

Towing and Seakeeping Tests OMAE2019-96207

Joost Sterenborg ${ }^{1}$ Nicola Grasso' Rogier Schouten ${ }^{1}$ Arjen Tjallema ${ }^{2}$

1. MARIN, Wageningen, Netherlands; 2. The Ocean Cleanup, Rotterdam, Netherlands

\section{Structures, Safety and Reliability}

\section{2-14-1 Risk Based Maintenance}

Thursday June 13

Room Crowne Plaza, Castle 2 | 13:30 - 15:00 Session Chair: Bernt Leira, Norwegian University of Science and Technology, Norway Session Co-Chair: Nianzhong Chen, Tianjin University, China

New Advances and Developments in Risk-based Inspection (RBI) of Marine Structures OMAE2019-95137 Mahmood Shafiee' Carlos Guedes Soares ${ }^{2}$

1. Cranfield University, Bedford, United Kingdom; 2. Instituto Superior Técnico, Universidade de Lisboa, Lisboa, Portugal

An Ontology-based Approach for Developing Offshore and Onshore Process Equipment Inspection Knowledge Base OMAE2019-95478 Andika Rachman, R.M. Chandima Ratnayake University of Stavanger, Stavanger, Norway

Asset Integrity Control: Prioritization of Offshore Topside Flange Openings during Preventive Maintenance Shutdowns OMAE2019-96576 R.M. Chandima Ratnayake

University of Stavanger, Stavanger, Norway

Bayesian Networks for Risk-based Decision Making 0MAE2019-96833 Sima Rastayesh

Aalborg University, Aalborg, Denmark

\section{Structures, Safety and Reliability}

\section{2-7-1 Reliability of Renewable Energy Systems}

Thursday June 13

Room Crowne Plaza, Castle 1 | 13:30 - 15:00

Session Chair: Zhen Gao, Norwegian University of Science and Technology, Norway Session Co-Chair: Athanasios Kolios, University of Strathclyde, United Kingdom

Sensitivity Analysis of a $5 \mathrm{MW}$ Bottom Fixed Offshore Wind Turbine using the Environmental Contour Method OMAE2019-95390 David Barreto ${ }^{1}$ Abdolmajid Moghtadaei ${ }^{2}$ Madjid Karimirad ${ }^{2}$ Arturo Ortega ${ }^{3}$ 1. Universidad Nacional de Ingeniería, Lima, Peru; 2. Queen's University Belfast, Belfast, United Kingdom; 3. The University of Edinburgh, Edinburgh, United Kingdom 
Impacts of Reliability on Operational Performance and Cost of Energy Evaluation of Multimegawatt, Far-offshore Wind Turbines OMAE2019-95561 Cuong Dao, Behzad Kazemtabrizi, Christopher Crabtree Durham University, Durham, United Kingdom

Structural Safety Assessment of Marine Operations from a Long-term Perspective - A Case Study of Offshore Wind Turbine Blade Installation OMAE2019-96686 Amrit Shankar Verma ${ }^{1}$ Zhen Gao ${ }^{1}$ Zhiyu Jiang ${ }^{2}$ Zhengru Ren ${ }^{1}$ Nils Petter Vedvik ${ }^{1}$ 1. Norwegian University of Science and Technology, Trondheim, Norway; 2. University of Agder, Grimstad, Norway

A Sensitivity Study for Operational Availability of Offshore Wind Energy Assets OMAE2019-96323 Juan Chiachio-Ruano, Mahé Hermile, Athanasios Kolios University of Strathclyde, Glasgow, United Kingdom

\section{Materials Technology}

\section{3-2-2 Analysis and Fatigue Performance of Tubular Joints (John Sharp Honorary Session)}

Thursday June 13

Room SEC, Boisdale 1 | 13:30 - 15:00

Session Chair: Alexander Stacey, Energy Division,

Health \& Safety Executive, United Kingdom

Session Co-Chair: Carol Johnston, TWI Ltd, United Kingdom

Large-scale Resonant Fatigue Testing of Welded Tubular

X-Joints for Offshore Jacket Foundations OMAE2019-96371

Jeroen Van Wittenberghe, Philippe Thibaux, Maarten Van Poucke

OCASNV, Gent, Belgium

Fatigue of Welded Tubular X-Joints in Offshore

Wind Platforms OMAE2019-95812

Theocharis Papatheocharis, Gregory C. Sarvanis, Philip C. Perdikaris, Spyros A. Karamanos University of Thessaly, Volos, Greece

Numerical Simulation and Result Interpretation of Large Scale Fatigue Testing of Tubular X-Joint Close to Resonance Frequency OMAE2019-96198 Philippe Thibaux, Jeroen Van Wittenberghe, Maarten Van Poucke OCASNV, Gent, Belgium

Study of Grouted Connections in Offshore Structures OMAE2019-95446 Efstathios Theotokoglou, Georgia Papaefthimiou National Technical University of Athens, Athens, Greece

\section{Pipelines, Risers, and Subsea Systems}

\section{4-4-1 Subsea Structures I}

Thursday June 13

Room Crowne Plaza, Staffa / Shuna | 13:30-15:00

Session Chair: Marcelo Igor Lourenço Souza, UFRJ, Brazil

Session Co-Chair: Ping Liu, INTECSEA, Netherlands

Challenges and Lessons Learnt from the Design, Fabrication and Installation of Rigid Tie-in Spools OMAE2019-95056

Curti Gianbattista' Pavone Diego ${ }^{1}$ Pirinu Gianluigi ${ }^{2}$ Qin Jianjun ${ }^{3}$

1. Saipem, Fano, Italy; 2. Saipem, Saint Quentin Yvelines, France;

3. Saipem, Kingston-upon-Thames, United Kingdom

Experimental Investigation of Over-trawlability of an Innovative Arctic Subsea Production Unit (SPU) OMAE2019-95503

Jie Wu' Chittiappa Muthanna' Hagbart Alsos' Rasmus Juhlin² Daniel Karunakaran²

1. SINTEF Ocean, Trondheim, Norway; 2. Subsea7, Stavanger, Norway
Subsea Rigid Jumper Design Optimization for

Sour Service Application 0MAE2019-95536

Ben Toleman ${ }^{1}$ Chaojun Huang ${ }^{1}$ Mahesh Sonawane ${ }^{1}$ Rohit Shankaran ${ }^{2}$ Danny Foster ${ }^{3}$ 1. 2 H Offshore Inc., Houston, TX, USA; 2. 2H Offshore Engineering Ltd.,

Woking, United Kingdom; 3. OneSubsea, Houston, TX, USA

A Study on the Validity of a One Degree of Freedom

Model for Deepwater Subsea Lifts OMAE2019-96047

Rodrigo Batista Tommasini' Leonardo de Oliveira Carvalho ${ }^{2}$ Renato Pavanello ${ }^{1}$

1. Unicamp, Campinas, SP, Brazil; 2. Petrobras, Rio de Janeiro, RJ, Brazil

\section{Pipelines, Risers, and Subsea Systems}

\section{4-6-1 Innovative Technologies for Deepwater Low-Cost Production I}

Thursday June 13

Room Crowne Plaza, Castle 3 | 13:30 - 15:00

Session Chair: Chris Timms, C-FER Technologies, Canada

Session Co-Chair: Ruud Selker, INTECSEA, Netherlands

Full Scale Thermal Testing of a New Flowline

Intervention System OMAE2019-95354

Stéphanie Harchambois ${ }^{1}$ Vincent Le Toux' Geoffrey Guindeuil ${ }^{1}$

Romain Vivet ${ }^{1}$ François-Xavier Pasquet ${ }^{2}$ Guillaume Tosi ${ }^{2}$

Thierry Palermo ${ }^{2}$ Christophe T'Joen ${ }^{3}$ Gilbert Herrera ${ }^{4}$

1. TechnipFMC, Rueil-Malmaison, France; 2. Total, Pau, France;

3. Shell, Riiswijk, Netherlands; 4. Shell, Houston, TX, USA

CFD Modelling of an Electrically Trace Heated Blanket OMAE2019-95493

Vincent Le Toux ${ }^{1}$ Stéphanie Harchambois ${ }^{2}$ Geoffrey Guindeuil'

Romain Vivet' François-Xavier Pasquet ${ }^{3}$ Guillaume Tosi ${ }^{3}$

Thierry Palermo $0^{3}$ Gilbert Herrera ${ }^{4}$ Christophe $\mathrm{T}^{\prime} \mathrm{Joen}^{5}$

1. TechnipFMC, Rueil-Malmaison, France; 2. TechnipFMC, Rueil Malmaison, France;

3. Total, Pau, France; 4. Shell, Houston, TX, USA; 5. Shell, Rijswijk, Netherlands

Active Control of Flexible Riser Vibration by Boundary

Control based on LQR Controller OMAE2019-95839

Jin Xin Yu' Weimin Chen ${ }^{2}$

1. Institute of Seismology, China Earthquake Administratio, Wuhan, China;

2. Institute of Mechanics, Chinese Academy of Sciences, Beijing, China

Hydrate Remediation Philosophy for a New Flowline

Intervention System based on Active Heating OMAE2019-96059

Geoffrey Guindeuil' Arnaud Sanchis' ${ }^{2}$ Stéphanie Harchambois ${ }^{1}$ Romain Vivet ${ }^{1}$

Thierry Palermo $0^{3}$ François-Xavier Pasquet ${ }^{3}$ Guillaume Tosi ${ }^{3}$

1. TechnipFMC, Rueil-Malmaison, France; 2. TechnipFMC, Lysaker, Norway; 3. Total, Pau, France

\section{Ocean Engineering}

\section{6-13-3 Numerical Methods}

Thursday June 13

Room SEC, M2 \& M3 | 13:30 - 15:00

Session Chair: D.C. Wan, Shanghai Jiao Tong University, China

RANS Based Resistance Prediction for Tumblehome Hull with Different Bow Appendages in Calm Water OMAE2019-95449

Shuzheng Sun, Xin Zhao

Harbin Engineering University, Harbin, China

CFD Simulation of a Twin-screw Ship Self-propulsion

using DDES-Overset Method OMAE2019-95527

Jianhua Wang, D.C. Wan

Shanghai Jiao Tong University, Shanghai, China 


\begin{abstract}
A Numerical Method for Calculation of Ship-Ship Hydrodynamics Interaction in Shallow Water Accounting for Sinkage and Trim OMAE2019-96151 Huilong Ren' Chen Xu' Xueqian Zhou' Serge Sutulo ${ }^{2}$ Carlos Guedes Soares 1. Harbin Engineering University, Harbin, China; 2. Lisbon University, Lisbon, Portugal; 3. Instituto Superior Técnico, Universidade de Lisboa, Lisboa, Portugal
\end{abstract}

Computations of Hydrodynamic Forces on Vessels Advancing in Waves by Four-node Higher-order Boundary Element Method OMAE2019-96792 Yuntao Yang, Renchuan Zhu, Shan Huang

Shanghai Jiao tong University, Shanghai, China

Improved Transient FSI Model in SPH Method

and its Applications OMAE2019-95257

Aman Zhang' Pingping Wang' Furen Ming ${ }^{1}$ Pengnan Sun ${ }^{2}$

1. Harbin Engineering University, Harbin, China; 2. Ecole Centrale Nantes, Nantes, France

\section{Ocean Engineering}

\section{6-7-3 Metocean Criteria I}

Thursday June 13

Room SEC, M4 | 13:30-15:00

Session Chair: Gus Jeans, Oceanalysis Ltd, United Kingdom

Session Co-Chair: Kevin Ewans, Met0cean Research Ltd, New Zealand

Including the Impact of Climate Change in Offshore and Onshore Metocean Design Criteria to Ensure Asset Robustness OMAE2019-95205 Alison Brown' Ag Stephens ${ }^{2}$ Ben Rabb ${ }^{3}$ Richenda Connell ${ }^{4}$ Jon Upton ${ }^{5}$ 1. Shell Research Ltd, Aberdeen, United Kingdom;2. STFC Centre for Environmental Data Analysi, Didcot, United Kingdom; 3. Acclimatise Group Ltd, Cardiff, United Kingdom; 4. Acclimatise Group Ltd, Oxford, United Kingdom; 5. Shell Research Ltd., Aberdeen, United Kingdom

Estimating Operational Weather Downtime:

A Comparison of Analytical Methods 0MAE2019-95367

David Lambkin, lan Wade, Robin Stephens

ABPmer, Southampton, United Kingdom

Decisional Criteria for Offshore Operations Interruption due to Adverse Weather OMAE2019-96086

Michele Drago ${ }^{1}$ Luigino Vitali' ${ }^{1}$ Andrea Del Guzzo ${ }^{2}$ Federico Gaggiotti ${ }^{1}$

1. Saipem S.p.A., Fano, Italy; 2. Saipem, Kuala Lumpur, Malaysia

A New Method for Deriving Soliton Design Criteria 0MAE2019-96637 Gus Jeans ${ }^{1}$ Oliver Jones ${ }^{2}$ Michael Zhang ${ }^{3}$ Chris Jackson ${ }^{4}$ Nataliya Stashchuk ${ }^{5}$ Alfred R. Osborne ${ }^{6}$ Ole Petersen ${ }^{7}$ Jose da Silva ${ }^{8}$

1. Oceanalysis Ltd, Wallingford, United Kingdom;2. BP, Sunbury-on-Thames, United Kingdom; 3. BP America Inc, Houston, TX, USA; 4. Global Ocean Associates, Alexandria, VA, USA; 5. Independent Author, Plymouth, United Kingdom; 6. Nonlinear Wave Research Corporation Alexandria, VA, USA; 7. DHI, Horsholm, Denmark; 8. University of Oporto, Oporto, Portugal

\section{CFD \& FSI}

\section{8-1-7 Propulsion}

Thursday June 13

Room SEC, Dochart 1 | 13:30 - 15:00 Session Chair: Karl Halse, Norwegian University of Science and Technology, Norway Session Co-Chair: Samuel Holmes, Red Wing Engineering, Inc, USA

An Investigation into the Effect of Biofouling on Full-scale

Propeller Performance using CFD OMAE2019-95315

Soonseok Song, Yigit Demirel, Mehmet Atlar

University of Strathclyde, Glasgow, United Kingdom
Research on The Blade Element Theory Coupled with Viscous Flow OMAE2019-95887

Zhiheng Li' Jiawei Yu ${ }^{2}$ Dakui Feng ${ }^{2}$ Kaijun Jiang ${ }^{2}$ Yujie Zhou ${ }^{2}$ 1. China Ship Design and Development Center, Wuhan, China;

2. Huazhong University of Science and Technology, Wuhan, China

Numerical Simulation of Submarine Self-propulsion based on Different Turbulent Simulation Models OMAE2019-95874 Tiechao Bai ${ }^{1}$ Yongfeng Wu ${ }^{2}$ Peng Wei ${ }^{2}$ Shuang Wang ${ }^{2}$ Liwei Liu $^{2}$ 1. China Ship Design and Development Center, Wuhan, China; 2. Huazhong University of Science and Technology, Wuhan, China

CFD Study of Propeller Cavitation with Hull-propeller Interaction 0MAE2019-95892

Chang Wei Kang, Xiuqing Xing

Institute of High Performance, A*STAR, Singapore, Singapore

\section{CFD \& FSI}

\section{8-4-4 VIV Suppression and Control}

Thursday June 13 Room SEC, Lomond Auditorium | 13:30 - 15:00 Session Chair: Decao Yin, SINTEF Ocean, Norway Session Co-Chair: Madhusuden Agrawal, BP, USA

Forced Vibration Tests for In-line VIV to Assess Partially Strake-covered Pipeline Spans OMAE2019-95970 Jie Wu' Decao Yin' Elizabeth Passano ${ }^{1}$ Halvor Lie' Ralf Peek ${ }^{2}$ Octavio Sequeiros ${ }^{3}$ Sze Yu Ang ${ }^{3}$ Chiara A. Bernardo ${ }^{4}$ Meliza Atienza ${ }^{4}$ 1. SINTEF Ocean, Trondheim, Norway; 2. Peek Solutions, St. Andreu de Llavaneres, Spain; 3. Shell Global Solutions International B.V., Rijswijk, Netherlands; 4. Shell Philippines Exploration B.V., Manila, Philippines

Laboratory Investigation of Helical Strakes with Serrated and Twisted Fins to Suppress VIV OMAE2019-95129

Gustavo R. S. Assi, Tommaso Crespi University of São Paulo, São Paulo, Brazil

Staggered Grooves for the Suppression of Vortex-induced Vibration in Flexible Cylinders OMAE2019-95649 Yun Zhi Law' Rajeev Kumar Jaiman ${ }^{2}$

1. National University of Singapore, Singapore, Singapore; 2. University of British Columbia, Vancouver, BC, Canada

The Investigation of a Circular Cylinder with a Detached Flexible Plate using Immersed Smoothed Point Interpolation Method OMAE2019-95610 Boqian Yan ${ }^{1}$ Shuangqiang Wang ${ }^{1}$ Guiyong Zhang ${ }^{2}$ Qihang Xiao Peng Wang ${ }^{1}$ 1. School of Naval Architecture, Dalian University of Technology, Dalian, China; 2. Dalian University of Technology, Dalian, China

\section{Ocean Renewable Energy}

\section{9-4-1 Power Take-offs and Experiments}

Thursday June 13 Room SEC, Carron 2 | 13:30 - 15:00 Session Chair: Masoud Hayatdavoodi, University of Dundee, United Kingdom Session Co-Chair: Jerica Nolte, Principle Power, France

Design and Construction of a 1/15th Scale Wave Tank Model of the Azura Commercial Wave Energy Converter OMAE2019-95538 Bradley A. Ling' Terry Lettenmaier ${ }^{2}$ Matthew Fowler ${ }^{3}$

Matthew Cameron ${ }^{3}$ Anthony M. Viselli ${ }^{3}$

1. Northwest Energy Innovations, Portland, OR, USA; 2. Williwaw Engineering, South Beach, OR, USA; 3. UMaine Advanced Structures and Composites Center, Orono, ME, USA 
Power Take-off Selection for a U-Shaped OWC

Wave Energy Converter OMAE2019-96368

Alessandra Romolo ${ }^{1}$ Joao C.C. Henriques ${ }^{2}$ Luis Gato ${ }^{3}$ Giovanni Malara ${ }^{1}$ Valentina Laface Rui P. F. Gomes ${ }^{2}$ Juan C. C. Portillo ${ }^{3}$ Antonio F.0. Falcão ${ }^{2}$ Felice Arena ${ }^{1}$

1. University Mediterranea, Reggio Calabria, Italy; 2. IDMEC, Instituto

Superior Técnico, Universidade de Lisboa, Lisboa, Portugal; 3. Instituto

Superior Técnico, Universidade de Lisboa, Lisboa, Portugal

A Critical Examination of the Hysteresis in Wells Turbines using CFD and Lumped Parameter Models OMAE2019-96518

Tiziano Ghisu ${ }^{1}$ Francesco Cambuli2 Pierpaolo Puddu ${ }^{1}$ Irene Virdis ${ }^{1}$ Mario Carta ${ }^{1}$ Fabio Licheri ${ }^{1}$ 1. University of Cagliari, Cagliari, Italy; 2. University of Cagliari, DIMCM, Cagliari, Italy

Experimental Study on Coupled Motions of a Spar-buoy

under Mathieu Instability OMAE2019-95937

Toshio Iseki, Peng Xu

Tokyo University of Marine Science and Technology, Tokyo, Japan

\section{Ocean Renewable Energy}

\section{9-5-4 Numerical Analysis II}

Thursday June 13

Room SEC, Carron 1 | 13:30 - 15:00

Session Chair: Madjid Karimirad, Queen's University Belfast, United Kingdom

Session Co-Chair: Milad Armin, Liverpool John Moores University, United Kingdom

Investigation of the Flow around a Tidal Stream Turbine OMAE2019-95722 Hassan el Sheshtawy, Ould el Moctar, Thomas Schellin, Satish Natarajan University of Duisburg-Essen, Duisburg, Germany

The Influence of Tidal Unsteadiness to a Tidal Turbine

Blade Flow-induced Vibration OMAE2019-96007

Nu Rhahida Arini' Stephen Turnock Mingyi Tan²

1. University of Exeter, Penryn, United Kingdom;

2. University of Southampton, Southampton, United Kingdom

An Actuator Disc Analysis of a Ducted High-solidity

Tidal Turbine in Yawed Flow OMAE2019-96014

Mitchell Borg' Qing Xia0 ${ }^{1}$ Atilla Incecik' Steven Allsop ${ }^{2}$ Christophe Peyrard ${ }^{3}$

1. University of Strathclyde, Glasgow, United Kingdom;

2. Electricite de France, Chatou, France; 3. EDF R\&D, Chatou, France

Hydrodynamic Performance of a Current Energy

Generator based on WIG OMAE2019-96378

Wang Jian, Guanghua He, Mo Weijie, Zhang Shijun, Jiangtao Man

Harbin Institute of Technology, Weihai, China

\section{Petroleum Technology}

\section{1-1-3 General Petroleum Technology - Drilling and Separation Enhancement}

Thursday June 13

Room Crowne Plaza, Jura | 13:30-15:00

Session Chair: Stephen Butt, Memorial University of Newfoundland, Canada

Session Co-Chair: Mohammad Rahman, Texas A\&M University at Qatar, Qatar

Study of the Relationship between Oriented Downhole

Dynamic Weight on Bit and Drilling Parameters in Coring

Isotropic Natural and Synthetic Rocks OMAE2019-96176

Abdelsalam Abugharara, John Molgaard, Charles Hurich, Stephen Butt

Memorial University of Newfoundland, St. John's, NL, Canada
Operational Behaviour of Supersonic Separators for Real Gas Mixtures of Methane and Carbon Dioxide, from the Homogeneous Nucleation Point of View OMAE2019-96315 Julian Restrepo ${ }^{1}$ Jose R. Simões-Moreira ${ }^{2}$

1. Universidade de São Paulo, São Paulo, DF, Brazil; 2. Universidade de São Paulo - SISEA Alternative Energy Systems Laboratory, São Paulo, SP, Brazil

Triaxial Testing of Gas Shale Permeability Dependence on Heterogeneous Stress with Respect to Bedding 0MAE2019-96707 Yufei Chen' Changbao Jiang' Guangzhi Yin' Andrew Wojtanowicz' Dongming Zhang' 1. Chongging University, Chongqing, China; 2. Louisiana State University, Baton Rouge, LA, USA

Improvement of the Method for Calculating Downhole Weight on Bit and its Application in Autodriller Systems OMAE2019-96784 Zebing Wu' Longlong Guo ${ }^{1}$ Shuai Zhang ${ }^{1}$ Yuanping Wu' Lantao Lv' Wenjuan Wang ${ }^{1}$ Yujie Pan' ${ }^{1}$ Yongyong Wang ${ }^{1}$ Adnane El Mokhtari' 1. Xi'an Shiyou University, Xi'an, China; 2. Drilling Research Institute of CNPC, Jingzhou, China

\section{Petroleum Technology}

\section{1-11-1 Innovations in Drilling, Production and Transport}

Thursday June 13 Room Crowne Plaza, Barra | 13:30 - 15:00 Session Chair: Ming Feng, Chongqing University, China Session Co-Chair: wenting Qin, China University of Petroleum Beijing, China

Optimizing Well Locations in Green Fields using Fast Marching Method: Optimize Well Locations for Millions of Cells using Hundreds of Scenarios and Realizations with High Accuracy in Seconds OMAE2019-95190 Abdulaziz AlQasim Mohan Kelkar ${ }^{2}$

1. Saudi Aramco, Dhahran, Saudi Arabia; 2. University of Tulsa, Tulsa, OK, USA

Architectures and Algorithms for a Smart Drilling Robot OMAE2019-95486 Suranga Geekiyanage ${ }^{1}$ Erik Andreas Løken ${ }^{2}$ Dan Sui' Tomasz Wiktorski' 1. University of Stavanger, Stavanger, Norway; 2. University of Stavanger, Tananger, Norway

Feature Analysis and Design for Kick Detection with Machine Learning using Laboratory Scale Rig Data OMAE2019-95496

Suranga Geekiyanage ${ }^{1}$ Adrian Ambrus ${ }^{2}$ Dan Sui ${ }^{1}$

1. University of Stavanger, Stavanger, Norway; 2. NORCE, Stavanger, Norway

The Experimental Investigation of Completion Fluid Working as a Good Thermal Insulation in the Deepwater Production Well OMAE2019-95725

Ming Feng' Boyun Guo ${ }^{2}$

1. Chongqing University, Chongqing, China;

2. University of Louisiana at Lafayette, Lafayette, LA, USA

\section{Takeshi Kinoshita Honoring} Symposium on Offshore Technology

\section{3-3-3 Ocean Current Energy, OTEC and Related Technology}

Thursday June 13 Room SEC, Boisdale 2 | 13:30-15:00 Session Organizer: Yusaku Kyozuka, Nagasaki University, Japan Session Co-Chair: Motohiko Murai, Yokohama National University, Japan

Optimization of a Horizontal Axis Tidal Current Turbine by Multi-objective Optimization OMAE2019-95829

Takumi Nagataki, Ko Kurokawa, Reiko Yamada, Daisaku Sakaguchi, Yusaku Kyozuka Nagasaki University, Nagasaki, Japan 
Stability Analysis of Free Hanging Riser Conveying Fluid for Ocean Thermal Energy Conversion (OTEC) Utilization OMAE2019-96749 Ristiyanto Adiputra, Tomoaki Utsunomiya

Kyushu University, Fukuoka, Japan

Numerical Estimation of Multiple Positions of Seepage of Dissolved Matter from Seafloor OMAE2019-95733 Shunsuke Kanao ${ }^{1}$ Toru Sato ${ }^{2}$

1. The University of Tokyo, Nagareyama, Japan; 2. The University of Tokyo, Kashiwa, Japan

BioFREE: A Novel System for Monitoring Biofouling and Testing Antifouling Coatings in High-energy Habitats used by the Offshore Renewable Energy Industry OMAE2019-96611 Andrew Want, Joanne Porter

Heriot-Watt University, Stromness, United Kingdom

\section{REFRESHMENT BREAK}

15:00 - 15:30

Location: Hall 5 (SEC)

\section{CONCURRENT SESSIONS}

$$
15: 30-17: 30
$$

\section{Offshore Technology}

\section{1-4-1 Experimental Design and Analysis}

Thursday June 13

Room SEC, Alsh 1 | 15:30 - 17:30

Session Chair: M A Hannan, Newcastle University, UK (Singapore Unit), Singapore

Session Co-Chair: David Molyneux, Memorial University of Newfoundland, Canada

Numerical Solutions and Model Test Design for

Anti-typhoon Drilling Riser OMAE2019-95196

Jinlong Wang ${ }^{1}$ Lihui Li' Frank Lim¹ Zhang Hui' Xu Liangbin² Sheng Leixiang² Ruijia Jin ${ }^{3}$

1.2H Offshore, Beijing, China; 2. CNOOC Research Institute, Beijing, China;

3. Tianjin Research Institute for Water Transport Engineering, Tianjin, China

Vortex-induced-vibration of Jack-ups with Cylindrical

Legs in Regular Waves OMAE2019-95764

Sudheesh Ramadasan ${ }^{1}$ Longbin $\mathrm{Ta}^{2} \mathrm{Arun} \mathrm{Dev}^{3}$

1. Newcastle University in Singapore (Cybermarine Technologies Pte Ltd),

Singapore, Singapore; 2. University of Strathclyde, Glasgow, United Kingdom;

3. Newcastle University in Singapore, Singapore, Singapore

Numerical Investigation of Wave-frequency Pontoon Responses of a Floating Bridge based on Model Test Results OMAE2019-96545 Yanlin Shao ${ }^{1}$ Xu Xiang ${ }^{2}$ Jianyu Liu ${ }^{3}$

1. Technical University of Denmark, Kongens Lyngby, Denmark; 2. Norwegian Public Roads Administration, Oslo, Norway; 3. Harbin Engineering University, Harbin, China

Lazy Wave Riser Design in High Current and

Mild Sour Environment OMAE2019-96588

Rupak Ghosh ${ }^{1}$ Carlo Pellegrini ${ }^{2}$ Tyler Visco

1. ExxonMobil, Spring, TX, USA;2. Saipem America, Houston, TX, USA

Investigations on Mode Localization of Offshore

Wind Turbine Blades OMAE2019-96616

Dongsheng Li' Yongpeng Zhang ${ }^{2}$ Xin Guo ${ }^{2}$ Xinglin Guo ${ }^{2}$

1. Shantou University, Shantou, China; 2. Dalian University of Technology, Dalian, China

\section{Structures, Safety and Reliability}

\section{2-5-1 Reliability of Marine Structures}

Thursday June 13

Room Crowne Plaza, Castle 1 | 15:30 - 17:30

Session Chair: Carlos Guedes Soares, Instituto Superior

Técnico, Universidade de Lisboa, Portugal

Session Co-Chair: Paulo M. Videiro, LACEO/COPPE/

Federal University of Rio De Janeiro, Brazil

An Approach for Estimating the Corrosion Rates on Aging FPSO Hull Structures OMAE2019-95067

Vicente Larangeira' Paulo M. Videiro' José Júnior ${ }^{3}$ Luis V.S. Sagrilo²

1. LACEO/Federal University of Rio de Janeiro, Rio de Janeiro, RJ,

Brazil; 2. LACEO/COPPE/Federal University of Rio de Janeiro, Rio de

Janeiro, RJ, Brazil; 3. Shell Brasil, Rio de Janeiro, RJ, Brazil

Remaining Useful Life Estimation of Subsea Pipelines under the Influences of Multiple Causes OMAE2019-95125 Baoping Cai' Xiaoyan Shao ${ }^{1}$ Yonghong Liu ${ }^{1}$ Xiangdi Kong ${ }^{1}$ Hongqi Xu ${ }^{2}$ Weifeng Ge ${ }^{3}$ 1. China University of Petroleum, Qingdao, China;

2. Rongsheng Machinery Manufacture Ltd. of Huabei Oiffiled, Renqiu, China; 3. CNOOC Safety \& Technology Services Co., Ltd, Tianjin, China

Mode Selection for Offshore Platform Damage Identification using CMSE Sensitivity OMAE2019-95550

Mingqiang Xu, Yuchi Liu, Yufeng Jiang, Shuqing Wang Ocean University of China, Qingdao, China

A Simple, Safe, and Reliable Nuclear Reactor Power System Concept for Deep Sea Missions OMAE2019-95857 Gu Hu, Weijian An, Xiaobo Sun China Institute of Atomic Energy, Beijing, China

Random Combination Factors for Still Water and Wave Bending Moments OMAE2019-96665

Wenbo Huang

Harbin Engineering University, Harbin, China

\section{Materials Technology}

\section{3-13-1 Dr. John Sharp Honorary Session}

Thursday June 13 Room SEC, Boisdale 1 | 15:30 - 17:30 Session Chair: Gerhard Ersdal, Petroleum Safety Authority, Norway Session Co-Chair: Alexander Stacey, Energy Division, Health \& Safety Executive, United Kingdom

Overview of Structural Integrity Research \& Development for the Safe Operation of Offshore Installations on the UKCS OMAE2019-95835 Alexander Stacey John Sharp ${ }^{2}$

1. Energy Division, Health \& Safety Executive, London, United Kingdom; 2. Cranfield University, Bedford, United Kingdom

The International Committee on Regulatory Authority Research and Development (ICRARD) an Early History OMAE2019-96598

Charles Smith

Consultant, Bay Roberts, NL, Canada

Inspection and Repair of Ageing Offshore Structures OMAE2019-96842 John Sharp

Cranfield University, Bedford, United Kingdom

Ageing and Life Extension of Offshore Structures OMAE2019-96841 Gerhard Ersdal

Petroleum Safety Authority, Stavanger, Norway 


\section{Pipelines, Risers, and Subsea Systems}

\section{4-4-2 Subsea Structures II}

Thursday June 13

Room Crowne Plaza, Staffa / Shuna | 15:30 - 17:30

Session Chair: Duane DeGeer, INTECSEA, USA

Session Co-Chair: Ruud Selker, INTECSEA, Netherlands

The Influence of Piping Arrangement on the Response of Vibration Isolation System under Underwater Explosion Loading OMAE2019-95603 Chen Pan' Wei Qiang' Liu Zhizhong Wang Guan'

1. China Ship Development and Design Center/National Key Laboratory on Ship Vibration \& Noise, Wuhan, China; 2. China Ship Development and Design Center, Wuhan, China

A PDE Model for Estimating the Life Time of a Riser OMAE2019-96185 Halvor Snersrud Gustad' Per T. Moe ${ }^{2}$ Elena Celledoni ${ }^{3}$

1. TechnipFMC, Trondheim, Norway; 2. TechnipFMC, Kongsberg, Norway;

3. Norwegian University of Science and Technology, Trondheim, Norway

CFD Simulation of a Flow Homogenizer for

Subsea Pumping Systems OMAE2019-96255

Nicolao Lima, Karla Holzmeister, Raphael Santos, Diener Volpin, Roberto Nunhez

State University of Campinas, Campinas, SP, Brazil

Computational Simulation of the Drilling Vessel Motion and

its Effects on the Riser/BOP Connection OMAE2019-96367

Xavier Castello ${ }^{1}$ José Luis Quispe ${ }^{1}$ Segen Estefen ${ }^{2}$

Marcelo Igor Lourenço Souza ${ }^{2}$ Nilo de Moura Jorge ${ }^{1}$

1. COPPE/Universidade Federal do Rio de Janeiro, Rio de Janeiro, RJ, Brazil;

2. Universidade Federal do Rio de Janeiro, Rio de Janeiro, RJ, Brazil

Multiphase Flow Induced Forces on Bend Structures 0MAE2019-96387

Stefan Belfroid, Nestor Gonzalez Diez, Hajo Pereboom, Can Tümer

TNO, Delft, Netherlands

\section{Pipelines, Risers, and Subsea Systems}

\section{4-6-2 Innovative Technologies for Deepwater Low-Cost Production II}

Thursday June 13

Room Crowne Plaza, Castle 3 | 15:30 - 17:30

Session Chair: Ping Liu, INTECSEA, Netherlands

Session Co-Chair: Doug Swanek, C-FER Technologies, Canada

Self-healing Concrete for under Water Cementitious Structures:

using Urea-decomposable and Urea Non-degradable

Microbial Co-cultures Capsule, Part 1 OMAE2019-96492

Muhammad Salman Haider, Wasif Muhammad, Sajjad Miran

University of Gujrat, Gujrat, Pakistan

Self-healing Concrete for under Water Cementitious Structures: using Urea-decomposable and Urea Non-degradable Microbial Co-cultures Capsule, Part 2 OMAE2019-96498 Muhammad Salman Haider, Wasif Muhammad, Sajjad Miran University of Gujrat, Gujrat, Pakistan

\section{Submerged Production Unit: Design and Method}

for Launch and Tow to Field OMAE2019-95291

Venkatesan Arumugam Elumalai' Sigbjørn Daasvatn ${ }^{1}$

Daniel Karunakaran ${ }^{1}$ Kjell Larsen² Bernt Leira ${ }^{3}$

1. Subsea 7 Norway AS, Stavanger, Norway; 2. Equinor ASA, Trondheim, Norway;

3. Norwegian University of Science and Technology, Trondheim, Norway

\section{Ocean Engineering}

\section{6-7-4 Metocean Criteria II}

Thursday June 13 Room SEC, M4 | 15:30-17:30

Session Chair: Gus Jeans, Oceanalysis Ltd, United Kingdom

Session Co-Chair: Kevin Ewans, Met0cean Research Ltd, New Zealand

Spatial and Seasonal Variability of Metocean Design

Criteria in Southern South China Sea from Covariate

Extreme Value Analysis OMAE2019-95913

Vadim Anokhin ${ }^{1}$ Emma Ross ${ }^{2}$ David Randell2 Philip Jonathan ${ }^{3}$

1. Sarwak Shell Bhd, Kuala Lumpur, Malaysia; 2. Shell Global Solutions BV,

Amsterdam, Netherlands; 3. Shell Research Ltd., London, United Kingdom

Metocean Criteria for the Fatigue Analysis

of Subsea Pipelines OMAE2019-96363

Richard Gibson, Marios Christou

Offshore Consulting Group, London, United Kingdom

The Derivation and Interpretation of Directional

Design Criteria OMAE2019-96586

Graham Feld' Philip Jonathan ${ }^{2}$ David Randell

1. Shell U.K. Limited, Aberdeen, United Kingdom; 2. Shell Research Ltd., London,

United Kingdom; 3. Shell Global Solutions BV, Amsterdam, Netherlands

On Environmental Contours for Marine Design OMAE2019-96587

Emma Ross' Ole Christian Astrup ${ }^{2}$ Elzbieta M. Bitner-Gregersen ${ }^{2}$ Nigel Bunn ${ }^{3}$ Graham Feld ${ }^{4}$ Ben Gouldby ${ }^{3}$ Arne Huseby ${ }^{5}$ Ye Liu ${ }^{3}$ David Randell' Erik Vanem ${ }^{2}$ Philip Jonathan ${ }^{6}$ 1. Shell Global Solutions BV, Amsterdam, Netherlands; 2. DNV GL, Høvik, Norway; 3. HR Wallingford Ltd, Wallingford, United Kingdom; 4. Shell U.K. Limited, Aberdeen, United Kingdom; 5. University of Oslo, Oslo, Norway; 6. Shell Research Ltd., London, United Kingdom

\section{CFD \& FSI}

\section{8-5-2 VIV Theory and CFD \& FSI Symposium Workshop}

Thursday June 13 Room SEC, Lomond Auditorium | 15:30 - 17:30 Session Chair: Yiannis Constantinides, Chevron, USA Session Co-Chair: Owen H. Oakley, Jr, Retired, USA

Dispersion Relation for Flow Induced Oscillations (VIV/Galloping)

Revealed at the Fluid-Structure Interface OMAE2019-96823

Michael Bernitsas, James Ofuegbe, Jau-Uei Chen, Hai Sun

University of Michigan, Ann Arbor, MI, USA

\section{Ocean Renewable Energy}

\section{9-2-6 Hybrid Systems and Farm Analysis}

Thursday June 13 Room SEC, Carron 1 | 15:30 - 17:30

Session Chair: Maurizio Collu, University of Strathclyde, United Kingdom Session Co-Chair: K A Abhinav, University of Strathclyde, United Kingdom

Characterizing Impacts of Atmospheric Turbulence on Wind Farms through Large Eddy Simulation (LES) OMAE2019-95837

Jahrul Alam ' Anton Afanassiev² Jagdeep Singh ${ }^{1}$

1. Memorial University of Newfoundland, St John's, NL, Canada;

2. University of British Columbia, Vancouver, $B C$, Canada

Development of a Hybrid Power Generation Platform Combining Floating Wind Turbine and Oscillating Water Column Wave Energy Converters OMAE2019-95968 Zheng Chen, Weijian Zeng, Ming Tan, Dahai Zhang, Yulin Si Zhejiang University, Zhoushan, China 
Design Methodology for a Floating Offshore Wind Turbine

Large-scale Outdoor Prototype OMAE2019-95979

Alessandro Fontanella, Federico Taruffi, Muggiasca Sara, Marco Belloli

Politecnico di Milano, Milano, Italy

Hybrid Renewable Energy Systems Configuration for Offshore Multi-purpose Platforms OMAE2019-96017

Luis Recalde' Hong Yue ${ }^{1}$ William Leithead' Olimpo Anaya-Lara' Hongda Liu' Jiang You ${ }^{2}$ 1. University of Strathclyde, Glasgow, United Kingdom; 2. Harbin University, Harbin, China

Progress on the Development of a Holistic Coupled Model of Dynamics for Offshore Wind Farms, Phase II: Study on a Data-driven Based Reduced-order Model for a Single Wind Turbine 0MAE2019-95542 Zi Lin ${ }^{1}$ Adrian-Mihai Stetco ${ }^{2}$ Jesus Carmona-Sanchez ${ }^{2}$ Debora Cevasco ${ }^{1}$ Maurizio Collu ${ }^{1}$ Goran Nenadic ${ }^{2}$ Ognjen Marjanovic ${ }^{2}$ Mike Barnes ${ }^{2}$

1. University of Strathclyde, Glasgow, United Kingdom;

2. Manchester University, Manchester, United Kingdom

\section{Ocean Renewable Energy}

\section{9-3-3 Wave Energy: CFD Simulations}

Thursday June 13

Room SEC, Carron 2 | 15:30 - 17:30

Session Chair: Jennifer van Rij, National Renewable Energy Laboratory, USA

A Validation of a Pivoted Point Absorber Type Wave

Energy Converter using CFD OMAE2019-96030

Injun Yang, Tahsin Tezdogan, Atilla Incecik

University of Strathclyde, Glasgow, United Kingdom

Extreme Load CFD Analysis and Verification for a

Multi-body Wave Energy Converter OMAE2019-96397

Jennifer van Rij' ${ }^{\mathrm{Y} i-H s i a n g} \mathrm{Yu}^{1}$ Alan McCall ${ }^{2}$ Ryan $\mathrm{Coe}^{3}$

1. National Renewable Energy Laboratory, Golden, CO, USA; 2. Ecomerit Technologies,

Santa Barbara, CA, USA; 3. Sandia National Laboratories, Albuquerque, NM, USA

A Study of Harbor Resonant Wave Energy Harvester OMAE2019-95325

Bang-Fuh Chen, Yun-Da Si

National Sun Yat-Sen University, Kaohsiung, Taiwan

Experimental and Numerical Analysis of Performance of Oscillating Water Column Wave Energy Converter

Applicable to Breakwaters OMAE2019-96500

Sewan Park, Kyong-Hwan Kim, Bo Woo Nam, Jeong-Seok Kim, Keyyong Hong

Korea Research Institute of Ships and Ocean Engineering, Daejeon, Korea

\section{Petroleum Technology}

\section{1-11-2 Innovations in Drilling, Production and Transport}

Thursday June 13

Room Crowne Plaza, Barra | 15:30-17:30

Session Chair: Ming Feng, Chongqing University, China

Session Co-Chair: Wenting Qin, China University of Petroleum Beijing, China

Optimization of Temporary Plugging Parameters

under Rough Fractures OMAE2019-95748

Lishan Yuan' Fujian Zhou' Jianbo Huang ${ }^{2}$ Ben Li' Junjie Gao Caizhong Wang ' Yanxin Tan'

1. China University of Petroleum-Beijing, Beijing, China;

2. PetroChina Xinjiang Oilfield, Xinjiang, China

Study on Acid Fracturing Technology for Carbonate

Reservoirs in Ordos Basin OMAE2019-95802

Lufeng Zhang, Fujian Zhou, Jianye Mou, Jie Wang, Jin Wang, Yuechun Wang

China University of Petroleum-Beijing, Beijing, China
The Tentative Case Study of Annulus Build Up Pressure in the Deepwater Gas Production Well in South China Sea OMAE2019-95847 Ming Feng

Chongqing University, Chongqing, China

Study on Desulfurization and Decarbonization Technology of Natural Gas on Bohai Offshore Platform OMAE2019-96733 Ping Lu, Zhaoguang Qu, Guogiang Gao, Yan Huang, Xin Qian, Lin Cheng Bohai Oilfield Research Institute, Tianjin Branch, CNOOC China Limited, Tanggu, China

\section{Takeshi Kinoshita Honoring Symposium on Offshore Technology}

\section{3-3-1 Wind Energy}

Thursday June 13 Room SEC, Boisdale 2 | 15:30 - 17:30

Session Organizer: Tomoaki Utsunomiya, Kyushu University, Japan Session Co-Organizer: Hideyuki Suzuki, University of Tokyo, Japan

At-sea Experiment on Durability and Residual Strength of Polyester Rope for Mooring of Floating Wind Turbine OMAE2019-95388 Tomoaki Utsunomiya' ${ }^{1}$ ku Sato ${ }^{2}$ Koji Tanaka ${ }^{2}$ 1. Kyushu University, Fukuoka, Japan; 2. TODA CORPORATION, Tokyo, Japan

Weathervane Performance and Stability Analysis of Single Point Moored FOWTs under Wind-current Coexisting Field OMAE2019-95404 Sharath Srinivasamurthy' Kazuki Hashimoto ${ }^{1}$ Kazuhiro lijima ${ }^{2}$ Yasunori Nihei ${ }^{1}$ 1. Osaka Prefecture University, Osaka, Japan; 2. Dept of NAOE, Osaka University, Osaka, Japan

New Spar Design for Floating Offshore Wind

Turbine with Damping Plates OMAE2019-95688

Shigesuke Ishida, Yasutaka Imai

Saga University, Saga, Japan

Comparison of Dynamic Response in a 2MW Floating Offshore Wind Turbine during Typhoon Approaches OMAE2019-95889 Koji Tanaka' Iku Sato ${ }^{1}$ Tomoaki Utsunomiya ${ }^{2}$ Hiromu Kakuya ${ }^{3}$ 1. Toda Corporation, Tokyo, Japan; 2. Kyushu University, Fukuoka, Japan; 3. Hitachi, Ltd, Hitachi-shi, Japan

Response Characteristics of a Floating Structure with Moon Pools Installed with Vertical-axis Wind Turbines 0MAE2019-96045 Mitsuru Nakamura' Tomoki Ikoma' Hiroaki Eto ${ }^{1}$ Yasuhiro Aida ${ }^{2}$ Koichi Masuda ${ }^{1}$ 1. Nihon University, Funabashi, Japan; 2. Nihon University, Chiba, Japan

\section{Farewell Reception \\ 17:30 - 19:00}

Location: Argyll Suite, Crowne Plaza

See Social Events, page 14 for more details.

Sponsored by Greater Ft. Lauderdale Convention Bureau

GREATER FORT LAUDERDALE CONVENTION \& VISITORS BUREAU 


\section{Technical Tours: Friday, June 14}

$\mathbf{T}$ he Local Organizing Committee has arranged two technical day tours that promise to be an excellent addition to your conference experience. On Friday, June 14th you have the option of a morning tour of either the University of Strathclyde's Advanced Forming Research Centre (AFRC) or the Glasgow Subsea 7 facilities. Both tours will join together for lunch and then visit the Falkirk Wheel for a boat tour of the world's only rotating boatlift. A stop to visit the iconic Kelpies is included on the way to the Wheel.

\section{Tour 1: Advanced Forming Research Centre (AFRC) and Falkirk Wheel}

Tour 2: Subsea 7 and Falkirk Wheel

Registration: Pre-purchased tickets for the tour are provided with your name badge. Additional tickets will be for sale at the Registration Desk if space is still available.

Meeting Point for Technical Tour: Main Entrance / Lobby of the Crowne Plaza Hotel

Technical Tour Departure Times:

- Subsea 7 and Falkirk Wheel Departure: 08:30

- Advanced Forming Research Centre (AFRC) and Falkirk Wheel Departure: 09:15

Approximate Return Time: 18:30

Technical Tour Ticket: $€ 65$ lincludes 20\% VAT, lunch and Falkirk Wheel boat admission)

\section{Advanced Forming Research Centre}

The University of Strathclyde's

Advanced Forming Research Centre

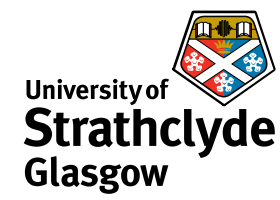

(AFRC) is a globally-recognised centre of excellence in innovative manufacturing technologies, engineering research and development, and metal forming and forging research.

For almost a decade the centre has been at the heart of manufacturing research in Scotland. It is the only High Value Manufacturing Catapult centre in the country, one of only 7 in the UK making it the critical link between manufacturers in Scotland and the rest of this world-class network of manufacturing innovation and expertise.

The AFRC helps to fill the gap between fundamental

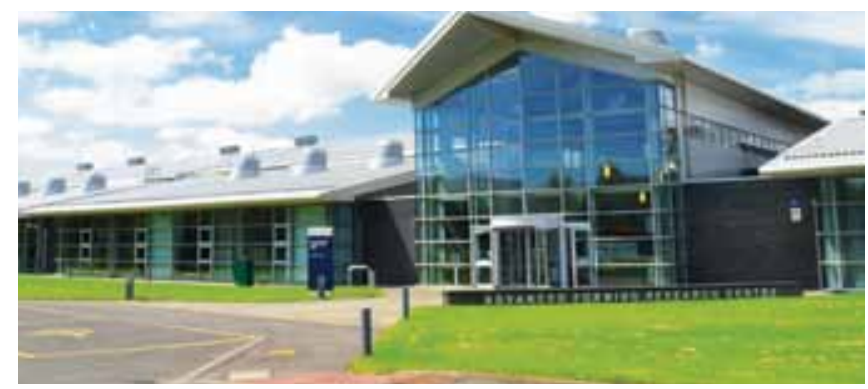

University of Strathclyde Advanced Forming Research Centre academic research and industry. We help companies to turn innovative technologies and ideas into a commercial reality that will increase their competitiveness, boost their business and secure the manufacturing sector in Scotland and the UK for generations to come.

We offer world-class expertise and cutting edge technologies that help firms develop solutions that bring about real business benefits for companies of all sizes from across the UK and internationally.

\section{Subsea 7}

Subsea 7 is a global leader in the delivery of offshore

\section{subsea 7}

projects and services for the evolving energy industry.

Subsea 7's Global Pipeline Welding Development Centre is a world-class facility that supports the continued development of leading-edge welding and material technology to meet demands of high-integrity pipelines for the subsea oil and gas industry. Built in 2013 it serves as a technical authority for the rolling-out of welding enhancements and polymer lining solutions to all Subsea 7 fabrication bases globally.

The centre provides and uses:

- Integrated R\&D welding and inspection capability

- Pre-fabrication welding qualification and procedures

- Wide range of welding technologies \& solutions

- Ultrasonic \& radiographic NDE testing

- Laboratory \& sectioning capability.

- Home to Swagelining, the world's leading polymer lining specialist for subsea pipelines

\section{Falkirk Wheel}

The Falkirk Wheel is a rotating boat lift in central Scotland, connecting the Forth and Clyde Canal with the Union Canal. The lift is named after Falkirk, the town in which it is located. It reconnects the two canals for the first time since the 1930s. It opened in 2002 as part of the Millennium Link project.

The wheel raises boats by 24 metres $(79 \mathrm{ft})$, but the Union Canal is still 11 metres ( $36 \mathrm{ft}$ ) higher than the aqueduct which meets the wheel. Boats must also pass through a pair of locks between the top of the wheel and the Union Canal. The Falkirk Wheel is the only rotating boat lift of its kind in the world, and one of two working boat lifts in the United Kingdom, the other being the Anderton Boat Lift.

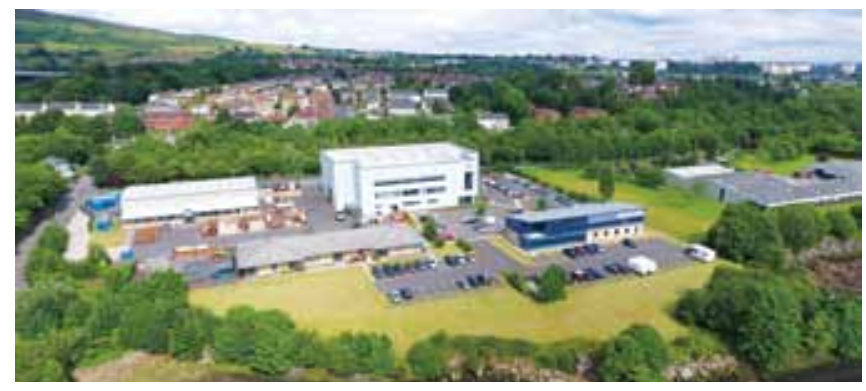

Subsea 7 Glasgow Facilities 


\section{3th Annual Outreach for Engineers Specialty Forum}

\section{"I have learned a lot on so many levels and I am so thankful to the Committee for having granted me a scholarship for this event. The forum has given me great insights on what working in industry could represent and thanks to that I am now considering new stimulating options for my future career."}

-Comment from an Outreach attendee.

\section{Overview}

The Ocean, Offshore and Arctic Engineering Division (OOAE) of ASME is hosting a specialty forum at the 2019 International Conference on Ocean, Offshore and Arctic Engineering (OMAE) in Glasgow, United Kingdom. The specialty forum is designed for students and early professionals who may not be familiar with the industry as well as those who have already specialized in this area.

This is the thirteenth year of the Outreach for Engineers Forum. Highlights of the Forum will include presentations of the various technologies required (e.g. from ocean and/or offshore engineering, civil engineering, petroleum engineering, aerospace engineering, mechanical/structural engineering and project management), types of job opportunities, possible career paths and a team building activity. As each year is different, a site tour or job fair may be included.

In addition, Outreach for Engineers Specialty Forum delegates will be provided with the opportunity to participate at OMAE 2019 as full conference delegates. This conference will showcase over 900 technical papers from engineers and scientists from around the world, with 13 Symposia representing the range of technologies.

\section{Attendee Profile}

- Senior Undergraduate Students enrolled in Engineering or Science Curricula

- Graduate Students (both Master and Doctoral levels) with specialization in fields such as ocean and/or offshore engineering, civil engineering, mechanical engineering, petroleum engineering, and aerospace engineering

- Early professionals with an interest in the oil \& gas industry and ocean, offshore \& arctic engineering

\section{Scholarships}

Through funding provided by the OOAE Division of ASME and corporate sponsors, the organizers of the Outreach to Engineers Specialty Forum will be offering scholarships to cover registration costs and a limited number of travel subsidies. The scholarships are open to students and early professionals from around the world. If you qualify and have not been a recipient yet, please feel free to apply for OMAE 2020 on the conference website.

\section{Conference Schedule with Outreach Events}

\begin{tabular}{|l|l|l|l|}
\hline Date & Event & Time & Location \\
\hline Saturday, June 8 & Outreach Team Building Exercise & $17: 00-19: 00$ & $\begin{array}{l}\text { Staffa/Shuna } \\
\text { (Crowne Plaza) }\end{array}$ \\
\hline \multirow{2}{*}{ Saturday, June 8 } & Outreach Welcome Dinner & $19: 00$ & Off-site \\
\hline \multirow{3}{*}{ Sunday, June 9 } & $\begin{array}{l}\text { Outreach Welcome \& Introductions plus } \\
\text { Industry Presentations }\end{array}$ & $08: 00-17: 00$ & $\begin{array}{l}\text { Castle } 1 \\
\text { (Crowne Plaza) }\end{array}$ \\
\cline { 2 - 4 } & OMAE 2019 Conference Registration & $13: 00-20: 00$ & Hall 5 (SEC) \\
\cline { 2 - 4 } & $\begin{array}{l}\text { OMAE 2019 Conference Welcome } \\
\text { Reception }\end{array}$ & $18: 30-20: 30$ & $\begin{array}{l}\text { Glasgow Science } \\
\text { Centre }\end{array}$ \\
\hline Monday, June 10 & OMAE 2019 Conference & See detailed program for session locations and times. \\
\hline Tuesday, June 11 & OMAE 2019 Conference & See detailed program for session locations and times. \\
\hline \multirow{2}{*}{ Wednesday, June 12 } & OMAE 2019 Conference & See detailed program for session locations and times. \\
\cline { 2 - 4 } & OMAE 2019 Conference Banquet & $19: 00-24: 00$ & Merchant Square \\
\hline \multirow{2}{*}{ Thursday, June 13 } & Outreach Breakfast / Feedback Session & $07: 30-10: 00$ & Alsh 2 (SEC) \\
\cline { 2 - 4 } & OMAE 2019 Conference & See detailed program for session locations and times. \\
\hline Friday, June 14 & OMAE Technical Tour (Optional) & See Technical Tour on page 87 for locations and times. \\
\hline
\end{tabular}




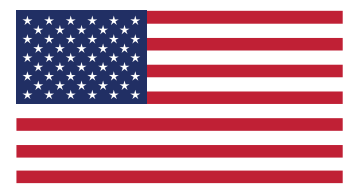

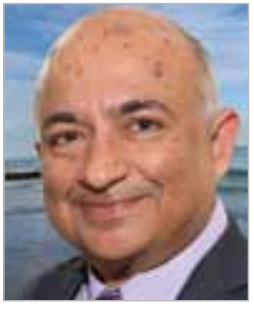

Professor Manhar R. Dhanak

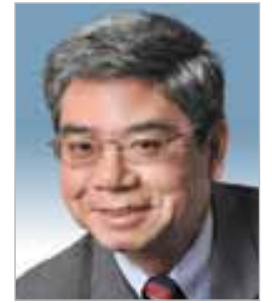

Professor Ronald W. Yeung

\section{Invitation to OMAE 2020}

$\mathbf{P}$ lease join us for the 39th International Conference on Ocean, Offshore and Arctic Engineering (OMAE 2020) in Fort Lauderdale, Florida, USA June 28 - July 3.

Located in southeast Florida, Fort Lauderdale is home to Port Everglades, one of the top three cruise ports in the world and among the most active containerized cargo ports in the United States. It is South Florida's main seaport for petroleum products including gasoline and jet fuel.

The City of Fort Lauderdale is famous for its award-winning, palm-fringed, golden-sand beaches, arts, culture and events. It provides a water-centric culture from the ocean and blue wave beaches to the exotic Everglades, and includes shopping malls, fine dining, entertainment, boat rides along canals, and golf courses. The City features a landscaped beachfront promenade, luxury hotels, a front to its historic New River, and mansions and yachts dotting its Millionaires Row. The Stranahan House is a preserved 1900s home furnished with antiques of the era. The "red brick road" of Riverwalk leads to the Arts and Entertainment District, where the Museum of Discovery and Science typically has substantial exhibits with thousands of visitors annually. The NSU Art Museum is home to diverse and innovative collections of art from around the world. The City's Flamingo Gardens features over 3000 species of tropical plants and a sanctuary of 90 native wildlife species.

OMAE 2020 will be hosted by the Department of Ocean and Mechanical Engineering (OME) and its Institute for Ocean and Systems Engineering (IOSE) at Florida Atlantic University, one of ten state universities in Florida with a student body of 30,000 students. The Department is part of FAU's College of Engineering and Computer Science and offers B.S., M.S. and $\mathrm{PhD}$ degrees in ocean engineering and mechanical engineering. The ocean engineering program, initiated in 1965 as the world's first undergraduate program of its kind, aims to provide an outstanding academic environment for education, research, and development of ocean technologies. IOSE, established in 1999 as an extension of the ocean engineering program, is located on eight acres of land between the Atlantic Ocean and the Intra Coastal water-way at FAU's SeaTech campus in Dania Beach. The Department and IOSE have been involved in a period of sustained sponsored research activity over the past twenty years, culminating in an aggregate of over $\$ 60$ million expenditures in ocean engineering research and technology development, together with granting of over 400 degrees in ocean engineering. The Institute has research focus areas in marine vehicles, hydrodynamics and physical oceanography, marine materials and corrosion, control and automation, nano-composites, and acoustics and vibrations. It provides the means for technology advancement, and transition of research products to applications. R\&D projects at IOSE have included unmanned underwater and surface vehicles, multi-domain, multi-vehicle maritime autonomy, ocean energy technologies, air-deployable buoys, durability of fiber-reinforced concrete and composite materials in seawater, nano-composites, underwater communication, and seabasing technologies. These projects complement basic research in ocean engineering as well as training and education of the next generation of ocean engineering workforce.

A strong technical program in offshore engineering is being put together for OMAE 2020, with thematic foci on renewable ocean energy and automation in maritime systems. The technical tours will include a visit to Port Everglades. The social program will include water and sports activities, shopping tours, everglades airboat adventure, Intracoastal boat tours, and much more.

We very much hope you can join us for OMAE 2020 and look forward to welcoming you to Fort Lauderdale next year.

-Professor Manhar R. Dhanak Conference Chair, OMAE 2020

Professor and Chair, Dept. of Ocean and Mechanical Engineering Director, Institute for Ocean and Systems Engineering Florida Atlantic University, USA

-Professor Ronald W. Yeung Conference Co-Chair, OMAE 2020

Distinguished Professor (E.) of Hydromechanics and Ocean Engineering University of California at Berkeley, USA 


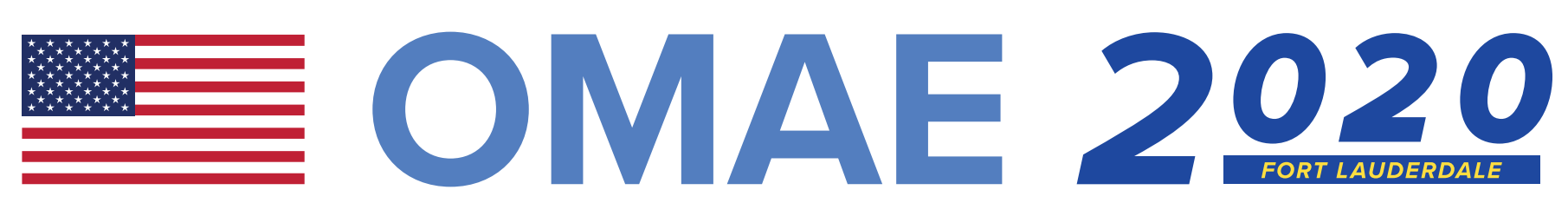

\section{OMAE 2020 Fort Lauderdale CALL FOR PAPERS}

\section{Abstract Submission is now open!}

Please visit the OMAE 2020 conference website (www.asme.org/ events/omae) to view the conference details.

Following OMAE 2019, we anticipate another successful conference showcasing the excellent technical content that OMAE has become known for internationally.

\section{Abstract/Paper Submission Guidelines}

Authors should submit a title/abstract to begin the paper submission process. Prior to the date noted below, authors should then submit full-length manuscripts for peer review. Draft manuscripts and final-paper submissions must conform to ASME publication guidelines.

\section{Important Dates and Information}

- Monday, November 4, 2019 - Deadline for Abstract Submission

NOTE: Abstracts submitted to individual topics will be automatically accepted by the system and assigned a paper number. Submission of the draft paper for review must be before the stated deadline. Presentation-only abstracts must be 400 650 words.

\section{- Monday, January 13, 2020 - Full-length Draft Paper} Submission

- Monday, February 17, 2020 - Notification of Draft

Paper Acceptance / Rejection

- Monday, March 30, 2020 - Final Paper Submission

For the full publications schedule and to submit your Abstract and Draft Paper, please visit www.asme.org/events/omae.
To submit your abstract, visit: www.asme.org/events/omae
PLEASE NOTE THAT THESE DEADLINES ARE FIRM AND WILL NOT BE EXTENDED. Due to the tremendous success of the OMAE conferences, the number of papers has increased steadily over the years hence we need to uphold firm deadlines to ensure proper management of the review and publication process. Your cooperation in adhering to the publication schedule and making OMAE 2020 a success will be greatly appreciated.

We ask that you return home from OMAE 2019 and start working on your Abstract and Full-length Draft Paper soon! We look forward to your contribution to a very successful OMAE 2020.

Sincerely,

Professor Manhar R. Dhanak Conference Chair, OMAE 2020 Professor and Chair, Department of Ocean and Mechanical Engineering Director, Institute for Ocean and Systems Engineering Florida Atlantic University, USA

Professor Ronald W. Yeung Conference Co-Chair, OMAE 2020 Distinguished Professor (E.) of Hydromechanics and Ocean Engineering University of California at Berkeley, USA

Professor Antonio C. Fernandes Technical Program Chair, OMAE 2020 Head, Ocean Engineering Program of COPPE/UFRJ LOC (Laboratório de Ondas e Correntes - Waves and Currents Laboratory), Coordinator LabOceano, Director for International Affairs 


\section{INTERNATIONAL OFFSHORE WIND TECHNICAL CONFERENCE}

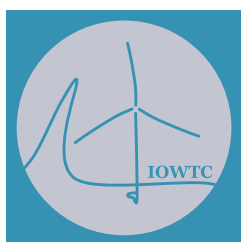

CONFERENCE

NOV $3-6,2019$

$2^{\text {nd }}$ International Offshore Wind Technical Conference

Corinthia St. George's Bay Hotel,

St. Julian's, Malta

\section{SCIENTIFIC TRACK}

» New Fixed Offshore Concepts

»New Floating Concepts

» Mooring \& Foundations

»Offshore Turbines Modeling

» Aero-Hydro modeling

» Structural Analysis

» Offshore Wind Turbine Drivetrains

»Metocean

» Model Testing

» Field Data

\section{PROJECT DEVELOPMENT TRACK}

» Farms / Layouts

»Permits

» Environmental Issues

» Fabrication \& Industrialization

» Installation / Commissioning

» O\&M

» Decommissioning

» Asian Projects

» American Projects

» European Projects

» LCOE \& Financing Challenges
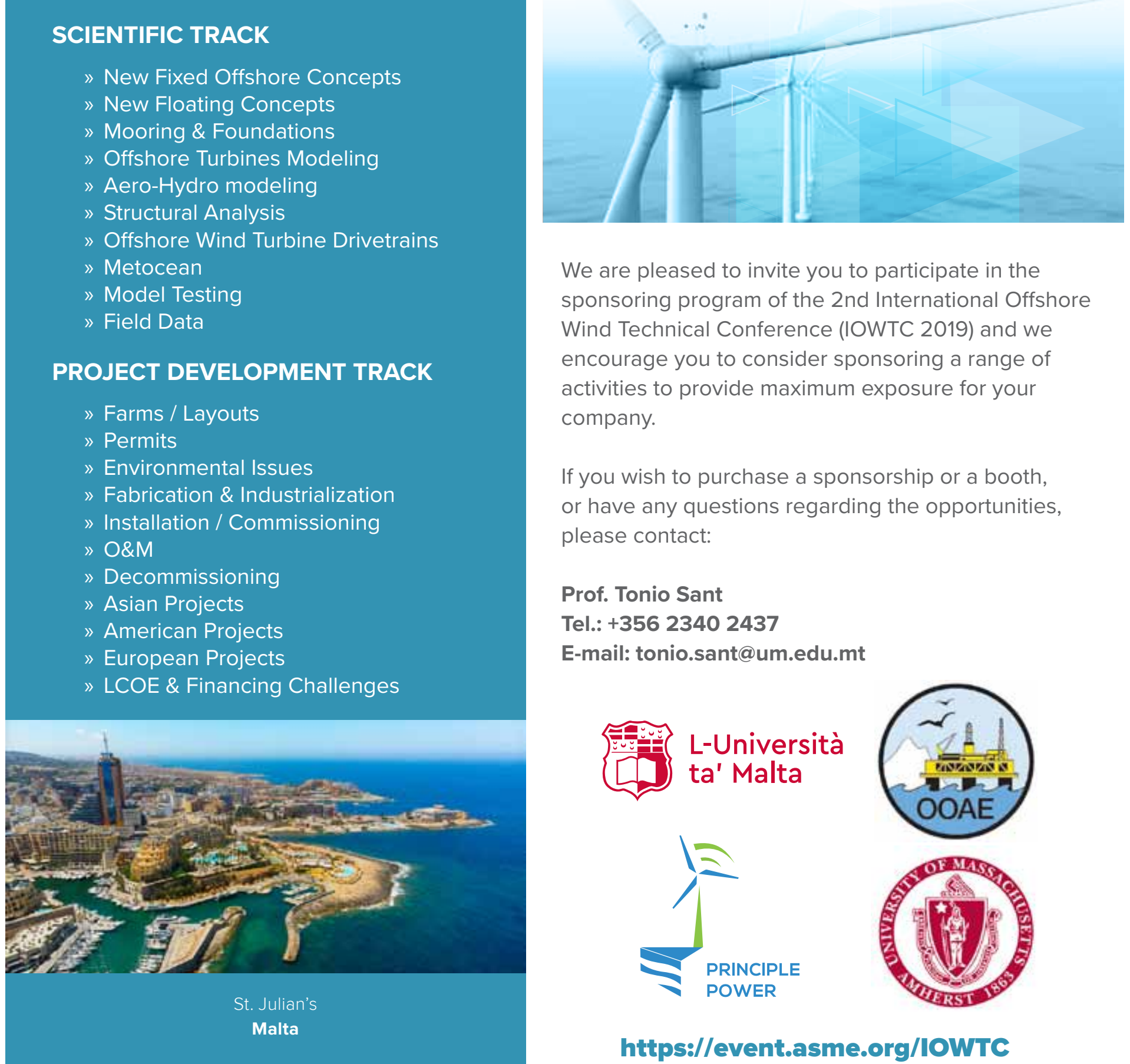

We are pleased to invite you to participate in the sponsoring program of the 2nd International Offshore Wind Technical Conference (IOWTC 2019) and we encourage you to consider sponsoring a range of activities to provide maximum exposure for your company.

If you wish to purchase a sponsorship or a booth, or have any questions regarding the opportunities, please contact:

\section{Prof. Tonio Sant}

Tel.: +356 23402437

E-mail: tonio.sant@um.edu.mt
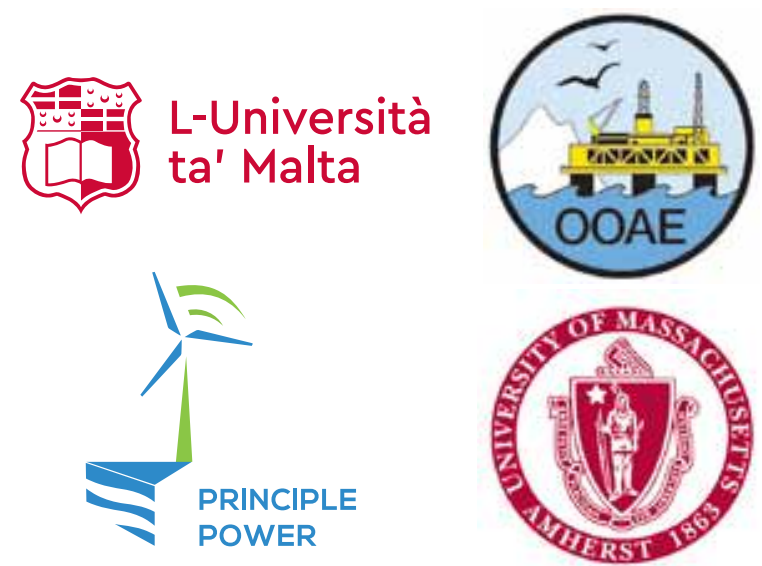

https://event.asme.org//OWTC 


\section{Listing of Committees}

\section{Conference Organizing Committee}

Professor Atilla Incecik, Conference Chair Professor Krish Thiagarajan Sharman, Technical Program Chair

\author{
Local Organizing Committee \\ Annabel Anderson \\ Feargal Brennan \\ Sandy Day \\ Julia Race \\ Willie Reid \\ Tahsin Tezdogan \\ Osman Turan \\ Dracos Vassalos \\ Qing Xiao
}

\section{Volunteers}

The Conference Organizing Committee would like to express their gratitude to all the OMAE 2019 volunteers. We sincerely appreciate all the support they provide!

\section{Technical Program Committee}

SYMP 1: Offshore Technology

Symposium Coordinator: R. Cengiz Ertekin, University of Hawaii

SYMP 2: Structures, Safety and Reliability Symposium Coordinator: Carlos Guedes Soares, Instituto Superior Técnico

SYMP 3: Materials Technology

Symposium Coordinator: Mamdouh Salama, ConocoPhillips

SYMP 4: Pipelines, Risers, and Subsea Systems

Symposium Coordinator: Theodoro A.

Netto, COPPE/ UFRJ

Symposium Co-Coordinator: Duane

DeGeer, INTECSEA

SYMP 5: Ocean Space Utilization

Symposium Coordinator: Tomoki Ikoma, Nihon University

Symposium Co-Coordinator: Dominique Roddier, Principle Power, inc.

SYMP 6: Ocean Engineering

Symposium Coordinator: Solomon Yim, Oregon State University

Symposium Co-Coordinator: Antonio

Carlos Fernandes, UFRJ/COPPE

SYMP 7: Polar and Arctic Sciences and Technology

Symposium Coordinator: Walter Kuehnlein, sea2ice Ltd. \& Co. KG

Symposium Co-Coordinator: Professor Dr. Sören Ehlers, Hamburg University of Technology
SYMP 8: CFD \& FSI

Symposium Coordinator: Yiannis

Constantinides, Chevron

Symposium Co-Coordinator: Owen $\mathrm{H}$.

Oakley, Jr, Retired

SYMP 9: Ocean Renewable Energy

Symposium Coordinator: Krish Thiagarajan

Sharman, University of Massachusetts

Symposium Co-Coordinator: Charles Smith, Consultant

SYMP 10: Offshore Geotechnics

Symposium Co-Coordinator: Horst Brandes, University of Hawaii at Manoa

SYMP 11: Petroleum Technology Symposium Co-Coordinator: Andrzej Wojtanowicz, Louisiana State University

SYMP 12: Honoring Symposium for Rodney Eatock Taylor on Marine and Offshore Hydrodynamics

Symposium Coordinator: Allan Magee, National University of Singapore

SYMP 13: Honoring Symposium for Takeshi Kinoshita on Offshore Technology Symposium Coordinator: Hideyuki Suzuki, University of Tokyo

\section{Topic Organizers}

Offshore Technology

1-1: ORGANIZER: Anil Sablok, TechnipFMC, United States

1-2: ORGANIZER: Allan Magee, National University of Singapore, Singapore

1-3: ORGANIZER: Longbin Tao, University of Strathclyde, United Kingdom

1-4: ORGANIZER: Olaf Waals, MARIN,

Netherlands; C0-ORGANIZER: Masoud

Hayatdavoodi, University of Dundee, United

Kingdom

1-5: ORGANIZER: Wenhua Zhao, University of

Western Australia, Australia; CO-ORGANIZER:

Marc Cahay, TechnipFMC, France

1-6: ORGANIZER: Zhenjia (jerry) Huang,

Exxonmobil Upstream Research Company,

United States; C0-ORGANIZERS: Jang Kim,

TechnipFMC, United States; Guangyu Wu,

Chevron, United States

1-7: ORGANIZER: Nuno Fonseca, SINTEF Ocean, Norway

Structures, Safety and Reliability

2-1: ORGANIZER: Elzbieta M. Bitner-Gregersen,

DNV GL AS, Norway; C0-ORGANIZER: Alexander

V. Babanin, University of Melbourne,

Australia

2-2: ORGANIZER: Carlos Guedes Soares, Instituto Superior Técnico, Universidade de Lisboa, Portugal; CO-ORGANIZER: Felice Arena, Univ Mediterranea, Italy
2-3: ORGANIZER: Lance Manuel, University of Texas at Austin, United States

2-4: ORGANIZER: Yordan Garbatov, University of Lisbon, Portugal

2-5: ORGANIZER: Carlos Guedes Soares, Instituto Superior Técnico, Universidade de Lisboa, Portugal

2-6: ORGANIZER: Ying Min Low, National University of Singapore, Singapore; CO-ORGANIZER: Luis V.S. Sagrilo, LACEO/ COPPE/Federal University of Rio De Janeiro, Brazil

2-7: ORGANIZER:Zhen Gao, Norwegian University of Science and Technology, Norway; CO-ORGANIZER: John Sorensen, Aalborg University, Denmark

2-8: ORGANIZER: Max Russo, Konsberg, Norway 2-9: ORGANIZER: Carlos Guedes Soares, Instituto Superior Técnico, Universidade de Lisboa, Portugal

2-10: ORGANIZER: Sören Ehlers, Hamburg University of Technology, Germany; CO-ORGANIZER: Zhiqiang Hu, Newcastle University, United Kingdom

2-11: ORganIZER: Masahiko Fujikubo, Osaka University, Japan

2-12: ORGANIZER: Jonas W. Ringsberg, Chalmers University of Technology, Sweden; CO-ORGANIZER: Paulo M. Videiro, LACEO/COPPE/Federal University of Rio De Janeiro, Brazil

2-13: ORGANIZER: Marcelo Ramos Martins, LabRisk - Analysis, Evaluation and Risk Management Laboratory - University of São Paulo, Brazil; C0-ORGANIZER: Ângelo Teixeira, Centre for Marine Technology and Ocean Engineering (CENTEC)/Instituto Superior Técnico, Portugal

2-14: ORGANIZER: Bernt Leira, NTNU, Norway 2-15: ORGANIZER: YeongAe Heo, Case Western Reserve University, United States

Materials Technology

3-1: ORGANIZER:Xin Wang, Carleton University, Canada; Co-ORGANIZER: Myung-Hyun Kim, Pusan National University, Korea (Republic) 3-2: ORGANIZER: Carol Johnston, TWI Ltd, United Kingdom

3-3: ORGANIZER: Jens Tronskar, Det Norske Veritas Pte Ltd, Singapore

3-4: ORGANIZER: Pedro Vargas, Chevron Energy Technology Company, United States

3-5: ORGANIZER: Koji Gotoh, Kyushu University, Japan

3-6: ORGANIZER: Satoshi Igi, JFE Steel

Corporation, Japan

3-9: ORGANIZER: Sheng Bao, Zhejiang University, China

3-11: ORGANIZER: Isabel Hadley, TWI Ltd, United Kingdom; C0-ORGaNIZER: Bostjan Bezensek, Shell Global Solutions UK, United Kingdom 
3-13: ORGANIZER: Gerhard Ersdal, Petroleum Safety Authority, Norway; CO-ORGANIZER: Alexander Stacey, Offshore Safety Division, Health \& Safety Executive, United Kingdom, CO-ORGANIZER: Charles Smith, Consultant, Canada

Pipelines, Risers, and Subsea Systems 4-1: ORGANIZER: Zhimin Tan, Baker Bughes, a GE company, United States; CO-ORGANIZER: Svein Saevik, NTNU, Norway

4-2: ORGANIZER: Basim Mekha, Cuneiform Offshore Consulting, LLC, United States; CO-ORGANIZER: olav fyrileiv, $D N V G L$, Norway 4-3: ORGANIZER: Julian Hallai, ExxonMobil, United States; CO-ORGANIZER: Theodoro Netto, COPPE/UFRJ, Brazil

4-4: ORGANIZER: Marcelo Igor Lourenço Souza, UFRJ, Brazil; CO-ORGANIZER: Duane DeGeer, INTECSEA, United States

4-5: ORGANIZER: Celso Morooka, University of Campinas, Brazil; CO-ORGANIZER: Daniel Carneiro, Wood, Brazil

4-6: ORGANIZER: Segen Estefen, Federal University of Rio de Janeiro, Brazil; CO-ORGANIZER: Chris Timms, C-FER Technologies, Canada

\section{Ocean Space Utilization}

5-1: ORGANIZER: Kazuhiro Iijima, Dept of NAOE, Osaka University, Japan

5-2: ORGANIZER: Dominique Roddier, Principle Power, inc., United States

5-3: ORGANIZER: Sotaro Masanobu, National Maritime Research Institute of Japan, Japan 5-4: ORGANIZER: Tomoya Inoue, JAMSTEC, Japan

5-5: ORGANIZER: Motohiko Murai, Yokohama National University, Japan

5-6: ORGANIZER: Koichi Masuda, Nihon University, Japan; CO-ORGANIZERS: Koji Takahashi, Japan Port Consultants, Ltd., Japan; Shigeru Tabeta, The University of Tokyo, Japan

5-7: ORGANIZER: Daisuke Kitazawa, The University of Tokyo, Japan

5-8: ORGANIZER: Yasuyuki Ikegami, Saga Univeristy, Japan

5-9: ORGANIZER: Shigeru Tabeta, The University of Tokyo, Japan

\section{Ocean Engineering}

6-1: ORGANIZER: Wei Qiu, Memorial University of Newfoundland, Canada; CO-ORGANIZER: Shuzheng Sun, Harbin Engineering University, China

6-2: ORGANIZER: Kuang-An Chang, Texas A\&M University, United States; CO-ORGANIZER: Mohammad-Reza Alam, University of California, Berkeley, United States 6-3: ORGANIZER: Pierre Ferrant, Ecole Centrale De Nantes/CNRS, France; CO-ORGANIZERS: Torgeir Kirkhorn Vada, DNV GL, Norway; Fonseca Nuno, SINTEF Ocean, Norway
6-4: ORGANIZER: Muk Chen Ong, University of Stavanger, Norway; C0-ORGANIZERS: Lin Li, University of Stavanger, Norway; Shuzheng Sun, Harbin Engineering University, China; Guang Yin, University of Stavanger, Norway 6-5: ORGANIZER: Sanne van Essen, MARIN, Netherlands; CO-ORGANIZER: Mohammad Rahmati, Northumbria University, United Kingdom

6-6: ORGANIZER: Muk Chen Ong, University of Stavanger, Norway

6-7: ORGANIZER: Gus Jeans, Oceanalysis Ltd, United Kingdom

6-8: ORGANIZER: Krishnankutty parameswaran, Indian Institute of Technology Madras, India; CO-ORGANIZER: David Molyneux, Memorial University of Newfoundland, Canada 6-9: ORGANIZER: Muk Chen Ong, University of Stavanger, Norway

6-10: ORGANIZER: Muk Chen Ong, University of Stavanger, Norway

6-11: ORGANIZER: Antonio Carlos Fernandes, UFRJ/COPPE, Brazil

6-12: ORGANIZER: Gus Jeans, Oceanalysis Ltd, United Kingdom

6-13: ORGANIZER: Ye Li, Shanghai Jiaotong University, China; C0-ORGANIZER: Jeffrey Falzarano, Texas A\&M University, United States

6-14: ORGANIZER: Muk Chen Ong, University of Stavanger, Norway

6-15: ORGANIZER: Eduardo Tannuri, Numerical Offshore Tank - University of São Paulo, Brazil; CO-ORGANIZER: Celso Morooka, University of Campinas, Brazil

6-16: ORGANIZER: Muk Chen Ong, University of Stavanger, Norway

6-17: ORGANIZER: Kostas Belibassakis, National Technical University of Athens, Greece; CO-ORGANIZER: Simen Ellingsen, Norwegian University of Science and Technology, Norway

Polar and Arctic Sciences and Technology 7-1: ORGANIZER: Sören Ehlers, Hamburg University of Technology, Germany 7-2: ORGANIZER: Sören Ehlers, Hamburg University of Technology, Germany 7-3: ORGANIZER: Sören Ehlers, Hamburg University of Technology, Germany 7-4: ORGANIZER: Sören Ehlers, Hamburg University of Technology, Germany

7-11: ORGANIZER: Walter Kuehnlein, sea2ice Ltd. \& Co. KG, Germany

7-12: ORGANIZER: Walter Kuehnlein, sea2ice Ltd. \& Co. KG, Germany

\section{CFD \& FSI}

8-1: ORGANIZER: Samuel Holmes, Red Wing Engineering, Inc, United States; CO-ORGANIZER: Steve Cosgrove, Altair Engineering, Inc. United States

8-2: ORGANIZER: Hans Bihs, NTNU Trondheim, Norway; CO-ORGANIZER: Tim Bunnik, MARIN, Netherlands
8-3: ORGANIZER: Rajeev Kumar Jaiman, University of British Columbia - Vancouver, Canada; CO-ORGANIZER: Guilherme Vaz, MARIN, Netherlands

8-4: ORGANIZER: Shixiao Fu, Shanghai Jiao Tong Univ, China; C0-ORGANIZERS: Svein Saevik, NTNU, Norway; Michael Tognarelli, $B P$ America Production Co., United States

Ocean Renewable Energy

9-1: ORGANIZER: Erin E. Bachynski, Norwegian University of Science and Technology, Norway; C0-ORGANIZER: Tonio Sant, Dept of Mechanical Engineering,University of Malta, Malta

9-2: ORGANIZER: Amy Robertson, National Renewable Energy Laboratory, United States; CO-ORGANIZER: Maurizio Collu, University of Strathclyde, United Kingdom

9-3: ORGANIZER: Yi-Hsiang Yu, National Renewable Energy Laboratory (NREL), United States; CO-ORGANIZER: Annie Dallman, Sandia National Laboratories, United States 9-4: ORGANIZER: Kelley Ruehl, Sandia National Laboratories, United States; CO-ORGANIZER: Masoud Hayatdavoodi, University of Dundee, United Kingdom

9-5: ORGANIZER: Madjid Karimirad, Queen's University Belfast, United Kingdom; CO-ORGANIZER: Marc Cahay, TechnipFMC, France

9-6: ORGANIZER: Pasquale Dinoi, Oceantec Marine Energy, Spain; C0-ORGANIZER: Claudie BENOIT, Bureau Veritas, France

9-7: ORGANIZER: Amir R. Nejad, Norwegian University of Science \& Technology, Norway; CO-ORGANIZER: Jan Helsen, Vrije Universiteit Brussel, Belgium

\section{Offshore Geotechnics}

10-1: ORGANIZER: Henry Milewski, TechnipFMC United Kingdom

10-2: ORGANIZER: Xuefei Wang, Hebei University of Technology, China

10-3: ORGANIZER: Fu-Ping Gao, Institute of Mechanics, Chinese Academy of Sciences, China

10-4: ORGANIZER: Susan Gourvenec, University of Southampton/Southampton Marine and Maritime Institute, United Kingdom

10-5: ORGANIZER: Severin Spill, Fraunhofer Institute for Wind Energy Systems IWES, Germany

10-6: ORGANIZER: Borana Kullolli, Bundesanstalt für Materialforschung -und prüfung, Germany

10-7: ORGANIZER: Manuela Kanitz, Hamburg University of Technology, Germany

Petroleum Technology

11-1: ORGANIZER: Stephen Butt, Memorial University of Newfoundland, Canada; CO-ORGANIZER: Mohammad Rahman, Texas A\&M Univ at Qatar, Qatar 
11-2: ORGANIZER: Jorge H B Sampaio Jr., Colorado School of Mines, United States 11-3: ORGANIZER: Arash Dahi Taleghani, The Pennsylvania State University, United States 11-4: ORGANIZER: Celso Morooka, University of Campinas, Brazil; CO-ORGANIZER: Sergio Bordalo, Unicamp, Brazil

11-5: ORGANIZER: Bernt Aadnoy, University of Stavanger, Norway

11-6: ORGANIZER: Jan David Ytrehus, SINTEF, Norway

11-7: ORGANIZER: Ergun Kuru, Univ Of Alberta, Canada

11-10: ORGANIZER: Mihail Minescu, University Oil And Gas Ploiesti, Romania

11-11: ORGANIZER: Ming Feng, Chongqing University, China; C0-ORGANIZER: wenting Qin, China University of Petroleum Beijing, China 11-12: ORGANIZER: Ian Frigaard, The University of British Columbia, Canada

11-13: ORGANIZER: Yuanhang Chen, Louisiana State University, United States; CO-ORGANIZER: Kjell Kåre Fjelde, University of Stavanger, Norway

11-15: ORGANIZER: Mahmoud Khalifeh, UiS, Norway; CO-ORGANIZER: Arild Saasen, UiS, Norway

Rodney Eatock Taylor Honouring Symposium on Marine and Offshore Hydrodynamics

12-2: ORGANIZER: Mamoun Naciri, Single Buoy Moorings Inc, Monaco

12-5: ORGANIZER: Kie Hian Chua, Technology Centre for Offshore and Marine, Singapore

12-7: ORGANIZER: Allan Magee, National

University of Singapore, Singapore

Takeshi Kinoshita Honoring Symposium on Offshore Technology

13-1: ORGANIZER: Takuji Waseda, The University of Tokyo, Japan

13-2: ORGANIZER: Rodolfo T. Gonçalves,

University of Tokyo, Japan; CO-ORGANIZER: Kazuo Nishimoto, University of San Paulo, Brazil; CO-ORGANIZER: Celso Morooka, University of Campinas, Brazil

13-3: ORGANIZER: Hideyuki Suzuki, University of Tokyo, Japan

13-7: ORGANIZER: Yasunori Nihei, Osaka

Prefecture University, Japan

\section{Session Organizers}

Offshore Technology

1-1-1: CHAIR: Johyun Kyoung, TechnipFMC, United States; C0-CHAIR: Sandeep Bukka Reddy, National University of Singapore, Singapore 1-1-2: CHAIR: Ewoud van Haaften, Shell Global Solutions International B.V., Netherlands; C0-CHAIR: Anil Sablok, TechnipFMC, United States
1-1-3: CHAIR: Bonjun Koo, TechnipFMC,

United States; CO-CHAIR: Mareike Leimeister, Fraunhofer IWES (Fraunhofer Institute for Wind Energy Systems), Germany

1-1-4: CHAIR: Partha Chakrabarti, Zentech Inc, United States; CO-CHAIR: Marc Cahay, TechnipFMC, France

1-1-5: CHAIR: Allan Magee, National University of Singapore, Singapore; CO-CHAIR: Erwan Auburtin, TechnipFMC, France

1-2-1: CHAIR: Allan Magee, National University of Singapore, Singapore; CO-CHAIR: Anil Sablok, TechnipFMC, United States

1-2-2: CHAIR: Djoni Sidarta, TechnipFMC, United States; C0-CHAIR: Jaakko Lehtonen, TechnipFMC Genesis, United States

1-2-3: CHAIR:Xinshu Zhang, Shanghai Jiaotong University, China; C0-CHAIRS: Dimitris Chalkias, GustoMSC, Netherlands; Xinliang Tian, Shanghai Jiao Tong University, China 1-2-4: CHAIR: David Molyneux, Memorial University of Newfoundland, Canada; CO-CHAIR: Allan Magee, National University of Singapore, Singapore

1-3-1: CHAIR: Longfei Xiao, Shanghai Jiao Tong University, China; CO-CHAIR: Zhenhua Huang, University of Hawaii at Manoa, United States 1-3-2: CHAIR: Tahsin Tezdogan, University of Strathclyde, United Kingdom; CO-CHAIR: Yibo Liang, University of Strathclyde, United Kingdom

1-4-1: CHAIR: M A Hannan, Newcastle University, UK (Singapore Unit), Singapore; CO-CHAIR: David Molyneux, Memorial University of Newfoundland, Canada 1-4-2: CHAIR: Yanlin Shao, Technical University of Denmark, Denmark; CO-CHAIR: Xu Xiang, Norwegian Public Roads Administration, Norway

1-4-3: CHAIR: Binbin zhao, Harbin Engineering University, China; CO-CHAIR: Masoud Hayatdavoodi, University of Dundee, United Kingdom

1-5-1: CHAIR: Wenhua Zhao, University of Western Australia, Australia; C0-CHAIR: Marc Cahay, TechnipFMC, France

1-6-1: CHAIR: Csaba Pakozdi, SINTEF Ocean, Norway

1-6-2: CHAIR: Arjen Koop, MARIN, Netherlands Antilles; CO-CHAIR: António Maximiano, WavEC - Offshore Renewables, Portugal 1-7-1: CHAIR: limin yang, dnvgl, Norway 1-7-2: CHAIR: Babak Ommani, SINTEF Ocean, Norway

\section{Structures, Safety and Reliability}

2-1-1: CHAIR: Elzbieta M. Bitner-Gregersen,

DNV GL AS, Norway; C0-CHAIR: Alexander V. Babanin, University of Melbourne, Australia 2-1-2: CHAIR: Alexander V. Babanin, University of Melbourne, Australia; C0-CHAIR: Elzbieta M. Bitner-Gregersen, DNV GL AS, Norway
2-2-1: CHAIR: Carlos Guedes Soares, Instituto Superior Técnico, Universidade de Lisboa, Portugal; CO-CHAIR: Felice Arena, Univ Mediterranea, Italy

2-2-2: CHAIR: Felice Arena, Univ Mediterranea, Italy; CO-CHAIR: Carlos Guedes Soares, Instituto Superior Técnico, Universidade de Lisboa, Portugal

2-3-1: CHAIR: Lance Manuel, University of Texas at Austin, United States; CO-CHAIR: SVERRE K. HAVER, University of Stavanger, Norway 2-4-1: CHAIR: Marcelo Igor Lourenço Souza, UFRJ, Brazil; CO-CHAIR: Fredhi Agung Prasetyo, Research \& Development Division, Biro Klasifikasi Indonesia, Indonesia

2-4-2: CHAIR: Nianzhong Chen, Tianjin University, China; C0-CHAIR: Fang Wang, Shanghai Ocean University, China

2-4-3: CHAIR: Yordan Garbatov, University of Lisbon, Portugal; CO-CHAIR: Myung-Hyun Kim, Pusan National University, Korea (Republic)

2-5-1: CHAIR: Carlos Guedes Soares, Instituto Superior Técnico, Universidade de Lisboa, Portugal; C0-CHAIR: Paulo M. Videiro, LACEO/ COPPE/Federal University of Rio De Janeiro, Brazil

2-8-1: CHAIR: Max Russo, Konsberg, Norway

2-6-1: CHAIR: Ying Min Low, National University of Singapore, Singapore; C0-CHAIR: Luis V.S. Sagrilo, LACEO/COPPE/Federal University of Rio De Janeiro, Brazil

2-6-2: CHAIR: Luis V.S. Sagrilo, LACEO/COPPE/ Federal University of Rio De Janeiro, Brazil; CO-CHAIR: Ying Min Low, National University of Singapore, Singapore

2-6-3: CHAIR: Ying Min Low, National University of Singapore, Singapore; CO-CHAIR: Luis V.S. Sagrilo, LACEO/COPPE/Federal University of Rio De Janeiro, Brazil

2-7-1: CHAIR: Zhen Gao, Norwegian University of Science and Technology, Norway; CO-CHAIR: Athanasios Kolios, University of Strathclyde, United Kingdom

2-9-1: CHAIR: Carlos Guedes Soares, Instituto Superior Técnico, Universidade de Lisboa, Portugal; CO-CHAIR: Luis V.S. Sagrilo, LACEO/ COPPE/Federal University of Rio De Janeiro, Brazil

2-9-2: CHAIR: Kazuhiro Iijima, Dept of NAOE, Osaka University, Japan; C0-CHAIR: Hans Bihs, NTNU Trondheim, Norway

2-9-3: CHAIR: Erik Vanem, DNV GL, Norway; C0-CHAIR: Guillaume de Hauteclocque, Bureau Veritas, France

2-9-4: CHAIR: YeongAe Heo, Case Western Reserve University, United States; CO-CHAIR: Deyu Wang, Shanghai Jiao Tong University, China

2-10-1: CHAIR: Sören Ehlers, Hamburg University of Technology, Germany; C0-CHAIR: Zhiqiang Hu, Newcastle University, United Kingdom 
2-10-2: CHAIR: Zhiqiang Hu, Newcastle University, United Kingdom; CO-CHAIR: Sören Ehlers, Hamburg University of Technology, Germany

2-11-1: CHAIR: Masahiko Fujikubo, Osaka University, Japan; C0-CHAIR: Yasuhira Yamada, Deyu Wang Shanghai Jiao Tong University, China

2-11-2: CHAIR: Yasuhira Yamada, Deyu Wang Shanghai Jiao Tong University, China; CO-CHAIR: Masahiko Fujikubo, Osaka University, Japan 2-11-3: CHAIR: Masahiko Fujikubo, Osaka University, Japan; C0-CHAIR: Yasuhira Yamada, Deyu Wang Shanghai Jiao Tong University, China

2-12-1: CHAIR: Jonas W. Ringsberg, Chalmers University of Technology, Sweden; C0-CHAIR: Paulo M. Videiro, LACEO/COPPE/Federal University of Rio De Janeiro, Brazil 2-12-2: CHAIR: Paulo M. Videiro, LACEO/ COPPE/Federal University of Rio De Janeiro, Brazil; CO-CHAIR: Jonas W. Ringsberg, Chalmers University of Technology, Sweden

2-12-3: CHAIR: Jonas W. Ringsberg, Chalmers University of Technology, Sweden; CO-CHAIR: Paulo M. Videiro, LACEO/COPPE/Federal University of Rio De Janeiro, Brazil

2-13-1: CHAIR: Marcelo Ramos Martins, LabRisk - Analysis, Evaluation and Risk Management Laboratory - University of São Paulo, Brazil; C0-CHAIR: Amir R. Nejad, Norwegian University of Science \& Technology, Norway

2-13-2: CHAIR: Marcelo Ramos Martins, LabRisk - Analysis, Evaluation and Risk Management Laboratory - University of São Paulo, Brazil; CO-CHAIR: Mahmood Shafiee, Cranfield University, United Kingdom

2-14-1: CHAIR: Bernt Leira, NTNU, Norway; CO-CHAIR: Nianzhong Chen, Tianjin University, China

2-15-1: CHAIR: YeongAe Heo, Case Western Reserve University, United States; C0-CHAIR: Bernt Leira, NTNU, Norway

\section{Materials Technology}

3-1-1: CHAIR:Xin Wang, Carleton University, Canada; CO-CHAIR: Myung-Hyun Kim, Pusan National University, Korea (Republic)

3-1-2: CHAIR: Carey L. Walters, Delft University of Technology, Netherlands; C0-CHAIR: Koji Gotoh, Kyushu University, Japan

3-2-1: CHAIR: Agnes Marie Horn, DNV GL, Norway; CO-CHAIR: Koji Gotoh, Kyushu University, Japan

3-2-2: CHAIR: Alexander Stacey, Energy Division, Health \& Safety Executive, United Kingdom; CO-CHAIR: Carol Johnston, TWI Ltd, United Kingdom

3-3-1: CHAIR: Yanhui Zhang, TWI Limited, United Kingdom; CO-CHAIR: Myung-Hyun Kim, Pusan National University, Korea (Republic)

3-3-2: CHAIR: Jens Tronskar, Det Norske Veritas Pte Ltd, Singapore; CO-CHAIR: Koji Gotoh, Kyushu University, Japan
3-3-3: CHAIR: Carol Johnston, TWI Ltd, United Kingdom; (0-CHAIR: Jens Tronskar, Det Norske Veritas Pte Ltd, Singapore

3-4-1: CHAIR: Agnes Marie Horn, DNV GL Norway; CO-CHAIR: Carol Johnston, TWI Ltd, United Kingdom

3-5-1: CHAIR: Carol Johnston, TWI Ltd, United Kingdom; C0-CHAIR: David A. Baker, Exxon Mobil Upstream Research Co, United States

3-6-1: CHAIR: Agnes Marie Horn, DNV GL, Norway; CO-CHAIR: Xin Wang, Carleton University, Canada

3-9-1: CHAIR: Sheng Bao, Zhejiang University, China; CO-CHAIR: Yanhui Zhang, TWI Ltd, United Kingdom;

3-11-1: CHAIR: Bostjan Bezensek, Shell Global Solutions UK, United Kingdom; C0-CHAIR: Isabel Hadley, TWI Ltd, United Kingdom 3-11-2: CHAIR: Isabel Hadley, TWI Ltd, United Kingdom; CO-CHAIR: Bostjan Bezensek, Shell Global Solutions UK, United Kingdom

3-13-1: CHAIR: Gerhard Ersdal, Petroleum Safety Authority, Norway; C0-CHAIR: Alexander Stacey, Energy Division, Health \& Safety Executive, United Kingdom

Pipelines, Risers, and Subsea Systems 4-1-1: CHAIR: Svein Saevik, NTNU, Norway; C0-CHAIR:Zhimin Tan, Baker Bughes, a GE company, United States

4-1-2: CHAIR:Zhimin Tan, Baker Bughes, a GE company, United States; C0-CHAIRS: Svein Saevik, NTNU, Norway; Adrian Connaire, Wood, Ireland

4-1-3: CHAIR: Murilo Augusto Vaz, COPPE/

UFRJ, Brazil; C0-CHAIR: Anh Tuan Do,

TechnipFMC, France

4-1-4: CHAIR: Anh Tuan Do, TechnipFMC,

France; (0-CHAIR: Murilo Augusto Vaz, COPPE/UFRJ, Brazil

4-1-5: CHAIR: Krassimir Doynov, ExxonMobil Upstream Integrated Solutions, United States; CO-CHAIR: Lin Zhao, Ocean university of China, China

4-1-6: CHAIR: Lin Zhao, Ocean university of China, China; C0-CHAIR: Krassimir Doynov, ExxonMobil Upstream Integrated Solutions, United States

4-1-7: CHAIR: José Renato M. de Sousa, Federal University of Rio de Janeiro, Brazil; C0-CHAIR: Farzan Parsinejad, Chevron, United States

4-1-10: CHAIR: Jun Yan, Dalian University of Technology, China; C0-CHAIR: Alan Dobson, Technip Umbilicals, United Kingdom

4-1-11: CHAIR: Alan Dobson, Technip Umbilicals, United Kingdom; CO-CHAIR: Jun Yan, Dalian University of Technology, China 4-2-1: CHAIR: Vishnu Vijayaraghavan, Aker Solutions Inc., United States

4-2-2: CHAIR: olav fyrileiv, DNV GL, Norway

4-2-3: CHAIR: Chenteh Alan Yu, ABS, United States

4-2-4: CHAIR: Alan Whooley, Wood Plc, United States
4-2-5: CHAIR: olav fyrileiv, $D N V G L$, Norway 4-3-1: CHAIR: Ilson Pasqualino, Coppe/ufrj, Brazil; C0-CHAIR: Spyros A. Karamanos, University of Thessaly, Greece

4-3-2: CHAIR: Julian Hallai, ExxonMobil, United States; CO-CHAIR: Chris Timms, C-FER Technologies, Canada

4-3-3: CHAIR: Theodoro Netto, COPPE/UFRJ, Brazil; C0-CHAIR: Duane DeGeer, INTECSEA United States

4-3-4: CHAIR: Rafael F. Solano, Petrobras, Brazil; C0-CHAIR: Celso Morooka, University of Campinas, Brazil

4-3-5: CHAIR: Daniel Carneiro, Wood, Brazil; C0-CHAIR: Rafael F. Solano, Petrobras, Brazil

4-3-6: CHAIR: Bostjan Bezensek, Shell, United Kingdom; CO-CHAIR: Andrew Cosham, Ninth Planet Engineering Ltd, United Kingdom 4-3-7: CHAIR: Celso Morooka, University of Campinas, Brazil; C0-CHAIR: Daniel Carneiro, Wood, Brazil

4-3-8: CHAIR: Doug Swanek, C-FER

Technologies, Canada; C0-CHAIR: Corey Drake, C-FER Technologies, Canada

4-4-1: CHAIR: Marcelo Igor Lourenço Souza, UFRJ, Brazil; CO-CHAIR: Ping Liu, INTECSEA, Netherlands

4-4-2: CHAIR: Duane DeGeer, INTECSEA United States; C0-CHAIR: Ruud Selker, INTECSEA, Netherlands

4-5-1: ORGANIZER: Daniel Carneiro, Wood, Brazil; CO-CHAIR: Hualei Yi, CNOOC Research Institute Co., Ltd, China; Paulo Paz, Federal University of Rio de Janeiro, Brazil

4-5-2: ORGANIZER: Daniel Carneiro, Wood, Brazil; C0-CHAIRS: Nestor Gonzalez Diez, TNO, Netherlands; Jongbae KIM, University of Ulsan, Korea (Republic)

4-6-1: CHAIR: Chris Timms, C-FER Technologies, Canada; CO-CHAIR: Ruud Selker, INTECSEA, Netherlands

4-6-2: CHAIR: Ping Liu, INTECSEA,

Netherlands; C0-CHAIR: Doug Swanek, C-FER Technologies, Canada

Ocean Space Utilization

5-1-1: CHAIR: Kazuhiro Iijima, Dept of NAOE, Osaka University, Japan

5-1-2: CHAIR: Fonseca Nuno, SINTEF Ocean, Norway; CO-CHAIR: Tomoki Ikoma, Nihon University, Japan

5-1-3: CHAIR: Maurizio Collu, University of Strathclyde, United Kingdom; CO-CHAIR: Tomoki Ikoma, Nihon University, Japan 5-2-1: CHAIR: Muk Chen Ong, University of Stavanger, Norway; CO-CHAIR: Lin Li, University of Stavanger, Norway

5-2-2: CHAIR: Yanlin Shao, Technical University of Denmark, Denmark; C0-CHAIR:Xu Xiang, Norwegian Public Roads Administration, Norway

5-3-1: CHAIR: Yoshiyasu Watanabe, Tokai University, Japan; C0-CHAIR: Marcio Yamamoto, National Maritime Research Institute, Japan 
5-4-1: CHAIR: Yoshitaka Watanabe, JAMSTEC, Japan; CO-CHAIR: Tomoya Inoue, JAMSTEC, Japan

5-5-1: CHAIR: Motohiko Murai, Yokohama National University, Japan; C0-CHAIR: Qiao Li, Institute of Industrial Science, the University of Tokyo, Japan

5-6-1: CHAIR: Koichi Masuda, Nihon University, Japan; CO-CHAIR: Koji Takahashi, Japan Port Consultants, Ltd., Japan

Ocean Engineering

6-1-2: CHAIR: Wei Qiu, Memorial University of Newfoundland, Canada

6-2-1: CHAIR: Masoud Hayatdavoodi, University of Dundee, United Kingdom

6-2-2:

6-2-3: CHAIR: Ian Robertson, University of Hawaii, United States

6-3-1: ORGANIZER: Pierre Ferrant, Ecole Centrale De Nantes/CNRS, France

6-3-2: CHAIR: Jeffrey Falzarano, Texas A\&M University, United States

6-3-3: ORGANIZER: Torgeir Kirkhorn Vada, DNV $G L$, Norway

6-3-4: ORGANIZER: Fonseca Nuno, SINTEF

Ocean, Norway

6-4-1: CHAIR: Lokukaluge Prasad Perera, UiT The Arctic University of Norway, Norway; CO-CHAIR: Yihan Xing, University of Stavanger, Norway

6-4-2: CHAIR: Lin Li, University of Stavanger, Norway; CO-CHAIR: Shuzheng Sun, Harbin Engineering University, China 6-4-3: CHAIR: Muk Chen Ong, University of Stavanger, Norway; C0-CHAIR: Guang Yin, University of Stavanger, Norway 6-4-4: CHAIR: Muk Chen Ong, University of Stavanger, Norway; CO-CHAIR: Zhenhui Liu, Aker Solutions AS, Norway

6-4-5: CHAIR: Zhengshun Cheng, Shanghai Jiao Tong University, China; CO-CHAIR: Xu Xiang, Norwegian Public Roads Administration, Norway

6-4-6: CHAIR: Muk Chen Ong, University of Stavanger, Norway; CO-CHAIR: Zhiyu Jiang, University of Agder, Norway

6-5-1: CHAIR: Gregor Macfarlane, Australian Maritime College, University of Tasmania, Australia; CO-CHAIR: Sanne van Essen, MARIN, Netherlands

6-5-2: CHAIR: Ilmas Bayati, MARIN Netherlands

6-5-3: CHAIR: Mohammad Rahmati, Northumbria University, United Kingdom; C0-CHAIR: Joop Helder, MARIN, Netherlands

6-5-4: CHAIR: Jule Scharnke, MARIN,

Netherlands; C0-CHAIR: Joost Sterenborg,

MARIN, Netherlands

6-7-1: CHAIR: Gus Jeans, Oceanalysis Ltd, United Kingdom; C0-CHAIR: Kevin Ewans, MetOcean Research Ltd, New Zealand
6-7-2: CHAIR: Gus Jeans, Oceanalysis Ltd, United Kingdom; CO-CHAIR: Kevin Ewans, MetOcean Research Ltd, New Zealand

6-7-3: CHAIR: Gus Jeans, Oceanalysis Ltd, United Kingdom; CO-CHAIR: Kevin Ewans, MetOcean Research Ltd, New Zealand

6-7-4: CHAIR: Gus Jeans, Oceanalysis Ltd, United Kingdom; C0-CHAIR: Kevin Ewans, MetOcean Research Ltd, New Zealand

6-8-1: CHAIR: Longbin Tao, University of Strathclyde, United Kingdom

6-8-2: CHAIR: Claudio A. Rodriguez C., Universidade Federal de Rio de Janeiro, Brazil 6-11-1: CHAIR: Celso Pesce, Univ. of S. Paulo - Escola Politecnica, Brazil; CO-CHAIR: Daniel Costa, COPPE/UFRJ, Brazil

6-11-2: CHAIR: Joel S. Sales Junior, Laboratory of Waves and Current - LOC - Universidade Federal do Rio de Janeiro, Brazil; CO-CHAIR: Milad shadman, COPPE/UFRJ, Brazil 6-12-1: CHAIR: Gus Jeans, Oceanalysis Ltd, United Kingdom

6-13-1: CHAIR: Atilla Incecik, University of Strathclyde, United Kingdom

6-13-2: CHAIR: Atilla Incecik, University of Strathclyde, United Kingdom

6-13-3: CHAIR: D.C. Wan, Shanghai Jiao Tong University, China

6-15-1: ORGANIZER: Eduardo Tannuri, Numerical Offshore Tank - University of São Paulo, Brazil; C0-CHAIR: Alexandre Immas, University of California, Berkeley, United States; Zhe Jiang, Shanghai Ocean University, China 6-15-2: ORGANIZER: Celso Morooka, University of Campinas, Brazil; C0-CHAIR: Zhe Jiang, Shanghai Ocean University, China; Alexandre Immas, University of California, Berkeley, United States

6-17-1: CHAIR: Sanne van Essen, MARIN, Netherlands

6-17-2: CHAIR: Thomas A.A. Adcock, University of Oxford, United Kingdom

Polar and Arctic Sciences and Technology 7-1-1: CHAIR: Walter Kuehnlein, sea2ice Ltd. \& Co. KG, Germany; CO-CHAIR: Sören Ehlers, Hamburg University of Technology, Germany 7-3-1: CHAIR: Michael Huisman, Hamburg University of Technology, Germany; C0-CHAIR: Walter Kuehnlein, sea2ice Ltd. \& Co. KG, Germany

7-4-1: CHAIR: Sören Ehlers, Hamburg University of Technology, Germany; CO-CHAIR: Walter Kuehnlein, sea2ice Ltd. \& Co. KG, Germany 7-11-1: CHAIR: Walter Kuehnlein, sea2ice Ltd. \& Co. KG, Germany; C0-CHAIR: Sören Ehlers, Hamburg University of Technology, Germany 7-12-1: CHAIR: Sören Ehlers, Hamburg University of Technology, Germany; C0-CHAIR: Walter Kuehnlein, sea2ice Ltd. \& Co. KG, Germany

\section{CFD \& FSI}

8-1-1: CHAIR: Allan Magee, National University of Singapore, Singapore; (0-CHAIR: Hyunchul Jang, TechnipFMC, United States

8-1-2: CHAIR: Luis Eca, Technical University of Lisbon, Portugal; CO-CHAIR: Owen H. Oakley, Jr, Retired, United States

8-1-3: CHAIR: Ould el Moctar, University of Duisburg-Essen, Germany; C0-CHAIR: Prasanta Sahoo, Florida Institute of Technology, United States

8-1-4: CHAIR: Prasanta Sahoo, Florida Institute of Technology, United States; C0-CHAIR: Ould el Moctar, University of Duisburg-Essen, Germany

8-1-5: CHAIR: Sandy Day, University of Strathclyde, United Kingdom; CO-CHAIR: Kie Hian Chua, Technology Centre for Offshore and Marine, Singapore

8-1-6: CHAIR: Steve Cosgrove, Altair

Engineering, Inc., United States; C0-CHAIR: Samuel Holmes, Red Wing Engineering, Inc, United States

8-1-7: CHAIR: Karl Halse, Norwegian University of Science and Technology, Norway; CO-CHAIR: Samuel Holmes, Red Wing Engineering, Inc, United States

8-2-1: CHAIR: Hans Bihs, NTNU Trondheim, Norway; CO-CHAIR: Arun Kamath, NTNU, Norway

8-2-2: CHAIR: Tim Bunnik, MARIN,

Netherlands; C0-CHAIR: Csaba Pakozdi, SINTEF Ocean, Norway

8-2-3: CHAIR: Arun Kamath, NTNU, Norway; CO-CHAIR: Csaba Pakozdi, SINTEF Ocean, Norway

8-3-1: CHAIR: Rajeev Kumar Jaiman, University of British Columbia - Vancouver, Canada; CO-CHAIR: Luis Eca, Technical University of Lisbon, Portugal

8-3-2: CHAIR: Luis Eca, Technical University of Lisbon, Portugal; CO-CHAIR: Rajeev Kumar Jaiman, University of British Columbia Vancouver, Canada

8-4-1: CHAIR: Michael Bernitsas, University of Michigan, United States; CO-CHAIR: Long Ge, $B P$, United States

8-4-2: CHAIR: Hai Sun, Harbin Engg Univ, China; CO-CHAIR: Aravind Nair, DNV GL, United States

8-4-3: CHAIR: Rajeev Kumar Jaiman, University of British Columbia - Vancouver, Canada; CO-CHAIR: Mohammed Abdul Hannan, Newcastle University, UK (singapore Campus), Singapore

8-4-4: CHAIR: Decao Yin, SINTEF Ocean, Norway; CO-CHAIR: Madhusuden Agrawal, BP, United States

8-5-1: CHAIR: Madhusuden Agrawal, BP, United States; CO-CHAIR: Yuwang Xu, Shanghai Jiao Tong University, China

8-5-2: CHAIR: Yiannis Constantinides, Chevron United States; C0-CHAIR: Owen H. Oakley, Jr, Retired, United States 
Ocean Renewable Energy

9-1-1: CHAIR: Wojciech Popko, Fraunhofer Institute for Wind Energy Systems IWES, Germany; CO-CHAIR: Tonio Sant, Dept of Mechanical Engineering,University of Malta, Malta

9-1-2: CHAIR: Alessandro Fontanella, Politecnico di Milano, Italy; CO-CHAIR: Ilmas Bayati, MARIN, Netherlands

9-1-3: CHAIR: Carlos Souza, NTNU, Norway; C0-CHAIR: Wei Shi, Dalian University of Technology, China

9-1-5: CHAIR: Tonio Sant, Dept of Mechanical Engineering, University of Malta, Malta; C0-CHAIR: Erin E. Bachynski, Norwegian University of Science and Technology, Norway 9-1-8: CHAIR: Jean-Christophe Gilloteaux, Ecole Centrale de Nantes, France

9-2-1: CHAIR: Jason Jonkman, National Renewable Energy Laboratory, United States 9-2-2: CHAIR: Kurt Delpeche, Pacifico Energy K.K., Japan; CO-CHAIR: Nigel Terry, Marine Renewable Engineering, United Kingdom 9-2-3: CHAIR: Amy Robertson, National Renewable Energy Laboratory, United States 9-2-6: CHAIR: Maurizio Collu, University of Strathclyde, United Kingdom; CO-CHAIR: K A Abhinav, University of Strathclyde, United Kingdom

9-3-1: CHAIR: John Ringwood, Maynooth University, Ireland

9-3-2: CHAIR: Kourosh Rezanejad, Centre for Marine Technology and Ocean Engineering (CENTEC), Instituto Superior Técnico(IST), Portugal; (0-CHAIR: Alessandra Romolo, University Mediterranea, Italy 9-3-3: CHAIR: Jennifer van Rij, National Renewable Energy Laboratory (NREL), United States

9-4-1: CHAIR: Masoud Hayatdavoodi, University of Dundee, United Kingdom; CO-CHAIR: Jerica Nolte, Principle Power, France; CHAIR: Kelley Ruehl, Sandia National Laboratories, United States

9-4-2: CHAIR: Kelley Ruehl, Sandia National Laboratories, USA; CO-CHAIR: Ryan Coe, Sandia National Laboratories, United States

9-4-3: CHAIR: Ryan Coe, Sandia National Laboratories, United States

9-4-4: CHAIR: Yi-Hsiang Yu, National Renewable Energy Laboratory (NREL), United States

9-5-2: CHAIR: Madjid Karimirad, Queen's University Belfast, United Kingdom; CO-CHAIR: Wei Shi, Dalian University of Technology, China

9-5-3: CHAIR: Marc Cahay, TechnipFMC, France; C0-CHAIR: Qing Xiao, University of Strathclyde, United Kingdom

9-5-4: CHAIR: Madjid Karimirad, Queen's University Belfast, United Kingdom; CO-CHAIR: Milad Armin, Liverpool John Moores University, United Kingdom

9-6-1: CHAIR: Fabio Licheri, University of Cagliari, Italy
9-7-1: CHAIR: Amir R. Nejad, Norwegian University of Science \& Technology, Norway; CO-CHAIR: Jan Helsen, Vrije Universiteit Brussel, Belgium

\section{Offshore Geotechnics}

10-1-1: CHAIR: Henry Milewski, TechnipFMC, United Kingdom

10-3-1: CHAIR: Fu-Ping Gao, Institute of Mechanics, Chinese Academy of Sciences, China

10-4-1: CHAIR: Susan Gourvenec, University of Southampton/Southampton Marine and Maritime Institute, United Kingdom

10-5-1: CHAIR: Tulio Quiroz, Fraunhofer Institute for Wind Energy Systems IWES, Germany

10-6-1: CHAIR: Borana Kullolli, Bundesanstalt für Materialforschung -und prüfung, Germany

10-7-1: CHAIR: Manuela Kanitz, Hamburg University of Technology, Germany

\section{Petroleum Technology}

11-1-1: CHAIR: Stephen Butt, Memorial University of Newfoundland, Canada; CO-CHAIR: Mohammad Rahman, Texas A\&M Univ at Qatar, Qatar

11-1-2: CHAIR: Stephen Butt, Memorial University of Newfoundland, Canada; CO-CHAIR: Mohammad Rahman, Texas A\&M Univ at Qatar, Qatar

11-1-3: CHAIR: Stephen Butt, Memorial University of Newfoundland, Canada; CO-CHAIR: Mohammad Rahman, Texas A\&M Univ at Qatar, Qatar

11-2-1: CHAIR: Jorge H B Sampaio Jr., Colorado School of Mines, United States

11-2-2: CHAIR: Jorge H B Sampaio Jr., Colorado School of Mines, United States

11-3-1: CHAIR: Arash Dahi Taleghani, The Pennsylvania State University, United States 11-4-1: ORGANIZER: Sergio Bordalo, Unicamp, Brazil; CO-CHAIR: Juliana Baioco, LAMCSO/ COPPE/UFRJ, Brazil; Lucas Sevillano, State University of Campinas, UNICAMP, Brazil 11-5-1: CHAIR: Bernt Aadnoy, University of Stavanger, Norway

11-6-1: CHAIR: Jan David Ytrehus, SINTEF Norway

11-6-2: CHAIR: Jan David Ytrehus, SINTEF, Norway

11-7-1: CHAIR: Arild Saasen, UiS, Norway; C0-CHAIR: Ergun Kuru, Univ Of Alberta,

Canada

11-7-2: CHAIR: Ergun Kuru, Univ Of Alberta, Canada; CO-CHAIR: Majid Bizhani, University of British Columbia, Canada

11-7-3: CHAIR: Ergun Kuru, Univ Of Alberta, Canada

11-10-1: CHAIR: Mihail Minescu, University Oi And Gas Ploiesti, Romania; CO-CHAIR: Catalin Teodoriu, Mewbourne School of Petroleum and Geological Engineering, United States
11-11-1: CHAIR: Ming Feng, Chongqing University, China; C0-CHAIR: wenting Qin, China University of Petroleum Beijing, China 11-11-2: CHAIR: Ming Feng, Chongqing University, China; C0-CHAIR: wenting Qin, China University of Petroleum Beijing, China 11-12-1: CHAIR: Ian Frigaard, The University of British Columbia, Canada; C0-CHAIR: Ali Etrati, University of British Columbia, Canada

11-12-2: CHAIR: Ian Frigaard, The University of British Columbia, Canada; CO-CHAIR: Majid Bizhani, The University of British Columbia, Canada

11-13-1: CHAIR: Yuanhang Chen, Louisiana State University, United States; C0-CHAIR: Kjell Kåre Fjelde, University of Stavanger, Norway 11-15-1: CHAIR: Mahmoud Khalifeh, UiS, Norway

11-15-2: CHAIR: Mahmoud Khalifeh, UiS, Norway

Rodney Eatock Taylor Honouring Symposium on Marine and Offshore Hydrodynamics

12-1-1: CHAIR: Paul Taylor, Univ Of Oxford, United Kingdom; C0-CHAIR: Kie Hian Chua, Technology Centre for Offshore and Marine, Singapore

12-1-2: CHAIR: Arne Løken, Dr. Ing. Arne E. Løken Engineering \& consulting, Norway; C0-CHAIR: Xinshu Zhang, Shanghai Jiaotong University, China

12-2-1: CHAIR: Wei Bai, Manchester Metropolitan University, United Kingdom; C0-CHAIR: Wei Qiu, Memorial University of Newfoundland, Canada

12-4-1: ORGANIZER:Xingya Feng, University of Oxford, United Kingdom; C0-CHAIR: Deborah Greaves, University of Plymouth, United Kingdom

12-5-1: CHAIR: Ronald W. Yeung, University of California at Berkeley, United States; CO-CHAIR: Mamoun Naciri, Single Buoy Moorings Inc, Monaco

12-5-2: CHAIR: Robert Beck, University of Michigan, United States

12-7-1: CHAIR: Allan Magee, National University of Singapore, Singapore; C0-CHAIR: Longbin Tao, University of Strathclyde, United Kingdom

Takeshi Kinoshita Honoring Symposium on Offshore Technology

13-1-1: CHAIR: Kevin Ewans, MetOcean Research Ltd, New Zealand

13-1-2: ORGANIZER: Takanori Hino, Yokohama National University, Japan

13-1-3: ORGANIZER: Alessandro Iafrati, CNR-INM (INstitute of Marine Engineering), Italy 13-1-4: ORGANIZER: Takuji Waseda, The University of Tokyo, Japan

13-2-1: CHAIR: Celso Morooka, University of Campinas, Brazil; C0-CHAIR: Yuri Coelho Del Sarto, Universidade Estadual de Campinas, Brazil 
13-2-2: CHAIR: Marcio Yamamoto, National Maritime Research Institute, Japan; CO-CHAIR: Celso Morooka, University of Campinas, Brazil

13-2-3: CHAIR: Rodolfo T. Gonçalves, University of Tokyo, Japan; C0-CHAIR: Longfei Xiao, Shanghai Jiao Tong University, China 13-2-4: CHAIR: Longfei Xiao, Shanghai Jiao Tong University, China; CO-CHAIR: Shinichiro Hirabayashi, The University of Tokyo, Japan 13-2-5: CHAIR: Shinichiro Hirabayashi, The University of Tokyo, Japan

13-3-1: ORGANIZER: Tomoaki Utsunomiya, Kyushu University, Japan; CO-ORGANIZER: Hideyuki Suzuki, University of Tokyo, Japan 13-3-2: ORGANIZER: Tomoki Ikoma, Nihon University, Japan; CO-ORGANIZER: Changkyu Rheem, The University of Tokyo, Japan; Yukitaka Yasuzawa, Kyushu University, Japan 13-3-3: ORGANIZER: Yusaku Kyozuka, Nagasaki University, Japan; C0-CHAIR: Motohiko Murai, Yokohama National University, Japan 13-3-4: CHAIR: Yasunori Nihei, Osaka Prefecture University, Japan

\section{Event Organizer}

Sea to Sky Meeting and Association

Management Inc.

206, 201 Bewicke Avenue

North Vancouver, BC V7M 3M7

Canada

Tel: +1-604-984-6455

www.seatoskymeetings.com

omae@seatoskymeetings.com

President: Sarah Lowis

Project Director: Ian Holliday

Project Manager: Bernadette Gessler

Registration Manager: Christina Gonzalez Fraser

\section{Co-sponsoring Organizations}

\begin{tabular}{|c|c|c|}
\hline Association/Institution & Country & Representative \\
\hline $\begin{array}{l}\text { American Society of Mechanical Engineers } \\
\text { International }\end{array}$ & USA & Denby Morrison \\
\hline American Concrete Institute & USA & Diane L. Baloh \\
\hline $\begin{array}{l}\text { American Society of Civil Engineers, Coasts, } \\
\text { Oceans, Ports, and Rivers Institute }\end{array}$ & USA & Zeki Demirbilek \\
\hline $\begin{array}{l}\text { Associazione Italiana di Ingegneria Offshore e } \\
\text { Marina }\end{array}$ & Italy & Elio Ciralli \\
\hline $\begin{array}{l}\text { Università degli Studi Mediterranea di Reggio } \\
\text { Calabria }\end{array}$ & Italy & Felici Arena \\
\hline Brazilian Society of Naval Architects & Brazil & Amte Bittencourt \\
\hline Canadian Association of Petroleum Producers & Canada & \\
\hline Canadian Society for Mechanical Engineering & Canada & \\
\hline Chinese Society of Ocean Engineers & China & J.M. Yang \\
\hline $\begin{array}{l}\text { Conseil de Liaison des Assoc. de Recherche } \\
\text { sur les Ouvrages en Mer }\end{array}$ & France & Jacques Burger \\
\hline Energy Rubber Group & & Steve Jagels \\
\hline Engineering Committee on Oceanic Research & & David Molyneux \\
\hline European Safety and Reliability Association & Portugal & Carlos Guedes Soares \\
\hline Gesellschaft fuer Maritime Technik & Germany & Walter Kuehnlein \\
\hline \multicolumn{3}{|l|}{ Institute of Materials } \\
\hline Institution of Engineers of Ireland & Ireland & Paddy Purcell \\
\hline Institution of Mechanical Engineers & UK & \\
\hline Instituto Brasileiro de Petróleo & Brazil & Alvaro Teixeira \\
\hline Japan Society of Mechanical Engineers & Japan & Shinobu Saitou \\
\hline $\begin{array}{l}\text { Japan Society of Naval Architects of and } \\
\text { Ocean Engineers }\end{array}$ & Japan & \\
\hline Korean Institute of Metals & Korea & \\
\hline Marine Technology Society & USA & \\
\hline National Association of Corrosion Engineers & USA & \\
\hline Norwegian Society of Chartered Engineers & Norway & Ketill Borge-Ask \\
\hline Office of Science and Technology, Australia & Australia & \\
\hline Portuguese Engineering Association & Portugal & Pedro Ponte \\
\hline $\begin{array}{l}\text { The Petroleum Society of CIM (Canadian } \\
\text { Institute of Mining) }\end{array}$ & Canada & Yvan Jacques \\
\hline Royal Flemish Engineers Association & Belgium & Hans Romaen \\
\hline Society of Danish Engineers & Denmark & Kristian Smestad \\
\hline $\begin{array}{l}\text { Society of Naval Architects and Marine } \\
\text { Engineers }\end{array}$ & USA & Michael Bernitsas \\
\hline $\begin{array}{l}\text { Society of Naval Architects and Marine } \\
\text { Engineers }\end{array}$ & $\begin{array}{l}\text { Taiwan, } \\
\text { China }\end{array}$ & Chang New Chen \\
\hline Society for Underwater Technology & UK & Ian Gallett \\
\hline TWI (formerly The Welding Institute) & UK & Carol Johnston \\
\hline
\end{tabular}




\section{PAST \& FUTURE CONFERENCES}

Past Conferences
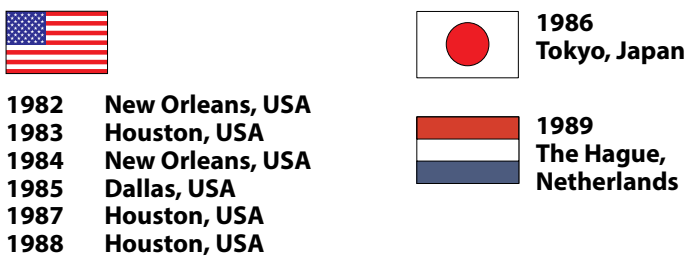

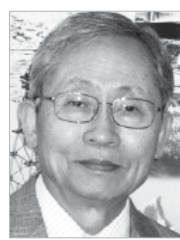

Jin S. Chung

Chair 1982-1989

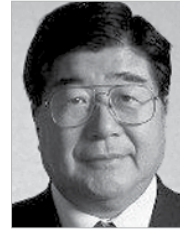

Hisaaki Maeda Virgil Lunardini

Co-chair Co-chair

$1986 \quad 1984 \& 1985$

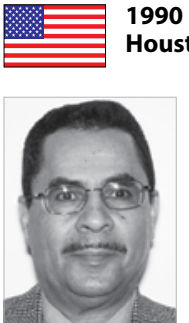

Mamdouh Salama Chair, 1990-1991

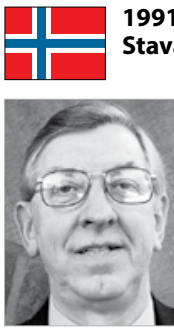

Bjorn Lian

Co-chair
1991 Stavanger, Norway

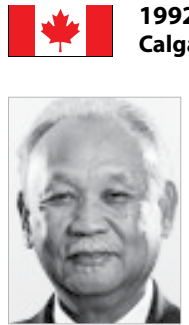

M. Idris Mansor R. Brawn Chair Co-chair
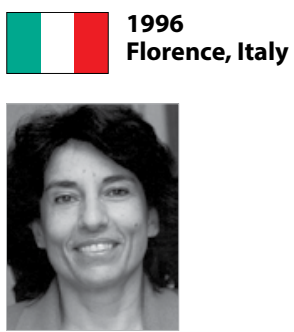

Daniela Mercati Chair

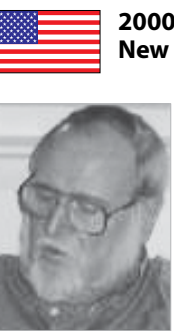

Stephen Jones Chair

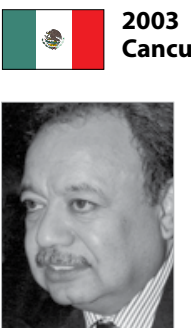

Oscar Valle Molina

Chair

Copenhagen

1999

St. John's, Canada

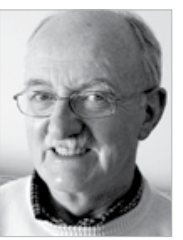

Carlos Guedes Soares Chair

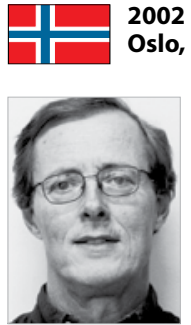

Arvid Naess

Chair
002

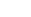
Denmark

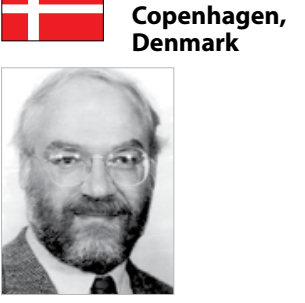

Christian Aage
Terry Jones (1934-2005) Chair

00

ew Orleans, USA

사 2004

Vancouver, Canada

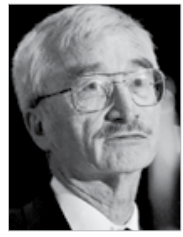

Sander Calisal

Chair

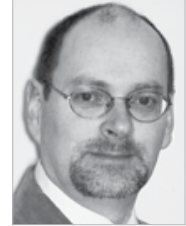

Jon Mikkelsen Co-chair
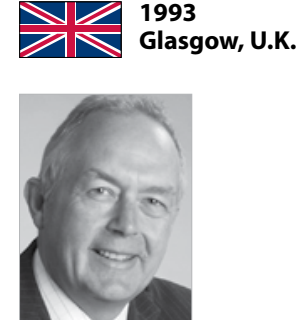

Geoff Booth

Chair 


\section{PAST \& FUTURE CONFERENCES}

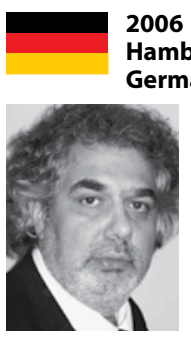

Walter L. Kuehnlein Chair

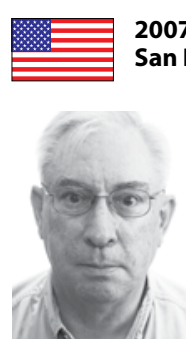

John Halkyard Chair

2007
Diego, USA

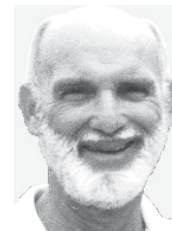

Richard Seymour

Co-chair
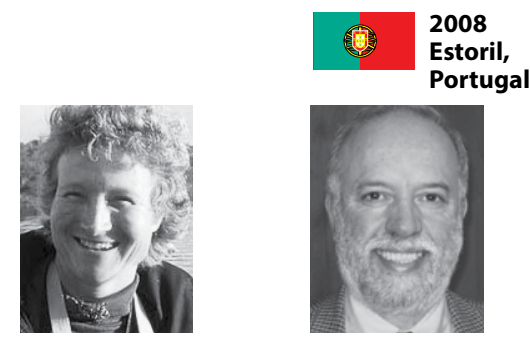

Barbara Fletcher Co-chair

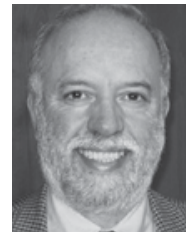

Carlos Guedes Soares Chair

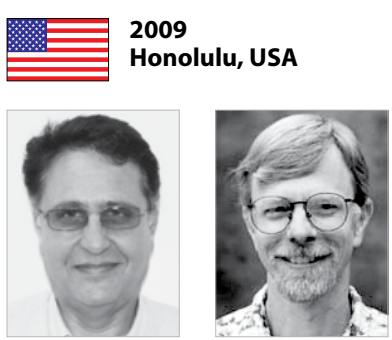

R. Cengiz Ertekin H. Ronald Riggs

Chair Co-chair

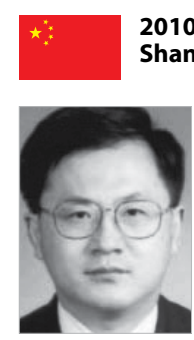

Gang Chen

Chair

2010

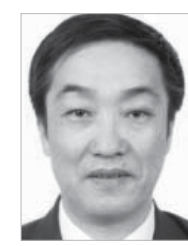

Jianmin Yang

Co-chair
Shanghai, China

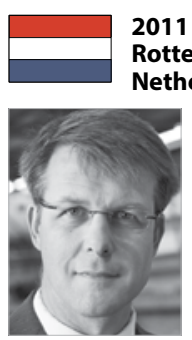

Bas Buchner Chair

2011 otterdam, therlands

2014

San Francisco, USA

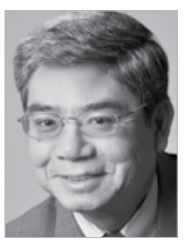

Ronald W. Yeung Chair

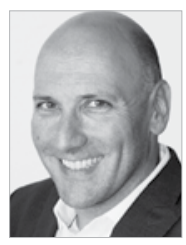

Dominique Roddier Co-chair

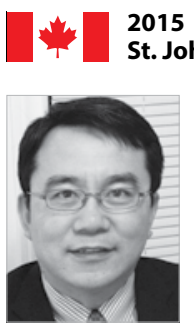

Wei Qiu

Chair

2015
John's, Canada

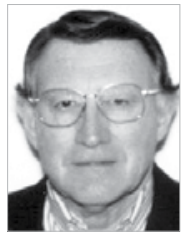

Charles Smith

Co-chair

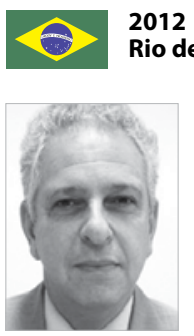

Segen F. Estefen Chair

2012 o de Janeiro, Brazil

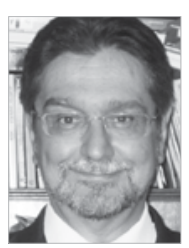

Antonio C. Fernandes Co-chair

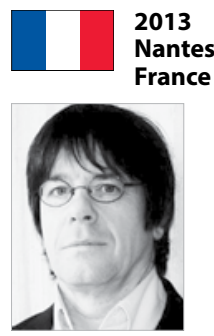

Pierre Ferrant Chair

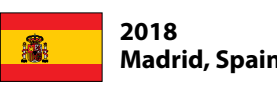

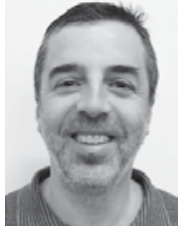

Antonio

Souto-Iglesias

Chair

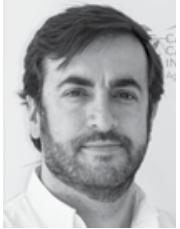

Raúl Guanche

Chair

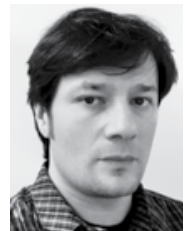

Francisco

Huera-Huarte Chair
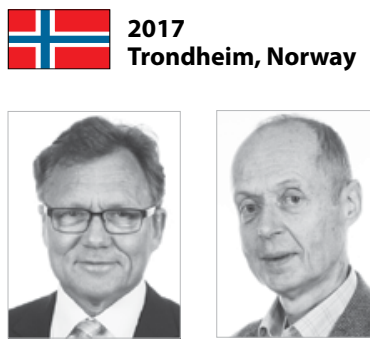

Bernt J. Leira Chair

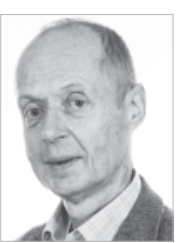

Atle Minsaas Co-chair

\section{Future Conferences}

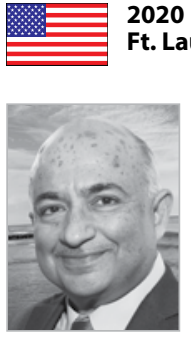

Manhar Dhanak Chair

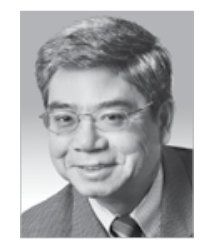

Ronald W. Yeung Co-Chair

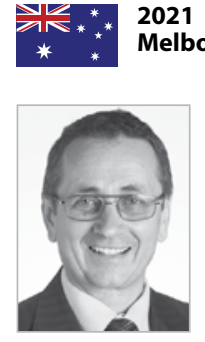

2021

elbourne, Australia

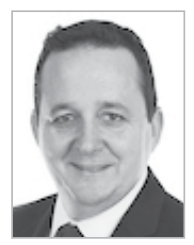

Alexander Babanin Hayden Marcollo Chair Co-Chair

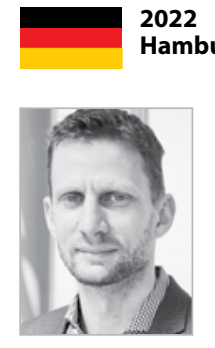

Soren Ehlers Chair

2022
Hamburg, Germany

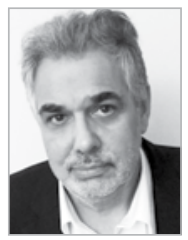

Walter Kuehnlein Co-Chair 


\section{Author Index}

\section{A}

Aadnoy, Bernt.

Aardal, Andreas Buvar

Aarnes, Ole Johan.

Aarsaether, Karl Gunnar

OMAE2019-95414

(5-2-1), OMAE2019-96079(5-2-2)

Abaei, Mohammad Mahdi ................. OMAE2019-95099 (9-6-1)

Abbad el Andaloussi, Hamza ................ OMAE2019-96239 (3-3-1)

Abbas, Muntazir ............................ OMAE2019-96227 (3-9-1)

Abdelrahman, Mustafa _..._......... OMAE2019-95619 (9-3-1)

Abdollahpour, Saeid ......................... OMAE2019-95380 (11-6-1)

Abhinav, K A ........................... OMAE2019-96212 (5-1-2)

Abobaker, Ekhwaiter ........................ OMAE2019-96311 (11-4-1)

Aborig, Amer................................... OMAE2019-95005 (4-5-1)

Abrahamsen-Prsic, Mia ....................... OMAE2019-96008 (6-3-3)

Abreu, Danilo T. M. P. _ _ _ _ _ _ . OMAE2019-96251 (2-13-2

OMAE2019-96269 (2-13-1), OMAE2019-96280 (2-8-1)

Abugharara, Abdelsalam ................... OMAE2019-96176 (11-1-3)

Achary, Gudimella G. S.,........................ OMAE2019-969-95467 (1-4-2)

OMAE2019-95467 (1-4-2)

Adami, Nasim .............................. OME2019-96802 (9-4-3)

Adcock, Thomas A.A. ...................... OMAE2019-95068 (6-17-2)

OMAE2019-95075 (9-6-1), OMAE2019-95161 (12-5-1)

OMAE2019-95172 (12-1-1), OMAE2019-95299 (6-17-2)

OMAE2019-95347 (9-5-3), OMAE2019-95767 (9-5-2)

OMAE2019-96082 (6-17-1), OMAE2019-96817 (12-5-1)

Addala, Mahesh Babu ...................... OMAE2019-95467 (1-4-2) OMAE2019-95470 (4-3-6)

Adedipe, Tosin ............................... OMAE2019-95539 (2-13-1)

Adiputra, Ristiyanto ............................... OMAE2019-96749 (13-3-3)

Aesoy, Lene OMAE2019-96689 (7-11-1)

Afanassiev, Anton

Afzali, Soroosh.....

Agarwal, Shagun

Aggarwal, Ankit.

Aguiar, Ludimar L.

Aguiari, Martina

Aguilar, Aaron

Aguilar, Ramon

Ahammad, Mohammad

Åhjem, Vidar R.

Aida, Yasuhiro

OMAE2019-95283 (6-4-6)

OMAE2019-95837 (9-2-6)

OMAE2019-96722 (7-12-1) OMAE2019-96745 (6-3-2) OMAE2019-95233 (8-3-1)

OMAE2019-96297 (4-2-5) OMAE2019-96592 (4-2-4) OMAE2019-96639 (11-2-2) OMAE2019-95130 (3-1-1) OMAE2019-96345 (3-5-1) OMAE2019-96345 (3-5-1) OMAE2019-96373 (11-1-2) OMAE2019-95469 (1-2-2) OMAE2019-95408 (5-6-1) OMAE2019-95925 (13-3-4), OMAE2019-95927 (5-6-1) OMAE2019-96045 (13-3-1), OMAE2019-96482 (13-2-5) OMAE2019-96493 (13-2-5) Akbari, Babak ……………........ OMAE2019-95330 (11-15-1) Aksnes, Vegard. Al Furati, Beder. Al Ramadan, Mustafa OMAE2019-96003 (11-5-1) OMAE2019-95589 (11-12-1) OMAE2019-95612 (11-15-1)

Al-Anjari, Noor.

Al-Houti, Dana...

Al-Osairi, Yousef.

Al-SAlem, Khaled.

Alam, Jahrul.

Alam, Jahrul.

Alam, Mohammad-Reza OMAE2019-95236 (6-2-2) OMAE2019-95236 (6-2-2) OMAE2019-95236 (6-2-2) OMAE2019-95236 (6-2-2) OMAE2019-95837 (9-2-6) OMAE2019-96373 (11-1-2) OMAE2019-96270 (6-15-1)

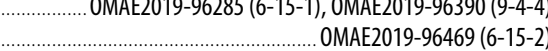
Alba, Kamran ............................... OMAE2019-95218 (11-12-2) Alem, Nasim ............................ OMAE2019-96421 (11-3-1) Alexandre, Armando ............................ OMAE2019-95429 (9-1-1) Alleva, Laura OMAE2019-95953 (3-6-1) Allix, Olivier..................................... OMAE2019-96165 (1-2-4) Allsop, Steven AlMudairis, Fahad.

Alom, Nur.

AlOtaibi, Fawaz.

Al0asim, Abdulaziz

Alrsai, Mahmoud OMAE2019-95002 (11-4-1) OMAE2019-95570 (9-2-2) OMAE2019-95191 (11-1-1) OMAE2019-95002 (11-4-1)
Alsos, Hagbart

Altomare, Corrado

Alver, Morten Omholt

Amaechi, Chiemela Victor

Amaro, Rubens Augusto Jr.

Amaroju, Kartheek

Ambrus, Adrian

Amendola, José

Amini, Shakib

Amirafshari, Peyman

An, Chen

An, Hongwei.

An, Song

An, Weijian..

An, Xiaoxue...

Anaya-Lara, Olimpo.

Andersen, Terje L.

Anderson, Mitchell

Anderson, Ted...

Anderson, Tim... OMAE2019-96556 (3-4-1)

Ando, Takahiro OMAE2019-96593 (3-4-1), OMAE2019-96614 (3-4-1)

Andrews, Robert

Ang, Kok Keng

Ang, Sze Yu

\section{Anokhin, Vadim}

Antonini, Alessandro

Antunes, Bruno R. OMAE2019-95289 (4-3-5)

OMAE2019-95389 (2-9-3) OMAE2019-95532 (3-11-2) .. OMAE2019-96789 (5-1-3) OMAE2019-95970 (8-4-4) . OMAE2019-95972 (8-4-1) OMAE2019-95913 (6-7-4) . OMAE2019-96262 (6-3-4) OMAE2019-95298 (4-3-5), OMAE2019-95343 (4-3-4) Anyfantis, Konstantinos .................... OMAE2019-96708 (2-11-2) Aoki, Masahiro .............................. OMAE2019-95912 (2-10-2) Araujo, Jairo _................................. OMAE2019-96575 (1-4-3) Arboleda Chavez, Carlos ..................... OMAE2019-95793 (10-4-1) Arena, Felice... OMAE2019-95880 (2-2-2)

OMAE2019-95973 (9-3-2), OMAE2019-96104 (5-1-2) OMAE2019-96212 (5-1-2), 0MAE2019-96282 (5-1-2) OMAE2019-96368 (9-4-1)

Arild, Øystein ............................... OMAE2019-96546 (11-15-2) Arini, Nu Rhahida ................................ OMAE2019-95099 (9-6-1) OMAE2019-95998 (9-5-2), OMAE2019-96007 (9-5-4) Armaoglu, Evren .............................. OMAE2019-95001 (1-3-1) Armesto, José................................. OMAE2019-96380 (9-1-2

Armin, Milad . $\ldots$ OMAE2019-95227 (9-5-2) Arnal, Vincent Arnout, Jonas

Arora, Vikas...

Arredondo, Alberto

Arslan, Haydar ....

Arumugam Elumalai, Venkatesan OMAE2019-96374 (9-1-2) OMAE2019-95793 (10-4-1) OMAE2019-95704 (1-4-2) OMAE2019-95618 (2-6-2) OMAE2019-96156 (4-2-4) OMAE2019-95291 (4-6-2) OMAE2019-96289 (2-1-1) OMAE2019-96395 (9-1-5) Assi, Gustavo R. S. OMAE2019-95353 (13-2-4), OMAE2019-95749 (13-2-3) Astrup, Ole Christian Atack, Timothy $\mathrm{H}$. Atienza, Meliza

OMAE2019-96587 (6-7-4) .. OMAE2019-96104 (5-1-2) OMAE2019-95970 (8-4-4) .. OMAE2019-95972 (8-4-1)

Atlar, Mehmet. OMAE2019-95315 (8-1-7) OMAE2019-96276 (6-5-4)

Aubeny, Charles Aubert, Jean-Michel

Aubrun, Sandrine.

Auburtin, Erwan

Augusto, Carlos

Aune, Robert.

Auriac, Bertrand

Awad, M. M. OMAE2019-96395 (9-1-5) OMAE2019-96631 (3-3-2) OMAE2019-96374 (9-1-2) OMAE2019-96090 (1-5-1) OMAE2019-95369 (3-3-2) OMAE2019-96329 (3-4-1)

Awe, Oluwaseun M OMAE2019-95429 (9-1-1) .. OMAE2019-95005 (4-5-1) OMAE2019-95219 (11-1-1) OMAE2019-95019 (4-5-1)
B

Babanin, Alexander V. OMAE2019-95633 (13-1-3) ... OMAE2019-95740 (2-1-2) Babarit, Aurelien ................................. OMAE2019-96296 (9-1-8) Bacelli, Giorgio ................................. OMAE2019-95216 (9-3-1) OMAE2019-96382 (9-4-3) Bachynski, Erin E................................ OMAE2019-95170 (9-1-1) OMAE2019-95929 (6-5-1), OMAE2019-96680 (6-3-2) Baessler, Matthias ............................ OMAE2019-96078 (10-7-1) Bahtui, Ali ....................................... OMAE2019-95274 (4-1-5) Bai, Tiecha0................................. OMAE2019-95874 (8-1-7) Bai, Wei ....................................... OMAE2019-96098 (12-5-2) Bai, Yong OMAE2019-95457 (4-1-4) OMAE2019-95458 (4-1-5), OMAE2019-95462 (4-1-6) OMAE2019-95631 (4-2-5), OMAE2019-95676 (4-1-5) OMAE2019-95903 (4-1-5), OMAE2019-95916 (4-1-7) OMAE2019-96596 (4-1-11), OMAE2019-96597 (4-1-6) Bai, Yong ………………………..... OMAE2019-95692 (4-1-6) OMAE2019-96490 (6-4-1) Bailey, Eleanor .................................. OMAE2019-96722 (7-12-1) Bailu, Luo _. OMAE2019-95630 (2-4-3) Bain, Dominique................................. OMAE2019-96466 (9-4-2) Baioco, Juliana ..................................... OMAE2019-96103 (11-4-1) Baker, David A . OMAE2019-95175 (4-2-1) OMAE2019-95618 (2-6-2), 0MAE2019-96191 (2-6-2) Balestra, Lorenzo. OMAE2019-96721 (9-7-1) Ballard, Jean-Christophe .................... OMAE2019-96174 (10-6-1) Bangalore, Gurudutt ................................ OMAE2019-96249 (4-2-2) Banik, Atul Krishna ............................ OMAE2019-96816 (6-11-2) Banumurthy, Surya ............................. OMAE2019-96249 (4-2-2) Bao, Sheng ...................................... OMAE2019-95275 (3-3-3) ... OMAE2019-95547 (3-5-1) Baquet, Aldric ................................. OMAE2019-96460 (1-6-1) OMAE2019-96465 (1-7-1), OMAE2019-96838 (1-6-1) Barnes, Mike ................................... OMAE2019-95542 (9-2-6) Barratt, Dylan .................................... OMAE2019-95299 (6-17-2) Barreto, David ................................... OMAE2019-95390 (2-7-1) Barzegar Valikchali, Saeed ...................... OMAE2019-96307 (1-2-1) Battistella, Tommaso ............................ OMAE2019-96380 (9-1-2) Bayati, IImas ..................................... OMAE2019-95967 (9-2-1) . OMAE2019-95976 (9-2-1)

Beck, Rober

Becker, Quenti

OMAE2019-96469 (6-15-2) Behrendt, Jasper OMAE2019-95063 (6-17-1) Bekhit, Adham S.............................. OMAE2019-95237 (8-2-2) OMAE2019-95237 (8-2-2) OMAE2019-95906 (4-1-3) OMAE2019-96155 (4-5-1), OMAE2019-96387 (4-4-2) Belloli, Marco ………….................. OMAE2019-95967 (9-2-1) OMAE2019-95976 (9-2-1), OMAE2019-95979 (9-2-6) Ben Rajeb, Faraj .................................. OMAE2019-95005 (4-5-1) Bento, A. Rute................................. OMAE2019-96179 (6-7-1) Bergua, Roger ................................... OMAE2019-95429 (9-1-1) Bernardo, Chiara A.................................. OMAE2019-95970 (8-4-4) .... OMAE2019-95972 (8-4-1) Bernier, Natacha ............................... OMAE2019-95842 (13-1-1) Bernitsas, Michael .............................. OMAE2019-96671 (9-5-2) ... OMAE2019-96823 (8-5-2) Bertolo, V.M. .................................. OMAE2019-95464 (3-1-2) Bezensek, Bostjan....._. OMAE2019-95787 (3-11-1) ..................................................... OMAE2019-96327 (3-11-2) Bhagtani, Dhruv …………………...... OMAE2019-96630 (2-3-1) Bhakta, Jai .................................. OMAE2019-95218 (11-12-2) Bhuiyan, Mohammad Hossain ............ OMAE2019-96547 (11-15-2) Bihs, Hans OMAE2019-95154 (5-2-2) OMAE2019-95193 (2-9-1), OMAE2019-95233 (8-3-1) OMAE2019-95921 (8-2-1), 0MAE2019-96004 (6-5-2) OMAE2019-96524 (8-2-1) Bin Omar, Abdulrahman ....................... OMAE2019-95002 (11-4-1) Bin Zhen, Zhou.................................... OMAE2019-96171 (5-1-2) 
Bingham, Harry B...

OMAE2019-95299 (6-17-2) OMAE2019-96383 (6-3-2)

Binns, Jonathan R.

Birchfield, Neal

Birkevold, Jens.

Bitner-Gregersen, Elzbieta M.

OMAE2019-95880 (2-2-2), OMAE2019-96061 (2-1-2) OMAE2019-96587 (6-7-4), OMAE2019-96837 (2-1-2)

Bittencourt Ferreira, Claudio Bizhani, Majid... OMAE2019-95163 (11-15-1) OMAE2019-96338 (11-7-1) OMAE2019-96214 (2-8-1)

Bjonnes, Paal

Bjørkevoll, Knut

Bjornoy, Palmar

Blanchard, Lisa.

Blasioli, Giuseppe

Blenkinsopp, Chris

Blundon, Andrew

Bocharov, Oleg..

Bohan, Paul...

Bonar, Paul A.J.

Bonnefoy, Felicien

Bonnet, Paul

Borg, Mitchell

Børvik, Tore

Bosma, Bret.

Bouchard, P John

Boulougouris, Evangelos

Bourdier, Sylvain.

Bouscasse, Benjamin

Bowo, Ludfi Pratiwi

Braga, Daniel

Brandão, Camila

Braun, Moritz.

Brechan, Bjorn

Bredmose, Henrik

Brekken, Ted.

Brennan, Feargal .

Brett, Alex

Breugem, Alexander.

Brito, Raphael M

Brizzi, Giulio.

Brown, Alison

Brown, Jason

Brown, Paris..

Brown, Scott

Bruaseth, Sindre

Bruno Ellwanger, Gilberto..

Bunn, Nigel

Bunnik, Tim..

Buruchenko, Sergey

Bussolati, Federico

Butt, Stephen

OMAE2019-95219 (11-1-1)

OMAE2019-96170 (11-2-2), OMAE2019-96176 (11-1-3) OMAE2019-96189 (11-1-2), 0MAE2019-96373 (11-1-2)

\section{C}

Caetano, Joao

Caglar, Egemen

Caharija, Walter

Cai, Baoping.

Cai, Jifeng.

Cai, Xiao.........

Cai, Zhisong........

Caire, Marcelo

Calisal, Sander

Calle Gonzales, Miguel Ange

Cambuli, Francesco

OMAE2019-95626 (1-1-4) OMAE2019-96804 (4-5-1) OMAE2019-95414 (5-2-1) OMAE2019-95125 (2-5-1) OMAE2019-95429 (9-1-1) OMAE2019-95714 (8-1-2)

MAE2019-96018 (8-1-1), OMAE2019-96831 (8-1-6) OMAE2019-95429 (9-1-1) OMAE2019-95826 (4-1-4) OMAE2019-96643 (6-4-3) OMAE2019-95772 (2-10-2) OMAE2019-96513 (9-6-1) OMAE2019-96518 (9-4-1) OMAE2019-95538 (9-4-1)

Cameron, Matthew OMAE2019-96372 (4-1-1)
Campos, Ricardo ...

OMAE2019-96626 (6-7-2) OMAE2019-96627 (6-7-2)

Canizares, Loris

Canut, Felipe..

Cao, Guanzhou.

Cao, Peimin

Cao, Qun

Capaldo, Matteo

Cappietti, Lorenzo

OMAE2019-96259 (1-1-1),

Carazza, Luca

Carballo, Manuel.

Carballo, Rodrigo .

Cardoso, Carlos 0 .

OMAE2019-95298 (4-3-5), OMAE2019-95343 (4-3-4)

Carmona-Sanchez, Jesus

Carneiro, Daniel

Caroee Kristoffersen, Julie.

Carpintero Moreno, Efrain

Carr, Malcolm

Carra, Christopher J.

Carreon, Kristian...

Carroll, James

Carta, Mario .

Carvalho, Leonardo de Oliveir

Cassidy, Mark J.

Castello, Xavier

Castillo Garcia, Pablo

Castro, Fabio.

Cavalcante, Gabriella

Cayeux, Eric......

Celledoni, Elena

Cenci, Fredi..

Cerasi, Pierre

Cerkovnik, Mark

Cerulli, Michele

Cevasco, Debora

Chagas, Felipe

Chakrabarti, Partha

Chakraborty, Arindam

Chan, Hoi-Sang.

Chang, Anteng.

Chang, Chih Hsin.

Chang, Rui.

Chang, Xing..

Chao, Wang..

Charnaux, Carlos

Chatziioannou, Konstantinos

Chaudhuri, Jay.

Chavan, Kishor

Chawan, Abhijeet

Chen, Bang-Fuh.

Chen, Can

Chen, Changhai

Chen, Changyuan

Chen, Chaohe

Chen, Chen..

Chen, Dengshuo

Chen, Ganchao

Chen, Hao.

Chen, Jau-Uei...

Chen, Jianyong

Chen, Jinbo

Chen, Jinlong

Chen, Ke

Chen, Lei .

Chen, Lifen

Chen, Limin

Chen, Ming.
OMAE2019-95024 (5-1-3)

OMAE2019-95369 (3-3-2)

OMAE2019-96181 (8-1-3)

OMAE2019-96422 (4-2-4)

OMAE2019-95661 (9-1-2)

OMAE2019-96243 (9-1-2)

OMAE2019-96123 (9-3-2)

OMAE2019-96703 (2-9-1)

OMAE2019-96347 (11-7-2)

2019-95804 (4-2-1)

OMAE2019-95542 (9-2-6)

OMAE2019-95359 (4-3-5)

OMAE2019-95577 (4-3-4)

OMAE2019-95179 (6-8-1)

OMAE2019-96756 (12-4-1)

OMAE2019-95529 (4-3-6)

OMAE2019-96411 (2-6-1)

OMAE2019-95130 (3-1-1)

OMAE2019-96720 (9-7-1)

OMAE2019-96518 (9-4-1)

OMAE2019-96047 (4-4-1)

OMAE2019-96698 (10-5-1)

OMAE2019-96367 (4-4-2)

OMAE2019-96096 (10-3-1)

OMAE2019-95369 (3-3-2)

OMAE2019-95131 (11-12-1)

OMAE2019-95049 (11-7-1)

OMAE2019-95062 (11-2-1)

OMAE2019-96185 (4-4-2)

OMAE2019-95250 (13-2-3)

OMAE2019-96773 (11-6-2)

OMAE2019-96826 (4-2-4)

OMAE2019-95505 (4-3-7)

OMAE2019-95542 (9-2-6)

OMAE2019-96678 (11-5-1)

OMAE2019-96736 (11-13-1)

OMAE2019-95537 (1-1-4)

OMAE2019-96754 (10-6-1)

OMAE2019-96553 (1-3-2)

OMAE2019-95490 (6-4-6)

OMAE2019-96321 (8-2-3)

OMAE2019-95966 (4-5-2)

OMAE2019-95051 (2-12-1)

OMAE2019-95814 (4-3-6)

OMAE2019-96154 (4-3-8)

OMAE2019-96137 (1-4-3)

OMAE2019-95537 (1-1-4

OMAE2019-95325 (9-3-3)

OMAE2019-96425 (8-1-5)

OMAE2019-96628 (2-12-2)

OMAE2019-95249 (6-13-2)

OMAE2019-95565 (6-11-2

OMAE2019-96759 (9-1-5)

OMAE2019-95173 (2-2-2)

OMAE2019-96490 (6-4-1)

OMAE2019-96628 (2-12-2)

OMAE2019-96098 (12-5-2)

OMAE2019-96823 (8-5-2)

OMAE2019-95924 (2-12-2)

OMAE2019-95800 (10-1-1)

OMAE2019-96491 (4-1-11)

OMAE2019-95891 (6-17-1)

OMAE2019-96029 (13-1-3)

OMAE2019-95347 (9-5-3)

OMAE2019-95198 (12-7-1)

OMAE2019-95865 (12-5-2)

OMAE2019-96383 (6-3-2)

OMAE2019-96802 (9-4-3)

OMAE2019-96803 (5-2-1)
OMAE2019-96581 (6-5-4)

OMAE2019-95001 (1-3-1)

OMAE2019-96706 (5-1-1)

Chen, Mingsheng

OMAE2019-95717 (12-2-1)

Chen, Mo.

Chen, Nianzhong .

OMAE2019-96193 (12-1-1)

hong ............................ OMAE2019-95108 (2-4-1)

Chen, Pengfei.

Chen, Pengfei..

Chen, Oiang

Chen, Shao-Yu.

Chen, Songgui

Chen, Tianyi...

Chen, Wei

Chen, Weimin..

OMAE2019-95473 (6-11-2)

OMAE2019-96716 (6-4-4)

OMAE2019-95739 (12-4-1)

OMAE2019-95321 (6-15-1)

OMAE2019-95039 (6-2-3)

OMAE2019-96001 (6-15-2)

OMAE2019-95462 (4-1-6) OMAE2019-95651 (4-1-4)

OMAE2019-95658 (6-4-5), OMAE2019-95839 (4-6-1)

Chen, Xi ..................................... OMAE2019-95727 (6-13-2)

Chen, Xiaolu ....................................... OMAE2019-95634 (1-1-3)

Chen, Xiqia ...................................... OMAE2019-95070 (6-3-2)

Chen, Xuanshu................................... OMAE2019-95563 (8-1-5)

OMAE2019-96791 (8-1-2)

OMAE2019-96703 (2-9-1)

Chen, Xuexue

Chen, Y.Y.

Chen, Yan

Chen, Yingchun

Chen, Yongping

Chen, Yuanhang

OMAE2019-96298 (2-1-1)

OMAE2019-95320 (2-13-2)

OMAE2019-95840 (6-11-2)

OMAE2019-95455 (4-5-2)

OMAE2019-96649 (11-13-1)

OMAE2019-96672 (11-13-1), 0MAE2019-96675 (11-3-1)

OMAE2019-96676 (11-3-1), 0MAE2019-96678 (11-5-1)

OMAE2019-96682 (11-7-3), OMAE2019-96683 (11-1-2)

OMAE2019-96767 (11-13-1)

Chen, Yufei

OMAE2019-96707 (11-1-3)

Chen, yunsai

Chen, Zheng..

Chen, Zhenhong

OMAE2019-96581 (6-5-4)

OMAE2019-95968 (9-2-6)

OMAE2019-96735 (6-2-2)

Cheng, Hui...

OMAE2019-95706 (13-2-1)

OMAE2019-96079 (5-2-2)

Cheng, Jingyun OMAE2019-96422 (4-2-4)

Cheng, Liang 
Colbourne, Bruce ...

Collins, Keri

Collu, Maurizio.

OMAE2019-95799 (8-2-3) OMAE2019-96573 (12-4-1) OMAE2019-95210 (1-1-3)

OMAE2019-95542 (9-2-6), 0MAE2019-96104 (5-1-2) OMAE2019-96171 (5-1-2), OMAE2019-96212 (5-1-2) OMAE2019-96282 (5-1-2)

Colombo, Danilo OMAE2019-95069 (11-4-1)

OMAE2019-96269 (2-13-1), 0MAE2019-96280 (2-8-1) Cong, Cui Connaire, Adrian Connell, Richenda Connellan, Michael Cooke, Nathan

Coppejans, Okko J.. Corbineau, Erwan.

Cordeiro, Wilson.

Corina, Anisa Noor

Corrignan, Hugues

Cosham, Andrew

Costa, Alvaro M.

Costa, Beethoven G.S

Coules, Harry..

Couñago, Bernardino

Cox, Daniel

Cozman, Fábio....

Crabtree, Christopher

Craidy, Pedro

Crema, Ilaria........

Crespi, Tommaso

Cross, Colin.. OMAE2019-95226 (2-11-1) OMAE2019-96516 (3-5-1) OMAE2019-95205 (6-7-3) OMAE2019-95337 (6-5-3) OMAE2019-95303 (4-1-3) OMAE2019-95368 (3-1-1) OMAE2019-95306 (8-1-5) OMAE2019-95363 (4-3-1) OMAE2019-95928 (11-15-2) OMAE2019-96261 (4-2-3) OMAE2019-95529 (4-3-6) OMAE2019-95532 (3-11-2 OMAE2019-96250 (2-13-2) OMAE2019-95069 (11-4-1) OMAE2019-96327 (3-11-2) OMAE2019-96380 (9-1-2) OMAE2019-96803 (5-2-1) OMAE2019-96120 (6-4-1) OMAE2019-95561 (2-7-1) OMAE2019-95175 (4-2-1) OMAE2019-96259 (1-1-1) OMAE2019-95129 (8-4-4) OMAE2019-95444 (4-3-7)

Crump, Jennifer OMAE2019-95448 (4-3-7), OMAE2019-95450 (4-3-6) Cuellar, Pablo

Cui, Luning.

Cui, Weicheng

Cunha, Bruno R. M. OMAE2019-96233 (3-2-1) OMAE2019-96078 (10-7-1) OMAE2019-96193 (12-1-1) OMAE2019-95327 (2-4-3) OMAE2019-96358 (3-2-1)

\section{D}

D, Adarsh.......

D'Agostini, Andressa

D'Arcy Evans, Nicholas

da Silva, Jose.

Daasvatn, Sigbjørn

Daems, Pieter-Jan

Dahi Taleghani, Arash

Dai, Saishuai.

Dai, Xiaojuan.

Dakshina, Moorthy

Dalane, Oddgeir.

Dale, Stein.

Dan, Saikat

Dankowski, Hendrik

Dao, Cuong.

Darcis, Philippe

-95953 (3-6-1), OMAE2019-96345 (3-5-1)

OMAE2019-96262 (6-3-4)

Datta, Indra...

Davidson, Michelle

Davies, Graham..

Davis, Michael

Day, Sandy OMAE2019-95172 (12-1-1)

OMAE2019-95784 (8-1-4), OMAE2019-95790 (6-13-1)

de Arruda Martins, Clovis...

de Hauteclocque, Guillaume. OMAE2019-95033 (4-1-10) OMAE2019-95993 (2-9-2) OMAE2019-96272 (2-9-2) OMAE2019-95938 (10-6-1)

de Leeuw, Lawrence

de Mello, Pedro. OMAE2019-95238 (12-2-1) OMAE2019-96670 (6-4-2) OMAE2019-96367 (4-4-2)

de Moura Jorge, Nilo de Oliveira Costa, Daniel

de Oliveira, Felipe $\mathrm{F}$

de Oliveira, Mauro C.

OMAE2019-96048 (9-3-2) OMAE2019-96051 (6-1-2) OMAE2019-96210 (2-6-1) OMAE2019-96334 (6-8-2)

de Ridder, Erik-Jan OMAE2019-95481 (1-7-1)

de Sousa, José Renato M

OMAE2019-95506 (4-1-7)

OMAE2019-95510 (4-1-2), OMAE2019-96372 (4-1-1) de Souza Mendes, Paulo .................. OMAE2019-95131 (11-12-1) de Souza, Ana Paula França .................. OMAE2019-95145 (4-3-1) OMAE2019-95768 (4-3-1) de Vroom, Joris ................................. OMAE2019-96549 (2-15-1) Decrop, Boudewijn _..................... OMAE2019-95789 (8-1-1) Degroot, Don J. Deguchi, Junichi Deguchi, Mitsuyasu Del Guzzo, Andrea

Delefortrie, Guillaume OMAE2019-95565 (6-11-2) Demirel, Yigit MAE2019-96341 (6-5-2), OMAE2019-96346 (6-5-2)

Deng, Di.

Deng, Yanfei OMAE2019-95522 (8-4-1) OMAE2019-95070 (6-3-2) OMAE2019-95693 (1-7-2)

Derbanne, Quentin

Deschrijver, Dirk

Desmond, Cian.

Dev, Arun. OMAE2019-95993 (2-9-2) OMAE2019-96731 (9-7-1) OMAE2019-96295 (9-1-5) OMAE2019-95126 (1-3-1)

OMAE2019-95485 (1-2-1), OMAE2019-95764 (1-4-1) OMAE2019-96042 (2-6-3) Devaney, Ronan J................................. OMAE2019-96516 (3-5-1) Dewhurst, Tobias ............................... OMAE2019-96388 (6-4-5) Dhaene, Tom Dhanak, Manhar. Diambra, Andrea Diaz, Brian.

Diego, Pavone.. OMAE2019-96731 (9-7-1) OMAE2019-96674 (6-17-2) OMAE2019-95938 (10-6-1) OMAE2019-96395 (9-1-5) OMAE2019-95055 (4-3-5)

Dietz, Matt OMAE2019-95056 (4-4-1), OMAE2019-95057 (4-1-2) OMAE2019-95938 (10-6-1) Dimopoulos, Simon _..................... OMAE2019-95882 (2-6-3) Ding, Haoyu ................................... OMAE2019-95739 (12-4-1) Ding, Jun. OMAE2019-96122 (6-3-1) OMAE2019-96771 (6-1-2)

Ding, Qian . ding, Songxiong Ding, Yong Dinh, Van Nguyen Dinita, Alin OMAE2019-96719 (11-7-3) OMAE2019-96114 (2-6-3) . OMAE2019-95438 (8-1-2) OMAE2019-96778 (9-1-1) Dobashi, Daisuke OMAE2019-96279 (11-10-1) OMAE2019-96283 (11-10-1)

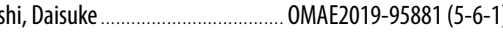
Dodbiba, Gjergj. ………………....... OMAE2019-96040 (5-3-1)

Doi, Yasuaki... Donatini, Luca... Dondapati, Varun Dong, Pingsha..

Dong, Qing

Dong, Sheng

Dong, Xingjian .

dos Reis Tagliari, Mariana

Dos Santos, Marcelo

Doshi, Karan.

Doynov, Krassimir

Drago, Michele.

Drake, Matthew

Draper, Scott OMAE2019-96405 (12-5-1) OMAE2019-96346 (6-5-2) OMAE2019-96287 (9-5-3) OMAE2019-96631 (3-3-2) OMAE2019-96578 (6-3-4) OMAE2019-95791 (6-7-1) OMAE2019-95915 (9-2-1) OMAE2019-96423 (4-1-1) OMAE2019-96592 (4-2-4) OMAE2019-95867 (2-11-2) OMAE2019-95303 (4-1-3) OMAE2019-96086 (6-7-3) OMAE2019-96270 (6-15-1) OMAE2019-95198 (12-7-1) OMAE2019-95557 (4-3-8), OMAE2019-95865 (12-5-2) OMAE2019-95933 (12-5-2) Draycott, Samuel ................................. OMAE2019-96405 (12-5-1) OMAE2019-96817 (12-5-1)

Driscol, Blake OMAE2019-95383 (9-4-2)

Du, Bo...

Du, Junfeng...

Du, Zhipeng

Du, Zunfeng...

Duan, Chuanjie

Duan, Menglan

Duan, Songchang

Duan, Wenhui

Duan, Wenyang OMAE2019-96052 (2-4-3) OMAE2019-96553 (1-3-2) OMAE2019-95776 (2-9-4) OMAE2019-95673 (6-4-2) OMAE2019-95392 (2-4-3) OMAE2019-95141 (4-1-3) OMAE2019-95876 (4-5-2) OMAE2019-95209 (8-2-2) OMAE2019-95860 (6-2-1) OMAE2019-95224 (8-2-1) OMAE2019-95675 (1-6-1), OMAE2019-95779 (6-7-1) OMAE2019-96181 (8-1-3), OMAE2019-96581 (6-5-4) Duarte Martins, Marcos Andre ............ OMAE2019-96592 (4-2-4) Dudek, Matthias OMAE2019-95063 (6-17-1) Duffy, Brian...

Dumitrescu, Andrei..

OMAE2019-96279 (11-10-1)

Duz, Bulent

Dvergsnes, Erik W.

Dwivedy, Santosha K. OMAE2019-96283 (11-10-1) OMAE2019-96201 (13-2-2) OMAE2019-96225 (13-2-2) OMAE2019-95062 (11-2-1)

E

E. Karam, Qusaie...

Eca, Luis.

Eckert-Gallup, Aubrey..

Eduardo, Decnop.

Edward, Ceasar

Efthymiou, Mike

Ehlers, Sören OMAE2019-95345 (6-15-1) OMAE2019-96740 (7-4-1), OMAE2019-96846 (7-1-1)

El Chahal, Ghassan .............................. OMAE2019-95312 (6-5-4) …............................................ OME2019-95313 (6-2-2) el Moctar, Ould …………………...... OMAE2019-95716 (8-1-6) OMAE2019-95722 (9-5-4), OMAE2019-95935 (8-1-1) OMAE2019-96138 (8-1-2) el Sheshtawy, Hassan ........................... OMAE2019-95722 (9-5-4) El-Chayeb, Abdul ................................. OMAE2019-96662 (4-3-3) Elsanoose, Abadelhalim ...................... OMAE2019-96311 (11-4-1) Elsherbiny, Khaled ........................... OMAE2019-95790 (6-13-1) Elusakin, Tobiloba ................................. OMAE2019-95539 (2-13-1) Endo, Yusuke _.......................... OMAE2019-95818 (3-6-1) Endresen, Per Christian ......................... OMAE2019-95414 (5-2-1) OMAE2019-96375 (5-2-1) Enet, Francois................................. OMAE2019-96549 (2-15-1) Erceg, Sandro Ercoli-Malacari, Luca OMAE2019-96740 (7-4-1) .... OMAE2019-96343 (4-3-3) Errotabehere, Xabier.......................... OMAE2019-96239(3-3-1) Ersdal, Gerhard Ertekin, R. Cengiz. Ervik, Anna Kringlen Esperança, Paulo de Tarso T. OMAE2019-96841 (3-13-1) OMAE2019-96013 (6-2-1) .1... OMAE2019-96334 (6-8-2) Estefen, Segen ................................... OMAE2019-96367 (4-4-2) 
Feld, Graham

OMAE2019-96586 (6-7-4) OMAE2019-96587 (6-7-4)

Feng, Bo

Feng, Dakui OMAE2019-95714 (8-1-2) OMAE2019-95727 (6-13-2), OMAE2019-95737 (8-5-1) OMAE2019-95887 (8-1-7), OMAE2019-96020 (8-1-4) OMAE2019-96831 (8-1-6) OMAE2019-95586 (7-11-1) OMAE2019-95777 (2-9-3) OMAE2019-96601 (2-12-3) OMAE2019-96661 (1-1-1)

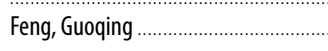

Feng, Jiaguo

Feng, Ming

Feng, Peiyuan

Feng, Qi.

Feng, Wei.

Feng, Xingya

Feng, Yan

Fernandes, Antonio Carlos OMAE2019-96349 (6-4-6), OMAE2019-96575 (1-4-3) Fernandez, Charles Fernandez, Jose

Fernando, Upul

Ferrant, Pierre

Ferrari, Victor

Ferreira Senra, Stae

Ferreira, Guilherme

Ferreira, Marcos

Ferreira, Marcus

Ferri, Francesco

Fischer, David

Fiskerstrand, Roy-Jostein

Fjelde, Kjell Kåre

Flamant, Nicolas

Foglia, Aligi

Fogliani, Antonino

Fonseca, Bruno

Fonseca, Ícaro A.

Fontana, Casey M.

Fontanella, Alessandro

Ford, Eric Patrick MAE2019-95976 (9-2-1), 0MAE2019-95979 (9-2-6)

Føre, Martin .

Fortis Kwietniewski, Carlos Eduardo

Foster, Danny

Fotland, Kristian

Fowler, Matthew

Franchek, Matthew

Francis, Vivek

Franciss, Ricardo

Franco, Luciano

Franzini, Guilherme

Frederic, Perrin..

Fredriksen, Arnt G.

Frigaard, lan. . OMAE2019-95163 (11-15-1)

OMAE2019-95164 (11-7-1), OMAE2019-95180 (11-12-2)

..OMAE2019-95218 (11-12-2), 0MAE2019-95415 (11-12-2)

Frimanslund, Erik.

Friswell, Michael.

Froyen, Johnny...

Fu, Pengcheng..

Fu, Shixiao

Fujarra, André L. C

Fujii, Toshihiro

Fujiki, Takashi

Fujikubo, Masahiko

Fujimatsu, Takuya

Fujimoto, Wataru

Fujita, Toyohisa.

Fujiwara, Tomo.

Fujiwara, Toshifumi

Fukui, Tsutomu

Fukunaga, Yuta.

Funada, Junki. OMAE2019-95485 (1-2-1) OMAE2019-96380 (9-1-2) OMAE2019-96310 (4-1-6) OMAE2019-96296 (9-1-8) OMAE2019-95513 (6-8-2) OMAE2019-96592 (4-2-4) OMAE2019-95369 (3-3-2) OMAE2019-96335 (6-4-1) OMAE2019-95363 (4-3-1) OMAE2019-95216 (9-3-1) OMAE2019-96556 (3-4-1) OMAE2019-95885 (6-4-2) OMAE2019-95214 (11-6-1) OMAE2019-95100 (11-12-2) OMAE2019-96109 (10-7-1) OMAE2019-95557 (4-3-8) OMAE2019-95131 (11-12-1) OMAE2019-96395 (9-1-5)

MAE2019-96546 (11-15-2) OMAE2019-95414 (5-2-1)

OMAE2019-96423 (4-1-1) OMAE2019-95536 (4-4-1) OMAE2019-96839 (6-4-3) OMAE2019-95538 (9-4-1) OMAE2019-96293 (4-5-1) OMAE2019-96305 (8-5-1) OMAE2019-95194 (6-2-1) OMAE2019-95204 (13-2-5) OMAE2019-95359 (4-3-5) OMAE2019-95150 (8-4-2) OMAE2019-95055 (4-3-5) OMAE2019-95057 (4-1-2) OMAE2019-95212 (2-9-2)

OMAE2019-96112 (8-4-1) OMAE2019-96486 (1-3-1) OMAE2019-95250 (13-2-3) OMAE2019-95817 (3-3-1) OMAE2019-95220 (6-12-1) OMAE2019-95896 (11-2-2) OMAE2019-95173 (2-2-2) OMAE2019-95949 (13-1-3) OMAE2019-96040 (5-3-1) OMAE2019-95990 (5-3-1) OMAE2019-95650 (9-4-3) OMAE2019-95121 (2-2-2) OMAE2019-95927 (5-6-1) OMAE2019-95400 (13-3-2) OMAE2019-95945 (9-2-3)

Furukawa, Yoshitaka.

.. OMAE2019-96056 (1-2-2)

Furusho, Masao

OMAE2019-96663 (2-13-2)

Gabrielsen, Øystein

OMAE2019-95083 (2-6-2)

OMAE2019-95084 (2-4-1), OMAE2019-95618 (2-6-2)

OMAE2019-96000 (2-4-1)

Gadelho, Jorge Filipe Marques

Galinos, Christos

Gallagher, Conor

Gallegillo, Martin

Gambarine, Dennis

Gao, Fu-Ping.

Gao, Guogiang.

$\mathrm{GaO}, \mathrm{He}$.

Gao, Hongtao

Gao, Jiancai..

Gao, Junjie.

Gao, Junliang

Gao, Yifan OMAE2019-96051 (6-1-2)

Gao, Yonghai

DAE2019-95462 (4-1-6), OMAE2019-96596 (4-1-11) Gao, Yu..................................... OMAE2019-96719 (11-7-3) Gao, Zhen OMAE2019-96686 (2-7-1) OMAE2019-96793 (6-4-5)

Garbatov, Yordan OMAE2019-96666 (2-4-2)

Garmbis, Alexandre G OMAE2019-96297 (4-2-5)

Garner, Alistai MAE2019-96358 (3-2-1), OMAE2019-96592 (4-2-4) OMAE2019-95001 (1-3-1) Gaspar, Henrique M O OMAE2019-96051 (6-1-2) Gatin, Inno ........................................ OMAE2019-95293 (8-1-3 OMAE2019-96026 (8-2-3)

Gato, Luis Gaudin, Christophe

Gavriilidis, llias.

Gavrilov, Andrey

Gayen, Rupanwita

Ge, Han.

Ge, Weifeng.

Geekiyanage, Suranga

Gemilang, Gilang Muhammad Geng, Baolei.

Georgakis, Christos Thomas

Ghaedi, Parisa

Gheibi, Sohrab

Ghisu, Tiziano

OMAE2019-96368 (9-4-1) OMAE2019-96541 (10-6-1) .. OMAE2019-95743 (4-3-2) OMAE2019-95228 (11-7-1) OMAE2019-95498 (6-3-4) OMAE2019-96715 (5-5-1) ... OMAE2019-95125 (2-5-1) OMAE2019-95217 (11-2-1) OMAE2019-96386 (1-2-4) OMAE2019-95006 (6-3-4) OMAE2019-95179 (6-8-1) OMAE2019-96594 (11-7-2) OMAE2019-96319 (11-6-2) OMAE2019-96513 (9-6-1) OMAE2019-96518 (9-4-1) Ghorban Zadeh, Leila $\quad$ OMAE2019-96802 (9-4-3) Ghosh, Rupak OMAE2019-96156 (4-2-4)

OMAE2019-96329 (3-4-1), OMAE2019-96588 (1-4-1) OMAE2019-95055 (4-3-5) OMAE2019-95056 (4-4-1), OMAE2019-95057 (4-1-2)

Gianluigi, Pirinu OMAE2019-95055 (4-3-5)

... OMAE2019-95056 (4-4-1), OMAE2019-95057 (4-1-2)

Giannoulis, Andreas. OMAE2019-95339 (8-1-4) OMAE2019-96363 (6-7-4) Gibson, Richard

Gilbert, Philippe

Gilloteaux, Jean-Christophe OMAE2019-95429 (9-1-1)

Gioia, Nicoletta

Gish, Andy

Giske, Finn-Idar G

Glennon, David .

Gomes, Dalila

Gomes, Rui P. F.
Gomes, Victor

Gonçalves, Rafaela

Gonçalves, Rodolfo T OMAE2019-95353 (13-2-4), OMAE2019-95749 (13-2-3) OMAE2019-95785 (13-3-2), OMAE2019-96727 (13-2-1)

Gong, Fuxian

Goniva, Christoph

Gonzalez, Gabriel.
Gonzalez Diez, Nestor

Gotoh, Koji... OMAE2019-95817 (3-3-1) OMAE2019-95818 (3-6-1), OMAE2019-95822 (3-3-2) OMAE2019-96750 (5-5-1) Gou, Ying....................................... OAE2019-95421 (12-1-2) Gouldby, Ben ..................................... OMAE2019-96587 (6-7-4) Gourvenec, Susan ................................. OMAE2019-95800 (10-1-1) Gowri Shankar, C................................. OMAE2019-95895 (6-2-1) Grabe, Jürgen.. OMAE2019-95337 (6-5-3) Michael R. OMAE2019-95629 (6-3-3) Gramstad, Odin OMAE2019-95352 (2-9-3) OMAE2019-95357 (2-1-2), OMAE2019-96061 (2-1-2) Granholt, Jason D. D. A. O OMAE2019-95885 (6-4-2) Grant, Hazel ..................................... OMAE2019-96021 (6-12-1) Grasso, Nicola. OMAE2019-96207 (1-7-2)

Gray-Stephens, Angus G D OMAE2019-95784 (8-1-4) Greaves, Deborah................................ OMAE2019-95998 (9-5-2)

... OMAE2019-96573 (12-4-1) Greene, John ................................... OMAE2019-96100 (2-13-1) Grey, Stephen OMAE2019-96245 (6-7-1) Griffiths, Terry ................................... OMAE2019-95557 (4-3-8)

.... OMAE2019-95585 (1-3-2) OMAE2019-96293 (4-5-1) OMAE2019-96305 (8-5-1)

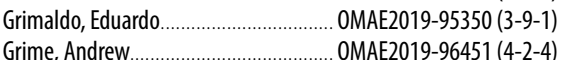
Grime, Andrew.................................. OMAE2019-96451 (4-2-4)
Grindheim, Jan Vidar .......................... OMAE2019-95038 (6-4-6) ........ OMAE2019-96349 (6-4-6) Growcock, Frederick B ....................... OMAE2019-96675 (11-3-1) OMAE2019-96676 (11-3-1) Gu, Xiechong................................ OMAE2019-95562 (13-2-1) Gu, Yibin... Guan, Wang. Guanche, Raúl. .. OMAE2019-95547 (3-5-1) OMAE2019-95603 (4-4-2) OMAE2019-96380 (9-1-2) Guedes Soares, Carlos OMAE2019-95137 (2-14-1) OMAE2019-95287 (2-2-2), OMAE2019-96151 (6-13-3) OMAE2019-96408 (9-3-2), OMAE2019-96510 (12-1-2) OMAE2019-96618 (6-11-1), 0MAE2019-96626 (6-7-2) OMAE2019-96627 (6-7-2)

Guidault, Pierre-Alain ............................. OMAE2019-96165 (1-2-4) Guillaume, Patrick .............................. OMAE2019-96731 (9-7-1) Guindeuil, Geoffrey............................ OMAE2019-95354 (4-6-1) OMAE2019-95493 (4-6-1), OMAE2019-96059 (4-6-1) Guiton, Martin _........................... OMAE2019-96165 (1-2-4) Gumley, Jonathan M........................... OMAE2019-96411 (2-6-1) Guo, Anxin ..................................... OMAE2019-95831 (6-17-1) .... OMAE2019-96657 (6-5-3) 
H H. Dadmarzi, Fatemeh
Ha, Yoon-Jin

Hadley, Isabel...

\section{Haeberle, Hunter}

Haghighi, Ali

Haider, Muhammad Salman

Haldorsen, Lars M.

Hall, Wayne

Hallowell, Spencer Halse, Karl. OMAE2019-96388 (6-4-5), OMAE2019-96395 (9-1-5) … OMAE2019-95339 (8-1-4) Han, Jialin ... Han, Mengmeng Han, Peihua..

Han, Rong-Gui Han, So Lyoung. Han, Young Soo.

Han, Zhaolong

Handa, Tsunehisa

Hann, Martyn

Hao, Yun

Hara, Naoyuki

Harchambois, Stéphanie OMAE2019-95354 (4-6-1) Harit, Naik OMAE2019-95493 (4-6-1), ON

Harries, Robert

Harrold, Magnus

Harrop, Andrew

Hartmann, Moritz.

Haselsteiner, Andreas F

Hashi, Kunihiko.

Hashimoto, Kazuki

Hashimoto, Noriaki

Håskoll, Lars.

Hauge, Mons

Haugen, Joakim.

Haver, Sverre K.

Hayatdavoodi, Masoud..

He, Daqian

He, Guanghua...

\section{He, Haiping ...}

He, Jiawei.

He, Ning.

$\mathrm{He}$, Shi.......

He, Xiaohan

He, Xuhong

He, Yangye.

Hejazi, Rasoul

Hekkenberg, Robert

Helder, Joop ...

Hellan, Oyvind..

\section{Hellum, Vidar}

Helmers, Jens B.

Helsen, Jan...

Henriques, Joao C.C

Heo, YeongAe

Hermawan, Yuda Apri

Hermile, Mahé..

Hermundstad, Elin Marita H..

Hernández-Fontes, Jassiel V.

Hernandez, Sergio.

Herrera, Gilbert.

Herrnring, Hauke

Hilbert, Renan.

Hill, Tricia...
OMAE2019-95929 (6-5-1) OMAE2019-95406 (1-7-1) OMAE2019-95934 (3-11-2) OMAE2019-96619 (3-11-1) OMAE2019-96409 (4-2-2) OMAE2019-95505 (4-3-7) OMAE2019-96492 (4-6-2) OMAE2019-96498 (4-6-2) OMAE2019-95175 (4-2-1) OMAE2019-95821 (4-3-1) OMAE2019-96289 (2-1-1) OMAE2019-95670 (5-2-2) OMAE2019-95827 (13-7-1) OMAE2019-95860 (6-2-1) OMAE2019-95457 (4-1-4) OMAE2019-95458 (4-1-5) OMAE2019-95675 (1-6-1) OMAE2019-96236 (1-3-2) OMAE2019-96053 (6-15-2) OMAE2019-96477 (4-2-2) OMAE2019-95923 (3-9-1) OMAE2019-96573 (12-4-1) OMAE2019-95177 (4-5-2) OMAE2019-95401 (13-7-1) OMAE2019-95160 (4-2-1) OMAE2019-95429 (9-1-1) OMAE2019-96080 (9-1-5) OMAE2019-95475 (4-3-2) OMAE2019-95116 (7-4-1) OMAE2019-96523 (2-2-1) OMAE2019-95816 (3-9-1) OMAE2019-95404 (13-3-1) OMAE2019-95220 (6-12-1) OMAE2019-95084 (2-4-1) OMAE2019-95344 (4-2-5) OMAE2019-95146 (6-4-2) OMAE2019-95414 (5-2-1) OMAE2019-95144 (2-3-1) OMAE2019-96013 (6-2-1) OMAE2019-96034 (9-1-3) OMAE2019-95530 (6-4-4) OMAE2019-96378 (9-5-4) OMAE2019-96383 (6-3-2) OMAE2019-95541 (3-3-2) OMAE2019-95525 (8-3-2) OMAE2019-95251 (6-4-5) OMAE2019-95868 (6-13-2) OMAE2019-96231 (11-7-3) OMAE2019-95853 (2-13-2) OMAE2019-95826 (4-1-4) OMAE2019-96451 (4-2-4) OMAE2019-96321 (8-2-3) OMAE2019-96761 (5-1-3) OMAE2019-96789 (5-1-3) OMAE2019-96114 (2-6-3) OMAE2019-95352 (2-9-3) OMAE2019-96669 (12-5-2) OMAE2019-96713 (9-7-1) OMAE2019-96731 (9-7-1) OMAE2019-96368 (9-4-1) OMAE2019-96573 (12-4-1) OMAE2019-96396 (2-15-1) OMAE2019-96056 (1-2-2) OMAE2019-96323 (2-7-1) OMAE2019-95921 (8-2-1) OMAE2019-96747 (6-13-1) OMAE2019-95354 (4-6-1) OMAE2019-95493 (4-6-1) OMAE2019-95085 (7-4-1) OMAE2019-96095 (8-3-2) .. OMAE2019-96426 (4-2-3) OMAE2019-95076 (8-1-3) OMAE2019-96380 (9-1-2)
Hino, Akinori

Hino, Takanori..

Hinostroza, Miguel

Hippert, Eduardo .

Hirabayashi, Shinichiro

Hirai, Shota

Hirata, Fausto....

Hisamatsu, Rikito.

Hizir, Olgun .

Hodne, Helge

Hoffmann, Norbert

Hofland, Bas

Holden, Christian

Holloway, Damien

Holmes, Samuel

Holzmeister, Karla

Hong Doan, Dinh

Hong, Keyyong

Hong, Sa Young

Hongtao, Yuan.

Honma, Yuta.

Hoo, Eric

Hopman, Hans

Hopperstad, Odd Sture.

Horel, Boris

Horn, Agnes Marie

Horte, Torfinn

Hosseini, Matin

Hosseinzadeh, Foroogh

Hou, Xiaonan

Hou, Yucheng

Houlsby, Guy T.

Hovland, Erlend

Howey, Ben...

Høyland, Knut Vilhelm

Hsu, Wei-Ting

Hsu, Wen-Yang

$\mathrm{Hu}, \mathrm{Gu}$

$\mathrm{Hu}$, Guanyu ..

Hu, Haitao ... OMAE2019-95426 (4-1-3)

Hu, Haoliang

$\mathrm{Hu}$, Kaiye.

Hu, Kang

$\mathrm{Hu}$, Ke.

$\mathrm{Hu}$, Lei

$\mathrm{Hu}, \mathrm{Qi}$.

Hu, Wenjia

$\mathrm{Hu}$, Wenwei.

Hu, Xiaofei

Hu, Xuecong.

Hu, Yuxia .

Hu, Zhihuan

$\mathrm{Hu}$, Zhiqiang

Huang, Chai-Cheng

Huang, Chaojun

Huang, Chi-Wen

Huang, Dekai

Huang, Jianbo...

Huang, Kai

Huang, Limin

Huang, Liuyi

OMAE2019-95225 (13-1-2), OMAE2019-95308 (13-2-5) OMAE2019-95706 (13-2-1)

Huang, Shan OMAE2019-96792 (6-13-3) OMAE2019-95596 (13-1-1) OMAE2019-95946 (13-1-4) OMAE2019-96618 (6-11-1) OMAE2019-96358 (3-2-1) OMAE2019-96592 (4-2-4) OMAE2019-95250 (13-2-3) OMAE2019-95749 (13-2-3) OMAE2019-95925 (13-3-4) .. OMAE2019-96358 (3-2-1) OMAE2019-95336 (13-1-1) OMAE2019-95596 (13-1-1) OMAE2019-95316 (6-5-1) OMAE2019-96594 (11-7-2) OMAE2019-95063 (6-17-1) OMAE2019-95116 (7-4-1) .. OMAE2019-96703 (2-9-1) OMAE2019-95054 (6-11-1) OMAE2019-95474 (1-4-2) OMAE2019-96684 (8-1-1) ... OMAE2019-96255 (4-4-2) OMAE2019-96096 (10-3-1) OMAE2019-96500 (9-3-3) OMAE2019-96429 (1-6-2) OMAE2019-95966 (4-5-2) . OMAE2019-95816 (3-9-1) OMAE2019-96032 (2-6-1) OMAE2019-95642 (4-1-7) OMAE2019-95746 (3-3-1) OMAE2019-96786 (2-9-4) OMAE2019-96272 (2-9-2) OMAE2019-96685 (3-3-3) OMAE2019-96214 (2-8-1) OMAE2019-96321 (8-2-3) OMAE2019-96615 (3-6-1) OMAE2019-96755 (4-1-4) OMAE2019-95118 (4-1-1) OMAE2019-95767 (9-5-2) .. OMAE2019-96322 (1-1-2) OMAE2019-96573 (12-4-1) OMAE2019-95139 (7-3-1) OMAE2019-96424 (13-2-2) OMAE2019-96424 (13-2-2) OMAE2019-95857 (2-5-1) .. OMAE2019-96826 (4-2-4) .. OMAE2019-96805 (6-1-2) OMAE2019-95438 (8-1-2) .. OMAE2019-95230 (2-4-2) .. OMAE2019-95039 (6-2-3) OMAE2019-96726 (6-4-4) OMAE2019-95192 (13-1-2) OMAE2019-95586 (7-11-1) .. OMAE2019-96136 (6-8-1) OMAE2019-95491 (2-12-1) OMAE2019-96020 (8-1-4) OMAE2019-95777 (2-9-3) .. OMAE2019-96136 (6-8-1) OMAE2019-96698 (10-5-1) OMAE2019-96739 (12-1-2) OMAE2019-95094 (2-10-1) OMAE2019-95730 (2-6-1) .. OMAE2019-95536 (4-4-1) OMAE2019-96826 (4-2-4) OMAE2019-96706 (5-1-1) .. OMAE2019-96715 (5-5-1) OMAE2019-96716 (6-4-4) OMAE2019-95748 (11-11-2) OMAE2019-96603 (1-4-2 OMAE2019-96181 (8-1-3) OMAE2019-96581 (6-5-4)

Huang, Wei-Po

OMAE2019-96706 (5-1-1) OMAE2019-96844 (3-3 
Jal, Eric .

OMAE2019-95875 (2-6-3) OMAE2019-95882 (2-6-3)

Jalon Ramirez, Maria Lourdes Jamrozik, Aleksandra

Jang, Hyunchul..... OMAE2019-95795 (1-1-3) OMAE2019-95380 (11-6-1) OMAE2019-96401 (1-6-2) OMAE2019-96404 (8-4-1), 0MAE2019-96429 (1-6-2) OMAE2019-96460 (1-6-1), OMAE2019-96838 (1-6-1) Jang, Youn-Young. OMAE2019-96480 (2-4-1) OMAE2019-96483 (3-1-2) OMAE2019-96010 (3-1-1)

Janin, Yin Jin Jaouën, Frédérick.

Jasak, Hrvoje

Jeans, Gus

Jegannathan, Manoj

Jenne, Dale

Jensen, Atle

Jensen, Jacob Fiske

Ji, Chunning..

Jia, Xu

Jian, Cao

Jian, Wang.

Jiang, Changbao

Jiang, Changqing.

Jiang, Fan.

Jiang, Kaijun

Jiang, Meirong..

Jiang, Quanxin ..

Jiang, Sheng-Chao

Jiang, Wei..

Jiang, Xiaoli...

Jiang, Yichen

Jiang, Yufeng.

Jiang, Zhe..

OMAE2019-95171 (8-1-5)

OMAE2019-95306 (8-1-5)

OMAE2019-95293 (8-1-3)

OMAE2019-96026 (8-2-3)

OMAE2019-96637 (6-7-3)

OMAE2019-96638 (1-1-2)

OMAE2019-96466 (9-4-2)

OMAE2019-96155 (4-5-1)

OMAE2019-95626 (1-1-4)

OMAE2019-95466 (8-4-3)

OMAE2019-96599 (10-1-1)

OMAE2019-96835 (6-15-2)

OMAE2019-96378 (9-5-4)

OMAE2019-96707 (11-1-3)

OMAE2019-95935 (8-1-1)

OMAE2019-95694 (7-1-1)

OMAE2019-95887 (8-1-7)

OMAE2019-96231 (11-7-3)

OMAE2019-95464 (3-1-2)

OMAE2019-95006 (6-3-4)

OMAE2019-95230 (2-4-2)

OMAE2019-95642 (4-1-7)

OMAE2019-95746 (3-3-1)

OMAE2019-95104 (9-2-2)

OMAE2019-95550 (2-5-1)

OMAE2019-95176 (6-15-2)

OMAE2019-95327 (2-4-3)

Jiang, Zhiyu . . . . . . OMAE2019-96221 (9-1-3), OMAE2019-95634 (1-1-3)

OMAE2019-95966 (4-5-2)

OMAE2019-95056 (4-4-1)

OMAE2019-96715 (5-5-1)

OMAE2019-96716 (6-4-4) OMAE2019-95541 (3-3-2) OMAE2019-95275 (3-3-3) OMAE2019-95200 (8-5-1) OMAE2019-96326 (13-1-4) OMAE2019-95196 (1-4-1) OMAE2019-95457 (4-1-4) OMAE2019-95911 (10-5-1) OMAE2019-96790 (6-17-2) OMAE2019-96699 (6-5-2) OMAE2019-95231 (9-5-3) OMAE2019-96055 (9-4-3) OMAE2019-95099 (9-6-1)

Jo, Chul Hee.

Johanning, Lars OMAE2019-95159 (9-1-2) OMAE2019-95759 (9-2-1), OMAE2019-95998 (9-5-2) OMAE2019-95759 (9-2-1), OMAE2019-95998 (9-5-2) ohansen, Sigrid Siksjø

Johlas, Hannah

John, Jeena Mary...

Johnston, Carol .

Johnstone, Daniel

Jonathan, Philip.

OMAE2019-95442 (13-1-1), OMAE2019-95913 (6-7-4) OMAE2019-96586 (6-7-4), OMAE2019-96587 (6-7-4) Jones, Colin ..

Jones, Justin..

Jones, Oliver...

Jonkman, Jason

Jorge, Gabriel

Josten, Michal

Juhlin, Rasmus.

Jun, HyunJo

Jung, Kwang Hyo

Júnior, José.

Jurdik, Erich. OMAE2019-96289 (2-1-1) OMAE2019-96092 (2-12-3) OMAE2019-96233 (3-2-1) OMAE2019-96426 (4-2-3) OMAE2019-96219 (4-3-2) OMAE2019-95276 (2-6-2) OMAE2019-96637 (6-7-3) OMAE2019-95429 (9-1-1) OMAE2019-95533 (9-1-8) OMAE2019-95145 (4-3-1) OMAE2019-96145 (1-4-3) OMAE2019-95503 (4-4-1) OMAE2019-95541 (3-3-2) OMAE2019-96557 (6-4-6) OMAE2019-95067 (2-5-1) OMAE2019-96154 (4-3-8)
$\mathbf{K}$

K A, Abhinav

Kabra, Saurabh

Kadakia, Ronak

Kagita, Gurumurthy

Kakuya, Hiromu

Kamal, Faris.

Kamath, Arun .......

OMAE2019-95154 (5-2-2)

OMAE2019-95921 (8-2-1), OMAE2019-96004 (6-5-2)

OMAE2019-96524 (8-2-1)

Kamizawa, Ken ……...................... OMAE2019-95828 (5-5-1)

Kana, Austin ................................... OMAE2019-95497 (2-9-3)

Kanada, Shige0................................ OMAE2019-95990 (5-3-1)

Kanao, Shunsuke .............................. OMAE2019-95733 (13-3-3)

Kanehira, Taiga OMAE2019-96405 (12-5-1)

Kang, Chang Wei............................... OMAE2019-95884 (9-5-3)

OMAE2019-95892 (8-1-7)

OMAE2019-95272 (1-1-1)

Kang, Daehoon

Kang, Ju-Yeon ...

Kang, Weili...

Kang, Youwei

OMAE2019-96480 (2-4-1)

OMAE2019-95473 (6-11-2)

OMAE2019-95070 (6-3-2)

OMAE2019-95072 (13-2-1), 0MAE2019-95251 (6-4-5)

OMAE2019-95490 (6-4-6)

Kang, Zhuang

OMAE2019-95251 (6-4-5)

OMAE2019-95490 (6-4-6)

Kanitz, Manuela

Kanno, Sota.

Kapoor, Kunal...

Karamanos, Spyros A

OMAE2019-95827 (13-7-1)

OMAE2019-96332 (4-3-3)

OMAE2019-95743 (4-3-2)

OMAE2019-95812 (3-2-2), 0MAE2019-95814 (4-3-6)

Karampour, Hassan

OMAE2019-95821 (4-3-1)

Karimirad, Madjid OMAE2019-95390 (2-7-1)

OMAE2019-96221 (9-1-3)

OMAE2019-95291 (4-6-2)

OMAE2019-95503 (4-4-1)

OMAE2019-95273 (6-5-1)

OMAE2019-95555 (12-7-1)

OMAE2019-95596 (13-1-1)

OMAE2019-95170 (9-1-1)

OMAE2019-95883 (11-2-1)

OMAE2019-96602 (13-2-2)

OMAE2019-95352 (2-9-3)

Kaufmann, Jan

Kawaguchi, Koji

Kawano, Seiya..

Kaynia, Amir M

OMAE2019-95220 (6-12-1)

OMAE2019-95270 (5-3-1)

OMAE2019-96168 (9-1-1)

Kazeminezhad, Mohammad Hossein .... OMAE2019-96827 (8-4-3)

Kazemtabrizi, Behzad.......................... OMAE2019-95561 (2-7-1)

$\mathrm{Ke}$, Sun

Kebadze, Elizbar B

Kejriwal, Vikas.

Kelkar, Mohan

Kellett, Paula

Kellner, Leon

Kelly, Michael

Kennedy, Justin

OMAE2019-96171 (5-1-2) OMAE2019-95523 (2-8-1) OMAE2019-96032 (2-6-1) OMAE2019-95190 (11-11-1) OMAE2019-95868 (6-13-2) OMAE2019-95085 (7-4-1) OMAE2019-96390 (9-4-4) OMAE2019-95505 (4-3-7) OMAE2019-95944 (10-4-1)

Keprate, Arvind

Khait, Anatoliy

Khalifeh, Mahmoud. OMAE2019-95155 (3-5-1) OMAE2019-95674 (11-15-1), OMAE2019-96594 (11-7-2) OMAE2019-96607 (11-15-2)

Khan, Ahsan

Khan, Zurwa OMAE2019-95330 (11-15-1)

Kim, Eung-su

OMAE2019-95272 (1-1-1)

Kim, Geon-Hong.

OMAE2019-95293 (8-1-3)

Kim, Hyun Joe. OMAE2019-95017 (1-6-2)

OMAE2019-95018 (1-6-2), OMAE2019-95272 (1-1-1)

OMAE2019-96236 (1-3-2), OMAE2019-96429 (1-6-2)

Kim, HyunS00 ............................... OMAE2019-95689 (7-3-1)

Kim, lk-Joong _ OMAE2019-96480 (2-4-1)

Kim, Jang ... OMAE2019-95675 (1-6-1)

OMAE2019-96139 (1-1-5), OMAE2019-96401 (1-6-2) OMAE2019-96404 (8-4-1), OMAE2019-96429 (1-6-2) OMAE2019-96460 (1-6-1), OMAE2019-96465 (1-7-1) OMAE2019-96838 (1-6-1) Kim, Jeong-Seok ............................ OMAE2019-96500 (9-3-3) Kim, Jo0-Sung ................................... OMAE2019-96236 (1-3-2) .... OMAE2019-96429 (1-6-2) Kim, Junbae ........... OMAE2019-95919 (9-1-3) Kim, Ki-Du. ..................................... OMAE2019-96778 (9-1-1) Kim, Ki-Seok ................................. OMAE2019-96483 (3-1-2) Kim, Kyong-Hwan................................. OMAE2019-96500 (9-3-3) Kim, Munsung .................................... OMAE2019-95272 (1-1-1) Kim, Myung-Hyun ........................... OMAE2019-95910 (3-5-1)

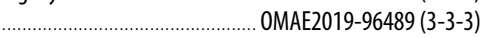
Kim, Nam-Woo ................................ OMAE2019-95406 (1-7-1) Kim, Sei-Hwan .................................... OMAE2019-95410 (8-1-6) Kim, Sooyoul.................................. OMAE2019-95336 (13-1-1) Kim, Sung-Jae................................... OMAE2019-96055 (9-4-3) Kim, Tae-min ................................... OMAE2019-95272 (1-1-1) Kim, Woo Sik ..................................... OMAE2019-95281 (3-3-1) Kim, Young Jun _ . ... OMAE2019-96278 (8-2-1) Kim, Young-Pyo …….......................... OMAE2019-96480 (2-4-1) Kim, Youngkook ................................. OMAE2019-95048 (2-6-1) Kim, Yun-Jae …............................ OMAE2019-96483 (3-1-2) Kimanzi, Raymos …........................ OMAE2019-95380 (11-6-1) .... OMAE2019-95614 (11-10-1) Kioka, Shinji ....................................... OMAE2019-95654 (7-3-1) .... OMAE2019-95901 (6-2-3)

Kirezci, Cagil.

Kirkemo, Finn.

Kisjes, Anton

Kitabatake, Yuichi OMAE2019-95740 (2-1-2) OMAE2019-96685 (3-3-3) OMAE2019-95513 (6-8-2)

Kitazawa, Daisuke OMAE2019-96493 (13-2-5) OMAE2019-95596 (13-1-1) OMAE2019-95670 (5-2-2), 0MAE2019-95672 (5-2-2)

Klein, Marco OMAE2019-95827 (13-7-1), 0MAE2019-95978 (5-5-1) OMAE2019-95063 (6-17-1) OMAE2019-95085 (7-4-1), OMAE2019-95116 (7-4-1) Knagenhjelm, Hans Olav. OMAE2019-95344 (4-2-5) Kodaira, Tsubasa . OMAE2019-95842 (13-1-1) OMAE2019-95949 (13-1-3)

Kogo, Bridget..

Kokal, Sunil.

Kolios, Athanasios OMAE2019-95191 (11-1-1) OMAE2019-95210 (1-1-3) OMAE2019-96323 (2-7-1)

Komariyah, Siti

Komoriyama, Yusuke

Kondo, Joe.....

Kong, Xiangdi..

Kong, Yue. OMAE2019-96760 (2-4-2) OMAE2019-95389 (2-9-3) OMAE2019-95923 (3-9-1) OMAE2019-95125 (2-5-1) OMAE2019-95651 (4-1-4) 


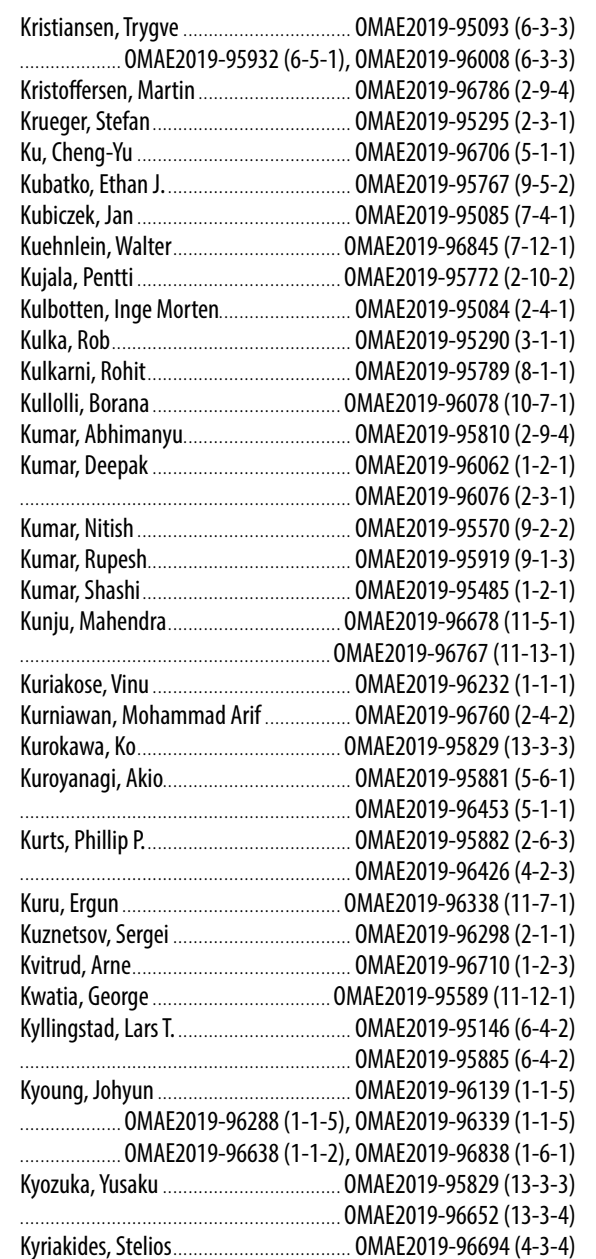

\section{L}

La Mura, Francesco

Lacan, Ludovic

Ladstein, Jarle

Laface, Valentina ..

Lagasco, Fabrizio.

Lal, Mayank

Lambkin, David

Lamei, Azin

Landon, Melissa E.

Lang, Donogh

Langøy, Morten

Langseth, Magnus

Larangeira, Vicente.

Larrea, Ibon...

Larsen, Kjell ..

Larsen, Mikkel L

Lassen, Tom.

Lataire, Evert OMAE2019-95565 (6-11-2) OMAE2019-96341 (6-5-2) OMAE2019-96346 (6-5-2) OMAE2019-96562 (6-8-2)

Lavroff, Jason

Lavrov, Alexandre

Law, Yun Zhi.

Le Dreff, Jean-Baptiste

Le Toux, Vincent

\section{Le Touze, David}

Lechner, Daniel.

Lee, Donghwan.

Lee, DongYeon..

Lee, Jae Sung

Lee, Jae-bin...

Lee, Jaeyong OMAE2019-96368 (9-4-1) OMAE2019-96104 (5-1-2) OMAE2019-95135 (4-2-5) OMAE2019-95367 (6-7-3) OMAE2019-96034 (9-1-3) OMAE2019-96395 (9-1-5) OMAE2019-96261 (4-2-3) OMAE2019-95260 (3-6-1) OMAE2019-95920 (2-10-1) OMAE2019-95067 (2-5-1) OMAE2019-96104 (5-1-2) OMAE2019-95083 (2-6-2 OMAE2019-95291 (4-6-2) OMAE2019-95704 (1-4-2) OMAE2019-96400 (11-7-2) OMAE2019-95649 (8-4-4) OMAE2019-95429 (9-1-1) OMAE2019-95354 (4-6-1) OMAE2019-95493 (4-6-1) OMAE2019-96278 (8-2-1) OMAE2019-95949 (13-1-3) OMAE2019-95046 (1-6-1) OMAE2019-95272 (1-1-1) OMAE2019-96236 (1-3-2 OMAE2019-96489 (3-3-3) OMAE2019-95689 (7-3-1) OMAE2019-96557 (6-4-6)

Lee, Jong Hun
Lee, Jung Min
Lee, Kang-Su
Lee, Kyung Ho
Lee, Myeong-Woo
Lee, Sang-Hun
Lee, Seung Jae
Lee, Young-Bum
Leen, Sean B.
Lefebvre, Timothee
Leimeister, Mareike

OMAE2019-96236 (1-3-2 OMAE2019-96053 (6-15-2) OMAE2019-95406 (1-7-1) OMAE2019-96053 (6-15-2) OMAE2019-96483 (3-1-2) OMAE2019-95410 (8-1-6) OMAE2019-96557 (6-4-6) OMAE2019-95410 (8-1-6) OMAE2019-96516 (3-5-1) OMAE2019-96288 (1-1-5) Leira, Bernt . _ OMAE2019-95139 (7-3-1)

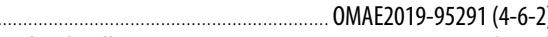
Leithead, William.................................. OMAE2019-96017 (9-2-6) Leixiang, Sheng ................................... OMAE2019-95196 (1-4-1) Lemmer, Frank.

\section{Lenn, Yueng-Djern}

Leong, Darrell

Lettenmaier, Terry

Li, Ben .

Li, Bo

Li, Chang-Fei.

Li, Chao.

Li, Chenfeng.

Li, Cheng.

Li, Chuntong

Li, Da

Li, Dongsheng..

Li, Hao Jin..

Li, Haoran.

Li, Harry..........

Li, Hui.

Li, Jiagang.............

Li, Jiale ........................................ OMAE2019-95637 (10-7-1)

OMAE2019-95641 (10-4-1)

Li, Jun... OMAE2019-95292 (4-2-2)

Li, Juyue ...................................... OMAE2019-95123 (4-1-10)

OMAE2019-95070 (6-3-2)

OMAE2019-95072 (13-2-1), OMAE2019-95693 (1-7-2) OMAE2019-95475 (4-3-2) Li, Liang...................................... OMAE2019-96212 (5-1-2) OMAE2019-95146 (6-4-2) OMAE2019-96282 (5-1-2) OMAE2019-95880 (2-2-2) Li, Lihui.......................................... OMAE2019-95196 (1-4-1)

Li, Li Li

Li, Min OMAE2019-96079 (5-2-2) OMAE2019-95651 (4-1-4) OMAE2019-95658 (6-4-5) Li, Minghui ................................... OMAE2019-95624 (11-3-1) Li, Ping ......................................... OMAE2019-96798 (6-8-2) Li, Qiao …………........................ OMAE2019-95400 (13-3-2) OMAE2019-95836 (13-3-2)

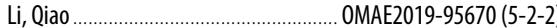

Li, Qinyuan

LI, Shancheng..

Li, Shengcang.

Li, Shuai

Li, Shuzhao .

Li, Xiao.......

Li, Xiaobin .

Li, Xin ........ OMAE2019-95634 (1-1-3) OMAE2019-95311 (6-8-2) OMAE2019-95438 (8-1-2) OMAE2019-96486 (1-3-1) OMAE2019-96599 (10-1-1) OMAE2019-95642 (4-1-7) OMAE2019-95745 (2-12-3) OMAE2019-95776 (2-9-4) OMAE2019-95744 (8-5-1)

OMAE2019-95635 (9-2-3)

OMAE2019-95638 (9-1-8)

Li, Yan ......................................... OMAE2019-96082 (6-17-1)

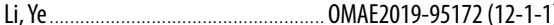

OMAE2019-95634 (1-1-3)

OMAE2019-95651 (4-1-4)

Li, Yilun.

OMAE2019-95658 (6-4-5)

Li, Yinggang.................................... OMAE2019-96193 (12-1-1)

Li, Yue . OMAE2019-95670 (5-2-2)

Li, Yugang ....................................... OMAE2019-95016 (1-2-2)

Li, Yuyan ....................................... OMAE2019-95308 (13-2-5)

OMAE2019-95706 (13-2-1)
Li, Zhaochuan

OMAE2019-96094 (11-7-3)

OMAE2019-95541 (3-3-2)

OMAE2019-95618 (2-6-2), OMAE2019-96191 (2-6-2)

Li, Zhigang ................................... OMAE2019-96599 (10-1-1)

$\mathrm{Li}$, Zhiheng ..................................... OMAE2019-95887 (8-1-7)

Li, Zhipeng .......................................... OMAE2019-95694 (7-1-1)

OMAE2019-95850 (8-3-1)

Li, Zhiyuan ....................................... OMAE2019-95128 (7-1-1)

Li, Zhun ....................................... OMAE2019-95106 (2-11-3)

LI, Zongchen OMAE2019-95746 (3-3-1)

Lian, Yushun ..................................... OMAE2019-96802 (9-4-3)

Liang, Shuxiu ...................................... OMAE2019-95942 (6-3-3)

Liang, Yibo _. OMAE2019-95203 (13-1-2)

OMAE2019-95597 (13-2-3), OMAE2019-95607 (13-2-4)

Liangbin, Xu ..................................... OMAE2019-95196 (1-4-1)

Liao, Kangping .................................... OMAE2019-95224 (8-2-1)

OMAE2019-95675 (1-6-1)

Liao, Yongqiang OMAE2019-96719 (11-7-3)

Licheri, Fabio .................................... OMAE2019-96513 (9-6-1)

OMAE2019-96518 (9-4-1)

Lie, Halvor $\quad$ OMAE2019-95798 (7-3-1)

OMAE2019-95083 (2-6-2)

Liebert, Maximilian …………....... OMAE2019-95248 (1-4-2)

Lillebø, Odd Arne ..................................... OMAE2019-96343 (4-3-3)

Lim, Frank OMAE2019-95196 (1-4-1)

Lim, Ho-Joon _............................ OMAE2019-96288 (1-1-5)

OMAE2019-96339 (1-1-5), OMAE2019-96465 (1-7-1)

OMAE2019-96638 (1-1-2), OMAE2019-96838 (1-6-1)

Lima, Nicolao ................................... OMAE2019-96255 (4-4-2)

Lin, Pengzhi .................................... OMAE2019-96790 (6-17-2)

Lin, Yu-Hsien _ OMAE2019-95321 (6-15-1)

Lin, Zaibin ................................... OMAE2019-96098 (12-5-2)

Lin, Zhiliang ...................................... OMAE2019-95172 (12-1-1)

Lin, Zi....................................... OMAE2019-95542 (9-2-6)

Lincoln, Ben ...................................... OMAE2019-96621 (6-7-2)

Ling, Bradley A. OMAE2019-95538 (9-4-1)

OMAE2019-95544 (9-3-1)

Ling, Shiun ...................................... OMAE2019-95541 (3-3-2)

Lirola, François ...................... OMAE2019-95057 (4-1-2)

Liu, Bin ........................................ OMAE2019-96699 (6-5-2)

Liu, Chang ........................................ OMAE2019-95692 (4-1-6)

OMAE2019-95903 (4-1-5), OMAE2019-95916 (4-1-7)

Liu, Cheng .................................... OMAE2019-95081 (6-11-1)

Liu, Dahui...................................... OMAE2019-95930 (7-12-1)

Liu, Feng....................................... OMAE2019-96001 (6-15-2)

Liu, Haixiao ..................................... OMAE2019-95778 (10-3-1)

OMAE2019-95960 (10-3-1), OMAE2019-96036 (10-5-1)

Liu, Hao ....................................... OMAE2019-95737 (8-5-1)

Liu, Hao ......................................... OMAE2019-95777 (2-9-3)

Liu, Haoxue .................................... OMAE2019-95915 (9-2-1)

Liu, Hongda ................................... OMAE2019-96017 (9-2-6)

Liu, Jiabin .............. OMAE2019-96657 (6-5-3)

Liu, Jianfei..................................... OMAE2019-95586 (7-11-1)

Liu, Jianyu ........................................ OMAE2019-96181 (8-1-3)

.... OMAE2019-96545 (1-4-1)

Liu, Jiaqi .................................... OMAE2019-95644 (2-12-2)

Liu, Jiaqi ....................................... OMAE2019-96013 (6-2-1)

Liu, Jing........................................ OMAE2019-95231 (9-5-3)

…… OMAE2019-95601 (13-2-3)

Liu, Jing ............................................ OMAE2019-96543 (8-3-1)

OMAE2019-96577 (1-2-3)

Liu, Jun ..................................... OMAE2019-95491 (2-12-1)

OMAE2019-95924 (2-12-2), 0MAE2019-96628 (2-12-2)

Liu, Junpeng ................................... OMAE2019-95141 (4-1-3)

Liu, Lei.............................................. OMAE2019-96578 (6-3-4)

Liu, Liqin _. OMAE2019-95207 (9-2-2)

Liu, Liwei........................................ OMAE2019-95563 (8-1-5)

OMAE2019-95874 (8-1-7), 0MAE2019-96791 (8-1-2)

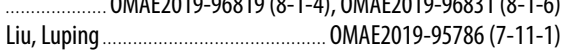

Liu, M ................ OMAE2019-95444 (4-3-7)

OMAE2019-95448 (4-3-7), OMAE2019-95450 (4-3-6)

Liu, Mingling ................................... OMAE2019-96791 (8-1-2)

Liu, Mingyue .................................. OMAE2019-95601 (13-2-3)

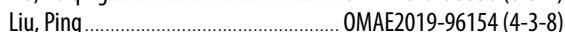

Liu, Peiqing ........................................ OMAE2019-95556 (6-3-1) 


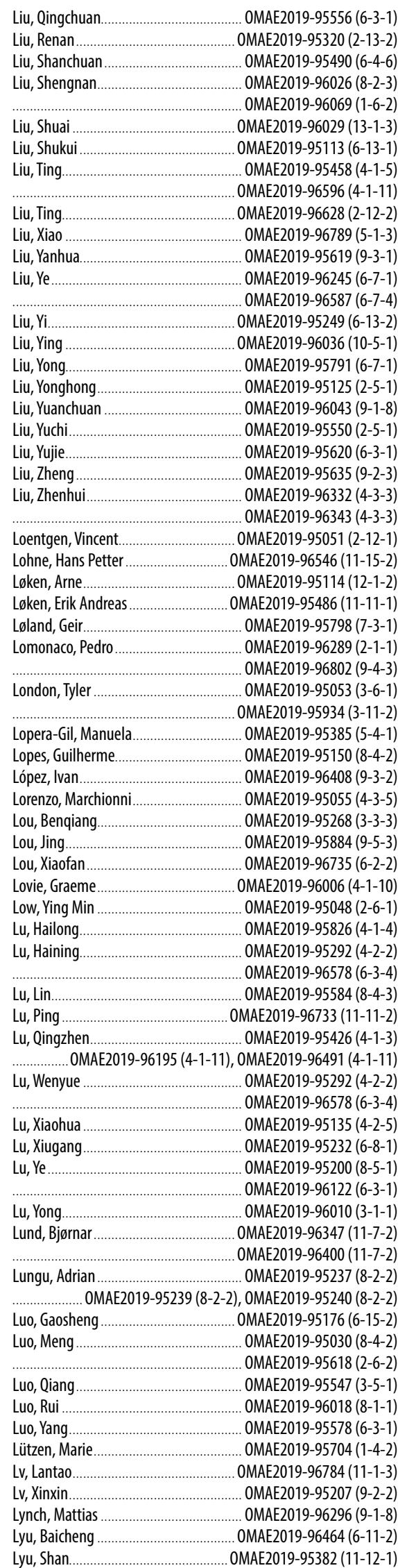

\section{M}

Ma, Chengqian $\mathrm{Ma}$, Chong.

$\mathrm{Ma}$, He.

$\mathrm{Ma}$, Jinsheng OMAE2019-95132 (13-1-4) OMAE2019-95781 (2-9-3) .. OMAE2019-95141 (4-1-3)

\section{Ma, Li...........}

$\mathrm{Ma}$, Ning..

\section{Ma, Qian...}

Ma, Qingwei

$\mathrm{Ma}$, Qingwei.

Ma, Shan.

Ma, Yuan

$\mathrm{Ma}$, Zhihua

Macchion, Olivier

Macdonald, Kenneth

Macfarlane, Gregor..

Machado Cruz, Leandro.

Mackay, Edward...

Macke, Michael

MacRae, Ian .

Maeda, Teruo

Magee, Allan.

OMAE2019-96543 (8-3-1)

OMAE2019-96577 (1-2-3), OMAE2019-96699 (6-5-2)

.. OMAE2019-96753 (5-1-3), OMAE2019-96761 (5-1-3)

OMAE2019-96789 (5-1-3)

Magluta, Carlos ................................ OMAE2019-96741 (4-3-3)

Magnusson, Anne Karin ........................ OMAE2019-96837 (2-1-2)

Maher, James................................... OMAE2019-95523 (2-8-1)

Maherault-Mougin, Stéphanie ............... OMAE2019-96239 (3-3-1)

Mai, SongYan OMAE2019-95320 (2-13-2)

Majed, Arya .................................... OMAE2019-95303 (4-1-3)

Mak, Bart ..................................... OMAE2019-96201 (13-2-2

OMAE2019-96225 (13-2-2)

Malara, Giovanni ................................ OMAE2019-95973 (9-3-2)

OMAE2019-96368 (9-4-1)

OMAE2019-95369 (3-3-2)

Malcher, Lucival

Maleki, Amir

Malenica, Sime

Malila, Mika

Mamiya, Edgar.

Man, Jiangtao

Mansour, Ahmed

Mansuy, Marc........

Manuel, Lance

Manzocchi, Mark

Mao, Baijin...

Mao, Wengang.

Marchesani, Furio

Marcollo, Hayden

OMAE2019-96411 (2-6-1), OMAE2019-96426 (4-2-3)

OMAE2019-96431 (1-3-2)

Marculino, Cinara F. G........................ OMAE2019-95069 (11-4-1)

Mariani, Alessio .............................. OMAE2019-95557 (4-3-8)

Marines-Garcia, Israel........................ OMAE2019-95130 (3-1-1) .. OMAE2019-96345 (3-5-1) . OMAE2019-96276 (6-5-4)

Marino, Alessandro

Marinov, Krasimir

Mariotti, Alessandra

Marjanovic, Ognjen

Marmonier, Geoffrey

Martin, Nevin...

Martin, Tobias. OMAE2019-95233 (8-3-1), OMAE2019-96004 (6-5-2)

OMAE2019-96524 (8-2-1)

Martinez Perez, Imanol ....................... OMAE2019-95084 (2-4-1) OMAE2019-96000 (2-4-1) OMAE2019-95510 (4-1-2)

Martins, Ricardo R

Mas Soler, Jordi.

Masanobu, Sotaro

Masud, Mamoon

Masuda, Koichi OMAE2019-96670 (6-4-2) OMAE2019-95270 (5-3-1) OMAE2019-95134 (5-4-1) OMAE2019-95408 (5-6-1) OMAE2019-95925 (13-3-4), OMAE2019-95927 (5-6-1) OMAE2019-96045 (13-3-1), OMAE2019-96159 (5-6-1) OMAE2019-96482 (13-2-5), OMAE2019-96493 (13-2-5)

Masuda, Mitsuhiro

Masuda, Norikazu

Mathieson, Scott

Matsumoto, Kazuyuki OMAE2019-96159 (5-6-1) OMAE2019-95401 (13-7-1) OMAE2019-96219 (4-3-2)
Maturana, Marcos C.

OMAE2019-96251 (2-13-2) OMAE2019-96269 (2-13-1)

Maximiano, António ............................... OMAE2019-96099 (8-2-3) May, Roland ................................... OMAE2019-95228 (11-7-1)

Mazadiego, Luis Felipe ....................... OMAE2019-96103 (11-4-1)

Mazhar, Suleman .................................... OMAE2019-95134 (5-4-1)

McAdam, Ross A............................... OMAE2019-95767 (9-5-2)

McAllister, Mark _........................... OMAE2019-96817 (12-5-1)

McCall, Alan ..................................... OMAE2019-96397 (9-3-3)

McCauley, Guy OMAE2019-95933 (12-5-2)

McConochie, Jason ............................. OMAE2019-95451 (2-2-1)

McDonald, Alasdair ……..................... OMAE2019-96720 (9-7-1)

McGuire, Leslie OMAE2019-95178 (1-4-3)

McKamey, Jacob ................................. OMAE2019-95101 (6-2-3)

McPhail, Finlay _......................... OMAE2019-96090 (1-5-1)

Medico, Roberto OMAE2019-96731 (9-7-1)

Meguro, Koji....................................... OMAE2019-96623 (5-4-1)

Mehmanparast, Ali _ OMAE2019-95290 (3-1-1)

Mehr, Javad...................................... OMAE2019-96562 (6-8-2)

Mei, Hongyuan ………................... OMAE2019-95683 (2-11-1)

Mei, Tianlong OMAE2019-95249 (6-13-2)

Melo, Luis _... OMAE2019-95363 (4-3-1)

Mendes, José Ricardo P D OMAE2019-95069 (11-4-1)

Mendoza, Edgar .................................. OMAE2019-96747 (6-13-1)

Meneses, Lucia OMAE2019-96380 (9-1-2)

Meng, Lida _.............................. OMAE2019-96716 (6-4-4)

Mentzoni, Fredrik ……………............. OMAE2019-95093 (6-3-3)

OMAE2019-96008 (6-3-3)

Merrien, Arnaud …………………….... OMAE2019-96272 (2-9-2)

Merxhani, Andi .................................. OMAE2019-95626 (1-1-4)

Meziou, Amine OMAE2019-96293 (4-5-1)

Miao, Yuji........................................ OMAE2019-96771 (6-1-2)

Michael, Martinez ................................ OMAE2019-95511 (4-3-2)

OMAE2019-96165 (1-2-4)

Michailides, Constantine ........................ OMAE2019-96221 (9-1-3)

Milano, Daniel _ OMAE2019-96243 (9-1-2)

Milewski, Henry................................. OMAE2019-95938 (10-6-1)

OMAE2019-95944 (10-4-1)

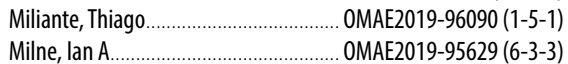

Milne, lan A.......

Min, Eun-Hong ..

Minami, Fumiyosh

Minami, Kiyokazu

Minescu, Mihail

Ming, Furen.

Mintu, Shafiul.

Miran, Sajjad...

OMAE2019-95664 (6-17-2)

OMAE2019-95816 (3-9-1) .. OMAE2019-96159 (5-6-1)

OMAE2019-96283 (11-10-1)

OMAE2019-95257 (6-13-3) . OMAE2019-95799 (8-2-3) OMAE2019-96492 (4-6-2) Miranda, Cristiane ............................ OMAE2019-95131 (11-12-1) Miratsu, Rei OMAE2019-95121 (2-2-2) Miriello, Davide ................................... OMAE2019-95024 (5-1-3) Mirzaee Sisan, Ali ................................. OMAE2019-96615 (3-6-1) OMAE2019-96622 (3-11-1)

Mitsui, Masao OMAE2019-95220 (6-12-1)

Miyanawala, Tharindu Pradeeptha ...... OMAE2019-95870 (8-3-1) Miyasawa, Shintaro ........................... OMAE2019-96493 (13-2-5) Miyazaki, Marcelo................................. OMAE2019-95506 (4-1-7) Mizukami, Yoichi................................. OMAE2019-95670 (5-2-2)

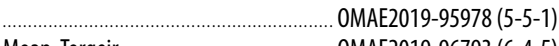
Moan, Torgeir .................................... OMAE2019-96793 (6-4-5) Mochizuki, Akito ............................ OMAE2019-95827 (13-7-1) Moe Føre, Heidi.................................... OMAE2019-95350 (3-9-1) Moe, Per T. OMAE2019-96185 (4-4-2) Moghtadaei, Abdolmajid ..................... OMAE2019-95390 (2-7-1) Mohammad Beigi Kasvaei, MohammadOMAE2019-96827 (8-4-3) Mohr, Henning OMAE2019-95557 (4-3-8) Mohr, William ……............................. OMAE2019-96748 (3-1-1) Mohtat, Ali.

Moideen, Rameeza Mole, Sindhu ............... OMAE2019-95312 (6-5-4) Molgaard, John …………………..... OMAE2019-96176 (11-1-3)

Molina Ruiz, Josep OMAE2019-96189 (11-1-2)

Molins, Climent

Molyneux, David. 


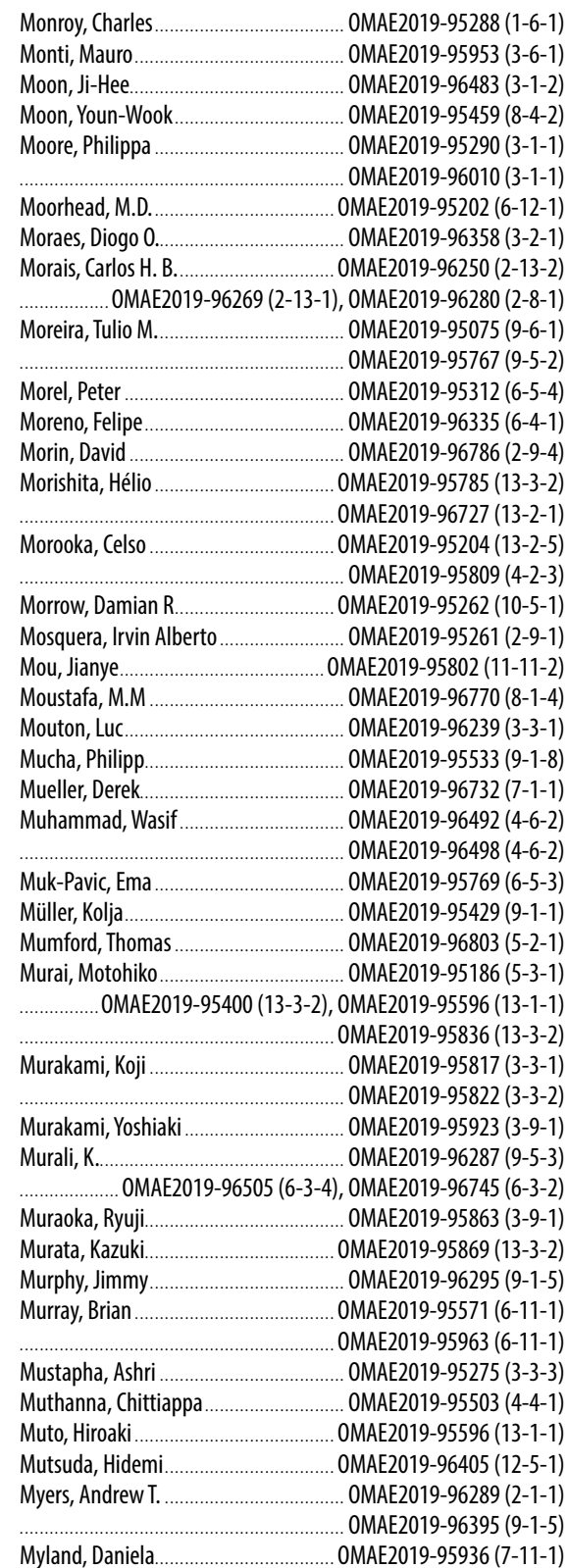

\section{$\mathbf{N}$}

$\mathrm{N}$, Vignesh Chellappan

Naccache, Mônica..

Nadathur, Varadarajan

Naeeni, Seyed Taghi Omid.

Nagataki, Takumi

Nahri, Syed Y. OMAE2019-96682 (11-7-3), OMAE2019-96683 (11-1-2)

Nakajima, Yasuharu. _.................... OMAE2019-95186 (5-3-1) OMAE2019-96040 (5-3-1) OMAE2019-96045 (13-3-1)

Nakamura, Mitsuru

Nakano, Kenji.

Nakayama, Shin

Naldi, Giovanni

Nam, Bo Woo

OMAE2019-95896 (11-2-2)

OMAE2019-95912 (2-10-2)

OMAE2019-96721 (9-7-1) OMAE2019-95406 (1-7-1)

OMAE2019-96429 (1-6-2), OMAE2019-96500 (9-3-3)

Nascimento, Leonardo _ OMAE2019-95577 (4-3-4)

Nasef, A.

Natarajan, Satish

Navarro, Jesus

Nedreberg, Mette Lokna

Nejad, Amir R.

Nenadic, Goran

Nestegaard, Arne...... OMAE2019-96770 (8-1-4) OMAE2019-96270 (6-15-1) OMAE2019-95283 (6-4-6) OMAE2019-95152 (9-7-1) OMAE2019-95542 (9-2-6) OMAE2019-95300 (6-3-1)
Netto, Theodoro

OAE2019-95768 (4-3-1) OMAE2019-95808 (4-5-2)

Neugebauer, Jens

Neumann, Karoline Mali

Nevarez, Victor.

Neves, Raniere

Newbury, Brian

Newell, Carter.. OMAE2019-96593 (3-4-1), OMAE2019-96614 (3-4-1)

Newsam, David

Nguyen, Hoai-Nam

Nguyen, Phong T. T.

Ni, Baoyu.

$\mathrm{Ni}$, Xinyun.

$\mathrm{Ni}, \mathrm{Yi}$

Nickerson, Brendon

Nicolosi, Eduardo Ribeiro

Nielsen, James L

Nihei, Yasunori OMAE2019-95403 (13-7-1), OMAE2019-95404 (13-3-1)

Niino, Eiji

Nikbin, Kamran

Ning, Dezhi...

OMAE2019-95165 (12-4-1), OMAE2019-95739 (12-4-1) OMAE2019-96221 (9-1-3)

Nishigochi, Ryo

Nishikawa, Tatsuo

Nishimoto, Kazuo

Nordam, Tor

Norén-Cosgriff, Karin

Norman, Rose...

Norvik, Carina

Nose, Takehiko.

Notani, Yusuke.

Nowé, Ann

Nuernberg, Martin

Nunes Filho, Eduardo

Nunhez, Roberto.

Nuno, Fonseca.

Nwaka, Nnamdi.............................. OMAE2019-96672 (11-13-1) OMAE2019-96138 (8-1-2)

OMAE2019-95907 (2-15-1) OMAE2019-96382 (9-4-3) OMAE2019-95369 (3-3-2) OMAE2019-96080 (9-1-5) OMAE2019-95197 (9-3-1) OMAE2019-96523 (2-2-1) OMAE2019-95694 (7-1-1) OMAE2019-95200 (8-5-1) , OMAE2019-96771 (6-1-2)
OMAE2019-95706 (13-2-1) OMAE2019-96746 (2-12-1) OMAE2019-96730 (4-1-2) OMAE2019-96678 (11-5-1) OMAE2019-95401 (13-7-1) OMAE2019-95818 (3-6-1) OMAE2019-95511 (4-3-2) OMAE2019-95739 (12-4-1) OMAE2019-96482 (13-2-5) OMAE2019-95403 (13-7-1) OMAE2019-95238 (12-2-1) OMAE2019-95674 (11-15-1) .. OMAE2019-96168 (9-1-1) OMAE2019-95142 (6-4-1) . OMAE2019-95485 (1-2-1) .. OMAE2019-96375 (5-2-1) OMAE2019-95949 (13-1-3) OMAE2019-95883 (11-2-1) OMAE2019-96602 (13-2-2) .. OMAE2019-96713 (9-7-1) OMAE2019-95244 (9-2-3) OMAE2019-95369 (3-3-2) OMAE2019-96255 (4-4-2) .. OMAE2019-96753 (5-1-3) OMAE2019-96761 (5-1-3) Nyhus, Bård.. OMAE2019-95344 (4-2-5)

\section{0}

0 'Dowd, Noel

$0^{\prime}$ Loughlin, Conleth

O'Brien Bromley, Stephen

$\mathrm{O}^{\prime}$ Donoghue, Padraic E.

O'Sullivan, Jim. OMAE2019-95722 (9-5-4)

\section{Oberhagemann, Jan.}

Odan, Mohamed..

Ofuegbe, James.

Ogbeifun, Achoyamen M.

Oh, Sho.

Okamoto, Ken

Oladosu, Olamide

Omair, Abdullatif

Omland, Tor Henry.

Ommani, Babak

\section{Ong, Lin Seng}

Ong, Muk Chen OMAE2019-96069 (1-6-2), OMAE2019-96079 (5-2-2 OMAE2019-96332 (4-3-3), OMAE2019-96839 (6-4-3)

Ono, Masao.

Onorato, Miguel

Ooshima, Teruo.

Opedal, Nils.. OMAE2019-95116 (7-4-1) OMAE2019-95394 (5-3-1) OMAE2019-95928 (11-15-2) OMAE2019-96773 (11-6-2)

Orsino, Renato Orszaghova, Jana.
OMAE2019-95990 (5-3-1)

Ortega, Arturo

Osawa, Naoki

Osborne, Alfred R. OMAE2019-95546 (2-2-1), 0MAE2019-96613 (13-1-3) OMAE2019-96637 (6-7-3) Osorio Santander, Elvis J........................ OMAE2019-96741 (4-3-3) Østby, Erling..................................... OMAE2019-96685 (3-3-3) Oterkus, Selda. Otten, Jeffrey D. Ozden, Erinc....... Ozekcin, Adnan Ozkan-Haller, Tuba Ozmutlu, Senol Ozturk, Burak... OMAE2019-95160 (4-2-1) OMAE2019-96232 (1-1-1) OMAE2019-95689 (7-3-1) OMAE2019-95541 (3-3-2) OMAE2019-96802 (9-4-3) OMAE2019-96395 (9-1-5) OMAE2019-96754 (10-6-1)

Paboeuf, Stéphane

OMAE2019-96239 (3-3-1) Paes, Marcelo. OMAE2019-96358 (3-2-1) Page, Ana. OMAE2019-95170 (9-1-1) OMAE2019-96168 (9-1-1)

Paggi, Alessandro

Pain, Christopher OMAE2019-95953 (3-6-1) …. OMAE2019-96814 (12-2-1) Paiva Nunes, Luis Manoel...................... OMAE2019-96592 (4-2-4) Pakozdi, Csaba

Palermo, Thierry OMAE2019-95921 (8-2-1) OMAE2019-95493 (4-6-1), OMAE2019-95059(4-6-1) Paltrinieri, Nicola ............................. OMAE2019-95622 (2-13-1) Pan, Chen ……………………...... OMAE2019-95603 (4-4-2) Pan, Guang...................................... OMAE2019-95106 (2-11-3) Pan, Jin.........

Pan, Yujie.... Pan, Zhiyuan

Pang, Dan.

Papaefthimiou, Georgia

Papanikolaou, Apostolos.

Papatheocharis, Theocharis..

Paquet, Stephane

Paravicini Bagliani, Emanuele..

Parihar, Yogendra..

Park, Jin Ho .

Park, Jong-Chun

Park, Sanggyu.

Park, Sewan

Park, Young-chan

Pasqualino, Ilson.

Pasquet, François-Xavier

Passano, Elizabeth

OMAE2019-95602 (2-10-1) OMAE2019-96784 (11-1-3) OMAE2019-95300 (6-3-1) OMAE2019-95584 (8-4-3) OMAE2019-95446 (3-2-2) OMAE2019-95113 (6-13-1) OMAE2019-95812 (3-2-2) OMAE2019-96840 (1-5-1) OMAE2019-95953 (3-6-1) OMAE2019-95867 (2-11-2) OMAE2019-96778 (9-1-1) OMAE2019-95459 (8-4-2) OMAE2019-95672 (5-2-2) OMAE2019-96500 (9-3-3) OMAE2019-95272 (1-1-1) OMAE2019-96730 (4-1-2) OMAE2019-95354 (4-6-1)

Patel, Harshkumar OMAE2019-95970 (8-4-4) OMAE2019-95972 (8-4-1) OMAE2019-95588 (11-12-2) OMAE2019-95614 (11-10-1), OMAE2019-96776 (11-6-2) Patino, Julio..

Patton, Ron

Pavanello, Renato

Paz, Paulo.

Pazouki, Kayvan.

Pedersen, Eilif.

Pedersen, Ronnie R.

Peek, Ralf OMAE2019-95144 (2-3-1) OMAE2019-95619 (9-3-1) OMAE2019-96047 (4-4-1) OMAE2019-95808 (4-5-2) OMAE2019-95142 (6-4-1) OMAE2019-95474 (1-4-2) OMAE2019-95704 (1-4-2) OMAE2019-95970 (8-4-4) OMAE2019-95972 (8-4-1) Peeters, Cédric …………………...... OMAE2019-96713 (9-7-1) Pelcastre Mauricio OMA..................... OMAE2019-96731 (9-7-1)

\section{Pelcastre, Mauricio}

Pelipenko, Sviatoslav OMAE2019-95100 (11-12-2)

Pellegrini, Carlo

Pena, Blanca.

Peng, Heather.

Peng, Peng...

Peng, Tao..

Peng, Xirui

Peng, Zhi-Ke.

Peng, Zhong

Peng, Ziya. .. OMAE2019-96588 (1-4-1) OMAE2019-95769 (6-5-3) OMAE2019-96775 (12-7-1) OMAE2019-95830 (4-2-5) OMAE2019-95292 (4-2-2) OMAE2019-95530 (6-4-4) OMAE2019-95915 (9-2-1) OMAE2019-96021 (6-12-1) OMAE2019-95226 (2-11-1)

Pepin, Aurelien

Perdikaris, Philip C MAE2019-95230 (2-4-2) 


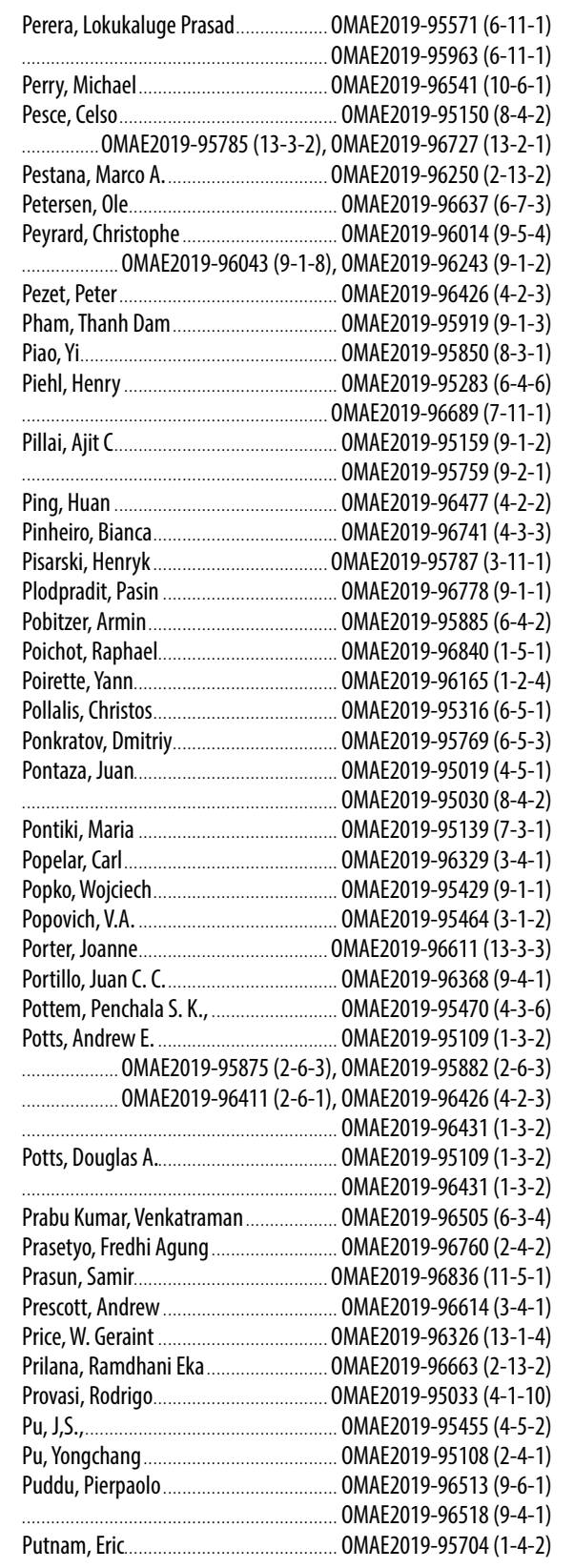

\section{Q}

Qian, Ling.

Qian, Xin

Qian, Yuan..

Qian, Zhaohui

\section{Qiang, Bin ...}

\section{Qiang, Wei}

Qiao, Dongsheng

\section{Qin, Wei}

Qiu, Hongyuan..

Qiu, Wei.

Qu, Qiulin.

Qu, Xiaoqi...

Qu, Yan.

\section{Qu, Zhaoguang}

Quadvlieg, Frans H. H. A.

Quah, Matthew.

Queija de Siqueira, Marcos.

Quiroz, Tulio .

Quispe, Carlos Abad Estrada

Quispe, José Luis

Qvist, Jacob...

OMAE2019-96131 (7-12-1)

OMAE2019-96715 (5-5-1)

OMAE2019-96716 (6-4-4)

OMAE2019-95517 (3-1-2)

OMAE2019-95603 (4-4-2)

OMAE2019-95016 (1-2-2)

OMAE2019-95148 (12-1-1)

OMAE2019-95631 (4-2-5)

OMAE2019-96170 (11-2-2

OMAE2019-96775 (12-7-1)

OMAE2019-95556 (6-3-1)

OMAE2019-95638 (9-1-8)

OMAE2019-95123 (4-1-10)

OMAE2019-96661 (1-1-1)

... OMAE2019-96733 (11-11-2)

OMAE2019-95513 (6-8-2)

OMAE2019-96541 (10-6-1)

OMAE2019-96372 (4-1-1)

OMAE2019-96109 (10-7-1)

OMAE2019-95768 (4-3-1)

OMAE2019-96367 (4-4-2) OMAE2019-95429 (9-1-1)

\section{$\mathbf{R}$}

Rabb, Ben

Raby, Alison

Race, Julia

Rachman, Andika

Rackwitz, Frank

Rade, Domingos A

Ragni, Raffaele

Rahman, Mohammad Azizur

OMAE2019-95219 (11-1-1), OMAE2019-96311 (11-4-1)

Rahmati, Mohammad .......................... OMAE2019-96256 (9-2-2)

\section{Rai, Christopher}

Rajaratnam, Sabesan

Ramadasan, Sudheesh.

Ramakrishnan, Balaji.

Ramos Martins, Marcel

OMAE2019-96251 (2-13-2), 0MAE2019-96269 (2-13-1)

Ramos, Rafaela ................................ OMAE2019-96730 (4-1-2)

Rampazzo, Fabiano............................ OMAE2019-95353 (13-2-4)

Ramzanpoor, Iman _ OMAE2019-95244 (9-2-3)

Randell, David ................................ OMAE2019-95913 (6-7-4)

OMAE2019-96586 (6-7-4), OMAE2019-96587 (6-7-4)

Randolph, Mark F._..................... OMAE2019-95393 (10-1-1)

Ransley, Edward

Rasheed, Adil

Rastayesh, Sima

Rathore, Devvrat.

Ratnayake, R.M. Chandima ................... OMAE2019-95155 (3-5-1) OMAE2019-95478 (2-14-1), OMAE2019-96576 (2-14-1)

Rauffus, René Bettencourt.................. OMAE2019-96099 (8-2-3)

Ravinthrakumar, Senthuran................... OMAE2019-95932 (6-5-1)

Read, Robert..

Reagan, Perdinan

Reali Costa, Anna...

Recalde, Luis

Reddy, Sandeep Bukka

Reed, Philippa.

Reinas, Lorents

Reinholdtsen, Svein-Arne

Reis Leite França, Bruna

Reite, Karl J.

Ren, Bing

Ren, Haojie...

Ren, Huilong

.. OMAE2019-95777 (2-9-3), OMAE2019-95781 (2-9-3)

Ren, Nianxin

.. OMAE2019-96761 (5-1-3), 0MAE2019-96789 (5-1-3)

Ren, Xiudi.................................. OMAE2019-95203 (13-1-2)

Ren, Xuhe..................................... OMAE2019-95779 (6-7-1)

Ren, Zhengru .

Renac, Laury...

Restrepo, Julian

Revhaug, Inge.
Rezanejad, Kourosh..

Rheem, Changkyu

Riaz, Saad..

Ribeiro Machado da Silva, Vinicius

Ribeiro, Mario

Ribeiro, Paulo...

Rica, Shilton

Richardson, Josh

Rideout, Geoff.

Rijnsdorp, Dirk

Ringsberg, Jonas W.

Ringwood, John

Rippeth, Tom .. OMAE2019-95369 (3-3-2)
OMAE2019-96373 (11-1-2) OMAE2019-96769 (9-2-1) OMAE2019-96100 (2-13-1) OMAE2019-95051 (2-12-1)

OMAE2019-95764 (1-4-1)

OMAE2019-95194 (6-2-1)

OMAE2019-96250 (2-13-2)

OMAE2019-96280 (2-8-1) OMAE2019-96451 (4-2-4) .. OMAE2019-95998 (9-5-2)

OMAE2019-95815 (6-5-4)

OMAE2019-96833 (2-14-1)

OMAE2019-96754 (10-6-1)

OMAE2019-96002 (5-2-1)

OMAE2019-96006 (4-1-10)

OMAE2019-96120 (6-4-1)

OMAE2019-96017 (9-2-6)

OMAE2019-96543 (8-3-1)

OMAE2019-96386 (1-2-4)

OMAE2019-96214 (2-8-1)

OMAE2019-95083 (2-6-2)

OMAE2019-96626 (6-7-2)

OMAE2019-95146 (6-4-2)

OMAE2019-95414 (5-2-1)

OMAE2019-96582 (1-3-1)

OMAE2019-96112 (8-4-1)

.. OMAE2019-96486 (1-3-1)

OMAE2019-95586 (7-11-1)

OMAE2019-96151 (6-13-3)

OMAE2019-96753 (5-1-3)

.. OMAE2019-96686 (2-7-1)

Rita, Francisco Afonso

Rizzo, Cesare Mario..

Roberts, Andrew.

Roberts, Graeme

Robertson, Amy...

Robertson, lan

Robi, P.S.

Rocha, Djalene

Roddier, Dominique.

Rodrigues, José Miguel

Rodriguez Luis, Álvaro.

Rodríguez, Claudio Alexis.

Roitman, Ney

Rombado, Gabriel

Romolo, Alessandra

Roneid, Viggo

Rosche, John

Ross, Emma.

Rourke, Jonathan

Roy, Anamitra.

Roy, Shovan

Rudman, Murray

Ruehl, Kelley

Rung, Thomas

Russell, Andy

Ruud Hagen, Torbjørm

Ruzzo, Carlo.

OMAE2019-95128 (7-1-1) OMAE2019-96639 (11-2-2) OMAE2019-96310 (4-1-6) OMAE2019-95051 (2-12-1) OMAE2019-95429 (9-1-1) OMAE2019-95533 (9-1-8) OMAE2019-95101 (6-2-3) OMAE2019-95345 (6-15-1) OMAE2019-95510 (4-1-2) OMAE2019-95024 (5-1-3) OMAE2019-96761 (5-1-3) OMAE2019-96380 (9-1-2) OMAE2019-96334 (6-8-2) OMAE2019-96741 (4-3-3) OMAE2019-95175 (4-2-1) OMAE2019-95303 (4-1-3) OMAE2019-95880 (2-2-2) OMAE2019-96368 (9-4-1) OMAE2019-96685 (3-3-3) OMAE2019-95030 (8-4-2) OMAE2019-95913 (6-7-4) OMAE2019-96587 (6-7-4) OMAE2019-95519 (4-2-1) OMAE2019-95393 (10-1-1) OMAE2019-96816 (6-11-2) OMAE2019-95194 (6-2-1) OMAE2019-95216 (9-3-1) OMAE2019-96740 (7-4-1) OMAE2019-95277 (4-3-8) OMAE2019-95429 (9-1-1) OMAE2019-96212 (5-1-2) OMAE2019-96282 (5-1-2)

S

Saadali, Nadjib Saadat, Mohsen

OMAE2019-95312 (6-5-4) Saasen, Arild OMAE2019-96270 (6-15-1) OMAE2019-95674 (11-15-1) ... OMAE2019-96347 (11-7-2), 0MAE2019-96594 (11-7-2) OMAE2019-96595 (11-7-3), OMAE2019-96607 (11-15-2) Sabiron, Guillaume ............................. OMAE2019-95197 (9-3-1) Sablok, Anil....................................... OMAE2019-96322 (1-1-2) Sadjina, Severin ................................. OMAE2019-95885 (6-4-2) Safier, Elchanan ................................. OMAE2019-95900 (9-4-2) .... OMAE2019-96104 (5-1-2) OMAE2019-95067 (2-5-1) OMAE2019-95261 (2-9-1), 0MAE2019-95386 (2-12-1) OMAE2019-95510 (4-1-2), OMAE2019-96210 (2-6-1) Saha, Nilanjan ................................ OMAE2019-96092 (2-12-3) OMAE2019-96630 (2-3-1) Saha, Ujjwal K.................................. OMAE2019-95570 (9-2-2)
Sahoo, Avilash ............................ OMAE2019-95345 (6-15-1) Sahoo, Prasanta ...................................... OMAE2019-96190 (8-1-3) Sahoo, Trilochan ……........................ OMAE2019-95409 (4-3-7) Sainte-Rose, Bruno Sakaguchi, Daisaku OMAE2019-96095 (8-3-2) OMAE2019-95829 (13-3-3) OMAE2019-96549 (2-15-1) OMAE2019-96315 (11-1-3) OMAE2019-95038 (6-4-6) OMAE2019-96349 (6-4-6) OMAE2019-96408 (9-3-2) OMAE2019-96510 (12-1-2) OMAE2019-95841 (13-2-4) OMAE2019-95869 (13-3-2) OMAE2019-95687 (6-4-5) OMAE2019-95147 (4-1-4) OMAE2019-96678 (11-5-1) OMAE2019-96736 (11-13-1) OMAE2019-96078 (10-7-1) OMAE2019-95274 (4-1-5) OMAE2019-96170 (11-2-2) OMAE2019-95419 (9-4-4) OMAE2019-95128 (7-1-1) OMAE2019-95138 (9-4-4) OMAE2019-95216 (9-3-1) OMAE2019-96621 (6-7-2) OMAE2019-95401 (13-7-1) OMAE2019-95403 (13-7-1)

Sakamoto, Hiroshi OMAE2019-95923 (3-9-1)

Sakimoto, Takahiro

Sakurai, Noriaki

Salehi, Alireza ................................. OMAE2019-96698 (10-5-1)

Salehi, Saeed OMAE2019-95380 (11-6-1)

OMAE2019-95588 (11-12-2), OMAE2019-95589 (11-12-1)

OMAE2019-95612 (11-15-1), 0MAE2019-95614 (11-10-1) OMAE2019-96776 (11-6-2), OMAE2019-96820 (11-10-1) Sales Junior, Joel S. OMAE2019-96048 (9-3-2) OMAE2019-96349 (6-4-6), 0MAE2019-96575 (1-4-3) Samaria, Sagar OMAE2019-96139 (1-1-5) Sampaio, Jorge H B Jr. _................. OMAE2019-96385 (11-2-2) Sanchis, Arnaud................................ OMAE2019-96059 (4-6-1) Saneian, Mohsen ................................ OMAE2019-96596 (4-1-11) OMAE2019-96597 (4-1-6) OMAE2019-95809 (4-2-3) OMAE2019-95819 (11-6-1), OMAE2019-95928 (11-15-2) OMAE2019-95995 (11-6-1), 0MAE2019-96319 (11-6-2) Santoro, Anita. OMAE2019-96104 (5-1-2) Santos, Joaquim OMAE2019-96269 (2-13-1) OMAE2019-96280 (2-8-1)
Salcedo Fernandez, Fernando ................. OMAE2019-96104 (5-1-2) Sangesland, Sigbjørn 


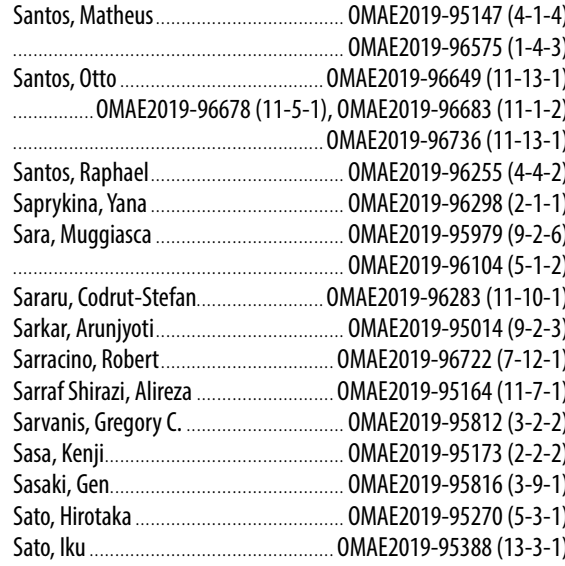

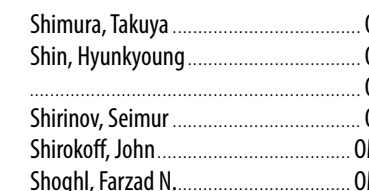

OAE2019-96623 (5-4-1) OMAE2019-95429 (9-1-1) OMAE2019-95919 (9-1-3) OMAE2019-95233 (8-3-1) .... OMAE2019-96311 (11-4-1) Shoji, Ruri Shoji, Ruri......................................... OMAE2019-95173 (2-2-2)

\section{Shugan, Igor}

Shuhaibar, Bassam

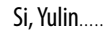
.. OMAE2019-95879 (1-1-5) OMAE2019-96298 (2-1-1) ... OMAE2019-95236 (6-2-2) ... OMAE2019-95945 (9-2-3) .. OMAE2019-95968 (9-2-6)

Si, Yun-Da.

Sidarta, Djoni... . OMAE2019-95325 (9-3-3) .. OMAE2019-96288 (1-1-5) OMAE2019-96339 (1-1-5)

Sidhar, Harpreet

OMAE2019-95889 (13-3-1), OMAE2019-96750 (5-5-1) Sato, Toru.

Sauvage, Jean-Pierre

Sayed Ahmad, Firas.

Scannell, Brian

Scharnke, Jule.

Schellin, Thomas OMAE2019-95722 (9-5-4), OMAE2019-95935 (8-1-1)

Schenk, Siegberto R. Jr...

Schnabl, Andrea M.

Schouten, Rogier

Scialò, Andrea.

Sclavounos, Paul

Sebastian, Abhilash

Seeninaidu, Nallayarasu.

Selker, Ruud.

Selvik, Jon Tømmerås

Seng, Sopheak . OMAE2019-96546 (11-15-2

Seo, Min-Guk

Sequeiros, Octavio OMAE2019-95972 (8-4-1)

Serna, José

Serret, Jordi.

Seubers, Henk

Sevillano, Lucas

Seyffert, Harleigh C

Shafiee, Mahmood Shahvaghar-Asl, Selda …………...... OMAE2019-95227 (9-5-2) Shankaran, Rohit ................................. OMAE2019-95536 (4-4-1) Shao, Qiangqiang ............................ OMAE2019-95458 (4-1-5) Shao, Wenbo.

Shao, Xiaoyan

Shao, Yanlin... OMAE2019-96383 (6-3-2), OMAE2019-96545 (1-4-1)

Shao, Yunliang

Sharman, Krish.

Sharp, John Sharples, John .............................. OMAE2019-96327 (3-11-2 Shemer, Lev...

Shen, Hui..

Shen, Julie.

Shen, Ke Chun

Shen, Yijun.

Shen, Yongming

Sheng, Ming.

Sheng, Weijia

Shi, Guangyu

Shi, Shuo...

Shi, Wei..

Shi, Weichao

Shi, Zhang..

Shijun, Zhang

Shimamura, Junji.

Shimomoto, Sena.
Silva-Casarín, Rodolfo OMAE2019-95733 (13-3-3) OMAE2019-96631 (3-3-2) OMAE2019-96239 (3-3-1) OMAE2019-96621 (6-7-2) OMAE2019-95004 (2-9-1) OMAE2019-95481 (1-7-1) OMAE2019-95716 (8-1-6) OMAE2019-96251 (2-13-2 OMAE2019-95767 (9-5-2) OMAE2019-96207 (1-7-2) OMAE2019-95973 (9-3-2) OMAE2019-96679 (12-5-1) OMAE2019-95135 (4-2-5) OMAE2019-96267 (2-11-3) OMAE2019-96154 (4-3-8) OMAE2019-95288 (1-6-1) OMAE2019-96278 (8-2-1) . OMAE2019-95406 (1-7-1)

OMAE2019-95970 (8-4-4) OMAE2019-96380 (9-1-2 OMAE2019-96321 (8-2-3) OMAE2019-95809 (4-2-3) OMAE2019-95497 (2-9-3) OMAE2019-95137 (2-14-1) OMAE2019-96181 (8-1-3) OMAE2019-95125 (2-5-1) OMAE2019-96002 (5-2-1) OMAE2019-96191 (2-6-2) OMAE2019-96424 (13-2-2) OMAE2019-95835 (3-13-1) OMAE2019-96842 (3-13-1)

\section{Silveira e Silva, Júlio Márcio}

Simão, Marina L.

Simms, Nigel.

Simões-Moreira, Jose R.

Simonetti, Irene

Simos, Alexandre N.

Sinclair, Jarrod

Singh, Jagdeep

Sireta, Francois-Xavier.

Skadsem, Hans Joakim.

.. OMAE2019-95541 (3-3-2

OMAE2019-96747 (6-13-1)

OMAE2019-95145 (4-3-1)

OMAE2019-96210 (2-6-1) OMAE2019-96227 (3-9-1) OMAE2019-96315 (11-1-3) .. OMAE2019-96123 (9-3-2) ... OMAE2019-96259 (1-1-1) OMAE2019-96670 (6-4-2) ... OMAE2019-95884 (9-5-3) .. OMAE2019-95837 (9-2-6) .. OMAE2019-95352 (2-9-3) OMAE2019-95700 (11-12-1) OMAE2019-96546 (11-15-2)

Skarbøvik, Reidar André

Skau, Kristoffer S.

Skjong, Stian.

Skorpa, Ragnhild

Skutle Høgsæt, Ingrid

Small, Andrew A.

Smedley, Philip.

Smith, Aaron.

Smith, Charles.

Smith, Rachael E.

Sobey, Adam.

Socariceanu, Mariana

Sogawa, Daisuke.

Sogawa, Yasuhiro.

Solaas, Frøydis

Solano, Rafael F. ... OMAE2019-95145 (4-3-1)

\section{Solomon, Kushal...}

Sonawane, Mahesh.

Song ... OMAE2019-95536 (4-4-1), OMAE2019-96409 (4-2-2)

Song, Soonseok ………......................... OMAE2019-95315 (8-1-7)

Song, Xingyu ................................ OMAE2019-96029 (13-1-3)

Song, Yuelin

Sørum, Stian Høegh

Sotoudeh, Kasra

Sousa, Fernando

Souto-Iglesias, Antonio

Souza Pinheiro da Silva, Leandro.

OMAE2019-96650 (1-2-4) OMAE2019-96270 (6-15-1) OMAE2019-95106 (2-11-3) OMAE2019-96759 (9-1-5) OMAE2019-95713 (6-2-1) OMAE2019-96715 (5-5-1) OMAE2019-96131 (7-12-1) OMAE2019-96136 (6-8-1) OMAE2019-95578 (6-3-1) .. OMAE2019-95619 (9-3-1) OMAE2019-96221 (9-1-3) OMAE2019-95607 (13-2-4) OMAE2019-96181 (8-1-3) OMAE2019-96581 (6-5-4) . OMAE2019-96378 (9-5-4) . OMAE2019-95863 (3-9-1) OMAE2019-96493 (13-2-5)
…….................................. OMAE2019-96727 (13-2-1)

Souza, Marcelo Igor Lourenço.............. OMAE2019-96367 (4-4-2)

Spencer, Steven J.................................. OMAE22019-96382 (9-4-3)

Spill, Severin ................................... OMAE2019-96109 (10-7-1)

Sproson, Richard ............................. OMAE2019-96021 (6-12-1)

Srigiriraju, Srikanth ............................... OMAE2019-96754 (10-6-1)

Srinil, Narakorn................................ OMAE2019-95459 (8-4-2) OMAE2019-95466 (8-4-3)

Srinivasamurthy, Sharath .................... OMAE2019-95401 (13-7-1)
OMAE2019-95403 (13-7-1). OMAE2019-95404 (13-3-1)

Srinivasan, Balaji ............................. OMAE2019-95470 (4-3-6) Sripada, Subramanyam V. R.,............... OMAE2019-95467 (1-4-2) Stacey, Alexander ............................. OMAE2019-95470 (4-3-6) OMAE2019-96161 (3-11-2), OMAE2019-96843 (3-11-2)

Stachyra, Leszek

Stansby, Peter.

Stark, Greg...

Stashchuk, Nataliya

Steen, Sverre

Stefek, Jeremy

Steinke, Dean..

Stephens, Ag

Stephens, Robin...

Sterenborg, Joost

Stetco, Adrian-Mihai

Stratford, Tim...

Stratigaki, Vasiliki

Streicher, Maximilian

Stroisz, Anna

Strømme, Svein Ole.

Su, Biao

\section{Su, Hang}

$\mathrm{Su}, \mathrm{Qi} \ldots$

Su, Yumin

Subramaniam, Neelamani

Subramanian, Rahul

Sugahara, Ryo

Sui, Dan...

OMAE2019-95486 (11-11-1), OMAE2019-95496 (11-11-1) Suja-Thauvin, Loup OMAE2019-95429 (9-1-1) Sun, Baojiang.................................. OMAE2019-96719 (11-7-3) Sun, Futai ..................................... OMAE2019-95911 (10-5-1) Sun, Hai ....................................... OMAE2019-96671 (9-5-2) Sun, Hai...................................... OMAE2019-96823 (8-5-2) Sun, Hanbing .................................... OMAE2019-96798 (6-8-2) Sun, Hao ........................................ OMAE2019-96739 (12-1-2) Sun, Hui...................................... OMAE2019-96669 (12-5-2) Sun, Jili......................................... OMAE2019-95945 (9-2-3) Sun, Jing ...................................... OMAE2019-95778 (10-3-1) Sun, Liang Sun, Longquan Sun, Mingyuan Sun, Peng.

Sun, Pengnan.

Sun, Shuzheng.

Sun, Tao...

Sun, Ting.

Sun, Wei.

Sun, Xiaobo

Sun, Xinyu......... OMAE2019-95717 (12-2-1) OMAE2019-95850 (8-3-1) OMAE2019-96474 (1-1-2) OMAE2019-95225 (13-1-2) OMAE2019-95257 (6-13-3) . OMAE2019-95449 (6-13-3) OMAE2019-95176 (6-15-2) OMAE2019-96649 (11-13-1) . OMAE2019-96767 (11-13-1) OMAE2019-95585 (1-3-2) ... OMAE2019-95857 (2-5-1) OMAE2019-95676 (4-1-5)

Sun, Yue ... OMAE2019-95903 (4-1-5), OMAE2019-95916 (4-1-7) OMAE2019-95714 (8-1-2) OMAE2019-96018 (8-1-1), 0MAE2019-96020 (8-1-4) Sun, Yuwei .................................... OMAE2019-95586 (7-11-1) 


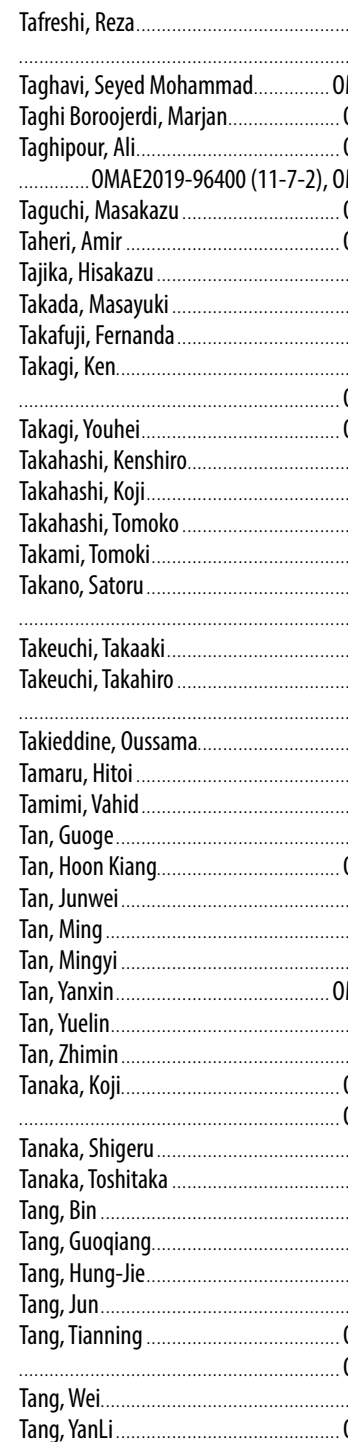

OMAE2019-96293 (4-5-1) OMAE2019-96305 (8-5-1) OMAE2019-95382 (11-12-1) OMAE2019-96722 (7-12-1) OMAE2019-96347 (11-7-2) MAE2019-96547 (11-15-2) OMAE2019-95555 (12-7-1) OMAE2019-96400 (11-7-2) OMAE2019-95923 (3-9-1) OMAE2019-95927 (5-6-1) OMAE2019-95150 (8-4-2) OMAE2019-95884 (9-5-3) OMAE2019-96443 (13-3-4) OMAE2019-95946 (13-1-4) OMAE2019-96190 (8-1-3) OMAE2019-96406 (5-6-1) OMAE2019-96040 (5-3-1) OMAE2019-95389 (2-9-3) OMAE2019-95270 (5-3-1) OMAE2019-95990 (5-3-1) OMAE2019-96750 (5-5-1) OMAE2019-95654 (7-3-1) OMAE2019-95901 (6-2-3) OMAE2019-96662 (4-3-3) OMAE2019-95879 (1-1-5) OMAE2019-95227 (9-5-2) OMAE2019-96799 (6-5-3) OMAE2019-95853 (2-13-2) OMAE2019-96444 (6-8-1) OMAE2019-95968 (9-2-6) OMAE2019-96007 (9-5-4) OMAE2019-95748 (11-11-2) OMAE2019-96002 (5-2-1) OMAE2019-95118 (4-1-1) OMAE2019-95388 (13-3-1) OMAE2019-95889 (13-3-1) OMAE2019-95269 (1-1-2) OMAE2019-95465 (3-9-1) OMAE2019-96034 (9-1-3) OMAE2019-95584 (8-4-3) OMAE2019-95730 (2-6-1) OMAE2019-95713 (6-2-1) OMAE2019-95068 (6-17-2) OMAE2019-95172 (12-1-1) OMAE2019-95016 (1-2-2) OMAE2019-95192 (13-1-2) ..0MAE2019-95225 (13-1-2), 0MAE2019-96019 (13-1-2)

Tang, Yougang OMAE2019-95031 (4-3-4) OMAE2019-95207 (9-2-2), OMAE2019-95635 (9-2-3) OMAE2019-95637 (10-7-1), OMAE2019-95638 (9-1-8) OMAE2019-95641 (10-4-1) OMAE2019-95945 (9-2-3) OMAE2019-95650 (9-4-3) OMAE2019-96120 (6-4-1)

Tang, Zecheng...

Tannuri, Eduardo OMAE2019-96335 (6-4-1), OMAE2019-96670 (6-4-2)

Tao, Longbin. OMAE2019-95179 (6-8-1), OMAE2019-95203 (13-1-2) OMAE2019-95244 (9-2-3), OMAE2019-95597 (13-2-3) OMAE2019-95607 (13-2-4), OMAE2019-95764 (1-4-1) Tao, Shanshan.. OMAE2019-95791 (6-7-1) OMAE2019-96191 (2-6-2)

Tao, Xing

Tardy, Philippe M.J. MAE2019-95133 (11-12-1) OMAE2019-95967 (9-2-1)

Taruffi, Federico OMAE2019-95979 (9-2-6) OMAE2019-95976 (9-2-1), OMAE2019-95970(9-2-6)

Tawfik, Adel A OMAE2019-95290 (3-1-1) OMAE2019-95172 (12-1-1)

Taylor, Paul

OMAE2019-95198 (12-7-1), 0MAE2019-96817 (12-5-1)

Taylor, Rocky OMAE2019-96722 (7-12-1) OMAE2019-96732 (7-1-1) OMAE2019-96288 (1-1-5)

Tcherniguin, Nicolas OMAE2019-96339 (1-1-5), OMAE2019-96838 (1-6-1) Teixeira, Pedro . OMAE2019-95369 (3-3-2) OMAE2019-95249 (6-13-2)

Tello Ruiz, Manases ), OMAE2019-96341 (6-5-2) OMAE2019-95565 (6-11-2), OMAE2019-96341 (6-5-2)
OMAE2019-96346 (6-5-2)

Temarel, Pandeli OMAE2019-95200 (8-5-1) OMAE2019-96326 (13-1-4)
Teng, Bin ...

OMAE2019-95165 (12-4-1) OMAE2019-95421 (12-1-2)

Teng, Yunfei.

Tengchao, Lu.

Tengesdal, Njaal

OMAE2019-95557 (4-3-8)

OMAE2019-95585 (1-3-2)

OMAE2019-95105 (7-4-1)

Teodoriu, Catalin OMAE2019-95474 (1-4-2)

OMAE2019-95589 (11-12-1), 0MAE2019-95612 (11-15-1) OMAE2019-95614 (11-10-1), OMAE2019-96283 (11-10-1) OMAE2019-96820 (11-10-1)

Terao, Takashi

Terazawa, Yusuke.

Terziev, Momchil...

Tett, Paul

Tezdogan, Tahsin OMAE2019-95270 (5-3-1) OMAE2019-95465 (3-9-1) OMAE2019-95445 (6-5-4) OMAE2019-96104 (5-1-2) OMAE2019-95445 (6-5-4)

OMAE2019-95477 (1-1-3), OMAE2019-95784 (8-1-4) OMAE2019-95790 (6-13-1), OMAE2019-96030 (9-3-3)

Theotokoglou, Efstathios ………..... OMAE2019-95446 (3-2-2) Thibaux, Philippe _............................ OMAE2019-96198 (3-2-2) OMAE2019-96371 (3-2-2)

Thibodeaux, Louis OMAE2019-96683 (11-1-2)

Thiele, Klaus. OMAE2019-96808 (2-4-2) OMAE2019-95099 (9-6-1)

Thies, Philipp R. OMAE2019-95159 (9-1-2), OMAE2019-95477 (1-1-3) OMAE2019-95759 (9-2-1), OMAE2019-95998 (9-5-2) OMAE2019-96080 (9-1-5) OMAE2019-95076 (8-1-3)

Thill, Cornel.

Thirumalai, Neeraj

Thomas, Della

Thomas, Giles

Thomas, Philipp OMAE2019-96593 (3-4-1) OMAE2019-96220 (6-8-2) OMAE2019-96562 (6-8-2) OMAE2019-95210 (1-1-3) OMAE2019-95429 (9-1-1)

Thomaz, Tianna

Thome, Michael

Thompson, Keith

Thomson, Jim

Thomson, Matthew.

Thornton, Blair.

Thurairajah, Sriskandarajah

Thurler, Ana

Thys, Maxime

Tian, Chao .

ing.... OMAE2019-95577 (4-3-4) OMAE2019-96138 (8-1-2) OMAE2019-95842 (13-1-1) OMAE2019-96802 (9-4-3) OMAE2019-95001 (1-3-1) OMAE2019-96040 (5-3-1) OMAE2019-95051 (2-12-1) OMAE2019-96575 (1-4-3) OMAE2019-95929 (6-5-1) OMAE2019-95200 (8-5-1)

Tian, Chenling MAE2019-96771 (6-1-2)

Tian, Xinliang OMAE2019-95601 (13-2-3) OMAE2019-95744 (8-5-1)

Tian, Zhenglin OMAE2019-95915 (9-2-1), OMAE2019-96739 (12-1-2)

Time, Rune Wiggo

Ting, Kee Chien

Tinoco, Francisco

Tjallema, Arjen

Tkach (WG), Yuri

Tkaczyk, Tomasz

Togawa, Sachio

Toleman, Ben OMAE2019-95942 (6-3-3) OMAE2019-95217 (11-2-1) .. OMAE2019-96137 (1-4-3) OMAE2019-96137 (1-4-3) OMAE2019-96207 (1-7-2) OMAE2019-96327 (3-11-2) OMAE2019-95511 (4-3-2) OMAE2019-96493 (13-2-5) OMAE2019-95519 (4-2-1) OMAE2019-95536 (4-4-1)

Tom, Joe

Tom, Nathan OMAE2019-95419 (9-4-4) ... OMAE2019-95216 (9-3-1) OMAE2019-96047 (4-4-1) Tona, Paolino ..................................... OMAE2019-95197 (9-3-1) Tong, Feifei OMAE2019-95585 (1-3-2) OMAE2019-95629 (6-3-3)

Torben, Sverre OMAE2019-95283 (6-4-6) OMAE2019-95885 (6-4-2) Torsæter, Malin OMAE2019-96400 (11-7-2) Tosi, Guillaume OMAE2019-95354 (4-6-1) OMAE2019-95493 (4-6-1), OMAE2019-96059 (4-6-1) Troch, Peter... Tronskar, Jens

Trubat, Pau

Trudel, Elizabeth

Trulsen, Karsten

Tsarau, Andre

Tsuchiya, Keita

Tsuji, Akiyoshi

Tsukamoto, Yoshiak

Tümer, Can .

Turan, Osman

Turnbull, lan

Turner, Trey...

Turnock, Stephen

Tveit, Mari R.

$\mathbf{U}$

Ueda, Keiji...

Ueda, Tetsuya

Umeda, Jun.

Unal, Ugur Oral

Unglaub, Julian

Upton, Jon.......

Urbano, Joaquin.

Utsunomiya, Tomoak ... OMAE2019-95388 (13-3-1)

OMAE2019-95482 (12-1-1), OMAE2019-95822 (3-3-2) OMAE2019-95889 (13-3-1), OMAE2019-96749 (13-3-3) OMAE2019-96750 (5-5-1)

V

Vachirapanyakun, Sorrasak OMAE2019-96778 (9-1-1) Vada, Torgeir Kirkhorn. OMAE2019-95300 (6-3-1) OMAE2019-95519 (4-2-1) OMAE2019-96409 (4-2-2)

Van Beek, Pieter... OMAE2019-95019 (4-5-1)

Van den Abeele, Filip. OMAE2019-96316 (3-1-2) van den Bremer, Ton S. OMAE2019-95172 (12-1-1) OMAE2019-96082 (6-17-1), OMAE2019-96817 (12-5-1) van der Heijden, G.H.M. _ OMAE2019-95031 (4-3-4) van der Plas, Peter .................................. OMAE2019-96321 (8-2-3) van Dijk, Radboud .............................. OMAE2019-95375 (1-2-3) van Essen, Sanne. van Haaften, Ewoud

Van Poucke, Maarten OMAE2019-95065 (6-17-1) OMAE2019-96090 (1-5-1) OMAE2019-96198 (3-2-2)

van Rij, Jennifer OMAE2019-96371 (3-2-2)

van Winsen, Ivan. Van Wittenberghe, Jeroen OMAE2019-96397 (9-3-3) OMAE2019-95375 (1-2-3) OMAE2019-96198(3-2-2) Vance, Charlene OMAE2019-95138 (9-4-4) Vanem, Erik. OMAE2019-95098 (2-9-2) OMAE2019-96587 (6-7-4)

Vantorre, Marc OMAE2019-95249 (6-13-2) OMAE2019-95565 (6-11-2), OMAE2019-96341 (6-5-2) OMAE2019-96346 (6-5-2) Vargas, Pedro ................................ OMAE2019-95618 (2-6-2) Varges, Priscilla.............................. OMAE2019-95131 (11-12-1) Vasa, Nilesh J. . Vasquez, Rafael E.. Vatinel, Lucas...

Vaz, Guilherme Vaz, Murilo Augusto OMAE2019-96220 (6-8-2) OMAE2019-95385 (5-4-1) OMAE2019-95171 (8-1-5) OMAE2019-96099 (8-2-3) OMAE2019-95141 (4-1-3) OMAE2019-95826 (4-1-4)

Vedvik, Nils Petter Veldman, Arthur. Venero, Germain. Venkatachalam, Sriram Venugopal, Vengatesan 
Visco, Tyler. OMAE2019-96588 (1-4-1) OMAE2019-95538 (9-4-1)

Viselli, Anthony M. Viswanathan, Savin Vitali, Luigino.

Vitola, Marcelo A

Vivet, Romain

Vivien, Guyon

Vladimir, Nikola

Vlasenko, Vasyl.

Vogel, Christopher

Volent, Zsolt...

Volpin, Diener

Von Bock Und Polach, Rüdiger Ulrich Fran OMAE2019-95054 (6-11-1) OMAE2019-96086 (6-7-3) OMAE2019-96569 (6-8-1) OMAE2019-95354 (4-6-1) AE2019-95493 (4-6-1), OMAE2019-96059 (4-6-1) OMAE2019-95055 (4-3-5) OMAE2019-96026 (8-2-3) OMAE2019-96621 (6-7-2) OMAE2019-95347 (9-5-3) OMAE2019-96375 (5-2-1) OMAE2019-96255 (4-4-2) OMAE2019-95085 (7-4-1) OMAE2019-95116 (7-4-1), OMAE2019-96740 (7-4-1) Vralstad, Torbjorn OMAE2019-95928 (11-15-2) OMAE2019-96319 (11-6-2), OMAE2019-96547 (11-15-2) . OMAE2019-96738 (11-6-2), 0MAE2019-96773 (11-6-2) Vukcevic, Vuko OMAE2019-95293 (8-1-3)

\section{W}

Wada, Ryota.

OMAE2019-95442 (13-1-1) OMAE2019-96443 (13-3-4)

Wadbled, Xavier

Wade, lan

Wadhwa, Hema

Wales, Stuart

Walker, Michael

Wallerand, Regis

Walters, Carey L.

Wamba, Lionel.

Wan, D.C.

OMAE2019-95526 (6-13-1), OMAE2019-95527 (6-13-3)

Wan, Decheng OMAE2019-95520 (9-6-1), OMAE2019-95522 (8-4-1)

Wan, Rong....... OMAE2019-95308 (13-2-5)

Wan, Yuxiang

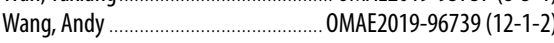

Wang, Bia0 ......................... OMAE2019-95176 (6-15-2)

Wang, Bin ....................................... OMAE2019-95638 (9-1-8)

Wang, Bin.

Wang, Bing

Wang, Caizhong

Wang, Chang .

Wang, Changhong

Wang, Chao...

Wang, Cheng...

Wang, Chien Ming

Wang, Congying

Wang, Deyang

Wang, Deyu... OMAE2019-96348 (3-2-1) OMAE2019-95072 (13-2-1) OMAE2019-95748 (11-11-2) OMAE2019-95840 (6-11-2) OMAE2019-95251 (6-4-5) OMAE2019-96582 (1-3-1) OMAE2019-96805 (6-1-2) OMAE2019-95860 (6-2-1) OMAE2019-95530 (6-4-4) OMAE2019-96650 (1-2-4) OMAE2019-95644 (2-12-2) OMAE2019-95683 (2-11-1) OMAE2019-96719 (11-7-3)

Wang, Di

Wang, Don .

Wang, Dong

Wang, Fa-Cheng

Wang, Fang

Wang, Feng...

Wang, Gang ..

Wang, Haojie

Wang, Hui

Wang, Huoping

Wang, Jianhua..

Wang, Jianhua

Wang, Jie...

OMAE2019-95402 (11-1-2), 0MAE2019-95802 (11-11-2)

Wang, Jin

Wang, Jinghua

Wang, Jinguang

Wang, Jinlong.

Wang, Jun-Qin

Wang, Jungao OMAE2019-95426 (4-1-3) OMAE2019-96755 (4-1-4) OMAE2019-95327 (2-4-3) OMAE2019-95135 (4-2-5) OMAE2019-95308 (13-2-5) OMAE2019-95706 (13-2-1) OMAE2019-95776 (2-9-4) OMAE2019-95876 (4-5-2) OMAE2019-95422 (10-4-1) OMAE2019-95752 (10-7-1) OMAE2019-95527 (6-13-3) OMAE2019-95326 (11-1-1) OMAE2019-95624 (11-3-1) OMAE2019-95247 (12-2-1) OMAE2019-96603 (1-4-2) OMAE2019-95196 (1-4-1) OMAE2019-96655 (10-3-1) OMAE2019-96112 (8-4-1)
Wang, Junrong

OMAE2019-95779 (6-7-1) OMAE2019-96553 (1-3-2)

Wang, Junxian

Wang, kai.

Wang, Kunpeng

Wang, Lei .

Wang, Lu.

Wang, Peng.

Wang, Pingping

Wang, Qing. ... OMAE2019-95515 (8-1-2)

OMAE2019-95737 (8-5-1), 0MAE2019-96020 (8-1-4) OMAE2019-96791 (8-1-2), OMAE2019-96819 (8-1-4) Wang, Rongquan ............................... OMAE2019-95165 (12-4-1) Wang, Shiping ................................. OMAE2019-95232 (6-8-1) Wang, Shuang .............................. OMAE2019-95563 (8-1-5) OMAE2019-95874 (8-1-7), OMAE2019-96425 (8-1-5)

Wang, Shuangqiang............................ OMAE2019-95610 (8-4-4) Wang, Shuqing .................................. OMAE2019-95550 (2-5-1) Wang, Sue ...................................... OMAE2019-96191 (2-6-2)

Wang, Weizhi

Wang, Wenda

Wang, Wenjuan

Wang, Wenming

Wang, Xi

OMAE2019-96803 (5-2-1) OMAE2019-96524 (8-2-1) OMAE2019-95840 (6-11-2) OMAE2019-96784 (11-1-3) OMAE2019-95840 (6-11-2) OMAE2019-95473 (6-11-2) OMAE2019-96617 (11-7-2)

Wang, Xianzhou OMAE2019-96791 (8-1-2) OMAE2019-96819 (8-1-4) OMAE2019-95126 (1-3-1) OMAE2019-95517 (3-1-2)

Wang, Xin

Wang, Xin...

Wang, Xinhua

Wang, Xinmeng

Wang, Xinxin OMAE2019-96019 (13-1-2) OMAE2019-95192 (13-1-2)

OMAE2019-95225 (13-1-2), OMAE2019-95308 (13-2-5) OMAE2019-95706 (13-2-1) .. OMAE2019-96777 (1-1-4)

Wang, Xu OMAE2019-96828 (6-17-1) OMAE2019-96461 (13-2-1) OMAE2019-96508 (6-2-2) OMAE2019-95637 (10-7-1) OMAE2019-95641 (10-4-1) OMAE2019-95215 (1-2-1) OMAE2019-95774 (11-1-1) OMAE2019-96486 (1-3-1) OMAE2019-96582 (1-3-1) OMAE2019-96784 (11-1-3) OMAE2019-95624 (11-3-1) OMAE2019-95802 (11-11-2) OMAE2019-96617 (11-7-2) OMAE2019-95039 (6-2-3) OMAE2019-95631 (4-2-5)

Wang, Yuezhi

Wang, Zeming OMAE2019-95676 (4-1-5), OMAE2019-95692 (4-1-6)
OMAE2019-95031 (4-3-4

Wang, Zhenkui

Wang, Zhifeng OMAE2019-95791 (6-7-1) OMAE2019-96650 (1-2-4) OMAE2019-95930 (7-12-1) OMAE2019-96719 (11-7-3) OMAE2019-96611 (13-3-3)

Wang, Zhiyuan.

Want, Andrew.

Ward, Phil.

Waseda, Takuji

OMAE2019-96409 (4-2-2)

OMAE2019-95442 (13-1-1)

OMAE2019-95949 (13-1-3)

Waskito, Kurniawan T. ......................... OMAE2019-95273 (6-5-1)

Wasson, Andrew OMAE2019-95541 (3-3-2)

OMAE2019-96556 (3-4-1), OMAE2019-96614 (3-4-1)

Watanabe, Keisuke OMAE2019-95394 (5-3-1)

Watanabe, Shogo

Watanabe, Yoshitaka

Watanabe, Yoshiyasu

Watson, Simon J..

Wei, Handi

Wei, Junkai..

Wei, Kai

Wei, Panfeng

Wei, Peng

Wei, Ran Feng

Wei, Zhang

Weijie, Mo.

Weiwen, Kong

Welker, Ken.

Wellens, Peter.

Wen, Binrong.

Wen, Li

Wen, Xiao.

Wen, Xueliang.

Wendt, Fabian.

Werner, Benjamin

Westgate, Zack

White, David J.

Whiteley, Elaine..

Wiktorski, Ekaterina

Wiktorski, Tomasz

Wilkins, Jonathan

Willden, Richard H.J.

Williams, Dara.

Williams, Wesley.

OMAE2019-96256 (9-2-2)

OMAE2019-96769 (9-2-1)

Wojtanowicz, Andrew OMAE2019-96707 (11-1-3)

OMAE2019-96836 (11-5-1)

Wolgamot, Hugh OMAE2019-95198 (12-7-1)

OMAE2019-95865 (12-5-2), OMAE2019-95933 (12-5-2)

Wong, Carlos.................................. OMAE2019-96034 (9-1-3)

Woo, Wai Lok OMAE2019-95485 (1-2-1)

Wood, Dylan ...................................... OMAE2019-95767 (9-5-2)

Wood, Geoffrey

Wrenger, Hendrik

Wright, Christopher

Wrobel, Luiz..

$\mathrm{Wu}, \mathrm{Bo}$

Wu, Guiyi...

Wu, Haoran OMAE2019-95503 (4-4-1)

OMAE2019-95970 (8-4-4), 0MAE2019-95972 (8-4-1)

Wu, Lei OMAE2019-95522 (8-4-1)

Wu, Meng...................................... OMAE2019-95694 (7-1-1)

Wu, Min ...................................... OMAE2019-96601 (2-12-3)

OMAE2019-96661 (1-1-1)

Wu, Minghao …….......................... OMAE2019-95793 (10-4-1)

$\mathrm{Wu}, \mathrm{Qi} \ldots \ldots \ldots \ldots . . . . .0$ OMAE2019-95292 (4-2-2)

Wu, Shanghua .................................... OMAE2019-95426 (4-1-3)

.. OMAE2019-96491 (4-1-11)

OMAE2019-95412 (2-15-1)

OMAE2019-96464 (6-11-2)

Wu, Xia ...................................... OMAE2019-95744 (8-5-1)

Wu, Xiao _... OMAE2019-95786 (7-11-1)

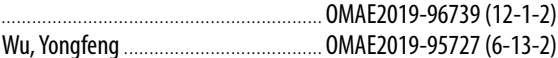

Wu, Yousheng ……...................... OMAE2019-95200 (8-5-1)

Wu, Youyou ...................................... OMAE2019-95500 (3-2-1)

Wu, Yuanping.................................. OMAE2019-96784 (11-1-3)

Wu, Zebing ..................................... OMAE2019-96784 (11-1-3)

Wuillaume, Pierre-Yves OMAE2019-96296 (9-1-8)

Wyatt, Lucy.................................... OMAE2019-95202 (6-12-1)

Xi, Huili ....................................... OMAE2019-95584 (8-4-3)

$X i$, Peng OMAE2019-95311 (6-8-2)

Xia, Andi........................................... OME2019-96719 (11-7-3) 


\begin{tabular}{|c|c|}
\hline Xiao, Yiqing .... & .... OMAE2019-96240 (9-1-3) \\
\hline Xiao, Zhong.... & .. OMAE2019-96036 (10-5-1) \\
\hline Xie, $0 \ldots \ldots \ldots \ldots$ & .... OMAE2019-95998 (9-5-2) \\
\hline Xie, Bin ........... & .... OMAE2019-96601 (2-12-3) \\
\hline Xie, Botao ....... & .... OMAE2019-95779 (6-7-1) \\
\hline Xie, Peng ......... & ..... OMAE2019-95638 (9-1-8) \\
\hline Xie, Qiang....... & ... OMAE2019-96698 (10-5-1) \\
\hline Xie, Tao ............. & ... OMAE2019-95192 (13-1-2) \\
\hline Xie, Wenhui.... & ... OMAE2019-96601 (2-12-3) \\
\hline & ..... OMAE2019-96661 (1-1-1) \\
\hline Xie, Xueshen .. & ..... OMAE2019-95737 (8-5-1) \\
\hline Xie, Zhitian...... & ..... OMAE2019-95620 (6-3-1) \\
\hline Xing, Xiuqing .. & ..... OMAE2019-95884 (9-5-3) \\
\hline & ..... OMAE2019-95892 (8-1-7) \\
\hline Xing, Yihan....... & ..... OMAE2019-96839 (6-4-3) \\
\hline Xiong, Ying....... & ..... OMAE2019-95311 (6-8-2) \\
\hline Xiong, Zhijian. & ..... OMAE2019-95585 (1-3-2) \\
\hline $\mathrm{Xu}$, Chen ........... & ... OMAE2019-96151 (6-13-3) \\
\hline Xu, Daqin ......... & ..... OMAE2019-95500 (3-2-1) \\
\hline Xu, Dong........... & ..... OMAE2019-95466 (8-4-3) \\
\hline & ..... OMAE2019-95673 (6-4-2) \\
\hline Xu, George...... & .... OMAE2019-95884 (9-5-3) \\
\hline Xu, Haitong .... & ... OMAE2019-96618 (6-11-1) \\
\hline Xu, Hongqi ..... & .... OMAE2019-95125 (2-5-1) \\
\hline $\mathrm{Xu}$, Jie............ & ..... OMAE2019-96799 (6-5-3) \\
\hline Xu, Lixin........ & .... OMAE2019-96474 (1-1-2) \\
\hline & ..... OMAE2019-96603 (1-4-2) \\
\hline Xu, Ming Cai .... & ... OMAE2019-95602 (2-10-1) \\
\hline Xu, Mingqiang & .... OMAE2019-95550 (2-5-1) \\
\hline Xu, Peng............ & ..... OMAE2019-95937 (9-4-1) \\
\hline $\mathrm{Xu}$, Qing Chang & ... OMAE2019-95308 (13-2-5) \\
\hline $\mathrm{Xu}$, Tang ............. & ..... OMAE2019-95926 (6-4-4) \\
\hline $\mathrm{Xu}$, Wei $\ldots \ldots \ldots$ & .... OMAE2019-95017 (1-6-2) \\
\hline & .... OMAE2019-95018 (1-6-2) \\
\hline Xu, Wei .. & .... OMAE2019-96543 (8-3-1) \\
\hline & .... OMAE2019-96577 (1-2-3) \\
\hline Xu, Weijun........ & ... OMAE2019-96001 (6-15-2) \\
\hline $\mathrm{Xu}$, Wentao $\ldots . . .$. & .. OMAE2019-95172 (12-1-1) \\
\hline Xu, Yuwang .... & .... OMAE2019-96112 (8-4-1) \\
\hline & .... OMAE2019-96486 (1-3-1) \\
\hline Xue, Xutian...... & .... OMAE2019-95108 (2-4-1) \\
\hline Xue, Yanpeng. & ... OMAE2019-95326 (11-1-1) \\
\hline & .. OMAE2019-95402 (11-1-2) \\
\hline Xue, Yanzhuo... & ..... OMAE2019-95694 (7-1-1) \\
\hline
\end{tabular}

\section{Y}

Yamabe, Yuta

Yamada, Reiko

Yamaguchi, Junko

Yamamoto, Joji.

\section{Yamamoto, Marcio}

Yamamoto, Morikazu

Yan, Boqian..

Yan, Jun.

OMAE2019-95426

OMAE2019-96195 (4-1-11), OMAE2019-96491 (4-1-11) Yan, Shiqiang

\begin{tabular}{|c|c|}
\hline rdil, Jiliqualig........ & . UIVIALZUIY-9JZ4/ $(1<-2-1)$ \\
\hline Yan, Xinkuan .......... & .. OMAE2019-96759 (9-1-5) \\
\hline Yang, Chunxue..... & .... OMAE2019-96179 (6-7-1) \\
\hline Yang, Guang............ & ... OMAE2019-96617 (11-7-2) \\
\hline Yang, Guangqiang & .... OMAE2019-95804 (4-2-1) \\
\hline & .... OMAE2019-96826 (4-2-4) \\
\hline Yang, Hezhen ........ & .... OMAE2019-95266 (4-3-8) \\
\hline Yang, Injun ............ & .... OMAE2019-96030 (9-3-3) \\
\hline Yang, Jianguo...... & .... OMAE2019-96726 (6-4-4) \\
\hline Yang, Jianmin .... & .... OMAE2019-95292 (4-2-2) \\
\hline & .... OMAE2019-96578 (6-3-4) \\
\hline Yang, Jianming ...... & $. .0 M A E 2019-96170(11-2-2)$ \\
\hline Yang, Jianye ........... & .. OMAE2019-95421 (12-1-2) \\
\hline Yang, Liang ............. & .... OMAE2019-96144 (4-1-7) \\
\hline Yang, Liang ............ & ... OMAE2019-96814 (12-2-1) \\
\hline Yang, Lijing ............. & ... OMAE2019-96655 (10-3-1) \\
\hline Yang, Lijun ......... & .. OMAE2019-95601 (13-2-3) \\
\hline Yang, Limin ............ & .... OMAE2019-95469 (1-2-2) \\
\hline Yang, Mingzheng.. & .. OMAE2019-96675 (11-3-1) \\
\hline & $. .0 M A E 2019-96676(11-3-1)$ \\
\hline
\end{tabular}

OMAE2019-96493 (13-2-5)

OMAE2019-95990 (5-3-1)

OMAE2019-96040 (5-3-1)

OMAE2019-95990 (5-3-1)

OMAE2019-96493 (13-2-5)

OMAE2019-95610 (8-4-4)
Yang, Ping ......................................... OMAE2019-95226 (2-11-1) OMAE2019-95230 (2-4-2) Yang, Ray-Yeng................................... OMAE2019-95730 (2-6-1) OMAE2019-96424 (13-2-2) Yang, Shuai ............. OMAE2019-96508 (6-2-2) Yang, Shun-Han.................................. OMAE2019-95138 (9-4-4) Yang, Wenjing ............................... OMAE2019-95727 (6-13-2) Yang, Xuda .................................. OMAE2019-95480 (11-5-1) Yang, Yunta0 .................................. OMAE2019-96792 (6-13-3) Yang, Zhaoging.......... OMAE2019-96803 (5-2-1) Yang, Zhengmao Yang, Zhijian . Yang, Zhixun OMAE2019-95500 (3-2-1) OMAE2019-95209 (8-2-2) OMAE2019-95426 (4-1-3) …........ OMAE2019-96144 (4-1-7), OMAE2019-96195 (4-1-11) Yao, Aifeng ........................................ OMAE2019-95618 (2-6-2) Yao, Erdong....... OMAE2019-95326 (11-1-1) OMAE2019-95402 (11-1-2) Yao, Ji........................................ OMAE2019-95412 (2-15-1)

Yao, Peng. Yao, Xiongliang Yasuda, Kyono..

Ye, Jianqiao.

Ye, Tianzhuang

Yeganeh-Bakhtiary, Abbas.

Yehia, Waleed

Yelland, Margaret J...

Yenduri, Anurag.

Yeon, SeongMo

Yeung, Ronald W.

Yi, Hualei.

Yiew, Lucas J.

Yim, Solomon..

OMAE2019-96464 (6-11-2)

OMAE2019-95455 (4-5-2)

OMAE2019-95850 (8-3-1)

OMAE2019-95863 (3-9-1)

OMAE2019-96755 (4-1-4) OMAE2019-96715 (5-5-1) OMAE2019-96827 (8-4-3) OMAE2019-96770 (8-1-4) OMAE2019-95068 (6-17-2) OMAE2019-96577 (1-2-3) OMAE2019-96429 (1-6-2 OMAE2019-96832 (6-1-2) OMAE2019-95177 (4-5-2) OMAE2019-96699 (6-5-2) OMAE2019-96802 (9-4-3) OMAE2019-96803 (5-2-1) OMAE2019-95970 (8-4-4) OMAE2019-95972 (8-4-1)

Yin, Decao

Yin, Fengjie

Yin, Guang .

Yin, Guangzhi

Yin, Yanju

Yin, Yuanchao

OMAE2019-96826 (4-2-4)

OMAE2019-96069 (1-6-2)

OMAE2019-96707 (11-1-3)

OMAE2019-96824 (4-1-1) OMAE2019-95426 (4-1-3) OMAE2019-96491 (4-1-11)

Yingcai, Huang

Yoo, Mintaek

.. OMAE2019-96666 (2-4-2)

OMAE2019-95845 (2-13-1)

Yoo, Seon Oh

Yordanova, Borislava

Yoshida, Ikuo.

OMAE2019-96236 (1-3-2)

OMAE2019-96010 (3-1-1)

OMAE2019-95431 (5-1-1)

OMAE2019-95670 (5-2-2)

Yoshida, Takero OMAE2019-95672 (5-2-2), OMAE2019-95978 (5-5-1)

Yoshimoto, Haruki .......................... OMAE2019-95828 (5-5-1) OMAE2019-96443 (13-3-4) OMAE2019-95818 (3-6-1) OMAE2019-96017 (9-2-6) OMAE2019-95891 (6-17-1) OMAE2019-95168 (6-7-2) OMAE2019-96347 (11-7-2)

Yotsuzuka, Takayuki.

You, Yunxiang

Young, lan

Ytrehus, Jan David OMAE2019-96400 (11-7-2), OMAE2019-96547 (11-15-2)

Yu, Chenteh Alan ............................... OMAE2019-95804 (4-2-1) Yu, Dongchi ..................................... OMAE2019-96832 (6-1-2) $Y_{u}$, Jiawei OMAE2019-95727 (6-13-2) . OMAE2019-95887 (8-1-7) OMAE2019-95839 (4-6-1)

Yu, Jin Xin

Yu, Tongxi

Yu, Wei............... OMAE2019-96134 (2-11-2) OMAE2019-96240 (9-1-3) OMAE2019-96360 (9-2-3)

Yu, Xianzhao

Yu, Xiong...

Yu, Yi OMAE2019-96835 (6-15-2) OMAE2019-95641 (10-4-1) OMAE2019-96601 (2-12-3) OMAE2019-96661 (1-1-1)

Yu, Yi-Hsiang OMAE2019-95216 (9-3-1)

OMAE2019-96397 (9-3-3), OMAE2019-96466 (9-4-2) Yu, Youngjae ................................ OMAE2019-95919 (9-1-3) Yu, Zuyao _ . OMAE2019-95926 (6-4-4) Yuan, Jiabei OMAE2019-95118 (4-1-1) OMAE2019-95326 (11-1-

Yuan, Lishan OMAE2019-95748 (11-11-2)
Yuan, Shuai .

OMAE2019-95458 (4-1-5) OMAE2019-95456 (2-11-3) Yuan, Ye ............................................. OMAE2019-96425 (8-1-5) Yuan, Zhenqin ................................ OMAE2019-95123 (4-1-10) Yuan, Zhiming . OMAE2019-95578 (6-3-1) OMAE2019-95868 (6-13-2), OMAE2019-96135 (1-5-1) Yuck, Raehyoung.............................. OMAE2019-95272 (1-1-1) Yue (Le), Jingxia................................. OMAE2019-95473 (6-11-2) Yue, Hong...................................... OMAE2019-96017 (9-2-6) Yue, Oianjin OMAE2019-95426 (4-1-3) OMAE2019-96195 (4-1-11), OMAE2019-96464 (6-11-2) OMAE2019-96491 (4-1-11) Yue, Xin ......................................... OMAE2019-96556 (3-4-1)

\section{2}

Zang, Jun. OMAE2019-95739 (12-4-1) Ze-cong, Chen .................................. OMAE2019-96461 (13-2-1) Zeinali Torbati, Reza............................. OMAE2019-96732 (7-1-1) Zeinoddini, Mostafa ............................ OMAE2019-95227 (9-5-2) Zeng, David .................................... OMAE2019-95637 (10-7-1) Zeng, HaO ..................................... OMAE2019-96617 (11-7-2) Zeng, Ji OMAE2019-95320 (2-13-2) Zeng, Qingsong.................................. OMAE2019-95076 (8-1-3) Zeng, Weijian _........................... OMAE2019-95968 (9-2-6) Zeng, Yi........................................... OMAE2019-96804 (4-5-1) Zeng, Yijin ....................................... OMAE2019-96617 (11-7-2) Zeng, Yuhao ……............................... OMAE2019-95926 (6-4-4) Zha, Ruosi ........................................ OMAE2019-96775 (12-7-1) Zhan, Jin ........................................... OMAE2019-96018 (8-1-1) Zhan, Xiaogin .......................... OMAE2019-96191 (2-6-2) Zhang, Aman .................................. OMAE2019-95232 (6-8-1) ..... OMAE2019-95257 (6-13-3) Zhang, Cheng ................................... OMAE2019-95251 (6-4-5) Zhang, Chi.................................... OMAE2019-95039 (6-2-3) Zhang, Chi OMAE2019-96753 (5-1-3) OMAE2019-96761 (5-1-3), OMAE2019-96789 (5-1-3) Zhang, Dahai...................................... OMAE2019-95530 (6-4-4) Zhang, Dahai _........................... OMAE2019-95945 (9-2-3) OMAE2019-95968 (9-2-6) Zhang, Donghai ................................. OMAE2019-96036 (10-5-1) Zhang, Dongming .............................. OMAE2019-96707 (11-1-3) Zhang, Fan ........................................ OMAE2019-96474 (1-1-2) Zhang, Feifei ................................. OMAE2019-96617 (11-7-2) OMAE2019-96675 (11-3-1) Zhang, Guiyong .................................. OMAE2019-95104 (9-2-2) ... OMAE2019-95610 (8-4-4) OMAE2019-96018 (8-1-1) OMAE2019-96020 (8-1-4) OMAE2019-96181 (8-1-3) OMAE2019-96581 (6-5-4) OMAE2019-95966 (4-5-2) OMAE2019-95687 (6-4-5) OMAE2019-95745 (2-12-3) OMAE2019-95891 (6-17-1) OMAE2019-95672 (5-2-2) OMAE2019-96735 (6-2-2) OMAE2019-95402 (11-1-2) OMAE2019-95123 (4-1-10) OMAE2019-96835 (6-15-2) OMAE2019-96221 (9-1-3) OMAE2019-95624 (11-3-1)

Zhang, Lufen OMAE2019-95802 (11-11-2) Zhang, Mengmeng Zhang, Michael. .................................... OMAE2019-96637 (6-7-3) Zhang, Min OMAE2019-96553 (1-3-2) Zhang, Pan ................................... OMAE2019-95491 (2-12-1) ... OMAE2019-95924 (2-12-2), 0MAE2019-96628 (2-12-2) Zhang, Peng …........................ OMAE2019-95988 (4-1-11) Zhang, Qi........................................ OMAE2019-95915 (9-2-1) Zhang, Qing ....................................... OMAE2019-96508 (6-2-2) Zhang, Ruoyu. Zhang, Shanli... Zhang, Shengming..

Zhang, Shuai.

Zhang, Shuhua..

Zhang, Weidong

Zhang, Weihan.. OMAE2019-95635 (9-2-3) ... OMAE2019-96699 (6-5-2) OMAE2019-96134 (2-11-2) OMAE2019-96784 (11-1-3) ... OMAE2019-95392 (2-4-3) OMAE2019-96739 (12-1-2) ... OMAE2019-96694 (4-3-4) 


\begin{tabular}{|c|}
\hline Zhang, Weijie ...... \\
\hline .. OMAE2019-95198 (12-7-1) \\
\hline .... OMAE2019-95744 (8-5-1) \\
\hline .... OMAE2019-95865 (12-5-2) \\
\hline .... OMAE2019-95602 (2-10-1) \\
\hline ..... OMAE2019-95631 (4-2-5) \\
\hline OMAE2019-95676 (4-1-5), 0MAE2019-95692 (4-1-6) \\
\hline 1-5), OMAE2019-95916 (4-1-7) \\
\hline Zhang, Xinshu .......... OMAE2019-95891 (6-17-1) \\
\hline .. OMAE2019-96029 (13-1-3) \\
\hline OMAE2019-96191 (2-6-2) \\
\hline OMAE2019-95327 (2-4-3) \\
\hline .... OMAE2019-95126 (1-3-1) \\
\hline .... OMAE2019-96448 (8-1-6) \\
\hline ..... OMAE2019-95005 (4-5-1) \\
\hline ..... OMAE2019-95984 (2-6-2) \\
\hline 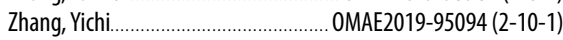 \\
\hline Zhang, Yizhao .......... OMAE2019-95271 (8-4-1) \\
\hline 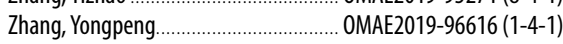 \\
\hline 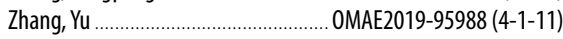 \\
\hline .... OMAE2019-96679 (12-5-1) \\
\hline ..... OMAE2019-95224 (8-2-1) \\
\hline OMAE2019-96122 (6-3-1) \\
\hline .... OMAE2019-96771 (6-1-2) \\
\hline ..... OMAE2019-96805 (6-1-2) \\
\hline .... OMAE2019-95563 (8-1-5) \\
\hline OMAE2019-95714 (8-1-2), OMAE2019-96425 (8-1-5) \\
\hline Zhang, Zhimeng ................................. OAE2019-95466 (8-4-3) \\
\hline .... OMAE2019-95602 (2-10-1) \\
\hline OMAE2019-95675 (1-6-1) \\
\hline .... OMAE2019-95779 (6-7-1) \\
\hline ... OMAE2019-95831 (6-17-1) \\
\hline Zhao, Fenfang .............................. OMAE2019-95192 (13-1-2) \\
\hline . OMAE2019-95225 (13-1-2), 0MAE2019-95308 (13-2-5) \\
\hline OMAE2019-95706 (13-2-1), 0MAE2019-96019 (13-1-2) \\
\hline Zhao, Jiannan $\ldots \ldots \ldots \ldots \ldots \ldots \ldots$ \\
\hline OMAE2019-95676 (4-1-5), 0MAE2019-95692 (4-1-6) \\
\hline OMAE2019-95903 (4-1-5), OMAE2019-95916 (4-1-7) \\
\hline Zhao, Jingrui ........... OMAE2019-96661 (1-1-1) \\
\hline OMAE2019-95911 (10-5-1) \\
\hline ..... OMAE2019-96444 (6-8-1) \\
\hline O.... OMAE2019-96824 (4-1-1) \\
\hline
\end{tabular}

Zhao, Peidong..

Zhao, Pengduo.

Zhao, Wei

Zhao, Wenhua..

Zhao, Xin.

Zhao, Xizeng

Zhao, Xuanlie

Zhao, Yakun.

Zhao, Yi.

Zhao, Yongsheng

Zhao, Zhanhua

Zhao, Zhengye .

Zhao, Zishu..

Zhaoqiang, Wang

Zheng, Guoping

Zheng, Jinhai

Zheng, KaiBo.

Zheng, Kaiyuan

Zheng, Lihui.

Zheng, Shaowen..

Zheng, Yi .

Zhiyun, Chen

Zhizhong, Liu

Zhong, Qian.

Zhou, Baoshun.

Zhou, Binzhen..

Zhou, Dai..

Zhou, Daocheng

Zhou, Fujian.

.. OMAE2019-95326 (11-1-1)

... OMAE2019-95402 (11-1-2), OMAE2019-95480 (11-5-1)

OMAE2019-95624 (11-3-1), OMAE2019-95748 (11-11-2) OMAE2019-95774 (11-1-1), OMAE2019-95802 (11-11-2)

\section{Zhou, Hanwei}

Zhou, Jian Guo.

Zhou, Jifu

Zhou, Jinxin

Zhou, Joe.

Zhou, Peilin.

Zhou, Peng...
.. OMAE2019-95104 (9-2-2) OMAE2019-95776 (2-9-4) OMAE2019-96682 (11-7-3) OMAE2019-95865 (12-5-2) OMAE2019-95449 (6-13-3) OMAE2019-95739 (12-4-1) OMAE2019-95744 (8-5-1) OMAE2019-95988 (4-1-11) OMAE2019-95915 (9-2-1) OMAE2019-96771 (6-1-2) OMAE2019-95275 (3-3-3) OMAE2019-95547 (3-5-1) OMAE2019-95412 (2-15-1) OMAE2019-95966 (4-5-2) OMAE2019-95081 (6-11-1) OMAE2019-95039 (6-2-3) OMAE2019-95930 (7-12-1) OMAE2019-95209 (8-2-2) OMAE2019-96094 (11-7-3) OMAE2019-95630 (2-4-3) OMAE2019-96193 (12-1-1) .. OMAE2019-96671 (9-5-2) OMAE2019-95603 (4-4-2) OMAE2019-96832 (6-1-2) OMAE2019-96144 (4-1-7) OMAE2019-95161 (12-5-1) OMAE2019-96477 (4-2-2) OMAE2019-95016 (1-2-2) OMAE2019-96098 (12-5-2) OMAE2019-96777 (1-1-4) OMAE2019-96828 (6-17-1) OMAE2019-95672 (5-2-2) .. OMAE2019-95978 (5-5-1) OMAE2019-96603 (1-4-2) OMAE2019-96716 (6-4-4) .. OMAE2019-96819 (8-1-4) OMAE2019-95209 (8-2-2)
Zhou, Shengtao

Zhou, Xiaohong

Zhou, Xueqian.

Zhou, Yang

Zhou, Yi

Zhou, Yujie.

Zhou, Zhongbing

Zhu, Gancheng..

Zhu, Haiming

Zhu, Haishan

Zhu, Jianjun ..

Zhu, Jin

Zhu, Linfang

Zhu, Ling

2019-96134 (2-11-2), OMAE2019-96193 (12-1-1)

Zhu, Renchuan $\quad$ OMAE2019-96792 (6-13-3)

Zhu, Tingyao ................................. OMAE2019-95121 (2-2-2)

Zhu, Weiquan .................................... OMAE2019-96650 (1-2-4)

Zhu, Wenbin ...................................... OMAE2019-95308 (13-2-5)

Zhu, Wencai ....................................... OMAE2019-96782 (6-4-3)

Zhu, Xiyang OMAE2019-95635 (9-2-3)

Zhuang, Jiayuan ................................ OMAE2019-96835 (6-15-2)

Ziguang, Zha0 ................................ OMAE2019-95072 (13-2-1)

Zong-quan, Ying ........................ OMAE2019-96461 (13-2-1)

OMAE2019-96508 (6-2-2)

Zong, Zhi .......................................... OMAE2019-95104 (9-2-2)

Zou, Jian OMAE2019-95777 (2-9-3)

OMAE2019-96136 (6-8-1)

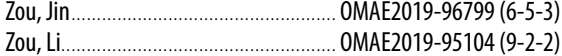

Zou, Meiyan ........................................ OMAE2019-95717 (12-2-1)

Zou, Qingping ............................. OMAE2019-95165 (12-4-1)

Zou, Zaojian ...................................... OMAE2019-95105 (7-4-1)

OMAE2019-95249 (6-13-2)

Z Carlos A........................... OMAE2019-95385 (5-4-1)

OMAE2019-96358 (3-2-1)

\title{
AIM-ALE 2020
}

\section{Asset Integrity Management -} Aging and Life Extension

\author{
March 23-26, 2020 \\ PSA offices, Stavanger, Norway
}




\section{Session Index}

\section{Offshore Technology}

1-1-1 Semi-Submersibles and TLPs

1-1-2 FPSO and Arctic Structures

1-1-3 Floating Wind Platforms

1-1-4 Fixed Platforms and Foundations

1-1-5 Artificial Intelligence and Advance Analysis

1-2-1 Dynamic Positioning I

1-2-2 Mooring System Design and Analysis I

1-2-3 Dynamic Positioning II

1-2-4 Mooring System Design and Analysis II

1-3-1 Nonlinear Wave and Wave Effects

1-3-2 Fluid-Structure Interaction

1-4-1 Experimental Design and Analysis

1-4-2 Numerical Design and Analysis

1-4-3 Design 0ptimization

1-5-1 FLNG

1-6-1 CFD Numerical Waves and Applications

1-6-2 Loads and Responses in Current and Wind I

1-7-1 Wave Loading and Motions in Extreme Seas

1-7-2 Wave Loading and Motions in Extreme Seas II

Structures, Safety and Reliability

2-1-1 Abnormal or Rogue Waves I

2-1-2 Abnormal or Rogue Waves II

2-2-1 Probabilistic and Spectral Wave Models I

2-2-2 Probabilistic and Spectral Wave Models II

2-3-1 Probabilistic Response Models

2-4-1 Fatigue and Fracture Reliability I

2-4-2 Fatigue and Fracture Reliability II

2-4-3 Fatigue and Fracture Reliability III

2-5-1 Reliability of Marine Structures

2-6-1 Reliability of Mooring and Riser Systems I

2-6-2 Reliability of Mooring and Riser Systems II

2-6-3 Reliability of Mooring and Riser Systems III

2-7-1 Reliability of Renewable Energy Systems

2-8-1 Well Integrity and Reliability Assessment

2-9-1 Extreme Loading and Responses I

2-9-2 Extreme Loading and Responses II

2-9-3 Extreme Loading and Responses III

2-9-4 Extreme Loading and Responses IV

2-10-1 Collision and Crashworthiness I

2-10-2 Collision and Crashworthiness II

2-11-1 Ultimate Strength I

2-11-2 Ultimate Strength II

2-11-3 Ultimate Strength III

2-12-1 Structural Analysis and Optimization I

2-12-2 Structural Analysis and Optimization II

2-12-3 Structural Analysis and Optimization III

2-13-1 Risk Analysis and Management I

2-13-2 Risk Analysis and Management II

2-14-1 Risk Based Maintenance

2-15-1 Data Driven Models

\section{Materials Technology}

3-1-1 Fracture Toughness measurement and Assessment

3-1-2 Formulation of the Fracture Parameter

3-2-1 Fabrication and Performance of Clad Pipes

3-2-2 Analysis and Fatigue Performance of Tubular Joints (John Sharp Honorary Session)

3-3-1 Fatigue Improvement and Repairs

3-3-2 Performance of Mooring Chains

3-3-3 Advances on Assessing Performance of Steel

3-4-1 Steel Performance in Sour Environment

3-5-1 Fatigue Assessment and Improvement

3-6-1 Advances in Materials Characterization

3-9-1 Advances in Materials Characterization
Thursday June 13

Monday June 10

Tuesday June 11

Tuesday June 11

Tuesday June 11

Monday June 10

Tuesday June 11

Tuesday June 11

Tuesday June 11

Wednesday June 12

Wednesday June 12

Thursday June 13

Wednesday June 12

Monday June 10

Wednesday June 12

Monday June 10

Tuesday June 11

Thursday June 13

Thursday June 13

Wednesday June 12

Wednesday June 12

Wednesday June 12

Wednesday June 12

Monday June 10

Tuesday June 11

Tuesday June 11

Tuesday June 11

Thursday June 13

Wednesday June 12

Thursday June 13

Thursday June 13

Thursday June 13

Tuesday June 11

Tuesday June 11

Tuesday June 11

Tuesday June 11

Tuesday June 11

Monday June 10

Monday June 10

Wednesday June 12

Wednesday June 12

Wednesday June 12

Tuesday June 11

Tuesday June 11

Tuesday June 11

Thursday June 13

Thursday June 13

Thursday June 13

Monday June 10

Tuesday June 11

Monday June 10

Thursday June 13

Thursday June 13

Wednesday June 12

Tuesday June 11

Tuesday June 11

Tuesday June 11

Thursday June 13

Wednesday June 12

Monday June 10
3-11-1 Developments in BS 7910 and other Fitness-for-service Procedures: Session I

Wednesday June 12

3-11-2 Developments in BS 7910 and other Fitness-for-service Procedures: Session II

3-13-1 Dr. John Sharp Honorary Session

Wednesday June 12

Thursday June 13

Pipelines, Risers, and Subsea Systems

4-1-1 Flexible Pipes I

4-1-2 Flexible Pipes II

4-1-3 Flexible Pipes III

4-1-4 Flexible Pipes IV

4-1-5 Flexible Pipes V

4-1-6 Flexible Pipes VI

4-1-7 Flexible Pipes VII

4-1-10 Umbilicals and Cables I

4-1-11 Umbilicals and Cables II

4-2-1 General Design and Analysis I

4-2-2 General Design and Analysis II

4-2-3 Drilling Risers I

4-2-4 SCRs and SLWRs I

4-2-5 SCRs and SLWRs II

4-3-1 Collapse

4-3-2 Installation

4-3-3 Mechanics I

4-3-4 Thermo-Mechanical I

4-3-5 Thermo-Mechanical II

4-3-6 ECA

4-3-7 Thermo-Mechanical III

4-3-8 Mechanics II

4-4-1 Subsea Structures I

4-4-2 Subsea Structures II

4-5-1 Flow Assurance I

4-5-2 Flow Assurance II

Monday June 10

Tuesday June 11

Tuesday June 11

Monday June 10

Tuesday June 11

Wednesday June 12

Wednesday June 12

Thursday June 13

Thursday June 13

Tuesday June 11

Tuesday June 11

Wednesday June 12

Wednesday June 12

Monday June 10

Monday June 10

Tuesday June 11

Wednesday June 12

Wednesday June 12

Wednesday June 12

Tuesday June 11

Thursday June 13

Thursday June 13

Thursday June 13

Thursday June 13

Tuesday June 11

Wednesday June 12

4-6-1 Innovative Technologies for Deepwater Low-Cost Production I Thursday June 13

4-6-2 Innovative Technologies for Deepwater Low-Cost Production II Thursday June 13

\section{Ocean Space Utilization}

5-1-1 Marine Utilization and Marine Spatial Planning

5-1-2 Hybrid and Complex Use of Floating Systems I

5-1-3 Hybrid and Complex Use of Floating Systems II

5-2-1 Aquaculture I: Design and Modeling I

5-2-2 Aquaculture II: Design and Modeling II

5-3-1 Development of Deep Sea Mining and Resources

5-4-1 Underwater Vehicle and Technology

5-5-1 Floating Systems for Renewable Energy

5-6-1 High Tide and Tsunamis

Monday June 10

Wednesday June 12

Wednesday June 12

Tuesday June 11

Tuesday June 11

Tuesday June 11

Tuesday June 11

Wednesday June 12

Monday June 10

\section{Ocean Engineering}

6-1-2 Floating Body Technology

6-2-1 Coastal Engineering I

6-2-2 Coastal Engineering II

6-2-3 Coastal Engineering III

6-3-1 Fluid-Structure Interaction/Hydroelasticity

6-3-2 Wave-Body Interactions/CFD

6-3-3 Damping and Viscous Effects

6-3-4 Wave-body interactions: Special Problems

6-4-1 Marine Control and Automation

6-4-2 Marine Operations and Vessel Motions

6-4-3 Marine Engineering and Applications I

6-4-4 Marine Engineering and Applications II

6-4-5 Very Large Floating Structures

6-4-6 Towed Cables, Ropes and Mooring Systems

6-5-1 Advanced Marine Hydrodynamics I

6-5-2 Advanced Marine Hydrodynamics II

6-5-3 Advanced Marine Hydrodynamics III
Monday June 10

Tuesday June 11

Tuesday June 11

Tuesday June 11

Wednesday June 12

Wednesday June 12

Wednesday June 12

Wednesday June 12

Monday June 10

Monday June 10

Tuesday June 11

Tuesday June 11

Tuesday June 11

Tuesday June 11

Wednesday June 12

Wednesday June 12

Wednesday June 12 
Ocean Engineering (Continued)

6-5-4 Advanced Marine Hydrodynamics IV

6-7-1 Regional Metocean I

6-7-2 Regional Metocean II

6-7-3 Metocean Criteria I

6-7-4 Metocean Criteria II

6-8-1 Wave Loads

6-8-2 Ship Hydrodynamics

6-11-1 Autonomous Vehicle Technology

6-11-2 Floating Bodies Technology

6-12-1 Ocean Measurement and Data Interpretation

6-13-1 Ship Resistance and Wave Loads

6-13-2 Ship Manoeuverability and Motion

6-13-3 Numerical Methods

6-15-1 Underwater Vehicles Control

6-15-2 Underwater Vehicles Design Technology and Hydrodynamics

6-17-1 Wave loads on structures

6-17-2 Nonlinear and Breaking Waves

\section{Polar and Arctic Sciences and Technology}

7-1-1 Arctic Frontiers and Manoeuvring in Ice

7-3-1 Structures in lce

7-4-1 Vessels in Ice and Waves

7-11-1 Ice Model Tests and Structure-Ice-Interactions

7-12-1 Numerical Ice Modeling

\section{CFD \& FS}

\section{8-1-1 FSI}

8-1-2 Surface Waves

8-1-3 Ship Performance I

8-1-4 Ship Performance ll

8-1-5 Seakeeping I

8-1-6 Seakeeping II

8-1-7 Propulsion

8-2-1 Free Surface Modeling

8-2-2 Free Surface Loading and Structure Interaction I

8-2-3 Free Surface Loading and Structure Interaction II

8-3-1 Data-Driven Modeling and Machine Learning

8-3-2 Code Development and V\&V

8-4-1 Cylinder VIV

8-4-2 Risers, Jumpers and Pipelines

8-4-3 Interference, Proximity and Geometry Effects

8-4-4 VIV Suppression and Control

8-5-1 Wave CFD modeling Applications

8-5-2 VIV Theory and CFD\&FSI Symposium Workshop

\section{Ocean Renewable Energy}

9-1-1 Bottom-fixed Wind Turbines

9-1-2 FWT - Numerical Analysis I

9-1-3 FWT - Numerical Analysis II

9-1-5 FWT - Mooring Systems

9-1-8 FWT Hydrodynamics I

9-2-1 Aerodynamics I

9-2-2 Aerodynamics II

9-2-3 Floating Wind Designs

9-2-6 Hybrid Systems and Farm Analysis

9-3-1 Wave Energy Converter Control Systems Competition (WECCCOMP)

9-3-2 Wave Energy: Oscillating Water Column I

9-3-3 Wave Energy: CFD Simulations

9-4-1 Power Take-offs and Experiments

9-4-2 Wave Farms and Alternative Markets

9-4-3 Advanced Controls

9-4-4 Optimization and Load Analysis

9-5-2 Concepts and Design

9-5-3 Numerical Analysis I

9-5-4 Numerical Analysis II

9-6-1 Thermal, Hybrid and Others: Analysis, Design and Prediction

9-7-1 Drivetrain Design, Operation and Condition Monitoring I
Wednesday June 12

Thursday June 13

Thursday June 13

Thursday June 13

Thursday June 13

Wednesday June 12

Wednesday June 12

Monday June 10

Tuesday June 11

Tuesday June 11

Thursday June 13

Thursday June 13

Thursday June 13

Wednesday June 12

Wednesday June 12

Thursday June 13

Thursday June 13

Tuesday June 11 Wednesday June 12 Wednesday June 12 Wednesday June 12 Wednesday June 12

Monday June 10 Monday June 10 Wednesday June 12 Wednesday June 12 Thursday June 13

Thursday June 13

Thursday June 13

Tuesday June 11

Tuesday June 11

Tuesday June 11

Wednesday June 12

Wednesday June 12

Wednesday June 12

Thursday June 13

Thursday June 13

Thursday June 13

Tuesday June 11

Thursday June 13

Monday June 10

Wednesday June 12

Wednesday June 12

Thursday June 13

Thursday June 13

Monday June 10

Wednesday June 12

Tuesday June 11

Thursday June 13

Tuesday June 11

Wednesday June 12

Thursday June 13

Thursday June 13

Wednesday June 12

Thursday June 13

Tuesday June 11

Tuesday June 11

Thursday June 13

Thursday June 13

Wednesday June 12

Wednesday June 12
Offshore Geotechnics

10-1-1 Seabed Properties and Processes Monday June 10

10-3-1 Anchors

10-4-1 Pile Foundations I

10-5-1 Bucket Foundations, Suction Caissons and Spudcans

10-6-1 Pipeline Geotechnics

10-7-1 Pile Foundations II

Monday June 10

Tuesday June 11

Tuesday June 11

Tuesday June 11

Tuesday June 11

Petroleum Technology

11-1-1 General Petroleum Technology Production Enhancement

Thursday June 13

11-1-2 General Petroleum Technology Production and Drilling Enhancement Thursday June 13

11-1-3 General Petroleum Technology Drilling and Separation Enhancement Thursday June 13

11-2-1 Drilling Mechanics Session I

11-2-2 Drilling Mechanics Session II

11-3-1 Drilling Geomechanics

11-4-1 Petroleum Production Systems Design and Operation

11-5-1 Well Inflow Control and Reservoir Management

11-6-1 Integrity of Well Barriers I

11-6-2 Integrity of Well Barriers II

11-7-1 Well Drilling Fluids and Hydraulics I

11-7-2 Well Drilling Fluids and Hydraulics II

11-7-3 Well Drilling Fluid and Hydraulics III

11-10-1 New Materials for Well Construction

11-11-1 Innovations in Drilling, Production and Transport

11-11-2 Innovations in Drilling, Production and Transport

11-12-1 Cementing I

11-12-2 Cementing II

11-13-1 LSU Workshop on Riser Gas Management and Well Control

11-15-1 Well Abandonment I - Rules and Regulations

11-15-2 Well Abandonment II - Research and Operational Experiences Wednesday June 12

\section{Rodney Eatock Taylor Honouring Symposium on}

Marine and Offshore Hydrodynamics

12-1-1 Numerical and Experimental Methods in Hydrodynamics I

12-1-2 Numerical and Experimental Methods in Hydrodynamics II

12-2-1 Multi-Body Hydrodynamics

12-4-1 Hydrodynamic Aspects of Offshore Renewable Energy

12-5-1 Non-Linear Waves and Wave Effects I

12-5-2 Non-Linear Waves and wave Effects I

12-7-1 Large-Amplitude Non-Linear Ship Motions

Wednesday June 12

Wednesday June 12

Wednesday June 12

Wednesday June 12

Wednesday June 12

Tuesday June 11

Tuesday June 11

Monday June 10

Tuesday June 11

Monday June 10

Thursday June 13

Thursday June 13

Thursday June 13

Tuesday June 11

Wednesday June 12

Thursday June 13

Wednesday June 12

Takeshi Kinoshita Honoring Symposium on Offshore Technology

13-1-1 Extremes and Environmental Modelling

13-1-2 Fluid Body Interaction

13-1-3 Nonlinear Waves I

13-1-4 Nonlinear Waves II

13-2-1 Numerical Methods

13-2-2 Experiments and Numerical Validation

13-2-3 Flow-Induced Motions (FIM)

13-2-4 Fluid-Structure Interactions (FSI)

13-2-5 0thers

13-3-1 Wind Energy

13-3-2 Wave Energy 1

13-3-3 0cean Current Energy, OTEC and Related Technology

13-3-4 Wave Energy II

13-7-1 Small vessel and Related Technology

Monday June 10

Wednesday June 12

Monday June 10

Tuesday June 11

Tuesday June 11

Tuesday June 11

Tuesday June 11

Wednesday June 12

Wednesday June 12

Wednesday June 12

Wednesday June 12

Monday June 10

Tuesday June 11

Tuesday June 11

Tuesday June 11

Tuesday June 11

Thursday June 13

Thursday June 13

Thursday June 13

Thursday June 13

Monday June 10 


\section{Notes}




\section{Notes}




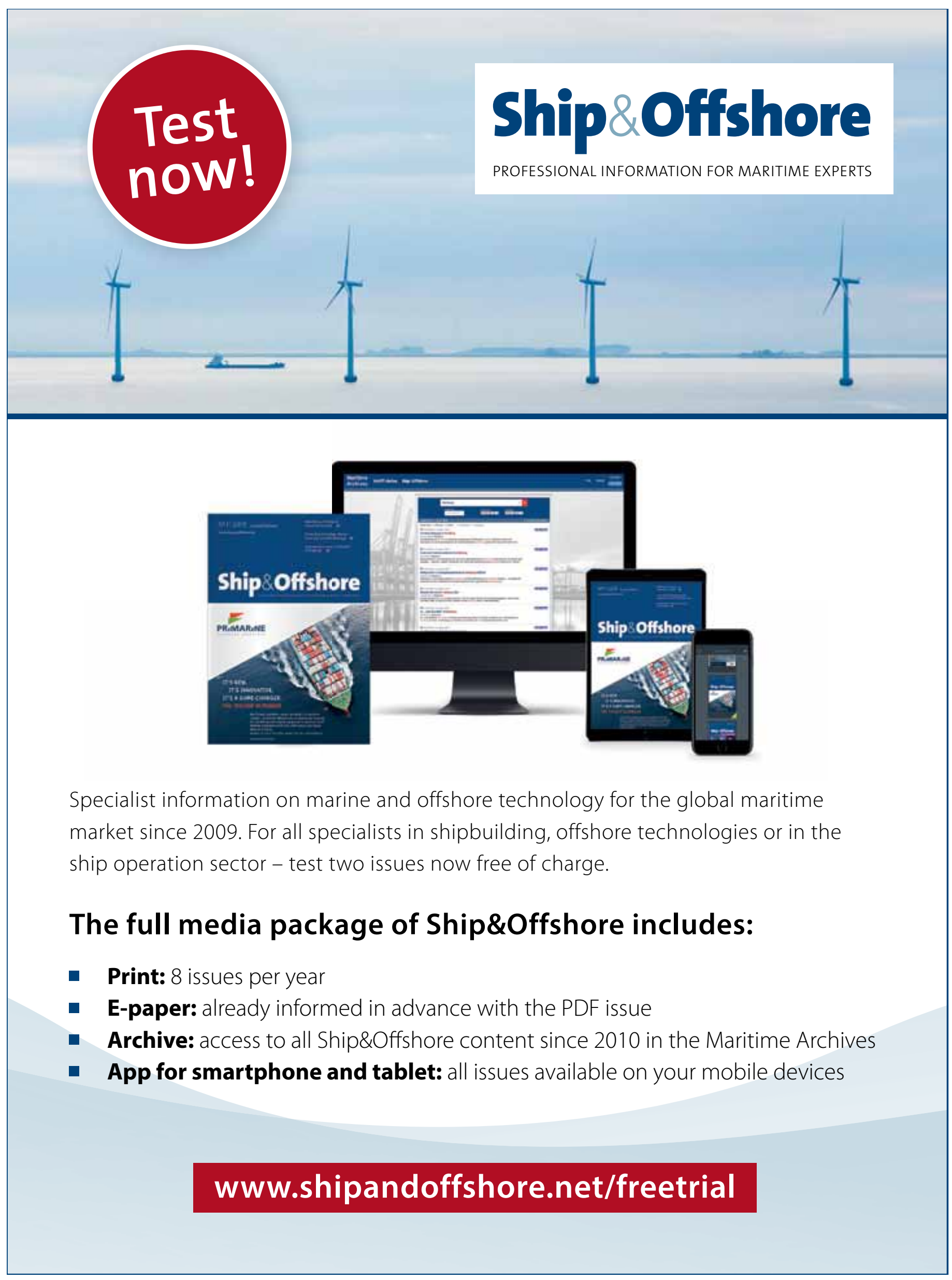




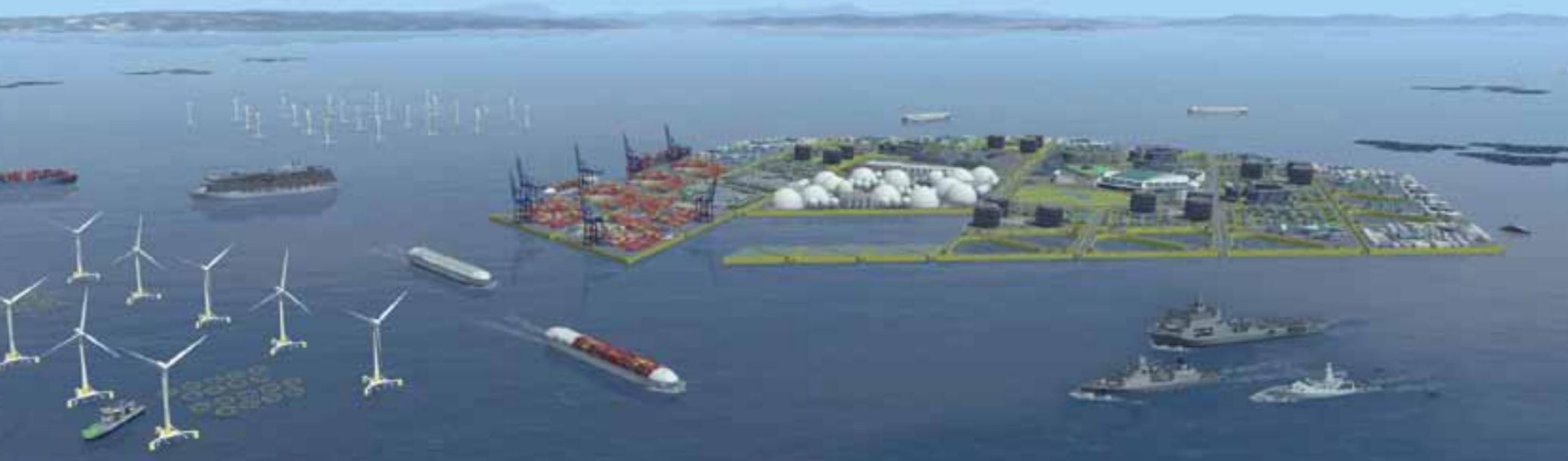

Our future is at sea. We believe that the oceans provide an opportunity to resolve the societal challenges of our time. This includes zero emission transport, renewable energy, sustainable food production and space to work and live.

The key to achieve this is maritime knowledge and innovation.

\section{BETTER SHIPS, BLUE OCEANS}

With 'Better Ships, Blue Oceans' we set course to make ships cleaner,

smarter and safer and to contribute to a sustainable use of the seas.

\section{MARTN www.marin.nl}

